\title{
GUIDE to ACCELERATOR PHYSICS PROGRAM SYNCH - CDC VERSION -
}

[This is an on line manual in]

BNLDAG: : DUA0: [PARSA1.SYNCH]

Accelerator Division

Technical Note No. 271

January 12, 1987

ZOHREH PARSA
\&
ERNEST COURANT

BROOKHAVEN NATIONAL LABORATORY ACCELERATOR DEVELOPMENT DEPARTMENT 


\author{
GUIDE to \\ ACCELERATOR PHYSICS \\ PROGRAM SYNCH \\ CDC Version
}

ZOHREH PARSA

and

ERNEST COURANT

BROOKHAVEN NATIONAL LABORATORY

Associated Universities, INC.

Upton, New York 11973

[January 1987]

This Guide is written to accommodate users of Accelerator Physics Data Base BNLDAG: DUA0:[PARSA1] . It describes the contents of the on Iine Accelerator Physics data base DUAO:[PARSAl.SYNCH]. SYNCH is a computer program used for the design and analysis of synchrotrons, storage rings and beamlines. 


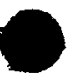<smiles>[18OH]</smiles> 
TABLE OF CONTENTS

I. Introduction $\ldots \ldots \ldots \ldots \ldots \ldots \ldots \ldots \ldots \ldots \ldots \ldots \ldots \ldots \ldots \ldots$

II. Directory Listings ......................

$>$ [PARSA1.SYNCH]READ.ME $\ldots \ldots \ldots \ldots \ldots \ldots \ldots \ldots \ldots$

$>$ HOW TO RUN THE CDC - VERSION OF SYNCH.........2

$>\quad$ CDCDEMO.DAT $\ldots \ldots \ldots \ldots \ldots \ldots \ldots \ldots \ldots \ldots \ldots \ldots \ldots \ldots \ldots \ldots \ldots$

$>\quad \operatorname{VAX}$ VERSION INFO....................4

$>$ HOW TO USE THE VAX - VERSION OF SYNCH $\ldots \ldots \ldots \ldots .4$

$>$ HOW TO RUN THE VAX VERSION OF SYNCH $\ldots \ldots \ldots \ldots \ldots 5$

$>$ WORK FILES $\ldots \ldots \ldots \ldots \ldots \ldots \ldots \ldots \ldots . \ldots \ldots$

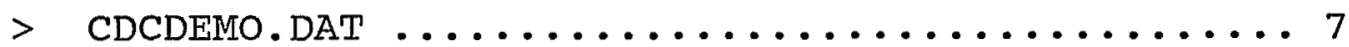

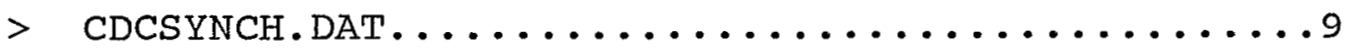

III. [PARSAI.SYNCH] CDCSYNCH.FOR. ...............

III. .........Paged $(1-500)$ 
0

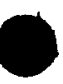




\section{INTRODUCTTON}

This guide describes the contents of the [PARSA1.SYNCH] Directory. In section II, the Directory listing of the files, and [PARSA1.SYNCH]READ.ME are shown.

You may note that, throughout the Data Base, READ.ME files provide you with detail information about the programs in the selected program directory.

Section III, shows [PARSA1.SYNCH] SYNCH.FOR, the source program.

A) Following are the contents of the [PARSA1.SYNCH]

Directory:

$\$$ DIR [PARSA1.SYNCH]

Directory DUA0: [PARSA1.SYNCH]

CDCDEMO.DAT; 1 CDCSHOW.COM; 1

CDCSYNCH.DAT; 1 D0.LOGA;1

FNLSYNCH.EXE;2 FOR011.DAT;1

F0R012.DAT; 1 INPUT.DIR;1

OUTPUT.DIR;1 READ.ME;12

SOURCE.DIR;1 SYN.COM;4

SYNBOOST10.DAT; 1 SYNBOOST9.DAT; 1

SYNCH.COM;2 SYNCH.EXE;52

SYNCH.EXE; $47 \quad$ SYNCH.GUIDE; 1

SYNCH.OUT; 6 SYNCH. RNO;1

SYNCHINC.TLB; 5 VAXSYN.COM;2

VAXSYN.COM; 1 VAXSYNBST9.DAT; 3

Total of 24 files.

B) $\$$ DIR [PARSA1.SYNCH. SOURCE]

Directory DUAO: [PARSA1.SYNCH. SOURCE]

BKLYIII.DIF ;2 BNLSYNIII.FOR ; 42

CYX.POL;1 FROM.ARDITH; 1

III.DIF ;2 IIIDIF.FOR ; 42

MAINS.FOR ; 8 SYNCHINC.TLB; 5

SYNDEMO.COM;2 SYNDEMO.DAT; 1

SYNDEMO.0UT; $1 \quad$ SYNI.FOR ; 39 
$\begin{array}{ll}\text { SYNII.FOR;27 } & \text { SYNIII.FOR;42 } \\ \text { SYNIIIA.FOR; } 6 & \text { SYNRHIC.DAT;1 }\end{array}$

VAXSYNCH. INF ;2

Total of 17 files.

C) \$ DIR [PARSA1.SYNCH.INPUT]

D) Following are the contents of [PARSA1.SYNCH] READ.ME:

Welcome to DUA0: [PARSA1.SYNCH]

LOG

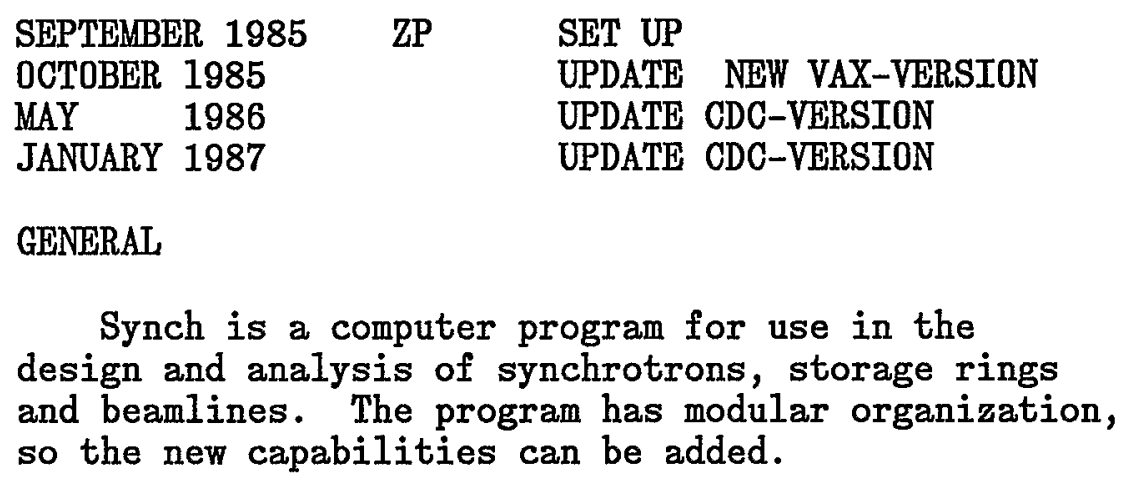

HOW TO RUN THE CDC - VERSION OF SYNCH FROM VAX

INPUT:

To run a SYNCH data file from the VAX on the CDC, you must include 1) The normal job cards (see example), 2)

The synch input data file.

Hence,i) create a file and call it SYNCH.DAT on VAX, ii) use the CYBERVAX to run it on CDC. So, [while on the VAX] type after the VAX $\$$ prompt

\$ MSUB SYNCH.DAT $\langle$ Cr $>$

[See Cybervax Guide for other commands]

OUTPUT:

I. When the job is finished on CDC, you will receive a mail message on Vax [that returns the output from CDC to VAX] .

EXAMPLE: (you are on vax and the prompt is \$) 
$\$$ edit Synch.dat

ZNAME,STMFZ. [your cdc outputfile will be ZNAME plus few more characters]

ACCOUNT, yourname, 123,123 .

COPYSP.

REWIND, INPUT.

ATTACH, MN , BSM85, ID=EDC.

ATTACH, L , BSL85, ID=EDC.

ATTACH,L1, PRINTLIB.

ATTACH,L2, CALCOMPLIB.

LIBRARY , L , L1, $\mathrm{L} 2$.

$\mathrm{MAP}, \mathrm{OFF}$.

MODE, 1 .

MN.

\%\%EOR

-

"SYNCH data file"

-

$\$$

[To compute depolarization resonance strength, you may use the programs POLARIZ, (which can give the plot of the result, but is limited to maximum of about 100 resonances), or POLRIZE (which can't give a plot of the result but can find as many resonances as you want). e.g. touse POLARIZE, you must add, and put the followng cards before \%\% $\% \mathrm{R}$ above.

Following are the contents of cdcdemo.dat:

ty cdcdemo.dat

...

$\cdots$

ATTACH, POLRIZE, ID=ZP.

POLRIZE.

REWIND, TAPE20.

COPY, TAPE20.

\%\%OR

.

"SYNCH DATA FILE"

•

$\$$ [now you have created your SYNCH.DAT on vax]

\$MSUB SYNCH.DAT [Via cybervax you submit synch.dat and run the program synch on cdc]

$\$$ YOU HAVE MAIL [you will receive a mail message when the cdc output is completed and returned to the VAX] 
Please note, that in this dir. we have a [FORTRAN (.FOR)], copy of SYNCH, that can run on VAX, (by using:

\$FOR SYNI, \$FOR SYNII, \$FOR SYNIIIA, \$FOR MAINS you make SYNI.OBJ, SYNII. OBJ, SYNIIIA.OBJ, MAINS.0BJ; then use \$LINK/EXECUTABLE=SYNCH.EXE SYNI, SYNII, SYNIIIA, MAINS and then, \$RUN SYNCH.

[Without the /EXECUTABLE=SYNCH.EXE, the LINK command would produce the same executable copy but would call it SYN1.EXE).

In addition you are given the information to run the synch on CDC (i.e.the CDC version of SYNCH) from vax through the CDC link. This allows you to edit your input data file on VAX, yet use the CDC version of the program (and the benefit of the CDC speed for running the program).

II. HOW TO USE THE VAX VERSION OF PROGRAM SYNCH:

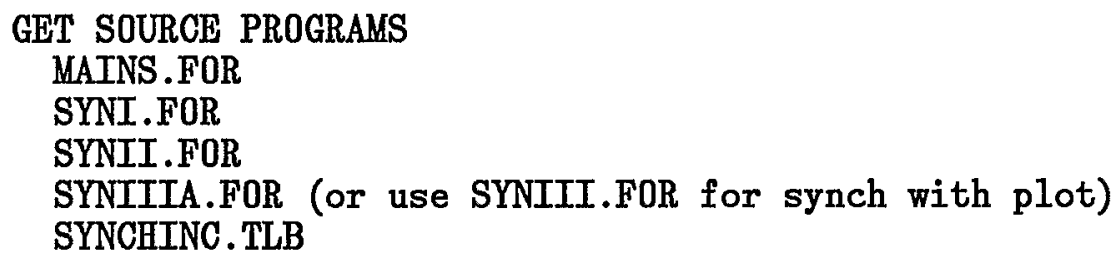

Compile MAINS, SYNI, SYNII, SYNIII. SYNCHINC.TLB is a table of the common blocks which are needed to satisfy the 'INCLUDE' STATEMENTS in the program. you never need to refer to SYNCHINC.TLB, But it has to be available for the compiler.

IF compiled OBJ files have the same names, then LINK/EXECUTABLE = SYNCH.EXE MAINS, SYNI, SYNII, SYNIIIA

Our current executable file that is equivalent to all of this is SYNCH.EXE.

A current test case is SYNDEMO.DAT and its output is SYNDEMO.OUT. 
You may run the vax version of SYNCH (with or without ploting) interactively by creating the following SYNCH.COM file. [Note that, you may add other DCL commands to the following example of SYNCH.COM file]

$\$$ ASSIGN SYNCH.DAT FOROO2

$\$$ ASSIGN DYNCH.OUT FOROO3

$\$$ RUN DUAO: [PARSA1.SYNCH] SYNCH.EXE

[Note, SYNCH.EXE, is the executable copy of SYNCH which can be obtained following the above description; for both cases, with or without the ploting options. additionally, the executable copies are provided for your convenience.]

SYNCH.DAT contains the input statements starting with a RUN command and ending with a STOP statement, (see example of input file; SYNDEMO.DAT). The output from the RUN goes to SYNCH.DAT, (for our example, SYNDEMO.OUT]

Having created the SYNCH.COM file, enter:

1. OSYNCH 〈return〉 [for interactiv run]

or to batch the job enter:

2. \$ SUBMIT/NOPRINT SYNCH 〈return〉 [here you are submitting SYNCH.COM file]

WORK FILES :

[PARSA1.SYNCH] READ.ME

[PARSA1.SYNCH] SYNCH.FOR

[PARSA1.SYNCH] SYNCH . EXE

[PARSA1.SYNCH] SYNCH.COM

READ.ME; gives general information about this directory and the program SYNCH.

SYNCH.FOR; is the source code from which SYNCH.EXE was obtained and a copy of it is given for convenience. 
Following are the contents of [PARSA1.SYNCH]*.com files:

DUA0: [PARSA1 . SYNCH] SYN.COM ; 4

$\$$ ASSIGN [PARSA1.SYNCH] SYNBOOST9. dat FOROO2

$\$$ ! SYNBO0ST9.DAT is a sample VAX input data.

$\$$ ASSIGN SYNCH.OUT FOROO3

$\$$ ! ASSIGN NL: FOR012

$\$$ ASSIGN DEBUG.DAT FORO01

$\$$ RUN [PARSA1.SYNCH] SYNCH

DUA0: [PARSA1.SYNCH] SYNCH.COM;2

$\$ \quad$ ASSIGN SYNCH.DAT FOROO2

$\$$ ASSIGN SYNCH.OUT FOROO3

$\$$ ! ASSIGN NL: FORO12

$\$$ ASSIGN DEBUG.DAT FORO01

$\$$ RUN SYNCH

DUAO: [PARSA1. SYNCH] VAXSYN. COM;2

$\$$ ASSIGN [PARSA1.SYNCH] VAXSYNBST9. dat FOR002

$\$$ ! VAXSYNBST9.DAT is a sample VAX input data.

$\$$ ASSIGN [PARSA1. SYNCH] SYNCH. OUT FORO03

$\$$ ! ASSIGN NL: FORO12

$\$$ ASSIGN DEBUG.DAT FORO01

$\$$ RUN [PARSA1.SYNCH] SYNCH.EXE; 47

DUAO: [PARSA1.SYNCH] VAXSYN.COM; 1

$\$$ ASSIGN [PARSA1.SYNCH] VAXSYNBST9. dat FOROO2

$\$$ ! VAXSYNBST9.DAT is a sample VAX input data.

$\$$ ASSIGN [PARSA1.SYNCH] SYNCH.OUT FOROO3

\$! ASSIGN NL: FOR012

$\$$ ASSIGN DEBUG.DAT FORO01

$\$$ RUN [PARSA1.SYNCH] SYNCH 
Following are the contents of [PARSA1.SYNCH]*.DAT; *:

DUAO : [PARSA1 . SYNCH] CDCDEMO .DAT; 1

ACCOUNT, YOURNAME , 123, 123.

COPYSP.

(123=YOUR PROB )

REWIND (INPUT)

REQQUEST (TAPE11, $*$ PF)

REQ̨UEST (TAPE20, $*$ PF)

FILE (TAPE20, RT $=W, \mathrm{MBL}=5120, \mathrm{BT}=\mathrm{I}$ )

REQUEST $(\mathrm{L}, * \mathrm{PF})$

REQQUEST (NN , $*$ PF)

ATTACH, MN , BSM85, ID=EDC .

ATTACH, L , BSL85, ID=EDC.

ATTACH (L1, PRINTLIB)

ATTACH (L2, CALCOMPLIB)

LIBRARY $(\mathrm{L}, \mathrm{L} 1, \mathrm{~L} 2)$

MAP (OFF)

$\operatorname{MODE}(1)$

MN.

ATTACH, POLRIZE , ID=ZP .

POLRIZE.

REWIND, TAPE20.

COPY, TAPE20.

\%\% EOR

AGSP RUN

$\mathrm{BR}=$

$\mathrm{BZ}=$

TUNE SUB

GFX1 =

GDX1 =

GFX2 =

GDX2 =

$\mathrm{GF}=$

GD =

S2 DRF

S2.5 DRF

S10 DRF

FS MAG

DS MAG

FL MAG

DL MAG

S5 DRF

HPER BML DS $\mathrm{S}$

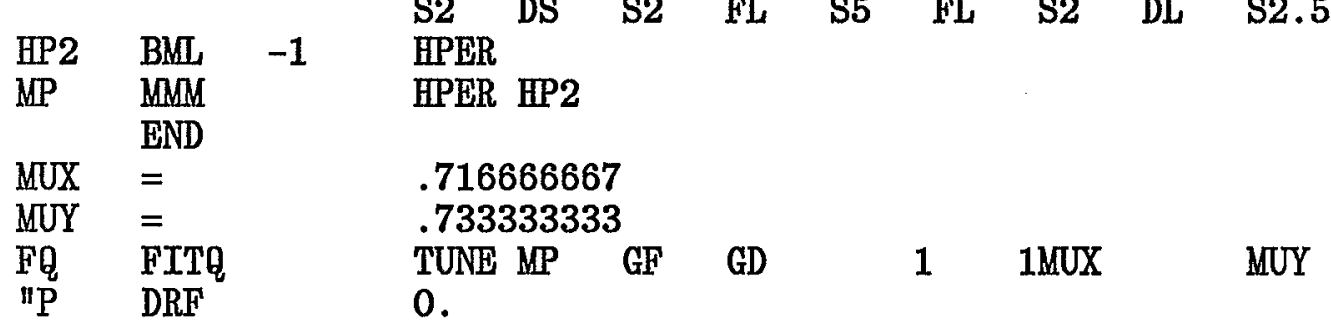

AGS WITH ERRORS FOR POLARIZATION CALCULATIONS

981.44

11.4952

7.5

$-7.5$

7.5

$-7.5$

47.421958

$-47.573705$

.6096

.762

3.048

$2.0066 \quad G F$

2.0066

2.3876

2.3876

1.524

S2.5 FL S2

GD

BR

$\mathrm{BR}$

$\mathrm{BR}$

BR

$\mathrm{BZ}$

BZ

$\mathrm{BZ}$

$\mathrm{BZ}$

$\$$
$\$$
$\$$
$\$$

DL $\quad \mathrm{S}$

S5 D

S2

FS S2 FS

S10

$\begin{array}{lllllllll}\text { S2 } & \text { DS } & \text { S2 } & \text { FL } & \text { S5 } & \text { FL } & \text { S2 } & \text { DL } & \text { S2.5 }\end{array}$

HPER

.716666667

.733333333

0 . 


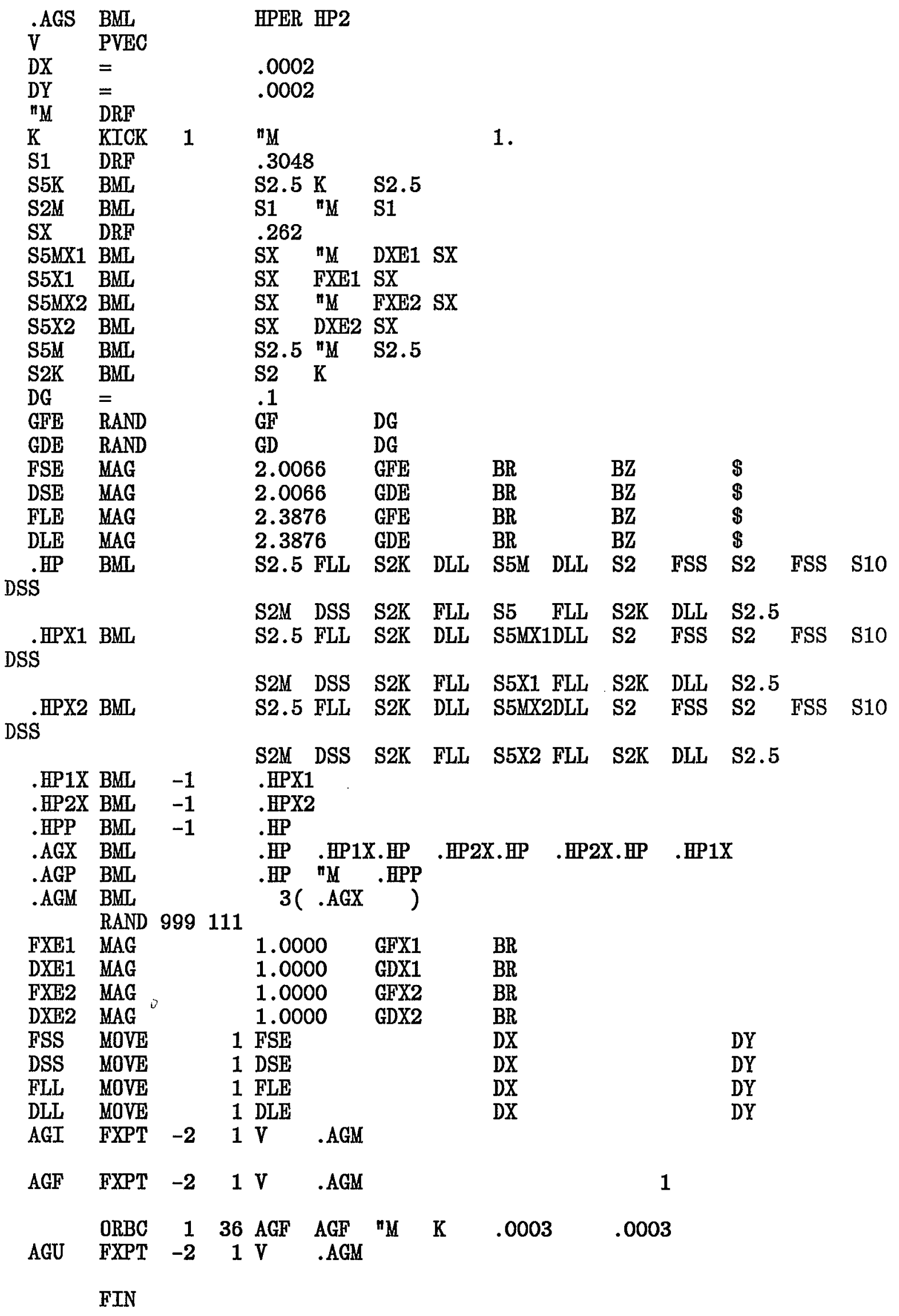




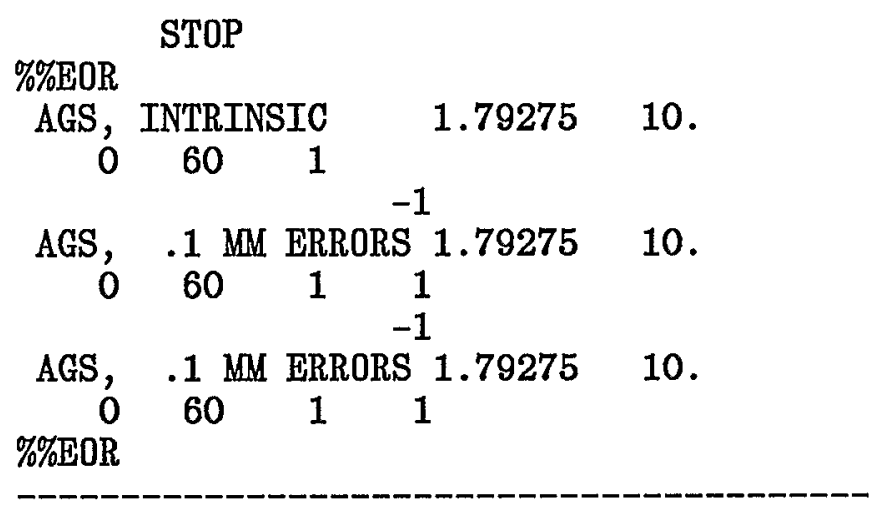

DUAO: [PARSA1 . SYNCH] CDCSYNCH .DAT; 1

ZNAME, STMFZ .

ACCOUNT, PARSA , 123, 123 .

COPYSP.

REWIND, INPUT .

ATTACH, MN , BSM85, ID=EDC .

ATTACH, L , BSL85, ID=EDC .

ATTACH, L1, PRINTLIB.

ATTACH, L2, CALCOMPLIB.

LIBRARY,L,L1,L2 .

MAP, OFF.

MODE, 1 .

MN.

\%\%OR

III. Following are the contents of the [parsa1.synch]cdcsynch.for

[this is the CDC version of prog. 


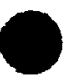


C SYNCH-

OVERLAY (SYNCH, $, 0,0$ ) PROGRAM SYNCH (INPUT=101, OUTPUT=1001, TAPE2=INPUT , TAPE3=OUTPUT , 1 TAPE5, TAPE6, TAPE11, TAPE12, TAPE98, TAPE99) analysis. by A.A.garRen and A.S. KenNey, lawrence berkeley lab. c

COMMON/CLC/REG(109)

LEVEL 2, STORE, INFF, IWORK

COMMON STORE(48000), IWORK(10)

DIMENSION INFF $(24,2000)$

EQUIVALENCE (INFF, STORE)

LEVEL 2, XX, Y1, Y2, Y3, Y4

COMMON XX(4000), Y1 (4000), Y2(4000), Y3(4000), Y4(4000)

COMMON/BPLTCOM/MN, KW, BXX, BYX, NPLT

COMMON/CCPOOL/XMIN, XMAX, YMIN, YMAX, CCXMIN, CCXMAX, CCYMIN, CCYMAX COMMON/CCFACT/FACTOR
MAIN 2

MAIN 3

MY3IMAIN 1

MAIN 5

MAIN 6

MAIN 7

MAIN 8

MAIN 11

MAIN 12

CLC 2

BLANK 2

BLANK 3

86MARSIZ 1

86MARSIZ 2

86MARSIZ 3

BLANK 5

BETPTS 2

RENAM8 31

RENAM83 2

PL6683 1

PL6683 2

BETPTS 5

$\begin{array}{rrr}\text { COMMON/INSTR/OPNAME, NAME, OP , KA, KB, OBJA, OBJB, NXTM, TRA, LQ, NQ, LIN, } & \text { INSTR } & 2 \\ \text { NIN, LFL, NFL, LBC, NBC, NTOT, IX, KIND, TYPE, ROWS, COLS, EXTR, } & \text { INSTR } & 3\end{array}$

$2 \quad M, N F, N B, N I, M S U B R, I S I G N$

INSTR $\quad 4$

INSTR 5

INSTR 6

CONTRL $\quad 2$

CONTRL 3

CONTRL 4

CONTRL 5

STORE 2

STORE 3

STORE 4

GRR 2

GRR 3

GRR 4

DIM 2

DIM 3

BMIL 1

BMIL 2

BMIL 3

BMIL 4

BMI 3

NV3BMI 1

BMI 1L 2

BMI 1L 3

BMIIL 4

BMI 1

MATCH3 2

MATCH3 3

MATCH2 2

MATCH2 3

MATCH2 4

FLTN 2


60

65

70

75

80

85

90

95

100

185

110
COMMON/SWTCH/BEND, MSIZE, CYCSWT, VCSW, PV, MSSW, VPR, MHS , MODS, MINZER LOGICAL CYCSWT, MSSW, VCSW, PV, VPR, MHS, MODS

COMMON/BCFD/PAR( 10), RMUX, RMUY, XGES, YGES , LAM

C

COMMON/TRKINT/G, EX, EY, OMSQ, SEND , VMX, VMN, DINT , BEG, DPR, JPR,

1 THET , PLT, NPT, NZ, PMAX, NSIZ, ITITLE(6)

LOGICAL BEG, DPR, PLT

COMMON/TSW/TRSW, MCY (20) , JM, LOCC

LOGICAL TRSW

COMMON/OPLIST/LIST(200)

COMMON/SVNAM/NAMRUN

COMMON/COPY/CPYSW

LOGICAL CPYSW

COMMON/CORB/TH(84), TV(84), NMON, NNH, NNV , I IH, I IV, NAMONH, NAMONV , NACORH, NACORV

DIMENSION IC(24)

EQUIVALENCE (IC (1), OPNAME)

DIMENSION IFM(15), IW(6)

COMMON/KINET/KNFLAG , TK, P, GAM, BETT, BETGAM, BRHO

LOGICAL KNFLAG

INTEGER HREM, HPAGE

DATA DWORD (1), DWORD2 (1), DWORD3 (1)/4HPRIN, 4HTOUT, 2H /

DATA DWORD (2), DWORD2(2), DWORD3(2)/4HMINI , 4HMIZE, $2 \mathrm{H} \mathrm{/}$

DATA DWORD (3), DWORD2 (3), DWORD3 (3)/4HEND , 4HRETU, 2HRN/

* To alter storage, change lmax, max and dimension store

* to increase matrix scratch space, change isav and ISAV7 for

* 3X3 OR 7X7 SIZES.

DATA LMAX, MAX, ISAV, ISAV7, KADD, KADDR/48000, 2000, 10, 12, 3, 7/

DATA MASK1 /77000000000000000000B/

DATA ( IW(I), I=1,6) $/ 0,0,0,-1,-1,-1 /$

DATA (IFM(J), J=1, 15)/1H-, 1H1, 1H2, 1H3, 1H4, 1H5, 1H6, 1H7, 1H8, $1 \mathrm{H} 9$,

$11 \mathrm{HO}, 1 \mathrm{H+}, 1 \mathrm{H}, 1 \mathrm{H} ., 1 \mathrm{H} / /$

DATA REG/109*0.0/

DATA MINZER/77777 7777777777 77777B/

CALL SYSTEMC $(3, I W)$

CALL SYSTEMC $(6, I W)$

CALL SYSTEMC $(13, I W)$

CALL SYSTEMC $(30, I W)$

CALL SYSTEMC $(36, I W)$

CALL SYSTEMC $(39, I W)$

CALL SYSTEMC $(42$, IW)

CALL RANGET(RS)

CALL RANSET(RS)

NPLT $=0$

$\mathrm{DD}=\mathrm{RANF}$ (DD)

DO $10 \mathrm{I}=1,84$

$\mathrm{TH}(\mathrm{I})=0$.

$10 \quad T V(I)=0$.

c

CALL SETOPS

$9 \quad$ FIN $=$.FALSE.

MINFLG $=1$

\begin{tabular}{|c|c|}
\hline FLTN & \\
\hline SWTCH & \\
\hline SWTCH & \\
\hline SWTCH & \\
\hline BCFD & \\
\hline BCFD & \\
\hline TRKINT & \\
\hline TRKINT & \\
\hline TRKINT & \\
\hline NOV3TSW & \\
\hline TSW & \\
\hline OPLIST & \\
\hline OPLIST & \\
\hline SVNAM & \\
\hline COPY & \\
\hline COPY & \\
\hline COPY & \\
\hline CORB613 & \\
\hline CORB613 & \\
\hline MAIN & \\
\hline MAIN & \\
\hline MAIN & \\
\hline KINET & \\
\hline KINET & \\
\hline MAIN & \\
\hline MAIN & \\
\hline MAIN & \\
\hline MAIN & \\
\hline MAIN & \\
\hline MAIN & \\
\hline MAIN & \\
\hline 86MARSIZ & \\
\hline MAIN & 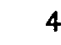 \\
\hline MAIN & $T$ \\
\hline MAIN & \\
\hline MAIN & \\
\hline MAIN & \\
\hline MAIN & \\
\hline MAIN & \\
\hline MAIN & 5 \\
\hline MAIN & \\
\hline MAIN & 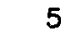 \\
\hline MAIN & \\
\hline MAIN & \\
\hline MAIN & \\
\hline MAIN & \\
\hline MAIN & \\
\hline MAIN & 6 \\
\hline MAIN & \\
\hline MN9JN83 & \\
\hline MN9JN83 & \\
\hline MN9JN83 & \\
\hline MAIN & \\
\hline MAIN & \\
\hline MAIN & \\
\hline MAIN & \\
\hline MAIN & \\
\hline
\end{tabular}


NONU $=$.FALSE.

MAIN

NOPR $=$. FALSE.

MAIN 69

MIFLG $=$.FALSE.

MAIN 70

MATFLG $=$.FALSE.

MAIN 71

GLOBAL $=$.FALSE.

MAIN $\quad 72$

120

LFILE $=1$

MAIN 73

LDFLG $=$. FALSE.

MAIN $\quad 74$

KNFLAG $=$. FALSE.

MAIN 75

EMPTY $=$.FALSE.

MAIN $\quad 76$

$I E R R=. F A L S E$.

MAIN 77

125

SMINCAL $=$. FALSE.

MAIN 78

CPYSW $=$. TRUE.

MAIN 79

DO $1 \mathrm{I}=1,7$

MAIN 80

DO $1 \mathrm{~J}=1,10$

MAIN 81

$1 \operatorname{DWORD7}(\mathrm{I}, \mathrm{J})=0.00000001$

MAIN 82

130

MINCOM=3

NOPR $=$. FALSE.

MAIN 83

$N O N U=. F A L S E$.

MAIN 84

LEND=LMAX

MAIN 85

MEND $=$ MAX

MAIN 86

$M I N=M A X$

MAIN 87

135

M7END = MEND - ISAV

MAIN 88

MAIN 89

* FILL BLANK PORTION OF ARRAY WITH ZEROES.
* IFL IS USED MAINLY TO DISTINGUISH SYMBOLIC FL.PT

MAIN 90

MAIN 91

DO $8 \mathrm{~J}=1,15$

MAIN 92

140

$\operatorname{IFL}(J)=$ MASK 1 . AND. IFM $(J)$

8 CONTINUE

2 CALL RDINST

4 CALL SWITCH

145

IF (FIN) GO TO 9

IF (ERROR) GO TO 6

C CHECK FOR REDEFINITION OF ELEMENT BY NAME

IF (MODE.EQ.1.OR.MODE.EQ.2) CALL NAMCHK(M)

MAIN 93

MAIN 94

MAIN 95

MAIN 96

MAIN 97

MAIN 98

MAIN 99

MAIN 100

MAIN 101

IF (MODE.EQ.3) GO TO 3

150

IF (.NOT.MSSW) GO TO 7

MAIN

102

CALL RDINF (IC,M)

MAIN 103

MAIN 104

CALL MESH(M, KA, OPNAME)

IF (MSSW) GO TO 3

C IF PAGE OR COMMENT OUTSIDE SUBROUTINE, REUSE INFF SPACE.

7 IF ((OPNAME. EQ.HPAGE.OR.OPNAME. EQ.HREM).AND. (MODE. EQ.1)) GO TO 2

MAIN

105

MAIN $\quad 106$

MAIN 107

MIN = MIN - 1

MAIN

108

NXTM $=$ MIN

CALL INFW( MIN, 8,M)

MAIN

109

MAIN

110

$M=M I N$

160

GO TO 2

$3 \quad N X T M=\operatorname{INFF}(8, M)$

$\mathrm{M}=\mathrm{N} X T \mathrm{M}$

IF (.NOT.TRASW) GO TO 5

$M=$ TRA

165

TRASW $=$. FALSE .

5 CALL RDINF( IC,M )

GO TO 4

6 CALL ERRSR(M)

GO TO 9

MAIN

111

MAIN 112

MAIN 113

MAIN 114

MAIN 115

MAIN 116

MAIN $\quad 117$

MAIN 118

MAIN $\quad 119$

MAIN 120

MAIN 121

END

MAIN

122

MAIN 


$\begin{array}{lr}\text { PLT } & 7 \\ \text { PLT } & 8 \\ \text { BLANK } & 2 \\ \text { BLANK } & 3 \\ \text { 86MARSIZ } & 1 \\ \text { 86MARSIZ } & 2 \\ \text { 86MARSIZ } & 3 \\ \text { BLANK } & 5 \\ \text { BETPTS } & 2 \\ \text { RENAM83 } & 1 \\ \text { RENAM83 } & 2 \\ \text { PL6683 } & 1 \\ \text { PL6683 } & 2 \\ \text { BETPTS } & 5 \\ \text { TRKINT } & 2 \\ \text { TRKINT } & 3 \\ \text { TRKINT } & 4 \\ \text { SVNAM } & 2 \\ \text { PLT } & 13 \\ \text { PLT } & 14 \\ \text { PLT } & 15 \\ \text { PLT } & 16 \\ \text { PLT } & 17 \\ \text { PLT } & 18 \\ \text { PLT } & 19 \\ \text { PLT } & 20 \\ \text { PLT } & 21 \\ & \end{array}$

LEVEL 2, STORE, INFF, IWORK

COMMON STORE(48000), IWORK(10)

DIMENSION INFF $(24,2000)$

EQUIVALENCE (INFF, STORE)

LEVEL. $2, X X, Y 1, Y 2, Y 3, Y 4$

COMMON XX(4000), Y1 (4000), Y2(4000), Y3(4000), Y4(4000)

COMMON/BPLTCOM/MN, KW, BXX, BYX, NPLT

COMMON/CCPOOL/XMIN, XMAX, YMIN, YMAX, CCXMIN, CCXMAX, CCYMIN, CCYMAX

COMMON/CCFACT/FACTOR

COMMON/TRKINT/G, EX, EY, OMSQ, SEND, VMX, VMN, DINT , BEG, DPR, JPR,

1 THET, PLT, NPT, NZ, PMAX, NSIZ, ITITLE(6)

LOGICAL BEG, DPR, PLT

COMMON/SVNAM/NAMRUN

DATA CCXMIN, CCXMAX, CCYMIN, CCYMAX, FACTOR/70 , 1070.,80., 1080 .,1./

PLT 
CALL FIN

PLT

23

RETURN

PLT

24

END

PLT

25 
1

10

15
SUBROUTINE CCLTR ( $X, Y, K O R, K S, K B C D, N C)$

DIMENSION KBCD(7)

$\mathrm{NCH}=10$

CALL NOARG(NA)

IF(NA.GT.4) GO TO 2

REWIND 98

$\operatorname{READ}(98,10) \mathrm{KTX}$

$5 \quad K B C D(1)=K T X$

10 FORMAT (A10)

REWIND 98

$2 \quad A N G=K O R * 90$.

$H T=K S / 10$.

IF (KS.LE. 1) $H T=.15$

$X Z=.01 * X \quad \$ Y Z=.01 * Y$

IF (NA.EQ.6) $\mathrm{NCH}=\mathrm{NC}$

CALL SYMBOL ( XZ, YZ, HT, KBCD , ANG ,NCH)

RETURN

END
PLT

PLT

PLT

PLT

PLT

PLT

PLT

PLT

PLT

PLT

PLT

PLT

PL2

PLT

PLT

PLT

PLT

PLT
26

27

28

29

30

31

32

33

34

35

36

37

1

38

39

40

41

42 
1

5

10

15

20

30

35

25
SUBROUTINE CCPLOT ( $X, Y, N, K B C D, N S, N T H, I S)$

LEVEL 2, STORE, INFF, IWORK

COMMON STORE(48000), IWORK(10)

DIMENSION INFF $(24,2000)$

EQUIVALENCE (INFF,STORE)

LEVEL 2, XX, Y1 $, Y 2, Y 3, Y 4$

COMMON XX(4000), Y1(4000), Y2(4000), Y3(4000), Y4(4000)

COMMON/BPLTCOM/MN, KW, BXX, BYX, NPLT

COMMON/CCPOOL/XMIN, XMAX, YMIN, YMAX, CCXMIN, CCXMAX, CCYMIN, CCYMAX

COMMON/CCFACT/FACTOR

DIMENSION KBCD(1),X(1),Y(1)

CALL INTENSE( $1 \mathrm{H} *)$

$D X=(X M A X-X M I N) * 100 . /($ CCXMAX-CCXMIN $)$

$D Y=(Y M A X-Y M I N) * 100 . /($ CCYMAX-CCYMIN)

$X(N+1)=X M I N-.01 * C C X M I N * D X$

$Y(N+1)=Y M I N-.01 *$ CCYMIN*DY

$X(N+2)=D X$

$Y(N+2)=D Y$

ISYM=3

$J=0$

$\mathrm{K}=1$

CALL NOARG(NA)

IF (NA.LE. 4) GO TO 1

$J=1$

IF (KBCD (1).EQ. 6HNOJOIN) $J=-1$

IF (NA.EQ.6) $J=J * N T H$

CONT INUE

IF(N.GT.2) GO TO 3

CALL LINE(X, Y,N, K,J, ISYM)

RETURN

3 IF(NA.EQ.7) CALL INTENSE(IS)

CALL LINE $(X, Y, N, 1,0,3)$

RETURN

END

\begin{tabular}{|c|c|}
\hline PRCH & \\
\hline BLANK & \\
\hline BLANK & \\
\hline 86MARSIZ & \\
\hline 86MARSIZ & \\
\hline 86MARS IZ & \\
\hline BLANK & \\
\hline BETPTS & \\
\hline RENAM83 & \\
\hline RENAM83 & \\
\hline PL6683 & \\
\hline PL6683 & \\
\hline BETPTS & \\
\hline PLT & \\
\hline PRCH & \\
\hline PLT & \\
\hline PLT & \\
\hline PLT & \\
\hline PLT & \\
\hline PLT & \\
\hline PLT & \\
\hline PLT & \\
\hline PLT & \\
\hline PLT & \\
\hline PLT & \\
\hline PLT & \\
\hline PLT & \\
\hline PLT & \\
\hline PLT & \\
\hline PLT & \\
\hline PL2 & \\
\hline PLT & \\
\hline PLT & \\
\hline $\mathrm{PRCH}$ & \\
\hline $\mathrm{PRCH}$ & \\
\hline PL2 & \\
\hline PLT & \\
\hline
\end{tabular}


LEVEL 2, STORE, INFF, IWORK

BLANK 2 COMMON STORE (48000), IWORK(10)

BLANK 86MARSIZ DIMENSION INFF $(24,2000)$ 86MARSIZ EQUIVALENCE (INFF,STORE) 86MARSIZ

LEVEL 2, XX, Y1 $, Y 2, Y 3, Y 4$ COMMON XX(4000), Y1 (4000), Y2(4000), Y3(4000), Y4(4000)

BLANK

BETPTS

RENAM83 COMMON/BPLTCOM/MN, KW, BXX, BYX, NPLT COMMON/CCPOOL/XMIN, XMAX, YMIN, YMAX, CCXMIN, CCXMAX, CCYMIN, CCYMAX RENAM83

PL6683 COMMON/CCFACT/FACTOR

PL6683

BETPTS

PLT

PLT

PLT

PLT 


$\begin{array}{rrl}\text { ADDRESS } & \text { LENGTH } & \text { BINARY CONTROL CARDS. } \\ 0 & 12 & \text { IDENT NOARG } \\ 12 & & \text { END }\end{array}$

ENTRY POINTS.

NOARG $1+$
- 16170122070000000001

100000000000000000000

$25120000001+$ 21236

$3 \quad 5232777776$ 53230 73720

$40317000006+$ 53710

$50400000001+$

654200 6110000001

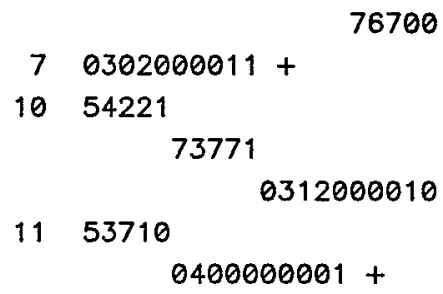

12
IDENT NOARG

*

* noarg returns the number of parameters in a fortran call

* statement. Since compiler calling CONVENTIONS DifFER, NoARg

* is assembled conditionally to produce code for either fTN or run

* (COMPass calls assemble ftn code).

*

* cern library program zozo

*

* A. yUle/L. pope CERN DECember 1971

* ENTRY NOARG

* SUBroutine nOARG(N)

VFD 42/OLNOARG, $18 / 1$

NOARG DATA 0

* CODE FOR FTN FOLLOWS - OR - SKIP TO RUN CODE RUNARG IFNE *F, 1

* FTN CODE

* routine for zero number of arguments

$\begin{array}{lll}\text { SA2 } & \text { NOARG } & \text { EQDOAAAAAA } 000000000 \\ \text { AX2 } & 30 & \text { ALIGN ADDRESS OF CALL+1 } \\ \text { SA3 } & \times 2-1 & \text { GO BACK TO +RJ-TRACE } \\ \text { SA2 } & \times 3 & \text { POINT TO TRACE WORD } \\ \text { SX7 } & \times 2 & \text { LOOK AT LAST 18 BITS } \\ \text { NZ } & \times 7, \text { ARGS } & \\ \text { SA7 } & \times 1 & \text { STORE AT ARG ADDRESS } \\ \text { ZR } & \text { BO,NOARG } & \text { RETURN }\end{array}$

* FTN CODE FOR NON-ZERO NUMBER OF ARGUMENTS

ARGS SA2 AO POINT TO ARG LIST

$\begin{array}{lll}\text { SB1 } & 1 & \text { INIT INCREMENT REG }\end{array}$

SX7 BO ZERO OUT $X 7$ FOR COUNT

ZR $X 2$,ENDSCAN IF FIRST ADDRESS ZERO - END

VFDSCAN SA2 A2+B1 NEXT ARG ADDRESS

SX7 $X 7+B 1 \quad$ INCREMENT COUNTER

NZ $\quad X 2, V F D S C A N$ SCAN UNTIL ZERO

ENDSCAN SA7 $X 1 \quad$ STORE AT ARG ADDRESS

ZR BO,NOARG RETURN

RUNARG ELSE

RUNARG ENDIF

END 
SYMBOLIC REFERENCE TABLE.

ARGS

ENDSCAN

NOARG

VFDSCAN

$\begin{array}{rllllll}6 & \text { PROGRAM* } & 9 / 38 & 9 / 42 & \mathrm{~L} & & \\ 11 & \text { PROGRAM* } & 9 / 45 & 9 / 49 \mathrm{~L} & & & \\ 1 & \text { PROGRAM* } & 9 / 25 \mathrm{E} & 9 / 28 \mathrm{~L} & 9 / 33 & 9 / 40 & 9 / 50 \\ 10 & \text { PROGRAM* } & 9 / 46 \mathrm{~L} & 9 / 48 & & & \end{array}$


LEVEL 2, STORE, INFF, IWORK

COMMON STORE (48000), IWORK(10)

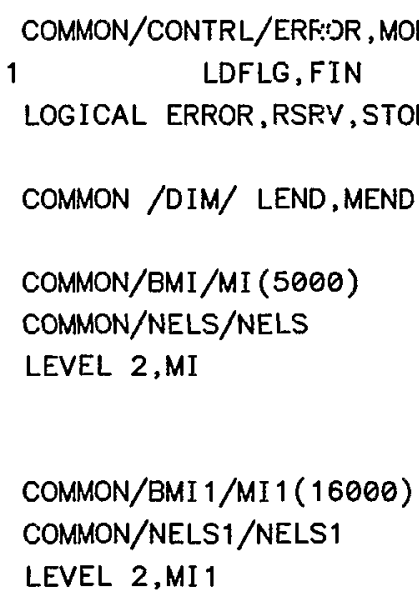

COMMON/BMI 1/MI 1 ( 16000) COMMON/NELS1/NELS1

LEVEL 2, MI 1

COMMON/SWTCH/BEND, MSIZE, CYCSWT, VCSW, PV, MSSW, VPR, MHS, MODS, MINZER LOGICAL CYCSWT, MSSW, VCSW, PV, VPR, MHS, MODS

COMMON/GRR/IERR, BSW, VSW, RSW, QSW, XSW

LOGICAL IERR, BSW, VSW, RSW, QSW, XSW

COMMON/TRKINT/G, EX, EY, OMSQ, SEND, VMX, VMN, DINT , BEG, DPR, JPR,

1 THET , PLT, NPT, NZ, PMAX, NSIZ, ITITLE(6)

LOGICAL BEG, DPR, PLT

LEVEL 2, XX,Y1,Y2,Y3,Y4

COMMON XX(4000), Y1 (4000), Y2(4000), Y3(4000), Y4(4000)

COMMON/BPLTCOM/MN, KW, BXX, BYX, NPLT

COMMON/CCPOOL/XMIN, XMAX, YMIN, YMAX, CCXMIN, CCXMAX, CCYMIN, CCYMAX

COMMON/CCFACT/FACTOR

COMMON/SVNAM/NAMRUN

COMMON/COPY/CPYSW

LOGICAL CPYSW

BLANK

BLANK

86MARSIZ

86MARSIZ

86MARSIZ

BLANK

STORE

STORE

STORE

CONTRL

CONTRL

CONTRL

CONTRL

DIM

DIM

BMIL

BMIL

BMIL

BMIL

BMI

NV3BMI 1

BMI 1L

BMI $1 L$

BMI $1 \mathrm{~L}$

BMI 1

SWTCH

SWTCH

SWTCH

GRR

GRR

GRR

TRKINT

TRKINT

TRKINT

BETPTS

RENAM83

RENAM83

PL6683

PL6683

BETPTS

SVNAM

COPY

COPY

COPY 
DIMENSION PAR(100), KREM(100), MATNM(20)

SW1 23

INTEGER R7, ROT,PRD

SW1

EQUIVALENCE (OPNAME, INF(1)), (PAR, KREM)

SW1

DIMENSION BX(20)

SW1

LOGICAL TABSW

SW1

DATA IBLNK/4H /

SW1

DATA ISCL/9/

SW1

65

DATA IBLNK, MXPMY, PVECS, R7, ROT, PRD/1H , 1, 2, 5, 6,8/

SW1

DATA (MATNM(I), I=1,12)/2HF, $2 \mathrm{HD}, 2 \mathrm{HS}, 2 \mathrm{HT}, 2 \mathrm{HE}, 2 \mathrm{HK}, 2 \mathrm{HIA}, 2 \mathrm{HIB}, 2 \mathrm{HB}$ SW1

$1,2 \mathrm{HA}, 2 \mathrm{HWD}, 2 \mathrm{HWF} /$

SW1

C

IF OPNAME $=$ NULL, IT IS A P OR C CARD. NEEDS SPECIAL

HANDLING TO CONSTRUCT A SYNCH PAGE OR REM INSTRUCTION

SW1

SW1

GO TO STOP, FIN, RUN, SUB, END, CALL, INCR, REPL, MESH, VPAR $=$, CRD, BML, DRF, MAG, MAGV, EQU, INV, SHF, REF

**, MMM, CYA, CYB, CYC, CELL, ALTC, TAB, PTAB, FITQ

SW1

SW1

SW1

:

GO TO $(1,2,3,4,5,6,19,20,74,74$,

FITB, FITR, FITV, PAGE, REM, WMA, SIZE, WBE, FIT, COPY, NCOPY, BEST, BE SW1

SW1

SW1

SW1

,BETA, KICK, RAND, SHF7, SOL, VAR, PRNT

SW

SW1

SW1

1

$27,7,86,10,13,42,11,12,16,18$,

$21,14,23,24,23,29,30,81,82,25$,

$25,25,25,34,35,15,61,26,31,32$,

$33,23,43,44,45,46,47,48,49,50) \mathrm{OP}$

SW1

SW1

SW1

SW1

SW1

SW1

85

$$
\text { * } 1
$$

STOP ENTRY

IF (NPLT.GE.1) CALL FINPL

STOP

FIN ENTRY

S1PLT

S1PLT

SW1

SW1

CALL SRUN(2)

90

RETURN

SW1

SW1

CALL SRUN(1)

RUN ENTRY

RETURN

SW1

SW1

SW1

CALL SUBR(1)

SUB ENTRY

SW1

RETURN

*

CALL SUBR(2)

END ENTRY

SW1

SW1

SW1

RETURN

100

$$
\begin{aligned}
& * \\
& 6 \\
& *
\end{aligned}
$$

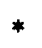

6

CALL SUBR(3)

CALL ENTRY

SW1

SW1

SW1

RETURN

105

CALL INP ( $M, M X P M Y, M I N Z E R$,

.)

IF (MODE.EQ. 2) RETURN

IF (XSW) RETURN

SW1

SW1

SW1

SW1

SW1

SW1

SW1

SW1

SETS UP DRIFT MATRICES IF IN EXEC.MODE.

C PROCESSES DRIFT DATA.

CALL DATA $(M, 1,1,12$, PAR $)$ 
CALL DRFRSV( $M, N A M E, K A, K B, \theta)$

If (KB.EQ.0) CALL $\operatorname{LOAD}(M, 1,1,0,0,0,0,0,0)$

IF (KB.GT.0) CALL $\operatorname{LOAD}(M, 2,1, \mathrm{~KB}, 2,0,0,0,0)$

IF (KA.EQ.0) GO TO 102

* Set up MATRIX SPACE for ka NUMBER of DRIFTS, WhOSE LENGTHS WiLL

* be elements of inPUt VEctor.

SW 
CALL $\operatorname{INV}(M, N)$

SW1

RETURN

C

175

185

190

195

200

205

210

215

220

225

C MAGNET ENTRY

C PROCESSES MAG DATA. SETS UP MAGNET MATRICES IF IN EXEC. MODE.

13 IF ( MODE.EQ.3) GO TO 131

CALL MAGRSV( $M, N A M E, K A, K B, 0)$

CALL LOAD $(M$,

- $\quad 6,1,0,0,0,0,0,0$

.)

IF (KA.EQ.0) GO TO 132

SET UP MATRIX SPACE FOR KA NUMBER OF MAGNETS. SOME OF THE

* input parameters derive from previously defined Vector.

CALL RSVMAT

$M I N=M I N-K A$

132 IF (MODE.EQ.2) RETURN

IF (XSW) RETURN

IF (ERROR) RETURN

131 IF (KA.GT.0) GO TO 133

CALL DATA $(M, 1,1,6$, PAR $)$

CALL MAGNET $(M, P A R)$

RETURN

133 CALL MAGMATS(M,KA)

RETURN

C

C KICK ENTRY

45 IF (MODE.EQ.3) GO TO 451

CALL INP(M, R7, MINZER, 1, 2, 3, 1, 0,0,0,0)

IF ( (MODE.EQ.2).OR. (XSW).OR. (ERROR) ) RETURN

451 CALL KICK $(M)$

RETURN

C

C MMM ENTRY

C PROCESSES MMM DATA. MULTIPLIES MATRICES IF IN EXEC. MODE

14 IF (MODE.EQ.3) GO TO 141

IF (MSIZE.NE.3) $\mathrm{KN}=\mathrm{R} 7$

CALL INP(M, KN, MINZER $, 1,-2,0,0,0,0,0,0)$

IF (MODE.EQ.2) RETURN

IF (XSW) RETURN

$141 \operatorname{NDAT}=\operatorname{INFF}(17, M)$

CALL MIFILL(M, 1, NDAT, NELS, MI)

$\mathrm{KB}=\operatorname{INFF}(5, M)$

IF (KB.NE.0.AND.KB.NE.1) GO TO 142

CALL MMM(M,NELS,MI)

RETURN

$142 \operatorname{CALL} \operatorname{EXECMM}(M, N E L S, K B, M I)$

RETURN

C

C WMA ENTRY

C PROCESSES WMA DATA. WRITES MATRICES IF IN EXEC.MODE.

$15 \operatorname{CALL} \operatorname{INP}(M, 0,0$,

- $K A, 2,0,0,0,0,0,0$

.)

IF (MODE.EQ.2) RETURN

IF (XSW) RETURN

$\operatorname{CALL} \operatorname{MLIST}(M, 1, K A, M I)$
SW1 $\quad 137$

SW1 138

SW1 139

SW1 140

SW1 141

SW1 142

SW1 143

SW1 144

SW1 $\quad 145$

SW1 146

SW1 $\quad 147$

SW1 148

SW1 149

SW1 150

SW1 151

SW1 152

SW1 153

SW1 154

SW1 155

SW1 156

SW1 $\quad 157$

SW1 158

SW1 $\quad 159$

SW1 160

SW1 161

SW1 162

SW1 163

SW1 164

SW1 165

SW1 $\quad 166$

SW1 $\quad 167$

SW1 168

SW1 169

SW1 $\quad 170$

SW1 $\quad 171$

SW1 172

SW1 173

SW1 174

SW1 $\quad 175$

SW1 176

SW1 $\quad 177$

SW1 178

SW1 $\quad 179$

SW1 180

SW1 181

SW1 182

SW1 183

SW1 $\quad 184$

SW1 185

SW1 186

SW1 $\quad 187$

SW1 188

SW1 189

SW1 190

SW1 191

SW1 192 
C SHIFT ENTRY

C PROCESSES SHF DATA. SETS UP SHIFT MATRIX IF IN EXEC. MODE 16 CALL INP (M, MXPMY, MINZER, - $4,1,0,0,0,0,0,0$

.)

IF (MODE.EQ.2) RETURN

IF (XSW) RETURN

CALL DATA $(M, 1,1,4$, PAR $)$

SW1

206

C

CALL SHF (M,PAR)

SW1

207

C REFLECT ENTRY

245

C PROCESSES REF DATA. COMPUTES MATRIX REFLECTION IF IN EXEC. MODE

SW1

208 $18 \mathrm{KN}=$ IBLNK

IF (MSIZE.EQ.7) $\mathrm{KN}=\mathrm{R7}$

IF (MSIZE.EQ.3) KN $=$ MXPMY

SW1

209

210

CALL INP $(M, K N, M I N Z E R$, RETURN

C

C INCR ENTRY

SW1 220

SW1 221

* DETERMINE TYPE TO BE INCREMENTED- FL.PT., INTEGER, KA OR KB.

- $2,2,2, K E, 0,0,0,0$

CALL INCR(M,KO) 
RETURN

$$
\begin{aligned}
& 193 \text { CALL INFW(MSUBR, 19, M) } \\
& \text { RETURN }
\end{aligned}
$$
$\mathrm{KE}=0$

*

IF (KT.EQ.1H ) KE $=1$

*

\section{ILLEGITIMATE TYPE}

203 FORMAT ( $5 \mathrm{X}, 1 \mathrm{H} *, *$ ERROR*,2H*, A5, * IS AN ILLEGITIMATE TYPE OF REPLAC SW 1

CONT INUE

.)

IF (MODE.EQ.2) RETURN

RETURN

C PROCESSES STAR DATA. COMPUTES IF IN EXEC. MODE

C

c

C CYA AND CYC ENTRY 
CALL INP $(M, K N, M I N Z E R$,

307

- $4,2,2,1,0,0,0,0)$

SW1

NOV3SW

1

NDAT $=1$

NOV 3 SW

2

IF (KN.EQ. IBLNK) KN $=\operatorname{INFF}(20, M)$

SW1

311

IF (KN.EQ.2HR7) KN $=R 7$

SW1

312

IF (KN.EQ. 3HMXY) KN = MXPMY

SW1 $\quad 313$

IF (OPNAME.NE.3HCYA.AND.KA.NE.21) GO TO 521

SW

314

350

IF (KA.GT.999) GO TO 545

SW

315

DECODE $(1,520$, NAME) NM

520 FORMAT (A1)

CALL MIFILL(M, 1 , NDAT, NELS1, MI 1)

IF ( OPNAME.EQ.3HCYA) GO TO 522

355

NELS2=NELS1

SW

316

SW1 317

SW1 318

SW1 $\quad 319$

SW1 320

IF (KB.LT.0) NELS2=NELS1+1

SW1 321

NQ3 $=14 *($ NELS $2+1)+18$

SW

322

LQ3 = LFILE + 1

SW

323

LFILE $=\mathrm{LQ3}+\mathrm{NQ3}$

SW

324

360

NTOT $=$ NTOT + NQ3

SW

325

$\operatorname{INFF}(18, M)=$ NTOT

SW

326

$\operatorname{INFF}(24, M)=L Q 3$

SW

327

IF (MODE.EQ.2) GO TO 542

SW

328

IF (XSW) RETURN

SW

329

365

GO TO 544

522 CALL DEFSET (M, NM, NELS1, MATNM, $0, K N)$

SWT

330

521 IF (MODE.EQ.2) GO TO 542

IF (XSW) RETURN

SW

331

SW

332

543 NDAT $=1$

CALL MIFILL(M, 1, NDAT, NELS1, MI 1)

544 CALL CYX(M)

IF (MODE.EQ.3) RETURN

542 IF (OPNAME. NE. 4HCYA) RETURN

MIN = MIN - NELS1

375

RETURN

545 WRITE $(3,546)$

546 FORMAT (23H CYA COUNT EXCEEDS 999.)

ERROR $=$. TRUE

RETURN

380

C

C CYB ENTRY

24 IF (MODE.EQ.3) GO TO 240

$K N=M X P M Y$

CALL $\operatorname{INP}(M, \theta, \theta$,

385

$1,2,1,3,0,0,0,0$

.)

IF (MODE.EQ.2) RETURN

IF (XSW) RETURN

C RETRIEVE ELEMENT NAME FROM DATA AND OBTAIN ITS INDEX.

390

240 CONTINUE

CALL SETCYB(M,KA)

SW1 333

NOV3SW1 3

SW1 $\quad 335$

SW1 $\quad 336$

SW1 $\quad 337$

SW1 $\quad 338$

SW1 $\quad 339$

SW1 340

SW1 341

SW1 342

SW1 343

SW1 344

SW1 345

SW1 346

SW1 $\quad 347$

SW1 348

SW1 $\quad 349$

SW1 $\quad 350$

SW1 351

SW1 352

SW1 353

SW1 354

SW1 $\quad 355$

SW1 356

SW1 357

245 CALL CYX(M)

SW1 358

SW1 359

395 RETURN

SW1 360

SW1 361

25 CALL SETFIT

FITQ, FITB, FITR, FITV ENTRY

SW1

362

RETURN 
C

C WBE ENTRY

C PROCESSES WBE DATA. CALLS WBE TO WRITE BETATRON FUNCTIONS

CALL INP $(M, \theta, 0$,

C KIND AND TYPE ARE SCALAR AND SINGLE $\mathrm{KN}=\mathrm{ISCL}$ 
c

CALL DATA $(M, 1,1, I Q, P A R)$

SW1 422

CALL AROP (M,PAR, IOP)

SW1

423

460

$277 \operatorname{KREM}(1)=\operatorname{BDAT}(M, 1)$

SW1

424

$\operatorname{KREM}(2)=\operatorname{BDAT}(M, 3)$

SW1

425

$I O P=\operatorname{BDAT}(M, 2)$

SW1

426

CALL AROP (M, KREM, IOP)

427

RETURN

SW1

428

SW1

429

C CELL ENTRY

29 IF(MODE. EQ.3) GO TO 293

C MAKE RESERVATIONS FOR FIRST CYCLED MATRIX AND STORE INPUT DATA

SW1

430

SW1

431

C RESERVE SPACE IN DATA FOR THE MI LIST

SW1

432

SW1 433

470

CALL RESRV ( $M$, MXPMY, MINZER,

SW1

434

- $7,1,12,3,0,0,0,0$

SW1

435

.)

CALL $\operatorname{LOAD}(M$,

SW1

436

SW1 437

SW1 438

- $7,1,0,0,0,0,0,0$

SW1

439

475

.)

C SET UP SPACE FOR CYCLED MATRICES

SW1

440

DECODE $(1,520$, NAME) NM

CALL DEFSET (M,NM, 12, MATNM, 4, MXPMY)

SW1

SW1

441

IF (MODE.EQ.2) GO TO 294

480

IF (XSW) RETURN

293 CALL DATA $(M, 1,1,7$, PAR $)$

CALL CELL(M,PAR)

IF (MODE.EQ.3) RETURN

SW1

SW1

SW1

SW1

SW1

SW1

294 MIN=MIN-16

SW1

SW1

C

RETURN

SW1

C ALTC ENTRY

30 IF (MODE.EQ.3) GO TO 301

C MAKE RESERVATIONS FOR FIRST CYCLED MATRIX AND STORE INPUT

490

C RESERVE SPACE IN DATA FOR MI LIST

CALL RESRV $(M, M X P M Y, M I N Z E R$,

$8,1,12,3,0,0,0,0$

.)

CALL LOAD $(M$,

495

$.8,1,0,0,0,0,0,0$

.)

DECODE $(1,520$, NAME $)$ NM

C SET UP SPACE FOR CYCLED MATRICES AND QF, QD,QS,QT

CALL DEFSET (M,NM, 12, MATNM 4, MXPMY)

500

IF (MODE.EQ.2) GO TO 302

IF (XSW) RETURN

301 CALL DATA $(M, 1,1,8$, PAR $)$

CALL ALTC (M, KA, PAR)

IF (MODE.EQ.3) RETURN

505

302 MIN=MIN-16

RETURN

510

C

*

31 IF (MODE, EQ.3) GO TO 311

$K 1=K A * 2$

$\mathrm{K} 2=\mathrm{KB} * 2$

$K=K 1+K 2+2$

CALL RESRV $(M, 0,0$, 
520

525

535

540

545

550

555

560

565
$K, 2,1,3,0,0,0,0$

.)

CALL LOAD $(M$,

- $2,2,1,3,0,0,0,0$

.)

CALL LOAD(M, - $K 1,2,0,0,0,0,0,0$

.)

CALL $\operatorname{LOAD}(\mathrm{M}$,

- $K 2,2,0,0,0,0,0,0$

.)

IF (MODE.EQ.2) RETURN

IF (XSW) RETURN

311 CALL DOFIT(M)

RETURN

C COPY ENTRY

32 IF (MODE.EQ.3) GO TO 320

$\operatorname{CALL} \operatorname{INP}(M, 0,0,0,0,0,0,0,0,0,0)$

320 IF (MODE.EQ.2) RETURN

IF (XSW) RETURN

CPYSW $=$. TRUE.

RETURN

C

33 IF (MODE.EQ.3) GO TO 330

$\operatorname{CALL} \operatorname{INP}(M, 0,0,0,0,0,0,0,0,0,0)$

330 IF (MODE.EQ.2) RETURN

IF (XSW) RETURN

CPYSW $=$.FALSE.

RETURN

C PAGE ENTRY

C RESTORES PAGE IF IN EXECUTION MODE

34 IF (MODE.EQ.3) GO TO 341

*

STORE DATA FIELD TO PRINT AS A COMMENT

$\operatorname{CALL} \operatorname{INP}(M, 0,0$,

- $12,2,0,0,0,0,0,0$

.)

IF (MODE.EQ.2) RETURN

IF (XSW) RETURN

$341 K \theta=\operatorname{INFF}(17, M)$

CALL DATA $(M, 2,1, K \theta, K R E M)$

IF (CPYSW) WRITE $(3,342)(\operatorname{KREM}(I), I=1, K \theta)$

342 FORMAT $(1 \mathrm{H} 1 / 8 \mathrm{X}, 16 \mathrm{A5})$

RETURN

C

C REM ENTRY

C PRINTS REMARKS

35 IF (MODE, EQ.3) GO TO 352

CALL INP $(M, \theta, \theta$,

- $12,2,0,0,0,0,0,0$

.)

352 IF (XSW) RETURN

$K \theta=\operatorname{INFF}(17, M)$

CALL DATA $(M, 2,1, K 0, K R E M)$

IF (CPYSW) WRITE $(3,353)(\operatorname{KREM}(I), I=1, K \theta)$

353 FORMAT $(/ 8 X, 16 A 5)$

RETURN

\begin{tabular}{|c|c|}
\hline SW1 & 479 \\
\hline SW1 & 480 \\
\hline SW1 & 481 \\
\hline SW1 & 482 \\
\hline SW1 & 483 \\
\hline SW1 & 484 \\
\hline SW1 & 485 \\
\hline SW1 & 486 \\
\hline SW1 & 487 \\
\hline SW1 & 488 \\
\hline SW1 & 489 \\
\hline SW1 & 490 \\
\hline SW1 & 491 \\
\hline SW1 & 492 \\
\hline SW1 & 493 \\
\hline SW1 & 494 \\
\hline SW1 & 495 \\
\hline SW1 & 496 \\
\hline SW1 & 497 \\
\hline SW1 & 498 \\
\hline SW1 & 499 \\
\hline SW1 & 500 \\
\hline SW1 & 501 \\
\hline SW1 & 502 \\
\hline SW1 & 503 \\
\hline SW1 & 504 \\
\hline SW1 & 505 \\
\hline SW1 & 506 \\
\hline SW1 & 507 \\
\hline SW1 & 508 \\
\hline SW1 & 509 \\
\hline SW1 & 510 \\
\hline SW1 & 511 \\
\hline SW1 & 512 \\
\hline SW1 & 513 \\
\hline SW1 & 514 \\
\hline SW1 & 515 \\
\hline SW1 & 516 \\
\hline SW1 & 517 \\
\hline SW1 & 518 \\
\hline SW1 & 519 \\
\hline SW1 & 520 \\
\hline SW1 & 521 \\
\hline SW1 & 522 \\
\hline SW1 & 523 \\
\hline SW1 & 524 \\
\hline SWI & 525 \\
\hline SW1 & 526 \\
\hline SW1 & 527 \\
\hline SW1 & 528 \\
\hline SW1 & 529 \\
\hline SW1 & 530 \\
\hline SW1 & 531 \\
\hline SW1 & 532 \\
\hline SW1 & 533 \\
\hline SW1 & 534 \\
\hline SW1 & 535 \\
\hline
\end{tabular}


C

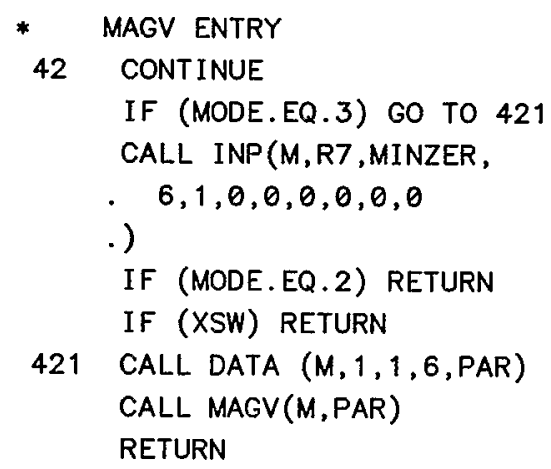


$.

C ZERO ARRAY AT LQ

C SET UP INDICES TABLE

CALL TABULAT ( $M$, TABSW)

810 TABSW $=$. TRUE. RETURN

C PTAB ENTRY

CALL INP $(M, \theta, \theta$,

.)

$\mathrm{KA}, 2,0,0,0,0,0,0$

650

IF (MODE.EQ.2) RETURN

820 CALL PRNTAB(M) RETURN

*

CALL INP $(M, \theta, \theta$,

- $1,-2,0,0,0,0,0,0$

.)

SW1

SW1

SW1

SW1

SW1

SW1

SW1

SW1

SW1

598

RETURN
C BETA ENTRY
44 IF(MODE. EQ.3) GO TO 441
CALL INP( $M, I S C L, M I N Z E R$, - $1,2,1,1,0,0,0,0$

665

.)

IF (MODE. EQ . 2) RETURN

SW1

SW1

SW1

SW1

SW1

SW1

SW1

SW1

SW1

SW1

SW1

SW1

SW1

SW1

SW1

SW1 
CALL INP ( $M, R 7, M I N Z E R$,

IF (MODE.EQ.2) RETURN

SW1

471 CALL DATA $(M, 1,1,6$, PAR)

SW1 657

CALL SHF7 ( $M, P A R)$

695

RETURN

SW1

\section{SOLENOID ENTRY}

48 IF (MODE.EQ.3) GO TO 481
CALL INP (M,R7,MINZER,

SW1 661

SW1 662

700

. $4,1,0,0,0,0,0,0$

SW1 663

SW1 664

.)

IF (MODE.EQ. 2) RETURN

SW1 665

SW1 666

481 CALL DATA (M, 1, 1, 4,PAR)

SW1 667

SW1 668

CALL SOL( $M, P A R)$

705

RETURN

SW1 669

SW1 670

C VAR ENTRY

SW1 671

C DEFINES VARIABLE BY INSTRUCTION NAME AND POSITION.

SW1 672

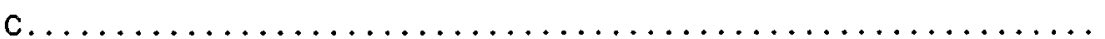

SW1 673

C VNAM VAR KA NAME

SW1 674

710

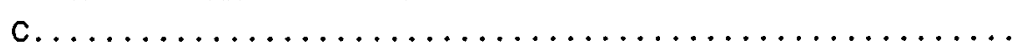

SW1 675

C EXAMPLE 1

SW1 676

$\begin{array}{lllllll}C & \text { QF } & \text { MAG } & 1.5 & 0.06 & 1 . & \text { SW1 }\end{array}$

$\begin{array}{lllllll}\text { C } & \text { GQF } & \text { VAR } & 1 & \text { QF } & S W 1 & 678\end{array}$

C QFH MAG $\quad 0.75$ GQF $\quad 1.2 .579$

715

720

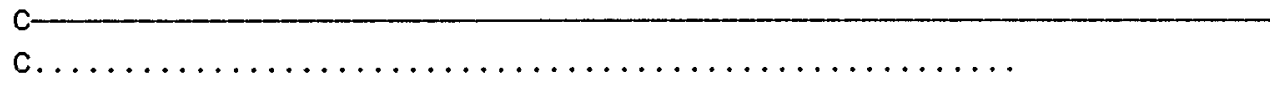

SW1

680

681

c

C IN THIS EXAMPLE QFH HAS HALF THE LENGTH OF QF, BUT THE SAME

SW1

SW1

c GRADIENT, 0.06 .

SW1

\section{EXAMPLE 2 - A MORE USEFUL EXAMPLE}

SW1

L DRF

2.

SW1

C SR SUB

725

QF MAG

FITQ

$$
\text { SR } \quad \text { C } \quad \text { QF } \quad \text { QD }
$$

2

2

SW1

SW1

SW1

SW1

695

SW1

C

IF (MODE.EQ.3) GO TO 491

CALL INP $(M, 0,0,1,2,0,0,0,0,0,0)$

IF (MODE.EQ. 2) RETURN 
WRITE $(3,490)$ NAM, FVAR

C

RETURN

SW

745

\author{
C PRNT ENTRY - PRINT SELECTED DATA \\ 50 IF (MODE.EQ.3) GO TO 51 \\ $\operatorname{CALL} \operatorname{INP}(M, 0,0,1,-2,0,0,0,0,0,0)$ \\ IF (MODE.EQ.2) RETURN \\ 51 IF (XSW) RETURN \\ CALL PRNT (M) \\ RETURN
}

SW1 $\quad 710$

SW1 $\quad 711$

SW1 $\quad 712$

750

SW1 $\quad 713$

SW1 $\quad 714$

SW1 $\quad 715$

SW1 $\quad 716$

SW1 $\quad 717$

END

SW1 $\quad 718$

SW1 719

CARD NR. SEVERITY DETAILS DIAGNOSIS OF PROBLEM

$\begin{array}{rr}90 & \text { I } \\ 93 & \text { I } \\ 96 & \text { I } \\ 99 & \text { I } \\ 102 & \text { I } \\ 107 & \text { I } \\ 108 & \text { I } \\ 111 & \text { I } \\ 124 & \text { I } \\ 125 & \text { I } \\ 141 & \text { I } \\ 143 & \text { I } \\ 144 & \text { I } \\ 146 & \text { I } \\ 154 & \text { I } \\ 155 & \text { I } \\ 158 & \text { I } \\ 168 & \text { I } \\ 169 & \text { I } \\ 173 & \text { I } \\ 187 & \text { I } \\ 188 & \text { I } \\ 189 & \text { I } \\ 193 & \text { I } \\ 195 & \text { I } \\ 200 & \text { I } \\ 202 & \text { I } \\ 210 & \text { I } \\ 211 & \text { I } \\ 217 & \text { I } \\ 219 & \text { I } \\ 226 & \text { I } \\ 227 & \text { I } \\ 229 & \text { I } \\ 231 & \text { I } \\ 238 & \text { I } \\ 239 & \text { I } \\ 242 & \text { I } \\ & \end{array}$

RETURN STATEMENT APPEARS IN MAIN PROGRAM. RETURN STATEMENT APPEARS IN MAIN PROGRAM. RETURN STATEMENT APPEARS IN MAIN PROGRAM. RETURN STATEMENT APPEARS IN MAIN PROGRAM. RETURN STATEMENT APPEARS IN MAIN PROGRAM. RETURN STATEMENT APPEARS IN MAIN PROGRAM. RETURN STATEMENT APPEARS IN MAIN PROGRAM. RETURN STATEMENT APPEARS IN MAIN PROGRAM. RETURN STATEMENT APPEARS IN MAIN PROGRAM. RETURN STATEMENT APPEARS IN MAIN PROGRAM. RETURN STATEMENT APPEARS IN MAIN PROGRAM. RETURN STATEMENT APPEARS IN MAIN PROGRAM. RETURN STATEMENT APPEARS IN MAIN PROGRAM. RETURN STATEMENT APPEARS IN MAIN PROGRAM. RETURN STATEMENT APPEARS IN MAIN PROGRAM. RETURN STATEMENT APPEARS IN MAIN PROGRAM. RETURN STATEMENT APPEARS IN MAIN PROGRAM. RETURN STATEMENT APPEARS IN MAIN PROGRAM. RETURN STATEMENT APPEARS IN MAIN PROGRAM. RETURN STATEMENT APPEARS IN MAIN PROGRAM. RETURN STATEMENT APPEARS IN MAIN PROGRAM. RETURN STATEMENT APPEARS IN MAIN PROGRAM. RETURN STATEMENT APPEARS IN MAIN PROGRAM. RETURN STATEMENT APPEARS IN MAIN PROGRAM. RETURN STATEMENT APPEARS IN MAIN PROGRAM. RETURN STATEMENT APPEARS IN MAIN PROGRAM. RETURN STATEMENT APPEARS IN MAIN PROGRAM. RETURN STATEMENT APPEARS IN MAIN PROGRAM. RETURN STATEMENT APPEARS IN MAIN PROGRAM. RETURN STATEMENT APPEARS IN MAIN PROGRAM. RETURN STATEMENT APPEARS IN MAIN PROGRAM. RETURN STATEMENT APPEARS IN MAIN PROGRAM. RETURN STATEMENT APPEARS IN MAIN PROGRAM. RETURN STATEMENT APPEARS IN MAIN PROGRAM. RETURN STATEMENT APPEARS IN MAIN PROGRAM. RETURN STATEMENT APPEARS IN MAIN PROGRAM. RETURN STATEMENT APPEARS IN MAIN PROGRAM. RETURN STATEMENT APPEARS IN MAIN PROGRAM. 
CARD NR. SEVERITY DETAILS 252 I

253 I

256 I

270 I

274 I

283 I

286 I

288 I

312 I

317 I

318 I

322 I

332 I

333 I

335 I

364 I

368 I

372 I

373 I

375 I

379 I

387 I

388 I

392 I

394 I

398 I

407 I

$408 \quad 1$

410 I

412 I

425 I

437 I

445 I

447 I

453 I

459 I

464 I

480 I

483 I

485 I

501 I

504 I

506 I

525 I

526 I

528 I

533 I

534 I

536 I

540 I

541 I
DIAGNOSIS OF PROBLEM

RETURN STATEMENT APPEARS IN MAIN PROGRAM. RETURN STATEMENT APPEARS IN MAIN PROGRAM. RETURN STATEMENT APPEARS IN MAIN PROGRAM.

TAPE3\# I/O FILE NOT DEFINED.

RETURN STATEMENT APPEARS IN MAIN PROGRAM. RETURN STATEMENT APPEARS IN MAIN PROGRAM. RETURN STATEMENT APPEARS IN MAIN PROGRAM. RETURN STATEMENT APPEARS IN MAIN PROGRAM. RETURN STATEMENT APPEARS IN MAIN PROGRAM. RETURN STATEMENT APPEARS IN MAIN PROGRAM. RETURN STATEMENT APPEARS IN MAIN PROGRAM. RETURN STATEMENT APPEARS IN MAIN PROGRAM. RETURN STATEMENT APPEARS IN MAIN PROGRAM. RETURN STATEMENT APPEARS IN MAIN PROGRAM. RETURN STATEMENT APPEARS IN MAIN PROGRAM. RETURN STATEMENT APPEARS IN MAIN PROGRAM. RETURN STATEMENT APPEARS IN MAIN PROGRAM. RETURN STATEMENT APPEARS IN MAIN PROGRAM. RETURN STATEMENT APPEARS IN MAIN PROGRAM. RETURN STATEMENT APPEARS IN MAIN PROGRAM. RETURN STATEMENT APPEARS IN MAIN PROGRAM. RETURN STATEMENT APPEARS IN MAIN PROGRAM. RETURN STATEMENT APPEARS IN MAIN PROGRAM. RETURN STATEMENT APPEARS IN MAIN PROGRAM. RETURN STATEMENT APPEARS IN MAIN PROGRAM. RETURN STATEMENT APPEARS IN MAIN PROGRAM. RETURN STATEMENT APPEARS IN MAIN PROGRAM. RETURN STATEMENT APPEARS IN MAIN PROGRAM. RETURN STATEMENT APPEARS IN MAIN PROGRAM. RETURN STATEMENT APPEARS IN MAIN PROGRAM. RETURN STATEMENT APPEARS IN MAIN PROGRAM. RETURN STATEMENT APPEARS IN MAIN PROGRAM. RETURN STATEMENT APPEARS IN MAIN PROGRAM. RETURN STATEMENT APPEARS IN MAIN PROGRAM. RETURN STATEMENT APPEARS IN MAIN PROGRAM. RETURN STATEMENT APPEARS IN MAIN PROGRAM. RETURN STATEMENT APPEARS IN MAIN PROGRAM. RETURN STATEMENT APPEARS IN MAIN PROGRAM. RETURN STATEMENT APPEARS IN MAIN PROGRAM. RETURN STATEMENT APPEARS IN MAIN PROGRAM. RETURN STATEMENT APPEARS IN MAIN PROGRAM. RETURN STATEMENT APPEARS IN MAIN PROGRAM. RETURN STATEMENT APPEARS IN MAIN PROGRAM. RETURN STATEMENT APPEARS IN MAIN PROGRAM. RETURN STATEMENT APPEARS IN MAIN PROGRAM. RETURN STATEMENT APPEARS IN MAIN PROGRAM. RETURN STATEMENT APPEARS IN MAIN PROGRAM. RETURN STATEMENT APPEARS IN MAIN PROGRAM. RETURN STATEMENT APPEARS IN MAIN PROGRAM. RETURN STATEMENT APPEARS IN MAIN PROGRAM.

*** DUE TO THE MANY ERRORS NOTED, ONLY THOSE WHICH ARE FATAL WILL BE LISTED HEREAFT 
OVERLAY (SYNCH, 3,0)

PROGRAM SW3

SW3

SW3

C PROCESSES DATA FOR CURRENT INSTR. IF IN MODE 1 OR 2.

SW3

C PROCEEDS WITH CALC.BY CALLING APPROPRIATE RÖUTINES IF MODE=1 OR 3 .

SW3

5

20

30

\section{DIMENSION INF (24)}

COMMON/INSTR/OPNAME, NAME, OP, KA, KB, OBJA, OBJB, NXTM, TRA, LQ, NQ, LIN, 1

2 NIN, LFL, NFL, LBC, NBC, NTOT, IX, KIND, TYPE, ROWS, COLS, EXTR $M, N F, N B, N I, M S U B R, I S I G N$

INTEGER OPNAME, OP, OBJA, OBJB, TRA, TYPE, ROWS, COLS, EXTR

LEVEL 2, STORE, INFF, IWORK

COMMON STORE( 48000$),$ IWORK( 10)

DIMENSION INFF $(24,2000)$

EQUIVALENCE (INFF, STORE)

COMMON/STORE/LMAX, LINF, LF I LE, MAX, MIN, ICARD (11) :

\section{* LMAX AND MAX ARE DIMENSIONS OF STORE AND INFF}

COMMON/SWTCH/BEND, MSIZE, CYCSWT, VCSW, PV, MSSW, VPR, MHS, MODS, MINZER LOGICAL CYCSWT!,MSSW, VCSW, PV, VPR, MHS, MODS

COMMON/GRR/IERR, BSW, VSW, RSW, QSW, XSW

LOGICAL IERR, BSW, VSW, RSW, QSW, XSW

INTEGER BDAT, PVECS

INTEGER R7, ROT, PRD

EQUIVALENCE (OPNAME, INF(1)), (PAR, KREM)

DIMENSION MATNM(20), MXLIST(20)

DIMENSION MATNM(20), MXLIST(20)

DIMENSION PAR(100), KREM(100), NCELL(5), JMAG(7)

DATA (JMAG (K), K=1,7)/2HIF, 2HID, 2HOF , 2HOD , $1 \mathrm{HE}, 1 \mathrm{HS}, 1 \mathrm{HT} /$

DATA MXPMY,PVECS, R7, ROT ,PRD/1,2,5,6,8/

DATA (MATNM(I), I=1,12)/2HF ,2HD ,2HS , $2 \mathrm{HT}, 2 \mathrm{HE}, 2 \mathrm{HK}, 2 \mathrm{HIA}, 2 \mathrm{HIB}, 2 \mathrm{HB}$ $1,2 \mathrm{HA}, 2 \mathrm{HWD}, 2 \mathrm{HWF} /$

C
IF OPNAME = NULL, IT IS A P OR C CARD. NEEDS SPECIAL HANDLING TO CONSTRUCT A SYNCH PAGE OR REM INSTRUCTION

$N O P=O P-50$
SW3

SW3

INSTR

INSTR

INSTR

INSTR

INSTR

BLANK

BLANK

86MARSIZ

86MARSIZ

86MARSIZ

BLANK

STORE

STORE

STORE

CONTRL

CONTRL

CONTRL

CONTRL

DIM

DIM

BMIL

BMIL

BMIL.

BMIL

BMI

SWTCH

SWTCH

SWTCH

GRR

GRR

GRR

SW3

SW3

SW3

SW3

SW3

SW3

SW3

SW3

SW3

SW3

SW3

SW3

SW3

SW3

SW3

SW3

SW3

SW3

SW3

SW3
2

3

4

5

6

7

2

3

4

5

6

2

3

1

2

3

5

2

3

4

2

3

4

5

2

3

1

2

3

4

3

2

3 
60

*

*

GO TO SUM, ACT, DELE, SCOP, TEST, WFL, MAGS, CELC, CFD, DCFD STR2, STR4, STRP, STRN

70

75

80

281 CALL DATA $(M, 1,1,5$, PAR $)$

$N=\operatorname{MDAT}(M, 1)$

CALL MAGS $(M, N, P A R)$

RETURN

C SUM ENTRY

36 IF (MODE.EQ.3) GO TO 361

$\operatorname{CALL} \operatorname{INP}(M, 0,1$,

- $K A, 1,0,0,0,0,0,0$

.)

IF (MODE.EQ. 2) RETURN

85

90

C

IF (XSW) RETURN

361 CALL DATA (M, 1, 1, KA,PAR)

CALL SUM(KA,PAR, RES)

$\operatorname{CALL} \operatorname{STDAT}(M, 5,1,1, \mathrm{RES})$

RETURN

95

100

C CELC ENTRY

37 IF (MODE.EQ.3) GO TO 371

C MAKE RESERVATIONS FOR FIRST CYCLED MATRIX AND STORE INPUT DATA

C RESERVE SPACE IN DATA FOR THE MI LIST

CALL RESRV $(M, M X P M Y, M I N Z E R$,

- $8,1,12,3,0,0,0,0$

.)

CALL LOAD(M,

$7,1,0,0,0,0,0,0$

.)

$\operatorname{DECODE}(1,520$, NAME) NM

C SET UP SPACE FOR CYCLED MATRICES AND QF, QD, QS, QT, QE

CALL DEFSET (M,NM, 12, MATNM , 5, MXPMY)

IF (MODE.EQ.2) GO TO 372

105

110

371 CALL DATA $(M, 1,1,8$, PAR $)$

CALL CELC (M,PAR)

IF (MODE.EQ.3) RETURN

372 MIN=MIN-17

RETURN

C

C CFD ENTRY

38 IF (MODE.EQ.3) GO TO 381

C MAKE RESERVATIONS FOR FIRST CYCLED MATRIX AND STORE INPUT DATA

SW3 $\quad 36$

SW3 $\quad 37$

SW3 $\quad 38$

SW3 $\quad 39$

SW3 40

SW3 41

SW3 42

SW3 43

SW3 $\quad 44$

SW3 45

SW3 $\quad 46$

SW3 $\quad 47$

SW3 48

SW3 $\quad 49$

SW3 50

SW3 51

SW3 52

SW3 53

SW3 54

SW3 $\quad 55$

SW3 56

SW3 57

SW3 58

SW3 59

SW3 60

SW3 61

SW3 62

SW3 63

SW3 64

SW3 65

SW3 66

SW3 67

SW3 68

SW3 69

SW3 70

SW3 71

SW3 72

SW3 73

SW3 74

SW3 75

SW3 $\quad 76$

SW3 $\quad 77$

SW3 $\quad 78$

SW3 79

SW3 80

SW3 81

SW3 82

SW3 83

SW3 84

SW3 85

SW3 86

SW3 87

SW3 88

SW3 89

SW3 90

SW3 91

SW3 92 
CALL RESRV $(M, M X P M Y, M I N Z E R$,

. $8,1,12,3,0,0,0,0$

CALL LOAD(M, 
C

C STRN ENTRY.

46 IF (MODE.EQ.3) GO TO 462
IF (KA.NE. 2) KA=1

$\mathrm{KF}=4$

IF (KA.EQ.2) KF=8

CALL RESRV $(M, M X P M Y, M I N Z E R$,

CALL LADD (M.

- $2,2,4,1,0,0,0,0$

.)

IF (KA.EQ.2) CALL LOAD ( $M$,

.)

DECODE $(1,520$, NAME $)$ NM

SW3

SW3

DO $461 \mathrm{I}=1,6$

190

$461 \operatorname{MXLIST}(I)=\operatorname{MATNM}(I+6)$

SW3

164

$\operatorname{MXLIST}(7)=\operatorname{MATNM}(5)$

SW3

SW3

$\operatorname{MXLIST}(8)=\operatorname{MATNM}(3)$

$M 1=M-1$

SW3

169

DO $460 \quad I=1,6$

SW3

170

CALL MAGRSV(-M1,MXLIST(I), 0,0, NAME)

SW3

171

195

$460 M 1=M 1-1$

DO $464 I=7,8$

SW3

172

SW3

173

CALL DRFRSV(-M1,MXLIST (I), 0,0, NAME)

SW3

174

$464 M 1=M 1-1$

IF (MODE.EQ.2) GO TO 463

SW3

175

SW3 $\quad 176$

200

IF (XSW) RETURN

SW3

SW3

177

462 CALL DATA $(M, 2,1,2$, NCELL)

SW3

$\mathrm{KF}=4$

SW3

IF (KA.EQ.2) $K F=8$

SW3

CALL DATA $(M, 1,1, K F, P A R)$

SW3

CALL STRN (M,NCELL,PAR)

SW3

IF (MODE. EQ.3) RETURN

463 MIN $=$ MIN -8

SW3

SW3

RETURN

SW3

C STR2 ENTRY. DESIGN COLLINS STRAIGHT SECTION FOR INSERTION.

210

47 IF (MODE.EQ.3) GO TO 502

CALL RESRV ( $M, M X P M Y, M I N Z E R$,

SW

SW3

SW3

SW3

.)

$1,2,4,1,6,3,0,0$

SW3

SW3

CALL LOAD(M,

- $1,2,4,1,0,0,0,0$

SW3

SW3

SW3

SW3

SW3

SW3

SW3

SW3

SW3

SW3

SW3

SW3

SW3

SW3 
IF (MODE.EQ.2) GO TO 503

502 CALL STR2(M)

C STRPI ENTRY. DESIGN PI-TYPE STRAIGHT SECTION FOR INSERTION.

.)

.)

DECODE $(1,520$, NAME) NM

SW3

SW3

CALL DEFSET (M, NM, 0, MATNM , 4, MXPMY)

SW3

IF (MODE.EQ.2) GO TO 513

SW3

IF (XSW) RETURN

SW3

SW3

512 CALL STRPI $(M)$

IF (MODE.EQ.3) RETURN

SW3

SW3

513 MIN = MIN - 4

SW3

RETURN

SW3

C SCOPE ENTRY

$49 \operatorname{CALL} \operatorname{INP}(M, \theta, 0$,

- $\quad 0,0,0,0,0,0,0,0$

SW3

SW3

SW3

.)

255

IF (MODE.EQ.2) RETURN

SW3

SW3

IF (XSW) RETURN

SW3

CALL SCOPCN

SW3

RETURN

C ACT OR DELE ENTRY

SW3

SW3

SW3

SW3

IF (MODE.EQ.3) GO TO 800

C IF $K B=0$, INPUT CONTAINS KA ELEMENTS

C IF $K B=1$, KA ELEMENTS SHOULD BE CHANGED BEGINNING WITH ONE INPUT

IF (KB.NE.0) CALL INP $(M, 0,0,1,2,0,0,0,0,0,0)$

265

IF (KB.EQ. 0 ) CALL INP $(M, 0,0,1,-2,0,0,0,0,0,0)$

IF (MODE. EQ.2) RETURN

END

CARD NR. SEVERITY DETAILS DIAGNOSIS OF PROBLEM

$\begin{array}{llll}43 & \text { I } & \text { MATNM } & \text { PREVIOUSLY DIMENSIONED ARRAY. FIRST DIMENSIONS WILL BE RETAINED. } \\ 43 & \text { I } & \text { MXLIST } & \text { PREVIOUSLY DIMENSIONED ARRAY. FIRST DIMENSIONS WILL BE RETAINED. } \\ 62 & \text { I } & & \text { RETURN STATEMENT APPEARS IN MAIN PROGRAM. } \\ 65 & \text { I } & \text { RETURN STATEMENT APPEARS IN MAIN PROGRAM. } \\ 73 & \text { I } & \text { RETURN STATEMENT APPEARS IN MAIN PROGRAM. } \\ 74 & \text { I } & \text { RETURN STATEMENT APPEARS IN MAIN PROGRAM. } \\ 78 & \text { I } & \text { RETURN STATEMENT APPEARS IN MAIN PROGRAM. } \\ 84 & \text { I } & \text { RETURN STATEMENT APPEARS IN MAIN PROGRAM. }\end{array}$


CARD NR. SEVERITY DETAILS

$\begin{array}{rr}85 & \text { I } \\ 89 & \text { I } \\ 105 & \text { I } \\ 108 & \text { I } \\ 110 & \text { I } \\ 126 & \text { I } \\ 129 & \text { I } \\ 131 & \text { I } \\ 145 & \text { I } \\ 148 & \text { I } \\ 150 & \text { I } \\ 164 & \text { I } \\ 168 & \text { I } \\ 170 & \text { I } \\ 200 & \text { I } \\ 206 & \text { I } \\ 208 & \text { I } \\ 230 & \text { I } \\ 232 & \text { I } \\ 234 & \text { I } \\ 246 & \text { I } \\ 248 & \text { I } \\ 250 & \text { I } \\ 255 & \text { I } \\ 256 & \text { I } \\ 258 & \text { I } \\ 266 & \text { I } \\ 267 & \text { I } \\ 269 & \text { I } \\ & \end{array}$

DIAGNOSIS OF PROBLEM

RETURN STATEMENT APPEARS IN MAIN PROGRAM.

RETURN STATEMENT APPEARS IN MAIN PROGRAM.

RETURN STATEMENT APPEARS IN MAIN PROGRAM.

RETURN STATEMENT APPEARS IN MAIN PROGRAM.

RETURN STATEMENT APPEARS IN MAIN PROGRAM.

RETURN STATEMENT APPEARS IN MAIN PROGRAM.

RETURN STATEMENT APPEARS IN MAIN PROGRAM.

RETURN STATEMENT APPEARS IN MAIN PROGRAM.

RETURN STATEMENT APPEARS IN MAIN PROGRAM.

RETURN STATEMENT APPEARS IN MAIN PROGRAM.

RETURN STATEMENT APPEARS IN MAIN PROGRAM.

RETURN STATEMENT APPEARS IN MAIN PROGRAM.

RETURN STATEMENT APPEARS IN MAIN PROGRAM.

RETURN STATEMENT APPEARS IN MAIN PROGRAM.

RETURN STATEMENT APPEARS IN MAIN PROGRAM.

RETURN STATEMENT APPEARS IN MAIN PROGRAM.

RETURN STATEMENT APPEARS IN MAIN PROGRAM.

RETURN STATEMENT APPEARS IN MAIN PROGRAM.

RETURN STATEMENT APPEARS IN MAIN PROGRAM.

RETURN STATEMENT APPEARS IN MAIN PROGRAM.

RETURN STATEMENT APPEARS IN MAIN PROGRAM.

RETURN STATEMENT APPEARS IN MAIN PROGRAM.

RETURN STATEMENT APPEARS IN MAIN PROGRAM.

RETURN STATEMENT APPEARS IN MAIN PROGRAM.

RETURN STATEMENT APPEARS IN MAIN PROGRAM.

RETURN STATEMENT APPEARS IN MAIN PROGRAM.

RETURN STATEMENT APPEARS IN MAIN PROGRAM.

RETURN STATEMENT APPEARS IN MAIN PROGRAM.

RETURN STATEMENT APPEARS IN MAIN PROGRAM. 
C

COMMON/MOVTOG/TOG

40
LEVEL 2, STORE, INFF, IWORK

COMMON STORE (48000), IWORK (10)

COMMON/CONTRL/ERROR, MODE, RSRV, STOR, XEQ, TRASW, NFSW, EMPTY, INDEF, 1 LDFLG, FIN

LOGICAL ERROR, RSRV, STOR, XEQ, TRASW, NFSW, EMPTY, INDEF, LDFLG, FIN

COMMON /DIM/ LEND, MEND, ISAV, ISAV7, M7END, KADD , KADDR, MUNIT, MSYMPL

COMMON/BMI/MI (5000)

COMMON/NELS/NELS

LEVEL 2,MI.

COMMON/SWTCH/BEND, MSIZE, CYCSWT, VCSW, PV, MSSW, VPR, MHS, MODS, MINZER LOGICAL CYCSWT, MSSW, VCSW, PV, VPR, MHS, MODS

INSTR

INSTR

INSTR

BLANK

BLANK

86MARSIZ

86MARSIZ

86MARSIZ

BLANK

STORE

STORE

STORE

CONTRL

CONTRL

CONTRL

CONTRL

DIM

DIM

BMIL

BMIL

BMIL

BMIL

BMI

SWTCH

SWTCH

SWTCH

GRR

GRR

GRR

MOVTOG

MOVTOG

MOVTOG

MOVTOG

SW4

SW4

SW4

DIMENSION T(49), RW(3)

INTEGER R7, ROT, PRD, HSXTP, HMAP

EQUIVALENCE(OPNAME, INF (1)), (PAR, KREM)

SW4

SW4

SW4

SW4

SW4

SW4

SW4

SW4

SW4

SW4

SW4

C GO TO FXPT, TRK, PRD, SXTP, MAP, BMIS, EMIS, ROTZ, ROT, INV2, 


$$
\begin{array}{ll}
8 & \text { CALL BMIS } \\
10 & \text { RETURN } \\
9 & \text { CALL EMIS }
\end{array}
$$

$$
\text { RETURN }
$$

C

C

SXTP ENTRY
33 IF (MODE.EQ.3) GO TO 331

CALL INP(M, MAP, MINZER, - $4,1,0,0,0,0,0,0$

.)

IF (MODE. EQ.2) RETURN IF (XSW) RETURN

331 CALL DATA $(M, 1,1,4, P A R)$ IF (MODE, EQ.1) $\operatorname{PAR}(4)=0$. DO $332 \quad I=1,6$

$332 V O(I)=0$.

CALL SXTP(M,VO,PAR)

RETURN

C PRD ENTRY DEFINE A PRODUCT OF LINEAR AND NON-LINEAR TRANSFORMATION 34 IF (MODE.EQ.3) RETURN . CALL INP (M,PRD, MINZER, - $\mathrm{KA}, 2,0,0,0,0,0,0$

.)

RETURN

C TRK ENTRY

90

100

105
35 IF (MODE.EQ.3) GO TO 352

CALL INP(M, R7, MINZER , 4, 2, 6, 3, 1, 4, 4, 1)

IF (MODE.EQ. 2) RETURN

IF (XSW) RETURN

CALL TRK(M)

RETURN

C FXPT ENTRY

40 IF (MODE.EQ.3) GO TO 401

C RESERVE SETS UP SPECIAL LQ LOCATIONS FOR FXPT

CALL RESRV $(M, R 7,0$,

$.5,1,2,2,5,3,0,0$

.)

CALL $\operatorname{LOAD}(M$,

$.2,2,5,3,0,0,0,0$

.)

CALL LOAD $(M$,

- $5,1,0,0,0,0,0,0$

.)

IF (MODE.EQ.2) GO TO 402

IF (XSW) RETURN

401 CALL FXPT(M)

402 RETURN

C ROTZ ENTRY
SW4

SW4683 2

SW4 35

SW4 36

SW4 37

SW4 38

SW4 39

SW4 $\quad 40$

SW4 41

SW4 42

SW4 43

SW4 44

SW4 45

SW4 $\quad 46$

SW4 $\quad 47$

SW4 48

SW4 49

SW4 50

SW4 51

SW4 52

SW4 53

SW4 54

SW4 55

SW4 56

SW4 57

SW4 58

SW4 59

SW4 60

SW4 61

SW4 62

SW4 63

SW4 64

MY3SW4 2

SW4 66

SW4 67

SW4 68

SW4 69

SW4 70

SW4 71

SW4 72

SW4 73

SW4 $\quad 74$

SW4683 3

SW4 76

SW4 $\quad 77$

SW4683 4

SW4 79

SW4 80

SW4 81

SW4 82

SW4 83

SW4 84

SW4 85

SW4 86

SW4 87

SW4 88 
41 IF (MODE.EQ.3) RETURN

SW4

CALL INP( $M$, ROT, MINZER,

- $1,1,0,0,0,0,0,0$

SW4

SW4

.)

SW4

RETURN

120

C

C ROT ENTRY-ROTATE MATRIX

42 IF (MODE. EQ.3) GO TO 420

CALL INP( $M, R 7, M I N Z E R$,

125

.)

IF (MODE.EQ.2) RETURN

IF (XSW) RETURN

$420 \mathrm{MB}=\operatorname{MDAT}(M, 1)$

THETA $=$ FDAT $(M, 1)$

130

THETA $=$ THETA $*$ RADEG

CALL ROTM(M,MB, THETA)

RETURN

135

INV2 ENTRY ROTATE MATRIX 180 DEGREES AND REFLECT

SW4

SW4

SW4

SW4

SW4

SW4

SW4

SW4

SW4

SW4

SW4

SW4

SW4

SW4

SW4

SW4

43 IF (MODE.EQ.3) GO TO 430

CALL INP( $M, R 7, M I N Z E R$,

- $1,2,0,0,0,0,0,0$

.)

IF (MODE.EQ.2) RETURN

140

IF (XSW) RETURN

$430 \mathrm{MB}=\operatorname{MDAT}(M, 1)$

CALL INV2 $(M, M B)$

RETURN

145

C ELQ ENTRY

44 IF (MODE.EQ.3) RETURN

CALL INP ( $M, M A P, M I N Z E R$,

$1,2,5,1,0,0,0,0)$

RETURN

150

C

C MAP ENTRY

1000 IF (MODE.EQ.3) RETURN

CALL INP( $M, M A P, M I N Z E R$,

155

.)

$K A, 1,0,0,0,0,0,0$

SW4

SW4

SW4

SW4

SW4

SW4

SW4

SW4

SW4

SW4

SW4

SW4

SW4

SW4

SW4

SW4

SW4

SW4

SW4

SW4

SW4

SW4

SW4

SW4

SW4

SW4

SW4

SW4

SW4

SW4

SW4

SW4

SW4

SW4

MY3SW4

SW4

MY3SW4 
C

THIS INSTRUCTION MAY BE USED TO SEE EXPLICITLY THE EFFECT OF SUBROUTINE REVMAT, WHICH MAKES A NEW MATRIX WHIS IS THE TRANSFER MATRIX FOR RAYS IN THE NEIGHBORHOOD OF A REFERENCE TRAJECTORY. IF (XSW) RETURN

571 CALL ORBIT(M) RETURN

C

END
SW4 146

SW4 147

SW4 148

SW4 149

SW4 150

SW4 $\quad 151$

SW4 152

SW4 153

SW4 154

SW4 155

SW4 $\quad 156$

SW4 157

SW4 158

SW4 159

SW4 160

SW4 161

SW4 162

SW4 163

SW4 164

SW4 165

SW4 $\quad 166$

SW4 167

SW4 168

SW4 169

SW4 170

SW4 171

SW4 $\quad 172$

SW4 173

SW4 $\quad 174$

SW4 175

SW4 176

SW4 177

SW4 178

SW4 179

SW4 180

SW4 181

SW4 182

SW4 $\quad 183$

SW4 184

SW4 185

SW4 186

SW4 187

SW4683 5

SW4683 6

SW4JN983 1

SW4683 8

SW4683 9

SW4683 10

SW4683 11

SW4683 12

SW4 188 


\begin{tabular}{rr}
63 & I \\
65 & I \\
73 & I \\
74 & I \\
80 & I \\
83 & I \\
87 & I \\
92 & I \\
93 & I \\
95 & I \\
110 & I \\
112 & I \\
115 & I \\
119 & I \\
126 & I \\
127 & I \\
132 & I \\
139 & I \\
140 & I \\
143 & I \\
146 & I \\
149 & I \\
152 & I \\
156 & I \\
165 & I \\
166 & I \\
173 & I \\
188 & I \\
189 & I \\
194 & I \\
197 & I \\
199 & I \\
202 & I \\
204 & I \\
210 & I \\
211 & I \\
215 & I \\
218 & I \\
220 & I \\
221 & I \\
223 & I \\
\hline
\end{tabular}

RETURN STATEMENT APPEARS IN MAIN PROGRAM. RETURN STATEMENT APPEARS IN MAIN PROGRAM. RETURN STATEMENT APPEARS IN MAIN PROGRAM. RETURN STATEMENT APPEARS IN MAIN PROGRAM. RETURN STATEMENT APPEARS IN MAIN PROGRAM. RETURN STATEMENT APPEARS IN MAIN PROGRAM. RETURN STATEMENT APPEARS IN MAIN PROGRAM. RETURN STATEMENT APPEARS IN MAIN PROGRAM. RETURN STATEMENT APPEARS IN MAIN PROGRAM. RETURN STATEMENT APPEARS IN MAIN PROGRAM. RETURN STATEMENT APPEARS IN MAIN PROGRAM. RETURN STATEMENT APPEARS IN MAIN PROGRAM. RETURN STATEMENT APPEARS IN MAIN PROGRAM. RETURN STATEMENT APPEARS IN MAIN PROGRAM. RETURN STATEMENT APPEARS IN MAIN PROGRAM. RETURN STATEMENT APPEARS IN MAIN PROGRAM. RETURN STATEMENT APPEARS IN MAIN PROGRAM. RETURN STATEMENT APPEARS IN MAIN PROGRAM. RETURN STATEMENT APPEARS IN MAIN PROGRAM. RETURN STATEMENT APPEARS IN MAIN PROGRAM. RETURN STATEMENT APPEARS IN MAIN PROGRAM. RETURN STATEMENT APPEARS IN MAIN PROGRAM. RETURN STATEMENT APPEARS IN MAIN PROGRAM. RETURN STATEMENT APPEARS IN MAIN PROGRAM. RETURN STATEMENT APPEARS IN MAIN PROGRAM. RETURN STATEMENT APPEARS IN MAIN PROGRAM. RETURN STATEMENT APPEARS IN MAIN PROGRAM. RETURN STATEMENT APPEARS IN MAIN PROGRAM. RETURN STATEMENT APPEARS IN MAIN PROGRAM. RETURN STATEMENT APPEARS IN MAIN PROGRAM. RETURN STATEMENT APPEARS IN MAIN PROGRAM. RETURN STATEMENT APPEARS IN MAIN PROGRAM. RETURN STATEMENT APPEARS IN MAIN PROGRAM. RETURN STATEMENT APPEARS IN MAIN PROGRAM. RETURN STATEMENT APPEARS IN MAIN PROGRAM. RETURN STATEMENT APPEARS IN MAIN PROGRAM. RETURN STATEMENT APPEARS IN MAIN PROGRAM. RETURN STATEMENT APPEARS IN MAIN PROGRAM. RETURN STATEMENT APPEARS IN MAIN PROGRAM. RETURN STATEMENT APPEARS IN MAIN PROGRAM. RETURN STATEMENT APPEARS IN MAIN PROGRAM. 
C PROCESSES DATA FOR CURRENT INSTR. IF IN MODE 1 OR 2.

DIMENSION INF (24)

SW7

SW7

COMMON/INSTR/OPNAME, NAME, OP, KA, KB, OBJA, OBJB, NXTM, TRA, LQ, NQ, LIN,

INTEGER OPNAME, OP, OBJA, OBJB, TRA, TYPE, ROWS, COLS, EXTR

LEVEL 2, STORE, INFF, IWORK

COMMON STORE(48000), IWORK(10)

COMMON/STORE/LMAX, LINF , LFILE, MAX, MIN, ICARD (11)

COMMON/CONTRL/ERROR, MODE, RSRV , STOR, XEQ, TRASW, NFSW, EMPTY, INDEF, 1 LDFLG, FIN

LOGICAL ERROR, RSRV, STOR, XEQ, TRASW, NFSW, EMPTY, INDEF, LDFLG, FIN

COMMON /DIM/ LEND, MEND, ISAV, ISAV7, M7END, KADD, KADDR, MUNIT , MSYMPL

COMMON/BMI 1/MI 1 ( 16000)

COMMON/NELS1/NELS1

LEVEL 2 , MI 1

C

COMMON/SWTCH/BEND, MSIZE, CYCSWT, VCSW, PV , MSSW, VPR, MHS, MODS, MINZER LOGICAL CYCSWT, MSSW, VCSW, PV, VPR, MHS, MODS
COMMON/GRR/IERR, BSW, VSW, RSW, QSW, XSW

LOGICAL IERR, BSW, VSW, RSW, QSW, XSW

DIMENSION PAR(100), KREM(100)

INTEGER R7, ROT , PRD, HSXTP, HMAP, PVECS

INTEGER BDAT

DIMENSION ZZ(1)

EQUIVALENCE(OPNAME, INF(1)), (PAR, KREM)

DIMENSION VO(7)

DATA R7, ROT , MAP, PRD/5,6,7,8/

DATA HSXTP, HMAP/4HSXTP, 3HMAP/

DATA RADEG/.0174532925/

DATA (VO(I), I=1,7)/7*0./

DATA IBLNK, MXPMY, PVECS, R7, ROT, PRD/1H , 1,2,5,6,8/

C

IF OPNAME = NULL, IT IS A P OR C CARD. NEEDS SPECIAL HANDLING TO CONSTRUCT A SYNCH PAGE OR REM INSTRUCTION

NOP $=O P-160$

$Z Z(1)=0$.

GO TO $(36,37,38,39,40,41)$ NOP

C
INSTR

INSTR 4

INSTR 5

INSTR 6

BLANK 2

BLANK 3

86MARSIZ 1

86MARSIZ 2

86MARSIZ 3

BLANK 5

STORE : 2

STORE 3

STORE 4

CONTRL 2

CONTRL 3

CONTRL 4

CONTRL 5

DIM 2

DIM 3

NV3BMI 1

BMI1L 2

BMI1L 3

BMI 1L 4

BMI 1

SWTCH 2

SWTCH 3

SWTCH 4

GRR 2

GRR 3

GRR - 4

SW7 16

SW7 17

SW7 18

86SW7 1

86SW7 2

SW7 19

SW7 20

SW7 21

SW7 22

SW7 23

SW7 24

SW7 25

SW7 26

SW7 27

SW7 28

SW7 29

SW7 30

86SW7 3

86SW7 4

$86 S W 7 \quad 5$ 
C

C TRKB ENTRY

36 IF (MODE.EQ.3) GO TO 360

CALL RESRV (M, MXPMY, MINZER,

CALL LOAD (M,

. $4,2,4,3,2,1,0,0)$

$\operatorname{CALL} \operatorname{STDAT}(M, 3,6,1, Z Z)$

C 6TH INTEGER IS A FLAG TO TELL TRKB IF IT HAS BEEN CALLED YET.

$865 W 7$

$\operatorname{CALL~REPINT}(M, 1,0)$

CALL REPINT $(M, 2, N E L)$

C

C TRKE ENTRY

.) 
NDAT $=\operatorname{INFF}(17, \mathrm{MBML})$

CALL MIFILL(MBML, 1, NDAT, NEL, MI 1)

IF (KA.NE.0.OR.KB.NE. . ) GO TO 391

$\mathrm{NQ3}=\mathrm{NCOL} *(\mathrm{NEL}+1)$

$86 S W 7$

$\mathrm{KB}=\mathrm{NEL}$

120

GO TO 392

86SW7

$865 W 7$

391 IF (KA.LE.-1) GO TO 393

$86 \mathrm{SW} 7$

NQ3 $=$ NCOL $*(K B-K A+1)$

392 LQ3 = LFILE + 1

LFILE $=\mathrm{LQ3}+\mathrm{NQ3}$

$865 W 7$

$865 W 7$

86 SW7

NTOT $=$ NTOT + NQ3

$865 W 7$

$\operatorname{INFF}(5, M)=N E L$

$\operatorname{INFF}(18, M)=$ NTOT

$86 S W 7$

$865 W 7$

$\operatorname{INFF}(24, M)=L Q 3$

CALL REPINT $(M, 1,0)$

CALL REPINT $(M, 2, N E L)$

394 IF (MODE.EQ.2) RETURN

IF (XSW) RETURN

390 CALL TRKE(M)

135 RETURN

C

C TRKM ENTRY

40 IF (MODE. EQ.3) GO TO 400

86SW7 83

865W7 84

$86 S W 7 \quad 85$

CALL RESRV (M, MXPMY, MINZER,

.)

CALL $\operatorname{LOAD}(M$,

- $4,2,4,3,2,1,0,0)$

86SW7 90

CALL STDAT $(M, 3,6,1, Z Z)$

145

150

155

160

165
C 6TH INTEGER IS A FLAG TO TELL TRKM IF IT HAS BEEN CALLED YET.

$\operatorname{IP1}=\operatorname{IDAT}(M, 1)$

$\operatorname{IP2}=\operatorname{IDAT}(M, 2)$

NAMVEC $=\operatorname{BDAT}(M, 3)$

NAMOP $=\operatorname{INFF}(1, M)$

$\mathrm{NCOL}=20$

IF (NAMVEC.EQ. $4 \mathrm{H} \quad$ ) NCOL $=14$

$\operatorname{CALL} \operatorname{STDAT}(M, 3,5,1, \mathrm{NCOL})$

$\operatorname{MBML}=\operatorname{MDAT}(M, 1)$

NDAT $=\operatorname{INFF}(17, \mathrm{MBML})$

CALL MIFILL(MBML, 1 , NDAT, NEL, MI 1)

IF (KA.NE.0.OR.KB.NE.0) GO TO 401

$\mathrm{NQ3}=\mathrm{NCOL} *(\mathrm{NEL}+1)$

$K B=N E L$

GO TO 402

401 IF (KA.LE. -1) GO TO 403

$\mathrm{NQ3}=\mathrm{NCOL} *(K B-K A+1)$

402

$L Q 3=L F I L E+1$

$L F I L E=L Q 3+N Q 3$

NTOT $=$ NTOT + NQ3

$\operatorname{INFF}(5, M)=N E L$

$\operatorname{INFF}(18, M)=$ NTOT

$\operatorname{INFF}(24, M)=\mathrm{LQ3}$

403 IF (IP1.NE.0.OR. IP2.NE.0) GO TO 404

CALL REPINT $(M, 1,0)$

$\operatorname{CALL}$ REPINT $(M, 2, N E L)$

404 IF (MODE.EQ. 2) RETURN
86SW7 91

86SW7 92

$865 W 7 \quad 93$

86SW7 94

86SW7 95

86SW7 96

86SW7 97

86SW7 98

86SW7 99

86SW7 100

86SW7 101

86SW7 102

86SW7 103

86SW7 104

86SW7 105

86SW7 106

86SW7 107

86SW7 108

86SW7 109

865W7 110

86SW7 $\quad 111$

86SW7 112

86SW7 113

86SW7 114

86SW7 115

86SW7 116

86SW7 117

86SW7 118

86SW7 119 
180

185

190

195

200

205

210

215

220

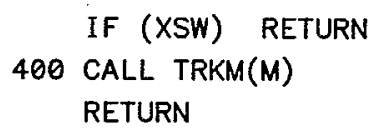

\begin{tabular}{|c|c|}
\hline $865 W 7$ & $12 e$ \\
\hline $86 \mathrm{SW7}$ & 12 \\
\hline 86SW7 & 12 \\
\hline 86 SW7 & 12 \\
\hline 86 SW7 & 12 \\
\hline $865 W 7$ & 12 \\
\hline $865 W 7$ & 126 \\
\hline 86 SW7 & 127 \\
\hline 86SW7 & 128 \\
\hline SW7 & 7 \\
\hline SW7 & 72 \\
\hline SW7 & 73 \\
\hline SW7 & 74 \\
\hline SW7 & 75 \\
\hline SW7 & $7 \epsilon$ \\
\hline SW7 & 77 \\
\hline SW7 & 78 \\
\hline SW7 & 79 \\
\hline SW7 & 80 \\
\hline SW7 & 81 \\
\hline SW7 & 82 \\
\hline SW7 & 83 \\
\hline SW7 & 84 \\
\hline SW7 & 85 \\
\hline SW7 & 86 \\
\hline SW7 & 87 \\
\hline SW7 & 88 \\
\hline SW7 & 89 \\
\hline SW7 & 90 \\
\hline SW7 & 91 \\
\hline SW7 & 92 \\
\hline SW7 & 93 \\
\hline SW7 & 94 \\
\hline SW7 & 95 \\
\hline SW7 & 96 \\
\hline SW7 & 97 \\
\hline SW7 & 98 \\
\hline SW7 & 99 \\
\hline SW7 & 100 \\
\hline SW7 & 101 \\
\hline SW7 & 102 \\
\hline SW7 & 103 \\
\hline SW7 & 104 \\
\hline SW7 & 105 \\
\hline SW7 & 106 \\
\hline SW7 & 107 \\
\hline sw7 & 108 \\
\hline SW7 & 109 \\
\hline SW7 & 110 \\
\hline SW7 & 111 \\
\hline SW7 & 112 \\
\hline SW7 & 113 \\
\hline
\end{tabular}


CARD NR. SEVERITY DETAILS DIAGNOSIS OF PROBLEM

$\begin{array}{rll}93 & \text { I } & \text { RETURN STATEMENT APPEARS IN MAIN PROGRAM. } \\ 94 & \text { I } & \text { RETURN STATEMENT APPEARS IN MAIN PROGRAM. } \\ 96 & \text { I } & \text { RETURN STATEMENT APPEARS IN MAIN PROGRAM. } \\ 132 & \text { I } \\ 133 & \text { I } & \text { RETURN STATEMENT APPEARS IN MAIN PROGRAM. } \\ 135 & \text { I } \\ 171 & \text { I } & \text { RETURN STATEMENT APPEARS IN MAIN PROGRAM. } \\ 172 & \text { I } & \text { RETURN STATEMENT APPEARS IN MAIN PROGRAM. } \\ 174 & \text { I } & \text { RETURN STATEMENT APPEARS IN MAIN PROGRAM. } \\ 177 & \text { I } & \text { RETURN STATEMENT APPEARS IN MAIN PROGRAM. } \\ 179 & \text { I } & \text { RETURN STATEMENT APPEARS IN MAIN PROGRAM. } \\ 203 & \text { I } & \text { RETURN STATEMENT APPEARS IN MAIN PROGRAM. } \\ 204 & \text { I } & \text { RETURN STATEMENT APPEARS IN MAIN PROGRAM. } \\ 206 & \text { I } & \text { RETURN STATEMENT APPEARS IN MAIN PROGRAM. } \\ 220 & \text { I } & \text { RETURN STATEMENT APPEARS IN MAIN PROGRAM. } \\ 222 & \text { I } & \text { RETURN STATEMENT APPEARS IN MAIN PROGRAM. } \\ & \text { RETURN STATEMENT APPEARS IN MAIN PROGRAM. }\end{array}$


C PROCESSES DATA FOR CURRENT INSTR. IF IN MODE 1 OR 2.

LEVEL 2, STORE, INFF, IWORK

COMMON STORE (48000), IWORK(10)

COMMON/SWTCH/BEND, MSIZE, CYCSWT , VCSW, PV, MSSW, VPR, MHS, MODS, MINZER LOGICAL CYCSWT, MSSW, VCSW, PV, VPR, MHS, MODS

COMMON/GRR/IERR, BSW, VSW, RSW, QSW, XSW

LOGICAL IERR, BSW, VSW, RSW, QSW, XSW

INTEGER BDAT, PVECS

DIMENSION T(49), RW(3)

DIMENSION PAR(100), KREM(100)

INTEGER R7

EQUIVALENCE (OPNAME, INF(1)), (PAR, KREM)

DATA PVECS, MAT, R $7 / 2,3,5 /$

DATA ISCL/9/

C
IF OPNAME $=$ NULL, IT IS A P OR C CARD. NEEDS SPECIAL HANDLING TO CONSTRUCT A SYNCH PAGE OR REM INSTRUCTION

IF (OP.EQ.123) GO TO 123

NOP $=$ OP -99

IF(OP.GT.111) GO TO 75

GO TO $(40,43,42,64,65,71,66,68,67,62,72,74)$ NOP

GO TO PBML, MAT, VEC, PVEC, MXV, CPLX, EQIL, EVEC, PRTV, PRV7, CVEC , LIST, SIN, COS, EXP, ALOG, SQ, SQRT, TAN, ASIN, ACOS, ATAN, ABS , CALC
INSTR

INSTR

INSTR

INSTR

BLANK

BLANK

86MARSIZ

86MARSIZ

86MARSIZ

BLANK

STORE

STORE

STORE

CONTRL

CONTRL

CONTRL

CONTRL

DIM

DIM

BMIL

BMIL

BMIL

BMIL

BMI

SWTCH

SWTCH

SWTCH

GRR

GRR

GRR

SW5

SW5

SW5

SW5

SW5

SW5

SW5

SW5

SW5

SW5

SW5

SW5

SW5

MY3SW5

SW5

MY3SW5

MY3SW5

SW5

SW5

SW5 
.)

IF (XSW) RETURN

680 CALL EVEC(M) RETURN 
$\mathrm{KN}=\mathrm{ISCL}$

CALL INP $(M, K N, \theta$,

C CVEC ENTRY -COMPLEX PARTICLE VECTOR, KA, IN NUMBER

C IF $K B=B L A N K$ OR 1 , ROW 7 IS 1 . IF $K B=0$, ROW7 IS 0 .

72 IF (MODE.EQ.3) RETURN

$\mathrm{VTR}=0$.

SW5

SW5

SW5

$V 7 I=0$.

IF (KB.EQ.1.OR.KB.EQ.1H) $V 7 R=1$.

SW5

IF (KA.EQ.0) $K A=1$

$\mathrm{KC}=14 * \mathrm{KA}$

ROWS $=7$

COLS $=\mathrm{KA}$

190

TYPE $=4 H C P L X$

CALL RESRV $(M$, PVECS, $\theta$,

- KC, $1,0,0,0,0,0,0$

.)

DO $721 \mathrm{I}=1, \mathrm{KA}$

195

CALL LOAD $(M$,

$$
\text { - } 12,1,0,0,0,0,0,0
$$

.)

$$
\mathrm{NF}=\mathrm{NF}+2
$$$$
N P L=14 *(I-1)+13
$$

200

CALL REPFLT(M,NPL, V7R)

$N P L=N P L+1$

CALL REPFLT (M,NPL,V7I)

SW5

SW5

SW5

SW5

SW5

SW5

SW5

SW5

SW5

SW5

SW5

SW5

SW5

SW5

SW5

SW5

SW5

SW5

721 CONTINUE

RETURN

205

\section{LIST ENTRY}

SW5

SW5

SW5

SW5

SW5

SW5

SW5

THE LIST INSTRUCTION SPECIFIES A SET OF ELEMENTS THAT ARE TO BE

$\begin{array}{lllll}C & L & \text { DRF } & 3 . & \\ C & . S & B M L & 5(Q \quad L \quad)\end{array}$

SW5

74 IF (MODE.EQ.3) GO TO 741

$\operatorname{CALL} \operatorname{INP}(M, 0,0,1,-2,0,0,0,0,0,0)$

741 IF (KB.NE.0) RETURN

$$
K B=1
$$$$
\operatorname{INFF}(5, M)=1
$$ 
230

235

245

250

255

265

260
C SCALAR FUNCTIONS

75 IF (MODE.EQ.3) GO TO 750

$\mathrm{KN}=\mathrm{ISCL}$

$\operatorname{CALL} \operatorname{INP}(M, K N, 1,1,1,0,0,0,0,0,0)$

IF (MODE.EQ.2.OR.XSW) RETURN

$750 X X=\operatorname{FDAT}(M, 1)$

NNOP $=$ OP -111

GO TO $(76,77,78,79,80,81,82,83,84,85,86)$ NNOP

$76 \quad Y Y=\operatorname{SIN}(X X)$

GO TO 99

$77 \quad Y Y=\cos (X X)$

GO TO 99

$78 \quad Y Y=\operatorname{EXP}(X X)$

GO TO 99

$79 \quad Y Y=A L O G(X X)$

GO TO 99

$80 Y Y=X X * X X$

GO TO 99

$81 Y Y=\operatorname{SQRT}(X X)$

GO TO 99

$82 \quad Y Y=\operatorname{TAN}(X X)$

GO TO 99

$83 Y Y=\operatorname{ASIN}(X X)$

GO TO 99

$84 \quad Y Y=A C O S(X X)$

$85 Y Y=\operatorname{ATAN}(X X)$

GO TO 99

$86 \quad Y Y=A B S(X X)$

$99 \operatorname{CALL} \operatorname{STDAT}(M, 5,1,1, Y Y)$

RETURN

C CALC ENTRY

123 IF (MODE.EQ.3) GO TO 1231

CALL INP $(M, 0,5,1,-2,0)$

IF (MODE.EQ.2.OR.XSW) RETURN

1231 CALL CALC $(M)$

RETURN

END
GO TO 99

\begin{tabular}{|c|c|}
\hline SW5 & 19 \\
\hline SW5 & \\
\hline SW5 & \\
\hline SW5 & \\
\hline SW5 & \\
\hline SW5 & 20 \\
\hline SW5 & $2 e$ \\
\hline SW5 & 20 \\
\hline SW5 & 20 \\
\hline SW5 & 20 \\
\hline SW5 & 20 \\
\hline SW5 & 21 \\
\hline SW5 & 21 \\
\hline SW5 & 21 \\
\hline SW5 & 21 \\
\hline SW5 & 21 \\
\hline SW5 & 21 \\
\hline SW5 & 21 \\
\hline SW5 & 21 \\
\hline SW5 & 218 \\
\hline SW5 & 21 \\
\hline SW5 & 22 \\
\hline SW5 & 22 \\
\hline SW5 & 222 \\
\hline SW5 & 223 \\
\hline SW5 & 22 \\
\hline SW5 & 225 \\
\hline SW5 & 226 \\
\hline SW5 & 227 \\
\hline SW5 & 228 \\
\hline SW5 & 229 \\
\hline SW5 & 230 \\
\hline SW5 & 231 \\
\hline SW5 & 232 \\
\hline SW5 & 233 \\
\hline SW5 & 234 \\
\hline SW5 & 235 \\
\hline SW5 & 236 \\
\hline SW5 & 23 \\
\hline
\end{tabular}

CARD NR. SEVERITY DETAILS . DIAGNOSIS OF PROBLEM

RETURN STATEMENT APPEARS IN MAIN PROGRAM. RETURN STATEMENT APPEARS IN MAIN PROGRAM. RETURN STATEMENT APPEARS IN MAIN PROGRAM. RETURN STATEMENT APPEARS IN MAIN PROGRAM. RETURN STATEMENT APPEARS IN MAIN PROGRAM. RETURN STATEMENT APPEARS IN MAIN PROGRAM. RETURN STATEMENT APPEARS IN MAIN PROGRAM. RETURN STATEMENT APPEARS IN MAIN PROGRAM. RETURN STATEMENT APPEARS IN MAIN PROGRAM. RETURN STATEMENT APPEARS IN MAIN PROGRAM. RETURN STATEMENT APPEARS IN MAIN PROGRAM. RETURN STATEMENT APPEARS IN MAIN PROGRAM. 
CARD NR. SEVERITY

127 I

141 I

149 I

150 I

152 I

158 I

159 I

161 I

167 I

168 I

170 I

173 I

179 I

182 I

204 I

224 I

227 I

233 I

259 I

263 I

264 I

266 I
DIAGNOSIS OF PROBLEM

RETURN STATEMENT APPEARS IN MAIN PROGRAM.

RETURN STATEMENT APPEARS IN MAIN PROGRAM.

RETURN STATEMENT APPEARS IN MAIN PROGRAM.

RETURN STATEMENT APPEARS IN MAIN PROGRAM.

RETURN STATEMENT APPEARS IN MAIN PROGRAM.

RETURN STATEMENT APPEARS IN MAIN PROGRAM.

RETURN STATEMENT APPEARS IN MAIN PROGRAM.

RETURN STATEMENT APPEARS IN MAIN PROGRAM.

RETURN STATEMENT APPEARS IN MAIN PROGRAM.

RETURN STATEMENT APPEARS IN MAIN PROGRAM.

RETURN STATEMENT APPEARS IN MAIN PROGRAM.

RETURN STATEMENT APPEARS IN MAIN PROGRAM.

RETURN STATEMENT APPEARS IN MAIN PROGRAM.

RETURN STATEMENT APPEARS IN MAIN PROGRAM.

RETURN STATEMENT APPEARS IN MAIN PROGRAM.

RETURN STATEMENT APPEARS IN MAIN PROGRAM.

RETURN STATEMENT APPEARS IN MAIN PROGRAM.

RETURN STATEMENT APPEARS IN MAIN PROGRAM.

RETURN STATEMENT APPEARS IN MAIN PROGRAM.

ARGUMENT COUNT INCONSISTENT WITH PRIOR USAGE.

RETURN STATEMENT APPEARS IN MAIN PROGRAM.

RETURN STATEMENT APPEARS IN MAIN PROGRAM. 
5
20

5

45

5

5
OVERLAY (SYNCH, 6,0)

PROGRAM SW6

C PROCESSES DATA FOR CURRENT INSTR. IF IN MODE 1 OR 2.

C PROCEEDS WITH CALC.BY CALLING APPROPRIATE ROUTINES IF MODE=1 OR 3 .

DIMENSION INF (24)

COMMON/INSTR/OPNAME, NAME, OP, KA, KB, OBJA, OBJB, NXTM, TRA, LQ, NQ, LIN, 1

2 NIN, LFL, NFL, LBC , NBC, NTOT , IX, KIND, TYPE, ROWS , COLS, EXTR,

INTEGER OPNAME, OP, OBJA, OBJB, TRA, TYPE, ROWS, COLS, EXTR

$M, N F, N B, N I, M S U B R, I S I G N$

LEVEL 2, STORE, INFF, IWORK

COMMON STORE (48000), IWORK (10)

DIMENSION INFF $(24,2000)$

EQUIVALENCE (INFF, STORE)

COMMON/STORE/LMAX, LINF, LF ILE, MAX, MIN, ICARD (11)

* LmaX AND maX aRE dimensions of STORE aND INFF

COMMON/CONTRL/ERROR, MODE, RSRV, STOR, XEQ, TRASW, NFSW, EMPTY, INDEF, 1

LDFLG, FIN

LOGICAL ERROR, RSRV, STOR, XEQ, TRASW, NFSW, EMPTY, INDEF , LDFLG , FIN

COMMON /DIM/ LEND, MEND, ISAV, ISAV7, M7END, KADD, KADDR, MUNIT, MSYMPL

COMMON/BMI/MI (5000)

COMMON/NELS/NELS

LEVEL 2,MI

COMMON/SWTCH/BEND, MSIZE, CYCSWT , VCSW, PV, MSSW, VPR, MHS, MODS, MINZER LOGICAL CYCSWT, MSSW, VCSW, PV, VPR, MHS, MODS

COMMON/GRR/IERR, BSW, VSW, RSW, QSW, XSW

LOGICAL IERR, BSW, VSW, RSW, QSW, XSW

EQUIVALENCE(OPNAME, INF(1))

* IF OPNAME $=$ NULL, IT IS A P OR C CARD. NEEDS SPECIAL

* handling to construct a synch page or rem instruction

$\mathrm{NOP}=\mathrm{OP}-150$

GO TO $(76,77,78,79,83,84,85)$ NOP

C GO TO BEAM, DKE, EDRF, SOL, CYEM, BVAL, CYAE

C BEAM ENTRY. DEFINE A BEAM PARTICLE

76 CONTINUE

IF (MODE.EQ.3) GO TO 761

CALL RESRV $(M, 0,10$,

- $8,1,1,3,0,0,0,0$

.)

C LOAD 4 COMPONENTS OF VECTOR FROM CARD 1

CALL $\operatorname{LOAD}(M$,

- $4,1,0,0,0,0,0,0$
SW6 2

SW6

SW6

SW6

SW6

SW6

INSTR

INSTR

INSTR

INSTR

INSTR

BLANK

BLANK

86MARSIZ

86MARSIZ

86MARSIZ

BLANK

STORE

STORE

STORE

CONTRL

CONTRL

CONTRL

CONTRL

DIM

DIM

BMI L

BMI L

BMIL

BMIL

BMI

SWTCH

SWTCH

SWTCH

GRR

GRR

GRR

SW6

SW6

SW6

SW6

SW6

SW6

SW6

SW6

SW6

SW6

SW6

SW6

SW6

SWG

SW6

SW6

SW6

SW6
4

5

6

7

2

3

4

5

6

2

3

1

2

3

5

2

3

4

2

3

4

5

2

3

1

2

3 
60

65

70

75

80

85

95

100

105

110

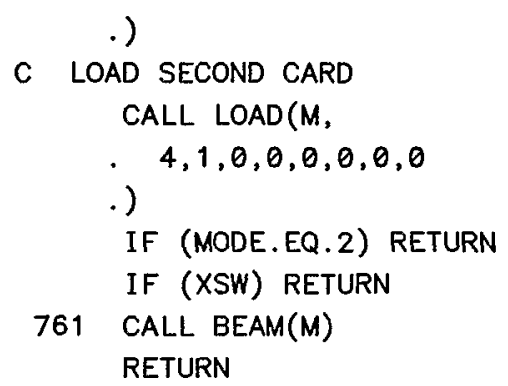




$\begin{array}{rr}63 & \text { I } \\ 64 & \text { I } \\ 66 & \text { I } \\ 69 & \text { I } \\ 73 & \text { I } \\ 76 & \text { I } \\ 80 & \text { I } \\ 83 & \text { I } \\ 87 & \text { I } \\ 95 & \text { I } \\ 96 & \text { I } \\ 98 & \text { I } \\ 102 & \text { I } \\ 103 & \text { I } \\ 105 & \text { I } \\ 112 & \text { I } \\ 113 & \text { I } \\ 115 & \text { I }\end{array}$

RETURN STATEMENT APPEARS IN MAIN PROGRAM. RETURN STATEMENT APPEARS IN MAIN PROGRAM. RETURN STATEMENT APPEARS IN MAIN PROGRAM. RETURN STATEMENT APPEARS IN MAIN PROGRAM. RETURN STATEMENT APPEARS IN MAIN PROGRAM. RETURN STATEMENT APPEARS IN MAIN PROGRAM. RETURN STATEMENT APPEARS IN MAIN PROGRAM. RETURN STATEMENT APPEARS IN MAIN PROGRAM. RETURN STATEMENT APPEARS IN MAIN PROGRAM. RETURN STATEMENT APPEARS IN MAIN PROGRAM. RETURN STATEMENT APPEARS IN MAIN PROGRAM. RETURN STATEMENT APPEARS IN MAIN PROGRAM. RETURN STATEMENT APPEARS IN MAIN PROGRAM. RETURN STATEMENT APPEARS IN MAIN PROGRAM. RETURN STATEMENT APPEARS IN MAIN PROGRAM. RETURN STATEMENT APPEARS IN MAIN PROGRAM. RETURN STATEMENT APPEARS IN MAIN PROGRAM. RETURN STATEMENT APPEARS IN MAIN PROGRAM. 
UNLABELED OLDPL

MODIFICATIONS / CONTROL CARDS

UPDATE $1.4-587$.

PAGE 1

*** YANK, SELYANK, OR YANKDECK IDENT MVR NOT KNOWN ***

*** YANK, SELYANK, OR YANKDECK IDENT EDC77 NOT KNOWN ***

*** YANK, SELYANK, OR YANKDECK IDENT EDC77 NOT KNOWN *** 


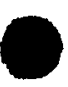

0 
SUBROUTINE AFD $(M, J, D E L T A)$

AFD

AFD

C INCREMENTS BY DELTA THE J-TH FL PT PARAMETER OF ELEMENT M AND

AFD

$F=F D A T(M, J)$

5

$F=F+D E L T A$

CALL REPFLT $(M, J, F)$

RETURN

END 
1

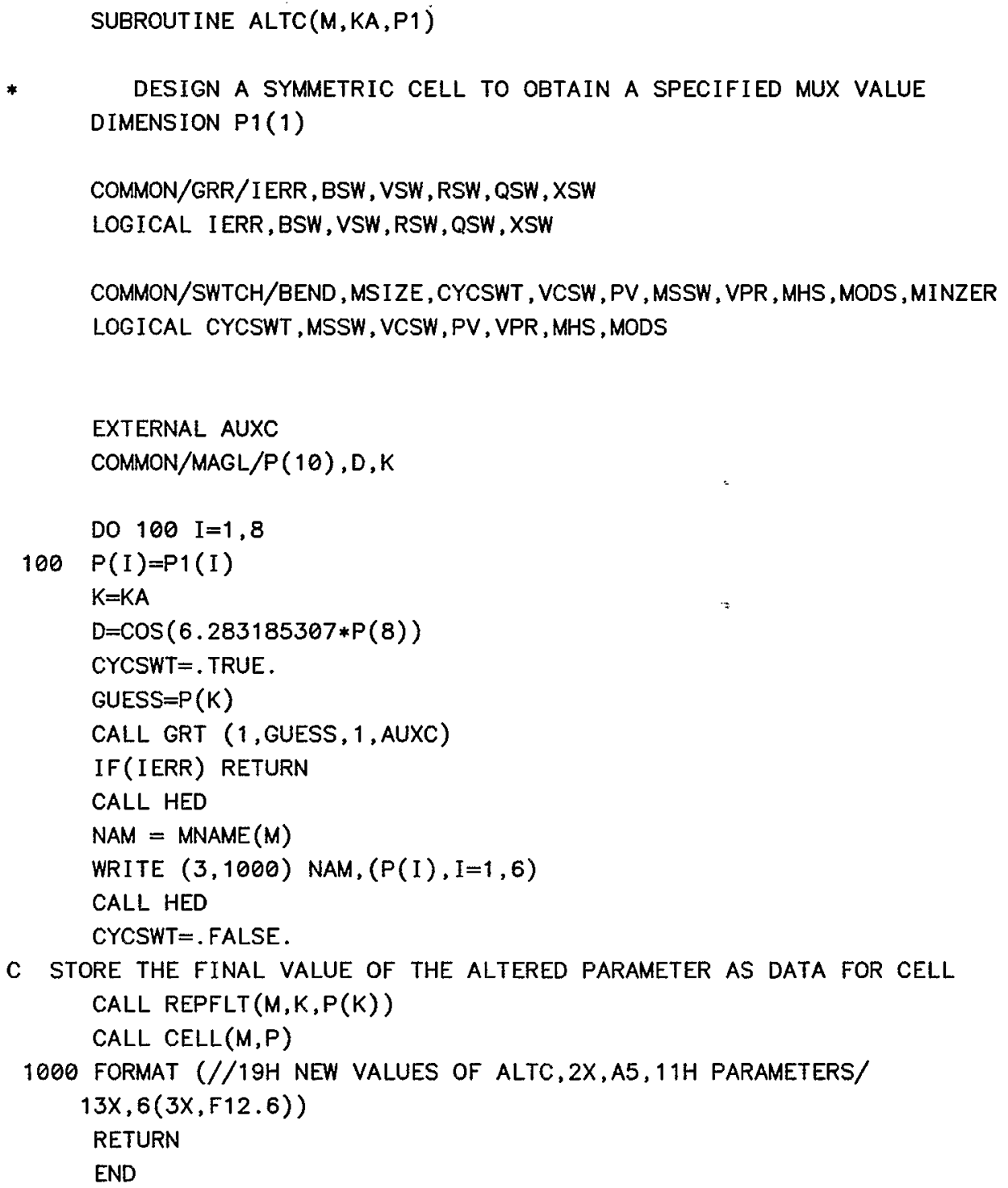

5

$\begin{array}{lr}\text { ALTC } & 2 \\ \text { ALTC } & 3 \\ \text { ALTC } & 4 \\ \text { ALTC } & 5 \\ \text { ALTC } & 6 \\ \text { GRR } & 2 \\ \text { GRR } & 3 \\ \text { GRR } & 4 \\ \text { SWTCH } & 2 \\ \text { SWTCH } & 3 \\ \text { SWTCH } & 4 \\ \text { ALTC } & 9 \\ \text { ALTC } & 10 \\ \text { ALTC } & 11 \\ \text { ALTC } & 12 \\ \text { ALTC } & 13 \\ \text { ALTC } & 14 \\ \text { ALTC } & 15 \\ \text { ALTC } & 16 \\ \text { ALTC } & 17 \\ \text { ALTC } & 18 \\ \text { ALTC } & 19 \\ \text { ALTC } & 20 \\ \text { ALTC } & 21 \\ \text { ALTC } & 22 \\ \text { ALTC } & 23 \\ \text { ALTC } & 24 \\ \text { ALTC } & 25 \\ \text { ALTC } & 26 \\ \text { ALTC } & 27 \\ \text { ALTC } & 28 \\ \text { ALTC } & 29 \\ \text { ALTC } & 30 \\ \text { ALTC } & 31 \\ \text { ALTC } & 32 \\ & \end{array}$


SUBROUTINE ARGINP(ND, I, NSETS, $N 1, I 1, N 2, I 2, N 3, I 3, N 4, I 4$

.)

DIMENSION ND(10), I (10)

5

NSETS $=0$

IF (N1.EQ.0) GO TO 8

NSETS $=1$

$\mathrm{ND}(1)=\mathrm{N} 1$

$I(1)=I 1$

10

IF (N2.EQ.0) GO TO 8

NSETS $=2$

$\mathrm{ND}(2)=\mathrm{N} 2$

$I(2)=I 2$

IF (N3.EQ.0) GO TO 8

15

NSETS $=3$

$\mathrm{ND}(3)=\mathrm{N} 3$

$I(3)=I 3$

IF (N4.EQ.O) GO TO 8

NSETS $=4$

20

$\begin{array}{lr}\text { ARGINP } & 2 \\ \text { ARGINP } & 3 \\ \text { ARGINP } & 4 \\ \text { ARGINP } & 5 \\ \text { ARGINP } & 6 \\ \text { ARG INP } & 7 \\ \text { ARGINP } & 8 \\ \text { ARGINP } & 9 \\ \text { ARGINP } & 10 \\ \text { ARGINP } & 11 \\ \text { ARGINP } & 12 \\ \text { ARGINP } & 13 \\ \text { ARGINP } & 14 \\ \text { ARG INP. } & 15 \\ \text { ARGINP } & 16 \\ \text { ARGINP } & 17 \\ \text { ARGINP } & 18 \\ \text { ARGINP } & 19 \\ \text { ARGINP } & 20 \\ \text { ARGINP } & 21 \\ \text { ARGINP } & 22 \\ \text { ARGINP } & 23 \\ \text { ARGINP } & 24\end{array}$


LEVEL 2, STORE, INFF, IWORK COMMON STORE(48000), IWORK(10)

DIMENSION INFF $(24,2000)$

EQUIVALENCE (INFF, STORE)

DIMENSION T(49), T1 (49)

AROP 4

AROP 5

AROP 6

AROPLL 1

AROPLL 2

AROPLL 3

DOUBLE DA, DB, DC 
60

8 IF (.NOT.MINUS) GO TO 9

AROP

51

$\mathrm{DB}=-\mathrm{DB}$

9 IF (MINUS.OR.PLUS) $D C=D A+D B$

AROP

52

IF (MULT) $D C=D A * D B$

AROP $\quad 53$

IF (DIV) DC $=D A / D B$

AROP

54

GO TO 11

C OPERATE ON MATRICES. CONSIDER ONLY,,$+- *$ FOR PRESENT

AROP

AROP

55

$10 \quad M A=\operatorname{ELNUM}(\operatorname{INAM}(1))$

$M B=\operatorname{ELNUM}(\operatorname{INAM}(2))$

AROP

AROP

AROP

IF (.NOT.MULT) GO TO 15

AROP

$M I(1)=M A$

AROP

$M I(2)=M B$

70

$\operatorname{CALL} \operatorname{MMM}(M, 2, M I)$

AROP

AROP

RETURN

15 IF (KN.NE.3HMXY) GO TO 30

CALL RXY(MA, RX, RY, RW)

CALL RXY(MB, RX1,RY1,RW1)

AROP

AROP

AROP

AROP

AROP

IF (.NOT.MINUS) GO TO 20

AROP

DO $17 \mathrm{~J}=1,3$

DO $16 \quad \mathrm{I}=1,2$

$R \times 1(I, J)=-R \times 1(I, J)$

AROP

AROP

$R Y 1(I, J)=-R Y 1(I, J)$

AROP

AROP

AROP

$R W_{1}(\mathrm{~J})=-R W_{1}(\mathrm{~J})$

17 CONTINUE

20 DO $25 \mathrm{~J}=1,3$

DO $22 I=1,2$

AROP

AROP

AROP

AROP

AROP

AROP

AROP

AROP

AROP

AROP

AROP

AROP

AROP

AROP

AROP

AROP

56

57

58

59

60

61

62

63

64

65

66

67

68

69

70

71

72

73

74

75

76

77

$R Y(I, J)=\operatorname{RY}(I, J)+\operatorname{RY} 1(I, J)$

78

79

$R W(J)=R W(J)+R W 1(J)$

CALL STXY (M,RX,RY, RW)

CALL RTRV7 (MA,T,RW)

CALL RTRV7(MB,T1,RW1)

95

DO $31 \mathrm{I}=1,49$

$31 \quad \mathrm{~T}_{1}(\mathrm{I})=-\mathrm{T}_{1}(\mathrm{I})$

DO $32 \mathrm{I}=1,3$

$32 \mathrm{RW1}$ (I) $=-\mathrm{RW} 1$ (I)

35 DO $37 \quad I=1,49$

AROP

AROP

AROP

AROP

AROP

AROP

AROP

105

$\mathrm{T}(\mathrm{I})=\mathrm{T}(\mathrm{I})+\mathrm{T} 1$ (I)

DO $38 \quad I=1,3$

$38 \quad R W(I)=R W(I)+R W 1(I)$

CALL STOR7 (M,T,RW)

RETURN

AROP

END 
COMMON/INSTR/OPNAME, NAME, OP, KA, KB, OBJA, OBJB, NXTM, TRA, LQ, NQ, LIN,

AUXC

INSTR 1

NIN, LFL, NFL, LBC, NBC , NTOT, IX, KIND, TYPE, ROWS, COLS, EXTR, INSTR

INSTR

INSTR

INSTR

AUXC

AUXC

AUXC

AUXC

AUXC

AUXC

AUXC

AUXC

AUXC

AUXC

AUXC
15

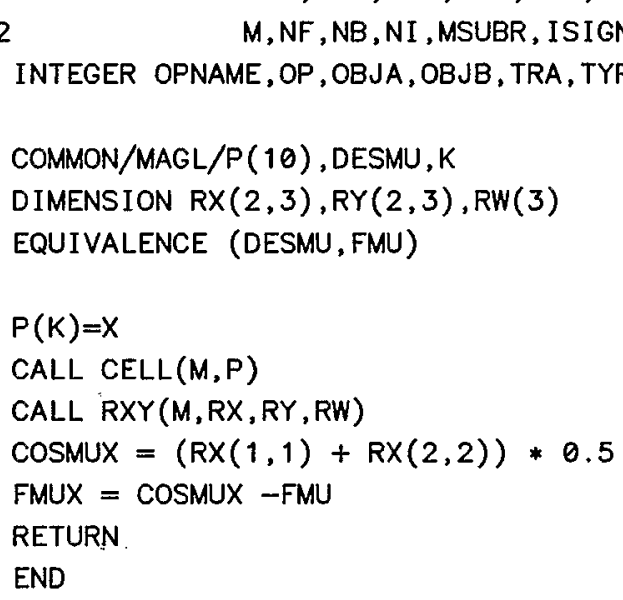

COMMON/MAGL/P (10), DESMU, K

END

$\operatorname{CosMUX}=(R X(1,1)+R X(2,2)) * 0.5$

FMUX $=$ COSMUX -FMU

RETURN

$P(K)=X$

$M, N F, N B, N I, M S$
$(10)$, DESMU, $K$
$(2,3), \operatorname{RY}(2,3)$

EQUIVALENCE (DESMU, FMU)

CALL CELL $(M, P)$ 
END

AUXD 
INTEGER FUNCTION BDAT $(M, J)$

BDAT

BDAT

C RETRIEVES ONE PIECE OF BCD DATA FROM INNDEX $J$ OF ELEMENT $J$

BLANK

LEVEL 2, STORE, INFF, IWORK

BLANK

COMMON STORE (48000), IWORK (10)

86MARSIZ

DIMENSION INFF $(24,2000)$

86MARSIZ

EQUIVALENCE (INFF, STORE)

86MARSIZ

BLANK

COMMON/CONTRL/ERROR, MODE, RSRV, STOR, XEQ, TRASW, NFSW, EMPTY, INDEF,

LOGICAL ERROR, RSRV, STOR, XEQ, TRASW, NFSW, EMPTY, INDEF, LDFLG, FIN

CONTRL

CONTRL

CONTRL

CONTRL

EQUIVALENCE(IDATA, FDATA)

BDAT

BDAT

$L O C=\operatorname{INFF}(16, M)+J-1$

BDAT

IF (LOC.GT.O) GO TO 1

BDAT

ERROR $=$. TRUE.

BDAT

BDAT

BDAT

BDAT

BDAT

BDAT

2

RETURN

FDATA = STORE (LOC)

BDAT = IDATA

RETURN 
C $3 \times 3$ MATRICES FOR RECTANGULAR, ZERO-GRADIENT MAGNET, MODIFIED TO ACT

C ON OFF-MOMENTUM ORBITS. TRANSFORMATION IS RELATIVE TO A RAY $V$,

C GIVEN IN THE ORIGINAL COORDINATES, WHICH WILL BE TRACKED THROUGH

C THE MAGNET. THE RESULTING MATRIX IS TO BE USED BY CYX TO FIND

C BETATRON FUNCTIONS RELATIVE TO A NEW CLOSED ORBIT.

C IF IOP $=1$, ACTS LIKE A MAG FOR THE SAME PARAMETERS.

$$
\text { c. }
$$$$
\text { C B BEND }
$$$$
\text { L }
$$$$
0 \text {. }
$$$$
\text { BRHO }
$$$$
\mathrm{BZ}
$$

BEND

BEND

C....


60

$$
\begin{aligned}
& \text { ARG3 }=\text { PIO2 - GAMO } \\
& R(4)=\text { RHO *CEXP }(X I * A R G 3)
\end{aligned}
$$

BEND

BEND

BEND

$Z=R(1)+R(2)+R(3)+R(4)$

BEND

$H=\operatorname{AIMAG}(Z)$

BEND

61

COSPHGM $=\operatorname{COS}($ PHIO-GAMO $)$

BEND

62

D4 $=\mathrm{H} *$ SINPHIO / COSPHGM

BEND

63

65

$X \theta F=H * \cos (G A M \theta) / \operatorname{COSPHGM}$

BEND

64

$A L O F=A L O+2 . * P H I O-$ THETA

BEND

65

$S=$ RHO * THETA

BEND

66

BEND

67

$\operatorname{TAR}(1)=S$

BEND

68

70

$\operatorname{TAR}(2)=0$.

BEND

69

$\operatorname{TAR}(3)=B R$

BEND

70

$\operatorname{TAR}(4)=B 0$

BEND

71

$\operatorname{TAR}(5)=A R G 1 *$ DEGRAD

BEND

BEND

72

$\operatorname{TAR}(6)=$ GAMO * DEGRAD

BEND

73

75

DO $1 \mathrm{I}=1,3$

BEND

74

75

BEND

76

$M L(I)=$ MEND - $2-I$

CALL DRIFT(MEND-3,D1)

CALL MAGNET(MEND-4, TAR)

80

CALL DRIFT(MEND-5, D4)

BENDLL 2

BENDLL 3

BENDLL 4

BENDLL 5

$\operatorname{CALL} \operatorname{MMM}(M, 3, M L)$

BEND

BEND

CALL RTRV7 ( $M, T, R W)$

BEND

85

CALL $M X V 7(T, V, V)$

BEND

$V(1)=X \otimes F$

BEND

$V(2)=A L \theta F$

BEND

84

$V(5)=V(5)-D 1-S-D 4+S 0$

BEND

85

IF (BO.GE.0.) GO TO 4

BEND

90

$$
V(1)=-X \otimes F
$$

BEND

$V(2)=-A L O F$

BEND

CARD NR. SEVERITY DETAILS DIAGNOSIS OF PROBLEM

$\begin{array}{lll}28 & \text { I } & \text { PAR } \\ 29 & \text { I } & \text { PAR } \\ 36 & \text { I } & \text { V } \\ 41 & \text { I } & \text { V } \\ 87 & \text { I } & \text { V } \\ 88 & \text { I } & \text { V } \\ 88 & \text { I } & \text { V } \\ 91 & \text { I } & \text { V }\end{array}$

ARRAY REFERENCE OUTSIDE DIMENSION BOUNDS. ARRAY REFERENCE OUTSIDE DIMENSION BOUNDS. ARRAY REFERENCE OUTSIDE DIMENSION BOUNDS. ARRAY REFERENCE OUTSIDE DIMENSION BOUNDS. ARRAY REFERENCE OUTSIDE DIMENSION BOUNDS. ARRAY REFERENCE OUTSIDE DIMENSION BOUNDS. ARRAY REFERENCE OUTSIDE DIMENSION BOUNDS. ARRAY REFERENCE OUTSIDE DIMENSION BOUNDS. 
1

SUBROUTINE BESIS ( $X, F I, N N)$

$C \gg$ FOR EVALUATION OF BESSEL FUNCTION \FI\ONLY

BEND

95

$c \gg$ DIMENSION OF FI IN CALLING PROGRAM MUST BE GE 2

BESIS

BESIS

BESIS

BESIS

BESIS

DIMENSION FI (1), PI (600)

BESIS

$N M A X=\operatorname{MAXO}((N N+1), 2)$

$K M A X=N M A X$

BESIS

SUM $=0$.

BESIS

BESIS

BESIS

$\mathrm{JMAX}=\mathrm{I}+31$

BESIS

$J M 2=J M A X+2$

BESIS

DO $10 \mathrm{~J}=\mathrm{JM} 2, \mathrm{NMAX}$

BESIS

BESIS

BESIS

BESIS

BESIS

BESIS

BESIS

BESIS

BESIS

BESIS

BESIS

BESIS

BESIS

BESIS

END 
SUBROUTINE BET (M,BETX,BETY, IF)

BET

c

C

COMPUTES BETATRON FUNCTIONS

$\operatorname{BETX}(I)=M U, B E T A, A L P H A$, GAMMA, XEQ, DXEQ FOR RX

$B E T Y(I)=M U, B E T A, A L P H A$, GAMMA, YEQ, DYEQ

FOR RY

COMMON/SWTCH/BEND, MSIZE, CYCSWT, VCSW, PV , MSSW, VPR, MHS, MODS, MINZER LOGICAL CYCSWT, MSSW, VCSW, PV, VPR, MHS, MODS

DIMENSION RX(2,3), RY(2,3), BETX(1), BETY (1), RXI $(2,3), \operatorname{RYI}(2,3)$

10

DIMENSION RW(3), RWI (3)

DATA PI /3.141592654/

TWOPI $=2 . * P I$

QINV $=0$.

$N N=-M$

CALL RXY (M,RX,RY,RW)

$T R=R \times(1,1)+R \times(2,2)$

$\mathrm{CS}=\mathrm{TR} / 2$.

$\operatorname{BETX}(9)=\mathrm{CS}$

IF(TR.EQ.2.)GO TO 1

IF(TR.EQ. $(-2)) G$.$O TO 2$

IF (ABS(TR).GT.2.) GO TO 3

GO TO 7

3 IF ( IF.EQ.0) GO TO 33

$\operatorname{BETX}(1)=0$.

GO TO 4

$1 \quad \operatorname{BETX}(1)=0$.

GO TO 4

$2 \quad \mathrm{BETX}(1)=\mathrm{PI}$

4 DO $5 \mathrm{I}=2,6$

$5 \quad \operatorname{BETX}(\mathrm{I})=0$.

GO TO 6

$33 \operatorname{SN}=\operatorname{SIGN}(1,, \operatorname{TR}) * \operatorname{SQRT}(\operatorname{CS} * * 2-1$.

$S=\operatorname{ALOG}(\operatorname{ABS}(\mathrm{CS})+\mathrm{SN})$

$\mathrm{BS}=\mathrm{RX}(1,2)$

GO TO 8

$7 \quad \mathrm{BS}=\mathrm{RX}(1,2)$

$\mathrm{SN}=\mathrm{SQRT}(1 .-\mathrm{CS} * \mathrm{CS}) * \mathrm{BS} / \mathrm{ABS}(\mathrm{BS})$

$S=A T A N 2(S N, C S)+$ TWOPI

$8 \mathrm{~B}=\mathrm{BS} / \mathrm{SN}$

IF (ABS(TR).GT.2.) $B=-A B S(B)$

$G=-R \times(2,1) / S N$

$A=(R \times(1,1)-R \times(2,2)) /(2 . * S N)$

$\operatorname{BETX}(1)=5$

$\operatorname{BETX}(2)=B$

$\operatorname{BETX}(3)=A$

$\operatorname{BETX}(4)=G$

CALL RXY(NN, RXI, RYI, RWI)

QINV $=1$.

$\operatorname{CSS}=2 . *(1 .-\operatorname{Cs})$

$\operatorname{BETX}(5)=(\operatorname{RX}(1,3)+R X I(1,3)) / \operatorname{csS}$

$\operatorname{BETX}(6)=(\operatorname{RX}(2,3)+\operatorname{RXI}(2,3)) / \operatorname{CSS}$

6

$T R=(R Y(1,1)+R Y(2,2))$

$\mathrm{CS}=\mathrm{TR} / 2$.

$\operatorname{BETY}(9)=C S$

IF(TR.EQ.2.)GO TO 11

IF(TR.EQ. (-2.))GO TO 12
BET

BET

BET

BET

SWTCH

SWTCH

SWTCH

BET

BET

BET

BET

BET

BET

BET

BET

BET

BET

BET

BET

BET

BET

BET

BET

BET

BET

BET

BET

BET

BET

BET

BET

BET

BET

BET

BET

BET

BET

BET

BET

BET

BET

BET

BET

BET

BET

BET

BET

BET

BET

BET

BET

BET

BET

BET

BET

BET 
60

65

70

75

80

85

90

95

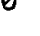

IF (ABS(TR).GT.2.)GO TO 13

GO TO 17

13 IF ( IF.EQ.0) GO TO 313 $\operatorname{BETY}(1)=0$.

C INDEX $M$ HAS ITS OWN INFO, WHICH REFERENCES DATA AND MATRIX LOC.OF $N$. GO TO 14

$11 \operatorname{BETY}(1)=0$.

GO TO 14

$12 \operatorname{BETY}(1)=P I$

14 DO $15 \quad I=2,6$

$15 \operatorname{BETY}(\mathrm{I})=0$.

$$
\text { GO TO } 16
$$

$313 \operatorname{SN}=\operatorname{SIGN}(1, . T R) * \operatorname{SQRT}(\operatorname{CS} * * 2-1$,

$S=\operatorname{ALOG}(\operatorname{ABS}(C S)+S N)$

$B S=R Y(1,2)$

GO TO 20

$17 \quad B S=\operatorname{RY}(1,2)$

$\mathrm{SN}=\mathrm{SQRT}(1 .-\mathrm{CS} * \mathrm{CS}) * \mathrm{BS} / \mathrm{ABS}(\mathrm{BS})$

$S=A T A N 2(S N, C S)+$ TWOPI

$20 \mathrm{~B}=\mathrm{BS} / \mathrm{SN}$

IF (ABS(TR).GT.2.) $B=-A B S(B)$

$G=-R Y(2,1) / S N$

$A=(R Y(1,1)-R Y(2,2)) /(2 . * S N)$

$\operatorname{BETY}(1)=S$

$\operatorname{BETY}(2)=B$

$\operatorname{BETY}(3)=A$

$\operatorname{BETY}(4)=G$

BET

BET

BET

BET

BET

BET

BET

BET

BET

BET

BET

BET

BET

BET

BET

BET

BET

BET

BET

BET

BET

BET

BET

BET

BET

18 CALL RXY(NN, RXI, RYI, RWI)

BET

BET

BET

BET

BET

$16 \quad J=2$

$\operatorname{BETY}(5)=(\operatorname{RY}(1,3)+\operatorname{RYI}(1,3)) /$ CSS

$\operatorname{BETY}(6)=(\operatorname{RY}(2,3)+\operatorname{RYI}(2,3)) /$ CSS

BET

BET

BET

BET

BET

BET

END

$\operatorname{BETX}(8)=\operatorname{BETX}(1) * 180 . / \mathrm{PI}$

$\operatorname{BETY}(8)=\operatorname{BETY}(1) * 180 . / P I$

RETURN

$\begin{array}{lll}19 & \text { I } & \text { BETX } \\ 45 & \text { I } & \text { BETX } \\ 46 & \text { I } & \text { BETX } \\ 47 & \text { I } & \text { BETX } \\ 51 & \text { I } & \text { BETX } \\ 52 & \text { I } & \text { BETX } \\ 55 & \text { I } & \text { BETY } \\ 82 & \text { I } & \text { BETY } \\ 83 & \text { I } & \text { BETY } \\ 84 & \text { I } & \text { BETY } \\ 88 & \text { I } & \text { BETY } \\ 89 & \text { I } & \text { BETY }\end{array}$

ARRAY REFERENCE OUTSIDE DIMENSION BOUNDS. ARRAY REFERENCE OUTSIDE DIMENSION BOUNDS. ARRAY REFERENCE OUTSIDE DIMENSION BOUNDS. ARRAY REFERENCE OUTSIDE DIMENSION BOUNDS. ARRAY REFERENCE OUTSIDE DIMENSION BOUNDS. ARRAY REFERENCE OUTSIDE DIMENSION BOUNDS. ARRAY REFERENCE OUTSIDE DIMENSION BOUNDS. ARRAY REFERENCE OUTSIDE DIMENSION BOUNDS. ARRAY REFERENCE OUTSIDE DIMENSION BOUNDS. ARRAY REFERENCE OUTSIDE DIMENSION BOUNDS. ARRAY REFERENCE OUTSIDE DIMENSION BOUNDS. ARRAY REFERENCE OUTSIDE DIMENSION BOUNDS. 
CARD NR. SEVERITY DETAILS

DIAGNOSIS OF PROBLEM

91 I BETX ARRAY REFERENCE OUTSIDE DIMENSION BOUNDS.

92 I BETY ARRAY REFERENCE OUTSIDE DIMENSION BOUNDS.

93 I BETX ARRAY REFERENCE OUTSIDE DIMENSION BOUNDS.

94 I BETY ARRAY REFERENCE OUTSIDE DIMENSION BOUNDS. 
SUBROUTINE BMIS $\quad$ BMIS

BMIS

C BEGIN MISALIGNMENT

COMMON/SWTCH/BEND, MS I ZE, CYCSWT, VCSW, PV, MSSW, VPR, MHS, MODS, MINZER

SWTCH

LOGICAL CYCSWT, MSSW, VCSW, PV, VPR, MHS, MODS

SWTCH

COMMON/INSTR/OPNAME, NAME, OP, KA, KB, OBJA, OBJB, NXTM, TRA, LQ, NQ, LIN,

INSTR

1 NIN, LFL, NFL, LBC, NBC, NTOT, IX, KIND, TYPE, ROWS, COLS, EXTR, INSTR

2

$M, N F, N B, N I, M S U B R, I S I G N$

INSTR

INTEGER OPNAME, OP, OBJA, OBJB, TRA, TYPE, ROWS, COLS, EXTR

INSTR

INSTR

COMMON/CONTRL/ERROR, MODE, RSRV, STOR, XEQ, TRASW, NFSW, EMPTY, INDEF, 1 LDFLG, FIN

LOGICAL ERROR, RSRV, STOR, XEQ, TRASW, NFSW, EMPTY, INDEF, LDFLG, FIN

15

$\mathrm{C}$

IF (MODE.EQ.3) GO TO 10

CONTRL

CONTRL

CONTRL

CONTRL

BMIS

BMIS

BMIS

ISGN $=1 \mathrm{H}$

BMIS

IF (OP.LT.O) ISGN=1H-

BMIS

WRITE $(3,20)$ ISGN

BMIS

BMIS

BMIS

BMIS

BMIS

BMIS

BMIS

END

IF (OP.LT.O) RETURN

IF (MODE. EQ.2) RETURN 
$\begin{array}{ll} & \text { SUBROUT INE BVAL }(M) \\ \text { C } & \text { PARTICLE BEAM DEFINITION } \\ \text { C COMPUTES KINEMATICS AND UNNORMALIZED EMITTANCES. } \\ \text { C IF INPUT EMITTANCES ARE NORMALIZED, UNNORMALIZED VALUES }\end{array}$

BVAL

BVAL

BVAL

BVAL

BVAL

BVAL

BVAL

BVAL

BVAL

BVAL

BVAL

BVAL

BVAL

BVAL

BVAL

BVAL

BVAL

BVAL

BVAL

BVAL

BVAL

BVAL

BVAL

BVAL

BVAL

BVAL

BVAL

BVAL

BVAL

BVAL

BVAL

BVAL

BVAL

BVAL

BLANK

BLANK

LEVEL 2, STORE, INFF, IWORK

COMMON STORE (48000), IWORK(10)

DIMENSION INFF $(24,2000)$

EQUIVALENCE (INFF,STORE)

86MARSIZ

86MARSIZ

86MARSIZ

BLANK

KINET

COMMON/KINET/KNFLAG, TK, P, GAM, BETT, BETGAM, ERHO

KINET

BVAL

BVAL

BVAL

BVAL

BVAL

BVAL

BVAL

BVAL

BVAL

BVAL

BVAL

BVAL

BVAL

BVAL

BVAL

C IST PARAM IS $P$

$$
\text { IF (KA.NE.O) GO TO } 15
$$

IF (A.EQ.O.) $A=R M E$

$A M P C 2=A * E O$

$A M P C 2 R=1 . / A M P C 2$ 
50

$P=P A R(1)$

BETGAM $=P * A M P C 2 R$

BVAL $\quad 53$

GAM = SQRT $($ BETGAM*BETGAM + 1.)

BVAL

$T K=A M P C 2 *(G A M-1$.

BVAL

GO TO 20

C 1ST PARAM IS T

BVAL $\quad 56$

BVAL $\quad 57$

15 TK $=\operatorname{PAR}(1)$

65

GAM $=T K * A M P C 2 R+1$.

BVAL

58

BVAL $\quad 59$

BVAL 60

BETGAM = SQRT $($ GAM*GAM - 1.)

BVAL 61

$P=$ AMPC $2 * B E T G A M$

BVAL 62

20

BETT $=$ BETGAM/GAM

BVAL

$B R H O=P * C O N K$

70

IF (KB.NE.O) KNFLAG = .TRUE.

BVAL

WRITE $(3,101)$

CALL DASH

WRITE $(3,100) \quad P$, TK, A, BRHO, BETT, GAM, BETGAM

BVAL $\quad 65$

BVAL $\quad 66$

BVAL $\quad 67$

BVAL 68

IF ((PAR(3).EQ.0.).AND. (PAR (4).EQ.0.) .AND. (PAR(5) .EQ.0.) . AND.

BVAL $\quad 69$

1 (PAR(6).EQ.0.)) RETURN

BVAL $\quad .70$

BETGAMR $=1 . /$ BETGAM

BVAL $\quad 71$

EPSX $=$ PAR (3)*BETGAMR

BVAL $\quad 72$

EPSY $=$ PAR $(4) * B E T G A M R$

BVAL $\quad 73$

EPSL $=$ PAR $(5) * B E T G A M R$

BVAL $\quad 74$

GO TO 25

80

C ALREADY UNNORMALIZED CASE

BVAL

BVAL $\quad 76$

22 EPSX $=$ PAR (3)

EPSY $=$ PAR (4)

BVAL

BVAL $\quad 78$

$E P S L=P A R(5)$

BVAL

25 SIGL $=$ PAR $(6)$

$S I G P=E P S L / S I G L$

CALL STDAT $(M, 5,1,4, E M)$

BVAL 80

BVAL 81

WRITE $(3,105)$ EPSX, EPSY, EPSL, SIGL, SIGP

BVAL $\quad 82$

CALL DASH

90

C

100 FORMAT $(8 X, * P=*, F 10.5, *$ GEV $/ \mathrm{C} *, 6 X, * T=*$,

$1 \mathrm{~F} 10.5, * \mathrm{GEV} *, 12 \mathrm{X}, * \mathrm{~A}=*, \mathrm{~F} 10.5,6 \mathrm{X}, * \mathrm{BRHO}=*, \mathrm{~F} 10.5, /$

$25 X, * B E T A=*, E 15.8,3 X, *$ GAMMA $=*, E 15.8,3 X$,

3 *BETAGAMMA $=*$, E15.8)

95

101 FORMAT (1H)

105 FORMAT (/5X,*EMITTANCES UNNORMALIZED (MM-MRAD)*/

$18 \mathrm{X}, * \mathrm{EPSX}=*, \mathrm{~F} 10.6,4 \mathrm{X}, * \mathrm{EPSY}=*, \mathrm{~F} 10.6,4 \mathrm{X}, * \mathrm{EPSL}=*$,

$2 \mathrm{~F} 10.6,10 \mathrm{X}, * \mathrm{SIGL}=*, \mathrm{~F} 10.6,1 \mathrm{X}, * \mathrm{MM} *, 4 \mathrm{X}, * \mathrm{SIGP}=*, \mathrm{~F} 10.6$,

RETURN

END

BVAL $\quad 83$

BVAL $\quad 84$

BVAL 85

BVAL 86

BVAL $\quad 87$

BVAL 88

BVAL $\quad 89$

BVAL 90

BVAL 91

BVAL 92

BVAL 93

BVAL 94

BVAL 95

BVAL 96 
1

SUBROUT INE STOREC ( $M, I$, NREG, NSY)

5

LEVEL 2, STORE, INFF, IWORK

COMMON STORE(48000), IWORK(10)

DIMENSION INFF $(24,2000)$

EQUIVALENCE (INFF, STORE)

INTEGER BDAT

DATA MASK/77000 $000000000000000 \mathrm{~B} /$

10

15

20
IREG $=\operatorname{BDAT}(M, I+1)$

$L C=I R E G \cdot A N D \cdot M A S K$

IF (LC.EQ.1H ) GO TO 1

NREG $=-1$

$M N=\operatorname{MDAT}(M, I+1)$

NSY $=\operatorname{INFF}(10$, MN) \$RETURN

$1 \operatorname{DECODE}(5,2$, IREG) NR

2 FORMAT(I5)

NREG $=N R+10$

RETURN

END

$\begin{array}{lr}\text { STOREC } & 2 \\ \text { BLANK } & 2 \\ \text { BLANK } & 3 \\ \text { 86MARSIZ } & 1 \\ \text { 86MARSIZ } & 2 \\ \text { 86MARSIZ } & 3 \\ \text { BLANK } & 5 \\ \text { STOREC } & 4 \\ \text { STOREC } & 5 \\ \text { STOREC } & 6 \\ \text { STOREC } & 7 \\ \text { STOREC } & 8 \\ \text { STOREC } & 9 \\ \text { STOREC } & 10 \\ \text { STOREC } & 11 \\ \text { STOREC } & 12 \\ \text { STOREC } & 13 \\ \text { STOREC } & 14 \\ \text { STOREC } & 15 \\ \text { STOREC } & 16\end{array}$


END 
SUBROUTINE CALC(M)

C INSTRUCTION CALC - SIMULATES AN H-P CALCULATOR.

C COMMANDS ARE ENTERED AS 5 CHARACTER BCD DATA:

COMMANDS ALLOWED -

CALC

CALC

CALC

$Y * * X \quad X * X \quad$ EXP

CALC RUP ASIN ACOS ATAN

CALC

CALC

CALC

CALC

ENTER CHS EEX CLX

$X=Y$

XLEY

XGTY

$X=0$

CALC

CALC

CALC

PI LASTX

$X G T Y$
$X=0$

KA CONTROLS PRINTING AS FOLLOWS -

$K A=0-$ NO PRINT
$K A=1$, PRINT STACK AT END
$K A=2$, PRINT STACK EVERY STEP

LEVEL 2, STORE, INFF, IWORK

COMMON STORE(48000), IWORK(10)

DIMENSION INFF $(24,2000)$

EQUIVALENCE (INFF, STORE)

COMMON/CONTRL/ERROR, MODE, RSRV, STOR, XEQ, TRASW, NFSW, EMPTY, INDEF, 1 LDFLG, FIN

LOGICAL ERROR, RSRV, STOR, XEQ, TRASW, NFSW, EMPTY, INDEF, LDFLG, FIN

COMMON /MATCH3/NOPR, NONU, FLAG, IPR, IPP, MINFLG, MIFLG, MATFLG, GLOBAL LOGICAL FLAG, NOPR, NONU, MIFLG, MATFLG, GLOBAL COMMON/CLC/REG (109)

DIMENSION CMND $(16,5), \operatorname{LX}(16,5), \operatorname{NUM}(14), \operatorname{STK}(5)$

LOGICAL LIFT, SKIP, NPRT

INTEGER CMND, ALPH, BDAT

COMPLEX CX

EQUIVALENCE (STK,REG), $(X, \operatorname{STK}(1)),(Y, \operatorname{STK}(2)),(Z, \operatorname{STK}(3)),(\operatorname{T}, \operatorname{STK}(4))$ $(X L, \operatorname{STK}(5)),(C X, X)$

DATA MASK/ $77000000000000000000 \mathrm{~B} /$. IBLANK/00555 $555555555555555 \mathrm{~B} /$

DATA NUM/1H0,1H1, $1 \mathrm{H} 2,1 \mathrm{H} 3,1 \mathrm{H} 4,1 \mathrm{H} 5,1 \mathrm{H} 6,1 \mathrm{H} 7,1 \mathrm{H} 8,1 \mathrm{H} 9,1 \mathrm{H}, 1 \mathrm{H}, 1 \mathrm{H}+, 1 \mathrm{H}-/$

DATA $(L X(1, L), L=1,5) / 0,1,1,0,1 /$

DATA $(L X(2, L), L=1,5) / 0,0,1,1,1 /$

DATA $(L X(3, L), L=1,5) / 0,0,0,0,1 /$

DATA $(L X(4, L), L=1,5) / 5 * \theta /$

DATA $(L X(5, L), L=1,5) / 1,4 * 0 /$

DATA $(L X(6, L), L=1,5) / 1,0,1,1,0 /$

DATA $(L X(7, L), L=1,5) / 1,4 * 0 /$

DATA $(L X(8, L), L=1,5) / 1,4 * 0 /$

DATA $(L X(9, L), L=1,5) / 0,1,1,0,1 /$

DATA $(\operatorname{LX}(10, L), L=1,5) / 0,0,1,1,1 /$

DATA $((L X(K, L), K=11,16), L=1,5) / 30 * 0 /$

DATA (CMND $(1, L), L=1,5) / 4 \mathrm{H} \quad, 4 \mathrm{H} 1 / X, 4 H S Q R T, 4 \mathrm{H} \quad, 4 \mathrm{HLN} /$,

$2 \quad(\operatorname{CMND}(2, L), L=1,5) / 4 H X-Y, 4 H R D N, 4 H S I N, 4 H C O S, 4 H T A N /$.

3 (CMND $(3, L), L=1,5) / 4 \mathrm{H} \quad, 4 \mathrm{H} \quad, 4 \mathrm{HSTO}, 4 \mathrm{HRCL}, 4 \mathrm{HABS} /$, (CMND $(4, L), L=1,4) / 5$ HENTER, 4HCHS , 4HEEX , 4HCLX $\operatorname{CMND}(5,1)$ $/ 4 \mathrm{H}-$ $(\operatorname{CMND}(6, L), L=1,4) / 4 \mathrm{H}+$ $\operatorname{CMND}(7,1)$

$/ 4 \mathrm{H} *$

, 4HP-R , 4HR-P

, $4 \mathrm{H}$

CALC

CALC

CALC

CALC

CALC

CALC

BLANK

BLANK

86MARSIZ

86MARSIZ

86MARSIZ

BLANK

CONTRL

CONTRL

CONTRL

CONTRL

MATCH3

MATCH3

CLC

CALC882

CALC

CALC

CALC

CALC

CALC

CALC

CALC

CALC882

CALC882

CALC882

CALC882

CALC882

CALC882

CALC882

CALC882

CALC882

CALC882

CALC882

CALC882

CALC

CALC

CALC

CALC

i.

CALC

1.

CALC

1

CALC

2

3

4

5

6

7

8

9

10

11

12

13

14

15

16

17

18

2

3

1

2

3

5

2

3

4

5

2

3

2

1

24

25

26

27

28

29

30

2

3

4

5

6

7

8

9

10

11

12 
60

65

70

75

80

85

90

95

100

105

110

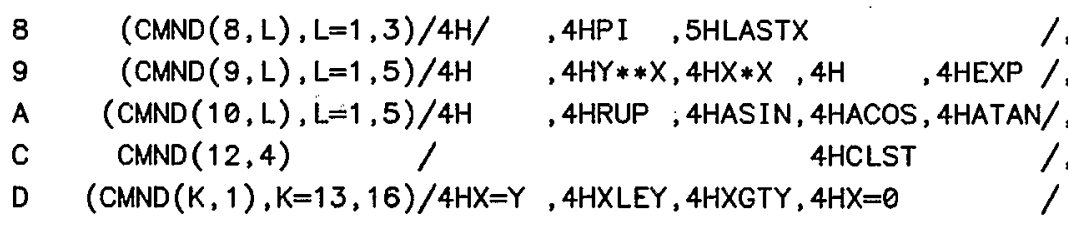

SKIP $=$. FALSE. $\quad \$ L I F T=$. TRUE. $\quad \$ N P R T=$. FALSE.

$\mathrm{NBC}=\operatorname{INFF}(17, M) \quad \$ \mathrm{KA}=\operatorname{INFF}(4, M)$

$P I=2 \cdot * A \operatorname{COS}(0) \quad \$ I=.0 \quad \$ I C M D=5 H$

IF (NOPR.OR.KA.EQ.0) NPRT = .TRUE.

IF (.NOT.NPRT) CALL DASH

IF (.NOT.NPRT) WRITE $(3,901)$

IF (.NOT.NPRT) CALL DASH

IF (.NOT.NPRT.AND.KA.EQ.2) WRITE(3,902) I, ICMD, X,Y,Z,T,XL

DO $300 I=1, N B C$

$\operatorname{ICMD}=\operatorname{BDAT}(\mathrm{M}, \mathrm{I})$

IF (ICMD. EQ. 5HLSTX ) ICMD $=5$ HLASTX

IF (ICMD. EQ. 5HENTR ) ICMD=5HENTER

IF (ICMD.EQ.5H ) 60 TO 300

IF (.NOT.SKIP) GO TO 2

SKIP $=$.FALSE. \$GO TO 3

2 DO $6 \mathrm{~K}=1,16$

DO $6 \mathrm{~L}=1,5$

$\mathrm{KL}=5 *(K-1)+\mathrm{L}$

$\operatorname{IF}(\operatorname{ICMD} . \operatorname{EQ} \cdot \operatorname{CMND}(K, L))$ GO TO 77

6 CONTINUE

$I C 1=($ MASK $\cdot A N D \cdot I C M D) \cdot O R \cdot I B L A N K$

DO $8 K=1,14$

IF(IC1.EQ.NUM(K)) GO TO 500

8 CONTINUE

9 WRITE $(3,10)$ ICMD

10 FORMAT $(1 X, 5 \mathrm{H} * * * *$, A5, $*$ IS NOT A RECOGNIZED CALC COMMAND*)

ERROR $=$. TRUE. $\$$ RETURN

C FLOATING POINT NUMBER

$500 \operatorname{DECODE}(5,900$, ICMD $) F$

900 FORMAT (F5.0)

IF (LIFT) CALL STKLFT $(X, Y, Z, T$ )

$X=F \quad \$ \quad$ GO TO 1

$77 \quad \operatorname{IF}(L X(K, L) . E Q, 1) \quad X L=X$

7 GO TO $(1,12,13,1,15,21,22,23,24,25,1,1,33,34,35$, $41,42,43,44,1,51,1,1,1,1,61,1,63,64,1$, $71,1,1,1,1,81,82,83,1,1,1,92,93,1,95$,

$1,102,103,104,105,1,1,1,1,1,1,1,1,124,1$, $131,1,1,1,1,141,1,1,1,1,151,1,1,1,1$, $161,1,1,1,1$

* $1 / x$

$12 \quad \mathrm{X}=1 . / \mathrm{X} \$$ GO TO 1

* SQRT
1. CALC $\quad 41$

CALC $\quad 42$

CALC $\quad 43$

CALC $\quad 44$

CALC $\quad 45$

CALC $\quad 46$

CALC $\quad 47$

CALC $\quad 48$

CALC $\quad 49$

CALC $\quad 50$

CALC $\quad 51$

CALC $\quad 52$

CALC $\quad 53$

CALC $\quad 54$

CALC 55

CALC $\quad 56$

CALC $\quad 57$

CALC $\quad 59$

86CALC 1

86CALC 2

CALC 60

CALC 61

CALC 62

CALC 63

CALC $\quad 64$

CALC $\quad 65$

CALC 66

CALC882 14

CALC 68

CALC $\quad 69$

CALC $\quad 70$

CALC $\quad 71$

CALC882 15

CALC882 16

CALC $\quad 74$

CALC $\quad 76$

CALC $\quad 77$

CALC $\quad 78$

CALC $\quad 79$

CALC 80

CALC 81

CALC 82

CALC 83

CALC 84

CALC 85

CALC 86

CALC882 17

CALC $\quad 87$

CALC 88

CALC $\quad 89$

CALC 90

CALC 91

CALC 92

CALC 93

CALC 94

CALC882 18

CALC 96 


$$
\begin{aligned}
& 13 X=\operatorname{SQRT}(X) \$ \text { GO TO } 1 \\
& { }^{*} \operatorname{LN} \quad \$=\operatorname{ALOG}(X) \$ \text { GO TO } 1 \\
& 15 \quad X-Y \\
& 21 \quad X X=X \$ X=Y \$ Y=X X \$ \text { GO TO } 1
\end{aligned}
$$

120

125

130

135

140

145

150

155

160

165

* RDN

* SIN

$23 x=\operatorname{SIN}(x)$ \$ GO TO 1

* $\cos$

$24 x=\cos (x)$ \$ GO TO 1

* TAN

$25 x=\operatorname{TAN}(X)$ \$ GO TO 1

* STO

33 CALL STOREC( $M, I$, NREG, NSY)

IF (NREG.LT.O) GO TO 331

REG(NREG) $=X \$$ GO TO 5

331 STORE(NSY) $=X$ \$ GO TO 5

* RCl

34 CALL STOREC(M, I , NREG, NSY)

IF (NREG.LT.O) GO TO 341

$X=$ REG(NREG) \$GO TO 5

$341 X=$ REALNUM(NSY)

5 SKIP $=$. TRUE. \$GO TO 1

* ABS

$35 X=\operatorname{ABS}(X) \quad \$$ GO TO 1

* ENT

41 CALL STKLFT $(X, Y, Z, T)$

LIFT $=$.FALSE. \$GO TO 3

* $\mathrm{CHS}$

$42 x=-X \$$ GO TO 1

* EEX

* CLX

* -

$51 X=Y-X \quad \$$ GO TO 4

* +

$61 X=Y+X \quad \$ 60$ TO 4

* P-R

* R-P

* *

$71 X=Y * X$ \$ GO TO 4

* 1

$81 X=Y / X \quad \$$ GO TO 4

* PI

$\$$ GO TO 1

$$
X=P I
$$

* LASTX

* $Y * * X$

$92 X=Y * * X \quad \$$ GO TO 4
$22 X X=X \$ X=Y \$ Y=Z \$ Z=T \$ T=X X \$$ GO TO 1

IF (LIFT) CALL STKLFT $(X, Y, Z, T$ )

$43 \operatorname{ICMD2}=\operatorname{BDAT}(M, I+1) \$ \operatorname{DECODE}(5,900, \operatorname{ICMD} 2) \times \mathrm{XEXP}$

$X=X * 10 . * * X E X P \quad \$$ SKIP $=$.TRUE. \$ GO TO 1

$44 X=0 . \$$ LIFT $=$.FALSE. \$ GO TO 3

$63 \quad X 1=X * \operatorname{Cos}(Y) \quad \$ Y=X * \operatorname{SIN}(Y) \quad \$ X=X 1 \quad \$$ GO TO 1

$64 T H=\operatorname{ATAN2}(Y, X) \quad \$ R=\operatorname{CABS}(C X)$

82 IF (LIFT) CALL STKLFT $(X, Y, Z, T$ )

83 IF(LIFT) CALL $\operatorname{STKLFT}(X, Y, Z, T) \$ X=X L \$$ GO TO 1

* $X * X$
CALC $\quad 97$

CALC $\quad 98$

CALC 99

CALC $\quad 100$

CALC882 19

CALC $\quad 102$

CALC882 20

CALC 104

CALC $\quad 105$

CALC 106

CALC $\quad 107$

CALC $\quad 108$

CALC 109

CALC $\quad 110$

CALC $\quad 111$

CALC $\quad 112$

CALC $\quad 113$

CALC : $\quad 114$

CALC $\quad 115$

CALC $\quad 116$

CALC $\quad 117$

CALC $\quad 118$

CALC $\quad 119$

JUN83CALC 1

CALC $\quad 121$

CALC $\quad 122$

CALC $\quad 123$

CALC $\quad 124$

CALC $\quad 125$

CALC $\quad 126$

CALC $\quad 127$

CALC882 21

CALC $\quad 129$

CALC 130

CALC $\quad 131$

CALC $\quad 132$

CALC - 133

CALC $\quad 134$

CALC $\quad 135$

CALC $\quad 136$

CALC $\quad 137$

CALC $\quad 138$

CALC $\quad 139$

CALC $\quad 140$

CALC 141

CALC $\quad 142$

CALC 143

CALC 144

CALC 145

CALC $\quad 146$

CALC $\quad 147$

CALC 148

CALC 149

CALC882 22

CALC $\quad 151$

CALC $\quad 152$

CALC $\quad 153$ 
175

180

185

190

195

200

205

210

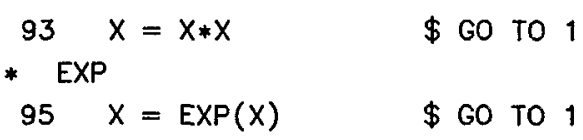

* RUP

$102 X X=X \quad \$ X=T \quad \$ T=Z \$ Z=Y \quad \$ Y=X X \$$ GO TO 1

* ASIN

$103 x=\operatorname{ASIN}(X) \quad \$$ GO TO 1

* ACOS

$104 X=A \operatorname{Cos}(X) \quad \$$ GO TO 1

* ATAN

$105 X=\operatorname{ATAN}(X) \quad \$$ GO TO 1

* CLST

$124 X=0 . \quad \$ Y=0 . \quad \$ Z=0 . \quad \$ T=0$.

GO TO 1

* $\quad X=Y$

131 IF (X.NE.Y) SKIP = .TRUE. \$GO TO 1

* XLEY

141 IF (X.GT.Y) SKIP = .TRUE. \$GO TO 1

* XGTY

151. IF $(X, L E . Y)$ SKIP $=$.TRUE. \$ GO TO 1

* $X=0$

161 IF (X.NE.O.)SKIP = .TRUE. \$ GO TO 1

C DROP STACK

$4 \quad Y=Z \quad \$ Z=T$

$1 \quad$ LIFT $=$. TRUE.

3 IF (.NOT.NPRT.AND.KA.EQ.2) WRITE(3,902) I, ICMD, X,Y,Z,T,XL

300 CONTINUE

$\operatorname{CALL~STDAT}(M, 5,1,5$, STK $)$

IF (.NOT.NPRT.AND.KA.EQ.1) WRITE(3,902) I , ICMD,X,Y,Z,T,XL

IF (.NOT.NPRT) CALL DASH

901 FORMAT ( $4 \mathrm{X}, 1 \mathrm{HI}, 5 \mathrm{X}, 5 \mathrm{HCMND}, 9 \mathrm{X}, 1 \mathrm{HX}, 14 \mathrm{X}, 1 \mathrm{HY}, 14 \mathrm{X}, 1 \mathrm{HZ}, 14 \mathrm{X}, 1 \mathrm{HT}, 14 \mathrm{X}, 1 \mathrm{HL}$ )

902

FORMAT ( $1 X, I 4,5 X, A 5,5 F 15.5)$

RETURN

END

\begin{tabular}{|c|c|}
\hline CALC & 154 \\
\hline CALC & 155 \\
\hline CALC & 156 \\
\hline CALC & 157 \\
\hline CALC882 & 23 \\
\hline CALC & 159 \\
\hline CALC & 160 \\
\hline CALC & 161 \\
\hline CALC & 162 \\
\hline CALC & 163 \\
\hline CALC & 164 \\
\hline CALC & 165 \\
\hline CALC & 166 \\
\hline CALC882 & 24 \\
\hline CALC & 168 \\
\hline CALC882 & 25 \\
\hline CALC & 170 \\
\hline CALC882 & 26 \\
\hline CALC & 172 \\
\hline CALC882 & 27 \\
\hline CALC & 174 \\
\hline CALC882 & 28 \\
\hline CALC & 176 \\
\hline CALC & 177 \\
\hline CALC & 178 \\
\hline CALC & 179 \\
\hline CALC & 180 \\
\hline CALC & 181 \\
\hline CALC & 182 \\
\hline CALC & 183 \\
\hline CALC & 184 \\
\hline CALC & 185 \\
\hline$C A L C$ & 186 \\
\hline CALC & 187 \\
\hline CALC & 188 \\
\hline CALC & 185 \\
\hline CALC & 19 \\
\hline CALC & 19 \\
\hline CALC & 19 \\
\hline
\end{tabular}


SUBROUTINE CDET5(C,DET)

CDET5

RETURN

CDET5

END

CDET5

2

3

4 
C MAGNET PARAMETER GAMMA IS DEPENDENT ON E. GAMMA IS P(5)

C SET UP MATRICES FOR AN FDDF CELL (WITH DRIFT SPACES).

CELL

CELL

CELL

COMMON/BMI/MI (5000)

COMMON/SWTCH/BEND, MSIZE, CYCSWT , VCSW, PV , MSSW, VPR, MHS , MODS, MINZER$$
P(5)=\operatorname{PAR}(6)
$$

CELL

CELL

CELL

CELL

CELL

CELL

CELL

CELL

CELL

CELL

CELL

CELL

C SET UP QD

$$
M I(2)=L
$$

$$
M I(11)=L
$$

CELL

$L=M-14$

$$
P(2)=-P(2)
$$

CELL

CELL

CELL

CELL

CELL

CELL

CELL

CELL

CELL

CELL
C SET UP QS

$M I(5)=L$

$L=M-15$

CALL DRIFT (L, SOV2)

$M I(1)=L$ 
60

$(6)=L$

$M I(7)=L$

$M I(12)=L$

C SET UP QT

$L=M-16$

CALL DRIFT (L, TOV2)

65

$M I(3)=L$

$M I(4)=L$

$M I(9)=L$

$M I(10)=L$

$\mathrm{L}=\mathrm{M}-1$

$\operatorname{CALL} \operatorname{MMM}(M, 12, M I)$

70

C IF CYCSWT IS ON, COMPUTE CELL MATRIX ONLY FOR TEMPORARY USE

(FOR DESIGN INSTRUCTIONS).

IF (CYCSWT) RETURN

C COMPUTE 12 CYCLED MATRICES

CALL CYCLE (M, $12, M I, 0,0)$

75

C STTORE THE MILIST AS DATA FOR Q

CALL MOVLEV (MI, MMI , 12)

$\operatorname{CALL} \operatorname{STDAT}(M, 3,1,12, M M I)$

RETURN

80

END

$\begin{array}{lr}\text { CELL } & 52 \\ \text { CELL } & 53 \\ \text { CELL } & 54 \\ \text { CELL } & 55 \\ \text { CELL } & 56 \\ \text { CELL } & 57 \\ \text { CELL } & 58 \\ \text { CELL } & 59 \\ \text { CELL } & 60 \\ \text { CELL } & 61 \\ \text { CELL } & 62 \\ \text { CELL } & 63 \\ \text { CELL } & 64 \\ \text { CELL } & 65 \\ \text { CELL } & 66 \\ \text { CELL } & 67 \\ \text { CELL } & 68 \\ \text { CELL } & 69 \\ \text { CELLL } & 2 \\ \text { CELLL } & 3 \\ \text { CELLL } & 4 \\ \text { CELL } & 71 \\ \text { CELL } & 72\end{array}$


5

10

15

20

30

35

40

45

50

55

25
SUBROUTINE CFD(M,PAR)

SET UP MATRICES FOR FDDF CELL (WITH DRIFT SPACES)

THE LENGTHS OF THE $F$ AND D MAGNETS MAY BE DIFFERENT

PAR $=$ INPUT PARAMETERS

INPUT CARDS...

Q

CFD

LFMAG GAMMOP

LDMAG K

K RHO

LSTR

LSEP

$\mathrm{C}$

THIS SUBROUTINE GENERATES 17 MATRICES AS FOLLOWS...

$Q F=$ THE FOCUSSING MAGNET $=$ MATRIX $M-13$

$Q D=$ THE DEFOCUSSING MAGNET $=$ MATRIX $M-14$

$Q S=$ HALF THE CENTER DRIFT SPACE = MATRIX M - 15

QT $=$ HALF THE DRIFT SPACE BETWEEN A D AND AN $F$ MAGNET = MATRIX $M$

THE FDDF CELL $=Q=$ THE PRODUCT OF QS QF QT QT QD QS QS QD QT QF QS

$=$ MATRIX $M$

QI $=$ THE I-TH CYCLED TRANSFER MATRIX = MATRIX $M-I$, WHERE

$I=1,12$, AND $Q 1=0$.

OMEGAX IS SET $=1$, AND DELTA IS SET $=0$. FOR THE CFD MAGNETS.

$\mathrm{c}$

COMMON/BMI/MI (5000)

COMMON/NELS/NELS

LEVEL 2,MI

COMMON/SWTCH/BEND, MSIZE, CYCSWT, VCSW, PV, MSSW, VPR, MHS, MODS, MINZER LOGICAL CYCSWT, MSSW, VCSW, PV, VPR, MHS, MODS

DIMENSION PAR( 8$), P(8)$

C

DIMENSION MMI(12)

DO $1 \quad I=1,8$

$1 \quad P(I)=\operatorname{PAR}(I)$

SLFMAG $=P(1)$

SLDMAG $=P(2)$

SOV $2=P(5) / 2$.

TOV2 $=P(6) / 2$.

$P(2)=A B S(P(3))$

$P(3)=P(4)$

$P(4)=P(8)$

$P(5)=P(7)$

$P(6)=P(5)$

C SET UP QF

$L=M-13$

CALL $\operatorname{STDAT}(L, 1,1,6, P)$

CALL MAGNET (L,P)

$M I(2)=L$

$\operatorname{MI}(11)=\mathrm{L}$

C SET UP QD

$L=M-14$

$P(1)=$ SLDMAG

$P(2)=-P(2)$

$\operatorname{CALL} \operatorname{STDAT}(L, 1,1,6, P)$

CALL MAGNET $(L, P)$

$M I(5)=L$

$\operatorname{MI}(8)=\mathrm{L}$

C SET UP QS
CFD 2

CFD

CFD

CFD

CFD

CFD

CFD

CFD

CFD

CFD

CFD

CFD

CFD

CFD

CFD

CFD

CFD

CFD

CFD

CFD

BMIL

BMIL

BMIL

BMIL

BMI

SWTCH

SWTCH

SWTCH

CFD

CFDL

CFD

CFD

CFD

CFD

CFD

CFD

CFD

CFD

CFD

CFD

CFD

CFD

CFD

CFD

CFD

CFD

CFD

CFD

CFD

CFD

CFD

CFD

CFD

CFD

CFD

CFD

CFD 
60

$L=M-15$

CFD

CALL DRIFT(L,SOV2)

CFD

$M I(1)=L$

CFD

$M I(6)=L$

CFD

$M I(7)=L$

CFD

$\operatorname{MI}(12)=\mathrm{L}$

CFD

C SET UP QT

CFD

65

$L=M-16$

CFD

59

CALL DRIFT(L, TOV2)

CFD

CFD

$\operatorname{MI}(3)=L$

$\operatorname{MI}(4)=L$

CFD

$\operatorname{MI}(9)=L$

70

$\operatorname{MI}(10)=\mathrm{L}$

CFD

CFD

CFD

CFD

CFD

CFD

CFD

CFD

CFD

CFD

CFD

CFD

CFD

CFD

CFD

CFDL

CFDL

CFDL

RETURN

CFD

CFD

CALL MOVLEV(MI, MMI , 12) 
LEVEL 2, STORE, INFF, IWORK

BLANK COMMON STORE(48000), IWORK(10)

86MARSIZ

DIMENSION INFF $(24,2000)$

EQUIVALENCE (INFF,STORE)

86MARSIZ 2

COMMON/FLTN/IFL(15)

86MARSIZ 3

BLANK

FLTN

FLTN

COMMON/INSTR/OPNAME, NAME, OP, KA, KB, OBJA, OBJB, NXTM, TRA, LQ, NQ, LIN,

INSTR

1 NIN, LFL, NFL, LBC , NBC, NTOT, IX, KIND, TYPE, ROWS, COLS, EXTR,

2 $M, N F, N B, N I, M S U B R, I S I G N$

\section{INSTR}

INSTR

INTEGER OPNAME, OP, OBJA, OBJB, TRA, TYPE, ROWS, COLS, EXTR

INSTR

\section{INTEGER ELNUM}

DATA MASKF/77000000000000000000B/

INSTR

CHKD

CHKD

DATA MASKL/ $77777777770000000000 \mathrm{~B} /$.

CHKD

. MASKBLK/ $00000000005555555555 \mathrm{~B} /$,

CHKD

CHKD

CHKD

CHKD

CHKD

CHKD

Go TO 12

CHKD

CHKD

CHKD

CHKD

CHKD

CHKD

CHKD

CHKD

CHKD

CHKD

CHKD

CHKD

CHKD

CHKD

CHKD

CHKD

CHKD

CHKD

CHKD

CHKD

CHKD

CHKD

RETURN

END

C FLOATING POINT. SCALAR AND SINGLE

$\mathrm{KN}=4 \mathrm{HSCAL}$

$I T P=4 H S N G L$ 
SUBROUTINE CHRM(GK,RI, SS, TAN1, TAN2, BETX, BETY, BXP, BYP, CRX, CRY)

CHRM

DIMENSION BETX(6),BETY(6), BXP(6),BYP(6),

$1 \mathrm{BX} 1(6), \mathrm{BY} 1(6), \mathrm{BX} 2(6), \mathrm{BY} 2(6)$

$C R X=0$.

$C R Y=0$.

IF(GK.EQ.0. .AND.RI.EQ.0.) RETURN

IF (RI.NE.O.) GO TO 11

$\mathrm{CRX}=(\operatorname{BETX}(4)+\mathrm{GK} * \mathrm{BETX}(2)) * S S+B E T X(3)-\operatorname{BXP}(3)$

$C R Y=(B E T Y(4)-G K * B E T Y(2)) * S S+B E T Y(3)-B Y P(3)$

10

15

20

25

30

35

40

45

50
RETURN

CONTINUE

DO $1 \mathrm{I}=1,6$

$B \times 1(I)=B \times P(I)$

$B Y 1(I)=B Y P(I)$

$B \times 2(I)=B E T X(I)$

$1 \quad B Y 2(I)=B E T Y(I)$

$B \times 1(3)=B \times 1(3)-T A N 1 * R I * B \times 1$ (2)

$B \times 2(3)=B \times 2(3)+T A N 2 * R I * B \times 2(2)$

$B Y 1(3)=B Y 1(3)+T A N 1 * R I * B Y 1$ (2)

BY2 (3) $=$ BY2 (3)-TAN2*RI $* B Y 2(2)$

$B \times 1(6)=B \times 1(6)+T A N 1 * R I * B \times 1(5)$

$\mathrm{B} \times 2(6)=\mathrm{B} \times 2(6)-\mathrm{TAN} 2 * \mathrm{RI} * \mathrm{~B} \times 2(5)$

$\mathrm{RI} 2=\mathrm{RI} * * 2$

$\mathrm{GKX}=\mathrm{GK}+\mathrm{R} I 2$

$B K G X=G K X * B X 1(2)+(1 .+B \times 1(3) * * 2) / B \times 1(2)$

$\mathrm{BKGY}=-\mathrm{GK} * \mathrm{BY} 1(2)+(1 .+\mathrm{BY} 1(3) * * 2) / B Y 1(2)$

$F 2=(S S * B K G Y+B Y 2(3)-B Y 1(3)) / 2$.

IF (GKX.NE.O.) GO TO 12

$F 5=S S *(B \times 1(5)+B \times 2(5)-S S * 2 * R I / 6) /$.2 .

$F 1=S S *(B \times 1(2)+B \times 2(2)+S S *(B \times 2(3)-B \times 1(3)) / 3) /$.2 .

$F 3=((B \times 1(2)+B \times 2(2)) *(B \times 1(5)+B \times 2(5))$

$1+B \times 1(2) * B \times 2(5)+B \times 2(2) * B \times 1(5)) / 3$.

$2+(B \times 2(3)-B \times 1(3)) *(5 *(B \times 1(5)+B \times 2(5))-R I * S S * * 2) * S S / 30$.

$3-(B \times 1(2)+B \times 2(2)) * R I * S S * * 2 / 12$.

$\mathrm{F3}=\mathrm{F} 3 * \mathrm{SS} / 2$.

GO TO 13

12

$F 1=(S S * B K G X+B \times 2(3)-B \times 1(3)) / G K X / 2$.

$F 5=(R I * S S-B \times 2(6)+B X 1(6)) / G K X$

$F 3=(2 . * B K G X * F 5-R I * F 1+B \times 2(6) * B \times 2(2)+2 . * B \times 2(3) * B \times 2(5)$

1

$-B \times 1(6) * B \times 1(2)-2 . * B \times 1(3) * B \times 1(5)) / 3 . / G K X$

$13 \mathrm{CRX}=-(\mathrm{GKX}+\mathrm{RI} 2) * \mathrm{~F} 1+\mathrm{RI} *(\mathrm{GK}+\mathrm{GK}) * \mathrm{F3}+\mathrm{RI} * \mathrm{BKGX} * \mathrm{F5}$

$1-\mathrm{RI} *(\mathrm{BXP}(2) * \mathrm{BXP}(6)-\mathrm{BETX}(2) * \mathrm{BETX}(6))$

$\mathrm{CRY}=-\mathrm{F} 2+\mathrm{RI} * \mathrm{BKGY} * \mathrm{F5}+\mathrm{RI} *(\mathrm{BYP}(2) * \mathrm{BXP}(6)-\mathrm{BETY}(2) * \mathrm{BETX}(6))$

IF (TAN1.EQ.0. .AND.TAN2.EQ.0.) GO TO 14

$\mathrm{T} 1=\mathrm{RI}$ *TAN1

$T 2=R I * T A N 2$

$C R X=C R X+B X P(2) *(T 1-T A N 1 *((G K+G K X) * B X P(5)-T 1 * B X P(6)))$

$1+\operatorname{BETX}(2) *(\operatorname{T2} 2-\operatorname{TAN} 2 *((G K+G K X) * B E T X(5)+T 2 * B E T X(6)))$

$2+(B X P(3)+B X 1(3)) * B X P(5) * T 1 * T A N 1-(B E T X(3)+B X 2(3)) * B E T X(5) * T 2 * T A N 2$

$\mathrm{CRY}=\mathrm{CRY}+\mathrm{BYP}(2) *(-\mathrm{T} 1+\mathrm{TAN} 1 *(2 . * \mathrm{GK} * \mathrm{BXP}(5)+\mathrm{T} 1 * \mathrm{BXP}$ (6)) )

$1+\operatorname{BETY}(2) *(-\operatorname{T} 2+\operatorname{TAN} 2 *(2 . * \mathrm{GK} * \mathrm{BETX}(5)-\mathrm{T} 2 * \mathrm{BETX}(6)))$

$2-(B Y P(3)+B Y 1(3)) * B X P(5) * T 1 * T A N 1+(B E T Y(3)+B Y 2(3)) * B E T X(5) * T 2 *$ TAN2

14 CONTINUE

$\mathrm{CRX}=-\mathrm{CRX}-\mathrm{CRX}$

$C R Y=-C R Y-C R Y$

RETURN

END
CHRM

CHRM

CHRM

CHRM

CHRM

CHRM

CHRM

CHRM

CHRM

CHRM

CHRM

CHRM

CHRM

CHRM

CHRM

CHRM

CHRM

CHRM

CHRM

CHRM

CHRM

CHRM

CHRM

CHRM

CHRM

CHRM

CHRM

CHRM

CHRM

CHRM

CHRM

CHRM

CHRM

CHRM

CHRM

CHRM

CHRM

CHRM

CHRM

CHRM

CHRM

CHRM

CHRM

CHRM

CHRM

CHRM

CHRM

CHRM

CHRM

CHRM

CHRM

CHRM

CHRM

CHRM

CHRM

CHRM 
END 
1

SUBROUTINE CMXDIV( $V$, IN, IM, DET, IDIM)
C REPLACES B BY (A INVERSE)*B. DESTROYS A.

C A IS $N$ BY $N$, B IS $N$ BY $M$. A AND B ARE STORED IN $V(N, N+M)$. COMPLEX SAVE, PIVOT, DETERM, DET , $V, C A B S$

5 DIMENSION $V(I D I M, 1)$

INTEGER $P$

$N=I N$

$M=I M$

C

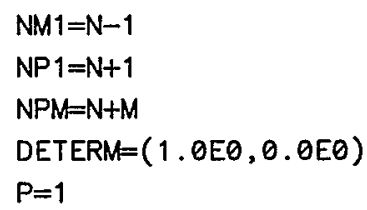

202 CONTINUE

IMIN $=P+1$

C

FIND PIVOT

CMXDIV

CMXDIV

CMXDIV

CMXDIV

CMXDIV

CMXDIV

CMXDIV

CMXDIV

CMXDIV

CMXDIV

CMXDIV

CMXDIV

CMXDIV

CMXDIV

CMXDIV

CMXDIV

CMXDIV

CMXDIV

RPIVOT $=0.0 \mathrm{EO}$

CMXDIV

CMXDIV

RSAVE $=\operatorname{CABS}(V(I, P))$

CMXDIV

IF (RSAVE . LE. RPIVOT) GO TO 209

CMXDIV

RPIVOT=RSAVE

IBIG $=I$

209 CONTINUE

IF (RPIVOT .GT. O.OEO) GO TO 210

CMXDIV

CMXDIV

CMXDIV

DETERM $=(\theta .0 E 0,0 . \theta E 0)$

GO TO 290

C

ROW EXCHANGE AND ROW REDUCTION

CMXDIV

CMXDIV

CMXDIV

CMXDIV

CMXDIV

CMXDIV

CMXDIV

CMXDIV

CMXDIV

CMXDIV

CMXDIV

CMXDIV

CMXDIV

BLOCK REDUCTION

C

$V(P, P)=(1.0 E \theta, 0.0 E \theta)$

DO 239 I=IMIN, N

DO $238 \mathrm{~J}=I M I N, N P M$

$V(I, J)=V(I, J)-V(I, P) * V(P, J)$

238 CONTINUE

$V(I, P)=(0.0 E 0,0.0 E 0)$

45

239 CONTINUE

$\mathrm{P}=\mathrm{P}+1$

249 GO TO 202

C

BACK SUBSTITUTION

250 CONTINUE

CMXDIV 40

CMXDIV $\quad 41$

CMXDIV $\quad 42$

CMXDIV 43

CMXDIV $\quad 44$

CMXDIV $\quad 45$

CMXDIV $\quad 46$

CMXDIV $\quad 47$

CMXDIV $\quad 48$

CMXDIV $\quad 49$

CMXDIV 50

CMXDIV 51

CMXDIV 52

DO $259 \mathrm{~K}=\mathrm{NP} 1$, NPM

DO $253 P=1$, NM1

$I=N-P$

DO $252 \mathrm{~J}=\mathrm{I}, \mathrm{NM} 1$

CMXDIV 53

CMXDIV 54

CMXDIV 55

CMXDIV $\quad 56$

CMXDIV 57

CMXDIV 58 
259 CONTINUE

CMXDIV

59

C

CMXDIV

60

60

290 CONTINUE

CMXDIV

61

DET $=$ DETERM

CMXDIV

62

RETURN

CMXDIV

63

END

CMXDIV

64 
END

CONJ 
DO $7 \mathrm{~J}=1,2$

$R X(I, J)=T 2(I, J)$

$R Y(I, J)=T 2(I+2, J+2)$

7 CONTINUE

8 CONTINUE

DO $9 \mathrm{I}=1,2$

$R X(I, 3)=T 2(I, 6)$

$\operatorname{RY}(I, 3)=T 2(I+2,6)$

9

CONT INUE

$\mathrm{RW}(3)=\mathrm{T} 2(5,6)$ 
60

65

22

*

$$
T 2(7,7)=1
$$$$
\text { SET } P 1, P 2, Q 1, Q 2
$$$$
\text { DO } 22 \mathrm{I}=1,2
$$$$
T 2(5, I)=T 2(1,6) * T 2(2, I)-T 2(2,6) * T 2(1, I)
$$$$
T 2(5, I+2)=T 2(3,6) * T 2(4, I+2)-T 2(4,6) * T 2(3, I+2)
$$$$
R P Q(I)=T 2(5, I)
$$$$
\text { DO } 26 \quad I=1,49
$$$$
T(I)=T 1(I)
$$

$$
\text { CONT INUE }
$$$$
\text { RETURN }
$$

70

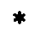

30 100

75
ERROR IF KN IS NOT MXY OR R7

WRITE $(3,100) \mathrm{KN}$

FORMAT $(5 \mathrm{H} * * *$ * *ERROR. CANNOT CONVERT MATRIX OF KIND *, I5) ERROR $=$. TRUE.

RETURN

END

$\begin{array}{ll}\text { CONVMAT } & 56 \\ \text { CONVMAT } & 57 \\ \text { CONVMAT } & 58 \\ \text { CONVMAT } & 59 \\ \text { CONVMAT } & 60 \\ \text { CONVMAT } & 61 \\ \text { CONVMAT } & 62 \\ \text { CONVMAT } & 63 \\ \text { CONVMAT } & 64 \\ \text { CONVMAT } & 65 \\ \text { CONVMAT } & 66 \\ \text { CONVMAT } & 67 \\ \text { CONVMAT } & 68 \\ \text { CONVMAT } & 69 \\ \text { CONVMAT } & 70 \\ \text { CONVMAT } & 71 \\ \text { CONVMAT } & 72 \\ \text { CONVMAT } & 73\end{array}$


USING BETATRON FUNCTIONS, COMPUTES BEAM ENVELOPES ALONG BEAM

CYAE

* $\quad B L=$ BEAMLINE NAME

* BEAM = NAME of BVAL OR CYEM THAT gIVES EMITTANCES AND DP/P

CYAE

CYAE

* KSQ = EPS/EPSO, WHERE EPSO IS EMITTANCE FROM BVAL (SIG**2/BETA)

CYAE AND EPS IS EMITTANCE TO BE USED BY CYAE.

CYAE XB AND XP ARE THUS MULTIPLIED BY SQRT(KSQ).

CYAE

CYAE

LEVEL 2, STORE, INFF, IWORK

COMMON STORE( 48000$), \operatorname{IWORK}(10)$

DIMENSION INFF $(24,2000)$

EQUIVALENCE (INFF, STORE)

COMMON/BMI/MI (5000)

COMMON/NELS/NELS

LEVEL 2, MI

COMMON /DIM/ LEND, MEND, ISAV, ISAV7, M7END, KADD, KADDR, MUNIT, MSYMPL

COMMON /MC/ ML \$ LEVEL 2, ML

DIMENSION P(4), BETX(9), BETY(9), ML(3)

EQUIVALENCE (EPX,P(1)), (EPY,P(2)), (EPL, P(3)) , (DPP,P(4))

EQUIVALENCE (BX,BETX(2)), (GX,BETX(4)), (DX,BETX(5)), (DDX,BETX(6)), 1

DATA IBLNK/1H /

*

$M D=\operatorname{MDAT}(M, 2)$

CALL DATA (MD, $5,1,4, P)$

* Ka determines calculation for xtot and xTOt PR $K A=\operatorname{INFF}(4, M)$

* get beam line, make milist and multiply matrices

* $\quad K$ is number of elements in beam

$\operatorname{MB}=\operatorname{MDAT}(M, 1)$

$K=\operatorname{INFF}(17, M B)$

MS $=$ MEND - 1

CALL MIFILL (MB, $1, K$, NELS, MI)

CALL MMM (MS, NELS,MI)

$\mathrm{KW}=\mathrm{NELS}+1$
CYAE 15

CYAE $\quad 16$

CYAE $\quad 17$

CYAE $\quad 18$

CYAE $\quad 19$

BLANK 2

BLANK 3

86MARSIZ 1

86MARSIZ 2

86MARSIZ 3

BLANK 5

BMIL 1

BMIL 2

BMIL 3

BMIL 4

BMI 3

DIM 2

DIM 3

CYAE 23

CYAEL 1

CYAEL 2

CYAE 24

CYAE 25

CYAE 26

CYAE 27

CYAE 28

CYAE $\quad 29$

CYAE $\quad 30$

CYAE 31

CYAE $\quad 32$

CYAE $\quad 33$

CYAE $\quad 34$

CYAE $\quad 35$

CYAE $\quad 36$

CYAE $\quad 37$

CYAE $\quad 38$

CYAE $\quad 39$

CYAE $\quad 40$

CYAE $\quad 41$

CYAE $\quad 42$

CYAE $\quad 43$

CYAE $\quad 44$

CYAE $\quad 45$

CYAE 46 
60

65

70

75

80

85

90

95

100

105

110
$M I(K W)=M I(1)$

$I=\theta$

$E L=0$.

$F L=0$.

MAT = IBLNK

$X K S Q=F D A T(M, 1)$

$\mathrm{RK}=\mathrm{SQRT}$ (XKSQ)

$\operatorname{EPXCO}=\operatorname{FDAT}(M, 2)$

$\operatorname{EPYCO}=\operatorname{FDAT}(M, 3)$

RX=SQRT (EPXCO/EPX)

RY $=S Q R T$ (EPYCO/EPY)

* print table heading and sigX and Sigy, as defined by factK CALL DASH

WRITE(3,1) $P(1), \operatorname{EPXCO}, P(3), P(2), \operatorname{EPYCO}, P(4)$

$I G=1$

IF (KA.EQ.0) IG $=2$

GO TO $(104,105)$ IG

104 WRITE $(3,6)$

GO TO 106

105 WRITE $(3,8)$

106 WRITE $(3,2)$ RK

WRITE $(3,11)$

115 CONTINUE

CALL BET(MS, BETX, BETY, 1)

COMPUTE AND PRINT BEAM ENVELOPES

$X=S Q R T(E P X * B X) * R K$

$Y=S Q R T(E P Y * B Y) * R K$

$X P=D X * D P P$

$Y P=D Y * D P P$

$X C O=R X * X$

$Y C O=R Y * Y$

$X P R=S Q R T(E P X * G X) * R K$

$Y P R=S Q R T(E P Y * G Y) * R K$

$X P P R=D D X * D P P * R K$

$Y P P R=D D Y * D P P * R K$

$X C O P R=R X * X P R$

$Y C O P R=R Y * Y P R$

GO TO $(116,117)$ IG

$116 \mathrm{XTOT}=\mathrm{X}+\mathrm{ABS}(\mathrm{XP})$

XPRTOT $=X P R+A B S(X P P R)$

YTOT $=Y+A B S$ (YP)

YPRTOT $=$ YPR + ABS (YPPR)

GO TO 118

117 CONTINUE

XTOT $=\operatorname{SQRT}(X * X+X P * X P)$

$X P R T O T=S Q R T(X P R * X P R+X P P R * X P P R)$

$Y T O T=\operatorname{SQRT}(Y * Y+Y P * Y P)$

YPRTOT = SQRT (YPR*YPR + YPPR*YPPR)

118

CONTINUE

$X$ XTOT $=X T O T+X C O$

$Y T O T=Y T O T+Y C O$

XPRTOT $=X P R T O T+X C O P R$

YPRTOT $=$ YPRTOT + YCOPR

$E L=E L+F L$

\begin{tabular}{|c|c|c|}
\hline CYAE & & 4 \\
\hline CYAE & & \\
\hline CYAE & & . \\
\hline CYAE & & 5 \\
\hline CYAE & & \\
\hline CYAE & & 3 \\
\hline CYAE & & \\
\hline CYAE & & \\
\hline CYAE & & \\
\hline CYAE & & \\
\hline CYAE & & \\
\hline CYAE & & 5 \\
\hline CYAE & & 5 \\
\hline CYAE & & 6 \\
\hline CYAE & 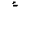 & \\
\hline CYAE & & 6 \\
\hline CYAE & & 6 \\
\hline CYAE & $\therefore$ & 6 \\
\hline CYAE & & 6 \\
\hline CYAE & & 6 \\
\hline CYAE & & 6 \\
\hline CYAE & & 6 \\
\hline CYAE & & 6 \\
\hline CYAE & & 7 \\
\hline CYAE & & 7 \\
\hline CYAE & & 7 \\
\hline CYAE & & 7 \\
\hline CYAE & & 7 \\
\hline CYAE & & 7 \\
\hline CYAE & & 7 \\
\hline CYAE & & 7 \\
\hline CYAE & & 7 \\
\hline CYAE & & 7 \\
\hline CYAE & & 8 \\
\hline CYAE & & 8 \\
\hline CYAE & & 8 \\
\hline CYAE & $\cdot$ & 8 \\
\hline CYAE & & 8 \\
\hline CYAE & & 8 \\
\hline CYAE & & 8 \\
\hline CYAE & & 8 \\
\hline CYAE & & 8 \\
\hline CYAE & & 8 \\
\hline CYAE & & 9 \\
\hline CYAE & & 9 \\
\hline CYAE & & 9 \\
\hline CYAE & & 9 \\
\hline CYAE & & 9 \\
\hline CYAE & & 9 \\
\hline CYAE & & 9 \\
\hline CYAE & & 9 \\
\hline CYAE & & 9 \\
\hline CYAE & & 9 \\
\hline CYAE & & 10 \\
\hline CYAE & & 10 \\
\hline CYAE & & 10 \\
\hline CYAE & & 10 \\
\hline
\end{tabular}




$\begin{array}{ll}\text { CYAE } & 104 \\ \text { CYAE } & 105 \\ \text { CYAE } & 106 \\ \text { CYAE } & 107 \\ \text { CYAE } & 108 \\ \text { CYAE } & 109 \\ \text { CYAE } & 110 \\ \text { CYAE } & 111 \\ \text { CYAE } & 112 \\ \text { CYAE } & 113 \\ \text { CYAE } & 114 \\ \text { CYAE } & 115 \\ \text { CYAE } & 116 \\ \text { CYAE } & 117 \\ \text { CYAE } & 118 \\ \text { CYAE } & 119 \\ \text { CYAE } & 120 \\ \text { CYAE } & 121 \\ \text { CYAE } & 122 \\ \text { CYAE } & 123 \\ \text { CYAE } & 124 \\ \text { CYAE } & 125 \\ \text { CYAE } & 126 \\ \text { CYAE } & 127 \\ \text { CYAE } & 128 \\ \text { CYAE } & 129 \\ \text { CYAE } & 130 \\ \text { CYAE } & 131 \\ \text { CYAE } & 132 \\ \text { CYAE } & 133 \\ \text { CYAE } & 134 \\ \text { CYAE } & 135 \\ \text { CYAE } & 136 \\ \text { CYAE } & 137 \\ \text { CYAE } & 138 \\ \text { CYAE } & 139 \\ \text { CYAE } & 140\end{array}$

120

CALL DATA(NI $, 5,1,1, F L)$

MAT = MNAME (NI)

$M L(1)=-N I$

$M L(2)=M S$

$M L(3)=N I$

125

CALL MMM(MS, 3, ML)

GO TO 115

CALL DASH

RETURN

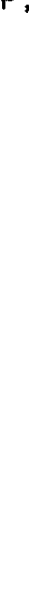

1 FORMAT (3X,*BEAM ENVELOPES (MM, MRAD)*/

$15 X, *$ EMITTANCES (MM-RAD) - EPSX $=*, F 10.6,5 X, *$ EPSXCO $=*$,

140

8 FORMAT $(1 H+, 13 X, 30 H X T O T=S Q R T(X * X+X P * X P+X C O), 5 X$,

$142 \mathrm{HXTOTPR}=\mathrm{SQRT}(X P R * X P R+X P P R * X P P R+X C O P R))$

9 FORMAT (I4,1X, A5, F9.4,12F9.4)

10 FORMAT ( $/ 2 X, * P O S *, 10 X, * S *, 8 X, * X *, 7 X, * X$ PR*, $6 X$, $1 * X P *, 5 X, * X P P R *, 4 X, * X T O T *, 3 X, * X T O T P R *, 5 X, * Y *, 7 X$,

145

$2 * Y P R *, 5 X, * Y P *, 6 X, * Y P$ PR* $5 X, * Y T O T *, 3 X, * Y T O T P R * / 1 X, 131(1 \mathrm{H}-))$

150

FORMAT (I 4, 1X, A5 , F9. 4, 5X, 4F9.4, 5X, 4F9.4 , 5X, 2F9.4)

END

140

CARD NR. SEVERITY DETAILS DIAGNOSIS OF PROBLEM

$76 \quad I$

98 I
AN IF STATEMENT MAY BE MORE EFFICIENT THAN A 2 OR 3 BRANCH COMPUTED GO TO STATEMENT AN IF STATEMENT MAY BE MORE EFFICIENT THAN A 2 OR 3 BRANCH COMPUTED GO TO STATEMENT 
1

SUBROUT INE CYCLE(M,K,MI , L,WS )

COMMON /DIM/ LEND, MEND, ISAV, ISAV7, M7END, KADD, KADDR, MUNIT, MSYMPL

5

DIMENSION M3(3)

DIMENSION MI (1)

COMMON/MD/M3

$\$$ LEVEL 2,MI, M3

INTEGER WS

10

15

20

25

30
IF (WS.EQ. O) GO TO 5

NO $=$ MEND-3

$\mathrm{NC}=\mathrm{K}$

GO TO 6

$5 \quad \mathrm{NO}=\mathrm{M}-1$

$\mathrm{NC}=\mathrm{K}-1$

6 IF (L.EQ.O) GO TO 1

$\operatorname{CALL} \operatorname{MMM}(M, K, M I)$

$1 \quad M I(K+1)=M I(1)$

DO $2 I=1, N C$

IF (I.NE.1) GO TO 11

$M 3(2)=M-1$

$\mathrm{NJ}=\mathrm{NO}-1$

GO TO 12

$11 \quad \mathrm{NJ}=\mathrm{NO}-\mathrm{I}$

$M 3(2)=N J+1$

$12 M 3(3)=M I(I)$

$M 3(1)=-M 3(3)$

CALL MMM (NJ,3, M3)

2 CONTINUE

RETURN

END

$\begin{array}{lr}\text { CYCLE } & 2 \\ \text { CYCLE } & 3 \\ \text { DIM } & 2 \\ \text { DIM } & 3 \\ \text { CYCLE } & 5 \\ \text { CYCLE } & 6 \\ \text { CYCLEL } & 1 \\ \text { CYCLEL } & 2 \\ \text { CYCLE } & 7 \\ \text { CYCLE } & 8 \\ \text { CYCLE } & 9 \\ \text { CYCLE } & 10 \\ \text { CYCLE } & 11 \\ \text { CYCLE } & 12 \\ \text { CYCLE } & 13 \\ \text { CYCLE } & 14 \\ \text { CYCLE } & 15 \\ \text { CYCLE } & 16 \\ \text { CYCLE } & 17 \\ \text { CYCLE } & 18 \\ \text { CYCLE } & 19 \\ \text { CYCLE } & 20 \\ \text { CYCLE } & 21 \\ \text { CYCLE } & 22 \\ \text { CYCLE } & 23 \\ \text { CYCLE } & 24 \\ \text { CYCLE } & 25 \\ \text { CYCLE } & 26 \\ \text { CYCLE } & 27 \\ \text { CYCLE } & 28 \\ \text { CYCLE } & 29 \\ \text { CYCLE } & 30\end{array}$


C $\quad K A=$ OUTPUT OPTION — SEE BELOW

CYEM

C $K B=$ NUMBER OF SUPERPERIODS

IF BL IS A FXPT INSTRUCTION, BEAM LINE AND KB ARE TAKEN FROM

CYEM THOSE OF THE FXPT INSTRUCTION.

CYEM

CYEM

$B L=$ NAME OF BEAM LINE (BML INSTRUCTION) OR OF FXPT INSTRUCTION

CYEM

$E=$ ELECTRON BEAM ENERGY (GEV)

C $\quad R=$ MACHINE RADIUS (M)

C $\quad F=$ RF FREQUENCY (MHZ)

C $\quad V=$ RF VOLTS/TURN (MV)

C $K=$ KAPPA $=$ COUPLING COEFFICIENT (SQRT (EPY/EPN)

CYEM

CYEM

CYEM

CYEM

CYEM

CYEM

KA OPTIONAL OUTPUT CAN ONLY BE DONE WHEN BL IS FXPT INSTR.

CYEM

CYEM

CYEM

$K A=0,1 \quad$ PRINT ONLY AT START OF BEAM LINE

CYEM

$K A=2,3,4$ PRINT AT EVERY ELEMENT

CYEM

$K A=$ EVEN, BETATRON PART OF SIGMA MATRIX

CYEM

$K A=O D D$, TOTAL SIGMA MATRIX

C $K A=2,3$ SIGMA MATRIX LONG OUTPUT

CYEM

CYEM

$K A=4 \quad$ AIJ, BIJ LONG OUTPUT

CYEM

CYEM

$\mathrm{c}$

C

C

C KA

NEGATIVE

$$
\text { PRINTS RII, TIJ. }
$$$$
\text { TIJ }=\text { ARCTAN ( SIJ/SII-SJJ ) } / 2
$$

RII IS THE PROJECTION OF BEAM ELLIPSE ON I AXIS TIJ IS TILT OF ELLIPSE PROJECTION IN I-J PLANE PRINTS SIJ $=\operatorname{SIGMA}(I, J) \quad$ FOR ALL ELEMENTS

CYEM

CYEM

CYEM

CYEM

CYEM

CYEM

LEVEL 2, STORE, INFF, IWORK COMMON STORE (48000), IWORK(10) DIMENSION INFF $(24,2000)$ EQUIVALENCE (INFF, STORE)

CYEM

CYEM

CYEM

CYEM

CYEM

CYEM

CYEM

CYEM

CYEM

BLANK

BLANK 8GMARSIZ 86MARSIZ 86MARSIZ BLANK

COMMON/BMI/MI (5000) COMMON/NELS/NELS LEVEL 2, MI 
C COMMON/MOVTOG/TOG

60 LOGICAL TOG

C

DIMENSION VWW(7)

LOGICAL TOG 1

DIMENSION BETX(9), BETY(9), PAR(5), P(6), EM(4), ML(3)

65

COMMON /MF/ ML \$ LEVEL 2,ML

DIMENSION EV $(4,8), \operatorname{VZ}(7,7), \operatorname{VW}(7), \operatorname{RW}(3), \operatorname{AA}(4,4), \mathrm{BB}(4,4), \mathrm{CC}(4,8)$

MOVTOG

MOVTOG

DIMENSION RX $(2,3), \operatorname{RY}(2,3), \operatorname{HMOV}(4)$

MOVTOG

MOVTOG

MYZCYEM

MY3CYEM

CYEM

CYEMLL

CYEMLL

CYEM

CYEM

DIMENSION EI (2)

70

COMPLEX GAMT

COMPLEX EV, DET, EJ1, EJ3, EK1, EK3, EI , XM2 I

CYEM

CYEM

CYEM

COMPLEX DETX, DETY

REAL I 41, I 43, I51, I53, J1, J3, J6, K, KSQ, LH

CYEM

CYEM

CYEM

CYEM

EQUIVALENCE (L,P(1)), (DB, $P(2)),(B R H O, P(3)),(B Z, P(4)),(O M, P(4))$

CYEM

EQUIVALENCE (PSI,XI)

CYEM

EQUIVALENCE (EPSX, EM(1)), (EPSY, EM(2)), (EPSL, EM(3)), (SIGP, EM(4))

CYEM

EQUIVALENCE $(B, \operatorname{BETX}(2)),(A, \operatorname{BETX}(3)),(\operatorname{E,BETX}(5)),(F, \operatorname{BETX}(6))$

EQUIVALENCE (GAM, BETX(4))

80

EQUIVALENCE (PC, PAR(1)), (R,PAR(2)), (FREQ, PAR(3)), (VO, PAR(4)),

1 (FKAP, PAR(5))

EQUIVALENCE (ANGO, IANGO), (ANG1, IANG1)

CYEM 62

CYEM 63

CYEM 64

CYEM 65

CYEM 66

REAL I1 I I , I3, I4, I5, L, KX, KK, N, LMTOT, K3R, JE, JX, JZ

CYEM $\quad 67$

85

LOGICAL BSW, MOVSW, LONG, FLAG, SIGFLG

CYEM $\quad 68$

DATA RME/.0005110041/

CYEM $\quad 69$

DATA PI, CGAM, CQP, C, JE, JX, JZ

CYEM $\quad 70$

1/3.1415926536,8.846002206E-5,1.467471313E-6, 2.997925E8, 2. .1 .,1./

CYEM

71

DATA RADEG/.0174532925/

90

DATA RW/3*0.0/

CYEM $\quad 72$

CYEM $\quad 73$

CALL RANGET(NRN)

CYEM

MY3CYEM

74

$X M 2 I=(0 .,-2$.

$\begin{array}{ll}* & \text { GET DATA P/C,R, FREQ, VRF, KAPPA } \\ * & \text { UNITS - GEV/C, METERS, MHZ, MV }\end{array}$

CALL DATA (M, $1,1,5$, PAR)

CYEM

CYEM

CYEM

CYEM

CYEM

$F M H Z=F R E Q$

VOMV $=$ VO

CYEM

CYEM

CYEM

CYEM

CYEM

CYEM

CYEM

CYEM

$M B=\operatorname{MDAT}(M, 1)$

105

* $K$ is number of elements in beam line

$K A=0$

$K B=\operatorname{INFF}(5, M)$

FLAG $=$.FALSE.

$M S=$ MEND -1

110

$\operatorname{IOPMB}=\operatorname{INFF}(1, \mathrm{MB})$

$\mathrm{NGO}=1$

IF (IOPMB. EQ. 4HFXPT) NGO=2

CALL DASH

WRITE $(3,13)$

CYEM

CYEM

CYEM

CYEM

CYEM

CYEM

CYEM

CYEM

CYEM

96 
$52 \quad M F X=M B$

$M B=\operatorname{MDAT}(M F X, 2)$

120

$\mathrm{NP}=\mathrm{MDAT}(\mathrm{MFX}, 1)$

$K A=\operatorname{INFF}(4, M)$

SIGFLG $=$.FALSE.

IF (KA.GE.O) GO TO 55

SIGFLG $=$. TRUE.

$K A=-K A$

$\mathrm{KAF}=\operatorname{INFF}(4, \mathrm{MFX})$

LONG $=$.FALSE.

IF (KA.GT. 1) LONG =. TRUE.

$K B=\operatorname{INFF}(5, M F X)$

$M S 7=$ M7END -3

53 CONT INUE

CALL DATA(MFX, $7,1,49, \mathrm{VZ})$

DO $60 \mathrm{I}=1,4$

$\operatorname{EV}(I, 1)=\operatorname{CMPLX}(\operatorname{VZ}(I, 2), \operatorname{VZ}(I, 3))$

135

$\operatorname{EV}(1,3)=\operatorname{CMPLX}(V Z(1,4), \operatorname{VZ}(1,5))$

$\operatorname{EV}(I, 2)=\operatorname{CONJG}(\operatorname{EV}(I, 1))$

$60 \quad \operatorname{EV}(I, 4)=\operatorname{CONJG}(E V(I, 3))$

$\operatorname{DETX}=\operatorname{EV}(1,1) * \operatorname{EV}(2,2)-\operatorname{EV}(2,1) * \operatorname{EV}(1,2)$

$1+\operatorname{EV}(3,1) * \operatorname{EV}(4,2)-\operatorname{EV}(4,1) * \operatorname{EV}(3,2)$

CYEM

CYEM

113

114

CYEM

115

CYEM

116

CYEM

117

CYEM

118

CYEM

119

CYEM

120

140

$\operatorname{DETY}=\operatorname{EV}(1,3) * \operatorname{EV}(2,4)-\operatorname{EV}(2,3) * \operatorname{EV}(1,4)$

CYEM

121

$1+\operatorname{EV}(3,3) * \operatorname{EV}(4,4)-\operatorname{EV}(4,3) * \operatorname{EV}(3,4)$

CYEM

122

CALL STOR7 (MS7,VZ, RW)

CALL DATA(MFX, 7, 1,7,WW)

CYEM

123

CYEM

124

CALL RXY(MFX,RX,RY,RW)

CYEM

125

145

CALL STXY(MS,RX,RY,RW)

CYEM

126

IF (.NOT.FLAG) GO TO 54

CYEM

127

$I I=0$

CALL DASH

IF (KA.EQ.1.OR.KA.EQ.3) GO TO 56

CYEM

128

CYEM 129

WRITE $(3,19)$

CYEM

130

150

GO TO 57

CYEM

131

WRITE $(3,20)$

CYEM 132

56

57 IF (SIGFLG) GO TO 58

CYEM

133

CYEM

134

WRITE $(3,16)$

155

GO TO 503

CYEM

135

CYEM 136

58 WRITE $(3,18)$

CYEM 137

GO TO 503

CYEM 138

54 WRITE $(3,14)$

CYEM 139

CYEM 140

160

51 CONTINUE

$I=\operatorname{INFF}(17, \mathrm{MB})$

CALL MIFILL (MB, 1, I, NELS, MI)

IF(NGO.EQ.2) GO TO 59

CALL MMM(MS, NELS,MI)

165

IF (KB.GE.0) GO TO 59

NELS $=N E L S+1$

$M I(N E L S)=M E N D-6$

CALL REF (MEND-6, MS)

$M L(1)=M S$

ML(2) $=$ MEND-6

CALL MMM(MS, 2,ML)

CYEM 
$K W=N E L S+1$

CYEM

$M I(K W)=M I(1)$

CYEM

$\mathrm{BSW}=$. FALSE.

CYEM

157

MOVSW $=$. FALSE.

CYEM

158

$\mathrm{I} 1=0$.

CYEM

159

$\mathrm{I} 2=0$.

CYEM

160

$I 3=0$.

CYEM

161

180

$14=0$.

CYEM

162

$15=0$.

CYEM

163

$\mathrm{I} 41=0$.

CYEM

164

$\mathrm{I} 43=0$.

CYEM

165

$I 51=0$.

CYEM

166

185

$\mathrm{I} 53=0$.

CYEM

167

II $=0$

CYEM

168

CYEM

169

170

$* * * * * * * * * * * * * * * * * * * * * * * * * * * * * * * * * * * * * * * * * * * * * * * * * * * * * * * * * * * * * * * * * * * * * * * *$

190

100 CALL BET(MS,BETX,BETY,1)

CYEM

171

IF (II.EQ.0) BXO=BETX(2)

CYEM

172

IF (NGO.EQ.2) GO TO 503

CYEM

173

101 II $=I I+1$

* IF I $=K W$, END OF BEAM LINE

CYEM

174

CYEM

175

195

IF (II.GT.KW) GO TO 400

CYEM

176

$N I=M I(I I)$

IF (FLAG) GO TO 121

* IF NOT AT BEgINNING OR END OF A BENDING MAGNET, CONTINUE

CYEM

177

CYEM

178

CYEM

179

$I O P=\operatorname{INFF}(1, N I)$

CYEM

180

200

IF (BSW) GO TO 300

111 IF (IOP.NE.4HMOVE) GO TO 115

DO 105 IV $=1,7$

$105 \mathrm{VWW}(\mathrm{IV})=\mathrm{VW}(\mathrm{IV})$

TOG1 = TOG

CALL RANGET(NRN1)

NN1 = M7END - 5

CALL MOVE(NI, NN1, VWW)

CALL RANSET (NRN1)

TOG = TOG1

CALL DATA(NI , 1,7,4, HMOV)

MOVSW $=$. TRUE.

$M Q=\operatorname{MDAT}(N I, 1)$

$\operatorname{IOP}=\operatorname{INFF}(1, \mathrm{MQ})$

IF (IOP.NE.4HMAG ) GO TO 120

CYEM

181

CYEM

MY3CYEM

CYEM

CALL DATA (MQ, $1,1,6, P)$

GO TO 116

115 IF (IOP.NE.4HMAG) GO TO 120

CYEM

188

CYEM

CYEM CALL DATA (NI, 1, 1,6,P)

116 IF ((BZ.NE.0.).OR. (NGO.EQ.2) ) GO TO 200

120 CONTINUE

CYEM

121 NF = M7END - 5 
IF(IPOS.NE.0) GO TO 901

CYEM

CYEM

901 IF (.NOT.LONG) GO TO 122

CYEM

$M K=M I$ ( IPOS)

MAT I $=\operatorname{INFF}(2, M K)$

IF (FLAG) GO TO 903

902 WRITE $(3,15)$ IPOS, MATI,

$1 \operatorname{AA}(1,1), A A(1,2), A A(2,2), A A(1,3), A A(1,4)$,

CYEM

CYEM

CYEM

CYEM

CYEM

CYEM

IF (.NOT.LONG.OR.KA.EQ.4) GO TO 400

CYEM 219

CYEM 220

CYEM 221

CYEM 222

CYEM 223

GO TO 503

122 CONTINUE

$$
M L(1)=-N I
$$

$M L(2)=M S$

CYEM

$M L(3)=N I$

CYEM

CALL MMM(MS, 3,ML)

CYEM

GO TO 100

CYEM

C CALCULATE BILINEAR FORMS — GENERALIZED ELLIPSE MATRICES

DO $501 \mathrm{I}=1,4$

$\operatorname{EV}(I, 1)=\operatorname{CMPLX}(\operatorname{VZ}(I, 2), \operatorname{VZ}(I, 3))$

CYEM

CYEM

CYEM

DO $502 \quad J=5,8$

$502 \operatorname{EV}(I, J)=\operatorname{CMPLX}(0 ., 0$.

$501 \operatorname{EV}(I, I+4)=\operatorname{CMPLX}(1,0$.

600 DO $601 \mathrm{~J}=1,2$

CYEM

$$
1+\operatorname{VZ}(3,6) * \operatorname{EV}(4, I)-\operatorname{VZ}(4,6) * \operatorname{EV}(3, I))
$$

CYEM

$\operatorname{EJ} 1=\operatorname{EI}(1) * \operatorname{EV}(1,1) * X M 2 I / D E T X$

CYEM 
290

295

300

305

310

315

320

325

330

335
$\operatorname{AA}(I, J)=4.0 * \operatorname{REAL}(\operatorname{CoNJG}(\operatorname{EV}(1, I+4)) * \operatorname{EV}(1, J+4))$

$\operatorname{BB}(I, J)=4.0 * \operatorname{REAL}(\operatorname{CoNJG}(\operatorname{EV}(3, I+4)) * \operatorname{EV}(3, J+4))$

IF (.NOT.FLAG) GO TO 505

$\operatorname{CC}(I, J)=\operatorname{REP} 1 * A A(I, J)+\operatorname{REP} 3 * B B(I, J)$

$\mathrm{UU}=0$.

IF (J.EQ.I) UU $=1$.

$\operatorname{Cc}(I, J+4)=U U$

505

CONTINUE

IF (.NOT.FLAG) GO TO 101

CALL MXDIV(CC, 4, 4,DCC, 4)

IF (.NOT.SIGFLG) GO TO 510

DO $511 \mathrm{I}=1,4$

DO $511 \mathrm{~J}=1,4$

$511 \operatorname{CC}(\mathrm{I}, \mathrm{J})=\mathrm{CC}(\mathrm{I}, \mathrm{J}+4)$

GO TO 101

510 DO $506 \quad \mathrm{I}=1,4$

DO $506 \mathrm{~J}=1,4$

SPII $=0$.

SPJJ $=0$.

SPIJ $=0$.

IF (KA.NE.0.AND.KA.NE. 3) GO TO 509

SPII $=V Z(I, 6) * V Z(I, 6) * S I G P S Q$

$S P J J=V Z(J, 6) * V Z(J, 6) * S I G P S Q$

$\mathrm{SPIJ}=\mathrm{VZ}(\mathrm{I}, 6) * \mathrm{VZ}(\mathrm{J}, 6) * \mathrm{SIGPSQ}$

509

CONTINUE

$S I I=C C(I, I+4)+S P I I$

IF (J.EQ.I) GO TO 507

$S I J=\operatorname{CC}(I, J+4)+\operatorname{SPIJ}$

$S J J=\operatorname{cc}(J, J+4)+$ SPJJ

$Y Y=2 \cdot * S I J$

$X X=S I I-S J J$

$T I J=0$.

IF (YY.EQ.0..AND.XX.EQ.0.) GO TO 508

TIJ $=\operatorname{ATAN2}(Y Y, X X) / 2$.

$508 \mathrm{CC}(\mathrm{I}, \mathrm{J})=\mathrm{TIJ}$

GO TO 506

$507 \mathrm{CC}(\mathrm{I}, \mathrm{I})=\operatorname{SQRT}(\mathrm{SII})$

506 CONTINUE

GO TO 101

C 200

$\mathrm{LH}=\mathrm{L} / 2$.

$\mathrm{BR}=\mathrm{P}(3)$

IF (NGO.EQ.2) $\quad B R=B R *(1 .+\mathrm{VW}(6))$

IF (BZ.EQ.0.) GO TO 262

$\mathrm{G}=\mathrm{BZ} / \mathrm{BR}$

$\mathrm{RHO}=1 . / \mathrm{G}$

$K X=P(2)+O M * G$

$K K=\operatorname{SQRT}(A B S(K X / P(3)))$

$\mathrm{PHI}=\mathrm{KK} * \mathrm{~L}$

$N=-P(2) * P(3) /(O M * O M)$

$\mathrm{G} 2=\mathrm{G} * \mathrm{G}$

$\mathrm{G} 3=\mathrm{G} * \mathrm{G} * \mathrm{G}$

$A G 3=A B S(G 3)$

$A G 3 L=A G 3 * L H$

$D F A C=G 3 *(1,-2, * N)$
CYEM 260

CYEM 261

CYEM 262

CYEM 263

CYEM 264

CYEM 265

CYEM 266

CYEM 267

CYEM 268

CYEM 269

CYEM 270

CYEM 271

CYEM 272

CYEM 273

CYEM 274

CYEM 275

CYEM 276

CYEM 277

CYEM 278

CYEM $\quad 279$

CYEM 280

CYEM 281

CYEM 282

CYEM 283

CYEM 284

CYEM 285

CYEM 286

CYEM 287

CYEM 288

CYEM 289

CYEM 290

CYEM 291

CYEM 292

CYEM 293

CYEM 294

CYEM 295

CYEM 296

CYEM 297

CYEM 298

CYEM 299

CYEM 300

CYEM 301

CYEM 302

CYEM 303

CYEM 304

CYEM 305

CYEM 306

CYEM 307

CYEM 308

CYEM 309

CYEM 310

CYEM 311

CYEM 312

CYEM 313

CYEM 314

CYEM 315

CYEM 316 
345

350

355

360

365

$\mathrm{GAM}=(1 .+A * A) / B$

$202 \mathrm{~K} 3 \mathrm{R}=1 . /(\mathrm{KK} * \mathrm{KK} * \mathrm{KK} * \mathrm{RHO})$

IF (KX) $230,210,220$

COMPUTE $V$. DO U AT END OF MAGNET

370

380

385
$E L 2 G=D F A C * L * L * L * G / 24$.

CYEM

CYEM

CYEM

CYEM

CYEM

CYEM

CYEM

CYEM

CYEM

CYEM

CYEM

CYEM

CYEM

CYEM

CYEM

CYEM

CYEM

CYEM

CYEM

CYEM

CYEM

CYEM

CYEM

CYEM

CYEM

CYEM

CYEM

CYEM

CYEM

CYEM

CYEM

CYEM

CYEM

CYEM

CYEM

CYEM

CYEM

CYEM

CYEM

CYEM

CYEM

CYEM

CYEM

CYEM

CYEM

CYEM

CYEM

CYEM

CYEM

CYEM

CYEM

CYEM

CYEM

CYEM

CYEM

CYEM

CYEM

VC6 $=1.5 * \mathrm{PHI}-2 . * \mathrm{~S}+.5 * \mathrm{SC}$

$V_{4}=(B * K 3 R / R H O) *(V C 5+G A M * V C 4 * V C 6 / K K)$ 
GO TO 250

FOR KX.LT.O

D MAGNET

CYEM

CONT INUE

CYEM

377

405

$E X=\operatorname{EXP}(P H I)$

CYEM

378

$\operatorname{EXM}=\operatorname{EXP}(-\mathrm{PHI})$

CYEM

379

$\mathrm{SH}=.5 *(E X-E X M)$

CYEM

380

$\mathrm{CH}=.5 *(\mathrm{EX}+\mathrm{EXM})$

CYEM

381

$\mathrm{SC}=\mathrm{SH} * \mathrm{CH}$

410

$\mathrm{VC} 1=1 .-\mathrm{CH}$

CYEM

382

CYEM 383

$\mathrm{VC2}=\mathrm{PHI}-\mathrm{SH}$

VC3 $=2 . * \mathrm{~K} 3 \mathrm{R}$

CYEM

384

$\mathrm{VC} 4=1 . /(\mathrm{KK} * \mathrm{~B})$

CYEM

385

$\mathrm{VC5}=\mathrm{PHI}-\mathrm{SC}$

415

VC6 $=\mathrm{VC1}+.5 * \mathrm{SH} * \mathrm{SH}$

$V C 7=1.5 * \mathrm{PHI}-2 . * \mathrm{SH}+.5 * \mathrm{SC}$

$V 1=L *(G A M * E * E+2 . * A * E * F+B * F * F)$

CYEM

386

CYEM

387

CYEM

388

CYEM

389

CYEM

390

$V 2=V C 3 * E *(K K * A * V C 1-G A M * V C 2)$

CYEM

391

$V 3=V C 3 * F *(K K * B * V C 1-A * V C 2)$

CYEM

392

420

$\mathrm{V} 4=(B * K 3 R / R H O) *(.5 * V C 5+2 . * A * V C 4 * V C 6-G A M * V C 4 * V C 7 / K K)$

CYEM

393

$V=V_{1}-V_{2}-V_{3}-V_{4}$

CYEM

394

GO TO 250

C

QUADRUPOLE

$262 K=D B / B R$

$E L 2 G=0$.

$\mathrm{KSQ}=\mathrm{K} * \mathrm{~K}$

$X K 2 L=K S Q * L H$

$A K 3 L=A B S(K * K S Q * L H)$

430

TKSQL $=2 . * K S Q * L H$

IF (.NOT.MOVSW) GO TO 265

$X=\operatorname{HMOV}(1)$

$Y=\operatorname{HMOV}(2)$

GO TO 266

435

440

250 GO TO $(251,260)$ NGO

260 IF (BZ.NE.0.) GO TO 264

IF (.NOT.MOVSW) GO TO 265

$X=\operatorname{HMOV}(3)$

$Y=\operatorname{HMOV}(4)$

GO TO 266

$265 X=V W(1)$

$Y=V W(3)$

$266 \mathrm{RSQ}=X * X+Y * Y$

445

$R Q=S Q R T(R S Q)$

$\mathrm{G} 2 \mathrm{~L}=\mathrm{RSQ} * X K 2 \mathrm{~L}$

$A G 3 L=R Q * R S Q * A K 3 L$

$I 2=I 2+G 2 L$

$I 3=I 3+A G 3 L$

CYEM

395

CYEM

396

CYEM 397

CYEM 398

CYEM 399

CYEM 400

CYEM

401

CYEM

402

CYEM

403

CYEM

404

CYEM

405

CYEM

406

CYEM

407

CYEM

408

CYEM

409

CYEM

410

CYEM

411

CYEM

412

CYEM

413

CYEM

414

CYEM

415

CYEM

416

CYEM

417

CYEM

418

CYEM

419

CYEM

CYEM

Q1 $=$ TKSQL $* X$

CYEM

$Q 3=T K S Q L * Y$

CYEM

GO TO 263

$\mathrm{Q} 1=\mathrm{Q} 10-\mathrm{TEO} * \mathrm{G} 2$ 
DO $261 \quad I=1,4$

CYEM

DO $261 \mathrm{~J}=1,4$

CYEM

$I 51=I 51+A A(I, J) * V Z(I, 6) * V Z(J, 6) * A G 3 L$

CYEM

433

$261 \quad I 53=153+B B(I, J) * V Z(I, 6) * V Z(J, 6) * A G 3 L$

CYEM

IF (BSW) GO TO 302

CYEM 435

CYEM $\quad 436$

251 CONTINUE

BSW $=$. TRUE.

SAVE E TO COMPUTE U AT END OF MAGNET

CYEM

$E S A V=E$

GO TO 120

CYEM 438

CYEM $\quad 439$

CYEM $\quad 440$

CYEM $\quad 441$

CYEM $\quad 442$

*

470

300 CONTINUE

BENDING MAGNET EXIT. CALCULATE U AND DO SUMMING.

CYEM

GO TO (301, 260) NGO

CYEM

302 IF (OM.EQ.0.) GO TO 550

301 IF (KX) $330,310,320$

310. $U=(E S A V+E) * .5 * L-(L * L * L) /(12, * R H O)$

475

GO TO 340

CYEM

CYEM $\quad 446$

CYEM $\quad 447$

CYEM $\quad 448$

CYEM $\quad 449$

$320 \mathrm{~T}=\operatorname{TAN}(.5 * \mathrm{PHI})$

$U=(E S A V+E) * T / K K+K 3 R *(P H I-2 . * T)$

CYEM

GO TO 340

$330 T=\operatorname{TANH}(.5 * \mathrm{PHI})$

480

$U=(E S A V+E) * T / K K-K 3 R *(P H I-2 . * T)$

340 CONTINUE

$\mathrm{I} 1=\mathrm{I} 1+\mathrm{G} * \mathrm{U}$

$\mathrm{I} 2=\mathrm{I} 2+\mathrm{G} * \mathrm{G} * \mathrm{~L}$

CYEM

451

CYEM 452

CYEM 453

CYEM 454

CYEM $\quad 455$

CYEM $\quad 456$

CYEM $\quad 457$

$I 3=13+A G 3 * L$

CYEM

458

485

$T R=(\operatorname{ESAV} * \operatorname{TAN}(E O)+E * \operatorname{TAN}(E 1)) /(\mathrm{RHO} * \mathrm{RHO})$

CYEM

459

$I 4=I 4+D F A C * U-T R$

$\mathrm{I} 5=\mathrm{I} 5+\mathrm{AG} 3 * \mathrm{~V}$

CYEM

460

550 CONTINUE

CYEM

461

MOVSW $=$.FALSE.

CYEM

462

490

$\mathrm{BSW}=$. FALSE.

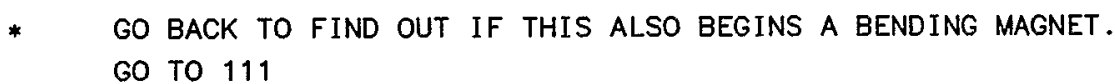

CYEM

463

CYEM

464

CYEM $\quad 465$

MY3CYEM 14

CYEM 467

CYEM 468

CYEM 469

495

* 400 CONTINUE RF OUTPUT

CYEM

470

CYEM

471

CALL RANSET(NRN)

IF (NGO.EQ.1) GO TO 561

MY3CYEM

15

500

IF (FLAG) GO TO 563

CYEM

472

$P C=P C *(1 .+W W(6))$

CYEM

473

FLAG $=$. TRUE.

CYEM

474

CYEM

475

561 CONTINUE

CYEM

476

$\mathrm{I} 1=\mathrm{I} 1 * \mathrm{~KB}$

CYEM

477

505

$\mathrm{I} 2=\mathrm{I} 2 * \mathrm{~KB}$

CYEM

478

$13=13 * K B$

CYEM

479

$\mathrm{I} 4=\mathrm{I} 4 * \mathrm{~KB}$

CYEM

480

$\mathrm{I} 5=\mathrm{I} 5 * \mathrm{~KB}$

CYEM

481

GO TO $(552,553)$ NGO

CYEM 
$\mathrm{D} 1=\mathrm{I} 41 / \mathrm{I} 2$

CYEM

$\mathrm{D} 3=\mathrm{I} 43 / \mathrm{I} 2$

CYEM

$\mathrm{J} 1=1.0-\mathrm{D} 1$

CYEM

$\mathrm{J} 3=1.0-\mathrm{D} 3$

CYEM

$\mathrm{J} 6=2.0+D 1+D 3$

CYEM

CONTINUE

CYEM 492

$\mathrm{PI} 2=2 . * \mathrm{PI}$

CYEM

493

LMTOT $=P I 2 * R H O$

CYEM

494

$\mathrm{EL}=\mathrm{PI} 2 * \mathrm{R}$

CYEM

495

$\mathrm{PC} 4=\mathrm{PC} * * 4$

CYEM

496

ALPHA $=I 1 / E L$

CYEM

497

525

GAMT $=(1 . /$ ALPHA $)$

CYEM

498

GAMT = CSQRT (GAMT)

CYEM

499

UO $=$ CGAM $*$ PC $4 * I 2 / P I 2$

CYEM

500

$\mathrm{UM}=\mathrm{U} 0 * 1 . \mathrm{E} 03$

CYEM

501

$D=I 4 / I 2$

CYEM

502

530

$\mathrm{JX}=1.0-\mathrm{D}$

CYEM

503

$J E=2.0+D$

CYEM

504

$\mathrm{TO}=\mathrm{EL} / \mathrm{C}$

TAUO $=4 . * P I *$ TO $/($ CGAM*PC $* * 3)$

CYEM

505

TAUY $=$ TAUO $/ 12$

535

TAUX $=$ TAUO $/(12-I 4)$

TAUE $=$ TAU日 $/(2, * I 2+I 4)$

CYEM

506

CYEM 507

CYEM 508

SQSIGE $=A B S(C Q P * P C 4 * I 3 /(J E * I 2))$

CYEM

509

SIGE $=$ SQRT $($ SQSIGE $)$

$F K=F R E Q * T O$

540

IF (VO.LT.U日) VO $=\mathrm{U} \theta / .86603$

CYEM

510

CYEM 511

CYEM 512

CPHIS $=$ UQ $/ V 0$

CYEM

513

PHIS $=A C O S(C P H I S)$

$T E=\operatorname{SQRT}(P I 2 * P C * T O * T 0 /($ ALPHA*FK*VO*SIN(PHIS)) $)$

CYEM

CYEM

$S Q E=2 . * U 0 *(T A N(P H I S)-P H I S) /(P I * F K * A L P H A * P C)$

CYEM

$A S Q E=A B S(S Q E)$

EPSM $=P C * S Q R T(A S Q E)$

$P S I=(E P S M / S I G E) * * 2 / 2$.

IF (PSI.LE.740.3) GO TO 556

PSITRU $=P S I$

$550 \quad$ PSI $=740.3$

WRITE $(3,1)$ PSITRU,PSI

556 CONTINUE

TAUQ $=(T A U E / 2). * \operatorname{EXP}(P S I) / P S I$

SIGPHI $=$ SIGE*SQRT $(P I 2 * F K * A L P H A /(V 0 * S I N(P H I S) * P C))$

555

$S I G L=S I G P H I * R / F K$

$\mathrm{PHISD}=\mathrm{PHIS} * 180 \cdot / \mathrm{PI}$

CYEM

CYEM 518

CYEM $\quad 519$

CYEM 520

CYEM $\quad 521$

CYEM 522

CYEM 523

CYEM 524

CYEM $\quad 525$

CYEM 526

CYEM 527

CYEM 528

CYEM 529

CYEM 530

CYEM 531

CYEM 532

$\operatorname{BETX}(2)=B \times 0$

COMPUTE EPSILONS FOR EMITTANCE AND STORE

560

IF (JX.GT.0.) GO TO 574

CYEM

533

$\operatorname{SIGXOB}=0$.

WRITE $(3,21)$

CYEM

GO TO 575

CYEM

574 CONTINUE

SIGXOB $=$ SQRT $(C Q P * P C * P C * I 5 /(J X * I 2))$

575 CONTINUE

CYEM

SIGX $=$ SIGXOB * SQRT $(B E T X(2))$

EPXO = SIGX*SIGX/BETX(2)

IF (FLAG.AND.FKAP.EQ.0.0) FKAP = 1.E-08

CYEM

CYEM

CYEM

$E P X=(1 .-F K A P * F K A P) * E P X O$

CYEM 
$E P Y=F K A P * F K A P * E P X O$

CYEM

GO TO $(554,555)$ NGO

CYEM

$\mathrm{CK}=\mathrm{CQP} * \mathrm{PC} * \mathrm{PC} / \mathrm{I} 2$

CYEM

$\mathrm{EP1}=\mathrm{CK} * 151 / \mathrm{J} 1$

CYEM

547

$E P 3=C K * I 53 / \mathrm{J} 3$

CYEM

548

$E P 3 S=E P 3$

CYEM

549

IF (EP3.EQ. Ө.) EP3S=EPY

CYEM

550

REP $1=1$. /EP1

CYEM

551

580

554

REP3 $=1$. /EP3S

CYEM

552

CONT INUE

SIGBG = SIGE/RME

$E P L N=S I G B G * S I G L$

CYEM

553

BETGAM = PC/RME

CYEM

554

$\mathrm{EPL}=\mathrm{EPLN} / \mathrm{BETGAM}$

585

C

SIGY $=$ SQRT $(\operatorname{EPY} * B E T Y(2))$

CYEM

555

CYEM

556

CYEM

557

$S I G D P=S I G E / P C$

CYEM

558

$S I G P S Q=S I G D P * S I G D P$

CYEM

559

C

$\mathrm{DPP}=\mathrm{SIGDP}$

CYEM

560

EPXN = EPX * BETGAM

CYEM

561

590

EPYN = EPY * BETGAM

CYEM

562

CYEM 563

CYEM $\quad 564$

C SCALE AND STORE

$E P S X=E P X * 1 . E 06$

CYEM

565

$E P S Y=E P Y * 1 . E O 6$

CYEM

566

595

$E P S L=E P L * 1 . E 06$

CYEM

567

$S I G P=S I G D P * 1 . E 03$

CYEM

568

$\operatorname{CALL} \operatorname{STDAT}(M, 5,1,4, E M)$

CYEM

569

IF (NGO.EQ.1) GO TO 562

CYEM

570

IF ((J1.GE.0.).AND.(J3.GE.0.).AND.(J6.GE.0.)) GO TO 562

CYEM

600

WRITE $(3,21)$

CYEM

$F L A G=$.FALSE.

CYEM 573

CYEM 574

562 IF (FLAG) GO TO 53

563 CONTINUE.

CYEM

CYEM 576

CYEM 577

605

CALL DASH

WRITE $(3,2) \quad$ PC, GAMT, R, UM

WRITE $(3,4)$ FMHZ, TO, VOMV, TAUX

CYEM

CYEM

WRITE $(3,6)$ FKAP, TAUY, TAUE

CYEM

CYEM 581

610

WRITE $(3,3)$ D, SIGX, PHISD, TAUQ

CYEM

WRITE $(3,5)$ TE, SIGPHI, EPSM,SIGL

CYEM

WRITE $(3,7) \times I$, SIGDP

WRITE $(3,11)$ SIGE, SIGBG

WRITE $(3,10)$ FK, BETGAM, ALPHA, BXO

615

WRITE $(3,9)$ I 1 , I 2, I 3 , I 4 , I 41 , I 43, I5 , I51, I53

CYEM

CYEM 585

CYEM 586

CYEM 587

CYEM 588

WRITE $(3,12) \mathrm{JX}, J Z, J E$

IF (NGO.EQ.2) WRITE $(3,22) \mathrm{J} 1, \mathrm{J3}, \mathrm{J} 6$

CYEM

WRITE $(3,8)$ EPXN, EPYN, EPLN, EPX, EPY, EPL, EPXO

CYEM

IF (NGO.EQ.2) WRITE $(3,23)$ EP1,EP3

CYEM

CALL DASH

CYEM

CYEM

CYEM

CYEM

1 FORMAT $(1 X, * P S I=* E 13.5 *$ - TAUQ IS GREATER THAN VALUE PRINTED*

CYEM 
630

635

640

645

650

655

660

665

670

675

680
* average Radius*,17X,* R=*,E12.5,

CYEM

2 * METERS*,9X, * SYNCHROTRON RADIATION*,7X,*U $\theta=*, E 12.5, *$ MEV/TURN*) CYEM 3 FORMAT (* DAMPING PARTITION*16X*D=*E12.5, 16X,

1 * SIGMAX (0-COUPLING)*,7X,*SIGXO=*,E12.5,* METERS*,

CYEM

A/,* PHASE ANGLE*,19X,

CYEM

CYEM

$2 *$ PHIS $=*, E 12.5, *$ DEGREES *,8X * QUANTUM LIFETIME*, 10X,$*$ TAUQ $=*$, E12.5, CYEM 3 * SECONDS*)

CYEM

4 FORMAT (*R F FREQUENCY*,18X,* F=*,E12.5,* MHZ*,12X,* CIRCULATION CYEM 1PERIOD*, 10X, *TO=*,E12.5,* SECONDS*,/,* R F PEAK VOLTS*, 18X,*VO=*, CYEM $2 E 12.5, *$ MV*, 14X,*HORIZONTAL DAMPING*,8X,*TAUX $=*, E 12.5, *$ SECONDS $*)$ CYEM FORMAT (* ENERGY OSCILLATION PERIOD*,7X,*TE=*,E12.5, * SECONDS*, $19 \mathrm{X}, *$ PHASE WIDTH*,13X,*SIGPHI $=*$, E12.5,* RADIANS*

2* ENERGY APERTURE*,15X, *EPSM $=*$, E12.5,* GEV *,10X, 3* BUNCH LENGTH*, 14X, *SIGL=*,E12.5,* METERS*)

6

FORMAT ( * COUPLING CONSTANT*,16X, *K=*,E12.5,17X,*VERTICAL *

1 *DAMPING*, 10X, *TAUY=*, E12.5,* SECONDS*/

$265 X, *$ ENERGY DAMPING $*, 12 X, *$ TAUE $=*, E 12.5, *$ SECONDS*)

7 FORMAT( $*($ EPSM $* 2 H * *, * 2 / S I G E *, 2 H * *, * 2) / 2 *, 11 X, * \quad X I=*, E 12.5,17 X$, $2 * \operatorname{SIGMA}(D P / P) *, 14 X, * \operatorname{SIGDP}=*, E 12.5)$

$9 \operatorname{FORMAT}(/ * I 1=*, E 12.5, /, * I 2=*, E 12.5, /, * I 3=*, E 12.5, /, * I 4=*, E 12.5$, A $14 X * I 41=* E 12.5,14 X, * I 43=* E 12.5$,

$1 / . * I 5=*, E 12.5,14 X, * I 51=*, E 12.5,14 X, * I 53=* E 12.5)$

11 FORMAT (* ENERGY SPREAD*,17X,*SIGE $=*$, E12.5,* GEV*,13X, *MOMENTUM *, $1 *$ SPREAD* $, 8 X, *$ SIGBGAM $=*, E 12.5$ )

12 FORMAT $(/ * \mathrm{JX}=*, \mathrm{~F} 12.5,14 \mathrm{X}, * \mathrm{JY}=*, \mathrm{~F} 12.5,14 \mathrm{X}, * \mathrm{JE}=*, \mathrm{~F} 12.5)$

22 FORMAT (

$1 \quad * \mathrm{~J} 1=* \mathrm{~F} 12.5,14 \mathrm{X}, * \mathrm{~J} 3=* \mathrm{~F} 12.5,14 \mathrm{X}, * \mathrm{~J} 6=* \mathrm{~F} 12.5)$

CYEM

CYEM

CYEM

CYEM

CYEM

CYEM

CYEM

CYEM

CYEM

CYEM

CYEM

CYEM

CYEM

CYEM

CYEM

CYEM

CYEM

10 FORMAT (* HARMONIC NUMBER*, 18X,*H=*,E12.5,17X,*MOMENTUM*,13X, $1 *$ BETAGAMMA $=*, E 12.5 /$

2 * MOMENTUM COMPACTION*,10X,*ALPHA=*,E12.5,17X,*BETA FUNCTION*, $311 X, * B E T A X 0=*, E 12.5)$

8 FORMAT $(/ 10 X, *$ EMITTANCES (METER RADIANS) $* / 10 X, *$ EPXN $=*, E 12.5$, $17 X, * E P Y N=*, E 12.5,7 X, * E P L N=*, E 12.5 / 10 X, * E P X=*, E 12.5,7 X$,

$2 * \mathrm{EPY}=*, \mathrm{E} 12.5,8 \mathrm{X}, * \mathrm{EPL}=*, \mathrm{E} 12.5,7 \mathrm{X}, * \mathrm{EPX} \theta=*, \mathrm{E} 12.5)$

23 FORMAT $(11 \mathrm{X}, * \mathrm{EP} 1=*, \mathrm{E} 12.5,7 \mathrm{X}, * \mathrm{EP} 3=*, \mathrm{E} 12.5 /)$

13 FORMAT $(1 X, *$ CYEM*/)

14 FORMAT $(2 X * P O S * 11 X * A 11 * 9 X * A 12 * 9 X * A 22 * 9 X * A 13 * 9 X * A 14 * 9 X * A 23 * 9 X * A 24 *$ $19 X * A 33 * 9 X * A 34 * 9 X * A 44 * /$

$216 X * B 11 * 9 X * B 12 * 9 X * B 22 * 9 X * B 13 * 9 X * B 14 * 9 X * B 23 * 9 X * B 24 * 9 X * B 33 * 9 X * B 34 *$ $39 X * B 44 * /)$

15 FORMAT $(/ 1 X, 14,1 X, A 5,1 X, 10 F 12.7 / 12 X, 10 F 12.7)$

16 FORMAT ( $1 X * B E A M$ ELLIPSE MATRIX $-\operatorname{SIJ}=\operatorname{SIGMA}(\mathrm{I}, \mathrm{J}), *$ $1 * \quad \operatorname{RII}=\operatorname{SQRT}(\operatorname{SII}), \quad \operatorname{TIJ}=\operatorname{ATAN}(2 . \operatorname{SIJ} /(\operatorname{SII}-\mathrm{SJJ})) / 2 * /$ 2 $2 X * P O S * 11 X * R 11 * 9 X * T 12 * 9 X * R 22 * 9 X * T 13 *$

18 FORMAT (1X*BEAM ELLIPSE MATRIX $-\operatorname{SIJ}=\operatorname{SIGMA}(I, \mathrm{~J}) * /$ $1 \quad 2 X * P O S * 11 X * S 11 * 9 X * S 12 * 9 X * S 22 * 9 X * S 13 *$ $29 X * S 14 * 9 X * S 23 * 9 X * S 24 * 9 X * S 33 * 9 X * S 34 * 9 X * S 44 * /)$

17 FORMAT $(1 X, I 4,1 X, A 5,1 X, 10 E 12,4)$

19 FORMAT ( $1 X *$ (BETATRON PART OF SIGMA MATRIX) * ) FORMAT $(1 X, *$ NEGATIVE DAMPING CONSTANT*)

CYEM

CYEM

CYEM

CYEM

CYEM

CYEM

CYEM

CYEM

CYEM

CYEM

CYEM

CYEM

CYEM

CYEM

CYEM

CYEM

CYEM

CYEM

CYEM

CYEM

CYEM

CYEM

CYEM

CYEM

CYEM

RETURN

CYEM

CYEM

CYEM

601

602

603

604

605

606

607

608

609

610

611

612

613

614

615

616

617

618

619

620

621

622

623

624

625

626

627

628

629

630

631

632

633

634

635

636

637

638

639

640

641

642

643

644

645

646

647

648

649

650

651

652

653

654

655 
AN IF STATEMENT MAY BE MORE EFFICIENT THAN A 2 OR 3 BRANCH COMPUTED GO TO STATEMENT. AN IF STATEMENT MAY BE MORE EFFICIENT THAN A 2 OR 3 BRANCH COMPUTED GO TO STATEMENT. AN IF STATEMENT MAY BE MORE EFFICIENT THAN A 2 OR 3 BRANCH COMPUTED GO TO STATEMENT. AN IF STATEMENT MAY BE MORE EFFICIENT THAN A 2 OR 3 BRANCH COMPUTED GO TO STATEMENT. $509 \quad I$ AN IF STATEMENT MAY BE MORE EFFICIENT THAN A 2 OR 3 BRANCH COMPUTED GO TO STATEMENT, AN IF STATEMENT MAY BE MORE EFFICIENT THAN A 2 OR 3 BRANCH COMPUTED GO TO STATEMENT. 
C THIS ROUTINE...

C 1. COMPUTES $K$ CYCLED PRODUCTS OF A MATRIX PRODUCT, PROD, WHOSE

CYX

CYX

crX

CYX

2. COMPUTES AND PRINTS OUT THE BETATRON FUNCTIONS OF THE CYCLED PRODUCTS.

* ITP - WRITE TAPES OPTION IF GT $\theta$.

C IF THE ORIGINAL INSTRUCTION WAS A CYB, THEN OPTIONAL OUTPUT MAY BE

C OBTAINED ON TAPE A5.

C IF KA NEGATIVE, ONLY PRINT POSITIONS OF ELEMENTS WHOSE FIRST

C CHARACTER IS "(4-8 PUNCH)

C FOR CYA,CYC WRITE TAPE 5 OPTIONS CONTROLLED BY KA AS FOLLOWS -

CYX

CYX

CYX

$C Y X$

$\mathrm{CYX}$

CYX

CYX

CYX

$C Y X$

$C Y X$

CrX

C - NPOS, POS, NAME, L, LP, LPP, THETA, KV, KVP , KV $2, B V, B H, A V, A H, Q V, Q H, X, X P$

C $A B S(K A)=13,14-X E Q, Y E Q$ IN BCD

C $A B S(K A)=12,14,16$ - SUPPRESS PRINTING

C ABS $(K A)=15,16$ POLARIZATION PARAMETERS ON TAPE 11

C FOR BEST PLOT PARAMETRS (BX, XEQ, BY, YEQ) ON TAPE 12

C FOR FXPT BX, XCO, BY, YCO ON 12 AND POLARIZATION PARAMETERS ON 11

C IF KB.LT. $\theta$, A MATRIX CORRESPONDING TO THE REFLECTION OF THE INPUT

C BEAM LINE IS ADDED TO THAT BEAM LINE. THUS ONLY HALF OF A SYMMETRIC

C SUPERPERIOD NEED BE SPECIFIED. TUNES AND CHROMATICITIES ARE

C CALCULATED FOR THE COMPLETE SUPERPERIOD.

C **NOTE** ROUTINE USES ALL 7 SPECIAL $3 \times 3$ LOCATIONS, MEND THRU MEND-6.

C AND M7END-5.

INTEGER OP, BDAT, ELNUM

COMMON/TSW/TRSW, MCY (20) , JM, LOCC

LOGICAL TRSW

LOGICAL PFLAG

LEVEL 2, STORE, INFF, IWORK

COMMON STORE (48000), IWORK( 10$)$

DIMENSION INFF $(24,2000)$

EQUIVALENCE (INFF,STORE)

LEVEL 2, XX,Y1,Y2,Y3,Y4

COMMON XX(4000), Y1 (4000), Y2(4000), Y3(4000), Y4(4000)

COMMON/BPLTCOM/MN, KW, BXX, BYX, NPLT

COMMON/CCPOOL/XMIN, XMAX, YMIN, YMAX, CCXMIN, CCXMAX, CCYMIN, CCYMAX COMMON/CCFACT/FACTOR

COMMON/CONTRL/ERROR, MODE, RSRV, STOR , XEQ, TRASW, NFSW, EMPTY, INDEF, $1 \quad$ LDFLG, FIN LOGICAL ERROR, RSRV, STOR, XEQ, TRASW, NFSW, EMPTY, INDEF, LDFLG, FIN 
C

60

65

70

75

80

85

90

95

105

100

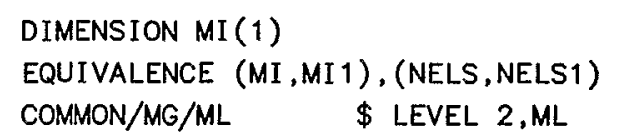

DATA IBLNK/1H /

DATA MASK/770000000000000000000B/,ZE/0./

DATA HMAGV/4HMAGV/

DATA II $/(0 ., 1$.

$\operatorname{PI} 2=8 . * A T A N(1$.

RADEG $=P I 2 / 360$.

FLG $=$. TRUE.

SKIP $=$.FALSE.

$\operatorname{ABX}(1,1)=A B \times(1,2)=A B \times(2,1)=A B \times(2,2)=0$.

$C H F L G=. F$.

IF (NOPR.AND. .NOT.GLOBAL) SKIP = .TRUE.

PRINT $=$. TRUE.

PFLAG $=$.FALSE.

\begin{tabular}{|c|c|}
\hline BMI 1 & 3 \\
\hline CYX & 42 \\
\hline CYX & 43 \\
\hline$C Y X L$ & . \\
\hline CYXL & 2 \\
\hline MATCH3 & 2 \\
\hline MATCH3 & 3 \\
\hline$c \gamma x$ & 45 \\
\hline crX & 46 \\
\hline CYX & 47 \\
\hline cyX & 48 \\
\hline CYX & 49 \\
\hline CYX & $5 e$ \\
\hline CYXMAYBS & 1 \\
\hline NOV3CYX & 1 \\
\hline NOV3CYX & 2 \\
\hline NOV3CYX & 3 \\
\hline CYX & 51 \\
\hline CYX & 52 \\
\hline DIM & 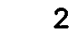 \\
\hline DIM & 2 \\
\hline SWTCH & 2 \\
\hline SWTCH & 3 \\
\hline SWTCH & 4 \\
\hline KINET & 2 \\
\hline KINET & 3 \\
\hline CYX & 56 \\
\hline CYXMPL & 1 \\
\hline cYX & 57 \\
\hline CYX & 58 \\
\hline CYX & 59 \\
\hline$C Y X$ & $6 e$ \\
\hline CYX & 61 \\
\hline CYX & 62 \\
\hline CYX & 63 \\
\hline CYX & 64 \\
\hline CYX & 65 \\
\hline CYX & 66 \\
\hline CYX & 67 \\
\hline CYX & 68 \\
\hline CYX & 69 \\
\hline CYX & 70 \\
\hline CYX & 71 \\
\hline CYX & 72 \\
\hline$C Y X$ & 73 \\
\hline CYX & 74 \\
\hline CYXMPL & 2 \\
\hline CYX & 75 \\
\hline CYX & 76 \\
\hline$C Y X$ & 77 \\
\hline CYX & 78 \\
\hline CYX & 79 \\
\hline NOV 3 CYX & \\
\hline NOV 3 CYX & 5 \\
\hline CYX & 80 \\
\hline$C Y X$ & 81 \\
\hline CYX & $8:$ \\
\hline
\end{tabular}


MVF $=$.FALSE.

CYX

83

BTABL $=$. FALSE.

CYX

IF(NELS.GT . 44) PFLAG = . TRUE.

JAN86CYX

1

ITP $=0$

CYX

86

$\mathrm{KS}=0$

120

I TAPE $=0$

CYXMAY85 2

CALL RANGET (NRN)

NOV3CYX 6

LNUM $=0$

CYX

87

CYX

IF (ERROR) RETURN

CYX

C USES WORKING STORAGE MEND-1

CYX

$K=\operatorname{INFF}(4, P R O D)$

CYX

IF (K.GE. O) GO TO 1

CYX

$\mathrm{K}=-\mathrm{K}$

CYX

FLG $=$. FALSE.

CYX

$1 \quad K A=K$

CYX

$\mathrm{KB}=\operatorname{INFF}(5, \mathrm{PROD})$

CYX

$O P=\operatorname{INFF}(1, \mathrm{PROD})$

CYX

IF (OP. NE. 3HCYB) K=NELS

CYX

98

$I P P=\operatorname{INFF}(6, P R O D)$

135

C ZERO TAB

CYX

CYX

CYX

101

DO 27 IC $=1,10$

CYX

102

$27 \mathrm{TAB}(\mathrm{IC})=0$.

CYX

103

$\mathrm{CHX}=0$.

CYX

104

$\mathrm{CHY}=0$.

CYX

105

140

$\mathrm{I} A=0$

CYX

106

$E L=0$.

CYX

107

THETH $=0$.

CYX

108

THETV $=0$.

CYX

109

$K K=K$

CYX

110

145

ISK $=0$

CYX

111

$\mathrm{NK}=0$

CYX

112

ETAST $=0$.

C $\quad \mathrm{FKK}=\mathrm{KK}$

CYX

113

$\mathrm{PR}=\mathrm{PROD}$

CYX

114

150

$\mathrm{NGO}=1$

CYX

115

$\mathrm{YCO}=\mathrm{DXCO}=\mathrm{DYCO}=0$.

CYX

$X C O=0$.

CYX620

116

CYX

NMVAR = HZEQ

CYX

117

IF (KNFLAG) NMVAR = HTEQ

CYX

118

155

IF (OP.EQ. 3 HCYB) GO TO 32

CYX

119

IF (OP.EQ. 4HFXPT) GO TO 33

CYX

120

CALL DATA(PR, 2,3,2, ISFD)

C SET CHFLG FOR SEXTUPOLE CHROM CORRECTION

IF (ISFD (1) . NE. IBLNK.OR. ISFD(2). NE. IBLNK) CHFLG=.T .

160

IF (CHFLG) CALL DATA(PR, 1,1,2,XI)

IF (OP.NE. 4HCYA) GO TO 29

NOV3CYX 7

NOV3CYX 8

NOV3CYX 9

NOV 3 CYX 10

CYX

122

CYX $\quad 123$

CYX $\quad 124$

CYX $\quad 125$

I $A=1$

CYA

CYX

126

165

$\mathrm{NK}=\mathrm{PR}-\mathrm{K}-1$

CYX

127

$29 \mathrm{MN}=$ MNAME $(\mathrm{PROD})$

CYX $\quad 128$

$I R=P R O D$

CYX

129

CALL MMM(PR, KK,MI)

CYX

130

$K W P=K K$

CYX

IF (KB.GE. O) GO TO 301

CYX

132 
175

180

185

190

195

200

205

210

215

220
IF (OP.EQ $3 \mathrm{HCYA}) \quad \mathrm{NK}=\mathrm{NK}-1$

CYX

$\mathrm{KK}=\mathrm{KK}+1$

$M I(K K)=M E N D-6$

CALL REF (MEND-6;PR)

$M L(1)=P R$

$M L(2)=M E N D-6$

CALL MMM(PR, 2,ML)

301 CONTINUE

IF (OP.EQ. 3HCYA) PR = PROD -1

IF (OP.EQ.3HCYC) GO TO 26

IF (OP.EQ.4HBEST) GO TO 28

310 CALL BET(PR, BETX,BETY, 1)

$V P(1)=\operatorname{BETX}(5)$

$\mathrm{VP}(2)=\operatorname{BETX}(6)$

$\operatorname{VP}(3)=\operatorname{BETY}(5)$

$\operatorname{VP}(4)=\operatorname{BETY}(6)$

$V P(5)=0$.

$V P(6)=1$.

$\operatorname{VP}(7)=0$.

$31 \quad K W=K K+1$

$M I(K W)=M I(1)$

IF (ITP.EQ.1) PRINT = .FALSE.

IF(ITP.EQ.2.OR. ITP.EQ.4.OR.ITP.EQ.6) PRINT=.FALSE.

$*$ SET UP PLOT OPTION

PLOT $=$. FALSE.

IF (OP.NE. 4HBEST. AND.OP.NE. 4HFXPT) GO TO 39

PLOT $=$. TRUE.

WRITE (12) MN, KW

GO TO 39

* CYB

32 ITP=IDAT (PROD, 1)

$M N=B D A T(P R O D, 1)$

$P R=E L N U M(M N)$

$\mathrm{IR}=\mathrm{PR}$

GO TO 310

C

26 IF (KA.NE.21) GO TO 28

$L Q 3=\operatorname{INFF}(24, P R)$

$\mathrm{LINE}=\mathrm{LQ3}$

BTABL $=$.TRUE.

c

$$
\text { BEST }
$$

28 CALL RXY(PR, RX, RY, RW)

$P R=M E N D-1$

CALL STXY (PR, RX,RY,RW)

GO TO 310

* 33

FXPT

$\mathrm{ISK}=1$

$\mathrm{NGO}=2$

$K A F=\operatorname{INFF}(4, P R)$

IF (KAF.LT.O) FLG = .FALSE.

$K A F=I A B S(K A F)$

CALL DATA(PR, 7, 1,7,VW)

IRAYS = IDAT $(P R, 4)$

ITAPE $=\operatorname{IDAT}(P R, 5)$

$F A C=1$.

IF (IRAYS.EQ.0.OR. IRAYS.EQ.2) $F A C=1000$.

$X O=V W(1)$

\begin{tabular}{|c|c|}
\hline CYX & \\
\hline CYX & \\
\hline CYX & \\
\hline CYX & \\
\hline CYX & \\
\hline CYX & \\
\hline CYX & \\
\hline CYX & \\
\hline CYX & \\
\hline CYX & \\
\hline CYX & \\
\hline CYX & \\
\hline CYX & 14 \\
\hline CYX & 14 \\
\hline CYX & 14 \\
\hline CYX & 14 \\
\hline CYX & 14 \\
\hline CYX & 15 \\
\hline CYX & \\
\hline CYX & \\
\hline CYX & 15 \\
\hline CYXMAY85 & \\
\hline CYX & 15 \\
\hline CYX & 15 \\
\hline cyX & 15 \\
\hline CYXNOV28 & \\
\hline cYX & \\
\hline CYX583 & \\
\hline CYX & 16 \\
\hline CYX & 161 \\
\hline CYX & 162 \\
\hline CYX & 16 \\
\hline CYX & 16 \\
\hline crx & $16 t$ \\
\hline CrX & $16 t$ \\
\hline NOV3CYX & 1 \\
\hline $\operatorname{crX}$ & 167 \\
\hline CYX & 168 \\
\hline CYX & $16 s$ \\
\hline CYX & 17 \\
\hline NOV 3 CYX & 1 \\
\hline crx & 17 \\
\hline CYX & 172 \\
\hline CYX & 173 \\
\hline CYX & 174 \\
\hline CYX & 175 \\
\hline CYX & $17 e$ \\
\hline CYX & 177 \\
\hline $\operatorname{crx}$ & 178 \\
\hline$C Y X$ & 179 \\
\hline CYX & 180 \\
\hline CYX & 18 \\
\hline CYX & 182 \\
\hline CYX683 & \\
\hline CYX & 18 \\
\hline CYX & \\
\hline CYX & 18 \\
\hline
\end{tabular}



GO TO 35

* turn on switch to terminate calculation of angle fit

CYX

CYX

CYX

CYX

CYX

CYX

CYX

CYX

CYX

CYX

CYX

CYX

CYX

CYX

CYX

CYX

CYX

CYX

CYX

CYX

CYX

cYX TRSW $=$. TRUE.

35 CONTINUE

$12 X, * X$ TRACE $=*, E 12.5,5 X, * Y$ TRACE $=*, E 12.5)$

CYX IF ((ABS (TRX).GE.2.), AND. (ABS(TRY).GE.2.).AND. (KA.GT.0)) GO TO 125 IF (IPP.GT.Q) GO TO 75

CYX

CYX

IF ((ISK.EQ.0).AND. (MODE.EQ.1).AND..NOT.NOPR) CALL DASH

CYX GO TO $(40,41)$ NGO

41 IF (.NOT.PRINT) GO TO 75

CYX

CYX

CYX

CYX

NAMFX $=\operatorname{INFF}(2$, PROD $)$

IF (PFLAG) WRITE $(3,1035)$ NAMFX

IF (.NOT.PFLAG) WRITE $(3,1029)$. NAMFX

WRITE $(3,1020)$

IF (IRAYS.NE.0.AND.IRAYS.NE.2) GO TO 75

CYX

CYX

CYX

WRITE $(3,1022)$

GO TO 75

C WRITE CYX HEADING

40 IF(PFLAG) WRITE $(3,1035)$ MN

IF(.NOT.PFLAG) WRITE $(3,1029)$ MN

IF (ITP.EQ.0.OR.MOD(ITP.2).EQ.1) WRITE(3, 1000)NMVAR

IF (ITP.EQ. 2) WRITE $(3,2002)$

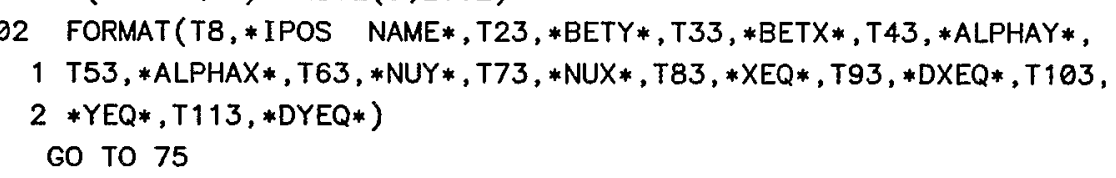

CYX

CYX

CYX

JUN27CYX

CYXJUL84

CYXJUL84

CYXJUL84

CYXJUL84

CYXJUL84

CYXJUL84

CYX

CYX 


$$
50
$$

$N I=M I(I)$

NISAVE $=\mathrm{NI}$

$M K N=\operatorname{INFF}(20, N I)$

GO TO $(505,506)$ NGO

506 NF $=$ M7END -5

$X C O=V W(1) * F A C$

$D X C O=V W(2) * F A C$

$Y C O=V W(3) * F A C$

295

300

305

310

315

320

325

330

335

340

$\operatorname{EX}=\mathrm{VP}(1)$

$\operatorname{EXP}=V P(2)$

$E Y=V P(3)$

$E Y P=V P(4)$

CALL RANGET(NRR)

$\mathrm{NI}=\mathrm{NF}$

CALL RTRV7(NI,T,RW)

CALL $M X V 7(T, V P, V P)$

$E S T=-V P(5)$

PSIX $=2 . * P S I X$

PSIY $=2 \cdot *$ PSIY

$I P O S=I-1$

MATI $=4 \mathrm{HREFL}$

GO TO $(52,508)$ NGO

$508 \mathrm{XCO}=X 0 * \mathrm{FAC}$

$D X C O=-X P O * F A C$

$Y C O=Y O * F A C$

DYCO $=-Y P O * F A C$

$E X=E X O$

EXP=-EXPO

$E Y=E Y O$

$E Y P=-E Y P O$

GO TO 52

507 CONTINUE

502 CONTINUE

504 CONTINUE
ITERATES FROM STATEMENTS 50 TO 90

$\operatorname{crX}$

crX

crx

crX

crx

crX

cyX

CYX

CYX

CYX

CYX

IF (I.GT.1) CALL RANSET(NRR)

CALL REVMAT (NF,NI, VW)

C CORRECT CALCULATION OF TRANSITION GAMMA

505 IF (I.EQ.KW) GO TO 5051

IF $($ KNFLAG $)$ EST $=$ EST $-(E L /(G A M * G A M))$

5051 CALL RXY(NI, RX, RY, RW)

IF ( ( KB .GE.0).OR. (I.LT.KW) ) GO TO 507

PSIX = PSIX + DELX/PI2

$P S I Y=P S I Y+D E L Y / P I 2$

$\operatorname{DENX}=\operatorname{BETX}(2) * \operatorname{RX}(1,1)-\operatorname{BETX}(3) * \mathrm{RX}(1,2)$

IF(DENX.EQ.0.) GO TO 502

$\operatorname{DELX}=\operatorname{ATAN2}(R X(1,2), D E N X)$

IF (DELX.LT . . . AND.RW(1).GE. O.) DELX = DELX+PI2

$\operatorname{DENY}=\operatorname{BETY}(2) * \operatorname{RY}(1,1)-\operatorname{BETY}(3) * \operatorname{RY}(1,2)$

IF (DENY.EQ.0.) GO TO 504

$\operatorname{DELY}=\operatorname{ATAN} 2(R Y(1,2), D E N Y)$

IF (DELY.LT.0. AND.RW(1).GE.0.) DELY = DELY+PI2

$\mathrm{IPOS}=\mathrm{I}-1$

IF (IPOS.NE.0) GO TO 51
CYX

cYX

crX

CYX

$\operatorname{crX}$

CYX

CYX

CYX

CYX

CYX

CYX

CYX

CYX

CYX

CYX

CYX

CYX

CYX

CYX

CYX

CYX

CYX

CYX

CYX

CYX

CYX

CYX

CYX

CYX

CYX

JUN27CYX

JUN27CYX

CYX

JUN27CYX

JUN27CYX

JUN27CYX

CYX

$C Y X$

CYX

JUN27CYX

JUN27CYX

JUN27CYX

CYX

CYX

CYX

CYX
238

239

240

241

242

243

244

245

246

247

248

249

250

251

252

253

254

255

256

257

258

259

260

261

262

263

264

265

266

267

268

269

270

271

272

273

274

275

276

277

278

3

4

281

5

6

7

292

293

294

8

9

10

303

304

305

306 
LINE $=$ LINE + 14

573 IF (NOPR) GO TO 574

CYX

357

WRITE (3, 1001) IPOS, MATI , EL,PSIX, PSIY,BETX(2), BETY (2),

CYX

358

$1 \operatorname{BETX}(5), \operatorname{BETY}(5), \operatorname{ETAST}, \operatorname{BETX}(3), \operatorname{BETY}(3), \operatorname{BETX}(6), \operatorname{BETY}(6)$

CYX

359

LNUM=LNUM+1

405

IF (MOD (LNUM, 5) . EQ.0.) WRITE $(3,1006)$

574 ETAST $=$ EST

GO TO 53

59 IF (IPOS.EQ.0) GO TO 58

591 WRITE $(3,1030)$

410

WRITE $(3,1020)$

592 IF (IRAYS.NE.O.AND. IRAYS.NE.2) GO TO 58

WRITE $(3,1022)$

58 WRITE $(3,1021)$ IPOS, MATI , EL, PSIX,PSIY,BETX(2), BETY(2),

$1 \quad \operatorname{BETX}(3), \operatorname{BETY}(3), E X, E X P, E Y, E Y P, X C O, D X C O, Y C O, D Y C O$

415

LNUM=LNUM+1

IF (MOD (LNUM, 5) .EQ.0.) WRITE $(3,1006)$

53 CONTINUE

IF (ITAPE. LT.1) GO TO 5333

$K V=0$.

420

IF (NAM.EQ. 3HMAG) $K V=-G K$

$\mathrm{DKV}=0$

IF (NAM. EQ . 4HSXTP) DKV=-GK2

WRITE (5) KK, IPOS, MATI, SLENI , ZE, ZE, THETI , KV, DKV, ZE,

425

1

BETY (2), BETX (2), BETY (3), BETX (3), PSIY, PSIX, A, AP

2 , AV, AVP

WRITE(5) EX, EXP, EY, EYP, XCO,DXCO, YCO, DYCO

5333 CONTINUE

IF (IPOS.EQ.0) GO TO 54

IF (SKIP) GO TO 54

430

CALL MAXMIN (BXMX,BXMN,BETX(2), MBX, IPOS)

CALL MAXMIN (BYMX, BYMN,BETY (2), MBY, IPOS)

CALL MAXMIN (XQMX, XQMN,BETX(5), MXQ, IPOS)

CALL MAXMIN (YQMX, YQMN,BETY(5), MYQ, IPOS)

CYX

360

CYX

361

DEC85CYX 2

CYX 362

CYX $\quad 363$

CYX 364

NOV $3 C Y X \quad 24$

NOV3CYX 25

MY3CYX 8

CYX $\quad 367$

CYX 368

CYX 369

CYX 370

DEC85CYX 3

CYXJN83 1

CYXNOV28 2

CYXNOV28 3

CYXNOV28 4

CYXNOV28 5

CYXNOV28 6

CYXNOV28 7

CYXNOV28 8

CYXNOV28 9

CYXNOV28 10

CYXNOV28 11

CYXJN83 2

CYX $\quad 372$

CYX $\quad 373$

CYX $\quad 374$

CYX $\quad 375$

CYX $\quad 376$

IF (NGO.EQ.1) GO TO 54

435

CALL MAXMIN(XCMX, XCMN, XCO, MCXX, IPOS)

CYX

377

CALL. MAXMIN (YCMX, YCMN, YCO, MCYY, IPOS)

CYX

378

$X X(I)=E L \$ Y 1(I)=B E T X(2) \$ Y 2(I)=X C O \$ Y 3(I)=B E T Y(2) \quad \$ Y 4(I)=Y C O$

CYX

379

$54 \quad E L L=E L$

IF(NGO.EQ.2) GO TO 622

CYX $\quad 380$

CYX 381

CYX $\quad 382$

440

STORE FOR PLOT FILE ON OPTION

IF (.NOT.PLOT) GO TO 56

$X X(I)=E L$

$Y 1(I)=B E T X(2)$

445

$Y 2(I)=\operatorname{BETX}(5)$

$Y 3(I)=\operatorname{BETY}(2)$

$Y 4(I)=\operatorname{BETY}(5)$

GO TO 55

56 CONTINUE

450

IF (ITP.EQ.0) GO TO 55

IF (ITP.GT.2) GO TO 62

CYX

382

CYX $\quad 384$

CYX $\quad 385$

CYX $\quad 386$

CYX $\quad 387$

CYX $\quad 388$

CYX $\quad 389$

CYX $\quad 390$

CYX $\quad 391$

CYX $\quad 392$

CYX $\quad 393$

$\mathrm{KV}=0$.

CYX $\quad 394$

IF (NAM.EQ. 3HMAG) $K V=-G K$

$\operatorname{crX}$

$D K V=0$.

CYX 396

IF (NAM. EQ . 4HSXTP) DKV $=-G K 2$

CYX

IF(NGO.EQ.2) GO TO 63 
460

465

470

475

485

495

500

505

510
$A=B E T X(5)$

$A P=B E T X(6)$

$A V=B E T Y(5)$

$A V P=\operatorname{BETY}(6)$

GO TO 64

$63 A=V W(1) / D P P$

$A P=V W(2) / D P P$

64 CONTINUE

WRITE (5) KK, IPOS, MATI , SLENI, ZE, ZE, THETI , KV, DKV, ZE,

1

2 $\operatorname{BETY}(2), \operatorname{BETX}(2), B E T Y(3), B E T X(3), P S I Y, P S I X, A, A P$ , AV, AVP

IF (ITP.EQ.2) GO TO 55

IF (.NOT.FLG.AND.MATJ.NE. 1 L". AND. IPOS.NE.KK) GO TO 55

WRITE $(3,2)$ KK, IPOS, MATI , BETY (2), BETX (2), BETY (3), BETX (3),

1 PSIY, PSIX, A, AP , AV, AVP

2 FORMAT (1X2I5, 1XA5, 10F10.4)

GO TO 55

62 IF(ITP.GE.5) GO TO 622

IF (I.EQ.KW) GO TO 55

WRITE $(5,1002)$ MN, IPOS, KK, EL, BETX(5), BETY (5), BETX(7),

$X$ BETY (7)

GO TO 55

C WRITE POLARIZATION PARAMETERS ON TAPE 11

$622 \mathrm{RHOI}=0$.

$\mathrm{GK}=0$.

$X I 1=0$.

$X I 2=0$.

IF (IPOS.EQ.0) GO TO 3333

$N A N=\operatorname{INFF}(1, M K)$

IF (NAM.EQ. 4HMOVE) GO TO 3335

IF (NAM.EQ. 3HMAG) GO TO 3336

GO TO 3333

3335 MMK=MDAT $(M K, 1)$

$\operatorname{IF}(\operatorname{INFF}(1, M M K)$.NE. 4HMAG ) GO TO 3333

$M K=M M K$

3336 CONTINUE

CALL DATA(MK, $1,1,6$, PRM)

$\mathrm{RHOI}=\mathrm{PRM}(4) / \mathrm{PRM}(3)$

$\mathrm{GK}=-\mathrm{PRM}(2) / \mathrm{PRM}(3)$

$X I 1=P R M(5) \quad \$ \quad X I 2=P R M(6)$

IF (IX1.EQ.1H\$.OR.IX2.EQ.1H\$) GO TO 3334

$X I 1=X I 1 * R A D E G$

$X I 2=X I 2 * R A D E G$

GO TO 3333

$3334 \times I 1=P R M(1) * R H O I / 2$.

$X I 2=X I 1$

3333 CONTINUE

IF (I.EQ.1) WRITE(11) KW

$Z Z=0$.

IF (NGO.EQ.2) ZZ = YCO/FAC

WRITE (11) ELL, PSIY,BETY(2), RHOI, GK, XI1, XI2, ZZ

$55 E L=E L+R W(1)$

COMPUTE LENGTH

$60 \quad I=I+1$

IF (I.GT.KW) GO TO 90

SLENI $=$ RW $(1)$

THETI $=$ RW(2)
CYX

CYX

CYX

CYX

CYX

CYX

CYX

CYX

CYX

CYX

CYX

CYXMAY85

CYXJUL84

CYXJUL84

CYXJUL84

CYXJUL84

CYX

CYX

CYX

CYX

CYX

CYX

CYX

CYX

CYX

CYX

CYX

CYX

CYX

CYX883

CYX

CYX

CYX

CYX

CYX

CYX

CYX

CYX

CYX

CYX

CYX

CYX

CYX

CYX

CYX

CYX

CYX

CYX

CYX

CYX

CYX

CYX

CYX

CYX

CYX

CYX

CYX
400

401

402

403

404

405

406

407

408

409

410

4

7

8

9

10

415

416

417

418

419

420

421

422

423

424

425

426

427

4

429

430

431

432

433

434

435

436

437

438

439

440

441

442

443

444

445

446

447

448

449

450

451

452

453

454

455 
$N A M=\operatorname{INFF}(1, N I)$

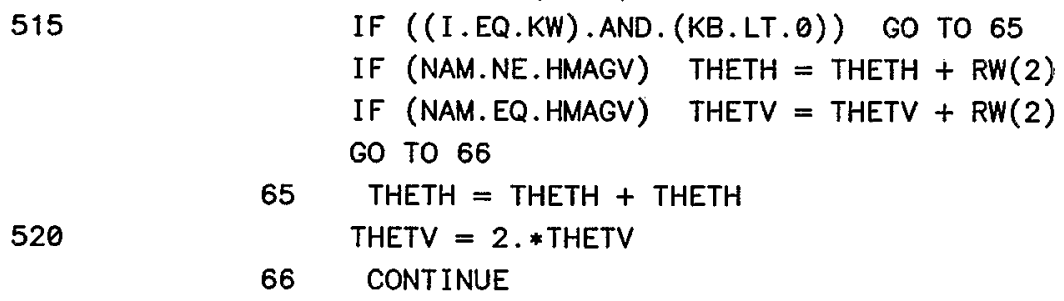

C COMPUTE BETATRON FUNCTIONS FOR THE I-TH CYCLED MATRIX

C IN CYA CASE, DO SIMILARITY TRANSFORMATION AND CALL BET

C IN OTHER CASES, COMPUTE NEW BETA FUNCTIONS DIRECTLY IF (OP.NE. 3HCYA) GO TO 7503

$$
\begin{aligned}
& M L(1)=-N I \\
& M L(2)=P R+I A \\
& M L(3)=N I
\end{aligned}
$$

7503 CONTINUE

DO $555 \mathrm{~J}=1,6$ $\operatorname{BXP}(J \mathrm{~J})=\operatorname{BETX}(\mathrm{J} J)$

$555 \operatorname{BYP}(J \mathrm{~J})=\mathrm{BETY}(\mathrm{J} J)$

$A X S=B E T X(3)$

$A Y S=B E T Y(3)$

$\mathrm{SS}=\mathrm{RW}(1)$

75 IF (I.EQ. 1.OR.OP.EQ. 3HCYA) CALL BET(PR, BETX,BETY, 1)

IF (I.EQ.1) GO TO 775

IF (SKIP) GO TO 775

IF (OP.EQ. 3HCYA) GO TO 7501

IF $(B X P(2) . E Q .0$.$) GO TO 7502$

$\operatorname{BETX}(3)=(1 .+2 . * \mathrm{RX}(1,2) * \mathrm{RX}(2,1)) * \operatorname{BXP}(3)-\mathrm{RX}(1,1) * \mathrm{RX}(2,1) * \mathrm{BXP}(2)$

$1-R \times(1,2) * R X(2,2) * \operatorname{BXP}(4)$

$\operatorname{BETX}(2)=(\mathrm{RX}(1,2) * * 2+(\mathrm{RX}(1,1) * \mathrm{BXP}(2)-\mathrm{RX}(1,2) * \mathrm{BXP}(3)) * * 2) / \operatorname{BXP}(2)$

7502 IF (BYP(2).EQ.0.) GO TO 7501

$\operatorname{BETY}(3)=(1 .+2 . * \mathrm{RY}(1,2) * \mathrm{RY}(2,1)) * \operatorname{BYP}(3)-\mathrm{RY}(1,1) * \mathrm{RY}(2,1) * \operatorname{BYP}(2)$

555

560

\section{1} $1-\mathrm{RY}(1,2) * \mathrm{RY}(2,2) * \mathrm{BYP}(4)$

$\operatorname{BETY}(2)=(\operatorname{RY}(1,2) * * 2+(\operatorname{RY}(1,1) * \operatorname{BYP}(2)-\operatorname{RY}(1,2) * \operatorname{BYP}(3)) * * 2) / \operatorname{BYP}(2)$

$\operatorname{BETY}(4)=(1 .+\operatorname{BETY}(3) * * 2) / \operatorname{BETY}(2)$

$\operatorname{BETY}(5)=\operatorname{RY}(1,1) * \operatorname{BYP}(5)+\operatorname{RY}(1,2) * \operatorname{BYP}(6)+\operatorname{RY}(1,3)$

$\operatorname{BETY}(6)=\operatorname{RY}(2,1) * \operatorname{BYP}(5)+R Y(2,2) * \operatorname{BYP}(6)+R Y(2,3)$

ONTINUE

$N A M=\operatorname{INFF}(1, N I)$

IF (NAM.EQ. 4HSXTP) GO TO 785

CONT INUE

IF (NAM.NE. 4HKICK.AND.NAM.NE.4HMOVE) GO TO 765

NKIK=MDAT $(N I, 1)$

NOP $=\operatorname{INFF}(1$, NKIK $)$

IF (NOP.NE. 3HMAG.AND.NOP.NE. 4HMAGV) GO TO 765

IF (MKN.EQ. 3HMAP) GO TO 775

CALL DATA(NKIK, 1, 1,6,PRM)

\begin{tabular}{lr} 
CYX & 456 \\
CYX & 457 \\
CYX & 458 \\
CYX & 459 \\
CYX & 460 \\
CYX & 461 \\
CYX & 462 \\
CYX & 463 \\
CYX & 464 \\
CYX & 465 \\
JUN27CYX & 13 \\
JUN27CYX & 14 \\
JUN27CYX & 15 \\
CYX & 466 \\
CYX & 467 \\
CYX & 468 \\
CYX & 469 \\
CYX & 470 \\
JUN27CYX & 16 \\
CYX & 471 \\
CYX & 472 \\
CYX & 473 \\
CYX & 474 \\
CYX & 475 \\
CYX & 476 \\
JUN27CYX & 17 \\
CYX & 478 \\
CYX & 479 \\
CYX & 480 \\
CYX & 481 \\
JUN27CYX & 18 \\
JUN27CYX & 19 \\
JUN27CYX & 20 \\
JUN27CYX & 21 \\
JUN27CYX & 22 \\
JUN27CYX & 23 \\
JUN27CYX & 24 \\
JUN27CYX & 25 \\
JUN27CYX & 26 \\
JUN27CYX & 27 \\
JUN27CYX & 28 \\
JUN27CYX & 29 \\
JUN27CYX & 30 \\
JUN27CYX & 31 \\
JUN27CYX & 32 \\
JUN27CYX & 33 \\
CYX & 482 \\
CYX & 483 \\
CYX & 484 \\
CYX & 488 \\
CYX & 489 \\
CYX & 490 \\
CYXX & 491 \\
CYX & 492 \\
& 493 \\
\hline
\end{tabular}


IF (NOP.EQ . 4HMAGV) MVF=.TRUE.

CYX

GO TO 770

765 IF (NAM.NE.4HMAG . AND.NAM.NE. 4HMAGV) GO TO 775

CALL DATA(NI $1,1,6$, PRM)

$\mathrm{GK}=\mathrm{PRM}(2) / \mathrm{PRM}(3)$

IF (NAM.EQ. 4HMAGV) MVF $=$. TRUE.

$N Z=N I$

IF (NAM.EQ.4HKICK.OR.NAM.EQ. 4HMOVE) NZ=NKIK

580

$R I=P R M(4) / P R M(3)$

$X I 1=P R M(5)$

$X I 2=P R M(6)$

IF (IX1.EQ.1H\$.OR.IX2.EQ.1H\$) GO TO 7701

$\mathrm{TH} 1=\mathrm{XI} 1 * \mathrm{RADEG}$

585

$\mathrm{TH} 2=X 12 * \mathrm{RADEG}$

GO TO 7702

$7701 \mathrm{TH} 1=\mathrm{PRM}(1) * \mathrm{RI} / 2$.

$\mathrm{TH} 2=\mathrm{TH} 1$

7702 TAN $1=$ TAN $($ TH1 $)$

590

TAN2=TAN $($ TH2)

IF (MVF) GO TO 7703

CALL CHRM(GK, RI, SS, TAN1, TAN2, BETX, BETY, BXP, BYP, CRX, CRY)

GO TO 786

595

$7703 \mathrm{MVF}=$.FALSE.

CALL CHRM(GK, RI, SS, TAN1, TAN2, BETY, BETX, BYP, BXP, CRY, CRX)

GO TO 786

785 CALL DATA(NI, 1,1,4,PRM)

SXLEN=PRM (1)

GK2=PRM(2)/PRM(3)

600

IF (NAM.NE. 4HSXTP) GO TO 5601

C STORE PARAMETERS FOR NONLINEAR AMPLITUDE CALCULATION

$\mathrm{DKV}=-\mathrm{GK} 2$

$K S=K S+1$

$\mathrm{AF}=\mathrm{DKV} * \mathrm{SQRT}(\mathrm{BETX}(2))$

605

610

615

IF (SLENI .NE. 0.$) \quad A F=A F * S L E N I$

$A S(K S)=A F * B E T X(2) \$ B S(K S)=A F * B E T Y(2)$

5601 CONTINUE

$\operatorname{PSX}(K S)=P S I X \$ P S Y(K S)=P S I Y$

CRX $=2 . *(B E T X(3)-A X S) \quad \$ \quad C R Y=2 . *(B E T Y(3)-A Y S)$

$\operatorname{KAS}=\operatorname{INFF}(4, N I) \$ \operatorname{KBS}=\operatorname{INFF}(5, N I)$

IF (KAS.EQ.0) KBS $=0 \$$ IF (KAS.EQ.0) KAS $=3$

$M P=K A S-3$

IF (MP.LT.O) GO TO 786

$Z C O=((X C O+S X L E N * D X C O / 2)+.I I *(Y C O+S X L E N * D Y C O / 2)) / 1000$.

$\mathrm{ZP}=\mathrm{BETX}(5)-\mathrm{SXLEN} * \mathrm{BETX}(6) / 2$. + II $*(\operatorname{BETY}(5)-\operatorname{SXLEN} * B E T Y(6) / 2$.)

$B X C=B E T X(2)+\operatorname{SXLEN} *(B E T X(3)+B E T X(4) * S X L E N / 4$.

$B Y C=B E T Y(2)+S X L E N *(B E T Y(3)+B E T Y(4) * S X L E N / 4$.

$Z G=-2 . * Z P * G K 2 \$ I F(K B S . E Q .1) \quad Z G=I I * Z G$

IF (SXLEN.NE.0.) ZG=ZG*SXLEN

620

IF (MP.EQ.0) GO TO 7852

DO $7851 \mathrm{MM}=1, \mathrm{MP}$

$7851 \mathrm{ZG}=\mathrm{ZG} * \mathrm{ZCO} / \mathrm{MM}$

$7852 \mathrm{CRX}=\mathrm{CRX}$

$$
+\operatorname{REAL}(Z G) * B \times C
$$

$C R Y=C R Y-R E A L(Z G) * B Y C$

$\mathrm{CHX}=\mathrm{CHX}+\mathrm{CRX}$

$\mathrm{CHY}=\mathrm{CHY}+\mathrm{CRY}$

CONT INUE

cYX

CYX 498

CYX $\quad 499$

CYX 500

CYX 501

CYX $\quad 502$

CYX 503

CYX 504

CYX $\quad 505$

CYX $\quad 506$

CYX $\quad 507$

CYX $\quad 508$

CYX $\quad 509$

CYX $\quad 510$

CYX $\quad 511$

CYX $\quad 512$

CYX $\quad 513$

CYX $\quad 514$

CYX $\quad 515$

CYX $\quad 516$

CYX $\quad 517$

CYX $\quad 518$

CYX $\quad 519$

CYX 520

CYX 521

CYX $\quad 522$

CYX $\quad 523$

CYX 526

CYXMAY85 5

CYXMAY85 6

CYXMAY85 7

CYXMAY85 8

CYXMAY85 9

CYXMAY85 10

CYXMAY85 11

CYXMAY85 12

CYXMAY85 13

CYXMPL 3

CYXMPL 4

CYXMPL 5

CYXMPL 6

CYXMPL 7

CYXMPL 8

CYXMPL 9

CYXMPL 10

CYXMPL 11

CYXMPL $\quad 12$

CYXMPL 13

CYXMPL 14

CYXMPL 15

CYXMPL $\quad 16$

CYXMPL 17

CYXMPL 18

CYX 530

CYX $\quad 531$

CYX $\quad 532$ 
C CHANGE INDEX TO SAVE CYCLED MATRICES IF CYA INSTRUCTION.

CYX

CYX

COMPLETES LOOP FROM STATEMENTS 50 TO 90

CYX

CYX

91 CONTINUE

$\operatorname{TKB}=\operatorname{IABS}(K B)$

$S(I)=0$.

$$
\text { DO } 99 \mathrm{~J}=1,2
$$

$99 S(I)=S(I)+A B X I(I, J) * B(J)$

C

APPLY CHROM CORRECTION TO SECOND SEXT PARAMETER

DO $95 \mathrm{I}=1,2$

$\operatorname{MSFD}(I)=\operatorname{ELNUM}(\operatorname{ISFD}(I))$

MSS=MSFD (I) \$ FF=FDAT(MSS, 1)

IF (FF.EQ.0.) $F F=1$. 


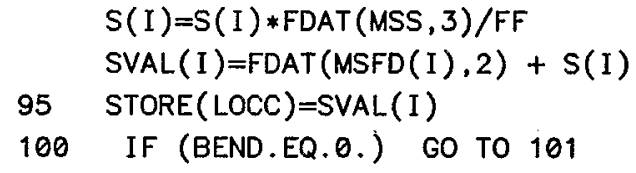

690

IF (ALPHA.EQ.0.) GO TO 101

TGAM2 $=1 . / A L P H A$

TGAM = CSQRT (TGAM2)

TTGAM = AIMAG $($ TGAM $)$

IF (TTGAM.NE.0.) TAB(6) = -TTGAM

695

TTGAM = REAL (TGAM)

IF (TTGAM.NE.0.) TAB(6) = TTGAM

GO TO 102

$101 \mathrm{TGAM}=0$.

102 IF (IPP.GT.0) GO TO 103

IF (NOPR) GO TO 104

WRITE $(3,1008)$ CIRC, THETH, XNUX, CHRX, RLEN, THETV, YNUY, CHRY

CY4AP

NOV3CYX

NOV $3 C Y X$

CYX

CYX

CYX

CYX

CYX

CYX

CYX

CYX

CYX

CYX

CYX

CYX

CYX

CYX

CYX

IF (BEND.NE.0) WRITE $(3,1026)$ ALPHA, TGAM

IF (BEND.EQ.0) WRITE $(3,1025)$ ALPHA

705

104 IF (.NOT.KNFLAG) GO TO 105

CCBET $=\mathrm{CC} * \mathrm{BETT}$

$T 0=0$.

IF (CCBET.NE.O.) TO = ELL/CCBET

$E T A=A L P H A-(1 . /($ GAM*GAM $))$

IF (NOPR) GO TO 210

710

715

720

WRITE $(3,1028)$ ETASTOT,TO,ETA

105 IF (NOPR) GO TO 210

LABEL = HMAX

WRITE $(3,1005)$

WRITE $(3,1007)$ LABEL, MBX (1), BXMX,MBY (1),BYMX,MXQ(1), XQMX,MYQ(1),

1 YQMX

LABEL $=$ HMIN

WRITE $(3,1007)$ LABEL, MBX(2), BXMN, MBY (2), BYMN, MXQ(2), XQMN, MYQ(2),

1 YQMN

IF(NGO.EQ.2) WRITE(3,1051) $\operatorname{MCXX(1),XCMX,MCYY(1),YCMX,MCXX(2),XCMN,~}$ $1 \mathrm{MCYY}(2), Y C M N$

1051 FORMAT (* MAXIMA*6X*XCO $(* I 4, *)=* F 10.5,5 X * Y C O(* I 4, *)=* F 10.5 /$

$1 * \operatorname{MINIMA} * 6 \mathrm{X} * X \mathrm{CO}(* \mathrm{I} 4, *)=* \mathrm{~F} 10.5,5 \mathrm{X} * \mathrm{YCO}(* I 4, *)=* \mathrm{~F} 10.5)$

IF(CHFLG) WRITE( 3,1045$) \mathrm{S}(1), \mathrm{S}(2), \operatorname{SVAL}(1), \operatorname{SVAL}(2)$

1045 FORMAT $(/ / *$ SEXTUPOLE CORRECTIONS - DKSF $=*$ E15.8,

$1 * \quad \mathrm{DKSD}=* \mathrm{E} 15.8, * \quad \mathrm{KSF}=* \mathrm{E} 15.8, * \quad \mathrm{KSD}=* \mathrm{E} 15.8)$

IF (ISK.EQ.O) CALL DASH

IF(KS.EQ.0) GO TO 210

IF (KB.GE.0) GO TO 410

DO $411 \mathrm{I}=1, \mathrm{KS}$

$$
K T=K S+K S+1-I
$$

$A S(K T)=A S(I) \$ B S(K T)=B S(I)$

$P S X(K T)=P S I X-P S X(I) \quad \$ P S Y(K T)=P S I Y-P S Y(I)$

411 CONTINUE

$\mathrm{KS}=\mathrm{KS}+\mathrm{KS}$

$410 \quad P I=P I 2 / 2$.

$\mathrm{PX}=\mathrm{PI} * \mathrm{PSIX} \$ \mathrm{PY}=\mathrm{PI} * \mathrm{PSIY}$

$\mathrm{PX}=3 . * \mathrm{PX} \$ \mathrm{PCP}=\mathrm{PY}+\mathrm{PY}+\mathrm{PX} \$ \mathrm{PCM}=\mathrm{PY}+\mathrm{PY}-\mathrm{PX}$

$\operatorname{SX} 1=\operatorname{SIN}(P X) \$ S \times 3=\operatorname{SIN}(P X 3) \$ \operatorname{SP}=\operatorname{SIN}(P C P) \$ S M=S I N(P C M)$

$\mathrm{H} 11=\mathrm{H} 12=\mathrm{H} 22=0$.

DO $402 \mathrm{I}=1$, KS

CYX

DO $402 \mathrm{~J}=1, \mathrm{KS}$

CYX $\quad 584$

CYX 585

CYX $\quad 586$

CYX $\quad 587$

CYX 588

CYX $\quad 589$

CYX 590

CYX $\quad 591$

CYX 592

CYX 593

CYX $\quad 594$

CYX 595

CYX 596

CYX $\quad 597$

CYX 598

CYX $\quad 599$

CYX 600

CYX 601

CYX 602

CYX 603

NOV3CYX 47

NOV3CYX 48

NOV 3 CYX $\quad 49$

604

CYXMAY85 14

CYXMAY85 15

CYXMAY85 16

CYXMAY85 17

CYXMAY85 18

JAN86CYX 2

CYXMAY85 20

CYXMAY85 21

CYXMAY85 22

CYXMAY85 23

CYXMAY85 24

CYXMAY85 25

CYXMAY85 26

CYXMAY85 27

CYXMAY85 28 
$402 \mathrm{H} 22=\mathrm{H} 22+\mathrm{BB} *(\mathrm{CP}-\mathrm{CM}+4 . * \mathrm{C} 1)$

CYXMAY85 34

CYXMAY85 35

CYXMAY85 36

$\mathrm{UU}(1)=\mathrm{TKB} * \mathrm{H} 11 / \mathrm{PI} 2 / 64$.

UU(2) $=$ TKB $* H 12 /$ PI $2 / 32$.

CYXMAY85

$\mathrm{UU}(3)=\mathrm{TKB} * \mathrm{H} 22 / \mathrm{PI} 2 / 64$.

CYXMAY85

DO $420 \mathrm{I}=1,3$

CYXMAY85

WRITE $(3,404)$ XNUX, LS(1),UU(1), LS(2), UU(2), YNUY, LS(2),UU(2), LS(3) $1, \mathrm{UU}(3)$

404 FORMAT (* AMPLITUDE DEPENDENCE OF TUNES DUE TO SEXTUPOLES*/

GO TO 210

C PRINT TAB VALUES

103 IF (NOPR) GO TO 210

WRITE $(3,1112) \operatorname{TAB}(1), \operatorname{TAB}(3), \operatorname{TAB}(4), \operatorname{TAB}(2), \operatorname{TAB}(5), \operatorname{TGAM}$

$210 \mathrm{KNFLAG}=$.FALSE.

IF (.NOT.BTABL) GO TO 216

LINE = LINE - 1

DO 215 ICY $=1,18$

215 STORE (LINE+ICY) = GL (ICY)

216 IF (NGO.EQ.2) RETURN

CALL STDAT(PROD, 7, 1, 10, TAB)

RETURN

C ERROR EXIT

125 CONTINUE

IF (IPP.GT.0) GO TO 210

WRITE $(3,1009)$ MN, TRX, TRY

* COMPUTE AND PRINT LENGTH

$K E=K W-1$

$\mathrm{EL}=0$.

780

$$
\text { DO } 127 \text { IK }=1, K E
$$

$M 1=M I(I K)$

CALL DATA $(M 1,5,1,1, F L)$

$E L=E L+F L$

CYX

CYX

CYX

CYX

CYX

CYX

$c Y X$

CYX

CYX

CYX

CYX

CYX

$\mathrm{CYX}$

CYX

CYX

CYX

cYX

CYX

cYX

CYX

CYX

CYX

CYX

CYX

CYX

WRITE $(3,1010)$ EL

GO TO 210

CYX

CYX

CYX

CYX

$*$

1009 FORMAT $(/ / 1 \mathrm{H}, 4 \mathrm{X}, 43 \mathrm{H} * * *$ * BETATRON FUNCTIONS UNSTABLE THROUGH ,

CYX

$1 \mathrm{~A} 5 / 5 \mathrm{X}, * \mathrm{X}$ TRACE $=*, E 12.5,10 \mathrm{X}, * Y$ TRACE $=*, E 12.5)$

CYX

CYX

1010 FORMAT ( $10 \mathrm{X}, *$ TOTAL BEAM LENGTH $=*, \mathrm{~F} 10.5)$

1000 FORMAT (

$12 X, * P O S *, 10 X, * S(M) *, 4 X, * N U X *, 5 X, * N U Y *, 6 X, * B E T A X(M) *, 4 X$,

CYX

CYX

2 *BETAY $(M) *, 5 X, * X E Q(M) *, 5 X, * Y E Q(M) *, 3 X, A 4, *(M) *, 3 X, * A L P H A X *$,

$34 X, * A L P H A Y *, 5 X, * D X E Q *, 5 X, * D Y E Q * / 1 X, 131(1 \mathrm{H}-))$

CYX

CYX

CYXMAY85

CYX

1002 FORMAT (A5, 213,5 F 14.8$)$

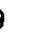

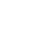

(2)

(2)

35

7

8

0

2

3

45

6


800

805

810

815

820

825

830
1005 FORMAT $(2 \mathrm{H})$

1006 FORMAT $(1 X)$

1007 FORMAT (2X,A10,*-- *, 1X,*BETX $(*, I 4, *)=*, \mathrm{~F} 12.5,4 X, * \operatorname{BETY}(*, \mathrm{I} 4$,

$1 *)=*, F 12.5,5 X, * X E Q(*, I 4, *)=*, F 12.5,5 X, * Y Y E Q(*, I 4$,

*) $=*, F 12.5$ )

1008 FORMAT (/

$12 X, *$ CIRCUMFERENCE $=* \mathrm{~F} 11.4, * \mathrm{M} *, 9 \mathrm{X}, *$ THETX $=*, \mathrm{~F} 12.8$,

$21 \mathrm{X}, * \mathrm{RAD} *, 6 \mathrm{X}, * \mathrm{NUX}=*, \mathrm{~F} 10.5,6 \mathrm{X}, * \mathrm{DNUX} /(\mathrm{DP} / \mathrm{P})=*, \mathrm{~F} 10.5 /$

$39 X *$ RADIUS $=* F 10.4, * M * 10 X *$ THETY $=* F 12.8$,

$41 \mathrm{X}, * \mathrm{RAD} *, 6 \mathrm{X}, * \mathrm{NUY}=*, \mathrm{~F} 10.5,6 \mathrm{X}, * \mathrm{DNUY} /(\mathrm{DP} / \mathrm{P})=*, \mathrm{~F} 10.5)$

1111 FORMAT $(15 X, *$ TGAM $=(*, F 10.5, *, *, F 10.5, *) *)$

1112 FORMAT (5X,*BETATRON FUNCTIONS*,7X, *MAX BETX $=*, F 10.5,4 X, *$ MAX XEQ CYX $1=*, F 10.5,4 X, * N U X=*, F 10.5 / 30 X, *$ MAX BETY $=*, F 10.5,28 X, * N U Y=*$,

2 F10.5/30X, *TRANSITION GAMMA $=(*, F 10.5, *, *, F 10.5, *) *)$

1900 FORMAT (2X,*CHROMATICITIES FROM QUADRUPOLES EXPLICITLY APPEARING* $1 *$ IN *A5, *-DNUX/(DP/P) $=*, F 10.4,3 X, * D N U Y /(D P / P)=*, F 10.4)$

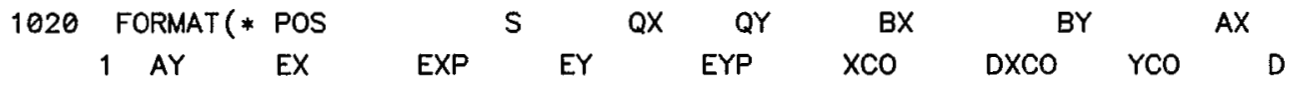
2YCO*)

1022 FORMAT (*

1

(M)

(M)

(M)

(M)

(MM)

(M)

2(MR)*)

1021 FORMAT (1XI4, 1XA5, F8.2,2F6.2,2F10.4, 2F6.2,8F8.4)

1025 FORMAT (* (DS/S) $/(D P / P)=*, F 10.7)$

1026 FORMAT $(* \quad(D S / S) /(D P / P)=*, F 10.7$

$14 \mathrm{X}, *$ TGAM $=(*, \mathrm{~F} 10.5,1 \mathrm{H}, \mathrm{F} 10.5,1 \mathrm{H}))$

1028 FORMAT $(2 X, *(D T / T) /(D P / P)=*, F 10.5,14 X, * T 0=*, E 12.5, * S E C *$,

$16 \mathrm{X}, * \mathrm{ETA}=1 /$ TGAMSQ $-1 / \mathrm{GAMSQ}=*, \mathrm{~F} 10.5)$

1029 FORMAT $(/ 1 X, *$ BETATRON FUNCTIONS OF *,A5/)

1030 FORMAT (1H1)

1035 FORMAT ( 1 H1, *BETATRON FUNCTIONS OF *,A5)

1040 FORMAT ( $1 \mathrm{H} 1,1 \mathrm{X}$,

$11 X, * P O S *, 10 X, * S(M) *, 4 X, * N U X *, 5 X, * N U Y *, 6 X, * \operatorname{BETAX}(M) *, 4 X$,

$2 * \operatorname{BETAY}(M) *, 5 X, * X E Q(M) *, 5 X, * Y E Q(M) *, 3 X, A 4, *(M) *, 3 X, * A L P H A X *$,

$34 X, *$ ALPHAY*, 5X, *DXEQ*, 4X,*DYEQ*/1X,131(1H-))

END
CYX

CYX

CYX

CYX

CYX

CYX

CYXMAY85

CYX

CYX

CYX

CYX

CYX

CYX

CYX

643

644

645

646

647

648

49

650

651

652

653

654

655

656

657

658

CYXF 1

CYXF.

CYXF

CYXF

CYXF

CYXF

CYXF

CYX

CYX

CYX

CYX

CYX

CYX

CYX

CYX

CYX

CYX

CYX

CYX

cYX

NOT ALL ITEMS IN THIS COMMON BLOCK OCCUR IN LEVEL STATEMENTS. AN IF STATEMENT MAY BE MORE EFFICIENT THAN A 2 OR 3 BRANCH COMPUTED GO TO STATEMENT AN IF STATEMENT MAY BE MORE EFFICIENT THAN A 2 OR 3 BRANCH COMPUTED GO TO STATEMENT AN IF STATEMENT MAY BE MORE EFFICIENT THAN A 2 OR 3 BRANCH COMPUTED GO TO STATEMENT AN IF STATEMENT MAY BE MORE EFFICIENT THAN A 2 OR 3 BRANCH COMPUTED GO TO STATEMENT AN IF STATEMENT MAY BE MORE EFFICIENT THAN A 2 OR 3 BRANCH COMPUTED GO TO STATEMENT AN IF STATEMENT MAY BE MORE EFFICIENT THAN A 2 OR 3 BRANCH COMPUTED GO TO STATEMENT 
1

$$
\text { SUBROUTINE DASH }
$$

CYX

C WRITES ONE LINE OF DASHES

DASH WRITE $(3,2)$

5

FORMAT $(1 \mathrm{X}, 131(1 \mathrm{H}-))$

DASH

DASH

DASH

DASH

RETURN

DASH

7


SUBROUTINE DATA $(M, I, J, K, A)$

C READS $K$ DATA OF TYPE I STARTING AT INDEX J INTO ARRAY A

IF I=4,SKIP. I=5, PICK UP FROM LQ, I=7 FROM LQ2, I=8 FROM LQ3

C IF I=6, PICK UP FROM FL. PT. STORAGE, BUT RETURN NUMBER IN

DATA

DATA

* STORAGE FORM. DO NOT TEST FOR FL. PT. OR SYMBOLIC.

LEVEL 2, STORE, INFF, IWORK

COMMON STORE(48000), IWORK(10)

DIMENSION INFF $(24,2000)$

EQUIVALENCE (INFF, STORE)

DATA

DATA

BLANK

COMMON/FLTN/IFL(15)

15

DIMENSION $A(1), \operatorname{LOC}(10)$

BLANK

FLTN

FLTN

DATA

DATA

IF (I.EQ.4) RETURN

DATA

$\operatorname{LOC}(1)=\operatorname{INFF}(14, M)$

20

$\operatorname{LOC}(2)=\operatorname{INFF}(16, M)$

DATA

DATA

DATA

DATA

DATA

DATA

DATA

DATA

DATA

DATA

DATA

DATA

DATA

DATA

DATA

DATA

DATA

END

10

$$
\text { CONT INUE }
$$$$
\text { RETURN }
$$

35

8


1

5

10

15

20

25

30

35
SUBROUTINE DCFD $(M, P)$

COMMON/BCFD/PAR(10), RMUX, RMUY, XGES, YGES, LAM

C

COMMON/GRR/I ERR, BSW, VSW, RSW, QSW, XSW

LOGICAL IERR, BSW, VSW, RSW, QSW, XSW

COMMON/SWTCH/BEND, MSIZE, CYCSWT, VCSW, PV, MSSW, VPR, MHS, MODS, MINZER LOGICAL CYCSWT, MSSW, VCSW, PV, VPR, MHS, MODS

C

DIMENSION $\mathrm{P}(9)$

EQUIVALENCE (FM,PAR(2)), (DM,PAR(3))

REAL LAM

EXTERNAL AUXF

C

DO $1 \mathrm{I}=1,6$

$1 \quad \operatorname{PAR}(I+2)=P(I)$

$\operatorname{PAR}(1)=\operatorname{PAR}(3)$

CYCSWT $=$. TRUE.

$\operatorname{PAR}(9)=P(7)$

RMUY $=\cos (6.283185307 * P(9))$

RMUX $=\cos (6.283185307 * P(8))$

C INITIAL GUESS FOR LAMBDA $=1 / 2$.

$X$ GES $=0.5$

YGES $=$ PAR( 4$)$

CALL GRT $(1$, XGES, 7, AUXF)

IF (IERR) RETURN

CALL HED

WRITE(3, 1000) $\operatorname{PAR}(1),(\operatorname{PAR}(I), I=4,8), F M, D M$

CALL HED

CYCSWT $=$. FALSE.

CALL CFD (M, FM)

C STORE FINAL VALUE OF PROFILE PARAMETER AS DATA FOR Q.

CALL REPFLT(M, 2,PAR(4))

RETURN

1000 FORMAT $(1 \mathrm{X}, *$ NEW VALUES $\ldots *, 6 \mathrm{~F} 14.8 / 9 \mathrm{X}, * \mathrm{LF}=*, \mathrm{~F} 12.8,9 \mathrm{X}$,

$1 *$ LD $=*, F 12.8)$

END
DCFD 2

BCFD 2

BCFD $\quad 3$

GRR 2

GRR $\quad 3$

GRR $\quad 4$

SWTCH 2

SWTCH 3

SWTCH 4

DCFD 6

DCFD 7

DCFD 8

DCFD 9

DCFD 10

DCFD 11

DCFD 12

DCFD 13

DCFD 14

DCFD 15

DCFD 16

DCFD 17

DCFD 18

DCFD 19

DCFD 20

DCFD 21

DCFD 22

DCFD 23

DCFD 24

DCFD 25

DCFD 26

DCFD 27

DCFD 28

DCFD 29

DCFD 30

DCFD 31

DCFD 32

DCFD 33

DCFD 34 
SUBROUTINE DEFSET ( $M, N M, K C, M X L I S T, I O P, K N)$

DEFSET

RESERVES SPACE FOR KC NUMBER OF CYCLED MATRICES WHOSE NAMES

DEFSET

CONSIST OF THE FIRST LETTER OF NM , FOLLOWED BY NUMBERS8 IN SEQUENCE

SET UP SPACE FOR TWO MAGNETS AND OPTIONAL NUMBER OF DRIFTS.

* IOP = TOTAL NUMBER OF MAGS AND DRIFTS.

5

* $\quad \mathrm{KN}=$ KIND FOR INFO

COMMON/BMI/MI (5000)

COMMON/NELS/NELS

LEVEL 2, MI

15

COMMON/SWTCH/BEND, MSIZE, CYCSWT, VCSW, PV, MSSW, VPR, MHS, MODS, MINZER

LOGICAL CYCSWT, MSSW, VCSW, PV, VPR, MHS, MODS

DEFSET

DEFSET

DEFSET

DEFSET

DEFSET

BMIL

BMIL

BMIL

BMIL

BMI

SWTCH

SWTCH

SWTCH

DIMENSION LIST (100)

DEFSETL

DIMENSION MXLIST(1)

DEFSET

DEFSET

IF (KC.EQ.0) GO TO 536

$M 1=M-1$

CALL MOVLEV(MI, LIST, KC)

CALL EQU $(M 1, M)$ GENERATE NUMBERED NAMES.

CALL GENNUM(NM, KC, LIST) STORE NAMES AND RESERVE SPACE FOR MATRIX AND LENGTH

DO $535 \mathrm{JA}=1, \mathrm{KC}$

CALL INFW(LIST (JA), 2,M1)

30

530 IF (JA.EQ.1) GO TO 531

CALL RESRV $(M 1, K N, M I N Z E R$,

$0,0,0,0,0,0,0,0$

.)

$531 \quad M 1=M 1-1$

535 CONTINUE

35

C IF IOP $=0$, RESERVE FOR CYCLED MATRICES ONLY

536 IF (IOP.EQ.0) RETURN

$M 1=M-K C$

40

RESERVE FOR 2 MAGNETS

$$
\begin{aligned}
& I 1=2 \\
& I F(I O P . E Q .1) I 1=1 \\
& \text { DO } 292 I=1, I 1 \\
& M 1=M 1-\quad 1 \\
& \text { CALL MAGRSV }(-M 1, M X L I S T(I), 0,0, N M)
\end{aligned}
$$

DEFSET

DEFSET 16

DEFSETL 2

DEFSETL 3

DEFSET 17

DEFSET 18

DEFSET 19

DEFSET 20

DEFSET 21

DEFSET 22

DEFSET 23

DEFSET 24

DEFSET 25

DEFSET 26

DEFSET 27

DEFSET 28

DEFSET 29

DEFSET 30

DEFSET 31

DEFSET $\quad 32$

DEFSET 33

DEFSET 34

DEFSET ' 35

DEFSET 36

DEFSET 37

DEFSET 38

DEFSET 39

DEFSET 40

DEFSET 41

DEFSET 42

DEFSET 43

DEFSET 44

DEFSET 45

DEFSET $\quad 46$

$$
\mathrm{I} 1=\mathrm{I} 1+1
$$

$M 1=M 1-1$

CALL DRFRSV (-M1,MXLIST(I), $0,0, N M)$ 
5

10

15

20

25

30
SUBROUTINE DELQ(S,W,DW)

LEVEL 2, STORE, INFF, IWORK

COMMON STORE (48000), IWORK(10)

DIMENSION INFF $(24,2000)$

EQUIVALENCE (INFF,STORE)

COMMON/CDERIV/IFLAG, MR, NR, DP, NV(11), DAT(20), UZ, STOT,

1 DAT2(7), MP2FLG, NF, HO, LOCALPH, NUMALPH

EQUIVALENCE (L,DAT(1)), (NU,DAT(2)), (EPS,DAT(3)), (Q,DAT(4))

EQUIVALENCE $(X, T(1)),(Y, T(2)),(Z, T(3))$,

1

DIMENSION $T(3), G(4)$

$(G X, G(1)),(G Y, G(2)),(G Z, G(3)),(G V, G(4))$

DIMENSION $W(4,1), \operatorname{DW}(4,1)$

C

C ENVELOPES

$E P S 2=E P S * E P S$

$A X=W(1,1) \$=A Y=W(3,1) \$ A X 3=A X * A X * A X \$ A Y 3=A Y * A Y * A Y$

$A X Y=A X+A Y \$ E X=E P S 2 / A X 3 \$ E Y=E P S 2 / A Y 3 \$ Q X Y=Q / A X Y$

$F X=E X+Q X Y \$ F Y=E Y+Q X Y$

C EL. QUAD.

$$
X=A X \$ \quad Y=A Y \$ \quad Z=S
$$

CALL ELQ (LOCALPH, NUMALPH, NU, L, T,G)

C

$$
\begin{aligned}
& \operatorname{DW}(1,1)=W(2,1) \\
& \operatorname{DW}(3,1)=W(4,1) \\
& \operatorname{DW}(2,1)=F X+G X \\
& \operatorname{DW}(4,1)=F Y+G Y
\end{aligned}
$$

C

RETURN

END

\begin{tabular}{lr} 
DELQ & 2 \\
BLANK & 2 \\
BLANK & 3 \\
86MARSIZ & 1 \\
86MARSIZ & 2 \\
86MARSIZ & 3 \\
BLANK & 5 \\
CDERIV & 2 \\
CDERIV & 3 \\
DELQ & 5 \\
DELQ & 6 \\
DELQ & 7 \\
DELQ & 8 \\
DELQ & 9 \\
DELQ & 10 \\
DELQ & 11 \\
DELQ & 12 \\
DELQ & 13 \\
DELQ & 14 \\
DELQ & 15 \\
DELQ & 16 \\
DELQ & 17 \\
DELQ & 18 \\
DELQ & 19 \\
DELQ & 20 \\
DELQ & 21 \\
DELQ & 22 \\
DELQ & 23 \\
DELQ & 24 \\
DELQ & 25 \\
DELQ & 26 \\
& \\
& \\
\hline
\end{tabular}


COMMON/CDERIV/IFLAG, MR , NR, DP , NV(11), DAT(20), UZ, STOT,

10

$$
\begin{array}{ll}
1 & \text { IF }(\text { IFLAG }) 1,1,2 \\
& J=0 \\
& \text { DO } 12 \text { I=2,6,2 } \\
& J=J+1 \\
& A(J)=\operatorname{DAT}(5+I) \\
12 \quad & B(J)=\operatorname{DAT}(6+I)
\end{array}
$$

15

$$
D X=D W(1)
$$

C $\quad D P X=D W(2)$

$$
D Y=D W(3)
$$

$$
\begin{aligned}
& D P Y=D W(4) \\
& U Y=0 .
\end{aligned}
$$$$
U Z=A \theta * Z
$$

$E I=1 \cdot / E$

$$
\begin{aligned}
& \mathrm{CH}=(E+E I) / 2 . \\
& \mathrm{SH}=(E-E I) / 2 .
\end{aligned}
$$$$
U Z=U Z+A B * C H / X K
$$

45

$$
U Y=U Y-A B * S H
$$

$U Z=U Z * B V B R$

$$
U Y=U Y * B V B R
$$

$$
P X M=P X-U Z
$$

$$
H A M=-S Q R T(1 .-P X M * P X M-P Y * P Y)
$$

$D X=-P X M / H A M$

$D Y=-P Y / H A M$

$D P Y=P X M * U Y / H A M$

$D W(1)=D X$

$D W(3)=D Y$

$D W(4)=D P Y$

5

7

8


RETURN 
1

$$
\text { SUBROUTINE DER2 }(S, W, D W)
$$

ENVELOPES AND DISPERSIONS OF IMPLODING BUNCH

$11 / 79$

LO = TOTAL LENGTH OF SYSTEM

C $\quad$ ZT $=$ BUNCH HALF LENGTH AT TARGET

C DELT $=(D P / P)$ HALF WIDTH OF TARGET

C QT $=4 * Z S Q * R P * N /(A * B E T A S Q * G A M A C U B E) \quad$ AT TARGET

C EPS = TRANSVERSE EMITTANCE/PI — NOT NORMALIZED

C $E=E T A=$ DISPERSION FOR BUNCH CENTER

C $E Q=E T A=$ DISPERSION FOR BUNCH END

C

COMMON/CDERIV/IFLAG, MR , NR , DP , NV(11) ,DAT (20), UZ, STOT, 1 DAT2(7), MP2FLG

REAL L, LO, K, KBAR, LMAG

C

IF (IFLAG) $1,1,2$

$1 \quad K=G R A D / B R H O$

$\mathrm{RHOI}=\mathrm{BZ} / \mathrm{BRHO}$

$Z T G 2=Z T * G A M * G A M$

QTDT $=$ QT/DELT

$E P S 2=E P S * E P S$

c

RETURN

$2 \quad A X=W(1,1)$

$A Y=W(3,1)$

$E=W(5,1)$

$E \theta=W(7,1)$

$L=L O-S-S T O T$

IF (L.LT.ZT.OR.LO.LT.O.) L = ZT

$D E L \theta=Z T G 2 / L$

$Q=Q T D T * D E L \theta$

$A P=E * D E L \theta$

$A A X=A X+A P$

40

$A X 3=A X * A X * A X$

$A Y 3=A Y * A Y * A Y$

$A=A A X+A Y$

$\mathrm{QA}=\mathrm{Q} / \mathrm{A}$

$Q A X A=Q A / A X$

45

$E P S A X=E P S 2 / A \times 3$

$E P S A Y=E P S 2 / A Y 3$

$K B A R=K-Q A X A$

50

C

$\operatorname{DW}(1,1)=W(2,1)$

$\operatorname{DW}(3,1)=W(4,1)$

$\operatorname{DW}(5,1)=W(6,1)$

$\operatorname{DW}(7,1)=W(8,1)$

$\operatorname{DW}(2,1)=-\operatorname{KBAR} * A X+\operatorname{EPSAX}$

$D W(4,1)=K * A Y+Q A+E P S A Y$

$\operatorname{DW}(6,1)=-\mathrm{KBAR} * \mathrm{E}+\mathrm{RHOI}$

$\operatorname{DW}(8,1)=-K * E O+R H O I$

RETURN

DER1

DER2

DER2

DER2

DER2

DER2

DER2

DER2

DER2

DER2

DER2

DER2

DER2

DER2

DER2

DER2

DER2

DER2

DER2

DER2

DER2

DER2

DER2

DER2

DER2

DER2

DER2

DER2

DER2

DER2

DER2

DER2

DER2

DER2

DER2

DER2

DER2

DER2

DER2

DER2

DER2

DER2

DER2

DER2

DER2

DER2

DER2

DER2

DER2

DER2

DER2

DER2

DER2

DER2

DER2

DER2

DER2
61

2

3

4

5

6

7

8

9

10

11

12

13

14

15 
1

SUBROUT INE DER3 ( $, W, D W)$

C INTEGRATE TRANSVERSE AND LONGITUDINAL ENVELOPES.

COMMON/CDERIV/IFLAG, MR, NR, DP, NV(11), DAT (20), UZ, STOT,

5

1 DAT2(7), MP2FLG

EQUIVALENCE (L,DAT(1)), (GRAD,DAT(2)), (BRHO,DAT(3)),

DER2

DER3

59

$1 \quad(\operatorname{EPS}, \operatorname{DAT}(4)), \quad(\mathrm{Q} 1, \operatorname{DAT}(5)), \quad(\mathrm{HO}, \operatorname{DAT}(6))$.

DER3

DER3

DER3

DER3

$(K Z, D A T(8)), \quad(\operatorname{EPSL}, \operatorname{DAT}(10)),(G, D A T(11))$

DER3

2

$(K Z, \operatorname{DAT}(8))$
$7,1), \operatorname{DW}(7,1)$

DER3

DIMENSION $W(7,1), \operatorname{DW}(7,1)$

10 REAL $K, K Z, L$

DER3

DER3

DER3

DER3

DER3

DER3

DER3

DER3

DER3

DER3

DER3

DER3

DER3

DER3

DER3

DER3

DER3

DER3

DER3

DER3

DER3

DER3

DER3

DER3

DER3

DER3

DER3

DER3

DER3

DER3

DER3

DER3

DER3

DER3

DER3

END

$\operatorname{DW}(2,1)=-K * A X+E A X 3+$ QAXYZ

$D W(4,1)=K * A Y+E A Y 3+$ QAXYZ

C

RETURN 
COMMON/CDERIV/IFLAG, MR, NR, DP , NV (11), DAT (20), UZ, STOT,

CDERIV

1 DAT2(7), MP2FLG, NF, HO, LOCALPH, NUMALPH

CDERIV

EQUIVALENCE (L,DAT(1)), (GRAD, DAT(2)), (BRHO,DAT(3)),

DER4

(EPS,DAT (4)), (Q,DAT (5))

DIMENSION W(4,1), DW $(4,1)$

REAL L, K, KX, KY, KBX, KBY

10

IF (IFLAG) $1,1,2$

$1 \quad B R H O=B R H O *(1 .+D P)$

$\mathrm{K}=\mathrm{GRAD} / \mathrm{BRHO}$

EPS2 $=$ EPS $* E P S$

RETURN

DER4

DER4

DER4

DER4

DER4

DER4

DER4

DER4

DER4

15

C ENVELOPE EQUATIONS

$2 \quad A X=W(1,1)$

$A Y=W(3,1)$

$A X 3=A X * A X * A X$

$A Y 3=A Y * A Y * A Y$

$A=A X+A Y$

$E A X 3=E P S 2 / A X 3$

$E A Y 3=E P S 2 / A Y 3$

$Q A=Q / A$

DER4

DER4

DER4

DER4

DER4

DER4

DER4

DER4

DER4

DER4

$D W(1,1)=W(2,1)$

$\operatorname{DW}(3,1)=W(4,1)$

$D W(2,1)=-K * A X+E A X 3+Q A$

$\operatorname{DW}(4,1)=K * A Y+E A Y 3+Q A$

30

GO TO $(7,3,3,5)$ IFLAG

C LINEARIZED ENVELOPE EQUATIONS

35

$3 \quad E A X 4=3, * E A X 3 / A X$

EAY 4=3. *EAY3/AY

QA2 $=Q A / A$

$K B X=-K-E A X 4-Q A 2$

$K B Y=K-E A Y 4-Q A 2$

DER4

DER4

DER4

DER4

DER4

DER4

DER4

DER4

DER4

DER4

DER4

DER4

DER4

DER4

DER4

DER4

DO $4 J=2, N R$

$\operatorname{DW}(1, J)=W(2, J)$

$\operatorname{DW}(3, J)=W(4, J)$

$\mathrm{DW}(2, \mathrm{~J})=\mathrm{KBX} * W(1, \mathrm{~J})-\mathrm{QA} 2 * W(3, \mathrm{~J})$

45

$4 \quad D W(4, J)=K B Y * W(3, J)-Q A 2 * W(1, J)$

DER4

DER4

DER4

DER4

DER4

7 RETURN

DER4

DER4

C SINGLE PARTICLE EQUATIONS

50

$5 \quad Q A X=Q A / A X$

$Q A Y=Q A / A Y$

$K X=-K+Q A X$

$K Y=K+Q A Y$

DO $6 \mathrm{~J}=2$, NR

55

$D W(1, J)=W(2, J)$

$\operatorname{DW}(3, \mathrm{~J})=W(4, \mathrm{~J})$

$\mathrm{DW}(2, \mathrm{~J})=K X * W(1, J)$

DER4

DER4

DER4

DER4

DER4

DER4

DER4

DER4

DER4

DER4 
$6 \quad D W(4, J)=K Y * W(3, J)$

$\begin{array}{ll}\text { DER4 } & 58 \\ \text { DER4 } & 59 \\ \text { DER4 } & 60 \\ \text { DER4 } & 61\end{array}$

END 
5

10

15

20

25

$$
\begin{aligned}
& 1 \mathrm{BRHO}=(1,+\mathrm{DP}) * \mathrm{DAT}(3) \\
& \mathrm{K}=\mathrm{DAT}(2) / \mathrm{BRHO}
\end{aligned}
$$$$
\text { RETURN }
$$

C

$2 \operatorname{DW}(1,1)=W(2,1)$

$\operatorname{DW}(2,1)=-K *(W(1,1) * W(1,1)-W(3,1) * W(3,1)) / 2$.

$\operatorname{DW}(3,1)=W(4,1)$

$\operatorname{DW}(4,1)=K * W(1,1) * W(3,1)$

IF (IFLAG.EQ. 1) RETURN

DO $10 \mathrm{~J}=2,5$

$\operatorname{DW}(1, J)=W(2, J)$

$\operatorname{DW}(2, J)=-K *(W(1,1) * W(1, J)-W(3,1) * W(3, J))$

$\operatorname{DW}(3, \mathrm{~J})=W(4, \mathrm{~J})$

$10 \quad \operatorname{DW}(4, J)=K *(W(1,1) * W(3, J)+W(3,1) * W(1, J))$

C

RETURN

END

$\begin{array}{lr}\text { DER5 } & 2 \\ \text { DER5 } & 3 \\ \text { DER5 } & 4 \\ \text { DER5 } & 5 \\ \text { CDERIV } & 2 \\ \text { CDERIV } & 3 \\ \text { DER5 } & 7 \\ \text { DER5 } & 8 \\ \text { DER5 } & 9 \\ \text { DER5 } & 10 \\ \text { DER5 } & 11 \\ \text { DER5 } & 12 \\ \text { DER5 } & 13 \\ \text { DER5 } & 14 \\ \text { DER5 } & 15 \\ \text { DER5 } & 16 \\ \text { DER5 } & 17 \\ \text { DER5 } & 18 \\ \text { DER5 } & 19 \\ \text { DER5 } & 20 \\ \text { DER5 } & 21 \\ \text { DER5 } & 22 \\ \text { DER5 } & 23 \\ \text { DER5 } & 24 \\ \text { DER5 } & 25 \\ \text { DER5 } & 26 \\ \text { DER5 } & 27 \\ \text { DER5 } & 28 \\ \text { DER5 } & 29\end{array}$


1

$$
\text { SUBROUTINE DERIV(S,Y,DY) }
$$

DERIV DIMENSION $Y(1), D Y(1)$

COMMON/TRKINT/G, EX, EY, OMSQ, SEND, VMX, VMN, DINT , BEG , DPR , JPR,

5 LOGICAL BEG, DPR, PLT

C ENVELOPE EQUATIONS

TRKINT

DERIV

$\operatorname{DY}(1)=Y(2)$

$F 1=G /(Y(1)+Y(3))$

$F 2=E X * * 2 / Y(1) * * 3$

DERIV

DERIV

DERIV

$\operatorname{DY}(2)=F 1+F 2-\operatorname{OMSQ} * Y(1)$

DERIV

DERIV

$\operatorname{DY}(3)=Y(4)$

DERIV

$\mathrm{F} 2=E Y * * 2 / Y(3) * * 3$

DERIV

RETURN

DERIV

END

DERIV

DIAGNOSIS OF PROBLEM

CARD NR. SEVERITY DETAILS

ARRAY REFERENCE OUTSIDE DIMENSION BOUNDS.

7 I $Y$

8 I $Y$

10 I DY

11 I DY

11 I $Y$

12 I $Y$

13 I DY

13 I $Y$
ARRAY REFERENCE OUTSIDE DIMENSION BOUNDS.

ARRAY REFERENCE OUTSIDE DIMENSION BOUNDS.

ARRAY REFERENCE OUTSIDE DIMENSION BOUNDS.

ARRAY REFERENCE OUTSIDE DIMENSION BOUNDS.

ARRAY REFERENCE OUTSIDE DIMENSION BOUNDS.

ARRAY REFERENCE OUTSIDE DIMENSION BOUNDS.

ARRAY REFERENCE OUTSIDE DIMENSION BOUNDS. 
1

SUBROUTINE DET $(\mathrm{C}, \mathrm{X})$

C CALCULATE THE DETERMINANT OF A $3 \times 3$ COMPLEX MATRIX.

COMPLEX $C, X$

DET

DET

DIMENSION $C(3,3)$

5

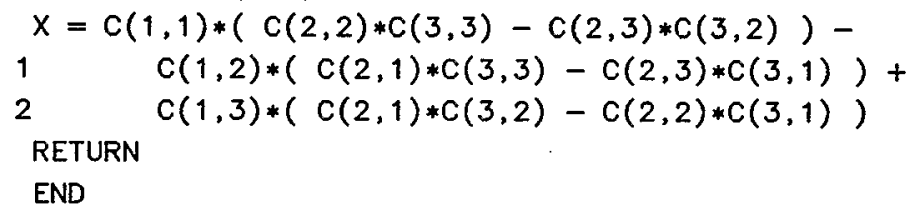


LEVEL 2, STORE, INFF, IWORK COMMON STORE(48000), IWORK(10)

DIMENSION $T(8,28)$

DOUBLE PRECISION $\mathrm{H}, \mathrm{T}$

EXTERNAL DERI

COMMON/CDERIV/IFLAG, MR, NR, DP, NV(11), DAT(20), UZ, STOT ,

1 DAT2(7), MP2FLG, NF, HO, LOCALPH, NUMALPH

INTEGER HDRF, HELQ

DATA HDRF, HELQ/3HDRF, 3HELQ/

DIFEQ

DIFEQ

DIFEQ

DIFEQ

DIFEQ

CDERIV

CDERIV

DIFEQ

DIFEQ

DIFEQ

C INTEGRATE FIRST ORDER DIFFERENTIAL EQUATIONS USING ZAM.

DIFEQ

C S=INDEPENDENT VARIABLE, GOES FROM $\theta$ TO SMAX.

DIFEQ

C HO=MAXIMUM INTEGRATION STEP SIZE (WILL BE ADJUSTED TO BE AN INTGRAL

DIFEQ

C FRACTION OF SMAX).

C NPR=NUMBER OF INTERVALS HO BETWEEN PRINTING OF $S, W$.

DIFEQ

DIFEQ

DIFEQ

DIFEQ

DIFEQ

DIFEQ

DIFEQ

DIFEQ

DIFEQ

DIFEQ

DIFEQ

DIFEQ

DIFEQ

DIFEQ

DIFEQ

DIFEQ

DIFEQ

DIFEQ

DIFEQ

DIFEQ

DIFEQ

DI FEQ

DIFEQ

DIFEQ

DIFEQ

DIFEQ

DIFEQ

DIFEQ

DIFEQ

DIFEQ

DIFEQ

DIFEQ

DIFEQ

DIFEQ

DIFEQ

DIFEQ

DIFEQ 
OUT $=$.FALSE.

IF (NPR.GT. O) OUT=. TRUE.

DIFEQ

DIFEQ

60

$$
\text { IFLAG }=-I F L A G
$$

DIFEQ

$U Z=0$.

DIFEQ

CALL DERI $(S, W, D W)$

DIFEQ

$W(2)=W(2)+U Z$

DIFEQ

IFLAG $=$-IFLAG

DIFEQ

CALL ZAMO(MN, S, DERI ,W,DW, T , H)

DIFEQ

DIFEQ

IF (.NOT.OUT) GO TO 3

DIFEQ

PRINT 101, ISTEP, $S, \quad(W(I), I=1, M N)$

DIFEQ

DIFEQ

DIFEQ

DIFEQ

DIFEQ

ISTEP $=$ ISTEP+1

IF (SWPR) GO TO 4

DIFEQ

GO TO 5

DIFEQ

DIFEQ

DIFEQ

DIFEQ

$I O S T P=I O S T P+1$

IF (IOSTP.LT.NPR) GO TO 5

80

PRINT 101, ISTEP, $S,(W(I), I=1, M N)$

DIFEQ

DIFEQ

DIFEQ

DIFEQ

DIFEQ

DIFEQ

DIFEQ

DIFEQ

DIFEQ

DIFEQ

DIFEQ

DIFEQ

DIFEQ

DIFEQ

DIFEQ

DIFEQ

DIFEQ

DIFEQ

END

RETURN

101 FORMAT $(5 X, 15,10 F 11.6 /(10 X, 10 F 11.6))$ 
1

5

10

15

20

25

30

35

40

45

50
SUBROUTINE DOIT(MSR)

INTEGER OPNAME,BDAT

LEVEL 2, STORE, INFF, IWORK

COMMON STORE (48000), IWORK(10)

DIMENSION INFF $(24,2000)$

EQUIVALENCE (INFF, STORE)

COMMON/BMI/MI (5000)

COMMON/NELS/NELS

LEVEL 2,MI

COMMON/BMI 1/MI1 (16000)

COMMON/NELS1/NELS1

LEVEL 2,MI 1

C

COMMON/CONTRL/ERROR, MODE, RSRV, STOR, XEQ, TRASW, NFSW, EMPTY, INDEF, 1 LDFLG, FIN

LOGICAL ERROR, RSRV, STOR, XEQ, TRASW, NFSW, EMPTY, INDEF, LDFLG, FIN

COMMON /MATCH3/NOPR, NONU, FLAG, IPR, IPP, MINFLG, MI FLG, MATFLG, GLOBAL LOGICAL FLAG, NOPR, NONU, MIFLG, MATFLG, GLOBAL

C

COMMON/MOVTOG/TOG

LOGICAL TOG

C

EQUIVALENCE( INDEF, I INDEF)

DIMENSION RW(3), VO(7), T(50), PAR(50), KREM(50), MIM(100)

COMMON/MIM/MIM \$ LEVEL 2, MIM

EQUIVALENCE (PAR, KREM)

COMMON/TSW/TRSW, MCY (20), JM, LOCC

LOGICAL TRSW

LOGICAL TABSW

COMMON/SWTCH/BEND, MSIZE, CYCSWT, VCSW, PV, MSSW, VPR, MHS, MODS, MINZER LOGICAL CYCSWT, MSSW, VCSW, PV, VPR, MHS, MODS

DIMENSION $N(200)$

DATA $N(5), N(51), N(161), N(102), N(104)$

$1 / 1,17,18,19,20 /$

DATA $N(47), N(78) / 21,22 /$

DATA $N(28), N(123) / 23,24 /$

DATA (N(I) $, I=11,25) / 2,3,4,5,6,7,8,9,10,11,12,13,14,15,16$ /

$\mathrm{JMT}=0$

$M S=M S R-1$

100 OPNAME $=\operatorname{INFF}(1$, MS $)$

IF (ERROR) RETURN

IOPMS $=\operatorname{INFF}(3, M S)$

C SCALAR OPERATIONS - GO TO 76

IF (IOPMS.GE. 112. AND. IOPMS. LE. 122) GO TO 76

IF (IOPMS.LT.0) GO TO 200

NSTAT $=$ N(IOPMS)
DOIT

BLANK

BLANK

86MARSIZ

86MARSIZ 2

86MARSIZ 3

BLANK 5

BMIL

BMIL

BMIL

BMIL

BMI

BMI1L 2

BMI 1L 3

BMI1L 4

BMI 1

CONTRL 2

CONTRL 3

CONTRL 4

CONTRL 5

MATCH3 2

MATCH3 3

MOVTOG 2

MOVTOG 3

MOVTOG 4

MOVTOG 5

DOIT 9

DOIT 10

DOITL 1

DOITL 2

DOIT 11

DOIT 12

NOV3TSW 1

TSW 3

NOV3ODOIT 2

DOIT $\quad 15$

SWTCH 2

SWTCH 3

SWTCH 4

DOIT 17

DOIT 18

DOIT 19

DOIT 20

DOIT 21

DOIT 22

DOIT 23

DOIT 24

DOIT 25

DOIT 26

DOIT 27

DOIT 28

DOIT 29

DOIT 30

DOIT 31

DOIT 32 
IF(NSTAT, EQ. I INDEF) GO TO 201

DOIT

60

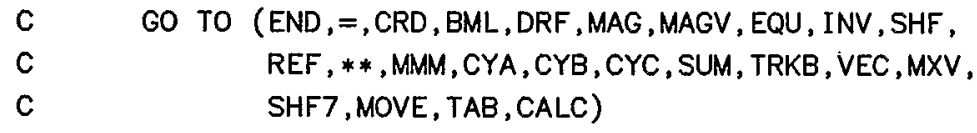

5 RETURN

DOIT 42

C ERROR ENCOUNTERED. OPERATION CALLED IS NOT ALLOWED.

1 FORMAT (13H***ERROR*** A5,57H IS AN OPERATION THAT CANNOT BE CALL DOIT IED BY SUBROUTINE DOIT) RETURN

C

C MMM ENTRY

$$
\begin{aligned}
& 22 \text { NDAT }=\operatorname{INFF}(17, M S) \\
& \text { CALL MIFILL (MS, } 1, \text { NDAT, NELM, MIM) }
\end{aligned}
$$


C MOVE ENTRY

DOIT 92

78 DO $781 \quad \mathrm{I}=1,7$

DOIT 93

$781 \mathrm{VO}(\mathrm{I})=0$.

DOIT

TOG $=$. TRUE.

DOIT 95

GO TO 200

MY3DOIT 2

C

C MXV ENTRY

125

$$
104 M 1=\operatorname{MDAT}(M S, 1)
$$

$M 2=\operatorname{MDAT}(M S, 2)$

$\mathrm{LOC}=1$

IF (.NOT.VCSW) GO TO 105

DECODE $(10,106, M 2)$ NUM

130

106 FORMAT $(1 \mathrm{X}, \mathrm{I} 1,8 \mathrm{X})$

LOC $=(N U M-1) * 7+1$

105 CALL DATA $(M 2,5, \operatorname{LOC}, 7, \operatorname{PAR}(8))$

C

$$
=\text { ENTRY }
$$

CALL RTRV7(M1, T, RW)

CALL MXV7(T,PAR(8),PAR(1))

CALL STDAT(MS, 5, 1,7,PAR)

GO TO 200

C REF ENTRY

140

$20 \quad$ NJ $=\operatorname{MDAT}(M S, 1)$

CALL REF (MS, NJ)

DOIT

DOIT

DOIT

DOIT

DOIT

DOIT

DOIT

DOIT

DOIT

DOIT

DOIT

DOIT

DOIT

DOIT

DOIT

DOIT

DOIT

DOIT

DOIT

GO TO 200

C SHF ENTRY

19 CALL DATA(MS, 1, 1, 4,PAR)

CALL SHF(MS,PAR)

145

GO TO 200

C SHF7 ENTRY

47 CALL DATA (MS, 1, 1,6,PAR)

CALL SHF7 (MS,PAR)

GO TO 200

150

C

C INV ENTRY

18 NJ $=\operatorname{MDAT}(\mathrm{MS}, 1)$

CALL INV(MS, NJ)

GO TO 200

155

C STAR ENTRY

$21 \quad \mathrm{KS}=\operatorname{INFF}(4, \mathrm{MS})$

CALL STAR(MS,KS)

GO TO 200

C SUM ENTRY

160

$51 \quad \mathrm{KS}=\operatorname{INFF}(4, \mathrm{MS})$

CALL DATA(MS, $1,1, \mathrm{KS}$, PAR)

CALL SUM(KS, PAR, RES)

CALL STDAT(MS $5,1,1$, RES)

GO TO 200

165

$C=$ ENTRY

$11 \mathrm{KN}=\operatorname{INFF}(20, M S)$

IF (KN.NE. 4HSCAL) GO TO 266

$I O P=\operatorname{BDAT}(M S, 1)$

DOIT 115

DOIT 116

DOIT 117

DOIT 118

DOIT 119

DOIT 120

DOIT 121

DOIT 122

DOIT 123

DOIT 124

DOIT 125

DOIT 126

DOIT 127

DOIT 128

DOIT 129

DOIT 130

DOIT 131

DOIT 132

DOIT 133

DOIT 134

DOIT 135

DOIT 136

DOIT 137

DOIT 138

DOIT 139

DOIT 140

DOIT 141

DOIT 142

DOIT 143

170 IF (IOP.EQ.1H) GO TO 200

DOIT 144

$\mathrm{IQ}=2$

DOIT 
$I T P=\operatorname{INFF}(21, M S)$

DOIT $\quad 146$

IF (ITP.NE. 4HSNGL) IQ $=4$

DOIT

CALL DATA(MS, 1, 1, IQ,PAR)

DOIT

CALL AROP (MS, PAR, IOP)

DOIT

GO TO 200

DOIT

C SCALAR OPERATIONS

DOIT

$76 \quad \mathrm{XX}=$ FDAT $(M S, 1)$

NOPMS = IOPMS -111

180

GO TO $(86,87,88,89,90,91,92,93,94,95,96)$ NOPMS

DOIT

152

DOIT 153

DOIT $\quad 154$

DOIT 155

DOIT $\quad 156$

DOIT 157

DOIT 158

185

$86 \quad Y Y=\operatorname{SIN}(X X) \quad \$$ GO TO 99

$87 \quad Y Y=\cos (X X) \quad \$$ GO TO 99

$88 \quad Y Y=\operatorname{EXP}(X X) \quad \$ G O$ TO 99

$89 \quad Y Y=A L O G(X X) \quad \$$ GO TO 99

DOIT $\quad 159$

$90 \quad Y Y=X X * X X \quad \$$ GO TO 99

DOIT 160

$91 \quad Y Y=\operatorname{SQRT}(X X) \quad \$$ GO TO 99

DOIT 161

$92 \quad Y Y=\operatorname{TAN}(X X) \quad \$$ GO TO 99

DOIT 162

$93 \quad Y Y=\operatorname{ASIN}(X X) \quad \$$ GO TO 99

DOIT 163

190

$94 \quad Y Y_{L}=\operatorname{ACOS}(X X) \quad \$$ GO TO 99

DOIT 164

$95 Y Y=\operatorname{ATAN}(X X) \quad \$$ GO TO 99

$96 \quad Y Y=\operatorname{ABS}(X X) \quad \$$ GO TO 99

DOIT 165

99 CALL STDAT(MS $, 5,1,1, Y Y)$

GO TO 200

DOIT 166

DOIT 167

DOIT $\quad 168$

195

$266 \operatorname{KREM}(1)=\operatorname{BDAT}(M S, 1)$

$\operatorname{KREM}(2)=\operatorname{BDAT}(M S, 3)$

DOIT 169

DOIT $\quad 170$

$I O P=\operatorname{BDAT}(M S, 2)$

DOIT 171

CALL AROP (MS, KREM, IOP)

GO TO 200

200

205

* Cya and cyc entries

23 CONTINUE

$25 \quad$ NDAT $=\operatorname{INFF}(17, M S)$

$\mathrm{KA}=\operatorname{INFF}(4, \mathrm{MS})$

IF (MINFLG.NE.4.OR.MIFLG) CALL MIFILL(MS, 1, NDAT, NELS1, MI 1)

DOIT

DOIT 173

DOIT 174

DOIT 175

DOIT 176

DOIT 177

DOIT 178

DOIT 179

DOIT 180

C GO TO 280

DOIT 181

GO TO 200

DOIT 182

* CYB ENTRY

210 C 24 CALL SETCYB(MS)

C CALL CYX(MS)

C $280 \mathrm{JMT}=\mathrm{JMT}+1$

* if tRSW is on, angle fit will Be REPLACEd by trace fit

C 122 IF (TRSW) RETURN

215 C GO TO 200

DOIT 183

DOIT 184

DOIT 185

DOIT 186

DOIT 187

DOIT 188

DOIT 189

DOIT 190

C TRKB ENTRY

75 CALL TRKB(MS)

DOIT 191

DOIT 192

GO TO 200

220

C TAB ENTRY

810 TABSW $=$. TRUE.

CALL TABULAT (MS, TABSW)

GO TO 200

DOIT 193

DOIT 194

DOIT 195

DOIT 196

DOIT 197

DOIT 198

DOIT 199

C CALC ENTRY

DOIT 200

77 CALL CALC(MS)

DOIT

GO TO 200

DOIT 
FORM KA NUMBER OF DRIFT MATRICES USING ELEMENTS OF A VECTOR AS DRFMATS

LENGTHS. NAME OF VECTOR REPLACED LENGTH ON DRF INPUT CARD.

5

10

15

LEVEL 2, STORE, INFF, IWORK

COMMON STORE(48000), IWORK (10)

DIMENSION INFF $(24,2000)$

EQUIVALENCE (INFF, STORE)

COMMON/CONTRL/ERROR, MODE, RSRV , STOR , XEQ, TRASW, NFSW, EMPTY, INDEF,

LOGICAL ERROR, RSRV, STOR, XEQ, TRASW, NFSW, EMPTY, INDEF, LDFLG, FIN

EQUIVALENCE (IVAR, FVAR)

INTEGER ELNUM

DATA MASKL/7777777777B/

DATA MASK1/77777777770000000000B/

RETRIEVE NAME OF VECTOR FROM DRF INPUT. CHECK THAT IT IS A

* Vector with at least ka elements.

* Vector name is pRESUMEd to be in SPECial internal fl. pt.

* SYMBOLIC FORM — OgOgONAMEV

25

$\operatorname{LF}=\operatorname{INFF}(14, M)$

FVAR=STORE (LF)

$N V=I V A R$

$N R=N V \cdot A N D \cdot M A S K L$

$N L=N V \cdot A N D$. NOT.MASKL

IF (NL.EQ.O.AND.NR.NE.O) GO TO 20

*

WRITE $(3,1)$ NV

ERROR. NOT SYMBOLIC FORM

GO TO 50

FORMAT $(5 X, 3 H * * *, 1 X, *$ THE FOLOOWING OCTAL NUMBER IS NOT ACCEPTABLE 1 AS A VECTOR, */9X,*WHOSE ELEMENTS ARE NEEDED FOR DEFINING MULTIPLE 2 DRIFTS. *2X,020)

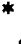

GET VECTOR NAME, INDEX AND OPNAME.

$N E W=\operatorname{SHIFT}(N V, 30)$

$N E W=N E W \cdot$ AND $\cdot$ MASK 1

NMVEC $=$ NEW.OR.5555555555B

$M V=E L N U M(N M V E C)$

$\mathrm{NMOP}=\operatorname{INFF}(1, \mathrm{MV})$

IF (NMOP.NE.3HVEC) GO TO 40

NK $=\operatorname{INFF}(4, M V)$

IF (KA.GT.NK) GO TO 45

$M 1=M-1$

50

DO $25 \mathrm{I}=1, \mathrm{KA}$

$E L=\operatorname{FDAT}(M V, I)$

* Store length in FL. PT. inPut

CALL REPFLT (M1, 1,EL)

CALL DRIFT $(M 1, E L)$
DRFMATS

BLANK

BLANK

86MARSIZ

86MARSIZ

86MARSIZ

BLANK

CONTRL

CONTRL

CONTRL

CONTRL

DRFMATS

DRFMATS

DRFMATS

DRFMATS

DRFMATS

DRFMATS

DRFMATS

DRFMATS

DRFMATS

DRFMATS

DRFMATS

DRFMATS

DRFMATS

DRFMATS

DRFMATS

DRFMATS

DRFMATS

DRFMATS

DRFMATS

DRFMATS

DRFMATS

DRFMATS

DRFMATS

DRFMATS

DRFMATS

DRFMATS

DRFMATS

DRFMATS

DRFMATS

DRFMATS

DRFMATS

DRFMATS

DRFMATS

DRFMATS

DRFMATS

DRFMATS

DRFMATS

DRFMATS

DRFMATS

DRFMATS

DRFMATS

DRFMATS

DRFMATS
2

3

4

5

2

3

1

2

3

5

2

3

4

5

8

9

10

11

12

13

14

15

16

17

18

19

20

21

22

23

24

25

26

27

28

29

30

31

32

33

34

35

36

37

38

39

40

41

42

43

44

45

46

47 
60 RETURN 1 DRIFT MATRICES.*) GO TO 50

65

2 FORMAT (5X,3H***,1X, A5, 1X,*HAS ONLY*1X,I5, 1X,*ELEMENTS.*/9X, I5, 1X,

DRFMATS $1 *$ ARE NEEDED TO DEFINE CURRENT DRIFT MATRICES.*)

DRFMATS

58

50 ERROR $=$.TRUE

DRFMATS

59

RETURN

DRFMATS

END

DRFMATS 
1

SUBROUT INE DRFRSV( MO, NAMEO, KAO, KBO, NAME1 )

DRFRSV

DRFRSV 2

* SETS UP INFO AND STORAGE FOR a DRIFT

* Reserves ofr inPut of 1 parameter, outPut of $3 \times 3$ matrix

DRFRSV

DRFRSV

DRFRSV

OPNAME IS DRF

DRFRSV

DRFRSV

SWTCH

SWTCH

SWTCH

DRFRSV

DRFRSV

DRFRSV

DRFRSV

DRFRSV

DRFRSV

DRFRSV

COMMON/SWTCH/BEND, MSIZE, CYCSWT, VCSW, PV, MSSW, VPR, MHS, MODS, MINZER

DATA MXPMY/1/

CALL STINFO( MO, NAMEO, 5HDRF ,KAO,KBO,NAME1)

IF (KBO.EQ.0) CALL RESRV(M0, MXPMY, MINZER, $1,1,0,0,0,0,0,0)$

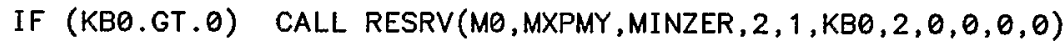

RETURN

END 
C SETS UP DRIFT SPACE MATRICES RX AND RY,DIMENSIONED $(2,3) \quad$ RY=RX

C THERE ARE 3 WAYS TO DEFINE DRIFTS -

DRIFT

C

C -STANDARD METHOD, EXAMPLES -

DRIFT

C $2-8--15-19$
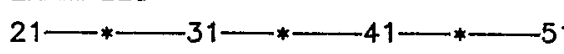

DRIFT

C D

3.85

DRIFT

10

$$
\text { C- }
$$

C LD4 =

3.85

C D4 DRF LD4

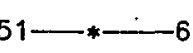

$61-7-71$ DRIFT

DRIFT

DRIFT

DRIFT

$$
\text { C- }
$$

C

D4 IS THE NAME OF A DRIFT OF LENGTH 3.85

DRIFT

15

C

C -METHOD 2, CREATE A SET OF DRIFTS -

DRIFT

DRIFT

DRIFT

C $2-8--15-19$
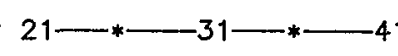

C L VEC

.54

2. 14

3.85

L

DRIFT

C D

DRF

-61
25

22.67

DRIFT

DRIFT

DRIFT

C 5 DRIFTS ARE CREATED. D1,D2,D3,D4,D5 OF LENGTHS $=.54,2.14,-25$.

DRIFT

DRIFT

DRIFT

C -METHOD 3 IS USED TO ADJUST THE LENGTH OF THE DRIFT TO MAINTAIN THE

DRIFT

DRIFT

C SUM OF A SET OF DRIFTS AND/OR MAGNETS AT CONSTANT LENGTH.

C $2-8--15-1921-*-3$

KB LDDD

LTOT

D1

D2 2 DKB

$61-71$ DRIFT

$C$

C IF LTOT .NE. $\theta$, LDDD IS ADJUSTED TO MAKE LDDD+LD $1+\ldots+L D K B=L T O T$

DRIFT

DRIFT

DRIFT

C IF LTOT .EQ. 0 , LTOT IS STORED AS LTOT $=$ LDDD+LD $1+\ldots+$ LDKB

DRIFT

30

C ON SUBSEQUENT CALLS, LDDD WILL BE ADJUSTED TO MAINTIAN CONSTANT LTOT. c

DO $1 \mathrm{I}=1,2$

DO $1 \mathrm{~J}=1,3$

$R \times(I, J)=0.0$

35

1

CONT INUE

$\operatorname{RX}(1,1)=1$.

$\mathrm{RX}(1,2)=E L$

$\operatorname{RX}(2,2)=1.0$

$R W(1)=E L$

DRIFT

DRIFT

DRIFT

DRIFT

DRIFT

DRIFT

DRIFT

DRIFT

DRIFT

40

CALL STXY (M,RX,RX,RW)

DRIFT

RETURN

DRIFT

END

DRIFT

DRIFT 
INTEGER FUNCTION ELNUM(NAME)

C SEARCHES FOR INDEX OF ELEMENT NAMED NAME

LEVEL 2, STORE, INFF, IWORK

5

COMMON STORE(48000), IWORK(10)

DIMENSION $\operatorname{INFF}(24,2000)$

EQUIVALENCE (INFF, STORE)

COMMON/STORE/LMAX, LINF, LFILE, MAX, MIN, ICARD (11)

* LMAX AND maX aRE DIMENSIONS OF STORE AND INFF

COMMON/CONTRL/ERROR, MODE, RSRV, STOR, XEQ, TRASW, NFSW, EMPTY, INDEF,

1 LDFLG, FIN

LOG ICAL ERROR, RSRV, STOR, XEQ, TRASW, NFSW, EMPTY, INDEF, LDFLG, FIN

COMMON/SWTCH/BEND, MSIZE, CYCSWT, VCSW, PV, MSSW, VPR, MHS, MODS, MINZER LOGICAL CYCSWT, MSSW, VCSW, PV, VPR, MHS, MODS

COMMON/FLTN/IFL(15)

20

COMMON /MATCH/ KAT, KBT, MPAR(30), IPAR(30), MSR, MTR, LTRTAB, FNORM, $1 \operatorname{ICON}(30), \operatorname{ITYPE}(30), \operatorname{NPOS}(30), \operatorname{IPOS}(7,30), \operatorname{BETO}(30), \operatorname{WT}(30)$,

$2 \operatorname{SIG}(30), \operatorname{NAMBFC}(42), \operatorname{ERPR}, \operatorname{IVSAV}, \operatorname{LVAR}(30), \operatorname{WTVAR}(30), \operatorname{VAL}(30)$,

3 VARWTS, IPRPOS $(6,30)$

LOGICAL ERPR, LVAR, VARWTS

DIMENSION INB(10)

LOGICAL MINV

DATA IBLNK/1H /

$\begin{array}{llllllll}\text { DATA(NAMBFC(I), I=1,42)/5HNUX } & , 5 \mathrm{HBX} & , 5 \mathrm{HAX} & , 5 \mathrm{HGX} & , 5 \mathrm{HX} \\ 1 & 5 \mathrm{HDX} & , 5 \mathrm{HNUY}, 5 \mathrm{HBY} & , 5 \mathrm{HAY} & , 5 \mathrm{HGY} & , 5 \mathrm{HY} & , 5 \mathrm{HDY} \\ 2 & 5 \mathrm{HS} & 5 \mathrm{HTHET} & & & & & \end{array}$

2

35 5HBXMX ,5HBYMX ,5HXMAX,5HQX ,5HQY ,5H ,5HGAMT , 5 HCHRX, 5 HCHRY, 5HALPH., 5 HYMAX, 5 HBXMN ,5HBYMN ,5HXMIN ,

$\begin{array}{lllllll}5 & 5 H Y M I N & , 5 H C I R C & , 5 H T H T X & , 5 H T H T Y & , 5 \mathrm{H} & , 5 \mathrm{HMTRX}, 5 \mathrm{HRES} ; \\ 6 & 5 \mathrm{HRESN}, 5 \mathrm{HBXBY}, 5 \mathrm{HRND2}, 5 \mathrm{HRND}, 5 \mathrm{HWST}, 5 \mathrm{HXDX}, 5 \mathrm{HAXAY} ;\end{array}$ DATA MASK/77000 $000000000000000 \mathrm{~B} /$, . (INB(I) , I=1 , 10)/33B, 34B, 35B, 36B, 37B , 40B , 41B, 42B, 43B, 44B/

$$
M I N V=. \text { FALSE. }
$$

VCSW=. FALSE.

$M S K=M A S K$. AND. NAME

MSK $1=$ MASK . AND . IBLNK

IF (MSK.EQ.MSK1) GO TO 30

IF (MSK.NE.IFL(15)) GO TO 15

MINV $=$. TRUE.

$$
L A G=\text { SHIFT }(\text { NAME, } 6)
$$

DECODE $(10,3$, LAG $)$ NAM

3 FORMAT $(A 5,5 X)$

$$
\text { GO TO } 16
$$

$N A M=N A M E$

$16 \quad \mathrm{NM}=$ NAM

17 DO 20 I=MIN, MAX

IF (NM.EQ. $\operatorname{INFF}(2, \mathrm{I}))$ GO TO 25

20 CONTINUE

C IF MODE IS 2, ELEMENT NOT YET DEFINED. SET ELEMENT NO. TO 0, RETURN IF (MODE.NE.2) 60 TO 22

$E L N U M=0$

\begin{tabular}{lr} 
& \\
ELNUM & 2 \\
ELNUM & 3 \\
BLANK & 2 \\
BLANK & 3 \\
86MARSIZ & 1 \\
86MARSIZ & 2 \\
86MARSIZ & 3 \\
BLANK & 5 \\
STORE & 2 \\
STORE & 3 \\
STORE & 4 \\
CONTRL & 2 \\
CONTRL & 3 \\
CONTRL & 4 \\
CONTRL & 5 \\
SWTCH & 2 \\
SWTCH & 3 \\
SWTCH & 4 \\
FLTN & 2 \\
FLTN & 3 \\
MATCH & 2 \\
MATCH & 3 \\
MATCH & 4 \\
MATCH & 5 \\
MATCH & 6 \\
ELNUM & 10 \\
ELNUM & 11 \\
ELNUM & 12 \\
MATCHD & 2 \\
MATCHD & 3 \\
MATCHD & 4 \\
MATCHD & 5 \\
MATCHD & 6 \\
MATCHD & 7 \\
MATCHD & 8 \\
ELNUM & 14 \\
ELNUM & 15 \\
ELNUM & 16 \\
ELNUM & 17 \\
ELNUM & 18 \\
ELNUM & 19 \\
ELNUM & 20 \\
ELNUNUM & 21 \\
ELNUM & 32 \\
ELNUM & 23 \\
ELNUM & 24 \\
ELNUM & 25 \\
ELNUM & 26 \\
ELNUM & 27 \\
ELNUM & 28 \\
ELNUM & 39 \\
\hline
\end{tabular}



VCSW $=$. TRUE.

23 ERROR $=$. TRUE.

$$
\begin{aligned}
& \text { WRITE }(3,11) \text { NAME } \\
& \operatorname{ELNUM}=-0
\end{aligned}
$$$$
\text { RETURN }
$$

11 FORMAT $(15 \mathrm{H} * * *$ ERROR ***, A5,30H IS AN UNDEFINED ELEMENT NAME.)

$$
I I=I
$$

IF (.NOT.VCSW) GO TO 26

ELNUM

ELNUM

$N O P=\operatorname{INFF}(1, I I)$

IF ((NOP.EQ.3HCYC) . OR. (NOP.EQ.3HCYA)) GO TO 26

90 
1

5

10

15

20

25

30

35

40

45
SUBROUTINE ELQ(LOC, MM, VO, L,W, E)

LEVEL 2, STORE, INFF, IWORK

COMMON STORE( 48000$)$, IWORK (10)

DIMENSION INFF $(24,2000)$

EQUIVALENCE (INFF,STORE)

C

COMMON PI, R, TH, ER, ET

REAL L

DIMENSION AL(1),BI(4)

DIMENSION $W(3), E(4)$

C

DATA PI/3.1415926536/

$X=W(1) \quad \$ \quad Y=W(2) \quad \$ \quad Z=W(3)$

$R=S Q R T(X * X+Y * Y)$

TH=ATAN2 $(Y, X)$

$V O L=P I * V \theta / L$

$V O R=2 . * V \ominus / R$

$\mathrm{RL}=\mathrm{PI} * \mathrm{R} / \mathrm{L}$

$\mathrm{ZL}=\mathrm{PI} * \mathrm{Z} / \mathrm{L}$

$V=0 . \$ E R=0 . \$ E T=0 . \$ E Z=0$.

$\mathrm{TH} 2=2 . * \mathrm{TH}$

$\mathrm{CT}=\operatorname{COS}(\mathrm{TH}) \$ \quad \mathrm{ST}=\mathrm{SIN}(\mathrm{TH})$

$C T 2=\operatorname{COS}(T H 2) \$ S T 2=\operatorname{SIN}(T H 2)$

C

DO $1 M=1, M M$

$A L M=S T O R E(L O C+M)$

$F M=2 * M-1$

$B L M=A L M * F M$

$R M=F M * R L$

$Z M=F M * Z L$

$S Z=S I N(Z M) \$ C Z=\operatorname{COS}(Z M)$

CALL BESIS(RM,BI, 3)

$V=A L M * B I(3) * S Z+V$

$E R=B L M *(B I(2)+B I(4)) * S Z+E R$

$E Z=B L M * B I(3) * C Z+E Z$

C

$E R=-(V \theta L / 2) * C T 2 * E$.

$\mathrm{ET}=\mathrm{VQR} * \mathrm{ST} 2 * \mathrm{~V}$

$E Z=-V \theta L * C T 2 * E Z$

$V=V O * C T 2 * V$

C

$E X=E R * C T-E T * S T$

$E Y=E R * S T+E T * C T$

c

$E(1)=E X \$ \quad E(2)=E Y \quad \$ \quad E(3)=E Z \quad \$ \quad E(4)=V$ RETURN

END

\begin{tabular}{|c|c|}
\hline ELQ & \\
\hline BLANK & \\
\hline BLANK & \\
\hline 86MARSIZ & \\
\hline 86MARSIZ & \\
\hline 86MARSIZ & \\
\hline BLANK & \\
\hline ELQ & \\
\hline ELQ & \\
\hline ELQ & \\
\hline ELQ & \\
\hline ELQ & \\
\hline ELQ & 0 \\
\hline ELQ & 10 \\
\hline ELQ & 11 \\
\hline ELQ & 12 \\
\hline ELQ & 13 \\
\hline ELQ & 14 \\
\hline ELQ & 15 \\
\hline ELQ & 16 \\
\hline ELQ & 17 \\
\hline ELQ & 18 \\
\hline ELQ & 19 \\
\hline ELQ & 20 \\
\hline ELQ & 21 \\
\hline ELQ & 22 \\
\hline ELQ & 23 \\
\hline ELQ & 24 \\
\hline ELQ & 25 \\
\hline ELQ & 26 \\
\hline ELQ & 27 \\
\hline ELQ & 28 \\
\hline ELQ & 29 \\
\hline ELQ & 30 \\
\hline ELQ & 31 \\
\hline ELQ & 32 \\
\hline ELQ & 33 \\
\hline ELQ & 34 \\
\hline ELQ & 35 \\
\hline ELQ & 36 \\
\hline$E L Q$ & 37 \\
\hline$E L Q$ & 38 \\
\hline ELQ & 39 \\
\hline ELQ & 40 \\
\hline ELQ & 41 \\
\hline ELQ & 42 \\
\hline$E L Q$ & 43 \\
\hline ELQ & 44 \\
\hline
\end{tabular}




$\begin{array}{lr}\text { EMIS } & 2 \\ \text { EMIS } & 3 \\ \text { EMIS } & 4 \\ \text { INSTR } & 2 \\ \text { INSTR } & 3 \\ \text { INSTR } & 4 \\ \text { INSTR } & 5 \\ \text { INSTR } & 6 \\ \text { SWTCH } & 2 \\ \text { SWTCH } & 3 \\ \text { SWTCH } & 4 \\ \text { CONTRL } & 2 \\ \text { CONTRL } & 3 \\ \text { CONTRL } & 4 \\ \text { CONTRL } & 5 \\ \text { EMIS } & 8 \\ \text { EMIS } & 9 \\ \text { EMIS } & 10 \\ \text { EMIS } & 11 \\ \text { EMIS } & 12 \\ \text { EMIS } & 13 \\ \text { EMIS } & 14 \\ \text { EMIS } & 15 \\ \text { EMIS } & 16 \\ \text { EMIS } & 17 \\ \text { EMIS } & 18\end{array}$

15

C

IF (MODE.EQ.3) GO TO 10

ISGN $=1 \mathrm{H}$

IF (OP.LT.O) ISGN $=1 \mathrm{H}-$

20

WRITE $(3,21)$ ISGN 
1

10

15

END

\section{RETURN}

SUBROUTINE ENVTRAC

LEVEL 2, STORE, INFF, IWORK

COMMON STORE (48000), IWORK(10)

DIMENSION INFF $(24,2000)$

EQUIVALENCE (INFF,STORE)

COMMON/TRKINT/G, EX, EY , OMSQ, SEND, VMX, VMN, DINT , BEG, DPR, JPR,

1 THET , PLT, NPT , NZ, PMAX, NSIZ, ITITLE(6)

LOGICAL BEG,DPR,PLT

COMMON/BMI/MI (5000)

COMMON/NELS/NELS

LEVEL 2,MI
ENVTRAC 2

BLANK 2

BLANK 3

8GMARSIZ 1

86MARSIZ 2

86MARSIZ 3

BLANK 5

TRKINT 2

TRKINT 3

TRKINT 4

BMIL 1

BMIL 2

BMIL 3

BMIL 4

BMI 3

ENVTRAC 6

ENVTRAC 7 
EQIL

EQIL

C CALCULATE 3 CLOSED ORBITS. SYNCHRONOUS, $D P / P=0, D P / P=1$.

EQIL

C $M$ IS A $7 \times 7$ MATRIX PARTITIONED AS FOLLOWS.

5

10

15

20

25

10

COMMON/CONTRL/ERROR, MODE, RSRV, STOR, XEQ, TRASW, NFSW, EMPTY, INDEF,

1

LOGICAL ERROR, RSRV, STOR, XEQ, TRASW, NFSW, EMPTY, INDEF, LDFLG, FIN

DIMENSION $T(49), R W(3), \operatorname{TN}(6,7), \operatorname{TT}(7,7), V(7,3), U(7,3)$

EQUIVALENCE (T,TT)

INTEGER BDAT

$M 1=\operatorname{MDAT}(M, 1)$

$\operatorname{IOP}=\operatorname{BDAT}(M, 2)$

CALL RTRV7 (M1, T, RW)

IF (ERROR) RETURN

$N=6$

DO $25 K=1,3$

$\mathrm{JJ}=7$

IF (K.EQ.3) $J J=6$

DO $10 \mathrm{~J}=1, \mathrm{~N}$

DO 10 I $=1, N$

$\operatorname{TN}(I, J)=\operatorname{TT}(I, J)$

CONTINUE

DO $15 \mathrm{I}=1, \mathrm{~N}$

$\operatorname{TN}(I, I)=\operatorname{TN}(I, I)-1$.

$T N(I, N+1)=-T T(I, J J)$

15 CONTINUE

CALL MXDIV(TN,N, 1,D,6)

30

35

40

45

20

DO $20 I=1, N$

$\mathrm{V}(\mathrm{I}, \mathrm{K})=\mathrm{TN}(\mathrm{I}, \mathrm{N}+1)$

CONTINUE

$N=4$

25 CONTINUE

$V(7,1)=1$.

$M N=\operatorname{MNAME}(M)$

$M T=\operatorname{MNAME}(M 1)$

$V(7,2)=1$.

$V(6,3)=1$.

$V(5,2)=0$

$V(6,2)=0$.

$V(5,3)=0$.

$V(7,3)=0$.

IF (IOP.EQ. 4HNOPR) GO TO 27

CALL DASH

C PRINT VECTORS BEFORE MULTIPLYING BY MATRIX

WRITE $(3,100)$ MN, MT

WRITE $(3,105)((V(I, J), I=1,7), J=1,3)$

C MULTIPLY BY MATRIX

$50 \quad 27$ DO $30 \quad J=1,3$

CALL MXV7(TT,V(1,J),U(1,J))

30

CONTINUE

IF (IOP.EQ.4HNOPR) GO TO 32

C PRINT VECTORS AFTER MULTIPLICATION

55

WRITE $(3,110)((U(I, J), I=1,7), J=1,2)$

WRITE $(3,115)$ MT, $(U(I, 3), I=1,7)$

EQIL

EQIL $\quad 6$

CONTRL 2

CONTRL 3

CONTRL 4

CONTRL $\quad 5$

EQIL 8

EQIL 9

EQIL 10

EQIL 11

EQIL 12

EQIL 13

EQIL 14

EQIL 15

EQIL 16

EQIL 17

EQIL 18

EQIL $\quad 19$

EQIL 20

EQIL $\quad 21$

EQIL $\quad 22$

EQIL 23

EQIL 24

EQIL 25

EQIL 26

EQIL 27

EQIL 28

EQIL 29

EQIL 30

EQIL 31

EQIL $\quad 32$

EQIL 33

EQIL $\quad 34$

EQIL $\quad 35$

EQIL 36

EQIL 37

EQIL 38

EQIL $\quad 39$

EQIL 40

EQIL 41

EQIL $\quad 42$

EQIL 43

EQIL $\quad 44$

EQIL 45

EQIL $\quad 46$

EQIL $\quad 47$

EQIL 48

EQIL 49

EQIL 50

EQIL 51

EQIL 52

EQIL 53

EQIL 54

EQIL $\quad 55$ 
60

CALL DASH

EQIL

$\operatorname{CALL} \operatorname{STDAT}(M, 5,1,21, V)$

EQIL

EQIL

RETURN

EQIL

100 FORMAT ( $/ 20 \mathrm{X}, 15 \mathrm{H}$ CLOSED ORBITS A6, 12H DEFINED BY A6/29X, $1 \mathrm{HX}, 10 \mathrm{X}$, EQIL $15 \mathrm{HDX} / \mathrm{DS}, 8 \mathrm{X}, 1 \mathrm{HY}, 10 \mathrm{X}, 5 \mathrm{HDY} / \mathrm{DS}, 7 \mathrm{X}, 4 \mathrm{HS}-\mathrm{S0}, 8 \mathrm{X}, 4 \mathrm{HDP} / \mathrm{P}, 7 \mathrm{X}, 6 \mathrm{HMISVAR} / /$ )

105 FORMAT (25H SYNCHRONOUS C.0. VS , $7(F 10.5,1 X) / 25 \mathrm{H} \quad \mathrm{DP} / \mathrm{P}=0$.

EQIL

EQIL

65 2/) $1 \mathrm{G}$

$$
\begin{aligned}
& \text { Vo }, 7(\mathrm{~F} 10.5,1 \mathrm{X})) \\
& \text { VALUES AFT }
\end{aligned}
$$

115 FORMAT ( $5 X, 3 \mathrm{H}$ BY, $1 \mathrm{X}, \mathrm{A} 4,7 \mathrm{X}, 2 \mathrm{HVP}, 3 \mathrm{X}, 7(\mathrm{~F} 10.5,1 \mathrm{X})$ )

EQIL

EQIL

EQIL 
1

SUBROUTINE EQU(M,N)

EQU

EQU

EQUATE COMPUTED DATA STORAGE OF $M$ TO THAT OF $N$.

C 1H* IS A FLAG TO LEAVE THE PRESENT VALUES OF $M$ UNTOUCHED,

EQU

C I.E. KEEP THE ORIGINALS SET FOR $M$. OTHERS WILL BE CHANGED TO

EQU

EQU

C THOSE READ IN FROM N OR RESET.

EQU

EQU

EQU

DIMENSION INF(24)

EQU

EQU

EQU

DO $1 \mathrm{I}=1,9$

EQU

EQU

EQU

EQU

EQU

EQU

EQU

EQU

EQU

EQU

2

3

4

5

6

7

8

9

10

11

$1 \operatorname{INF}(\mathrm{I})=1 \mathrm{H} *$

C USE LQ AND NQ OF $N$. COMPUTE NEW NTOT

$2 \quad \operatorname{INF}(\mathrm{I})=1 \mathrm{H} *$

$\operatorname{INF}(18)=\operatorname{INF}(11)+\operatorname{INF}(17)$

20 RETURN

END 
1

SUBROUTINE ERRSR(M)

C ERROR HAS BEEN ENCOUNTERED. EXECUTION OF RUN HALTED.

ERRSR

ERRSR

C READS CARDS TO FIND END OF RUN

C RETURNS TO MAIN FOR NEXT RUN

ERRSR

INTEGER OPNAME

DIMENSION ICARD (8)

ERRSR

ERRSR

CALL WFLSR

$1 \operatorname{READ}(2,5)($ ICARD $(I W), I W=1,8)$

5 FORMAT $(8 A 10)$

10

DECODE $(12,6$, ICARD $)$ OPNAME

ERRSR

ERRSR

ERRSR

ERRSR

ERRSR

ERRSR

ERRSR

IF (OPNAME.NE. 3HFIN) GO TO 1

ERRSR

WRITE $(3,20)$

15

FORMAT ( $30 \mathrm{H} * * *$ ERROR *** RUN TERMINATED. $/ 1 \mathrm{X}, 119(1 \mathrm{H}$.$) )$

ERRSR

ERRSR

RETURN

ERRSR 
1

SUBROUTINE EXCHM(M,N)

EXCHM

EXCHM

2

C RX(N) IS SET EQUAL TO RY(M), AND RY(N) IS SET EQUAL TO RX(M)

EXCHM

EXCHM

CALL RXY (M, RX,RY, RW)

EXCHM

EXCHM

EXCHM

RETURN

END

8 


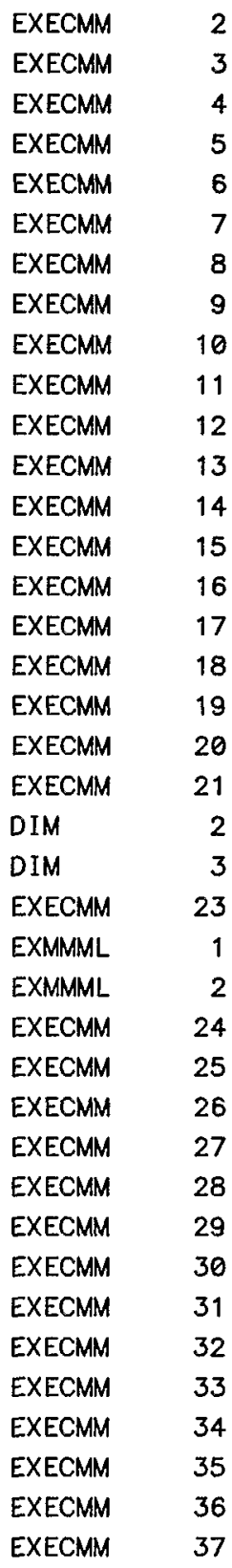

CARD NR. SEVERITY DETAILS DIAGNOSIS OF PROBLEM

31 I MI ARRAY REFERENCE OUTSIDE DIMENSION BOUNDS. 
1

5

10

15

20

25

30

35

40

45

50

55
SUBROUTINE FCN(NPAR, G, F, X, IFLAG)

FCN

LEVEL 2, STORE, INFF, IWORK

COMMON STORE( 48000 ), IWORK (10)

DIMENSION INFF $(24,2000)$

EQUIVALENCE (INFF, STORE)

COMMON/PAREXT/ U(30), NAM(30), DDD(32)

COMMON /MATCH/ KAT, KBT, MPAR(30), IPAR(30), MSR, MTR, LTRTAB, FNORM,

$1 \quad \operatorname{ICON}(30), \operatorname{ITYPE}(30), \operatorname{NPOS}(30), \operatorname{IPOS}(7,30), \operatorname{BETO}(30), \operatorname{WT}(30)$,

$2 \operatorname{SIG}(30), \operatorname{NAMBFC}(42), \operatorname{ERPR}, \operatorname{IVSAV}, \operatorname{LVAR}(30), \operatorname{WTVAR}(30), \operatorname{VAL}(30)$,

3 VARWTS, IPRPOS $(6,30)$

LOGICAL ERPR, LVAR, VARWTS

COMMON /MATCH3/NOPR, NONU, FLAG, IPR, IPP, MINFLG, MI FLG, MATFLG, GLOBAL

LOGICAL FLAG, NOPR, NONU, MIFLG, MATFLG, GLOBAL

LOGICAL BETFLG, BXBYFLG

DIMENSION X(30), BETX(9), BETY(9),T(7,,7), RW(3), NPS(6)

DATA NN, IBLNK/6,1H /

C EVALUATION OF F, IE CHISQUARE, FOR MINUIT

MINFLG=IFLAG

$\mathrm{NGO}=2$

IF (IFLAG.EQ.4) GO TO 4

$\mathrm{NGO}=1$

NOPR $=$. FALSE,

IF (IFLAG.EQ.1.AND.IPP.NE. O) NOPR = .TRUE.

IF (IFLAG. EQ.3.AND. IPP.GT.1) NOPR=.TRUE.

NONU $=$. FALSE.

$\mathrm{N}=0$

$\mathrm{RTPI}=1 . /(4 . * \operatorname{ACOS}(\theta)$.

4 CONTINUE

C PUT PARAMETER VALUES IN DATA ARRAYS

BETFLG $=$.FALSE.

NVARS $=$ NPAR

DO $1 J=1$,NVARS

MEL $=\operatorname{MPAR}(J)$

$\operatorname{INTEL}=\operatorname{IPAR}(J)$

CALL REPFLT(MEL, INTEL, $X(J)$ )

CONTINUE

C CALL SYNCH SUBROUTINE

CALL DOIT(MSR)

C CALCULATE CHISQUARE

GO TO $(8,9)$ NGO

8 WRITE $(3,100)$

$9 \quad F=0.0$

DO $2 K=1$, KAT

$I$ ITYPK $=I$ TYPE $(K)$

IF (ITYPK.EQ. -1) GO TO 2

$\mathrm{NPOSK}=\mathrm{NPOS}(\mathrm{K})$

$I C O N K=I C O N(K)$

BXBYFLG $=$.FALSE.

IF (ICONK.EQ.37) BXBYFLG = . TRUE.

$W T K=W T(K)$

$B F I T K=B E T O(K)$

\section{FCN}

BLANK

BLANK

8GMARSIZ

86MARSIZ 2

86MARSIZ 3

BLANK 5

FCN 5

MATCH 2

MATCH 3

MATCH 4

MATCH 5

MATCH 6

MATCH3 2

MATCH3 3

FCN 8

FCN 9

FCN 10

FCN 11

FCN 12

FCN 13

FCN 14

FCN $\quad 15$

FCN 16

FCN 17

FCN $\quad 18$

FCN 19

FCN 20

FCN 21

FCN 22

FCN 23

FCN 24

FCN 25

FCN 26

FCN 27

FCN 28

FCN 29

FCN $\quad 30$

FCN 31

FCN $\quad 32$

FCN 33

FCN $\quad 34$

FCN $\quad 35$

FCN 36

FCN $\quad 37$

FCN $\quad 38$

FCN $\quad 39$

FCN 40

FCN 41

FCN $\quad 42$

FCN $\quad 43$

FCN $\quad 44$

FCN $\quad 45$

FCN $\quad 46$

FCN $\quad 47$

FCN $\quad 48$ 
60

65

70

75

80

85

90

95

100

105
IF ((ITYPK.EQ.1).OR. (ITYPK.EQ.2)) GO TO 10

IF (ICONK.EQ.34) GO TO 22

IF (MATFLG.OR. ICONK.EQ.35) GO TO 3

IF (BXBYFLG) GO TO 21

LTRTBK $=$ LTRTAB $-1+$ ICONK

GO TO 28

21 LBXK $=$ LTRTAB +1

LBYK = LTRTAB +7

28 CONTINUE

IF(NPOSK.EQ.1) GO TO 17

$F I K=0.0$

DO $6 \mathrm{~L}=1$, NPOSK

$\operatorname{IPLK}=14 * \operatorname{IPOS}(\mathrm{L}, \mathrm{K})$

IF (.NOT.BXBYFLG) GO TO 25

NIKL1 $=$ LBXK + IPLK

NIKL2 = LBYK + IPLK

$B X=\operatorname{STORE}$ (NIKL1)

$B Y=S T O R E(N I K L 2)$

$B E T K L=B X / B Y$

GO TO 26

25 NIKL $=$ LTRTBK + IPLK

BETKL $=$ STORE $($ NIKL $)$

26 DEL $=$ BETKL - BFITK

$F I K=F I K+D E L * D E L$

GO TO $(11,6)$ NGO

$11 \quad \mathrm{NTYP}=5 \mathrm{H}$

$N=N+1$

WRITE $(3,101) N, N A M B F C$ (ICONK), NTYP, IPRPOS $(L, K), B E T K L, B F I T K, D E L$,

6 CONTINUE SIG (K)

GO TO 5

17 CONT INUE

$\operatorname{IPLK}=14 * \operatorname{IPOS}(1, \mathrm{~K})$

IF (.NOT.BXBYFLG) GO TO 27

NIKL1 = LBXK + IPLK

NIKL2 = LBYK + IPLK

$B X=$ STORE (NIKL1)

$B Y=S T O R E(N I K L 2)$

$B E T 1 K=B X / B Y$

GO TO 23

27 NIK1 $=$ LTRTBK + IPLK

BET $1 K=S T O R E$ (NIK1)

23 CONTINUE

DEL $=B E T 1 K-B F I T K$

$F I K=D E L * D E L$

GO TO $(12,5)$ NGO

$12 \mathrm{NTYP}=5 \mathrm{H}$

$\mathrm{N}=\mathrm{N}+1$

IF (NPOSK.EQ.1) GO TO 36

WRITE $(3,104)$ N,NAMBFC (ICONK), NTYP, (IPRPOS $(L, K), L=1, N N), B E T 1 K$,

\section{1} BFITK,DEL, SIG(K)

GO TO 5
FCN

FCN

FCN

FCN

FCN

FCN

FCN

FCN

FCN

FCN

FCN

FCN

FCN

FCN

FCN

FCN

FCN

FCN

FCN

FCN

FCN

FCN

FCN

FCN

FCN

FCN

FCN

FCN

FCN

FCN

FCN

FCN

FCN

FCN

FCN

$\mathrm{FCN}$

FCN

FCN

FCN

FCN

FCN

FCN

FCN

FCN

FCN

FCN

FCN

FCN

FCN

FCN

FCN

FCN

FCN

FCN

FCN

FCN

FCN 
36 WRITE $(3,101) \mathrm{N}, \operatorname{NAMBFC}(\mathrm{ICONK}), \operatorname{NTYP}, \operatorname{IPRPOS}(1, K), \operatorname{BET} 1 K, \mathrm{BFITK}, \mathrm{DEL}$, 1 $\operatorname{SIG}(K)$ GO TO 5

120

10 CONTINUE

$F I K=0.0$

DO $14 \mathrm{~L}=1$, NPOSK

NIKL $=L T R T A B+14 * I P O S(L, K)-1+I C O N K$

$B E T K L=S T O R E(N I K L)$

IF (L.EQ.1) GO TO 140

125

$D B K J=B E T K L-B E T K J$

$D E L=D B K J-B F I T K$

$F I K=F I K+D E L * D E L$

140 IF (ITYPK.EQ.2) BETKL = -BETKL

$B E T K J=B E T K L$

IF (L.EQ.1) GO TO 14

GO TO $(13,14)$ NGO

$13 \quad N T Y P=5 H D I F$

IF (ITYPK.EQ.2) NTYP $=5$ HSUM

$\mathrm{N}=\mathrm{N}+1$

WRITE $(3,101)$ N, NAMBFC (ICONK), NTYP, IPRPOS (L, K), DBKJ , BFITK,

1 DEL,SIG(K)

14 CONTINUE

GO TO 5

140

3 CONTINUE

IF (BETFLG) GO TO 33

CALL BET(MTR, BETX, BETY, 0$)$

BETFLG $=$. TRUE.

33 IF (ICONK.EQ.35) GO TO 24

IF (BXBYFLG) GO TO 29

IF (ICONK.GT.6) GO TO 31

$B E T 1 K=B E T X(I C O N K)$

GO TO 32

150

31 BET1K = BETY $($ ICONK-6)

32 IF (ICONK.EQ.1.OR. ICONK.EQ.7) BETIK = RTPI * BETIK

GO TO 23

$\mathrm{BX}=\mathrm{BETX}(2)$

$\mathrm{BY}=\mathrm{BETY}(2)$

$B E T 1 K=B X / B Y$

GO TO 23

160

24

CONTINUE

$\operatorname{APQN}=0.5 *(\operatorname{IPOS}(1, K) * \operatorname{BETX}(1)+\operatorname{IPOS}(2, K) * \operatorname{BETY}(1)) * \operatorname{IPOS}(3, K)$

$1 / \operatorname{IPOS}(4, K)$

$\mathrm{BET} 1 \mathrm{~K}=1 . /(\mathrm{ABS}(\operatorname{SIN}(\mathrm{APQN}))+.0000000001)$

GO TO 23

CONT INUE

CALL RTRV7 (MTR, T, RW)

$I I=I P O S(1, K)$

$\mathrm{J} J=\operatorname{IPOS}(2, \mathrm{~K})$

BET1K $=T(I I, J J)$

FCN $\quad 106$

FCN 107

FCN $\quad 108$

FCN 109

FCN 110

FCN $\quad 111$

FCN $\quad 112$

FCN $\quad 113$

FCN $\quad 114$

FCN $\quad 115$

FCN $\quad 116$

FCN $\quad 117$

FCN $\quad 118$

FCN $\quad 119$

FCN 120

FCN $\quad 121$

FCN 122

FCN 123

FCN 124

FCN 125

FCN $\quad 126$

FCN 127

FCN 128

FCN 129

FCN 130

FCN 131

FCN 132

FCN 133

FCN 134

FCN 135

FCN 136

FCN 137

FCN 138

FCN 139

FCN 140

FCN 141

FCN 142

FCN 143

FCN 144

FCN 145

FCN 146

FCN 147

FCN 148

FCN 149

FCN 150

FCN 151

FCN 152

FCN 153

FCN 154

FCN 155

FCN 156

FCN 157

FCN 158

FCN $\quad 159$

FCN 160

FCN 161

FCN 162 
175

$5 \quad F=F+F I K * W T K * W T K$

FCN $\quad 163$

2 CONTINUE

180

IF (.NOT.VARWTS) GO TO 20

FCN

DO $18 \mathrm{~J}=1$, NVARS

FCN 167

$\operatorname{IF}($. NOT. LVAR(J)) GO TO 18

FCN 168

$D I F=(X(J)-V A L(J))$

FCN 169

DIFWT $=D I F * W T V A R(J)$

FCN 170

$F=F+D I F W T * D I F W T$

FCN 171

GO TO $(19,18)$ NGO

$19 \mathrm{~N}=\mathrm{N}+1$

SIGV $=1 . / W T V A R(J)$

185

WRITE $(3,103) N, \operatorname{NAM}(J), X(J), \operatorname{VAL}(J), D I F, S I G V$

$\mathrm{FCN}$

172

FCN 173

FCN 174

FCN $\quad 175$

18 CONTINUE

FCN $\quad 176$

FCN $\quad 177$

$20 \quad F=F * F N O R M$

FCN $\quad 178$

FCN 179

FCN 180

$190 \quad$ GO TO $(15,16)$ NGO

FCN 181

15 WRITE $(3,102)$ N,F

16 IF(IFLAG.NE. 1) RETURN

FCN $\quad 182$

NOPR $=$. TRUE.

FCN 183

NONU $=$. TRUE.

$195 \quad$ DO $7 \mathrm{~K}=1$, KAT

IF (ICON(K).EQ.1) NONU=. FALSE.

FCN $\quad 185$

FCN $\quad 186$

IF (ICON(K).EQ.7) NONU=.FALSE.

FCN $\quad 187$

7 CONTINUE

FCN 188

FCN 189

FCN 190

200

RETURN

FCN

191

100 FORMAT $(/ 3 X, *$ CONSTRAINT $*, 3 X, *$ FUNCTION $*, 13 X, *$ POSITIONS OR INDICES $*$

FCN

1 13X,*PRESENT VALUE*, 4X,*DESIRED VALUE*, 4X,*ERROR*,11X,

FCN

193

2 *TOLERANCE*/)

205

101 FORMAT $(/ 3 X, 15,8 X, A 5,2 X, A 5,2 X, A 5,30 X, 4 F 16.7)$

$104 \operatorname{FORMAT}(/ 3 \mathrm{X}, 15,8 \mathrm{X}, \mathrm{A5}, 2 \mathrm{X}, \mathrm{A} 5,2 \mathrm{X}, 6 \mathrm{A5}, 5 \mathrm{X}, 4 \mathrm{~F} 16.7)$

FCN

103 FORMAT $(3 X, I 5,8 X, A 10,38 X, 4 F 16.7)$

102 FORMAT $(/ 5 X, 27 \mathrm{HFCN}=\operatorname{SUM}[($ ERROR/TOL. $) * * 2] /, 12,2 \mathrm{H}=, \mathrm{E} 15.7)$

FCN

197 END

FCN

198

FCN

199

CARD NR. SEVERITY DETAILS DIAGNOSIS OF PROBLEM

$\begin{array}{rr}45 & \text { I } \\ 84 & \text { I } \\ 108 & \text { I } \\ 131 & \text { I } \\ 182 & \text { I } \\ 190 & \text { I }\end{array}$

AN IF STATEMENT MAY BE MORE EFFICIENT THAN A 2 OR 3 BRANCH COMPUTED GO TO STATEMENT. AN IF STATEMENT MAY BE MORE EFFICIENT THAN A 2 OR 3 BRANCH COMPUTED GO TO STATEMENT. AN IF STATEMENT MAY BE MORE EFFICIENT THAN A 2 OR 3 BRANCH COMPUTED GO TO STATEMENT. AN IF STATEMENT MAY BE MORE EFFICIENT THAN A 2 OR 3 BRANCH COMPUTED GO TO STATEMENT. AN IF STATEMENT MAY BE MORE EFFICIENT THAN A 2 OR 3 BRANCH COMPUTED GO TO STATEMENT. AN IF STATEMENT MAY BE MORE EFFICIENT THAN A 2 OR 3 BRANCH COMPUTED GO TO STATEMENT. 
C

C RETRIEVES ONE PIECE OF FL. PT. DATA FROM INDEX J OF FL PT

FDAT

C STORAGE OF ELEMENT $M$. IF DATA IS SYMBOLIC, THE VALUE (IN FL PT) IS

FDAT

C RETRIEVED. SYMBOLICS MAY BE NESTED 10 DEEP.

FDAT

FDAT

FDAT

C INTERNAL STORAGE OF SYMBOLIC IS OONNNINAME

FDAT

C WHERE

$\mathrm{C}$

00 ARE PURE ZEROS

NNN IS AN INTEGER OR BCD BLANK INDICATING WHICH VARIABLE OF

10

C ELEMENT NAMED INAME IS TO BE RETRIEVED.

C

C

15

20

LEVEL 2, STORE, INFF, IWORK

COMMON STORE(48000), IWORK(10)

DIMENSION INFF $(24,2000)$

EQUIVALENCE (INFF, STORE)

C

$\operatorname{LOC}=\operatorname{INFF}(14, M)+J-1$

FDAT = REALNUM(LOC)

C

RETURN

END
FDAT

FDAT

FDAT

FDAT

FDAT

BLANK

BLANK

86MARSIZ

86MARSIZ

86MARSIZ

BLANK

FDAT

FDAT

FDAT

FDAT

FDAT

FDAT
2

3

4

5

6

7

8

9

10

11

12

13

2

3

1

2

3

5

15

16

17

18

19

20 
FITS FITQ -PHASE ADVANCE OVER 2PI

FITB - BETATRON FUNCTIONS WHOSE INDECES ARE KA AND KB.

FIT

FITR — MATRIX ELEMENTS INDEXED BY KA AND KB.

FITV - VECTOR ELEMENTS INDEXED BY KA AND KB. SUB IS NAME OF SUBROUTINE TO EXECUTE.

VARY THE I1-TH PARAMETER OF QF AND I2-TH OF QD, COMPUTE $C$, ITERATE UNTIL THEY MATCH, BUT NOT MORE THAN 100 TIMES.

LEVEL 2, STORE, INFF, IWORK

COMMON STORE(48000), IWORK (10)

DIMENSION INFF $(24,2000)$

EQUIVALENCE (INFF,STORE)

COMMON/GRR/IERR, BSW, VSW, RSW, QSW , XSW

LOGICAL IERR, BSW, VSW, RSW, QSW, XSW

COMMON/CONTRL/ERROR, MODE, RSRV, STOR, XEQ, TRASW, NFSW, EMPTY, INDEF,

\section{1} LDFLG, FIN

LOGICAL ERROR, RSRV, STOR, XEQ, TRASW, NFSW, EMPTY, INDEF, LDFLG, FIN

30

DIMENSION $H(2), D(2), A(2,2), B(2,2), R X(2,3), R Y(2,3), S(2), P H I(5)$

DIMENSION MV1 (1), IV1(1),V(2),U(2),W(2), VAR(2), FC(2),F(2),G(2)

DIMENSION RXRY(12), VC(7), BXBY(20), RW(3), Q(2)

LOGICAL IND, INF

LOGICAL INDEFF, INF INN

INTEGER P1,P2, ELNUM

FIT 15

FIT : 16

FIT 17

FIT 18

BLANK : 2

BLANK 3

86MARSIZ 1

86MARSIZ 2

86MARSIZ 3

BLANK 5

GRR 2

GRR 3

GRR 4

CONTRL 2

CONTRL 3

CONTRL 4

CONTRL 5

FIT 22

FIT 23

FIT 24

FIT 25

FIT 26

LOGICAL SINGL

FIT - 28

SINGL $=$. FALSE.

IF (QSW) GO TO 99

IF(RSW.AND.KB.NE.3H ) GO TO 99

FIT 31

IF (KA.GE.100.OR.KB.NE.0) GO TO 99

SINGL=. TRUE.

$K B=K A$

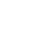

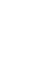

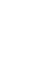

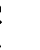

4

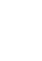


DECODE $(3,21, K B) J 1, J 2, J 3$

21 FORMAT (3I1)

$\mathrm{K} 1=\mathrm{I} 1 * 6+(\mathrm{I} 3-1) * 2+\mathrm{I} 2$

FIT

$\mathrm{K} 2=\mathrm{J} 1 * 6+(\mathrm{J} 3-1) * 2+\mathrm{J} 2$

FIT

51

GO TO 16

FIT

52

$60 \quad \mathrm{~K} 1=\mathrm{KA}$

FIT

$K 2=K B$

65

$16 \quad M 1=\operatorname{ELNUM}(M V 1(1))$

FIT

FIT

FIT

$M 2=\operatorname{ELNUM}(M V 1(2))$

$P 1=I V 1(1)$

$P 2=I V 1(2)$

C LOOK UP INPUT GUESS FOR VARIABLES

FIT

FIT

FIT

FIT

FIT

FIT

FIT

FIT

FIT

FIT

FIT

FIT

FIT

FIT

FIT

FIT

FIT

FIT

FIT

FIT

FIT

FIT

FIT

FIT

FIT

FIT

FIT

FIT

FIT

FIT

FIT

FIT

FIT

FIT

FIT

FIT

FIT

FIT

FIT

FIT

FIT

FIT

FIT

C FITQ

CALL DOIT(MSR)

IF (ERROR) RETURN

IF (VSW) GO TO 110

IF (RSW) GO TO 112

FIT

CALL RXY (MC, RX, RY, RW)

$F C(1)=(R X(1,1)+R X(2,2)) * .5$

FIT

FIT

$F C(2)=(R Y(1,1)+R Y(2,2)) * .5$

FIT

GO TO 106 
105 CALL 'BET(MC, BXBY (1), $\operatorname{BXBY}(11), 0)$

$F C(1)=B X B Y(K 1) / 100$

$\mathrm{FC}(2)=\operatorname{BXBY}(K 2) / 100$.

GO TO 106

110 CALL DATA $(M C, 5,1,7, V C)$

$F C(1)=V C(K 1) / 100$.

$F C(2)=V C(K 2) / 100$.

FIT

GO TO 106

112 CALL RXY (MC, RXRY (1), RXRY(7),RW)

FIT

$F C(1)=R X R Y(K 1) / 100$

$\mathrm{FC}(2)=\mathrm{RXRY}(K 2) / 100$.

106 GO TO $(107,108,109)$, I

$107 \mathrm{G}(1)=F C(1)$

$G(2)=F C(2)$

GO TO 115

$108 H(1)=F C(1)$

$H(2)=F C(2)$

GO TO 115

$109 F(1)=F C(1)$

$F(2)=F C(2)$

135

$I N D=\operatorname{INDEFF}(F(1))$

$I N F=\operatorname{INFINN}(F(1))$

IF(IND.OR. INF) GO TO 130

$\operatorname{IND}=\operatorname{INDEFF}(F(2))$

$I N F=\operatorname{INFINN}(F(2))$

140

IF (IND.OR. INF) GO TO 130

115 CONTINUE

$D(1)=P H I(1)-F(1)$

$D(2)=P H I(2)-F(2)$

IF (ABS(D(1)) . LE.DEL.AND.ABS(D(2)).LE.DEL) GO TO 120

FIT

FIT

FIT

FIT

FIT

FIT

FIT

FIT

FIT

FIT

FIT

FIT

FIT

FIT

FIT

FIT

FIT

FIT

FIT

FIT

FIT

FIT

FIT

145

C

FIT NOT CLOSE ENOUGH. ITERATE AGAIN UNLES LIMIT HAS BEEN REACHED

FIT

$K=K+1$

IF (K.EQ.KI) GO TO 125

FIT

FIT

C SET UP NEXT GUESS

$H D=1 . / H S T E P$

150

$A(1,1)=(G(1)-F(1)) * H D$

$A(1,2)=(H(1)-F(1)) * H D$

$A(2,1)=(G(2)-F(2)) * H D$

$A(2,2)=(H(2)-F(2)) * H D$

IF(SINGL) GO TO 999

155

DET $=(A(1,1) * A(2,2))-(A(1,2) * A(2,1))$

$B(1,1)=A(2,2) / D E T$

$B(1,2)=-A(1,2) / D E T$

$B(2,1)=-A(2,1) / D E T$

$B(2,2)=A(1,1) / D E T$

160

$S(1)=B(1,1) * D(1)+B(1,2) * D(2)$

$S(2)=B(2,1) * D(1)+B(2,2) * D(2)$

$\operatorname{VAR}(1)=\operatorname{VAR}(1)+(S(1) * X L A M)$

$\operatorname{VAR}(2)=\operatorname{VAR}(2)+(S(2) * X L A M)$

GO TO 75

165

$999 \operatorname{VAR}(1)=\operatorname{VAR}(1)+\operatorname{XLAM} * \mathrm{D}(1) / A(1,1)$

$\operatorname{VAR}(2)=\operatorname{VAR}(1)$

GO TO 75

FIT

FIT

FIT

FIT

FIT

FIT

FIT

FIT

FIT

FIT

FIT

FIT

FIT

FIT

FIT

FIT

FIT

FIT

FIT

FIT

FIT

FIT

FIT

FIT

120 WRITE $(3,1) P 1, \operatorname{MV} 1(1), \operatorname{VAR}(1), P 2, \operatorname{MV} 1(2), \operatorname{VAR}(2)$

RETURN 
1 FORMAT ( $/ / 39 H$ PARAMETER REPLACEMENTS MADE BY FITTING/5X, I5, $1 \mathrm{X}$,

$14 \mathrm{H}$ OF $, A 5,3 \mathrm{H}=, \mathrm{E} 16.9,7 \mathrm{X}, \mathrm{I5}, 1 \mathrm{X}, 4 \mathrm{H}$ OF $, \mathrm{A5}, 3 \mathrm{H}=, \mathrm{E} 16.9)$

* IteRATION LIMIT REACHED. FIT FAILED:

FIT

125 WRITE $(3,7) \mathrm{K}$, VAR

FIT

GO TO 135

7 FORMAT ( $39 \mathrm{H}$ NUMBER OF ITERATIONS REACHED LIMIT OF $15 / 31 \mathrm{H}$ CURRENT

10 FORMAT (32H ROOT IS INDEFINITE OR INFINITE.//)

* If FITQ, tRY OtHER fit ROUTINE

IF (QSW) CALL FITE(MSR, MC, MV1, IV1, Q, KA, KB)

END

CARD NR. SEVERITY DETAILS DIAGNOSIS OF PROBLEM

$\begin{array}{rrr}45 & \text { I } & \text { MV1 } \\ 46 & \text { I } & \text { IV1 } \\ 66 & \text { I } & \text { MV1 } \\ 68 & \text { I } & \text { IV1 } \\ 94 & \text { I } & \\ 126 & \text { I } & \\ 170 & \text { I } & \text { MV1 }\end{array}$

ARRAY REFERENCE OUTSIDE DIMENSION BOUNDS.

ARRAY REFERENCE OUTSIDE DIMENSION BOUNDS.

ARRAY REFERENCE OUTSIDE DIMENSION BOUNDS.

ARRAY REFERENCE OUTSIDE DIMENSION BOUNDS.

AN IF STATEMENT MAY BE MORE EFFICIENT THAN A 2 OR 3 BRANCH COMPUTED GO TO STATEMENT AN IF STATEMENT MAY BE MORE EFFICIENT THAN A 2 OR 3 BRANCH COMPUTED GO TO STATEMENT. ARRAY REFERENCE OUTSIDE DIMENSION BOUNDS. 
1

5

10

15

20

25

30

35

40

45

50

55
SUBROUTINE FITE(MSR, MC, MV1, IV1, Q, KA, KB)

LEVEL 2, STORE, INFF, IWORK

COMMON STORE(48000), IWORK(10)

DIMENSION INFF $(24,2000)$

EQUIVALENCE (INFF, STORE)

COMMON/QUE/V1, V2, G, CQXZ, CQYZ, CQX, CQY, M1 , M2, P1 ,P2, MS, ME, VV1, VV2

$1, K 1, K 2$

INTEGER P1,P2, ELNUM

DIMENSION MV1(1), IV1(1),Q(2)

COMMON/GRR/IERR, BSW, VSW, RSW, QSW, XSW

LOGICAL IERR, BSW, VSW, RSW, QSW, XSW

EXTERNAL QUEX

IF (.NOT.RSW) GO TO 2

$\operatorname{DECODE}(3,10, \mathrm{KA}) \mathrm{I} 1, \mathrm{I} 2, \mathrm{I} 3$

$\operatorname{DECODE}(3,10, \mathrm{~KB}) \mathrm{J} 1, \mathrm{~J} 2, \mathrm{~J} 3$

10 FORMAT (3I1)

$\mathrm{K} 1=\mathrm{I} 1 * 6+(\mathrm{I} 3-1) * 2+\mathrm{I} 2$

$\mathrm{K} 2=\mathrm{J} 1 * 6+(\mathrm{J} 3-1) * 2+\mathrm{J} 2$

GO TO 4

$2 \quad K 1=K A$

$\mathrm{K} 2=\mathrm{KB}$

$4 \quad M S=M S R$

$M E=M C$

$M 1=\operatorname{ELNUM}(M V 1(1))$

$M 2=\operatorname{ELNUM}(M V 1(2))$

$P 1=\operatorname{IV} 1(1)$

$P 2=\operatorname{IV} 1(2)$

C $\quad M N=\operatorname{INFF}(1, M 1)$

$V_{1}=\operatorname{FDAT}(M 1, P 1)$

$V 2=\operatorname{FDAT}(M 2, P 2)$

$\mathrm{V} 1=\mathrm{V} 1$

$\mathrm{V} 2=V_{2}$

IF (.NOT.QSW) GO TO 3

CQXZ $=\cos (6.283185307 * Q(1))$

$\operatorname{CQYZ}=\cos (6.283185307 * Q(2))$

GO TO 5

$3 \quad \operatorname{CQXZ}=Q(1)$

$\mathrm{CQYZ}=\mathrm{Q}(2)$

CQXZ $=$ CQXZ/100.

CQYZ $=$ CQYZ/100.

$5 \quad \operatorname{CALL} \operatorname{GRT}(1, \mathrm{~V} 1,7, \mathrm{QUEX})$

IF (IERR) GO TO 7

6 WRITE $(3,1) \mathrm{P} 1, \mathrm{MV} 1(1), \mathrm{V} 1, \mathrm{P} 2, \mathrm{MV} 1(2), \mathrm{V} 2$

1 FORMAT (//39H PARAMETER REPLACEMENTS MADE BY FITTING/5X, I5, 1X,

$14 \mathrm{H}$ OF $, A 5,3 \mathrm{H}=, E 16.9,7 \mathrm{X}, \mathrm{I5}, 1 \mathrm{X}, 4 \mathrm{H}$ OF $, A 5,3 \mathrm{H}=, \mathrm{E} 16,9)$

RETURN

$7 \quad \mathrm{~V} 1=\mathrm{V} 1$

$\mathrm{V} 2=\mathrm{V} 2$

CALL REPFLT( $M 1, P 1, V 1)$

CALL REPFLT (M2,P2, V2)

GO TO 6

END

\begin{tabular}{|c|c|}
\hline FITE & 2 \\
\hline BLANK & \\
\hline BLANK & \\
\hline 86MARSIZ & \\
\hline 86MARSIZ & \\
\hline 86MARSIZ & \\
\hline BLANK & \\
\hline FITE & \\
\hline FITE & \\
\hline FITE & \\
\hline FITE & \\
\hline GRR & \\
\hline GRR & \\
\hline GRR & \\
\hline FITE & \\
\hline FITE & 10 \\
\hline FITE & 11 \\
\hline FITE & 12 \\
\hline FITE & 13 \\
\hline FITE & 14 \\
\hline FITE & 15 \\
\hline FITE & 16 \\
\hline FITE & 17 \\
\hline FITE & 18 \\
\hline FITE & 19 \\
\hline FITE & 20 \\
\hline FITE & 21 \\
\hline FITE & 22 \\
\hline FITE & 23 \\
\hline FITE & 24 \\
\hline FITE & 25 \\
\hline FITE & 26 \\
\hline FITE & 27 \\
\hline FITE & 28 \\
\hline FITE & 29 \\
\hline FITE & 30 \\
\hline FITE & 31 \\
\hline FITE & 32 \\
\hline FITE & 33 \\
\hline FITE & 34 \\
\hline FITE & 35 \\
\hline FITE & 36 \\
\hline FITE & 37 \\
\hline FITE & 38 \\
\hline FITE & 39 \\
\hline FITE & 40 \\
\hline FITE & 41 \\
\hline FITE & 42 \\
\hline FITE & 43 \\
\hline FITE & 44 \\
\hline FITE & 45 \\
\hline FITE & 46 \\
\hline FITE & 47 \\
\hline FITE & 48 \\
\hline FITE & 49 \\
\hline FITE & 50 \\
\hline
\end{tabular}


CARD NR. SEVERITY DETAILS

DIAGNOSIS OF PROBLEM

29 I $\quad$ MV1

IV1

ARRAY REFERENCE OUTSIDE DIMENSION BOUNDS.

31 I IV1

ARRAY REFERENCE OUTSIDE DIMENSION BOUNDS.

47 I MV1 ARRAY REFERENCE OUTSIDE DIMENSION BOUNDS. 
1

5

10

15

20

25
FUNCTION FLDAT $(M, J)$

C DATA RETRIEVED MUST BE FLOATING POINT. SYMBOLIC NOT ALLOWED.

C RETRIEVES ONE FLOATING POINT NUMBER FROM INDEX $J$ OF LQ OF ELEMENT $M$

C

C IF RETRIEVING FROM A VAR SYNCH INSTRUCTION, FETCH VALUE

C IN FL. PT. STORAGE FROM POSITION DEFINED BY KA.

LEVEL 2, STORE, INFF, IWORK

COMMON STORE(48000), IWORK(10)

DIMENSION INFF $(24,2000)$

EQUIVALENCE (INFF, STORE)

INTEGER HVAR

DATA HVAR/3HVAR/

C

$\operatorname{LOC}=\operatorname{INFF}(10, M)+J-1$

NAMOP $=\operatorname{INFF}(1, M)$

IF (NAMOP.NE.HVAR) GO TO 25

$N=\operatorname{MDAT}(M, 1)$

$K A=\operatorname{INFF}(4, M)$

IF (KA.EQ.O) $K A=1$

$\operatorname{LOC}=\operatorname{INFF}(14, N)+K A-1$

25

FLDAT $=$ STORE $($ LOC $)$

RETURN

END
FLDAT 2

FLDAT 3

FLDAT 4

FLDAT 5

FLDAT 6

FLDAT 7

BLANK 2

BLANK 3

86MARSIZ 1

86MARSIZ 2

86MARSIZ 3

BLANK 5

FLDAT 9

FLDAT 10

FLDAT 11

FLDAT 12

FLDAT 13

FLDAT 14

FLDAT 15

FLDAT 16

FLDAT 17

FLDAT 18

FLDAT 19

FLDAT 20

FLDAT 21 
1

5

10

15

20
SUBROUTINE FLQ(M,L,J,F)

C FETCH F FROM INDEX OF LQ STORAGE OF ELEMENT $M$

C $L=1$ FOR LQ $L=2$ FOR $L Q 2 \quad L=3$ FOR $L Q 3$

LEVEL 2, STORE, INFF, IWORK

COMMON STORE(48000), IWORK(10)

DIMENSION INFF $(24,2000)$

EQUIVALENCE (INFF, STORE)

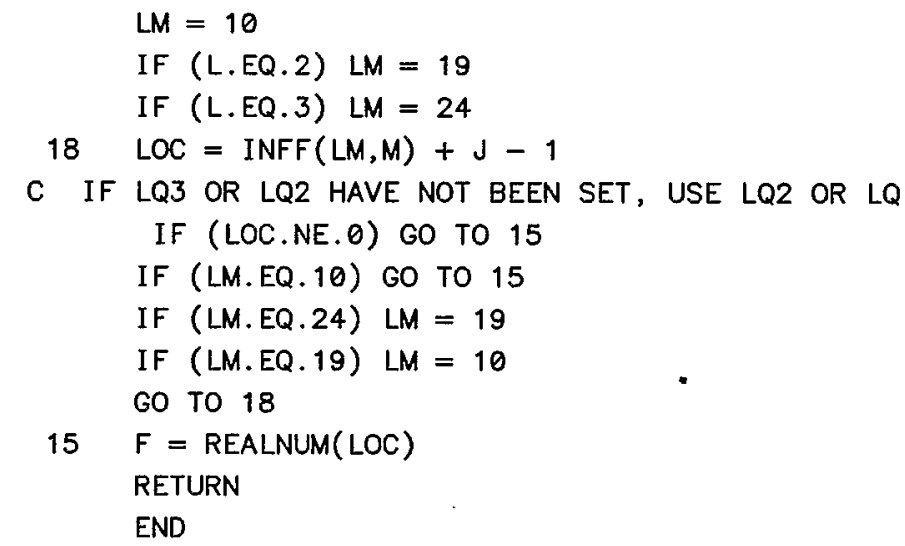

FLQ

FLQ

FLQ

BLANK

BLANK

86MARSIZ

86MARSIZ

86MARSIZ

BLANK

FLQ

FLQ

FLQ

FLQ

FLQ

FLQ

FLQ

FLQ

$F L Q$

$F L Q$

FLQ

FLQ

FLQ
2

3

4

2

3

1

2

3

5

6

7

8

9

10

11

12

13

14

15

16

17

18 
EQUILIBRIUM ORBITS UNDER LINEAR AND/OR ARBITRARY EXTERNAL TRANSFOR-

C MATIONS, LINEARIZED 4X4 MATRIX NEAR FIXED POINT (M4), EIGENVALUES

FXPT

AND EIGENVECTORS OF M4, ETC.

C

c s

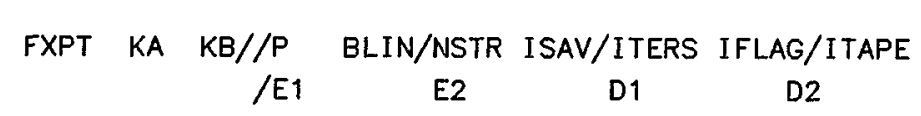

E2 D1

E1

FXPT

FXPT

C

FXPT

FXPT

FXPT

$K A=$ OUTPUT OPTION -

FXPT

$K A=0 \quad$ MATRIX, EIGENVALUES, CLOSED ORBIT, ETA-ORBIT AT POSITION $\theta$

$K A=1 \quad$ OPTION $\theta$ AND EIGENVECTORS AT POSITION 0

$K A=2 \quad$ OPTION 1 AND TRACK VCO, ETA

$K A=3 \quad$ OPTION 1 AND TRACK EIGENVECTORS

FXPT

FXPT

$K A=4$ OPTIONS 2 AND 3 TOGETHER

IF KA IS NEGATIVE, PRINT OF ORBITS IS SUPPRESSED

FXPT

FXPT

AT ALL POSITIONS EXCEPT THOSE OF ELEMENTS WHOSE

FXPT

FXPT

FIRST CHARACTER IS ":( 4-8)

FXPT

FXPT

FXPT

$K B=$ NUMBER OF SUPERPERIODS

$P=$ PREV. DEF. PART. WHOSE INPUT CONTAINS INITIAL REF. RAY

FXPT

FXPT

$B L I N=B E A M$ LINE DEFINED BY BML INSTRUCTION

IF KB IS NEGATIVE, THEN

THE REFLECTION OF BLIN IS ADDED TO BLIN TO FORM COMPLETE SP.

THIS FEATURE WILL NOT WORK IF NSTR .GT. 1

FXPT

FXPT

FXPT

FXPT

FXPT

FXPT

FXPT

FXPT

FXPT

FXPT

FXPT

FXPT

FXPT

C IFLAG $=3$ PLUS 4 RAYS OF LINEARIZED EQUATIONS,

FIRST FOR THE ENVELOPE AND SECOND FOR THE IONS OF A BEAM.

ITAPE $=0,1$

DO NOT, DO WRITE ETA AND CLOSED ORBITS ON TAPE 12

E1, $E 2=$ CONVERGENCE CRITERIA FOR DISPLACEMENTS, SLOPES

D1,D2 = DISP. FROM REF, RAY OF NEIGHBOR RAYS FOR CALCULATING M4.

$$
\mathrm{D} 3=\mathrm{DISP}
$$

$$
\begin{aligned}
& \text { IN DP/P FOR CALCULATING LINEARIZED } 3 \times O \text { MATRICES } \\
& \text { OUT }=\text { OUTPUT OPTION IN BCD }
\end{aligned}
$$

IF $D 1=0$, NEIGHBORING RAYS NOT CALCULATED, LINEARIZATION WITH REVMAT.

IF OUT IS BLANK, EIGENVECTORS NOT CALCULATED, NO TRACKING.

IF OUT $=$ 5HEVTRK, EIGENVECTOR ORBITS ARE TRACKED.

LINEARIZED $7 \times 7$ MATRIX STORED AT LQ+KADD OF FXPT INSTRUCTION

C LINEARIZED $4 \times 4$ MATRIX STORED AT LQ+KADD +53

C EIGENVALUES STORED AT AT LQ3, EIGENVECTORS AT LQ3+8

C $L Q 2=L Q+K A D D+49+16, L Q 3=L Q 2+7, \quad K A D D=3$. KIND IS $2 H R 7$

LEVEL 2, STORE, INFF, IWORK 
60

65

70

75

80

85

90

95

100

105

110
COMMON/BMI 1/MI 1 (16000)

COMMON/NELS1/NELS1

LEVEL 2,MI 1

C

COMMON/MH/ML \$ LEVEL 2,ML

DIMENSION MI (1)

EQUIVALENCE (MI, MI1), (NELS, NELS1)

COMMON/CONTRL/ERROR, MODE, RSRV, STOR, XEQ, TRASW, NFSW, EMPTY, INDEF,

1

LDFLG, FIN

LOGICAL ERROR, RSRV, STOR, XEQ, TRASW, NFSW, EMPTY, INDEF, LDFLG, FIN

COMMON /DIM/ LEND, MEND, ISAV, ISAV7, M7END, KADD, KADDR, MUNIT, MSYMPL

COMMON/CDERIV/IFLAG, MR, NR, DP, NV(11), DAT(20), UZ, STOT,

1 DAT2(7), MP2FLG, NF, HO, LOCALPH, NUMALPH

COMMON/CORB/TH(84), TV(84), NMON, NNH, NNV, I IH, I IV, NAMONH, NAMONV, NACORH, NACORV

DIMENSION RW(3)

DIMENSION $P(5), W W(112), V(7,16), E(4), F(4), V V(7)$,

$\operatorname{RM}(4,4), \operatorname{RMI}(4,5), \operatorname{LAM}(4), \operatorname{BLAM}(4), C(4), \operatorname{CM}(3,3), X(4,4)$,

$2 \operatorname{VZ}(7,7), \operatorname{RX}(2,3), \operatorname{LABELA}(4), \operatorname{LABELB}(4), \mathrm{MU}(4)$

DIMENSION LBL(6), $Q(4), R 7(7,7), \operatorname{ML}(2), \operatorname{VP}(7), \operatorname{EMOD}(4,2), \operatorname{EPHI}(4,2)$

EQUIVALENCE $(V, V W),(V V(1), V W(78)),(V Z(1,1), V W(85))$

COMPLEX CTR, CSQ, LAM, BLAM, CABS , CSQRT, CM, X, EX1, EX 3

REAL MU

INTEGER TRKR, R5J

DATA (NV(I) $I=1,11) / 1,2,3,4,5,6 * 0 /$

DATA ( LABELA(I), I $=1,4) / 5 \mathrm{HOX} \ldots, 1 \mathrm{H}, 5 \mathrm{HOY} \ldots, 1 \mathrm{H} /$,

$1 \quad(\operatorname{LABELB}(\mathrm{I}), \mathrm{I}=1,4) / 6 \mathrm{HLMD} 1,6 \mathrm{H} 1 / \mathrm{LMD} 1,6 \mathrm{HLMD} 3$,

$26 \mathrm{H} 1 / \mathrm{LMD} 3 /$

DATA (RW(I), I=1,3)/3*0.0/

$\operatorname{DATA}(\mathrm{VP}(\mathrm{I}), \mathrm{I}=5,7) / 0,1,0 . /$

$\operatorname{DATA}(\operatorname{LBL}(\mathrm{J}), \mathrm{J}=1,6) / 10 \mathrm{H} \operatorname{REAL}(X 1), 10 \mathrm{H} \operatorname{IMAG}(X 1)$,

1

2

DATA IBLNK/1H /

$10 \mathrm{H}$ REAL $(X 3), 10 \mathrm{H}$ IMAG $(X 3), 10 \mathrm{H}$ EQ ORBIT , 10H ETA ORBIT/

CALL RANGET (NRN)

$I I H=1$

I IV $=1$

$\mathrm{TPI}=4 . * A \cos (0$.

$K A=\operatorname{INFF}(4, M)$

$K A=I A B S(K A)$

$K B=\operatorname{INFF}(5, M)$

SPER $=$ FLOAT $(I A B S(K B))$

$\operatorname{NSTR}=\operatorname{IDAT}(M, 1)$

IF (NSTR.EQ.0) NSTR=1

$\operatorname{IVSV}=\operatorname{IDAT}(M, 2)$

$\operatorname{ITERS}=\operatorname{IDAT}(M, 3)$

$\operatorname{IFLAG}=\operatorname{IDAT}(M, 4)$

$\operatorname{ITAPE}=\operatorname{IDAT}(M, 5)$

IF (ITERS. EQ. $\theta$ ) ITERS $=10$

C RETRIEVE FLOATING POINT INPUT E1,E2,D1,D2

$\operatorname{CALL} \operatorname{DATA}(M, 1,1,5, P)$

$E P S W=P(1)$

\begin{tabular}{|c|c|}
\hline BLANK & \\
\hline NV3BMI 1 & \\
\hline BMI 1L & \\
\hline BMI $1 \mathrm{~L}$ & \\
\hline BMI 1L & \\
\hline BMI 1 & \\
\hline FXPTL & \\
\hline FXPT & 5 \\
\hline FXPT & 5 \\
\hline CONTRL & \\
\hline CONTRL & \\
\hline CONTRL & \\
\hline CONTRL & \\
\hline DIM & \\
\hline DIM & \\
\hline CDERIV & \\
\hline CDERIV & \\
\hline CORB613 & \\
\hline CORB 613 & \\
\hline FXPT & 6 \\
\hline FXPT & 6 \\
\hline FXPT & 6 \\
\hline FXPT & 6 \\
\hline FXPT & 6 \\
\hline FXPT & 6 \\
\hline FXPT & 6 \\
\hline FXPT & 6 \\
\hline FXPT & 6 \\
\hline FXPT & 6 \\
\hline FXPT & 7 \\
\hline FXPT & 7 \\
\hline FXPT & 7 \\
\hline FXPT & 7 \\
\hline FXPT & 7 \\
\hline FXPT & 7 \\
\hline FXPT & 7 \\
\hline FXPT & 7 \\
\hline FXPT & 7 \\
\hline FXPT & 7 \\
\hline FXPT & 8 \\
\hline FXPT683 & \\
\hline FXPT683 & \\
\hline FXPT & 81 \\
\hline FXPT & 82 \\
\hline FXPT & 8 \\
\hline FXPT & 8 \\
\hline FXPT & 85 \\
\hline FXPT & 86 \\
\hline FXPT & 87 \\
\hline FXPT & 88 \\
\hline FXPT & $8 s$ \\
\hline FXPT & 98 \\
\hline FXPT683 & , \\
\hline FXPT & 9 \\
\hline FXPT & 92 \\
\hline FXPT & 93 \\
\hline FXPT & 9 \\
\hline
\end{tabular}


$D E L W=P(3)$

$D E L T=P(4)$

FXPT

$D E L P=P(5)$

FXPT

IF (EPSW.EQ.0.) $E P S W=1 . E-08$

120

IF (EPST.EQ.0.) EPST=1.E-08

FXPT

IF (DELP.EQ.0) DELP $=1 . E-08$

FXPT

$N G O=2$

FXPT

IF (IFLAG.EQ.0) GO TO 2

FXPT

$\mathrm{NGO}=1$

IF (DELW.EQ.0.) DELW=1.E-08

FXPT

FXPT

IF (DELT.EQ.0.) DELT=1.E-08

FXPT

FXPT

2

$M L(1)=M 7 E N D-3$

$M L(2)=M 7 E N D-4$

130

C SET UP BEAM SYSTEM ARRAY MI

FXPT

FXPT

$M B M L=\operatorname{MDAT}(M, 2)$

NDAT $=\operatorname{INFF}(17, \mathrm{MBML})$

CALL MIFILL(MBML, 1 , NDAT, NELS, MI)

FXPT

FXPT

FXPT

IF (ERROR) RETURN

C OPTIONS ON ITERATIONS AND PRINT

135

C SET UP ARRAY FOR PREVIOUSLY DEFINED PARTICLE

FXPT

FXPT

FXPT

FXPT

FXPT

C SET UP INITIAL REFERENCE RAY

C ZERO VW ARRAY

DO $3 \mathrm{I}=1,112$

$V W(I)=0.0$

140

3

CONTINUE

$N P=\operatorname{MDAT}(M, 1)$

CALL DATA (NP, $1,1,7, V W)$

$N V(1)=1$

145

$M B=1$

$M E=N E L S$

NITS $=0$

C

$$
\text { IF ( ITERS.EQ. } 0 \text { ) ITERS }=25
$$

150

155

160

$E(1)=E P S W$

FXP

FXPT

FXPT

FXPT

FXPT

FXPT

FXPT

FXPT

FXPT

FXPT

FXPT

FXPT

FXPT

$E(2)=E P S T$

$E(3)=E P S W$

$E(4)=$ EPST

$F(1)=D E L W$

$F(2)=D E L T$

FXPT

FXPT

FXPT

FXPT

FXPT

$F(3)=D E L W$

$F(4)=D E L T$

CALL HED

$N P M=\operatorname{INFF}(2, N P)$

$\operatorname{NAM}=\operatorname{INFF}(2, M)$

WRITE $(3,1018)$

WRITE $(3,1000)$ NAM,NPM, (WW(I), I=1,7)

FXPT

FXPT

FXPT

FXPT

FXPT

FXPT

FXPT

$M M=1$

$I O P=I F L A G$

FXPT

FXPT

GO TO $(31,32)$ NGO

FXPT

FXPT

FXPT

FXPT

FXPT

FXPT

FXPT

FXPT

$N R=1$

GO TO 4

170

$31 \quad M N=9$ 
175

185

190

195

200

205

210

215

220

225
ASSIGN 4 TO NRS

IF (IFLAG.EQ.1) GO TO 800

$N R=5$

$\mathrm{MN}=1$

GO TO 802

39 IF (IFLAG.NE.1) GO TO 802

GO TO 800

C SAVE INITIAL CONDITIONS OF THE REFERENCE RAY.

4 DO $5 \quad \mathrm{I}=1,7$

$5 V V(I)=V W(I)$

IF (IFLAG.EQ.4) CALL DATA $(M, 7,1,7, V(1,8)$ )

C TRACK PARTICLES MM TO MN THROUGH BEAMLINE ELEMENTS MB TO ME. ASSIGN 510 TO TRKR

DO 1 ISTR=1,NSTR

CALL RANSET (NRN)

GO TO 900

510 CONTINUE

1 CONTINUE :

GO TO $(51,52)$ NGO

C CALCULATE THE 4X4 MATRIX, M.

51 IF (IFLAG.EQ.1) GO TO 511

DO $512 \mathrm{~J}=1,4$

DO $512 I=1,4$

$512 \operatorname{RM}(I, J)=V(I, J+1)$

GO TO 513

511 DO $55 \mathrm{~J}=1,4$

$N=J+1$

$\mathrm{DN}=.5 / \mathrm{F}(\mathrm{J})$

DO $55 \quad I=1,4$

$55 R M(I, J)=(V(I, N)-V(I, N+4)) * D N$

513 IF (KB.GE.Q) GO TO 56

$\operatorname{RX}(1,1)=\operatorname{RM}(2,1)$

$\operatorname{RX}(2,1)=\operatorname{RM}(4,1)$

$\operatorname{RX}(1,2)=\operatorname{RM}(2,3)$

$\operatorname{RX}(2,2)=\operatorname{RM}(4,3)$

$\operatorname{RX}(1,3)=-V(2,1)$

$R \times(2,3)=-V(4,1)$

GO TO 56

52 CALL RTRV7(M,R7, RW)

IF (KB.GE.0) GO TO 57

$\operatorname{RX}(1,1)=\operatorname{R7}(2,1)$

$\operatorname{RX}(2,1)=R 7(4,1)$

$\operatorname{RX}(1,2)=R 7(2,3)$

$\operatorname{RX}(2,2)=R 7(4,3)$

$\operatorname{RX}(1,3)=-V(2,1)$

$R X(2,3)=-V(4,1)$

57 DO $53 \mathrm{~J}=1,4$

DO $53 \mathrm{I}=1,4$

$53 \quad R M(I, J)=R 7(I, J)$

56 CONTINUE

IF (KB.GE.Q) GO TO 58

CALL MXDIV(RX, 2,1,D,2)

$V(1,1)=R X(1,3)+V V(1)$

$V(3,1)=R \times(2,3)+V V(3)$

$D X=V(2,1)$

$\mathrm{DY}=\mathrm{V}(4,1)$

$V(2,1)=0$.
FXPT

FXPT

FXPT

FXPT

FXPT

FXPT

FXPT

FXPT

FXPT

FXPT

FXPT

FXPT

FXPT

FXPT

FXPT

FXPT

FXPT

FXPT

FXPT

FXPT

FXPT

FXPT

FXPT

FXPT

FXPT

FXPT

FXPT

FXPT

FXPT

FXPT

FXPT

FXPT

FXPT

FXPT

FXPT

FXPT

FXPT

FXPT

FXPT

FXPT

FXPT

FXPT

FXPT

FXPT

FXPT

FXPT

FXPT

FXPT

FXPT

FXPT

FXPT

FXPT

FXPT

FXPT

FXPT

FXPT

FXPT
152

153

154

155

156

157

158

159

160

161

162

163

164

165

166

167

168

169

170

171

172

173

174

175

176

177

178

179

180

181

182

183

184

185

186

187

188

189

190

191

192

193

194

195

196

197

198

199

200

201

202

203

204

205

206

207

208 
$V(4,1)=0$.

FXPT

NITS $=$ NITS +1

FXPT

210

IF ( (ABS(DX).LT.E(2)).AND. (ABS(DY).LT.E(2)) ) GO TO 146

FXPT

211

IF (NITS.GT.ITERS) GO TO 146

FXPT $\quad 212$

GO TO $(39,391)$ NGO

FXPT 213

235

146 GO TO $(141,142)$ NGO

FXPT 214

58 CONTINUE

C SET UP N - I AND REF RAY JUMP (RHS).

FXPT $\quad 215$

DO $7 \mathrm{I}=1,4$

FXPT 216

DO $6 \mathrm{~J}=1,4$

FXPT 217

$6 \operatorname{RMI}(I, J)=\operatorname{RM}(I, J)$

FXPT 218

240

$7 \operatorname{RMI}(I, I)=\operatorname{RMI}(I, I)-1.0$

FXPT $\quad 219$

DO $61 \mathrm{I}=1,4$

FXPT 220

$61 \operatorname{RMI}(I, 5)=V V(I)-V(I, 1)$

FXPT 221

C SOLVE FOR EQ.-ORBIT-INITIAL CONDITIONS ( IN TERMS OF REF. RAY).

FXPT 222

CALL MXDIV( RMI, 4, 1, D, 4)

FXPT 223

IF ( D.NE.O. ) GO TO 75

FXPT 224

WRITE $(3,1006)$

FXPT $\quad 225$

WRITE $(3,1001)((\operatorname{RM}(I, J), J=1,4), I=1,4)$

FXPT $\quad 226$

1001 FORMAT $(/ 10 \mathrm{X}, 4 \mathrm{~F} 16.8)$

FXPT $\quad 227$

FXPT 228

CALL HED

250

RETURN

C CALCULATE EQ.-ORBIT-INITIAL-CONDITIONS.

FXPT 229

FXPT 230

FXPT 231

75 DO $8 I=1,4$

$8 \quad V(I, 1)=\operatorname{RMI}(I, 5)+V V(I)$

C CHECK FOR CONVERGENCE.

NITS $=$ NITS +1

IF (KA.LT.2) GO TO 14

WRITE $(3,1013)$ NITS, $(V(I, 1), I=1,4)$

14 CONTINUE

DO 9 I $=1,4$

IF( $\operatorname{ABS}(\operatorname{RMI}(I, 5)) . L T . E(I))$ GO TO 9

IF (NITS.GT.ITERS) GO TO 9

GO TO $(39,391)$ NGO

9 CONTINUE

GO TO $(141,142)$ NGO

265

141 CALL RTRV7(MUNIT,R7, RW)

$I O P=1$

DO $200 \mathrm{~J}=1,4$

DO $200 \quad \mathrm{I}=1,4$

$200 R 7(I, J)=R M(I, J)$

$M M=10$

$M N=11$

$N R=1$

IF (IFLAG.EQ.1) GO TO 138

IF (IFLAG.EQ.4) GO TO 142

275

$$
M M=6
$$

$$
\mathrm{MN}=7
$$

138 DO $143 \quad I=1,7$

$V(I, M M)=V W(I)$

$143 \mathrm{~V}(I, M N)=\mathrm{VW}(\mathrm{I})$

280

$V(6, M M)=V W(6)+D E L P$

$V(6, M N)=V W(6)-D E L P$

FXPT 232

FXPT 233

FXPT 234

FXPT 235

FXPT 236

FXPT $\quad 237$

FXPT 238

FXPT $\quad 239$

FXPT 240

FXPT 241

FXPT 242

FXPT 243

FXPT 244

FXPT 245

FXPT 246

FXPT $\quad 247$

FXPT 248

FXPT 249

FXPT 250

FXPT 251

FXPT 252

FXPT 253

FXPT 254

FXPT 255

FXPT 256

FXPT 257

FXPT 258

FXPT 259

FXPT 260

ASSIGN 144 TO TRKR

FXPT 261

GO TO 900

FXPT 262

144 DO $145 \quad I=1,5$ 


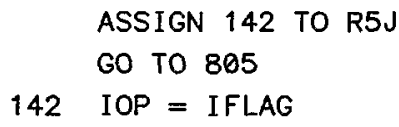

142 IOP = IFLAG

CALL ROW5(R7)

FXPT

FXPT

FXPT 270

FXPTL 2

FXPTL 3

DO $139 \mathrm{~J}=1,4$

DO $139 \quad I=1,4$

FXPTL

FXPT

C REPLACE INITIAL CONDITIONS OF PARTICLE WITH COMPUTED VALUES IF ((IVSV.NE.0).AND. (IFLAG.NE.4))

1 CALL STDAT(NP, 1, 1, 4,WW)

C STORE CLOSED ORBIT VECTOR AT LQ2 $V W(5)=0$. 


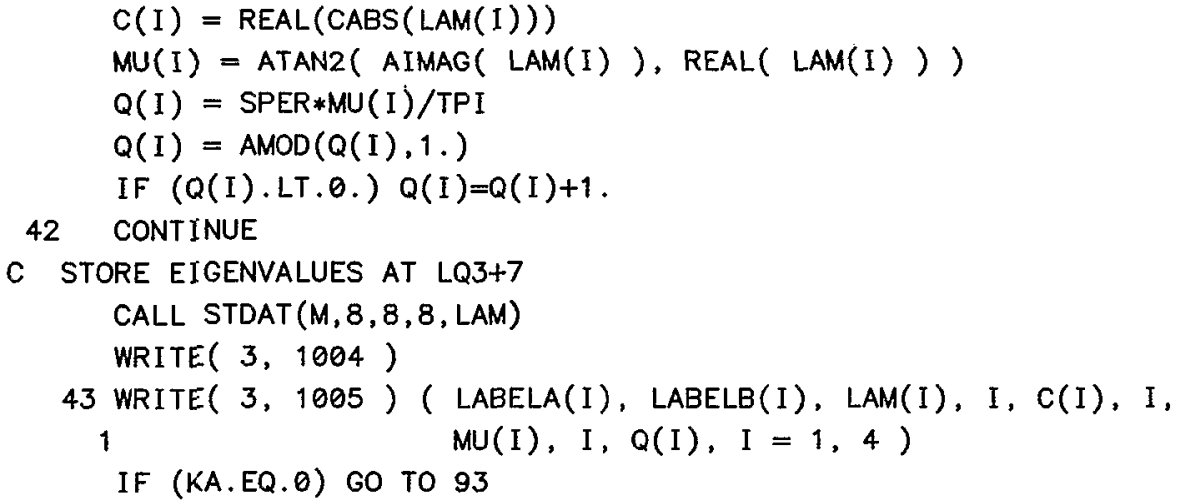

C COMPUTE EIGENVECTORS.

C FIRST AND SECOND EIGENVECTORS.

C FIRST COMPONENTS.

$$
35 \text { DO } 45 \mathrm{~L}=1,3
$$

$$
\begin{array}{rl} 
& \text { DO } 44 J=1,3 \\
& R T=\operatorname{RM}(L+1, J+1) \\
44 \quad & C M(L, J)=C M P L X(R T, 0.0) \\
45 & C M(L, L)=C M(L, L)-\operatorname{LAM}(1) \\
& C A L L \operatorname{DET}(C M, X(1,1))
\end{array}
$$

C SECOND THROUGH FOURTH COMPONENTS.

$\mathrm{D}=-1.0$

DO $50 \mathrm{~L}=2,4$

$I=L-1$

DO $46 \mathrm{~J}=1,3$

$R T=\operatorname{RM}(J+1, I)$

$46 \quad \mathrm{CM}(\mathrm{J}, \mathrm{I})=\mathrm{CMPLX}(\mathrm{RT}, 0.0)$

$\operatorname{IF}(\operatorname{L.EQ.3}) \operatorname{CM}(1,2)=\operatorname{CM}(1,2)-\operatorname{LAM}(1)$

$\operatorname{IF}(\operatorname{L.EQ} .4) \operatorname{CM}(2,3)=\operatorname{CM}(2,3)-\operatorname{LAM}(1)$

CALL DET $(C M, X(L, 1))$

50 CONTINUE

$X(L, 1)=D * X(L, 1)$

$D=-D$

$$
C 1=\operatorname{CABS}(\times(1,1))
$$

$\mathrm{EX} 1=X(1,1) / \mathrm{C} 1$

$$
\text { DO } 536 \quad I=1,4
$$

$536 \times(I, 1)=X(I, 1) / E X 1$

RHO1 $=\operatorname{AIMAG}(\operatorname{CONJG}(X(1,1)) * X(2,1)+\operatorname{CONJG}(X(3,1)) * X(4,1))$

$F A C=1$.

IF (RHO1.NE. . .) FAC $=1 . / \mathrm{SQRT}(\mathrm{ABS}(\mathrm{RHO1}))$

FXPT

FXPT

FXPT

FXPT

FXPT

FXPT

FXPT

FXPT

FXPT

FXPT

FXPT

FXPT 


$$
\begin{aligned}
& \operatorname{DO} 532 \mathrm{~J}=2,4 \\
& \mathrm{RT}=\operatorname{RM}(4, \mathrm{~J}) \\
& \mathrm{CM}(3, \mathrm{~J}-1)=\operatorname{CMPLX}(\mathrm{RT}, 0.0) \\
& \operatorname{CM}(2,1)=\operatorname{CM}(2,1)-\operatorname{LAM}(3) \\
& \operatorname{CM}(3,3)=\operatorname{CM}(3,3)-\operatorname{LAM}(3) \\
& \operatorname{CALL} \operatorname{DET}(\operatorname{CM}, \times(1,3)) \\
& \times(1,4)=\operatorname{CONJG}(\times(1,3))
\end{aligned}
$$
GO TO 998

C 1. TRACE EQUILIBRIUM ORBIT AND EIGENVECTOR PARTS THROUGH SYSTEM,

C 2. LINEARIZE INDIVIDUAL NLT"S. 535 CONTINUE

C SET UP EIGENVECTOR INITIAL CONDITIONS.

DO $91 \mathrm{I}=1,3,2$

DO $91 \mathrm{~J}=1,4$

$V Z(J, I)=\operatorname{REAL}(X(J, I))$

91

$\operatorname{VZ}(J, I+1)=\operatorname{AIMAG}(X(J, I))$

445

C STORE 4 EIGENVECTORS AT LQ2+7, 7 COMPONENTS EACH.

C ORDER — REAL X1, IMAG X1, REAL X3, IMAG $\times 3$.

450

DO $92 \quad I=1,4$

92

$$
\text { DO } 92 \mathrm{~J}=5,7
$$

$V Z(J, I)=0$.

DO $97 \mathrm{I}=5,7$

DO $97 \mathrm{~J}=1,7$

$97 \quad V Z(J, I)=0$.

$\mathrm{IQ}=8$

DO $90 \quad I=1,4$

FXPT 383

FXPT $\quad 384$

FXPT $\quad 385$

FXPT $\quad 386$

FXPT $\quad 387$

FXPT $\quad 388$

FXPT $\quad 389$

FXPT 390

FXPT 391

FXPT 392

FXPT 393

FXPT 394

FXPT 395

FXPT 396

FXPT 397

FXPT 398

FXPT 399

FXPT $\quad 400$

FXPT $\quad 401$

FXPT $\quad 402$

FXPT $\quad 403$

FXPT $\quad 404$

FXPT 405

FXPT $\quad 406$

FXPT $\quad 407$

FXPT $\quad 408$

FXPT $\quad 409$

FXPT $\quad 410$

FXPT $\quad 411$

FXPT $\quad 412$

FXPT $\quad 413$

FXPT $\quad 414$

FXPT $\quad 415$

FXPT $\quad 416$

FXPT $\quad 417$

FXPT $\quad 418$

FXPT $\quad 419$

FXPT $\quad 420$

FXPT $\quad 421$

FXPT $\quad 422$

FXPT 423

FXPT $\quad 424$

FXPT $\quad 425$

FXPT $\quad 426$

FXPT $\quad 427$

FXPT $\cdot 428$

FXPT $\quad 429$

FXPT $\quad 430$

FXPT $\quad 431$

FXPT $\quad 432$

FXPT $\quad 433$

FXPT $\quad 434$

FXPT $\quad 435$

FXPT $\quad 436$

FXPT 437 
460

465

470

475

480

485

490

495

500

505

510

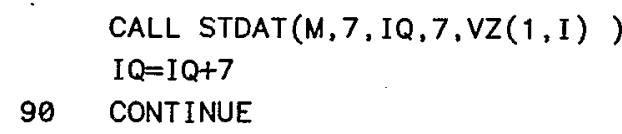

C RESTORE INITIAL CONDITIONS.

DO $111 \mathrm{~J}=1,7$

$F(J)=W W(J)$

$111 \mathrm{VW}(\mathrm{J})=\mathrm{V}(\mathrm{J})$

IF (IFLAG.NE.4) GO TO 105

DO $106 \mathrm{~J}=1,7$

$106 \mathrm{~V}(\mathrm{~J}, 8)=\mathrm{VP}(\mathrm{J})$

105 ASSIGN 112 TO NRS

ASSIGN 116 TO TRKR

$M M=1$

$M N=11$

$J P=10$

$J Q=11$

\begin{tabular}{|c|c|}
\hline FXPT & 438 \\
\hline FXPT & 439 \\
\hline FXPT & 440 \\
\hline FXPT & 441 \\
\hline FXPT & 442 \\
\hline FXPT & 443 \\
\hline FXPT & 444 \\
\hline FXPT & 445 \\
\hline FXPT & 446 \\
\hline FXPT & 447 \\
\hline FXPT & 448 \\
\hline FXPT & 449 \\
\hline FXPT & 450 \\
\hline FXPT & 451 \\
\hline FXPT & 452 \\
\hline FXPT & 453 \\
\hline FXPT & 454 \\
\hline FXPT & 455 \\
\hline FXPT & 456 \\
\hline FXPT & 457 \\
\hline FXPT & 458 \\
\hline FXPT & 459 \\
\hline FXPT & 460 \\
\hline FXPT & 461 \\
\hline FXPT & 462 \\
\hline FXPT & 463 \\
\hline FXPT & 464 \\
\hline FXPT & 465 \\
\hline FXPT & 466 \\
\hline FXPT & 467 \\
\hline FXPT & 468 \\
\hline FXPT & 469 \\
\hline FXPT & 470 \\
\hline FXPT & 471 \\
\hline FXPT & 472 \\
\hline FXPT & 473 \\
\hline FXPT & 474 \\
\hline FXPT & 475 \\
\hline FXPT & 476 \\
\hline FXPT & 477 \\
\hline FXPT & 478 \\
\hline FXPT & 479 \\
\hline FXPT & 480 \\
\hline FXPT & 481 \\
\hline FXPT & 482 \\
\hline FXPT & 483 \\
\hline FXPT & 484 \\
\hline FXPT & 485 \\
\hline FXPT & 486 \\
\hline FXPT & 487 \\
\hline FXPT & 488 \\
\hline FXPT & 489 \\
\hline FXPT & 490 \\
\hline FXPT & 491 \\
\hline FXPT & 492 \\
\hline FXPT & 493 \\
\hline FXPT & 494 \\
\hline
\end{tabular}


$\mathrm{NR}=5$

IF (IFLAG.NE.4) ASSIGN 114 TO TRKR

FXPT

FXPT

FXPT

$$
\text { GO TO } 802
$$

112 DO $113 \quad \mathrm{I}=1,7$

$V(I, J P)=V W(I)$

$113 V(I, J Q)=V W(I)$

$V(6, J P)=V W(6)+D E L P$

525

$V(6, J Q)=V W(6)-D E L P$

GO TO 900

$114 \quad M N=6$

$M N=7$

$N R=1$

$$
I O P=1
$$

ASSIGN 115 TO TRKR

$$
\text { GO TO } 900
$$

115 IOP = IFLAG

C PUT LINEARIZED $3 \times 3$ MATRIX REPRESENTING AN EXT IN ITS STORAGE

116 CALL RTRV7(MUNIT,R7,RW)

IF (IFLAG.EQ.1) GO TO 15

DO $16 \mathrm{~J}=1,4$

DO $16 \mathrm{I}=1,4$

540

FXPT

FXPT

FXPT

FXPT

FXPT

FXPT

FXPT

FXPT

FXPT

FXPT

FXPT

FXPT

FXPT

FXPT

FXPT

FXPT

FXPT

FXPT

FXPT

FXPT

FXPT

FXPT

FXPT

$$
\text { DO } 11 \mathrm{~J}=1,4
$$

$D=D E L W * 2$.

IF (J.EQ.2.OR.J.EQ.4) D=DELT*2.

545

DO $11 \mathrm{I}=1,4$

$11 R 7(I, J)=(V(I, J+1)-V(I, J+5)) / D$

17 DO $12 \quad I=1,4$

$12 R 7(I, 6)=0.5 *(V(I, J P)-V(I, J Q)) / D E L P$

ASSIGN 18 TO R5J

550

555

GO TO 805

18 CALL MXV7(R7,VV,VV)

DO $13 \mathrm{I}=1,5$

$13 \quad \mathrm{RT}(\mathrm{I}, 7)=\mathrm{WW}(\mathrm{I})-\mathrm{VV}(\mathrm{I})$

DO $19 \quad I=1,7$

$19 \mathrm{WW}(\mathrm{I})=F(\mathrm{I})$

$\mathrm{RW}(1)=\operatorname{FDAT}(\mathrm{NL}, 1)$

CALL STOR7 (NL, R7, RW)

40 IF (II.LT.NELS) GO TO 100

FXPT

FXPT

FXPT

FXPT

FXPT

FXPT

FXPT

FXPT

FXPT

FXPT

FXPT

FXPT

FXPT

FXPT

FXPT

FXPT

FXPT

FXPT

402 CONTINUE

FXPT

FXPT

C TRACK BETA FUNCTIONS, ETA AND CLOSED ORBITS THROUGH BEAM SYSTEM.

FXPT

FXPT

C BETA FUNCTIONS FORMED FROM $2 \times 2$ SUBMATRICES ARE ONLY APPROXIMATE 
WRITE $(3,1016)$

FXPT

552

1016 FORMAT ( $5 X, * X$ OR Y BETATRON MOTION UNSTABLE.*)

FXPT

553

405 CONTINUE

IF (KB.GE.O) GO TO 406

FXPT

554

NELS $=N E L S+1$

FXPT

555

$M I(N E L S)=M 7 E N D-4$

406

CONT INUE

$M L 1=M L(1)$ \$ CALL RTRV7(ML1,R7,RW)

580

CALL STOR7 (M,R7, RW)

IF (IFLAG.NE.4) GO TO 409

$\operatorname{CALL} \operatorname{DATA}(M, 7,1,7, \mathrm{~V}(1,8))$

$\operatorname{CALL} \operatorname{STDAT}(M, 7,1,7, \mathrm{VW})$

585

409

CALL RANSET(NRN)

CALL CYX(M)

CALL RANSET(NRN)

CALL HED

IF (IFLAG.NE.3) RETURN

IF (KB.LT.0) NELS=NELS-1

590

DO $408 \quad I=1,6$

$408 W W(I)=0$.

IFLAG $=4$

$\operatorname{NV}(1)=8$

GO TO 33

595

600

605

C SET UP THE EIGHT NEIGHBORING RAYS

800 DO $801 \mathrm{~J}=8,63$

$801 \quad W W(\mathrm{~J})=W W(\mathrm{~J}-7)$

$V(1,6)=V(1,6)-D E L W$

$V(2,7)=V(2,7)-D E L T$

$V(3,8)=V(3,8)-$ DELW

$V(4,9)=V(4,9)-D E L T$

$V(1,2)=V(1,2)+$ DELW

$V(2,3)=V(2,3)+$ DELT

$V(3,4)=V(3,4)+D E L W$

$V(4,5)=V(4,5)+$ DELT

FXPT

556

FXPT $\quad 557$

FXPT $\quad 558$

FXPTL 6

FXPTL 7

FXPT $\quad 560$

FXPT $\quad 561$

FXPT $\quad 562$

FXPT $\quad 563$

FXPT $\quad 564$

FXPT $\quad 565$

FXPT $\quad 566$

FXPT $\quad 567$

FXPT $\quad 568$

FXPT $\quad 569$

FXPT $\quad 570$

FXPT $\quad 571$

FXPT $\quad 572$

FXPT $\quad 573$

FXPT $\quad 574$

FXPT $\quad 575$

FXPT $\quad 576$

FXPT $\quad 577$

FXPT $\quad 578$

FXPT $\quad 579$

FXPT $\quad 580$

FXPT $\quad 581$

FXPT $\quad 582$

FXPT $\quad 583$

FXPT $\quad 584$

FXPT $\quad 585$

FXPT $\quad 586$

GO TO NRS, $(4,112)$

FXPT $\quad 587$

802 DO $803 \mathrm{~J}=8,35$

$803 \mathrm{WW}(\mathrm{J})=0$.

610

DO $804 I=1,4$

$804 V(I, I+1)=1$.

CALL STOR7 $(M, V(1,2), R W)$

GO TO NRS, $(4,112)$

805. DO $806 \mathrm{~J}=1,4$

615

$806 R 7(5, J)=R 7(2, J) * R 7(1,6)-R 7(1, J) * R 7(2,6)$

FXPT $\quad 588$

FXPT $\quad 589$

FXPT $\quad 590$

FXPT 591

FXPT $\quad 592$

FXPT $\quad 593$

FXPT $\quad 594$

FXPT $\quad 595$

$1+R 7(4, J) * R 7(3,6)-R 7(3, J) * R 7(4,6)$

FXPT $\quad 596$

GO TO R5J, $(142,18)$

FXPT $\quad 597$

C TRACK PARTICLES MM THROUGH MN THROUGH BEAM ELEMENTS MB THROUGH ME.

FXPT

598

$900 \mathrm{NLF}=0$

FXPT

599

620

DO $950 \mathrm{~J}=\mathrm{MB}, \mathrm{ME}$

FXPT

600

$N L=M I(J)$

FXPT

601

MATL $=\operatorname{INFF}(2, N L)$

FXPT

FXPT

602

DO $950 M T=M M, M N$

FXPT

603

CALL TRKCN ( $M, N L, V(1, M T), N P, I O P)$

FXPT $\quad 605$

625

$\operatorname{KIND}=\operatorname{INFF}(20, N L)$

FXPT $\quad 606$

950 CONTINUE

FXPT

607 
$\mathrm{NL}=\mathrm{NLF}$

GO TO TRKR, $(102,114,115,116,144,510)$

FXPT

C WRITE OUT AN EIGENVECTOR.

998 IF (IOUT.NE.5H ) WRITE $(3,1008)$ LAM(L), $(X(J, L), J=1,4)$

FXPT

609

1630

GO TO IWR, ( $467,468,534,535)$

FXPT $\quad 610$

FXPT 611

C

C PRINT EIGENFUNCTIONS IN POLAR FORM

FXPT $\quad 612$

FXPT 613

635

599 CONTINUE

FXPT

614

DO $601 \mathrm{~K}=1,2$

FXPT

615

DO $601 \mathrm{I}=1,4$

FXPT

616

$J=2 * K-1$

FXPT

617

IF ( (VZ(I,J).NE.0.) .OR. (VZ(I,J+1).NE.0.)) GO TO 602

FXPT

618

640

$\operatorname{EMOD}(I, K)=\operatorname{CMPLX}(0 ., 0$.

FXPT

619

$\operatorname{EPHI}(I, K)=\operatorname{CMPLX}(0,0$.

FXPT

620

GO TO 601

FXPT $\quad 621$

$602 \operatorname{EMOD}(I, K)=\operatorname{SQRT}(\operatorname{VZ}(I, J) * V Z(I, J)+V Z(I, J+1) * V Z(I, J+1))$

FXPT $\quad 622$

FXPT 623

645

$\operatorname{EPHI}(I, K)=\operatorname{ATAN2}(\operatorname{VZ}(I, J+1), \operatorname{VZ}(I, J))$

FXPT $\quad 624$

601 CONTINUE

WRITE $(3,1003)$ L,MATL, ( $\operatorname{EMOD}(I, 1), \operatorname{EPHI}(I, 1), I=1,4)$,

FXPT $\quad 625$

$1 \quad(\operatorname{EMOD}(I, 2), \operatorname{EPHI}(I, 2), I=1,4)$

FXPT $\quad 626$

GO TO IBET, $(600,604)$

FXPT $\quad 627$

FXPT 628

FXPT 629

650

1000 FORMAT(64HOCALCULATION OF THE EQUILIBRIUM ORBIT AND BETATRON FUNCT FXPT

1 IONS OF A5, $1 \mathrm{H} . / 34 \mathrm{H}$ INITIAL REFERENCE RAY DEFINED BY A5/7H $X=$,

$2 \mathrm{~F} 11.8,3 \mathrm{X}, * \mathrm{DX}=*, \mathrm{~F} 11.8,3 \mathrm{X}, * Y=*, \mathrm{~F} 11.8,3 \mathrm{X}, * \mathrm{DY}=*, \mathrm{~F} 11.8$,

$35 X, * D S=*, F 11.8,3 X, * D P / P=*, F 11.8,3 X, F 11.8)$

655

1002 FORMAT ( $1 \mathrm{H} 1, *$ EIGENVECTORS 1 AND 3 IN POLAR COORDINATES*)

$11 X * P O S * 23 X * X 1 * 22 X * D X 1 * 22 X * Y 1 * 22 X * D Y 1 * /$

$2 \quad 27 X * X 3 * 22 X * D X 3 * 22 X * Y 3 * 22 X * D Y 3 * /)$

㴶

FXPT

631

632

FXPT 633

1003 FORMAT $(1 \mathrm{X}, \mathrm{I} 4,1 \mathrm{X}, \mathrm{A} 5,1 \mathrm{X}, 4(2 \mathrm{~F} 11.6,3 \mathrm{X}) / 12 \mathrm{X}, 4(2 \mathrm{~F} 11.6,3 \mathrm{X}) /)$

FXPT

1004 FORMAT (*OEIGENVALUES OF THE $4 \times 4$ SUBMATRIX*)

FXPT $\quad 637$

1005 FORMAT $(2 \mathrm{~A} 6,4 \mathrm{H}=(, 2 \mathrm{~F} 12.8,5 \mathrm{X}, 6 \mathrm{H}), \mathrm{C}(\mathrm{I} 1,4 \mathrm{H})=\mathrm{F} 12.8,5 \mathrm{H}, \mathrm{MU}(\mathrm{I} 1$,

FXPT

638

660

$14 H)=F 12.8,12 H$ RAD, $\quad Q(I 1,4 H)=F 12.8)$

1006 FORMAT(21HOSINGULAR $4 \times 4$ MATRIX.)

1007 FORMAT (*07X7 MATRIX FOR *,A5/(/10X,7F16.8))

FXPT

639

FXPT 640

FXPT 641

1008 FORMAT $(16 \mathrm{HOEIGENVALUE}=(\mathrm{F} 16.8,2 \mathrm{H}, \mathrm{F} 16.8,21 \mathrm{H})$, EIGENVECTOR $=($

FXPT

642

$1 \mathrm{~F} 16.8,2 \mathrm{H}, \mathrm{F} 16.8,2 \mathrm{H}) /(69 \mathrm{X}, 2 \mathrm{H}(\mathrm{F} 16.8,2 \mathrm{H}, \mathrm{F} 16.8,2 \mathrm{H})))$

FXPT

643

665

1013 FORMAT $(12 \mathrm{H}$ ITERATION $=, \mathrm{I} 4,4 \mathrm{H} X \mathrm{XO}=, \mathrm{F} 16.8,5 \mathrm{H} \mathrm{DXO}=, \mathrm{F} 16.8,4 \mathrm{H} \mathrm{YO}=, \mathrm{F} 16.8$,

$15 \mathrm{H}$ DYO $=, F 16.8)$

1014 FORMAT (/1X,A10,7F15.8)

1018 FORMAT ( $1 \mathrm{H} 1)$

FXPT

644

645

$\begin{array}{ll}\text { FXPT } & 646 \\ \text { FXPT } & 647\end{array}$

1017 FORMAT $(/ 15 X, 7 X, * X *, 13 X, * D X *, 13 X, * Y *, 13 X, * D Y *, 13 X, * D S *, 13 X, * D P / P *$

FXPT

648

670

1)

FXPT

649

C

END

FXPT

CARD NR. SEVERITY DETAILS DIAGNOSIS OF PROBLEM

$\begin{array}{rll}\text { EQV/COMM } & \text { I } & \text { BMI1 } \\ 164 & \text { I } & \\ 190 & \text { I } & \\ 233 & \text { I } & \\ 234 & \text { I } \\ 262 & \text { I } & \end{array}$

NOT ALL ITEMS IN THIS COMMON BLOCK OCCUR IN LEVEL STATEMENTS.

AN IF STATEMENT MAY BE MORE EFFICIENT THAN A 2 OR 3 BRANCH COMPUTED GO TO STATEMENT. AN IF STATEMENT MAY BE MORE EFFICIENT THAN A 2 OR 3 BRANCH COMPUTED GO TO STATEMENT. AN IF STATEMENT MAY BE MORE EFFICIENT THAN A 2 OR 3 BRANCH COMPUTED GO TO STATEMENT. AN IF STATEMENT MAY BE MORE EFFICIENT THAN A 2 OR 3 BRANCH COMPUTED GO TO STATEMENT. AN IF STATEMENT MAY BE MORE EFFICIENT THAN A 2 OR 3 BRANCH COMPUTED GO TO STATEMENT. 
CARD NR. SEVERITY DETAILS 264 496
DIAGNOSIS OF PROBLEM

AN IF STATEMENT MAY BE MORE EFFICIENT THAN A 2 OR 3 BRANCH COMPUTED GO TO STATEMENT. AN IF STATEMENT MAY BE MORE EFFICIENT THAN A 2 OR 3 BRANCH COMPUTED GO TO STATEMENT. 
1

* generate numbered names, e.g. , N1, N2, N3 . . . NKC in BCD Where

* $N$ IS FIRST LETER OF NM. PUT NAMES INTO ARRAY LIST.

5 DIMENSION LIST(1)

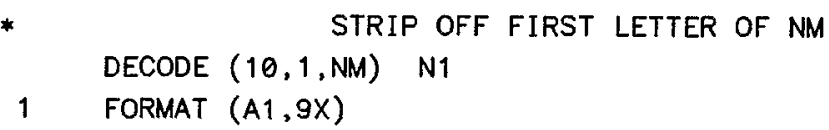

10

$$
\mathrm{IJ}=1
$$

$\mathrm{K} 1=27$

$\mathrm{K} 2=28$

GENNUM

GENNUM

GENNUM

GENNUM

GENNUM

GENNUM

GENNUM

GENNUM

GENNUM

GENNUM

$\mathrm{K} 3=28$

GENNUM

DO $100 \mathrm{JA}=1, \mathrm{KC}$

15

$\mathrm{K} 1=\mathrm{K} 1+1$

GO TO $(25,30,35)$ IJ

GENNUM

GENNUM

GENNUM

GENNUM

GENNUM

GENNUM

GENNUM

GENNUM

GENNUM

GENNUM

GENNUM

GENNUM

GENNUM

GENNUM

GENNUM

GENNUM

GENNUM

GENNUM

GENNUM

GENNUM

GENNUM

GENNUM

GENNUM

GENNUM

GENNUM

GENNUM

GENNUM

GENNUM

GENNUM

GENNUM

GENNUM

END

44

CARD NR, SEVERITY DETAILS DIAGNOSIS OF PROBLEM 
10

LOGICAL IND, INF

LOGICAL INDEFF, INFINN

DATA FC /1.0E-10/

15

DO $100 \mathrm{~L}=1, N$

IERR=. FALSE.

$J K=0$

IF $(C(L)) 45,46,45$

20

$45 \mathrm{RT}=.9 * \mathrm{C}(\mathrm{L})$

ASSIGN 1 TO NN

GO TO 80

$1 \quad X 0=F P R T$

$R T=1.1 * C(L)$

ASSIGN 2 TO NN

25

GO TO 80

$2 \times 1=F P R T$

$\mathrm{RT}=\mathrm{C}(\mathrm{L})$

ASSIGN 3 TO NN

GO TO 80

30

$3 \quad \times 2=F P R T$

$\mathrm{H}=-.1 * \mathrm{C}(\mathrm{L})$

GO TO 50

$46 \mathrm{RT}=-1$.

ASSIGN 4 TO NN

35

GO TO 80

$4 \quad X \theta=F P R T$

$\mathrm{RT}=1$.

ASSIGN 5 TO NN

GO TO 80

40

$5 \times 1=F P R T$

$\mathrm{RT}=0$.

ASSIGN 6 TO NN

GO TO 80

$6 \times 2=F P R T$

45

$\mathrm{H}=-1$.

$50 \mathrm{D}=-.5$

$49 \quad \mathrm{DD}=1 .+\mathrm{D}$

$\mathrm{BI}=(X 0 * \mathrm{D} * \mathrm{D})-(\mathrm{X} 1 * \mathrm{DD} * \mathrm{DD})+(X 2 *(\mathrm{DD}+\mathrm{D}))$

$\mathrm{DEN}=\mathrm{BI} * \mathrm{BI}-(4 . * X 2 * \mathrm{D} * \mathrm{DD}) *(X \theta * \mathrm{D}-(\mathrm{X} 1 * \mathrm{DD})+\mathrm{X} 2)$

50

IF (DEN) $52,52,51$

52 IF (IN) $36,35,36$

35 WRITE $(3,25)$ L,DEN

$36 \quad \mathrm{DEN}=0$.

51 DEN=SQRT (DEN)

55

$53 \mathrm{DN}=\mathrm{BI}+\mathrm{DEN}$

$D M=B I-D E N$

If (ABS(DN)-ABS(DM)) $57,57,56$

CONTRL 5

GRT

GRR

GRR

GRR

GRT

GRT

GRT

GRT

GRT

GRT

GRT

GRT

GRT

GRT

GRT

GRT

GRT

GRT

GRT

GRT

GRT

GRT

GRT

GRT

GRT

GRT

GRT

GRT

GRT

GRT

GRT

GRT

GRT

GRT

GRT

GRT

GRT

GRT

GRT

GRT

GRT

GRT

GRT

GRT

GRT

GRT

GRT

GRT

GRT

GRT

GRT
2

3

2

3

4

5

2

3

4

7

8

9

10

11

12

13

14

15

16

17

18

19

20

21

22 
60

$56 \mathrm{DEN}=\mathrm{DN}$

GO TO 58

GRT

GRT

54

57 DEN=DM

GRT

GRT

GRT

GRT

GRT

GRT

GRT

GRT

GRT

GRT

GRT

GRT

GRT

GRT

GRT

GRT

GRT

GRT

GRT

GRT

GRT

GRT

GRT

GRT

74 WRITE $(3,28)$ RT

GRT

GRT

GRT

GRT

GRT

GRT

GRT

GRT

GRT

GRT

GRT

GRT

GRT

GRT

GRT

GRT

GRT

GRT

GRT

GRT

GRT

GRT

GRT

GRT

GRT

GRT

GRT

GRT

GRT

83 WRITE $(3,25)$ L, RT, FRT, FPR

89 IF (ABS(FRT)-FC) $90,84,84$

90 IF (ABS(FPRT)-FC) 76,84,84

GRT

GRT

84 GO TO NN, $(1,2,3,4,5,6,7)$ 
115

$85 \quad R T=R T+.001$

IF (IN) $88,87,88$

87 WRITE $(3,25)$ L

88 GO TO 80

25 FORMAT (I3, 3E20.8)

120

26 FORMAT (I3, 2E20.8/1H)

27 FORMAT $(/ /)$

END

GRT

GRT

GRT

GRT

GRT

GRT

GRT

GRT
111

112

113

114

115

116

117

118 
1

SUBROUTINE HED

HED

2

C PRINTS A LINE OF DOTS.

HED

3

5 WRITE $(3,1000)$

HED 4

RETURN

HED

HED

5

1000 FORMAT $(1 \times, 130(1 \mathrm{H})$.

HED 
1

0

15

20
FUNCTION IDAT $(M, J)$

C RETRIEVES ONE PIECE OF INTEGER DATA FROM INDEX $\downarrow$ OF ELEMENT $M$

LEVEL 2, STORE, INFF, IWORK

COMMON STORE (48000), IWORK (10)

DIMENSION INFF $(24,2000)$

EQUIVALENCE (INFF,STORE)

COMMON/CONTRL/ERROR, MODE, RSRV, STOR, XEQ, TRASW, NFSW, EMPTY, INDEF, 1 LDFLG, FIN

LOGICAL ERROR, RSRV, STOR , XEQ, TRASW, NFSW, EMPTY, INDEF, LDFLG, FIN

EQUIVALENCE(IDATA, FDATA)

$\operatorname{LOC}=\operatorname{INFF}(12, M)+J-1$

IF (LOC.GT.O) GO TO 1

ERROR $=$.TRUE.

RETURN

$1 \quad$ FDATA $=$ STORE $($ LOC $)$

IDAT = IDATA

RETURN

END

$\begin{array}{lr}\text { IDAT } & 2 \\ \text { IDAT } & 3 \\ \text { BLANK } & 2 \\ \text { BLANK } & 3 \\ \text { 86MARSIZ } & 1 \\ \text { 86MARSIZ } & 2 \\ \text { 86MARSIZ } & 3 \\ \text { BLANK } & 5 \\ \text { CONTRL } & 2 \\ \text { CONTRL } & 3 \\ \text { CONTRL } & 4 \\ \text { CONTRL } & 5 \\ \text { IDAT } & 6 \\ \text { IDAT } & 7 \\ \text { IDAT } & 8 \\ \text { IDAT } & 9 \\ \text { IDAT } & 10 \\ \text { IDAT } & 11 \\ \text { IDAT } & 12 \\ \text { IDAT } & 13 \\ \text { IDAT } & 14 \\ \text { IDAT } & 15\end{array}$


* card image incr $K$ // $Q$ type delta

* INCREMENT THE KTH PARAMETER OF Q bY DELTA

* tYPE MAY be F, I, Ka OR KB.

* delta will be fl. pt. if type is f or omitted.

* It will be INTEger if I, ka or kB.

C IF MODE $=1$, THE NEW VALUE REMAINS IN DATA STORAGE

C IF MODE $=3$, THE ORIGINAL VALUE IS REPLACED AFTER REQUIRED ITERATIONS

C HAVE BEEN COMPLETED.

LEVEL 2, STORE, INFF, IWORK

COMMON STORE(48000), IWORK(10)

DIMENSION INFF $(24,2000)$

EQUIVALENCE (INFF,STORE)

15

COMMON/CONTRL/ERROR, MODE, RSRV, STOR , XEQ, TRASW, NFSW, EMPTY, INDEF, 1 LDFLG, FIN

LOGICAL ERROR, RSRV, STOR, XEQ, TRASW, NFSW, EMPTY, INDEF, LDFLG, FIN

INTEGER BDAT

INTEGER ELNUM

C

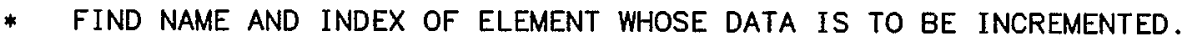


* SHOULD NOT GET HERE

63 WRITE $(3,5)$ IGO ERROR $=$. TRUE. RETURN

$65 \mathrm{JI}=5$

$66 \operatorname{INFF}(J I, N)=I S F$ INEW $=$ ISF

$$
I D F=0
$$

INCR

INCR

INCR

INCR

INCR

INCR

INCR

INCR

INCR

INCR

INCR

INCR

INCR

INCR

INCR

INCR

INCR

INCR

INCR

INCR

INCR

INCR

INCR

INCR

INCR

INCR

INCR

INCR

INCR

INCR

INCR

INCR

INCR

INCR

INCR

INCR

GO TO 40

INCR

INCR

INCR

INCR

1

FORMAT (6H $* * *, 8 X, 5 \mathrm{HINCR}, 1 \mathrm{X}, \mathrm{I3}, 4 \mathrm{X}, 5 \mathrm{H} / / \quad \mathrm{A} 5,5 \mathrm{X}, \mathrm{F} 10,6,5 \mathrm{X}$,

$19 H$ VALUE $=, F 14,6)$

2 FORMAT (6H *** ,8X,5HINCR , 1X, I3, 4X,5H // , A5, 2X, I10,5X,

$19 H$ VALUE $=,(10)$

INCR

INCR

3 FORMAT ( $6 \mathrm{H} * * *, 8 X, 5 \mathrm{HINCR}, 2 \mathrm{X}, 2 \mathrm{HKA}, 4 \mathrm{X}, 5 \mathrm{H} / /, \mathrm{A} 5,5 \mathrm{X}, 110,5 \mathrm{X}$,

51

52

53

54

55

56

57

58

59

60

61

62

63

64

65

66

67

$=68$

69 
5 FORMAT $(6 \mathrm{H} * * *$, *ERROR IN INCR ROUTINE, *, I 10,*IS INVALID COMPUTE INCR 1ED GO TO.*) 
STORAGE ALLOCATION.

$\begin{array}{rrl}\text { ADDRESS } & \text { LENGTH } & \text { BINARY CONTROL CARDS. } \\ 0 & 5 & \text { IDENT INDEFF } \\ 5 & & \text { END }\end{array}$

ENTRY POINTS.

INDEFF $\quad 1+$

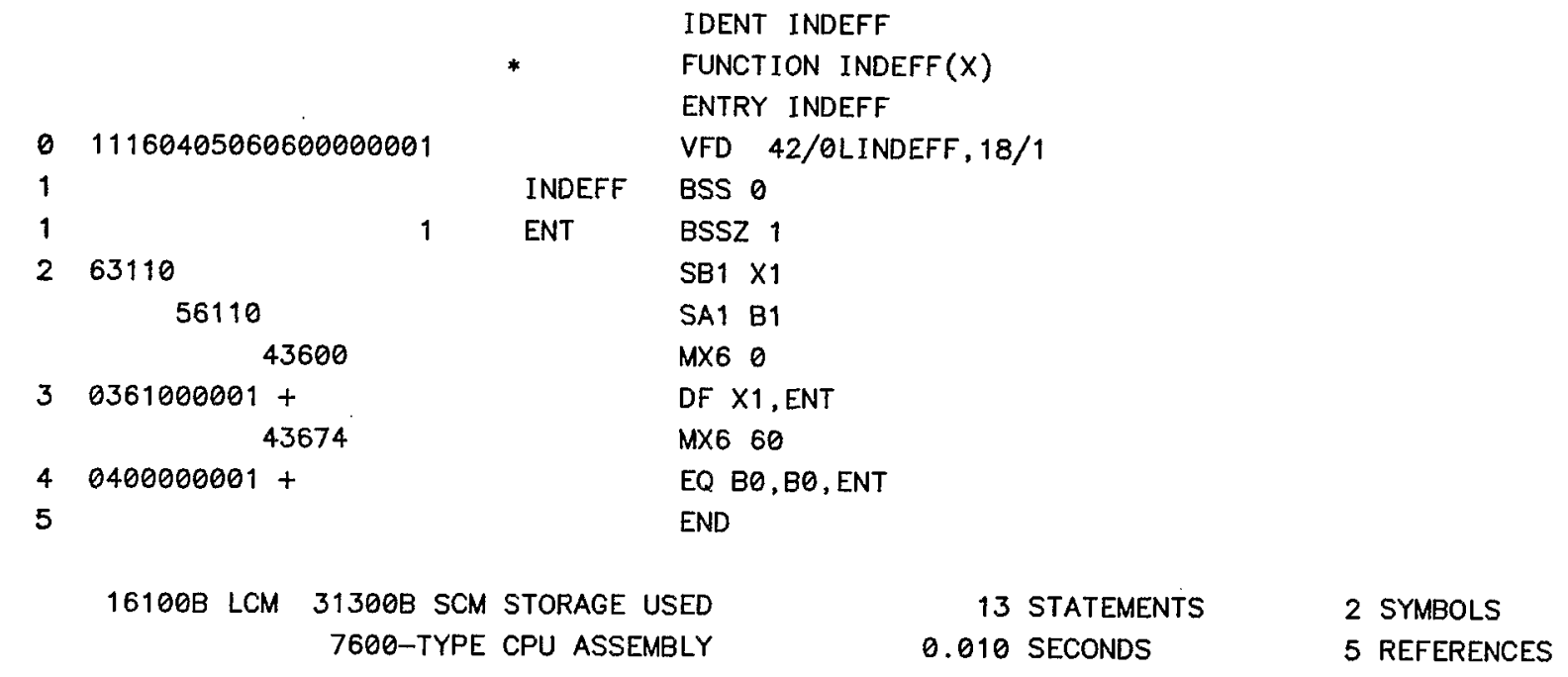

SYMBOLIC REFERENCE TABLE.

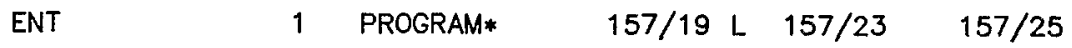

$\begin{array}{llllll}\text { INDEFF } & 1 \text { PROGRAM* } & 157 / 16 \text { E } & 157 / 18 \text { L }\end{array}$ 
STORAGE ALLOCATION.

$\begin{array}{rrl}\text { ADDRESS } & \text { LENGTH } & \text { BINARY CONTROL CARDS. } \\ 0 & 5 & \text { IDENT INFINN } \\ 5 & & \text { END }\end{array}$

ENTRY POINTS.

INFINN $1+$

$\begin{array}{ll} & \text { IDENT INFINN } \\ & \text { FUNCTION INFINN }(X) \\ & \text { ENTRY INFINN } \\ & \text { VFD } 42 / 0 \text { ININF INN, 18/1 } \\ \text { INFINN } & \text { BSS } 0 \\ \text { ENT } & \text { BSSZ } 1 \\ & \text { SB1 } \times 1 \\ & \text { SA1 B1 } \\ \text { MX6 } 0 \\ \text { IR X1, ENT } \\ \text { MX6 } 60 \\ \text { EQ B0,B0, ENT } \\ \text { END }\end{array}$

16100B LCM 31300B SCM STORAGE USED

13 STATEMENTS

2 SYMBOLS

7600-TYPE CPU ASSEMBLY

0.010 SECONDS

5 REFERENCES

SYMBOLIC REFERENCE TABLE.

ENT

INF INN

PROGRAM*

$158 / 19$ L $158 / 23$

$158 / 25$

1 PROGRAM*

$158 / 16$ E $158 / 18 \mathrm{~L}$ 
1

10
SUBROUTINE INFW(IA, J,M)

C WRITES ONE INTEGER WORD FROM I INTO $\operatorname{INFF}(J, M)$

LEVEL 2, STORE, INFF, IWORK

COMMON STORE(48000), IWORK(10)

DIMENSION INFF $(24,2000)$

EQUIVALENCE (INFF, STORE)

$\operatorname{INFF}(J, M)=I A$

RETURN

END

$\begin{array}{ll}\text { INFW } & 2 \\ \text { INFW } & 3 \\ \text { BLANK } & 2 \\ \text { BLANK } & 3 \\ \text { 86MARSIZ } & 1 \\ \text { 86MARSIZ } & 2 \\ \text { 86MARSIZ } & 3 \\ \text { BLANK } & 5 \\ \text { INFW } & 5 \\ \text { INFW } & 6 \\ \text { INFW } & 7\end{array}$


1

10

15
SUBROUTINE INP( $M, K I N D, N Q$,

. $N 1, I 1, N 2, I 2, N 3, I 3, N 4, I 4$

.)

C RESERVES AND STORES INPUT

COMMON/CONTRL/ERROR, MODE, RSRV, STOR, XEQ, TRASW, NFSW, EMPTY, INDEF,

1 LDFLG, FIN

LOGICAL ERROR, RSRV, STOR, XEQ, TRASW, NFSW, EMPTY, INDEF, LDFLG, FIN

IF (RSRV) CALL RESRV(M,KIND,NQ,

. N1,I1,N2,I2,N3,I3,N4, I4

.)

IF(ERROR) RETURN

IF (STOR) CALL LOAD (M,

- N1,I1,N2,I2,N3,I3,N4,I4

.)

RETURN

END
INP

INP

INP

INP

CONTRL

CONTRL

CONTRL

CONTRL

INP

INP

INP

INP

INP

INP

INP

INP

INP
2

3

4

5

2

3

4

5

7

8

9

10

11

12

13

14

15 


$\begin{array}{lr}\text { INTCON } & 2 \\ \text { INTCON } & 3 \\ \text { INTCON } & 4 \\ \text { INTCON } & 5 \\ \text { INTCON } & 6 \\ \text { INTCON } & 7 \\ \text { INTCON } & 8 \\ \text { INTCON } & 9 \\ \text { INTCON } & 10 \\ \text { INTCON } & 11 \\ \text { INTCON } & 12 \\ \text { INTCON } & 13 \\ \text { INTCON } & 14 \\ \text { INTCON } & 15 \\ \text { INTCON } & 16 \\ \text { INTCON } & 17 \\ \text { INTCON } & 18 \\ \text { INTCON } & 19 \\ \text { INTCON } & 20 \\ \text { INTCON } & 21 \\ \text { INTCON } & 22 \\ \text { INTCON } & 23 \\ \text { INTCON } & 24 \\ \text { INTCON } & 25 \\ \text { INTCON } & 26\end{array}$

* Na is an aRRay Of LENGTH N. It CONTAINS NO MORE THAN 4 BCD * characters, REPRESENTING an INTEgER Within PARENTHESES.

5

10

15

25

20
* ROUTINE EXTRACTS THE INTEgER AND RETURNS IT IN THE ARRAY IN

* INTEgER form.

DIMENSION NA(1)

FORMAT $(3 X, A 1,6 X)$

IF (NB.EQ.1H)) GO TO 50 ASSUMES 1 DIGIT INTEGER

DECODE $(10,2, N A(I))$ NB

FORMAT $(1 \mathrm{X}, \mathrm{I} 1,8 \mathrm{X})$

GO TO 70

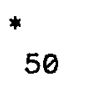

CONT INUE

ASSUMES 2 DIGIT INTEGER

DECODE $(10,3, N A(I))$ NB

3 FORMAT (1X, I2, 7X)

$70 \quad \mathrm{NA}(\mathrm{I})=\mathrm{NB}$

100 CONTINUE

RETURN

END 
LEVEL 2, STORE, INFF, IWORK

COMMON STORE (48000), IWORK (10)

DIMENSION INFF $(24,2000)$

EQUIVALENCE (INFF, STORE)

BLANK

BLANK

86MARSIZ

86MARSIZ

86MARSIZ

BLANK

COMMON/SWTCH/BEND, MSIZE, CYCSWT, VCSW, PV, MSSW, VPR, MHS, MODS, MINZER

SWTCH

SWTCH

LOGICAL CYCSWT, MSSW, VCSW, PV, VPR, MHS, MODS

SWTCH

DIMENSION $T(49), R W(3), R X(2,3), R Y(2,3), R P Q(4)$

15

$\mathrm{K} 1=\operatorname{INFF}(20, M)$

INV

INV

INV

INV

INV

INV

INV

INV

INV

INV

INV

INV

INV

INV

INV

END 
1

SUBROUTINE INV2(M,MB)

* Rotate matrix MB by 180 Degrees, REFlect it and Store in M.

INV2

* M AND MB aRE INDECES of MATRICES

INV2

COMMON /DIM/ LEND, MEND, ISAV, ISAV7, M7END, KADD, KADDR, MUNIT, MSYMPL

INV2

5

DIM

CALL ROTM(M7END, MB, PI)

CALL REF $(M, N N)$

RETURN

END 
C $B K=K I C K E R$ FIELD

.

BK

C $K=1,2$ FOR FIELD IN $1,2(X, Y)$ DIRECTION

C $C=$ PREVIOSLY DEFINED DRF OR MAG

C IF $C$ IS A DRF OF ZERO LENGTH, BK = KICKER LENGTH $X$ FIELD

LEVEL 2, STORE, INFF, IWORK

COMMON STORE(48000), IWORK(10)

EQUIVALENCE (INFF, STORE)

DIMENSION T (7,7), RW(3)

15

INTEGER OPNAME

$N=\operatorname{MDAT}(M, 1)$

CALL RTRV7(N,T,RW)

$B K=F D A T(M, 3)$

$B K K=B K$

C IF (KB.GE. 1) ERROR IS RANDOM WITH GIVEN RANGE

IF (BK.EQ.O.) GO TO 4

$K=\operatorname{INFF}(4, M)$

OPNAME $=\operatorname{INFF}(1, N)$

IF (OPNAME.NE. 3HDRF) GO TO 3

$2 \operatorname{S}=\operatorname{FDAT}(\mathrm{N}, 1)$

$\operatorname{BRHO}=F D A T(M, 2)$

IF (S.NE.O.) GO TO 5

$\mathrm{RHO}=0$.

THETA=BK/BRHO

$\mathrm{XK}=0$.

GO TO 6

5

CONT INUE

$\mathrm{RHO}=\mathrm{BRHO} / \mathrm{BK}$

35

THETA=S/RHO

$X K=-R H O *$ THETA $*$ THETA $/ 2$.

6

DXK $=-$ THETA

$T(5,7)=X K *$ THETA $/ 3$.

$T(5,6)=-2 . * T(5,7)$

40

$\operatorname{RW}(3)=T(5,6)$

IF (K.NE.1) GO TO 1

$T(3,6)=X K$

$T(3,7)=-X K$

$T(4,6)=$ DXK

45

$T(4,7)=-D X K$

$T(5,3)=-D X K$

$\mathrm{T}(5,4)=-\mathrm{XK}$

GO TO 4

50

1

$T(1,7)=X K$

$T(1,6)=-X K$

$T(2,6)=-D X K$

$T(2,7)=D X K$

$T(5,1)=$ DXK

$T(5,2)=X K$

GO TO 4

KICK

KICK

KICK

KICK

KICK

KICK

BLANK

BLANK

86MARSI

86MARSIZ 2

86MARSIZ 3

BLANK

KICK

KICK

KICK

KICK

KICK

KICK

KICK

$\mathrm{KICK}$

KICK

$\mathrm{KICK}$

KICK

KICK

KICK

KICK

$\mathrm{KICK}$

KICK

KICK

KICK

KICK

KICK

$\mathrm{KICK}$

$\mathrm{KICK}$

KICK

KICK

KICK

KICK

KICK

KICK

KICK

KICK

KICK

$\mathrm{KICK}$

KICK

KICK

$\mathrm{KICK}$

KICK

KICK

KICK

KICK

KICK

KICK

KICK

KICK

KICK

KICK

2

3

4

5

6

7

2

3

1

5

9

10

11

12

13

14

15

16

17

18

19

20

21 
60

IF ( (BO.EQ.0.).OR.(K.EQ.1)) GO TO 2

KICK

$\mathrm{DBB}=\mathrm{BK} / \mathrm{B} 0$

KICK

55

$T(1,7)=-T(1,6) * D B B$

KICK

56

4

$T(2,7)=-T(2,6) * D B B$

KICK

57

$\operatorname{CALL} \operatorname{STOR7}(M, T, R W)$

KICK

58

$B K=B K K$

KICK

59

65

RETURN

KICK

60

END

KICK

61 
1

5

10

15

25

20
SUBROUTINE KIN(PAR)

COMMON/KINET/KNFLAG , TK, P , GAM, BETT , BETGAM, BRHO

LOGICAL KNFLAG

LOGICAL KNFLAG

DIMENSION PAR(3)

KNFLAG $=$.TRUE.

$E K=P A R(1)$

$P K=P A R(2)$

BETRO $=$ PAR(3)

IF (EK.EQ.0.) GO TO 5

GAMA $=1 .+(E K / E O P)$

BGAMA = SQRT (GAMA*GAMA -1.)

$\mathrm{PK}=\mathrm{EOP} * \mathrm{BGAMA}$

GO TO 10

C

5 IF (PK.EQ.0.) $\quad P K=B E T R O / 33.356405$

$B G A M A=P K / E O P$

GAMA $=$ SQRT $($ BGAMA $*$ BGAMA +1.$)$

10 BETK = BGAMA/GAMA

C

PRINT 20, EOP, PK, GAMA, BGAMA, EK, BETRO, BETK

20 FORMAT $(/ 5 X, * E O=*, F 12.8,2 X, * P \quad=*, F 12.8,2 X, *$ GAMMA $=*, F 12.8$,

$12 \mathrm{X}, * \mathrm{BETGAM}=*, \mathrm{~F} 12.8 / 5 \mathrm{X}, * \mathrm{EK}=*, \mathrm{~F} 12.8,2 \mathrm{X}, * \mathrm{BRHO}=*, \mathrm{~F} 12.8,2 \mathrm{X}$,

$2 * B E T A=*, F 12.8 / /$ )

RETURN

END
KIN

KINET

KINET

KIN

KIN

KIN

KIN

KIN

KIN

KIN

KIN

KIN

KIN

KIN

KIN

KIN

KIN

KIN

KIN

KIN

KIN

KIN

KIN

KIN

KIN

KIN
2

2

3

4

5

6

7

8

9

10

11

12

13

14

15

16

17

18

19

20

21

22

23

24

25

26

CARD NR. SEVERITY DETAILS DIAGNOSIS OF PROBLEM

4 I KNFLAG A TYPE WAS DECLARED PREVIOUSLY FOR THIS VARIABLE OR FUNCTION. THIS DECLARATION IGNC 
1

10
SUBROUTINE KINEM(KP, T,P)

C KP IS TYPE OF PARTICLE, $T$ IS KE, $P$ IS BETGAM DIMENSION ER(2)

DATA ER(1), ER(2)/.511006,938.256/

$E=E R(K P)+T$

$G A M=E / E R(K P)$

$P=\operatorname{SQRT}(G A M * * 2-1$.

$B E T=P / G A M$

RETURN

END
KINEM

KINEM

KINEM

KINEM

KINEM

KINEM

KINEM

KINEM

KINEM

KINEM
2

3

4

5

6

7

8

9

10

11 
1

15

20

25

35

40

45

50

55

SUBROUTINE LOAD (MO,

- $N 1, I 1, N 2, I 2, N 3, I 3, N 4, I 4$

LOAD

LOAD

.)

LOAD

LOAD

LEVEL 2, STORE, INFF, IWORK

BLANK

COMMON STORE(48000), IWORK(10)

BLANK

DIMENSION INFF $(24,2000)$

86MARSIZ 1

EQUIVALENCE (INFF,STORE)

86MARSIZ 2

86MARSIZ 3

BLANK 5

COMMON/INSTR/OPNAME, NAME, OP, KA, KB, OBJA, OBJB, NXTM, TRA, LQ, NQ, LIN, INSTR

1 NIN, LFL, NFL, LBC, NBC, NTOT, IX, KIND, TYPE, ROWS, COLS, EXTR, INSTR 3

2

$M, N F, N B, N I, M S U B R, I S I G N$

INTEGER OPNAME, OP , OBJA, OBJB, TRA, TYPE, ROWS, COLS, EXTR

INSTR 4

INSTR $\quad 5$

INSTR 6

COMMON/STORE/LMAX, LINF, LFILE, MAX, MIN, ICARD (11)

STORE 2

* lMaX AND MAX aRE DIMENSIONS OF STORE AND INFF

STORE 3

STORE $\quad 4$

COMMON/CONTRL/ERROR, MODE, RSRV, STOR, XEQ, TRASW, NFSW, EMPTY, INDEF,

CONTRL 2

1

LDFLG, FIN

LOGICAL ERROR, RSRV, STOR, XEQ, TRASW, NFSW, EMPTY, INDEF, LDFLG, FIN

CONTRL 3

CONTRL $\quad 4$

COMMON/FLTN/IFL(15)

CONTRL 5

FLTN 2

FLTN 3

COMMON/COPY/CPYSW

LOGICAL CPYSW

COPY

COPY

COMMON /DIM/ LEND, MEND, ISAV, ISAV7, M7END, KADD, KADDR, MUNIT, MSYMPL

COPY

DIM

DIM

LOAD

DIMENSION IFCD(10)

LOAD

INTEGER BDAT

LOAD

DIMENSION IF(6), IB(12), $\operatorname{ICD}(6), \operatorname{BCD}(12), \operatorname{ICD}(12), \operatorname{ND}(10), \operatorname{IT}(10)$,

1 I I (12)

LOAD

LOAD

EQUIVALENCE( IF, ICARD(3) )

LOAD

INTEGER BCD, OPN, RET

LOAD

EQUIVALENCE (FCD, IFCD)

LOAD

LOAD

LOAD

DATA HCOPY/4HCOPY/

DATA MASKF/77000000000000000000B/.

LOAD

LOAD

DATA IBLANK/1H /

LOAD

LOAD

LOAD

LOAD

LOAD

LOAD

LOAD

LOAD

LOAD

ASSIGN 2 TO RET

IF (.NOT.EMPTY) GO TO 1

LOAD

LOAD

LOAD

LOAD

LOAD

LOAD

LOAD

IT (NSETS) $=-I T$ (NSETS)

(n)

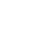

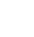

(n)

4


60

$$
\mathrm{NB}=0
$$

$N F=0$

65

EMPTY $=$.FALSE.

LOAD

LOAD

LDFLG $=$. TRUE.

LOAD

LOAD

GO TO 3

c

$1 \operatorname{READ}(2,100)(\operatorname{ICARD}(\mathrm{IW}), \mathrm{IW}=1,8)$

LOAD

LOAD

LOAD

LOAD

IF (ICARD (1). EQ. IBLANK) GO TO 201

LOAD

3 DECODE $(20,101$, ICARD (1)) ISGN, NM, OPN, KAM, KBM

LOAD

LOAD

IF (. NOT.CPYSW) GO TO 501

IF (IPRNT.NE.0) GO TO 501

WRITE $(3,106)$ ISGN, NM, OPN, KAM, KBM, ( ICARD (IW), IW=3,8)

FORMAT (6H ***, A1, A5, 2X, A5, 1X, A3, 1X, A3,5H // ,6A10)

LOAD

IPRNT=IPRNT+1

501 IF (.NOT. (NS.EQ.NSETS.AND. INDEF)) GO TO 202

GO TO 74

201 IF (.NOT.CPYSW) GO TO 202

WRITE $(3,107)$ (ICARD (IW), IW=3,8)

107 FORMAT $(6 \mathrm{H} *, 21 \mathrm{X}, 5 \mathrm{H} / /, 6 \mathrm{~A} 10)$

202 IF (NSETS.EQ.0) RETURN

$\operatorname{DECODE}(60,102, \operatorname{IF}(1))(\operatorname{IB}(J), J=1,12)$

80

102 FORMAT (12A5)

$\operatorname{DECODE}(60,105, \operatorname{IF}(1))(\operatorname{II}(\mathrm{J}), \mathrm{J}=1,12)$

105 FORMAT (12R5)

$\mathrm{NIO}=0$

LOAD

LOAD

LOAD

LOAD

LOAD

LOAD

LOAD

LOAD

LOAD

LOAD

LOAD

LOAD

LOAD

$\mathrm{NBO}=0$

LOAD

$\mathrm{NFO}=0$

LOAD

$I=1$

LOAD

$J=1$

LOAD

GO TO RET $(2,5,6,7,8,9)$

$$
\text { c.. }
$$

LOAD

$$
2
$$

DO $4 \mathrm{NS}=1$, NSETS

ASSIGN 5 TO RET

LOAD

IF ( J.GT.12) GO TO 1

95

5

$$
K=1
$$

LOAD

LOAD

$I$ TNS $=I T$ (NS)

GO TO $(6,7,8,9)$ ITNS

LOAD

LOAD

C

c FLOATING POINT

C TEST FOR SYMBOLIC FLOATING POINT

LOAD

LOAD

LOAD

$6 \quad M S K=M A S K F$. AND. IF (I)

C TEST FOR - AND +

IF (MSK.NE.IFL(1).AND.MSK.NE. IFL(12)) GO TO 12

LOAD

LOAD

LOAD

$M S K=\operatorname{SHIFT}(\operatorname{IF}(I), 6)$

LOAD

MSK = MASKF.AND.MSK

LOAD

LOAD

12 DO $10 \quad L=2,14$

IF (MSK.EQ.IFL(L)) GO TO 120

LOAD

LOAD

10 CONTINUE

LOAD

C SYMBOLIC. CIRCULAR LEFT SHIFT 5 CHARACTERS, FLAG BY FILLING FIRST

LOAD

LOAD

LOAD

$\operatorname{IFCD}(N F \theta+1)=\operatorname{SHIFT}(\operatorname{IF}(I), 30)$

$\operatorname{IFCD}(N F \theta+1)=\operatorname{IFCD}(N F \theta+1)$. AND. MASKL

LOAD

GO TO 110

LOAD

C FLOATING POINT NUMBER

$120 \operatorname{DECODE}(10,103, \operatorname{IF}(I)) \operatorname{FCD}(\mathrm{NF} \theta+1)$ 
$\mathrm{I}=\mathrm{I}+1$

LOAD

$\mathrm{J}=\mathrm{J}+2$

LOAD

100

$N F O=N F O+1$

LOAD

101

120

IF( K.GT.ND(NS) ) GO TO 61

LOAD

102

IF ( I.LE.6 ) GO TO 6

LOAD

103

$\operatorname{CALL} \operatorname{STDAT}(M, 1, N F+1, N F O, F C D)$

LOAD

104

$\mathrm{NF}=\mathrm{NF}+\mathrm{NFO}$

LOAD

105

ASSIGN 6 TO RET

LOAD

106

125

GO TO 1

$61 \operatorname{CALL} \operatorname{STDAT}(M, 1, N F+1, N F O, F C D)$

LOAD

107

$N F=N F+N F \theta$

$\angle O A D$

108

$N F \theta=0$

LOAD

109

NFO $=0$

LOAD

110

GO TO 4

LOAD

111

130

C

c $\mathrm{BCD}$

$7 \quad \mathrm{BCD}(\mathrm{NBO}+1)=\mathrm{IB}(\mathrm{J})$

$\mathrm{K}=\mathrm{K}+1$

LOAD

112

LOAD

113

LOAD

114

LOAD $\quad 115$

$72 \mathrm{~J}=\mathrm{J}+1$

LOAD $\quad 116$

135

$I=J / 2+1$

$\mathrm{NBO}=\mathrm{NBO}+1$

LOAD

117

IF(K.GT.ND(NS)) GO TO 73

LOAD

118

IF ( J.LE.12) GO TO 7

LOAD

119

LOAD $\quad 120$

$\operatorname{CALL} \operatorname{STDAT}(M, 2, N B+1, N B O, B C D)$

LOAD

121

140

$\mathrm{NB}=\mathrm{NB}+\mathrm{NBO}$

LOAD

122

ASSIGN 7 TO RET

LOAD

123

IF (NS.NE.NSETS) GO TO 1

LOAD

124

If (INDEF) $N D(N S)=N D(N S)+12$

LOAD

125

GO TO 1

LOAD

126

145

$73 \mathrm{~J}=2 *(\mathrm{~J} / 2)+1$

LOAD $\quad 127$

LOAD $\quad 128$

$\operatorname{CALL} \operatorname{STDAT}(M, 2, N B+1, N B O, B C D)$

LOAD

$\mathrm{NB}=\mathrm{NB}+\mathrm{NBO}$

$\mathrm{NBO}=0$

GO TO 4

LOAD $\quad 130$

LOAD $\quad 131$

LOAD $\quad 132$

150

C LAST SET WAS BCD OF INDEFINITE LENGTH

LOAD $\quad 133$

LOAD $\quad 134$

C WARNING - AS PRESENTLY CODED(7/1975) , NO INTEGER SETS MAY BE

LOAD $\quad 135$

C INCLUDED. TO BE SAFE, THERE SHOULD ONLY BE A SINGLE SET LOADED.

LOAD

C E.G. CALL INP( $M, M X P M Y,-0,1,-2,0)$

LOAD

74 EMPTY=. TRUE.

LOAD $\quad 138$

LOAD $\quad 139$

INDEF $=$. FALSE.

LOAD

$N B C=N B$

LOAD

$L F M X=L B C+N B C$

LOAD

NTOT $=$ LFMX-LFILE

LOAD

$\operatorname{INFF}(17, M)=N B C$

LOAD

$\operatorname{INFF}(18, M)=$ NTOT

LOAD

LFILE=LFMX

LOAD

IF (LFILE. LE. LMAX) RETURN

LOAD $\quad 147$

165

WRITE $(3,75)$

NSTO = LMAX - LFILE

LOAD

NINFF = MAX - MIN

LOAD 
$134 X$, *STORE (ELEMENT STORAGE)*, 14X, I 10, 1X,*(LMAX)*, 4X, I 10,5X, I 10/ LOAD

$234 \mathrm{X}, *$ INFF (ELEMENT DEFINITIONS) *,10X, I10,1X,*(MAX)*,5X, I10,5X, LOAD

3 I10)

LOAD

ERROR

LOAD

C

LOAD

C INTEGER

180

8 DECODE $(10,104, \mathrm{II}(\mathrm{J})) \operatorname{ICD}(\mathrm{NI \theta +1})$

LOAD

161

$$
104 \text { FORMAT }(5 X, I 5)
$$

LOAD

CALL STDAT $(M, 3, N I+1, N I \theta, I C D)$

LOAD

$N I=N I+N I \theta$

$N I \theta=0$

LOAD

C

c SKIP ND(NS) WORDS

LOAD

$$
9 \quad K=K+1
$$

$I=I+1$

$J=J+2$

LOAD

IF( K.GT.ND(NS)) GO TO 4

LOAD

IF (I.LE.6) GO TO 9

LOAD

GO TO 1

LOAD

IF (OPNAME. EQ. 4HEVEC.OR.OPNAME. EQ. 4HEQIL) GO TO 310

C KIND HAS TO BE DETERMINED BY BCD INPUT.

LOAD

$\mathrm{IJ}=1$

$\operatorname{NCD}=\operatorname{IABS}(\operatorname{MDAT}(M, 1))$

IF (NCD.NE. . ) KND=INFF (20,NCD)

LOAD

IF (OPNAME.EQ.1H=) IJ $=2$

LOAD

IF (OPNAME.EQ. $1 \mathrm{H}=$. AND.KND.EQ.4HSCAL) GO TO 315

LOAD

DO $305 \mathrm{~J}=1, \mathrm{NBC}, \mathrm{IJ}$

LOAD

$\operatorname{NCD}=\operatorname{IABS}(\operatorname{MDAT}(M, J))$

LOAD

C IF ELEMENT NO. $=0$, IT HAS NOT YET BEEN DEFINED

LOAD

IF (NCD.EQ.0) GO TO 320

$307 \mathrm{KND}=\operatorname{INFF}(20, \mathrm{NCD})$

IF (KND.EQ.2HR7.OR.KND.EQ.3HROT) GO TO 320

LOAD

ITE $=1$

$\operatorname{ITYP}=\operatorname{INFF}(21, N C D)$

LOAD 


$$
\mathrm{LBC}=\mathrm{LQ}
$$

LOAD

$\operatorname{INFF}(16, M)=\operatorname{LBC}$

LOAD

$\operatorname{CALL} \operatorname{STDAT}(M, 2,1,2$, ICD $)$

LOAD

$\mathrm{NQ}=0$

LOAD 220

$L F I L E=L B C+N B C-1$

LOAD

221

240

GO TO 330

LOAD

222

C KIND IS SCALAR

LOAD 223

$315 \operatorname{INFF}(20, M)=K N D$

LOAD

$\mathrm{NQ}=1$

LOAD 225

TYPE $=\operatorname{INFF}(21, N C D)$

LOAD 226

245 $\operatorname{INFF}(21, M)=$ TYPE

LOAD $\quad 227$

LOAD $\quad 228$

$\therefore$ IF (TYPE.NE.4HSNGL) NQ $=2$

LOAD

GO TO 326

LOAD 230

C SET KIND TO A $7 \times 7$

LOAD 231

$320 \operatorname{INFF}(20, M)=2 \mathrm{HR7}$

LOAD 232

250

ITE $=1$

LOAD 233

C IF ELEMENT UNDEFINED, LEAVE IT TYPE SINGLE

LOAD

234

IF (NCD.EQ.0) GO TO 324

LOAD

235

$\operatorname{ITYP}=\operatorname{INFF}(21, \mathrm{NCD})$

LOAD

236

$\operatorname{INFF}(21, M)=\operatorname{ITYP}$

255

IF (ITYP.NE.4HSNGL) ITE=2

LOAD $\quad 237$

LOAD $\quad 238$

324 IF (OPNAME. NE. 3HCYB) NQ=49*ITE+KADD

LOAD 239

$\operatorname{INFF}(22, M)=7$

$325 \operatorname{INFF}(23, M)=7$

LOAD 240

LOAD 241

$326 L Q=L F I L E+1$

LOAD 242

260

$L F I L E=L Q+N Q-1$

LOAD

243

$\operatorname{INFF}(10, M)=L Q$

$330 \operatorname{INFF}(11, M)=N Q$

LOAD 244

LOAD 245

NTOT $=N Q+N F L+N I N+N B C$

LOAD

246

$\operatorname{INFF}(18, M)=$ NTOT

LOAD

247

265

RETURN

LOAD

248

END

LOAD

249

CARD NR. SEVERITY DETAILS DIAGNOSIS OF PROBLEM

266 I 91 NON-INNER LOOP BEGINNING AT THIS CARD IS ENTERED FROM OUTSIDE ITS RANGE. 
LOC 2 c

C SET LOC EQUAL TO REPETITION NUM OF NAME IN LIST, WHERE LIST

LOC

LOC

LOC

LOC

c MORE PRECISELY, NAME=MNAME(LIST(LOC)).

C LIST CONTAINS NL M-NUMBERS.

C ALL OCCURRENCES OF NAME ARE PUT IN ARRAY LOCA,

C THERE ARE NLOC OF THEM.

10

C EXAMPLE -

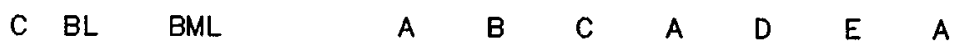


1

10

15

20
INTEGER FUNCTION LOCC (NAME, NUM, NL, LIST, LOCA, NLOC)

LEVEL 2, LOCA

DIMENSION LIST (1), LOCA(1)

LEVEL 2, LIST

c

DATA MAX/100/

DO $1 K=1$, MAX

$1 \quad \operatorname{LOCA}(K)=0$

C

$$
J=0
$$

DO $2 \mathrm{I}=1, \mathrm{NL}$

LL=LIST (I)

NAMEI $=\operatorname{MNAME}(L L)$

IF (NAME.NE. NAMEI) GO TO 2

$J=J+1$

$\operatorname{LOCA}(J)=I$

IF (J.EQ.MAX) GO TO 3

2 CONTINUE

c

$3 \quad N L O C=J$

LOCC $=$ LOCA(NUM)

RETURN

END

$\begin{array}{lr}\text { LOC2 } & 1 \\ \text { LOC2 } & 2 \\ \text { LOC2 } & 3 \\ \text { LOCLL } & 4 \\ \text { LOC2 } & 4 \\ \text { LOC2 } & 5 \\ \text { LOC2 } & 6 \\ \text { LOC2 } & 7 \\ \text { LOC2 } & 8 \\ \text { LOC2 } & 9 \\ \text { LOC2 } & 10 \\ \text { LOCLL } & 5 \\ \text { LOCLL } & 6 \\ \text { LOC2 } & 12 \\ \text { LOC2 } & 13 \\ \text { LOC2 } & 14 \\ \text { LOC2 } & 15 \\ \text { LOC2 } & 16 \\ \text { LOC2 } & 17 \\ \text { LOC2 } & 18 \\ \text { LOC2 } & 19 \\ \text { LOC2 } & 20 \\ \text { LOC2 } & 21\end{array}$


1

10

15

20

25
SUBROUT INE LOCS(NAMES, NUMT, NL, LIST, I LIST)

c

DIMENSION NAMES(1), LIST(1), ILIST(1), LOCA(100)

LEVEL 2, LIST

DATA MASK1/77000 $000000000000000 \mathrm{~B} /$

C

$\begin{array}{lr}\text { LOCS } & 2 \\ \text { LOCS } & 3 \\ \text { LOCS } & 4 \\ \text { LOCLL } & 7 \\ \text { LOCLL } & 8 \\ \text { LOCS } & 5 \\ \text { LOCS } & 6 \\ \text { LOCS } & 7 \\ \text { LOCS } & 8 \\ 86 \text { LOCS } & 1 \\ 86 \text { LOCS } & 2 \\ 86 \text { LOCS } & 3 \\ \text { LOCS } & 9 \\ \text { LOCS } & 10 \\ \text { LOCS } & 11 \\ \text { LOCS } & 12 \\ 86 L O C S & 4 \\ \text { LOCS } & 13 \\ \text { LOCS } & 14 \\ \text { LOCS } & 15 \\ \text { LOCS } & 16 \\ 86 L O C S & 5 \\ \text { LOCS } & 17 \\ \text { LOCS } & 18 \\ \text { LOCS } & 19 \\ \text { LOCS } & 20 \\ \text { LOCS } & 21\end{array}$


5

10

15

20

25

30

35

40

45

50
SUBROUTINE LPAR(THETA, ERL)

C FUNCTION EVALUATION FOR LONG STRAIGHT SECTION DESIGN

C

C

COMMON/AGS/ALPHA, BETA, RHO, BZ, AVAC , BMAX

COMMON/STR/GAM , A , LK, BK, F , LM, BL, LL, BQ, LTO, SMUD , CMUD

REAL LK, LM, LL, LTO

COMMON/CONTRL/ERROR, MODE, RSRV, STOR, XEQ, TRASW, NFSW, EMPTY, INDEF, 1 LDFLG, FIN

LOGICAL ERROR, RSRV, STOR, XEQ, TRASW, NFSW, EMPTY, INDEF , LDFLG, FIN

REAL LDRF, LT

INTEGER GAM

C

$I T S=\theta$
$C=\operatorname{COS}$ (THETA)
$S=\operatorname{SIN}$ (THETA)
$E=\operatorname{EXP}$ (THETA)

HTAN = TANH $($ THETA $)$

WOE $=1 . / \mathrm{E}$

$\mathrm{CH}=0.5 *(E+W O E)$

$S H=0.5 *(E-W O E)$

TANTH $=S / C$

TANDIF = TANTH - HTAN

100

$B L=(1 .-(B K *$ LSEP $/ 2)$.$* TANDIF ) /(B K *$ TANDIF+BK $* * 2 *$ LSEP $*$ TANTH $* H T A N)$

$\mathrm{LT}=4 . * \mathrm{BL}+2 . * \mathrm{LSEP}+4 . * \mathrm{LM}$

IF (GAM.EQ.7) GO TO 150

140

$F=B K * L S E P * C * S H$.

GO TO 160

150

$F=B K *$ LSEP $* C H * S$

160

$\mathrm{PWW}=\mathrm{C} * \mathrm{CH}+\mathrm{S} * \mathrm{SH}+\mathrm{F}$

$P W T=(C * S H+S * C H) / B K+(B L+L S E P) * C * C H+B L * S * S H+B L * F$

$A S N=A * S Q R T((B E T A / B E T M A X) *((P W W-A L P H A * P W T / B E T A) * * 2+$

1

(PWT/BETA)**2))

$A D=A S N-A S$

$A B=A B S(A D) / A$

IF $(A B-.00001) 120,110,110$

110

$I T S=I T S+1$

IF (ITS.GT.50) GO TO 130

$\mathrm{AS}=\mathrm{ASN}$

$L K=B Q /(A S * B 0)$

$B K=S Q R T(L K / R H O)$

$L M=$ THETA/BK

GO TO 100

120

$E R L=L T$ - LTO

RETURN

130 WRITE $(3,1000)$

ERROR $=$.TRUE.

RETURN

1000 FORMAT (*ODLSS COMPUTATIONS DO NOT CONVERGE.*)
LPAR

LPAR

LPAR

AGS

AGS

STR

STR

STR

CONTRL

CONTRL

CONTRL

CONTRL

LPAR

LPAR

LPAR

LPAR

LPAR

LPAR

LPAR

LPAR

LPAR

LPAR

LPAR

LPAR

LPAR

LPAR

LPAR

LPAR

LPAR

LPAR

LPAR

LPAR

LPAR

LPAR

LPAR

LPAR

LPAR

LPAR

LPAR

LPAR

LPAR

LPAR

LPAR

LPAR

LPAR

LPAR

LPAR

LPAR

LPAR

LPAR

LPAR

LPAR
2

3

4

2

3

2

3

4

2

3

4

5

8

9

10

11

12

13

14

15

16

17

18

19

20

21

22

23

24

25

26

27

28

29

30

31

32

33

34

35

36

37

38

39

40

41

42

43

44

45

46

47 
COMPUTE KA NUMBER OF MAGNET MATRICES.

5

* input parameters may be Fl. pt. nUmbers, SYMBolic fl. pt. or

* vector name from which element values are used.

COMMON/CONTRL/ERROR, MODE, RSRV, STOR, XEQ, TRASW, NFSW, EMPTY, INDEF, 1 LDFLG, FIN

LOGICAL ERROR, RSRV, STOR, XEQ, TRASW, NFSW, EMPTY, INDEF, LDFLG, FIN

10

10

DIMENSION ITYP(6), IP(6),PAR(6), IPAR(6)

EQUIVALENCE (PAR, IPAR)

CALL DATA $(M, 6 ; 1,6, I P)$

15

20

25

30

35

40
DETERMINE TYPE OF PARAMETER.

DO $10 \quad I=1,6$

CALL PARTYP(IP(I), ITYP(I ))

IF (ERROR) RETURN

CONT INUE
CALL MAGNET (M1,PAR)

25

CONT INUE

RETURN

END

MAGMATS

MAGMATS

MAGMATS

MAGMATS

CONTRL

CONTRL

CONTRL

CONTRL

MAGMATS

MAGMATS

MAGMATS

MAGMATS

MAGMATS

MAGMATS

MAGMATS

MAGMATS

MAGMATS

MAGMATS

MAGMATS

MAGMATS

MAGMATS

MAGMATS

MAGMATS

MAGMATS

MAGMATS

MAGMATS

MAGMATS

MAGMATS

MAGMATS

MAGMATS

MAGMATS

MAGMATS

MAGMATS

MAGMATS

MAGMATS

MAGMATS

MAGMATS

MAGMATS

MAGMATS

2

3

4

5

2

3

4

5

8

9

10

11

12

13

14

15

16

17

18

19

20

21

22

23

24

25

26

27

28

29

30

31

32

33

34

35

36

37

38 
C SET UP MAGNET MATRICES.

8

C

C OF CURVATURE.

c

C THETA $=$ L*OMEGA/RHO

* rectangular magnet

40

50

COMMON/SWTCH/BEND, MSIZE, CYCSWT, VCSW, PV, MSSW, VPR, MHS, MODS, MINZER LOGICAL CYCSWT, MSSW, VCSW, PV, VPR, MHS, MODS

DIMENSION RX(2,3), RY $(2,3), F(2,3), D(2,3), A(2,3), \operatorname{PAR}(1), \operatorname{RW}(3)$

REAL L, K, KP

EQUiVALENCE $(C, C H),(S, S H),(F, D, A),(R W(1), L),(R W(2), \operatorname{THETA})$,

C IN MISALIGNMENT MODE IF BEND $=0$.

IF (PAR(1).GE.0) GO TO 8

IVERT $=1$

$\operatorname{PAR}(1)=-\operatorname{PAR}(1)$

$$
L=\operatorname{PAR}(1)
$$

PROF = PAR (2)

$\mathrm{RHO}=\mathrm{PAR}(3)$

OMEGA $=$ PAR (4)

$\mathrm{RHOI}=1 . / \mathrm{RHO}$

C $\quad$ CURV = OMEGA/RHO = CURVATURE OF REFERENCE ORBIT

C OMEGA IS POSITIVE IF CENTER OF CURVATURE HAS A NEGATIVE $X$ VALUE

C RELATIVE TO REFERENCE ORBIT.

CURV $=$ OMEGA $*$ RHOI

C GAMMA, THE MAGNET WEDGE ANGLE, IS POSITIVE IF THE MAGNET FACES

C INTERSECT ON THE SIDE OF THE REFERENCE ORBIT OPPOSITE TO THE CENTER

THETA $=$ L*CURV

IF (CURV.EQ.0.) GO TO 100

* par(5) and (6) are entrance and exit angles in degrees.

* if either or both are \$ Sign, magnets are rectangular.

ANG1 = PAR(5)

ANG2 = PAR (6)

IF ((IANG1.EQ.1H\$).OR. (IANG2. EQ.1H\$)) GO TO 4

$E 1=\operatorname{PAR}(5) *$ RADEG

$\mathrm{E} 2=\mathrm{PAR}(6) * \mathrm{RADEG}$

GO TO 40

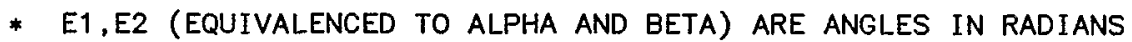

4 IF (PROF.EQ.O..AND.L.NE.0.) GO TO 200

$E 1=E 2=A B S(T H E T A) / 2$.

CONT INUE
MAGNET

MAGNET

MAGNET

SWTCH

SWTCH

SWTCH

MAGNET

MAGNET

MAGNET

MAGNET

MAGNET

MAGNET

MAGNET

MAGNET

MAGNET

MAGNET

MAGNET

MAGNET

MAGNET

MAGNET

MAGNET

MAGNET

MAGNET

MAGNET

MAGNET

MAGNET

MAGNET

MAGNET

MAGNET

MAGNET

MAGNET

MAGNET

MAGNET

MAGNET

MAGNET

MAGNET

MAGNET

MAGNET

MAGNET

MAGNET

MAGNET

MAGNET

MAGNET

MAGNET

MAGNET

MAGNET

MAGNET

MAGNET

MAGNET

MAGNET

MAGNET

MAGNET

MAGNET

MAGNET

MAGNET

MAGNET

MAGNET 
IF (KP.LT.O.) GO TO 3

MAGNET $\quad 57$ C FOCUSSING MATRIX

60

$$
\begin{aligned}
2 \quad S & =\operatorname{SIN}(\mathrm{PHI}) \\
\mathrm{C} & =\cos (\mathrm{PHI})
\end{aligned}
$$$$
F(1,1)=C
$$$$
F(1,2)=S / K
$$$$
F(1,3)=(\text { CURV } *(1,-C) /(K * K)) * \text { BEND }
$$

$F(2,1)=-K * S$

MAGNET

$F(2,2)=\mathrm{C}$

$F(2,3)=($ CURV $* S / K) * B E N D$

IF (ID.EQ.1) $\mathrm{PL}=-(\mathrm{PHI}-\mathrm{S}) * \mathrm{CURV} * \mathrm{CURV} /(\mathrm{K} * \mathrm{~K} * \mathrm{~K})$

MAGNET

$E I=1 . / E$

$\mathrm{CH}=0.5 *(E+E I)$

$\mathrm{SH}=0.5 *(\mathrm{E}-\mathrm{EI})$

$\mathrm{D}(1,1)=\mathrm{CH}$

$D(1,2)=S H / K$

$D(2,1)=S H * K$

$\mathrm{D}(1,3)=(\mathrm{CURV} *(\mathrm{CH}-1) /.(\mathrm{K} * \mathrm{~K})) * \mathrm{BEND}$

$\mathrm{D}(2,2)=\mathrm{CH}$

$A(2,2)=1$.

MAGNET

$A(2,3)=($ CURV $* L) * B E N D$

IF (ID.EQ.1) $P L=-L * L * L * C U R V * C U R V / 6$.

MAGNET

MAGNET

C CORRECT FOR EDGE FOCUSSING.

$10 \quad A(1,1)=A(1,1)+A(1,2) * E F I N$

$A(2,1)=A(1,1) *$ EFOUT $+A(2,1)+A(2,2) * E F I N$

MAGNET

MAGNET

$A(2,2)=A(1,2) *$ EFOUT $+A(2,2)$

$A(2,3)=A(1,3) *$ EFOUT $+A(2,3)$

MAGNET

IF (ID.EQ.2) GO TO 20

MAGNET

MAGNET

C SET UP RADIAL TRANSFER MATRIX.

MAGNET 96

DO $15 \mathrm{I}=1,2$

DO $15 \mathrm{~J}=1,3$

MAGNET 97

$R X(I, J)=A(I, J)$

15 CONTINUE

IF (IVERT.EQ.0) GO TO 16

MAGNET

MAGNET

MAGNET

MAGNET

IVERT $=0$

MAGNET

GO TO 26

105

16

CURV $=0$.

ID $=2$

EFIN $=-E F I N$

EFOUT $=-E F O U T$

MAGNET

MAGNET

MAGNET

MAGNET

MAGNET

$F C=-1$.

MAGNET

GO TO 1

C SET UP VERTICAL TRANSFER MATRIX.

MAGNET

MAGNET

MAGNET

MAGNET

DO $25 \mathrm{~J}=1,3$

$\operatorname{RY}(I, J)=A(I, J)$ 
115

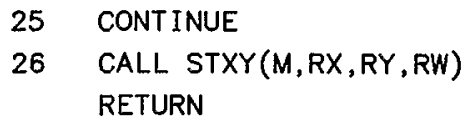

$\begin{array}{ll}\text { MAGNET } & 114 \\ \text { MAGNET } & 115 \\ \text { MAGNET } & 116 \\ \text { MAGNET } & 117 \\ \text { MAGNET } & 118 \\ \text { MAGNET } & 119 \\ \text { MAGNET } & 120 \\ \text { MAGNET } & 121 \\ \text { MAGNET } & 122 \\ \text { MAGNET } & 123 \\ \text { MAGNET } & 124 \\ \text { MAGNET } & 125 \\ \text { MAGNET } & 126 \\ \text { MAGNET } & 127 \\ \text { MAGNET } & 128 \\ \text { MAGNET } & 129 \\ \text { MAGNET } & 130 \\ \text { MAGNET } & 131 \\ \text { MAGNET } & 132 \\ \text { MAGNET } & 133 \\ \text { MAGNET } & 134 \\ \text { MAGNET } & 135 \\ \text { MAGNET } & 136 \\ \text { MAGNET } & 137 \\ \text { MAGNET } & 138 \\ \text { MAGNET } & 139 \\ \text { MAGNET } & 140 \\ \text { MAGNET } & 141 \\ \text { MAGNET } & 142 \\ \text { MAGNET } & 143 \\ \text { MAGNET } & 144 \\ \text { MAGNET } & 145 \\ \text { MAGNET } & 146 \\ \text { MAGNET } & 147 \\ \text { MAGNET } & 148 \\ \text { MAGNET } & 149\end{array}$

CARD NR. SEVERITY DETAILS DIAGNOSIS OF PROBLEM

$\begin{array}{llll}20 & \text { I } & \text { PAR } & \text { ARRAY REFERENCE OUTSIDE DIMENSION BOUNDS. } \\ 21 & \text { I } & \text { PAR } & \text { ARRAY REFERENCE OUTSIDE DIMENSION BOUNDS. } \\ 22 & \text { I } & \text { PAR } & \text { ARRAY REFERENCE OUTSIDE DIMENSION BOUNDS. } \\ 38 & \text { I } & \text { PAR } & \text { ARRAY REFERENCE OUTSIDE DIMENSION BOUNDS. } \\ 39 & \text { I } & \text { PAR } & \text { ARRAY REFERENCE OUTSIDE DIMENSION BOUNDS. } \\ 41 & \text { I } & \text { PAR } & \text { ARRAY REFERENCE OUTSIDE DIMENSION BOUNDS. } \\ 42 & \text { I } & \text { PAR } & \text { ARRAY REFERENCE OUTSIDE DIMENSION BOUNDS. }\end{array}$


1

SUBROUT INE MAGRSV( MO, NAMEO, KAO, KBO, NAME1 )

MAGRSV 2

* SetS up info AND storage for a magnet.

* RESERVES for INPUT of 6 PARAMETERS, OUtPUt of $3 \times 3$ MATRIX.

MAGRSV

MAGRSV

MAGRSV

MAGRSV

* $\quad M O=M$ INDEX, KAO = KA

$\mathrm{KBO}=\mathrm{KB}$

MAGRSV

* NAMEO = NAME, OPNAME WILL BE MAG

* if mo is negative, the first letter of name1 will precede nameo

* to fORM NAME.

COMMON/SWTCH/BEND, MSIZE, CYCSWT, VCSW, PV, MSSW, VPR, MHS, MODS, MINZER 
LEVEL 2, STORE, INFF, IWORK

COMMON STORE(48000), IWORK (10)

COMMON/SWTCH/BEND, MSIZE, CYCSWT, VCSW, PV, MSSW, VPR, MHS, MODS, MINZER LOGICAL CYCSWT, MSSW, VCSW, PV, VPR, MHS, MODS

COMMON /DIM/ LEND, MEND, ISAV, ISAV7, M7END, KADD , KADDR, MUNIT , MSYMPL

COMMON/MI /MLL(3) \$ LEVEL 2,MLL

LOGICAL BSW

DIMENSION RX( 2,3$), \operatorname{RY}(2,3), \operatorname{RW}(3), Z(5), Y(6), X(4), M L(3)$

DIMENSION ZZ(5)

EQUIVALENCE $(Y(2), X, M L)$

DATA RW/3*0.0/

C COMPUTE MATRIX WITH MISALIGNMENTS INCLUDED

C RETRIEVE LENGTH

C RETRIEVE MATRIX ELEMENTS OF INDEX $N$ AND STORE IN $M$.

C EQUATES $M$ TO $N$ INITIALLY. $N$ WILL BE PRESERVED. A NEW MATRIX WILL BE C COMPUTED FOR $M$

C MS AND MT ARE WORKING STORAGE LOCATIONS

86MARSIZ

86MARSIZ

86MARSIZ

BLANK

SWTCH

SWTCH

SWTCH

DIM

DIM

MAGSLL

MAGS

MAGS

MAGS

MAGS

MAGS

MAGS

MAGS

MAGS

MAGS

MAGS

MAGS

MAGS

MAGS

$$
M S=M E N D-1
$$

$M T=M E N D-2$

$$
K A=\operatorname{INFF}(4, M)
$$

MAGS

MAGS

IF (KA.EQ. $\theta) \mathrm{KA}=1$

C IF(KB,GE. 1) ERRORS ARE RANDOM WITH GIVEN RANGE

$\mathrm{KB}=\operatorname{INFF}(5, M)$

$$
\mathrm{DO} 21 \mathrm{I}=1,5
$$

$21 \quad Z Z(I)=Z(I)$

IF (KB.LE. $\theta)$ GO TO 20

$$
D O 22 \mathrm{I}=1,5
$$

$22 \quad Z(I)=Z Z(I) *($ RANF $(D)-.5)$

20 CONTINUE

$$
\mathrm{DBB}=\mathrm{Z}(5)
$$

$$
\begin{aligned}
& B S W=. F A L S E . \\
& I F(Z(5) . E Q .0) \text { GO TO } 10
\end{aligned}
$$

3 IF $(Y(4) \cdot E Q \cdot 0$.$) BSW = .TRUE.$

C COMPUTE MAGNET MATRIX WITH FIELD ERROR INCLUDED 
60

* where b not $\theta$.

$\operatorname{RX}(1,3)=-\operatorname{RX}(1,3) * \mathrm{DBB}$

MAGS

MAGS

$\mathrm{RX}(2,3)=-\mathrm{RX}(2,3) * \mathrm{DBB}$

MAGS

GO TO 9

WHERE $B=0$.

MAGS

MAGS

MAGS

CURV $=Y(4) / Y(3)$

$E L=Y(1)$

65

$X X=-(E L * E L / 2) * C U R$.

MAGS

MAGS

$X P=-E L * C U R V$

MAGS

$R X(1,3)=X X$

MAGS

$\mathrm{RX}(2,3)=X P$

MAGS

MAGS

MAGS

MAGS

MAGS

MAGS

MAGS

MAGS

MAGS

MAGS

MAGS

MAGS

MAGS

MAGS

MAGS

MAGS

MAGS

MAGS

MAGS

MAGS

MAGS

MAGS

MAGS

MAGSLL

90

$M L(2)=M$

$M L(3)=M S$

$\operatorname{MLL}(1)=M L(1) \$ M L L(2)=M L(2) \$ M L L(3)=M L(3) \$ C A L L M M M(M, 3, M L L)$

6 CONTINUE

D023 $I=1,5$

95

$23 \quad Z(I)=Z Z(I)$

RETURN

$10 \quad Y(1)=\operatorname{FDAT}(N, 1)$

CALL RXY (N,RX,RY,RW)

CALL STXY(M,RX,RY,RW)

100

GO TO 4

END

MAGSLL

MAGS 84

MAGS 85

MAGS 86

MAGS 87

MAGS 88

MAGS $\quad 89$

MAGS 90

MAGS 91

MAGS 92 
1

SUBROUTINE MAGSRV( MO,NAMEO, KAO,KBO,NAME1 ) COMMON/SWTCH/BEND, MSIZE, CYCSWT, VCSW, PV, MSSW, VPR, MHS, MODS, MINZER LOGICAL CYCSWT, MSSW, VCSW, PV, VPR, MHS, MODS

5

10
DATA MXPMY/1/

CALL STINFO( MO, NAMEO, 5HMAGS ,KAO, KBO, NAME1)

CALL INP (MO, MXPMY, MINZER,

$1,2,5,1,0,0,0,0$

.)

RETURN

END
MAGSRV

SWTCH

SWTCH

SWTCH

MAGSRV

MAGSRV

MAGSRV

MAGSRV

MAGSRV

MAGSRV

MAGSRV
2

2

3

4

4

4

5

6

7

8

9

10 
1

SUBROUT INE MAGV(M,PAR)

MAGV

SET UP MATRIX WITH VERTICAL BEND

5

* IF B IS + , THEN dO +PI/2 M -PI $/ 2$

MAGV

MAGV

MAGV

MAGV

MAGV

COMMON /DIM/ LEND, MEND, ISAV, ISAV7, M7END, KADD, KADDR, MUNIT, MSYMPL

DIM

DIM

DIMENSION T(49), TR(49), RW(3),PAR(1)

10

DATA PI2/1.5707963268/

MAGV

MAGV

MAGV

MAGV

MAGV

MAGV

MAGV

MAGV

MAGV

MAGV

MAGV

END

THETA = PI 2

CALL STOR7 (M,TR,RW)

RETURN 
1

SUBROUTINE MAP3(V,PAR)

C SIMULATE BEAM-BEAM FORCE WITH ROUND BEAM

MAP3

MAP3

MAP3

MAP3

DIMENSION $V(4), \operatorname{PAR}(3)$

MAP3

DATA TWOPI/6.2831853/

MAP3

$\mathrm{RS}=\mathrm{V}(3) * * 2 / \mathrm{PAR}(2) * * 2$

10

IF (V(3).EQ.0.) GO TO 2

MAP3

MAP3

$F=2 . * T W O P I * \operatorname{PAR}(1) *(1,-\operatorname{EXP}(-\mathrm{RS})) /(\operatorname{RS} * \operatorname{PAR}(3))$

MAP3

MAP3

$V(4)=V(4)+V(3) * F$

2 RETURN

MAP3

END

MAP3 
1

SUBROUTINE MAPG(V,PAR)

RETURN

END

$\begin{array}{ll}\text { MAP9 } & 2 \\ \text { MAP9 } & 3 \\ \text { MAP9 } & 4\end{array}$


1

SUBROUT INE MAXMIN(BMAX, BMIN, BET, MB, IP)

MAXMIN

MAXMIN

2

C DETERMINES ALGEBRAIC MAX OF BMAX,BET AND MINIMUM OF BMIN,BET

MAXMIN

MAXMIN

* if two are equal; the first REMAINS as min or max

5

* MB(1), MB(2) aRE indices of maX. AND min. VAlues

MAXMIN

MAXMIN

MAXMIN

MAXMIN

MAXMIN

MAXMIN

MAXMIN

MAXMIN

MAXMIN

MAXMIN

MAXMIN

MAXMIN

RETURN

3
4
5
6
7
8
9
10
11
12
13
14
15
16
17

CARD NR. SEVERITY DETAILS DIAGNOSIS OF PROBLEM

14 I MB ARRAY REFERENCE OUTSIDE DIMENSION BOUNDS. 
1

FUNCTION MDAT $(M, J)$

MDAT

MDAT

C IF THE JTH BCD STORAGE LOCATION OF M CONTAINS AN ELEMENT NAME,

MDAT

BLANK

BLANK

86MARSIZ

86MARSIZ

86MARSIZ

BLANK

EQUIVALENCE (IVAR, FVAR)

INTEGER ELNUM

MDAT

MDAT

$\operatorname{LOC}=\operatorname{INFF}(16, M)+J-1$

FVAR=STORE $(L O C)$

MDAT

MDAT

MDAT

MDAT

RETURN

END

MDAT 
LEVEL 2, STORE, INFF, IWORK COMMON STORE(48000), IWORK(10)

DIMENSION INFF $(24,2000)$

EQUIVALENCE (INFF,STORE)

COMMON/SWTCH/BEND, MSIZE, CYCSWT, VCSW, PV , MSSW, VPR, MHS , MODS, MINZER LOGICAL CYCSWT, MSSW, VCSW, PV, VPR, MHS, MODS

MESH

MESH

BLANK

BLANK

86MARSIZ

86MARSIZ

86MARSIZ

BLANK

SWTCH

SWTCH

SWTCH

COMMON/CONTRL/ERROR, MODE, RSRV, STOR, XEQ, TRASW, NFSW, EMPTY, INDEF,

LOGICAL ERROR, RSRV, STOR, XEQ, TRASW, NFSW, EMPTY, INDEF, LDFLG, FIN

INTEGER ELNUM

$\mathrm{JX}=1$

IF (MOP.EQ.4HVPAR) JX $=2$

IF (.NOT.MSSW) GO TO 5

IF (JX.GT.1) GO TO 4

IF (MHS) GO TO 46

GO TO 5

4 IF (VPR) GO TO 48

C INITIAL CALL

C RETRIEVE VARIABLE NAMES AND INDEX NUMBER

5 CONTINUE

$\operatorname{CALL} \operatorname{DATA}(M, 2,2, K A, N M(1, J X))$

CONTRL

CONTRL

CONTRL

CONTRL

MESH

MESH

MESH

MESH

MESH

MESH

MESH

MESH

MESH

MESH

MESH

MESH

MESH

C RETRIEVE MESH LIMITS THAT WERE INPUT

MESH

$K M=K A * 3$

$\operatorname{CALL} \operatorname{DATA}(M, 1,1, K M, F)$

MESH

$I 1=1$

35

$12=2$

$13=3$

MESH

MESH

MESH

MESH

c

DO $10 I=1, \mathrm{KA}$

$F M I N(I, J X)=F(I 1)$

$\operatorname{FMAX}(I)=F(I 2)$

MESH

MESH

MESH

$\operatorname{FDEL}(I, J X)=F(I 3)$

$I 1=I 1+3$

$12=12+3$

$13=13+3$ 
MESH

CALL $\operatorname{STDAT}(J N(I, J X), 1, \operatorname{IND}(I, J X), 1, \operatorname{FPAR}(I, J X))$

80

36 CONTINUE

GO TO 60

MESH

MESH

MESH

C FULL MESH

46 CONTINUE

$K E=K E J(J X)$

85

$K V=K E$

$$
I=1
$$

49 IF (LP(KV,JX).NE.LL(KV, JX)) GO TO 55

MESH

MESH

MESH

MESH

MESH

MESH

C LOOP COMPLETED. RESET

$$
L P(K V, J X)=1
$$

90

$$
\operatorname{FPAR}(K V, J X)=\operatorname{FMIN}(K V, J X)
$$

CALL STDAT (JN(KV,JX), 1, IND $(K V, J X), 1, \operatorname{FPAR}(K V, J X))$

MESH

MESH

MESH

MESH

$K V=K V-1$

IF (I.EQ.KE) GO TO 64

$I=I+I$

95

$$
\text { GO TO } 49
$$

$55 \quad L P(K V, J X)=L P(K V, J X)+1$

$\operatorname{FPAR}(K V, J X)=\operatorname{FPAR}(K V, J X)+\operatorname{FDEL}(K V, J X)$

CALL STDAT $(J N(K V, J X), 1, \operatorname{IND}(K V, J X), 1, \operatorname{FPAR}(K V, J X))$

100

$60 \quad K A=1$

WRITE $(3,100)$

DO $62 \quad I=1, K E$

WRITE $(3,101)$ IND $(I, J X), N M(I, J X), \operatorname{FPAR}(I, J X)$

62 CONTINUE

CALL SUBR(3)

105

MSSW $=$. TRUE.

MESH

MESH

MESH

MESH

MESH

MESH

MESH

MESH

MESH

MESH

MESH

MESH

MESH

MODS $=$. TRUE.

RETURN

C FINISHED. REPLACE ORIGINAL VALUES OF VARIABLES

MESH

MESH

MESH

MESH

MESH

MESH

MESH

MESH

MESH

MESH 
115

$F(I)=\operatorname{VSAV}(I, J X)$

CALL $\operatorname{STDAT}(J N(I, J X), 1, I N D(I, J X), 1, F(I))$

MESH

106

70 CONT INUE

IF (MHS) RETURN

MESH

107

IF (VPR) RETURN

MESH

108

MSSW $=$.FALSE.

MESH

109

120

MODE $=\operatorname{INFF}(19, M)$

MESH

110

RETURN

MESH

111

MESH

112

100 FORMAT (/15H CURRENT VALUES/)

MESH

113

101 FORMAT (5X, I5, 4H OF , A5, 3H =, F10.5)

MESH

114

125

END

MESH

115

MESH

116 
SUBROUTINE MFD $(M, J, F A C T O R)$

MFD

2

C MULTIPLIES BY FACTOR THE J-TH FL PT PARAMETER OF ELEMENT $M$ AND

MFD

3

C RETURNS NEW VALUE TO STORAGE.

MFD

4

5

$F=F D A T(M, J)$

MFD

MFD

MFD

MFD

MFD

CALL REPFLT $(M, J, F)$

RETURN

9 
DIMENSION ML(1), KBEG(10), NREP(10), NR(10)

MIEXP

LEVEL 2, ML

MIEXP

MIXL

5

INTEGER BK, BDAT

DATA IBLANK/10H

DATA MASK/00000077000000000000B/

DATA IPL/00000051000000000000B/

MIXL

MIEXP

MIEXP

MIEXP

MIEXP

MIEXP

MIEXP

MIEXP

MIEXP

MIEXP

MIEXP

MIEXP

MIEXP

MIEXP

1

$\mathrm{I}=0$
$\mathrm{LOOP}=0$

$K=1$

20

$B K=\operatorname{BDAT}(M, K+J-1)$

MIEXP

MIEXP

MIEXP

MIEXP

MIEXP

MIEXP

MIEXP

MIEXP

MIEXP

MIEXP

MIEXP

MIEXP

MIEXP

MIEXP

MIEXP

MIEXP

MIEXP

MIEXP

MIEXP

MIEXP

MIEXP

MIEXP

MIEXP

MIEXP

MIEXP

MIEXP

MIEXP

MIEXP

2

3

$5 \quad$ NELS $=$ I

RETURN

END 
1

5

10

15

20

25

30

35

40

45

50

55
SUBROUT INE MIFILL( $M, J, N D A T, N E L S, M I)$

LEVEL 2, STORE, INFF, IWORK

COMMON STORE(48000), IWORK(10)

DIMENSION INFF $(24,2000)$

EQUIVALENCE (INFF,STORE)

DIMENSION MI (1), ME(16000)

COMMON/ME/ME

LEVEL 2,MI,ME

INTEGER OPL

CALL MIEXP( $M, J, N D A T, N E L S, M E)$

KREF $=1$

$O P L=\operatorname{INFF}(1, M)$

IF (OPL.NE. 3HBML) GO TO 25

$\mathrm{KAL}=\operatorname{INFF}(4, M)$

IF (KAL.GE.O) GO TO 25

DO $20 \mathrm{~N}=1$, NELS

20

$M I(N)=M E(N E L S-N+1)$

KREF $=-K R E F$

GO TO 27

25 DO $26 \mathrm{~N}=1$, NELS

$26 \quad \operatorname{MI}(N)=\operatorname{ME}(N)$

$27 \quad K E=N E L S$

$L=0$

$1 \quad L=L+1$

IF (L.GT.KE) GO TO 2

$M L=M I(L)$

IF (ML.GT.999990) GO TO 3

$\operatorname{OPL}=\operatorname{INFF}(1, \mathrm{ML})$

$\operatorname{KAL}=\operatorname{INFF}(4, \mathrm{ML})$

IF (OPL.NE. 3HBML.AND.OPL.NE. 4HLIST) GO TO 1

$K L=\operatorname{INFF}(17, \mathrm{ML})$

CALL MIEXP(ML, $1, K L, N L, M E)$

IF (OPL.EQ.3HBML) GO TO 11

$\mathrm{KBL}=\operatorname{INFF}(5, \mathrm{ML})$

IF (KREF.LT.O.AND.KAL.NE.1) GO TO 12

$M I(L)=M E(K B L)$

GO TO 13

12

$M I(L)=M E(N L-K B L+1)$

13

$K B L=K B L+1$

IF (KBL.LE.NL) GO TO 14

$K B L=1$

14

$\operatorname{INFF}(5, M L)=K B L$
MIFILL 2

BLANK 2

BLANK 3

86MARSIZ 1

86MARSIZ 2

86MARSIZ 3

BLANK 5

NOV3MFLL 1

MIFILLL 2

MIFILLL 3

MIFILLL 4

MIFILL 5

MIFILL 6

MIFILL 7

MIFILL : 8

MIFILL $\quad 9$

MIFILL $\quad 10$

MIFILL : 11

MIFILL $\quad 12$

MIFILL 13

MIFILL $\quad 14$

MIFILL $\quad 15$

MIFILL $\quad 16$

MIFILL 17

MIFILL 18

MIFILL $\quad 19$

MIFILL 20

MIFILL 21

MIFILL 22

MIFILL 23

MIFILL 24

MIFILL 25

MIFILL 26

MIFILL 27

MIFILL 28

MIFILL 29

MIFILL - 30

MIFILL $\quad 31$

MIFILL 32

MIFILL 33

MIFILL 34

MIFILL $\quad 35$

MIFILL $\quad 36$

MIFILL $\quad 37$

MIFILL $\quad 38$

MIFILL $\quad 39$

MIFILL $\quad 40$

MIFILL $\quad 41$

MIFILL $\quad 42$

MIFILL $\quad 43$

MIFILL $\quad 44$

MIFILL $\quad 45$

MIFILL $\quad 46$

MIFILL $\quad 47$

MIFILL $\quad 48$

MIFILL $\quad 49$

MIFILL $\quad 50$ 
Go TO 1

60

65

70

$$
\begin{array}{ll}
4 & D O 5 N=1, K E L \\
5 & M I(K E+N L-N)=M I(K E-N+N O)
\end{array}
$$$$
\text { IF (KAL.GE. O) GO TO } 4
$$$$
N O=0
$$$$
K E=K E+1
$$$$
K R E F=-K R E F
$$$$
\text { IF (KREF.LT.0) GO TO } 7
$$$$
\text { DO } 6 \mathrm{~N}=1, \mathrm{NL}
$$$$
6 \quad M I(L+N-1)=M E(N)
$$$$
\text { GO TO } 9
$$

75

$$
\begin{array}{ll}
7 & \text { DO } 8 N=1, N L \\
8 & M I(L+N-1)=M E(N L-N+1)
\end{array}
$$

80

$9 \quad \mathrm{KE}=\mathrm{KE}+\mathrm{NL}-1$

IF (KAL.LT. O) MI $(L+N L)=999999$

$$
L=L-1
$$

$$
\text { GO TO } 1
$$

85

90

95
$3 \quad K E L=K E-L$

DO $10 \mathrm{~N}=1, \mathrm{KEL}$

$10 \quad M I(L+N-1)=M I(L+N)$

$K E=K E-1$

$L=L-1$

KREF $=-K R E F$

GO TO 1

$2 \quad N E L S=K E$

RETURN

END
MIFILL $\quad 51$

MIFILL $\quad 52$

MIFILL 53

MIFILL $\quad 54$

MIFILL $\quad 55$

MIFILL $\quad 56$

MIFILL $\quad 57$

MIFILL $\quad 58$

MIFILL $\quad 59$

MIFILL $\quad 60$

MIFILL $\quad 61$

MIFILL $\quad 62$

MIFILL 63

MIFILL 64

MIFILL 65

MIFILL $\quad 66$

MIFILL $\quad 67$

MIFILL 68 .

MIFILL 69

MIFILL $\quad 70$

MIFILL 71

MIFILL $\quad 72$

MIFILL $\quad 73$

MIFILL $\quad 74$

MIFILL $\quad 75$

MIFILL $\quad 76$

MIFILL $\quad 77$

MIFILL $\quad 78$

MIFILL $\quad 79$

MIFILL $\quad 80$

MIFILL 81

MIFILL $\quad 82$

MIFILL 83

MIFILL 84

MIFILL 85

MIFILL $\quad 86$

MIFILL $\quad 87$

MIFILL $\quad 88$

MIFILL $\quad 89$ 
1

10
SUBROUTINE MLIST ( $M, J, K, M I)$

C READS K ELEMENT NAMES FROM THE JTH BCD LOCATION OF $M$ AND STORES

C THEIR INDICES IN MI.

$$
\text { DIMENSION MI (1) }
$$

LEVEL 2,MI

DIMENSION ML(200)

INTEGER ELNUM

CALL DATA $(M, 2, J, K, M L)$

DO $1 \quad I=1, K$

$1 \quad M I(I)=E L N U M(M L(I))$

RETURN

END
MIFILL $\quad 90$

MLIST 2

MLIST 3

MLIST 4

MLIST 5

MLISTL 1

MLISTL 2

MLIST 6

MLISTL 3

MLIST 8

MLISTL 4

MLISTL 5

MLIST 10

MLIST 11 
C MATRIX MULTIPLICATION. $R(M)=R(M K) * \ldots R(M 2) * R(M 1)$

C IF $M I(I)$ IS NEGATIVE, $R(M)=R(M K) \ldots(1 / R(M I)) \ldots R(M 2) . R(M 1)$ ETC.

* SYNCH INSTRUCTION MMM:

* $X$ MMM A B C . . . .

* a,b, C, . . are previously defined matrices, or bML?S, or numbered

* PARENTHESES. THIS INPUT LIST IS EXPANDED BY MIFILL AND MIEXP TO

* Form a list of matrix indices, stored in mi(i). THESE are

* MUltiplied together to form MATRiX NAMED $X$.

C

C SEE EXECMM ROUTINE FOR OPTIONS OF MMM INSTRUCTION

LEVEL 2, STORE, INFF, IWORK

COMMON STORE(48000), IWORK(10)

DIMENSION INFF $(24,2000)$

EQUIVALENCE (INFF, STORE)

COMMON /DIM/ LEND, MEND, ISAV, ISAV7, M7END, KADD, KADDR, MUNIT, MSYMPL

$K N=\operatorname{INFF}(20, M)$

$\mathrm{KK}=3$

IF (KN.EQ.2HR7.OR.KN.EQ.3HROT.OR.KN.EQ.3HMAP) KK $=7$

$$
N T=M 7 E N D
$$

$$
\mathrm{K}=3 \$ \mathrm{NT}=\mathrm{MEND}
$$

DO $100 \mathrm{I}=1, \mathrm{KA}$

$\operatorname{IF}(\operatorname{INFF}(20, \operatorname{IABS}(M I(I))) \cdot$ EQ.3HMXY) GO TO 100

$K=7 \quad \$ N T=M 7 E N D$

GO TO 5

100 CONTINUE

$5 \quad M 1=M I(1)$

C IF FIRST INDEX IN MILIST IS - SET IT + AND INVERT

IF (M1) 1,2, 2

$1 \quad M 1=-M 1$

40

$$
\text { CALL INV(NT, M1) }
$$

$\mathrm{K} 1=2$

GO TO 4

2 CONTINUE

IF (KA.GE.2) GO TO 7

C ONLY ONE ELEMENT. MULTIPLY BY UNIT MATRIX.

$M 2=M U N I T$

GO TO 8

$7 \quad M 2=M I(2)$

IF (KA.GT.2) GO TO 12

C IF THERE ARE ONLY 2 MATRIX MULT., RESULT SHOULD BE STORED FOR $M$.

CALL $M X D(M, M 1, M 2, K K)$

GO TO 11

C ACCUM. RESULTS OF MULT. ARE STORED IN WORKING STORAGE OF NT

12 CALL MXD(NT,M1,M2,K)

$\mathrm{K} 1=3$

4 DO $10 \mathrm{I}=\mathrm{K} 1, \mathrm{KA}$

MMM

MMM

MMM

MMM

MMM

MMM

MMM

MMM

MMM

MMM

MMM

MMM

BLANK

BLANK

86MARSIZ

86MARSIZ

86MARSIZ

BLANK

DIM

DIM

MMM

MMML

MMML

MMM

MMM

MMM

MMM

MMM

MMM

MMMSPEED

MMMSPEED

MMMSPEED

MMMSPEED

MMMSPEED

MMMSPEED

MMM

MMM

MMM

MMM

MMM

MMM

MMM

MMM

MMM

MMM

MMM

MMM

MMM

MMM

MMM

MMM

MMM

MMM

MMM

MMM

MMM

MMM
2 


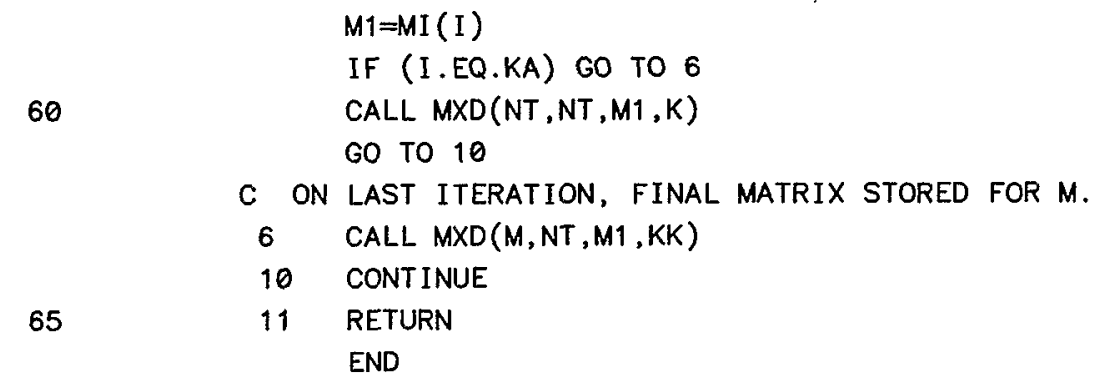

$\begin{array}{ll}\text { MMM } & 46 \\ \text { MMM } & 47 \\ \text { MMM } & 48 \\ \text { MMM } & 49 \\ \text { MMM } & 50 \\ \text { MMM } & 51 \\ \text { MMM } & 52 \\ \text { MMM } & 53 \\ \text { MMM } & 54\end{array}$

CARD NR. SEVERITY DETAILS DIAGNOSIS OF PROBLEM

48 I MI ARRAY REFERENCE OUTSIDE DIMENSION BOUNDS. 
COMMON/SWTCH/BEND, MSIZE, CYCSWT, VCSW, PV, MSSW, VPR, MHS, MODS, MINZER

MMMRSV

SWTCH

SWTCH

SWTCH

MMMRSV

MMMRSV

MMMRSV

MMMRSV

MMMRSV

MMMRSV

MMMRSV

MMMRSV

MMMRSV

MMMRSV

MMMRSV

MMMRSV

MMMRSV

MMMRSV

MMMRSV

MMMRSV

MMMRSV

MMMRSV

MMMRSV

MMMRSV

MMMRSV

.)

25

END RETURN

CALL INP(MO, KN, MINZER,

$K A O, 2,0,0,0,0,0,0$ 
C IF $M$ IS NEGATIVE, RETURN/NAME FOR INVERSE

MNAME

BLANK

LEVEL 2, STORE, INFF, IWORK

BLANK COMMON STORE (48000), IWORK(10)

DIMENSION INFF $(24,2000)$

EQUIVALENCE (INFF, STORE)

86MARSIZ

86MARSIZ

86MARSIZ

BLANK

10

COMMON/FLTN/IFL(15)

MNAME $=\operatorname{INFF}(2, \operatorname{IABS}(M))$

FLTN

FLTN

IF $(M . G T, 0)$ RETURN

15

$M N=$ SHIFT $(M N A M E,-6)$. AND .0077777777777777777777B

MNAME

MNAME

MNAME $=($ IFL $(15) \cdot$ OR.MN $)$

MNAME

MÑAME

MNAME

MNAME

RETURN 
1

5

10

15

20

25

30

35

40

45

50

55
SUBROUTINE MODQ $(M, N, V, W)$

C

COMMON/DIM/LEND, MEND, ISAV, ISAV7, M7END, KADD , KADDR, MUNIT , MSYMPL COMMON /MJ/ML \$ LEVEL 2, ML

DIMENSION RX(2,3), RY(2,3),R(2,3), RYW(3), RPQ(4), RW(3)

DIMENSION $V(1), W(7), \operatorname{PAR}(6), T(7,7), D(7,7), M L(3)$

EQUIVALENCE (BR, PAR(3))

DATA RADEG/.0174532925/

c

CALL DATA $(M, 1,1,6$, PAR $)$

$\mathrm{BR}=\operatorname{PAR}(3) *(1 .+\mathrm{V}(6))$

CALL MAGNET (N,PAR)

CALL RTRV7 (N,T,RW)

CALL $\operatorname{MXV7}(T, V, W)$

$S=\operatorname{PAR}(1)$

RHOIX $=(V(2)-W(2)) / S$

RHOIY $=(V(4)-W(4)) / S$

$M L(1)=M 7 E N D-8$

$M L(2)=N$

$M L(3)=M 7 E N D-9$

CALL RTRV7 (MUNIT,D, RW)

$D(2,1)=-V(4) *$ RHOIY

$D(4,3)=-V(2) *$ RHOIX

CALL STOR7 (M7END-8,D, RW)

$D(2,1)=W(4) *$ RHOIY

$D(4,3)=W(2) *$ RHOIX

CALL STOR7 (M7END-9,D,RW)

CALL RXY(N,RX,RY,RW)

IF (RHOIX.EQ.0.) GO TO 3

C

$\operatorname{SGN}=\operatorname{SIGN}(1,, \mathrm{RHOIX}) / \mathrm{RADEG}$

$\operatorname{PAR}(1)=-\operatorname{PAR}(1)$

$\operatorname{PAR}(4)=B R * R H O I X$

$\operatorname{PAR}(5)=S G N * V(2)$

$\operatorname{PAR}(6)=-S G N * W(2)$

CALL MAGNET(N,PAR)

CALL RXY (N,RX,R,RW)

3 IF (RHOIY.EQ.O.) GO TO 4

C

$\operatorname{SGN}=\operatorname{SIGN}(1$, RHOIY $) /$ RADEG

$\operatorname{PAR}(1)=-\operatorname{PAR}(1)$

$\operatorname{PAR}(2)=-\operatorname{PAR}(2)$

$\operatorname{PAR}(4)=\mathrm{BR} * \mathrm{RHOIY}$

$\operatorname{PAR}(5)=S G N * V(4)$

$\operatorname{PAR}(6)=-S G N * W(4)$

CALL MAGNET(N,PAR)

CALL RXY(N,RY,R,RYW)

$R W(3)=R W(3)+R Y W(3)$

C

4 CALL CONVMAT (7, T,RX,RY,RW, RPQ)

CALL STOR7 (N, T, RW)

CALL MMM(N, 3,ML)

RETURN

END
MODQ

MODQ

MODQ

MODQLL

MODQ

MODQ

MODQ

MODQ

MODQ

MODQ

MODQ

$M O D Q$

MODQ

MODQ

MODQ

MODQ

MODQ

MODQ

MODQ

MODQ

MODQ

MODQ

MODQ

MODQLL

MODQ

MODQ

MODQLL

MODQLL

MODQ

MODQ

MODQ

MODQ

MODQ

MODQ

MODQ

MODQ

MODQ

MODQ

MODQ

MODQ

MODQ

MODQ

MODQ

MODQ

MODQ

MODQ

MODQ

MODQ

MODQ

MODQ

MODQ

MODQ

MODQ

MODQ

MODQ
2

3

4

1

5

6

7

8

9

10

11

12

13

14

15

16

17

18

19

20

21

22

23

2

25

26

3

4

28

29

30

31

32 
CARD NR. SEVERITY DETAILS

$\begin{array}{lll}11 & \text { I } & \text { V } \\ 16 & \text { I } & \text { V } \\ 17 & \text { I } & \text { V } \\ 22 & \text { I } & \text { V } \\ 23 & \text { I } & \text { V } \\ 35 & \text { I } & \text { V } \\ 45 & \text { I } & \text { V }\end{array}$

DIAGNOSIS OF PROBLEM

ARRAY REFERENCE OUTSIDE DIMENSION BOUNDS. ARRAY REFERENCE OUTSIDE DIMENSION BOUNDS. ARRAY REFERENCE OUTSIDE DIMENSION BOUNDS. ARRAY REFERENCE OUTSIDE DIMENSION BOUNDS. ARRAY REFERENCE OUTSIDE DIMENSION BOUNDS. ARRAY REFERENCE OUTSIDE DIMENSION BOUNDS. ARRAY REFERENCE OUTSIDE DIMENSION BOUNDS. 
MOVE

LEVEL 2, STORE, INFF, IWORK

BLANK

BLANK COMMON STORE (48000), IWORK(10)

$\mathrm{C}$

COMMON/MOVTOG/TOG LOGICAL TOG DIMENSION PARR(6) DIMENSION PP( 6$)$

$$
\text { REAL L,K }
$$$$
\text { INTEGER BDAT }
$$

DIMENSION $T(7,7), R W(3), R W 1(3), \operatorname{PAR}(10), M L(7), V(7)$ COMMON /MK /ML \$ LEVEL 2,ML

\section{MOVE}

MOVE 
C MQ IS THE INDEX OF THE ELEMENT TO BE MOVED.

MOVE

C MPAR IS THE INDEX OF THE VEC COMMAND THAT CONTAINS THE MISALIGNMENTS. MOVE

C KB GE. 1 - PARAMETERS RANDOM

C KB GE. 2 - RANDOM SEED RESET SO NEXT PÁSS IS SAME

MOVE

MOVE

MOVE

$M Q=\operatorname{MDAT}(M, 1)$

$K B=\operatorname{INFF}(5, M)$

65

IF (KB.LT.2) GO TO 10

IF (TOG) CALL RANGET(NRN)

10

QDSW $=$.FALSE.

$\operatorname{KIND}=\operatorname{INFF}(20, M)$

IF (KIND.EQ.HMAP) GO TO 3

70

$N O P Q=\operatorname{INFF}(1, M Q)$

$S X S W=$.FALSE.

IF (NOPQ.NE.HMAG) GO TO 12

CALL DATA (MQ, 1, 1,6,PAR)

75

IF. (PAR(4).EQ.0.) QDSW = .TRUE.

$L=P A R(1)$

$K=\operatorname{PAR}(2) / \operatorname{PAR}(3) /(1 .+\mathrm{V}(6))$

GO TO 11

80

3

SXSW $=$.TRUE.

MOVE

MOVE

MY3MOVE

MYZMOVE

MY3MOVE

MOVE

MOVE

MOVE

MOVE

MOVE

MOVE

MOVE

MOVE

MOVE

MOVE

MOVE

MOVE

CALL DATA (MQ, 1, 1, 4,PAT)

$\mathrm{L}=\operatorname{PAT}(1)$

GO TO 11

85

12

CALL RTRV7 (MQ, T, RW)

$\mathrm{L}=\mathrm{RW}(1)$

$K=0$.

11 IF (M.NE.N) GO TO 7

$\operatorname{NAVEC}=\operatorname{BDAT}(M, 2)$

IF (NAVEC.EQ.4H ) GO TO 7

MOVE

MOVE

MOVE

MOVE

MOVE

MOVE

MOVE

MOVE

$\operatorname{MVEC}=\operatorname{MDAT}(M, 2)$

90

CALL DATA (MVEC $, 5,1,7, V$ )

MOVE

MOVE

MOVE

$\operatorname{NAPAR}=\operatorname{BDAT}(M, 3)$

MPAR $=M$

$H Z=0$.

THETZ $=0$.

95

100

NVARS $=4$

IF (NAPAR.EQ.4H ) GO TO 1

MOVE

MOVE

MOVE

MOVE

MOVE

MOVE

NVARS $=6$

MOVE

$\operatorname{MPAR}=\operatorname{MDAT}(M, 3)$

1 CALL DATA(MPAR, 1,1 , NVARS, PARR)

MOVE

MOVE

D0111 I=1, NVARS

MOVE

$\operatorname{PAR}(I)=P A R R(I)$

$\operatorname{IF}(K B . G E .1) \operatorname{PAR}(I)=\operatorname{PAR}(I) *(\operatorname{RANF}(D)-.5)$

MOVE

MOVE

MOVE

$T L X=L * D H X / 2$.

MOVE

$H X 1=H X-T L X$

MOVE

$H X 2=H X+T L X$

MOVE

$T L Y=L * D H Y / 2$.

MOVE

$H Y 1=H Y-T L Y$

MOVE

$H Y 2=H Y+T L Y$

MOVE

MOVE

MOVE

$M L(1)=M 7 E N D-1$

$M L(2)=M 7 E N D-2$

MOVE

$M L(3)=M 7 E N D-6$

MOVE

MOVE 
115

120

125

130

135

140

145

150

160

165

170

155
CALL RTRV7 (MUNIT, T, RW)

$T(1,7)=-H \times 1$

$T(2,7)=-D H X$

$T(3,7)=-H Y 1$

$T(4,7)=-D H Y$

CALL STOR7 (M7END-2,T,RW1)

IF (HZ.EQ.Ө. .AND.THETZ.EQ.๑.) GO TO 201

CALL DRIFT (M7END-1,HZ)

CALL ROTZ(THETZ,T)

CALL STOR7(M7END-6, T,RW1)

CALL MMM(M7END-2,3,ML)

201 CONTINUE

CALL RTRV7(M7END-2,T,RW)

CALL MXV7 $(T, V, V)$

IF (.NOT.QDSW) GO TO 20

CALL MODQ (MQ,N,V,W)

GO TO 8

20 IF (.NOT.SXSW) GO TO 21

CALL SXTP(N,V,PAT)

GO TO 14

21 CALL DATA(MQ, $1,1,6, \mathrm{PP})$

$\mathrm{PP}(3)=\mathrm{PP}(3) *(1 .+\mathrm{V}(6))$

$P P(4)=P P(4)+P P(2) *(V(1)+V(2) * L / 2$.

CALL MAGNET (N,PP)

CALL RTRV7 $(N, T, R W)$

CALL $M X V 7(T, V, W)$

C

$8 \quad X I N=V(1)$

$Y I N=V(3)$

XOUT $=W(1)$

YOUT $=W(3)$

DO 112 I=1, NVARS

$112 \operatorname{PAR}(I)=\operatorname{PARR}(\mathrm{I})$

CALL STDAT $(M, 1,7,4$, PAR $)$

DO $9 \mathrm{I}=1,7$

$9 \quad V(I)=W(I)$

14 CALL RTRV7 (MUNIT, T, RW)

$T(1,7)=-H \times 2$

$T(2,7)=-D H X$

$T(3,7)=-H Y 2$

$T(4,7)=-D H Y$

CALL STOR7 (M7END-7, T, RW)

$M L(1)=-(M 7 E N D-6)$

$M L(2)=-(M 7 E N D-7)$

$M L(3)=-(M 7 E N D-1)$

IF (HZ.EQ.0. .AND.THETZ.EQ.0.) $M L(1)=M L(3)=$ MUNIT

CALL MMM(M7END-7,3,ML)

$M L(1)=M 7 E N D-2$

$M L(2)=N$

$M L(3)=$ M7END -7
MOVE $\quad 98$

MOVE 99

MOVE $\quad 100$

MOVE $\quad 101$

MOVE $\quad 102$

MOVE 103

MOVELL 3

MOVE 105

MOV883 2

MOV883 3

MOVE $\quad 106$

MOVELL 4

MOVE $\quad 108$

MOVE $\quad 109$

MOVE 110

MOV883 4

MOVE $\quad 111$

MOVE 112

MOVE 113

MOVE 114

MOVE $\quad 115$

MOVE $\quad 116$

MOVE $\quad 117$

MOVE 118

MOV883 5

MOV883 6

MOV883 7

MOV883 8

MOV883 9

MOVE $\quad 120$

MOVE 121

MOVE 122

MOVE 123

MOVE 124

MOVE $\quad 125$

MOVE 126

MOVE $\quad 127$

MOVC 1

MOVE $\quad 129$

MOVE $\quad 130$

MOVE 131

MOVE $\quad 132$

MOVE 133

MOVE $\quad 134$

MOVE 135

MOVE $\quad 136$

MOVE 137

MOVE 138

MOVE $\quad 139$

MOVE $\quad 140$

MOVE $\quad 141$

MOV883 10

MOVE 142

MOVE 143

MOVE $\quad 144$

MOVE $\quad 145$

MOVE 146 
CALL RTRV7(M7END-7, T, RW)

$\begin{array}{lr}\text { MOVE } & 147 \\ \text { MOVELL } & 5 \\ \text { MOVELL } & 6 \\ \text { MOVE } & 149 \\ \text { MOVE } & 150 \\ \text { MOVE } & 151 \\ \text { MOVE } & 152 \\ \text { MOVE } & 153 \\ \text { MOVE } & 154 \\ \text { MOVE } & 155 \\ \text { MOVE } & 156 \\ \text { MY3MOVE } & 17 \\ \text { MY3MOVE } & 18 \\ \text { MY3MOVE } & 19 \\ \text { MY3MOVE } & 20 \\ \text { MY3MOVE } & 21 \\ \text { MY3MOVE } & 22 \\ \text { MOVE } & 158 \\ \text { MOVE } & 159\end{array}$

\begin{tabular}{|c|c|c|}
\hline 175 & & $\operatorname{CALL} \operatorname{MXV7}(T, V, V)$ \\
\hline & & IF (N.EQ.0) GO TO 113 \\
\hline & & $\operatorname{CALL} \operatorname{MMM}(N, 3, M L)$ \\
\hline & & IF (M.NE.N) GO TO 113 \\
\hline & & CALL RTRV7(N,T,RW) \\
\hline 180 & & CALL ROW5(T) \\
\hline & & CALL STOR7 (N, T, RW) \\
\hline & 113 & IF (KB.LT.2) RETURN \\
\hline & & IF (TOG) GO TO 115 \\
\hline 185 & & TOG $=$. TRUE \\
\hline & & RETURN \\
\hline & 115 & CALL RANSET(NRN) \\
\hline & & TOG $=$. FALSE \\
\hline & & RETURN \\
\hline 190 & & END \\
\hline
\end{tabular}


1 SUBROUTINE MPCONJ $(A, B, C, D C)$

RETURN

END 
COMMON/SWTCH/BEND, MSIZE, CYCSWT, VCSW, PV, MSSW, VPR, MHS, MODS, MINZER LOGICAL CYCSWT, MSSW, VCSW, PV, VPR, MHS, MODS

DIMENSION RX(2,3), RY $(2,3)$

DO $5 \mathrm{I}=1,3$

$5 \quad \mathrm{RW}(\mathrm{I})=0$.

IF(KD.EQ.3) GO TO 100

\section{*}

100

103

MULTIPLY MATRICES AS $7 \times 7$, THEN STORE ACCORDING TO KIND

CALL RTRV7(M1,T1, RW1)

CALL RTRV7 (M2,T2, RW2)

CALL MXM7 (T2,T1,T)

$\mathrm{RW}(1)=\mathrm{RW} 1$ (1) $+\mathrm{RW} 2(1)$

$R W(2)=R W 1(2)+R W 2(2)$

$\mathrm{RW}(3)=\mathrm{T}(40)$

IF (KD.EQ.3) GO TO 210

CALL $\operatorname{STOR7}(M, T, R W)$

RETURN

210 CALL CONVMAT (KD, T, RX, RY, RW, RPQ)

CALL STXY $(M, R X, R Y, R W)$

$\operatorname{CALL} \operatorname{STDAT}(M, 5,4,4, \mathrm{RPQ})$

RETURN

CONT INUE

CALL RXY (M1 , RX1,RY1,RW1)

CALL RXY (M2, RX2, RY2, RW2)

DO $102 \quad I=1,2$

DO $101 \mathrm{~J}=1,2$

$R X(I, J)=R \times 2(I, 1) * R \times 1(1, J)+R \times 2(I, 2) * R X 1(2, J)$

$\operatorname{RY}(I, J)=R Y 2(I, 1) * R Y 1(1, J)+R Y 2(I, 2) * R Y 1(2, J)$

$R X(I, 3)=R \times 2(I, 1) * R \times 1(1,3)+R \times 2(I, 2) * R \times 1(2,3)+R \times 2(I, 3)$

$\operatorname{RY}(I, 3)=\operatorname{RY} 2(I, 1) * R Y 1(1,3)+R Y 2(I, 2) * R Y 1(2,3)+R Y 2(I, 3)$

$102 R W(I)=R W 1(I)+R W 2$ (I)

DO $103 \quad \mathrm{I}=1,2$

$\mathrm{RPQ}(\mathrm{I})=\mathrm{RX}(1,3) * \mathrm{RX}(2, \mathrm{I})-\mathrm{RX}(2,3) * \mathrm{RX}(1, \mathrm{I})$

$R P Q(I+2)=R Y(1,3) * R Y(2, I)-R Y(2,3) * R Y(1, I)$

$\mathrm{RW}(3)=\mathrm{RW} 1(3)+\mathrm{RW} 2(3)-\mathrm{RX} 1(1,3) *(\mathrm{RX} 2(2,3) * \mathrm{RX} 2(1,1)-\mathrm{RX} 2(1,3) * \mathrm{R} \times 2(2,1)) \quad \mathrm{MXD}$

1

2

3

CALL STXY $(M, R X, R Y, R W)$ $-R Y 1(1,3) *(R Y 2(2,3) * R Y 2(1,1)-R Y 2(1,3) * R Y 2(2,1))$ MXD $-R Y 1(2,3) *(R Y 2(2,3) * R Y 2(1,2)-R Y 2(1,3) * R Y 2(2,2))$ MXD 45 $-R \times 1(2,3) *(R \times 2(2,3) * R \times 2(1,2)-R \times 2(1,3) * R \times 2(2,2))$ MXD 46

CALL STDAT $(M, 5,4,4, R P Q)$

RETURN

END

$M X D$

MXD

$M X D$

MXD

2

MXD

MXD

MXD

SWTCH

SWTCH

SWTCH

MXD

MXD

MXD

MXD

$M X D$

$M X D$

MXD

MXD

MXD

MXD

MXD

MXD

MXD

MXD

MXD

$M \times D$

MXD

$M \times D$

MXD

MXD

MXD

MXD

MXD

MXD

MXD

MXD

$M \times D$

MXD

MXD

MXD

MXD

MXD

MXD

MXD 
5

10

15

20

25

30

35

40

45

50

55
SUBROUTINE MXDIV(V,IN, IM,DET, IDIM)

MATRIX DIVISION

C REPLACES B BY (A INVERSE)*B. DESTROYS A:

C A IS $N$ BY $N$, B IS $N$ BY $M$. A AND B ARE STORED IN $V(N, N+M)$.

C N MUST NOT EXCEED 4 .

INTEGER PERM, $P$

DIMENSION PERM(4)

DIMENSION V(IDIM, 1)

$N=I N$

C

$M=\operatorname{MAX} \theta(I M, \theta)$

$\mathrm{NM} 1=\mathrm{N}-1$

$\mathrm{NP} 1=\mathrm{N}+1$

$N P M=N+M$

200 DÓ $201 \mathrm{I}=1, N$

$\operatorname{PERM}(I)=I$

201 CONTINUE

DETERM $=1.0$

$P=1$

202 CONTINUE

IMIN $=P+1$

C

$\mathrm{PIVOT}=0$.

FIND PIVOT

DO 209 I=P,N

DO $208 \mathrm{~J}=\mathrm{P}, \mathrm{N}$

$S A V E=\operatorname{ABS}(V(I, J))$

IF (SAVE . LE. PIVOT) GO TO 208

$P I V O T=S A V E$

$I B I G=I$

$J B I G=J$

208 CONTINUE

209 CONTINUE

IF (PIVOT .GT. 0.0 ) GO TO 210

DETERM $=0 . \theta$

GO TO 290

C

210 PIVOT $=V($ IBIG, JBIG)

DETERM=DETERM $* P I$ VOT

DO $219 \mathrm{~J}=\mathrm{P}, \mathrm{NPM}$

SAVE $=V($ IBIG , J $) / P I V O T$

$V(I B I G, J)=V(P, J)$

$V(P, J)=S A V E$

219 CONTINUE

IF (IBIG .NE. P) DETERM=-DETERM

220 CONTINUE

IF (P .GE. N) GO TO 250

C

COLUMN EXCHANGE

IF (JBIG .EQ. P) GO TO 230

ISAVE=PERM $(P)$

$\operatorname{PERM}(P)=P E R M(J B I G)$

PERM $(J B I G)=I S A V E$

DO $229 I=1, N$

SAVE $=V(I, P)$

$V(I, P)=V(I, J B I G)$

$V(I, J B I G)=S A V E$

229 CONTINUE

DETERM=-DETERM
MXDIV 2

MXDIV 3

MXDIV 4

MXDIV 5

MXDIV 6

MXDIV 7

MXDIV 8

MXDIV 9

MXDIV 10

MXDIV 11

MXDIV 12

MXDIV 13

MXDIV 14

MXDIV 15

MXDIV $\quad 16$

MXDIV 17

MXDIV $\quad 18$

MXDIV $\quad 19$

MXDIV 20

MXDIV 21

MXDIV 22

MXDIV 23

MXDIV 24

MXDIV 25

MXDIV 26

MXDIV 27

MXDIV 28

MXDIV 29

MXDIV 30

MXDIV 31

MXDIV 32

MXDIV 33

MXDIV 34

MXDIV $\quad 35$

MXDIV $\quad 36$

MXDIV $\quad 37$

MXDIV $\quad 38$

MXDIV $\quad 39$

MXDIV 40

MXDIV $\quad 41$

MXDIV $\quad 42$

MXDIV 43

MXDIV 44

MXDIV $\quad 45$

MXDIV $\quad 46$

MXDIV $\quad 47$

MXDIV $\quad 48$

MXDIV $\quad 49$

MXDIV $\quad 50$

MXDIV $\quad 51$

MXDIV $\quad 52$

MXDIV 53

MXDIV $\quad 54$

MXDIV 55

MXDIV $\quad 56$

MXDIV $\quad 57$

MXDIV $\quad 58$ 
60

65

70

75

80

85

90
230 CONT INUE

$$
V(P, P)=1.0
$$

C

DO $239 I=I M I N, N$

DO $238 \mathrm{~J}=I M I N, N P M$

$V(I, J)=V(I, J)-V(I, P) * V(P, J)$

238 CONTINUE

$V(I, P)=0.0$

239 CONTINUE

$$
\mathrm{P}=\mathrm{P}+1
$$

249 GO TO 202

C

250 CONTINUE

BACK SUBSTITUTION

BLOCK REDUCTION

MXDIV

MXDIV

MXDIV

MXDIV

MXDIV

MXDIV

MXDIV

MXDIV

MXDIV

MXDIV

MXDIV

MXDIV

MXDIV

MXDIV

MXDIV

MXDIV

MXDIV

MXDIV

MXDIV

MXDIV

MXDIV

MXDIV

MXDIV

MXDIV

MXDIV

MXDIV

MXDIV

MXDIV

MXDIV

MXDIV

MXDIV

MXDIV

MXDIV

59

60

61

62

63

64

65

66

67

68

69

70

71

72

73

74

75

76

77

78

79

80

81

82

83

84

85

86

87

88

89

DET $=$ DETERM

RETURN

END 


$\begin{array}{lr}\text { MXM6 } & 2 \\ \text { MXM6 } & 3 \\ \text { MXM6 } & 4 \\ \text { MXM6 } & 5 \\ \text { MXM6 } & 6 \\ \text { MXM6 } & 7 \\ \text { MXM6 } & 8 \\ \text { MXM6 } & 9 \\ \text { MXM6 } & 10 \\ \text { MXM6 } & 11 \\ \text { MXM6 } & 12 \\ \text { MXM6 } & 13 \\ \text { MXM6 } & 14 \\ \text { MXM6 } & 15 \\ \text { MXM6 } & 16 \\ \text { MXM6 } & 17 \\ \text { MXM6 } & 18\end{array}$

C MULTIPLES $6 \times 6$ MATRICES

5

DIMENSION $A(6,1), B(6,1), C(6,1), C C(6,6)$

DO $100 \mathrm{I}=1,6$

DO $90 \mathrm{~J}=1,6$

SUM $=0$.

DO 80 II $=1,6$

10

$80 S \mathrm{SUM}=\mathrm{SUM}+\mathrm{A}(\mathrm{I}, \mathrm{II}) * B(\mathrm{II}, \mathrm{J})$

$90 \operatorname{CC}(\mathrm{I}, \mathrm{J})=\mathrm{SUM}$

100 CONTINUE

DO $115 \quad I=1,6$

DO $115 \mathrm{~J}=1,6$

15

$C(I, J)=C C(I, J)$

115 CONTINUE

RETURN

END 
1

SUBROUTINE MXM7 $(A, B, C)$

MXM7

MXM7

MXM7

MXM7

MXM7

MXM7

MXM7

$M X M 7$

MXM7

MXM7

MXM7

MXM7

MXM7

MXM7

MXM7

MXM7

MXM7

2

DO $100 \quad 1=1,7$

SUM $=0$.

$80 \mathrm{SUM}=\mathrm{SUM}+\mathrm{A}(\mathrm{I}, \mathrm{II}) * \mathrm{~B}(\mathrm{II}, \mathrm{J})$

15

115 CONTINUE

RETURN

END 
1 SUBROUTINE MXVG(T,V,VN)

MXVG

MXV6

C MULTIPLES A $6 \times 6$ MATRIX BY A 6 COMPONENT VECTOR

MXV6

DIMENSION $T(6,6), V(6), V N(6), V V(6)$

5

DO $10 \mathrm{I}=1,6$

MXV6

MXV6

$\mathrm{VV}(\mathrm{I})=0$.

DO $10 \mathrm{~J}=1,6$

MXVE

MXV6

$V V(I)=V V(I)+T(I, J) * V(J)$

10

CONTINUE

10

DO $15 \mathrm{~J}=1,6$

MXV6

MXV6

MXV6

MXV6

MXV6

RETURN

END

MXV6 
C MULTIPLIES MATRIX BY A VECTOR

MXV7

DIMENSION $T(7,7), V(7), V N(7), V V(7)$

MXV7

DO $10 \quad I=1,7$

$V V(I)=0$.

DO $10 \mathrm{~J}=1,7$

$M \times V 7$

MXV7

$M \times V 7$

$V V(I)=V V(I)+T(I, J) * V(J)$

MXV7

MXV7

MXV7

DO $15 \mathrm{~J}=1,7$

MXV7

MXV7

MXV7 
1

5

10

20

25

30

15
SUBROUTINE NAMCHK(M)

C LOOKS FOR IDENTICAL NAME PREVIOUSLY DEFINED.

C BLANKS OR NO NAMES ARE NOT CONSIDERED IDENTICAL.

C INFO OF PREVIOUSLY DEFINED ELEMENTS IS REPLACED BY THAT OF THE

C LAST DEFINED ONE, EXCEPT FOR INFO $(3, \mathrm{I})$, WHICH CONTAINS NXTM

LEVEL 2, STORE, INFF, IWORK

COMMON STORE(48000), IWORK(10)

DIMENSION INFF $(24,2000)$

EQUIVALENCE (INFF,STORE)

COMMON/STORE/LMAX, LINF, LFILE, MAX, MIN, ICARD (11)

* LMAX AND MAX ARE DIMENSIONS OF STORE AND INFF

c

$$
\text { DIMENSION INF(24) }
$$

$\operatorname{NAME}=\operatorname{INFF}(2, M)$

$M 1=M I N+1$

DO $120 \mathrm{~J}=\mathrm{M} 1, \mathrm{MAX}$

$\operatorname{INAM}=\operatorname{INFF}(2, \mathrm{~J})$

IF (INAM.EQ.NAME.AND.NAME.NE.5H ) GO TO 105

GO TO 120

C DUPLICATE NAME, NOT BLANK HAS BEEN FOUND

C REPLACE INFO $(I, J)$ WITH THAT OF INFO $(I, M)$, EXCEPT FOR INFO $(3, J)$ WHICH

C CONTAINS NXTM AND TRA

105 CALL RDINF (INF,M)

$\operatorname{INF}(8)=1 \mathrm{H} *$

$\operatorname{INF}(9)=1 \mathrm{H} *$

CALL WRTINF (INF,J)

RETURN

120 CONTINUE

RETURN

END

$\begin{array}{lr}\text { NAMCHK } & 2 \\ \text { NAMCHK } & 3 \\ \text { NAMCHK } & 4 \\ \text { NAMCHK } & 5 \\ \text { NAMCHK } & 6 \\ \text { BLANK } & 2 \\ \text { BLANK } & 3 \\ \text { 86MARSIZ } & 1 \\ \text { 86MARSIZ } & 2 \\ \text { 86MARSIZ } & 3 \\ \text { BLANK } & 5 \\ \text { STORE } & 2 \\ \text { STORE } & 3 \\ \text { STORE } & 4 \\ \text { NAMCHK } & 9 \\ \text { NAMCHK } & 10 \\ \text { NAMCHK } & 11 \\ \text { NAMCHK } & 12 \\ \text { NAMCHK } & 13 . \\ \text { NAMCHK } & 14 \\ \text { NAMCHK } & 15 \\ \text { NAMCHK } & 16 \\ \text { NAMCHK } & 17 \\ \text { NAMCHK } & 18 \\ \text { NAMCHK } & 19 \\ \text { NAMCHK } & 20 \\ \text { NAMCHK } & 21 \\ \text { NAMCHK } & 22 \\ \text { NAMCHK } & 23 \\ \text { NAMCHK } & 24 \\ \text { NAMCHK } & 25 \\ \text { NAMCHK } & 26 \\ \text { NAMCHK } & 27\end{array}$


C $N=$ ORDER OF PERTURBATION IN HAMILTONIAN, $M=N-1$

5 C $J=0,1$ FOR NORMAL OR SKEW N-POLE LENS

C $\mathrm{L}=$ EEFFECTIVE LENGTH

NPOM

NPOM

NPOM

NPOM

NPOM

C $\quad C M=$ TAYLOR EXPANSION COEFFICIENT OF MEDIAN-PLANE EXPRESSION OF FIEL C

C $J=0: B Y+I * B X=\operatorname{SUM}(C M / M !) Z * * M \quad(Z=X+I * Y)$

C $J=1: B X-I * B Y=\operatorname{SUM}(C M / M !) Z * * M$

C PARTICLE $V(I)=X, P X, Y, P Y, O, O, D P P$

C $\quad \mathrm{N}=2$ QUAD, $\mathrm{N}=3$ SEXT, $\quad \mathrm{N}=4$ OCT, $\mathrm{N}=5, \mathrm{DEC}$

C

COMMON /ML/ ML $\$$ LEVEL 2, ML

LEVEL 2, STORE, INFF, IWORK

COMMON STORE(48000), IWORK(10)

DIMENSION INFF $(24,2000)$

EQUIVALENCE (INFF, STORE)

COMMON /DIM/ LEND, MEND, ISAV, ISAV7, M7END, KADD, KADDR, MUNIT, MSYMPL

DIMENSION $V(1), \operatorname{PAR}(1), R(7,7), R W(3), M L(3), \operatorname{PR}(3), W(6)$

REAL L, LH

EQUIVALENCE $(P R(1), L),(P R(2), C M),(P R(3), B R H O),(W(1), X)$,

$1 \quad(W(2), P X),(W(3), Y),(W(4), P Y),(W(6), D P P)$

30

COMPLEX I I , $Z, D P, Q$

C

DATA II $/(0 ., 1$.

35

40

$N N=M$

DO $5 \mathrm{I}=1,3$

$5 \quad \operatorname{PR}(\mathrm{I})=\operatorname{PAR}(\mathrm{I})$

DO $6 \quad I=1,6$
$W(I)=V(I)$

$\mathrm{BR}=(1 .+\mathrm{DPP}) * \mathrm{BRHO}$

$U M=C M / B R$

IF (L.EQ.O.) GO TO 7

$\mathrm{LH}=\mathrm{L} / 2$.

$X=X+P X * L H$

$Y=Y+P Y * L H$

$U M=L * U M$

$7 \quad N P=\operatorname{INFF}(4, N N)$

$J P=\operatorname{INFF}(5, N N)$

$M P=N P-1$

$M P M=N P-2$

$Z=X+I I * Y$

CALL RTRV7(MUNIT, R, RW)

50

c

55

NPOM 8

NPOL JUNB3

NPOLJUN83 3

NPOM 12

NPOM 13

NPOM 14

NPOLLL 1

NPOLLL 2

BLANK 2

BLANK 3

86MARSIZ 1

86MARSIZ 2

86MARSIZ 3

BLANK 5

DIM 2

DIM 3

NPOM 18

NPOM 19

NPOM 20

NPOM 21

NPOM 22

NPOL682 1

NPOL682 2

NPOM 23

NPOLJUN83 4

NPOM 24

NPOM 25

NPOM 26

NPOM 27

NPOM 28

NPOM 29

NPOM 30

NPOM 31

NPOM 32

NPOM 33

NPOLJUN83 5

NPOM $\quad 35$

NPOM 36

NPOM 37

NPOL682 3

NPOL682 4

NPOM 38

NPOM $\quad 39$

NPOL682 5

NPOL682 6

NPOL682 7

NPOL682 8

NPOL682 9

NPOL682 10

NPOL682 11 
60

65

70

75

80

C

C

$40 \quad R(4,3)=-R(2,1)$

$R(4,1)=R(2,3)$

$V(2)=P X+D P X$

$V(4)=P Y+D P Y$

IF (L.EQ.0) GO TO 41

$V(1)=X+V(2) * L H$

$V(3)=Y+V(4) * L H$

$41 \quad R(2,6)=-D P X$

$R(2,7)=-D P X$

$R(4,6)=-D P Y$

$R(4,7)=-D P Y$

$R(5,1)=D P X$

$R(5,3)=D P Y$

$R W(2)=-D P X$

IF (L.EQ.0) GO TO 45

$M L(1)=M E N D-3$

$M L(2)=M$

$M L(3)=M E N D-3$

CALL DRIFT(MEND-3, LH)

CALL STOR7 $(M, R, R W)$

$\operatorname{CALL} \operatorname{MMM}(M, 3, M L)$

RETURN

85

CALL STOR7 ( $M, R, R W)$

RETURN

END

$\begin{array}{lr}\text { NPOLJUN83 } & 6 \\ \text { NPOL682 } & 13 \\ \text { NPOL682 } & 14 \\ \text { NPOM } & 86 \\ \text { NPOM } & 87 \\ \text { NPOM } & 88 \\ \text { NPOM } & 89 \\ \text { NPOM } & 90 \\ \text { NPOM } & 91 \\ \text { NPOM } & 92 \\ \text { NPOLJUN83 } & 7 \\ \text { NPOM } & 94 \\ \text { NPOM } & 95 \\ \text { NPOM } & 96 \\ \text { NPOM } & 97 \\ \text { NPOM } & 98 \\ \text { NPOM } & 99 \\ \text { NPOM } & 100 \\ \text { NPOLJUN83 } & 8 \\ \text { NPOM } & 101 \\ \text { NPOM } & 102 \\ \text { NPOM } & 103 \\ \text { NPOM } & 104 \\ \text { NPOM } & 105 \\ \text { NPOM } & 106 \\ \text { NPOM } & 107 \\ \text { NPOM } & 108 \\ \text { NPOM } & 109 \\ \text { NPOM } & 110 \\ \text { NPOM } & 111 \\ & \end{array}$

CARD NR. SEVERITY DETAILS DIAGNOSIS OF PROBLEM

$\begin{array}{lll}64 & \text { I } & \text { V } \\ 65 & \text { I } & \text { V } \\ 67 & \text { I } & \text { V } \\ 68 & \text { I } & \text { V } \\ 68 & \text { I } & \text { V }\end{array}$

ARRAY REFERENCE OUTSIDE DIMENSION BOUNDS. ARRAY REFERENCE OUTSIDE DIMENSION BOUNDS. ARRAY REFERENCE OUTSIDE DIMENSION BOUNDS. ARRAY REFERENCE OUTSIDE DIMENSION BOUNDS. ARRAY REFERENCE OUTSIDE DIMENSION BOUNDS. 
* checks opName against list of acceptable names

* assigns an op number to opname.

OPNUM 3

OPNUM 4

OPNUM 5

OPNUM 6

COMMON/CONTRL/ERROR, MODE, RSRV, STOR, XEQ, TRASW, NFSW, EMPTY, INDEF, 1 LDFLG, FIN

LOGICAL ERROR, RSRV, STOR, XEQ, TRASW, NFSW, EMPTY, INDEF , LDFLG, FIN

COMMON/GRR/IERR, BSW, VSW, RSW, QSW, XSW

LOGICAL IERR, BSW, VSW, RSW, QSW, XSW

COMMON/OPLIST/LIST(200)

15

INTEGER OPNAME

$\operatorname{DECODE}(10,8$, OPNAME) KOP

8 FORMAT $(A 3,7 X)$

* if OPNAME IS ILlegal, OP IS SET TO $\theta$ AND ERROR SWITCH IS TURNED ON. OPNUM $=0$

CONTRL $\quad 2$

CONTRL $\quad 3$

CONTRL $\quad 4$

CONTRL $\quad 5$

GRR $\quad 2$

GRR $\quad 3$

GRR $\quad 4$

OPLIST 2

OPLIST 3

OPNUM 10

OPNUM 11

OPNUM 12

OPNUM 13

OPNUM 14

OPNUM 15

OPNUM 16

OPNUM 17

OPNUM 18

OPNUM $\quad 19$

OPNUM 20

OPNUM 21

OPNUM 22

OPNUM 23

OPNUM 24

OPNUM 25

OPNUM 26

OPNUM 27

OPNUM 28

OPNUM 29

OPNUM 30

OPNUM 31

OPNMPL 1

OPNMPL 2

OPNMPL 3

OPNUM 32

OPNUM 33

110 OPNUM $=69$

RETURN

OPNUM 34

END

OPNUM 35


SUBROUTINE ORBIT(MM)

ORBIT PROGRAM USES "MICADO" TO CALCULATE OPTIMIZED CORRECTORS FOR GIVEN ORBIT WITH FIELD ERRORS.

FORMAT IS

ORBC KA KB CY FX MON COR DELM DELY WHERE KA=0 FOR HORIZONTAL, 1 FOR VERTICAL CORRECTION,

FX = NAME OF A FXPT INSTRUCTION DEFINING THE INITIAL ORBIT (FX MUST HAVE A 1 IN COLUMNS 55)

CY IS REDUNDANT

MON IS NAME OF ELEMENTS IN BEAM LINE WHERE DISPLACEMENTS ARE ASSUMED TO BE MEASURED COR IS NAME OF CORRECTION ELEMENTS IN BEAM LINE

C (THERE SHOULD BE AMNY IDENTICAL MON-S AND COR-S IN BEAM LINE)

C DELM $=$ ASSUMED MEASUREMENT ERROR (+- DELM/2)

C DELY = TOLERABLE RANGE OF FINAL RESIDUAL ORBIT ERRORS

C (ITERATIONS WILL STOP WHEN THIS LEVEL IS REACHED)

C $A B S(K B)=$ MAX NO. OF ITERATIONS. 2F KB NEGATIVE, ALL INTER-

C MEDIATE ITERATIONS AS WELL AS THE MATRIX RELATING ORBIT

C DISPLACEMENTS TO CORRECTORS ARE PRINTED OUT,

C IF KB POSITIVE, ONLY FIRST AND LAST ITERATIONS ARE PRINTED

C AND MATRIX PRINT IS SUPPRESSED.

COMMON/CORB/TH(84), TV(84), NMON, NNH, NNV, I IH, I IV, NAMONH, NAMONV, NACORH, NACORV

LEVEL 2, STORE, INFF, IWORK

COMMON STORE (48000), IWORK(10)

DIMENSION INFF $(24,2000)$

EQUIVALENCE (INFF,STORE)

COMMON/BMI 1/MI 1 ( 16000 )

COMMON/NELS1/NELS1

LEVEL 2, MI 1

C

LEVEL 2, BETA, ALPHA, AP, $B, N B, R, X, R H O, N X, N V, X A, N A, N C, X X, A, A A, B B$ COMMON/AAA/BETA, ALPHA, AP, $B, N B, R, X, R H O, N X, N V, X A, N A, N C, X X, A, A A, B B$ DIMENSION BETA(2,84), ALPHA(84), AP(84), $B(84), N B(1), R(84), X(84)$, $. R H O(252), N X(84), N V(84), X A(1), N A(1), N C(1), X X(84), A(84,84), D U M(7)$, . $A A(84,84), B B(84)$

REAL MX,MY, MU(2,84),NU, K(84), L(84),M11, M12

LOGICAL KPR, KPP

C

INTEGER BDAT

\begin{tabular}{|c|c|}
\hline ORB I TC & \\
\hline ORBI TPRNT & \\
\hline ORBI TPRNT & \\
\hline ORBI TPRNT & \\
\hline ORB I TPRNT & \\
\hline NOV28ORB & \\
\hline ORB I TPRNT & \\
\hline ORB I TPRNT & \\
\hline ORBI TPRNT & \\
\hline NOV280RB & \\
\hline ORBI TPRNT & \\
\hline ORBI TPRNT & \\
\hline ORB I TPRNT & \\
\hline ORB I TPRNT & \\
\hline ORB I TPRNT & \\
\hline ORB I TPRNT & \\
\hline ORBI TPRNT & \\
\hline ORB I TPRNT & \\
\hline ORBI TPRNT & \\
\hline ORB I TPRNT & \\
\hline ORB I TPRNT & \\
\hline ORBI TPRNT & \\
\hline CORB613 & \\
\hline CORB 613 & \\
\hline BLANK & \\
\hline BLANK & \\
\hline 86MARSIZ & \\
\hline 86MARSIZ & \\
\hline 86MARS I Z & \\
\hline BLANK & \\
\hline NV3BMI 1 & \\
\hline BMI 1L & \\
\hline BMI $1 \mathrm{~L}$ & \\
\hline BMI 1L & \\
\hline BMI 1 & \\
\hline DEBUG & \\
\hline DEBUG & \\
\hline ORBIT & \\
\hline ORBIT & \\
\hline ORBIT & \\
\hline ORBIT & \\
\hline ORBI TPRNT & 22 \\
\hline ORBITD & \\
\hline ORBIT & 12 \\
\hline ORBIT & 13 \\
\hline ORBITC & \\
\hline ORBI TC & \\
\hline ORB I TC & \\
\hline ORBITC & \\
\hline NOV280RB & \\
\hline ORBIT & 19 \\
\hline ORBIT & 20 \\
\hline ORB I TD & \\
\hline DEBUG & \\
\hline ORBIT & 22 \\
\hline ORB I TPRNT & 23 \\
\hline ORBI TPRNT & 2 \\
\hline
\end{tabular}


60

65

70

75

80

85

90

95

100

105

110
$I T E R=I A B S(K B) \$ I F(K B, E Q .0) \quad I T E R=1 \$ K P P=K P R$

IFLAG $=K A$

$\operatorname{EPS}=\operatorname{FDAT}(M M, 1)$

$\operatorname{EPSB}=\operatorname{FDAT}(M M, 2)$

$E P S Z=E P S$

NACOR $=\operatorname{BDAT}(M M, 4)$

LCOR $=$ LOCC $(N A C O R, 1, N E L S 1, M I 1, N X, N)$

IF (KA.EQ.1) GO TO 9

NAMONH $=$ MON

$\mathrm{NACORH}=\mathrm{NACOR}$

$\mathrm{NNH}=\mathrm{N}$

GO TO 26

$9 \quad$ NAMONV $=$ MON

NACORV $=$ NACOR

$\mathrm{NNV}=\mathrm{N}$

26 CONTINUE

c

PRINT 3

3 FORMAT ( $1 \mathrm{H} 1)$

PRINT 106, EPSB, EPS

106 FORMAT ( $1 X *$ DISPERSION DE MESURE *,F10.5/

$1 X * C . A . C$. RESIDUEL $\bullet *, F 10.5 /$ )

PRINT 100

100 FORMAT ( $1 X *$ CORRECTEURS $* /$ )

PRINT $2,(N X(I), I=1, N)$

2

FORMAT (2I5)

PRINT 4

PRINT 101

101 FORMAT ( $1 \mathrm{X} *$ STATIONS*/)

PRINT $1,(N B(I), I=1, M)$

1 FORMAT (2I5)

PRINT 4

4 FORMAT (1HO)

$I B=1$

$\mathrm{IC}=1$

REWIND 5

IF (IFLAG.EQ.0) GO TO 10

$\mathrm{PX}=0$.

$\mathrm{PY}=1$.

GO TO 11

$10 P X=1$.

$P Y=0$.

11 READ (5) IEL

READ (5)

$P I=4$. *ATAN (1.)

ICC $=0$

DO $12 \mathrm{I}=1, \mathrm{IEL}$

READ (5) IE , NO, NAME, DUM, BY, BX, AY, AX, MY, MX

READ (5) EX, EXP,EY,EYP, XP, DXP, Y,DYP

IF (NO.EQ.NB(IB)) GO TO 13

IF(NO.EQ.NX(IC)) GO TO 14

GO TO 12

$13 \mathrm{IA}=1$

$I X=I B$

$I B=I B+1$

B $\quad(I X)=P R(X P, Y, P X, P Y)+(R G E N(\theta)-.5) * E P S B$

$\operatorname{AP}(I X)=0$.
ORBITPRNT 25

ORBIT 24

ORBITC 10

ORBITC $\quad 11$

ORBITPRNT 26

ORBIT613

ORBIT613 2

ORBIT613 3

ORBIT613 4

ORBIT613 5

ORBIT613 6

ORBIT613 7

ORBIT613 8

ORBIT613 9

ORBIT613 10

ORBIT613 11

ORBIT 35

ORBIT 36

ORBIT 37

ORBIT 38

ORBIT $\quad 39$

ORBIT 40

ORBIT 41

ORBIT 42

ORBIT 43

ORBITC 12

ORBIT 45

ORBIT 46

ORBIT 47

ORBIT 48

ORBITC 13

ORBIT 50

ORBIT $\quad 51$

ORBIT 52

ORBIT 53

ORBITC 14

ORBIT $\quad 54$

ORBIT 55

ORBIT $\quad 56$

ORBIT $\quad 57$

ORBIT 58

ORBIT 59

ORBIT 60

NOV280RB 4

ORBIT 61

ORBIT 62

ORBIT 63

ORBIT 64

NOV280RB 5

ORBIT 66

ORBIT 67

ORBIT 68

ORBIT 69

ORBIT 70

ORBIT 71

ORBIT $\quad 72$

ORBITC 16 
DO $118 \mathrm{~J}=1, \mathrm{~N}$

$D M=(M U(1, I)-M U(2, J)) * 2 . * P I+N U$

$\operatorname{IF}(\operatorname{MU}(2, J) \cdot L T \cdot M U(1, I)) \quad D M=D M-2 . * N U$

$\operatorname{SB}=\operatorname{SQRT}(\operatorname{BETA}(2, J))$

$\mathrm{CM}=\cos (\mathrm{DM})$

IF(I.GT.1) GO T0111

$N \times(J)=N X(J)-1$

111 CONTINUE

IF (L(J).EQ.0.) GO TO 116

$S M=S I N(D M)$

$17 A K=A B S(K(J))$

$S K=S Q R T(A K)$

$M 11=S I N H(S K * L(J)) / S K$

$M 12=(\operatorname{COSH}(S K * L(J))-1$. $) / A K$

GO TO 16

$18 M 11=L(J)$

$M 12=L(J) * L(J) / 2$.

GO TO 16

$19 \operatorname{SK}=\operatorname{SQRT}(K(J))$

M11=SIN(SK*L(J)) $/ S K$

$M 12=(1 .-\operatorname{Cos}(S K * L(J))) / K(J)$

$16 \mathrm{~A}(\mathrm{I}, \mathrm{J})=\operatorname{SQRT}(\mathrm{BETA}(1, \mathrm{I})) *(\mathrm{SB} * \mathrm{CM} * \mathrm{M} 11+(\mathrm{SM}-\mathrm{ALPHA}(\mathrm{J}) * \mathrm{CM}) * \mathrm{M} 12 / \mathrm{SB}) / \mathrm{SP}$ GO TO 118

$116 \mathrm{~A}(\mathrm{I}, \mathrm{J})=\mathrm{SBI} * \mathrm{SB} * \mathrm{CM} / \mathrm{SP}$

118 CONTINUE

IF (.NOT.KPP) GO TO 1181
ORBIT

ORBITD

ORBITD

ORBIT

ORBIT

ORBIT

ORBIT

ORBIT

ORBIT

ORBIT

ORBIT

ORBIT

ORBITC

ORBIT

ORBI TPRNT

ORBI TPRNT

ORBITPRNT

ORBITPRNT

ORBIT

ORBITC

ORBIT

ORBIT

ORBIT

ORBITD

ORBI TD

ORBITD

ORBIT

ORBIT

ORBIT

ORBIT

ORB I TD

ORBI TD

ORBI TD

ORBITD

ORBIT

ORBIT

ORBIT

ORBIT

ORBIT

ORBIT

ORBIT

ORBIT

ORBIT

ORBIT

ORBIT

ORBIT

ORBIT

ORBIT

ORBITD

ORBITD

ORBITD

ORBITPRNT 
175

180

185

190

195

200

205

210

215
PRINT 4

PRINT 103

103 FORMAT ( $1 X *$ MATRICE $A * /$ )

PRINT $8,((A(I, J), J=1, N), I=1, M)$

8 FORMAT $(6(1 \times 12 F 10.4 /) /)$

1181 CONTINUE

DO $22 \mathrm{~J}=1$, ITER

PRINT 4

DO $23 \quad I 1=1, M$

$\mathrm{BB}(\mathrm{I} 1)=\mathrm{B}(\mathrm{I} 1) / 1000$.

DO $23 \quad 12=1, N$

$23 A A(I 1, I 2)=A(I 1, I 2)$

$\mathrm{JB}=\mathrm{J}$

JTER $=\rfloor$

CALL MICADO(AA,BB, 84, M,N, AP, $0,0,0,0$, EPS, JTER, DP, X, NV, R, RHO)

IF (J.EQ.1.OR.J.EQ. ITER. OR. EPS. LT . EPSZ)KPP =.T.

IF (JTER.LT.JB) RETURN

IF (KPP) PRINT 104

104 FORMAT $(1 X *$ CORRECTIONS MRAD MRAD, MM*/)

DO $20 \quad I=1, \mathrm{~J}$

$X(I)=X(I) * 1000$.

$X X(I)=X(I)$

$N K=N V(I)$

$\operatorname{IF}(K(N K) . N E .0) \quad X X.(I)=X(I) / K(N K)$

IF (KPP) PRINT 5, NK,NX(NK),X(I),XX(I)

IF (IFLAG.EQ.0) TH(NV(I)) $=-X($ I $) / 1000$.

IF (IFLAG.EQ.1) $\operatorname{TV}(\mathrm{NV}(\mathrm{I}))=x(\mathrm{I}) / 1000$.

20 CONTINUE

5 FORMAT (2I5, 2F 10.5)

DO $24 I=1, M$

$24 \mathrm{R}(\mathrm{I})=\mathrm{R}(\mathrm{I}) * 1000$.

IF (.NOT.KPP) GO TO 22

$\operatorname{IMAX}=\operatorname{MXMIN}(R(1), M, 1)$

$\operatorname{IMIN}=\operatorname{MXMIN}(R(1), M, 0)$

$E P S 1=R(I M A X)-R(I M I N)$

EPS1 $=$ EPS $1 / 1000$.

PRINT $201, \mathrm{~J}$, EPS 1

201 FORMAT ( 1 HO , 5HAFTER, I3, 22H ITERAT IONS, RMAX-RMIN=, E13.4)

PRINT 4

PRINT 105

105 FORMAT ( $1 X *$ ORBITE CORRIGEE (MM)*/)

PRINT $6,(I, R(I), I=1, M)$

$22 \quad \mathrm{KPP}=\mathrm{KPR}$

6 FORMAT (7 (I5, F10.5))

RETURN

END

\begin{tabular}{|c|c|}
\hline ORBIT & 61 \\
\hline ORBIT & 118 \\
\hline RBIT & 115 \\
\hline RBIT & $12 e$ \\
\hline RBIT & 121 \\
\hline ORB I TPRNT & 32 \\
\hline ORBIT & 122 \\
\hline ORBIT & 123 \\
\hline ORBIT & 124 \\
\hline ORBITC & 21 \\
\hline RBIT & 126 \\
\hline RBIT & 127 \\
\hline RBIT & 128 \\
\hline ORBIT & 129 \\
\hline ORBIT & $13 e$ \\
\hline ORBI TPRNT & 33 \\
\hline ORBIT & 131 \\
\hline ORB I TPRNT & 34 \\
\hline ORBIT & 133 \\
\hline RBIT & 134 \\
\hline RBIT & 135 \\
\hline ORBIT & 136 \\
\hline ORBIT & 137 \\
\hline ORBIT & 138 \\
\hline ORBITPRNT & 35 \\
\hline ORBIT & 140 \\
\hline RBIT & 141 \\
\hline RBIT & 142 \\
\hline ORBIT & 143 \\
\hline ORBIT & 144 \\
\hline ORBIT & 145 \\
\hline ORB I TPRNT & 36 \\
\hline ORBITPRNT & 37 \\
\hline ORBI TPRNT & 38 \\
\hline ORBI TPRNT & 39 \\
\hline ORB I TPRNT & 48 \\
\hline ORB I TPRNT & 4 \\
\hline ORB I TPRNT & 42 \\
\hline ORBIT & 146 \\
\hline ORBIT & 147 \\
\hline ORBIT & 148 \\
\hline ORBI TPRNT & 43 \\
\hline ORBI TPRNT & 44 \\
\hline ORBIT & 150 \\
\hline ORBIT & 151 \\
\hline ORBIT & 15 \\
\hline
\end{tabular}


DIMENSION A(NDIM, 1), B(1),AP(1),XA(1),NA(1),NB(1),NC(1),X(1),NX(1) $1, R(1), R H O(1)$

C

C REDUCTION DE AP ET DE A

CALL NBR(NA,N1)

CALL NBR(NC,N3)

CALL NBR(NB,N2)

$M 1=M-N 3$

$M 2=N-N 2$

15

C CALL MATRED( $A, N D I M, N, N 3, N 2, N C, N B)$

C CALL MATRED(AP, $1, M, 0, N 3,0, N C)$

C

C***PRISE EN COMPTE DES CONDITIONS INITIALES

C

$$
\text { IF(N1.EQ.0)GO TO } 4
$$$$
\text { DO } 1 \mathrm{I}=1, \mathrm{~N} 1
$$

$\mathrm{K}=0$

IF (N2.EQ.0)GO TO 1

DO $2 J=1, N 2$

IF (NA (I ) . GT.NB (J))K=K+1

2 CONTINUE

$1 N A(I)=N A(I)-K$

DO $3 I=1, M 1$

DO $3 \mathrm{~J}=1, \mathrm{~N} 1$

$K=N A(J)$

$3 B(I)=B(I)-A(I, K) * X A(J)$

C

C CALCUL DE DP/P

c

$$
4 S 1=0.0
$$$$
S 2=0.0
$$$$
\text { DO } 5 \mathrm{I}=1, \mathrm{M} 1
$$$$
\mathrm{S} 1=\mathrm{S} 1+\mathrm{B}(\mathrm{I}) * \mathrm{AP}(\mathrm{I})
$$

$5 \mathrm{~S} 2=S 2+\mathrm{AP}(\mathrm{I}) * \mathrm{AP}(\mathrm{I})$

IF(S2.EQ.0.0)GO TO 6 $\mathrm{DP}=\mathrm{S} 1 / \mathrm{S} 2$

DO $21 \quad I=1, M 1$

$21 B(I)=B(I)-D P * A P(I)$

$6 \mathrm{NN}=\mathrm{N}+\mathrm{N}$

45

50

$$
\begin{gathered}
\text { DO } 7 \mathrm{I}=1, \mathrm{M} 1 \\
\mathrm{RHO}(\mathrm{NN}+\mathrm{I})=\mathrm{B}(\mathrm{I})
\end{gathered}
$$

$7 \mathrm{~B}(\mathrm{I})=-\mathrm{B}(\mathrm{I})$

CALL HTLS(A, B, NDIM,M1,M2, ITER, X,NX,R, RHO, EPS)

DO $8 \quad I=1, M$

$R(I)=-R(I)$

$8 \mathrm{~B}(\mathrm{I})=\mathrm{RHO}(\mathrm{NN}+\mathrm{I})$

IF (N2.EQ.0) RETURN

DO $9 \quad J=1, N 2$

DO $9 \quad I=1, M 2$

$9 \operatorname{IF}(\mathrm{NX}(\mathrm{I}) \cdot \mathrm{GE} \cdot \mathrm{NB}(\mathrm{J})) \mathrm{NX}(\mathrm{I})=\mathrm{NX}(\mathrm{I})+1$ RETURN

END
MICADO 5

MICADO 6

MICADO 7

MICADO 8

MICADO 9

MICADO 10

MICADO 11

MICADO 12

MICADO 13

MICADOC 2

MICADOC 3

MICADO 16

MICADO 17

MICADO 18

MICADO 19

MICADO 20

MICADO 21

MICADO 22

MICADO 23

MICADO 24

MICADO 25

MICADO 26

MICADO 27

MICADO 28

MICADO 29

MICADO 30

MICADO 31

MICADO $\quad 32$

MICADO 33

MICADO 34

MICADO $\quad 35$

MICADO $\quad 36$

MICADO $\quad 37$

MICADO $\quad 38$

MICADO $\quad 39$

MICADO 40

MICADO 41

MICADO 42

MICADO 43

MICADO $\quad 44$

MICADO $\quad 45$

MICADO 46

MICADO 47

MICADO 48

MICADO 49

MICADO 50

MICADO 51

MICADO 52

MICADO 53

MICADO 54

MICADO 55

MICADO 56

MICADO $\quad 57$ 
1

SUBROUT INE NBR( $L, N)$

MICADO

58

DIMENSION $L(12)$

MICADO

59

$\mathrm{N}=0$

MICADO

60

DO $1 \mathrm{I}=1,12$

MICADO

61

IF (L(I).EQ.O) RETURN

MICADO

62

IF(L(I).EQ.10H

)RETURN

MICADO

63

$\mathrm{N}=\mathrm{I}$

MICADO

64

END

MICADO

65 
LEVEL $2, A, B, X, I P I, R, A U X$

DIMENSION $A(M 1,1), B(1), \dot{X}(1), \operatorname{IPI}(1) ; R(1) ; A U X(1)$

C

CALCUL DU PREMIER PIVOT

c

$\mathrm{K} 2=\mathrm{N}+1$

$\mathrm{PIV}=0.0$

DO $4 K=1, N$

IPI $(K)=K$

$\mathrm{H}=0$.

$G=\theta$.

$$
\begin{aligned}
& \text { DO } 2 I=1, M \\
& H=H+\operatorname{DBLE}(A(I, K)) * \operatorname{DBLE}(A(I, K))
\end{aligned}
$$$$
G=G+D B L E(A(I, K)) * D B L E(B(I))
$$

HTLS

HTLS

HTLS

HTLS

HTLS

HTLS

HTLS

HTLS

HTLS

HTLS

HTLS

HTLS

2 CONTINUE

$\operatorname{AUX}(K)=H$

$A U X(K 2)=G$

$P I V T=G * G / H$

IF (PIVT-PIV) 4, 4,3

HTLS

HTLS

HTLS

HTLS

HTLS

3 PIV = PIVT

$$
K P I V=K
$$

HTLS

HTLS

$4 K 2=K 2+1$

C BOUCLE POUR CHAQUE ITERATION

HTLS

HTLS

DO $21 \mathrm{~K}=1$, ITER

IF (KPIV.EQ.K)GO TO 8

30

C ON ECHANGE LES $K$ ET KPIV SI KPIVPLUS GRAND QUE K

HTLS

HTLS

HTLS

HTLS

$H=A U X(K)$

$\operatorname{AUX}(K)=A U X(K P I V)$

$A U X(K P I V)=H$

$\mathrm{K} 2=\mathrm{N}+\mathrm{K}$

$K 3=N+K P I V$

$G=A U X(K 2)$

$A U X(K 2)=A U X(K 3)$

$A \cup X(K 3)=G$

DO $7 \quad I=1, M$

$H=A(I, K)$

$$
A(I, K P I V)=H
$$

7 CONTINUE

45

C CALCUL DE BETA, SIGMA ET UK DANS HTUL

HTLS

HTLS

HTLS

HTLS

HTLS

HTLS

HTLS

HTLS

HTLS

HTLS

HTLS

HTLS

HTLS

HTLS

HTLS

8 CALL HTUL (A, M, N, K, SIG, BETA, M1)

HTLS

HTLS

C

c ON GARDE SIGMA DANS AUX $(N+K)$

HTLS

$J=N+K$

50

$$
\operatorname{AUX}(\mathrm{J})=-\mathrm{SIG}
$$

$$
\begin{aligned}
& I P=\operatorname{IPI}(K P I V) \\
& I P I(K P I V)=\operatorname{IPI}(K)
\end{aligned}
$$$$
\operatorname{IPI}(K)=I P
$$

IF (K.EQ.N) GO TO 13

HTLS

HTLS

HTLS

HTLS

HTLS

c

55

TRANSFORMATION DE A DANS HTAL

HTLS

HTLS

HTLS

HTLS

HTLS

CALL HTAL (A, M, N, K, BETA, M1) 
60

65

70

75

80

85

90

95

100

105

110
C

C TRANSFORMATION DE B DANS HTBL

C

13 CALL HTBL(A,B,M,N, K, BETA, M1)

c

C RECHERCHE DU PIVOT $(K+1)$

c

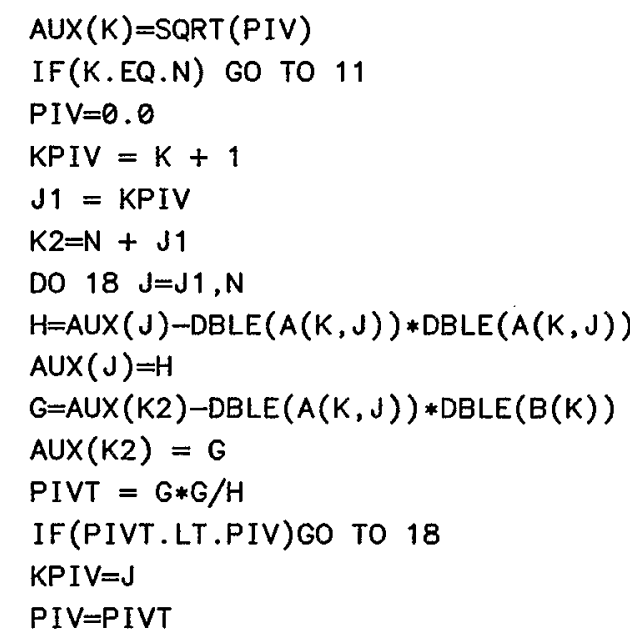

$8 K 2=K 2+1$

C

C CALCUL DES $X$

C

$11 \times(K)=B(K) / A U X(N+K)$

IF (K.EQ.1)GO TO 27

DO $15 \mathrm{I}=2, \mathrm{~K}$

$\mathrm{KK}=\mathrm{K}-\mathrm{I}+1$

$X(K K)=B(K K)$

$\mathrm{KI}=\mathrm{KK}+1$

DO $25 \mathrm{~J}=\mathrm{KI}, \mathrm{K}$

$25 X(K K)=X(K K)-A(K K, J) * X(J)$

$X(K K)=X(K K) / A U X(N+K K)$

15 CONTINUE

C 27 CALL UCOPY $(B, R, M)$

27 CONTINUE

DO 29 III $=1, M$

$R(I I I)=B(I I I)$

29 CONTINUE

c

C CALCUL DU VECTEUR RESIDUEL DANS HTRL

CALL HTRL(A, R, M,N,K,AUX,M1)

C IMAX $=\operatorname{MAXFZE}(R(1), M)$

C IMIN $=\operatorname{MINFZE}(R(1), M)$

$\operatorname{IMAX}=\operatorname{MXMIN}(R(1), M, 1)$

$\operatorname{IMIN}=\operatorname{MXMIN}(R(1), M, \theta)$

$E P S 1=R(I M A X)-R(I M I N)$

GOTO 1000

C PRINT OUT OF INTERMEDIATE CORRECTOR STRENGTHS/ORBITS

PRINT 6000,K, R(IMAX), IMAX, R(IMIN), IMIN

PRINT 6002, (IX, IPI (IX), X(IX), IX=1,K,1)

PRINT 6004

PRINT 6006, (IX,R(IX), IX=1,M,1)

1000 CONTINUE

IF (EPS1 . GT . EPS)GO TO 21

\begin{tabular}{|c|c|c|}
\hline HTLS & & 58 \\
\hline HTLS & & 59 \\
\hline HTLS & & $6 e$ \\
\hline HTLS & & 6 \\
\hline HTLS & & 6 \\
\hline HTLS & & 63 \\
\hline HTLS & & 6 \\
\hline HTLS & & 65 \\
\hline HTLS & & 66 \\
\hline HTLS & & 6 \\
\hline HTLS & & 68 \\
\hline HTLS & & 69 \\
\hline HTLS & & 70 \\
\hline HTLS & & 71 \\
\hline HTLS & & 72 \\
\hline HTLS & & 73 \\
\hline HTLS & & 74 \\
\hline HTLS & & 75 \\
\hline HTLS & & 76 \\
\hline HTLS & & 77 \\
\hline HTLS & & $7 \varepsilon$ \\
\hline HTLS & & $7 s$ \\
\hline HTLS & & $8 e$ \\
\hline HTLS & & 81 \\
\hline HTLS & & 82 \\
\hline HTLS & & 83 \\
\hline HTLS & & 8 \\
\hline HTLS & & 85 \\
\hline HTLS & & 86 \\
\hline HTLS & & 87 \\
\hline HTLS & & $8 \varepsilon$ \\
\hline HTLS & & $8 s$ \\
\hline HTLS & & 98 \\
\hline HTLS & & 91 \\
\hline HTLS & & 92 \\
\hline HTLS & & 93 \\
\hline HTLS & & 94 \\
\hline HTLS & & 95 \\
\hline HTLS & & 96 \\
\hline HTLS & & 97 \\
\hline HTLS & & 98 \\
\hline HTLS & & 99 \\
\hline HTLS & & 100 \\
\hline HTLS & & 101 \\
\hline HTLS & & 102 \\
\hline HTLS & & 103 \\
\hline HTLS & & 104 \\
\hline HTLS & & 105 \\
\hline HTLS & & 106 \\
\hline HTLS & & 107 \\
\hline HTLS & & 108 \\
\hline HTLS & & 109 \\
\hline HTLS & & 110 \\
\hline HTLS & & 111 \\
\hline HTLS & & 112 \\
\hline HTLS & & 113 \\
\hline HTLS & & \\
\hline
\end{tabular}


115

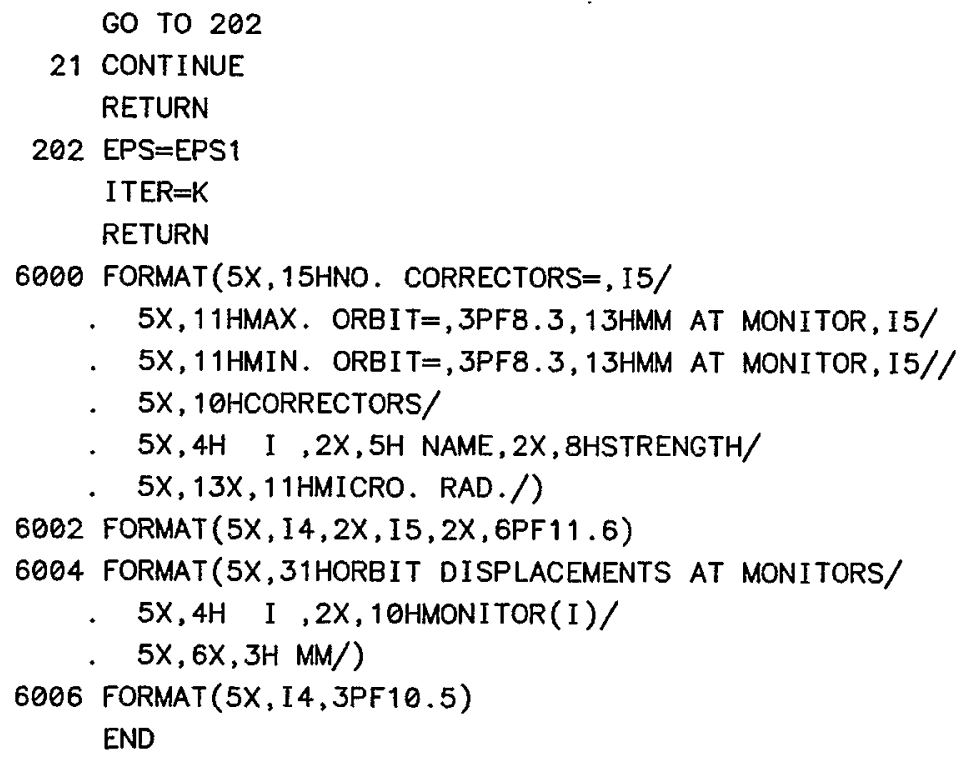

$\begin{array}{ll}\text { HTLS } & 115 \\ \text { HTLS } & 116 \\ \text { HTLS } & 119 \\ \text { HTLS } & 120 \\ \text { HTLS } & 121 \\ \text { HTLS } & 122 \\ \text { HTLS } & 123 \\ \text { HTLS } & 124 \\ \text { HTLS } & 125 \\ \text { HTLS } & 126 \\ \text { HTLS } & 127 \\ \text { HTLS } & 128 \\ \text { HTLS } & 129 \\ \text { HTLS } & 130 \\ \text { HTLS } & 131 \\ \text { HTLS } & 132 \\ \text { HTLS } & 133 \\ \text { HTLS } & 134\end{array}$

CARD NR. SEVERITY DETAILS DIAGNOSIS OF PROBLEM

109 I THERE IS NO PATH TO THIS STATEMENT. 
1

SUBROUTINE HTUL ( $A, M, N, K$, SIG, BETA, M1)

LEVEL 2,A

DIMENSION $A(M 1,1)$

DOUBLE PRECISION SIG

HTLS

DEBUG

135

HTLS

HTLS

$S I G=0.0$

HTLS

HTLS

HTLS

DO $10 \quad I=K, M$

$S I G=S I G+D B L E(A(I, K)) * \operatorname{DBLE}(A(I, K))$

HTLS

10 CONTINUE

10

$S I G=D S Q R T$ (SIG)

HTLS

HTLS

\section{C}

HTLS

HTLS

HTLS

HTLS

15

$\mathrm{H}=\mathrm{A}(\mathrm{K}, \mathrm{K})$

IF (H.LT. 0.0)SIG $=-S I G$

$B E T A=H+S I G$

$A(K, K)=B E T A$

BETA $=1 . /(S I G * B E T A)$

RETURN

END

HTLS

HTLS

HTLS

HTLS

HTLS

8
136

137

138

139

140

141

142

143

144

145

146

147

148

149

150

151

152 
1

10
SUBROUTINE HTAL(A, M, N, K, BETA, M1)

LEVEL 2, A

DIMENSION $A(M 1,1)$

DOUBLE PRECISION H

$N C=N-K$

DO $21 \mathrm{~J}=1, \mathrm{NC}$

$H=0.0$

DO $20 K 1=K, M$

$20 \mathrm{H}=\mathrm{H}+\operatorname{DBLE}(\mathrm{A}(\mathrm{K} 1, \mathrm{~K})) * \operatorname{DBLE}(A(K 1, K+J))$

$H=B E T A * H$

DO $21 K 1=K, M$

$21 A(K 1, K+J)=A(K 1, K+J)-A(K 1, K) * H$

RETURN

END
HTLS

DEBUG

HTLS

HTLS

HTLS

HTLS

HTLS

HTLS

HTLS

HTLS

HTLS

HTLS

HTLS

HTLS
153

9

154

155

156

157

158

159

160

161

162

163

164

165 
5

10
SUBROUT INE HTBL ( $A, B, M, N, K, B E T A, M 1)$

LEVEL 2, A,B

DIMENSION $A(M 1,1), B(1)$

DOUBLE PRECISION $H$

$\mathrm{H}=\mathrm{O}$.

DO $20 \mathrm{~K} 1=\mathrm{K}, \mathrm{M}$

$20 H=H+D B L E(A(K 1, K)) * D B L E(B(K 1))$

$\mathrm{H}=\mathrm{BETA} * \mathrm{H}$

DO $21 K 1=K, M$

$21 B(K 1)=B(K 1)-A(K 1, K) * H$

RETURN

END

$\begin{array}{lr}\text { HTLS } & 166 \\ \text { DEBUG } & 10 \\ \text { HTLS } & 167 \\ \text { HTLS } & 168 \\ \text { HTLS } & 169 \\ \text { HTLS } & 170 \\ \text { HTLS } & 171 \\ \text { HTLS } & 172 \\ \text { HTLS } & 173 \\ \text { HTLS } & 174 \\ \text { HTLS } & 175 \\ \text { HTLS } & 176\end{array}$


1

5

10

15
SUBROUTINE HTRL (A, B, M, N, K, AUX, M1)

LEVEL 2, A, B, AUX

DIMENSION $A(M 1,1), B(1), A U X(1)$

C CALL UZERO $(B, 1, K)$

DO $10 \quad I=1, K$

$\mathrm{B}(\mathrm{I})=0.0$

10 CONTINUE

DO $30 \mathrm{KK}=1, \mathrm{~K}$

$\mathrm{LV}=\mathrm{M}-\mathrm{K}+\mathrm{KK}$

$K N=N+K-K K+1$

$K L=K-K K+1$

C

C

$B E T A=-1 . /(A U X(K N) * A(K L, K L))$

CALL HTBL(A, B, M, N, KL, BETA, M1)

30 CONTINUE

RETURN

END

$\begin{array}{lr}\text { HTLS } & 177 \\ \text { DEBUG } & 11 \\ \text { HTLS } & 178 \\ \text { HTLS } & 179 \\ \text { HTLS } & 180 \\ \text { HTLS } & 181 \\ \text { HTLS } & 182 \\ \text { HTLS } & 183 \\ \text { HTLS } & 184 \\ \text { HTLS } & 185 \\ \text { HTLS } & 186 \\ \text { HTLS } & 187 \\ \text { HTLS } & 188 \\ \text { HTLS } & 189 \\ \text { HTLS } & 190 \\ \text { HTLS } & 191 \\ \text { HTLS } & 192 \\ \text { HTLS } & 193\end{array}$


1

10

15
FUNCTION MXMIN $(A ; N, M)$

C IF $M=0$, MAXMIN=LOWEST INDEX OF MINIMUM ELEMENT IN A

C IF $M=1$, MAXMIN=LOWEST INDEX OF MAXIMUM ELEMENT IN A

C IF $M Z 1$, MAXMIN=1

LEVEL 2,A

DIMENSION $A(N)$

MXMIN $=1$

IF (N.LT.1) RETURN

CURENT $=A(1)$

DO $10 \mathrm{I}=2, \mathrm{~N}$

IF ((M.EQ.0).AND. (A(I).GE.CURENT)) GO TO 10

IF ((M.EQ.1).AND. (A(I).LE.CURENT)) GO TO 10

CURENT $=A(I)$

MXMIN = I

10 CONTINUE

RETURN

END
HTLS

HTLS

HTLS

HTLS

DEBUG

HTLS

HTLS

HTLS

HTLS

HTLS

HTLS

HTLS

HTLS

HTLS

HTLS

HTLS

HTLS
194

195

196

197

12

198

199

200

201

202

203

204

205

206

207

208

209 
1

10

15

20

25

30
SUBROUTINE PARSR (THET, ERR)

C FUNCTION EVALUATION FOR COLLINS STRAIGHT SECTION DESIGN COMMON/AGS/ALPHA, BETA, RHO, BZ, AVAC, BMAX

C COMMON/STR/GAM, A , LK, BK, F , LM, BL, LL, BQ, LTO, SMUD , CMUD REAL LK, LM, LL, LTO

c

REAL KL

C

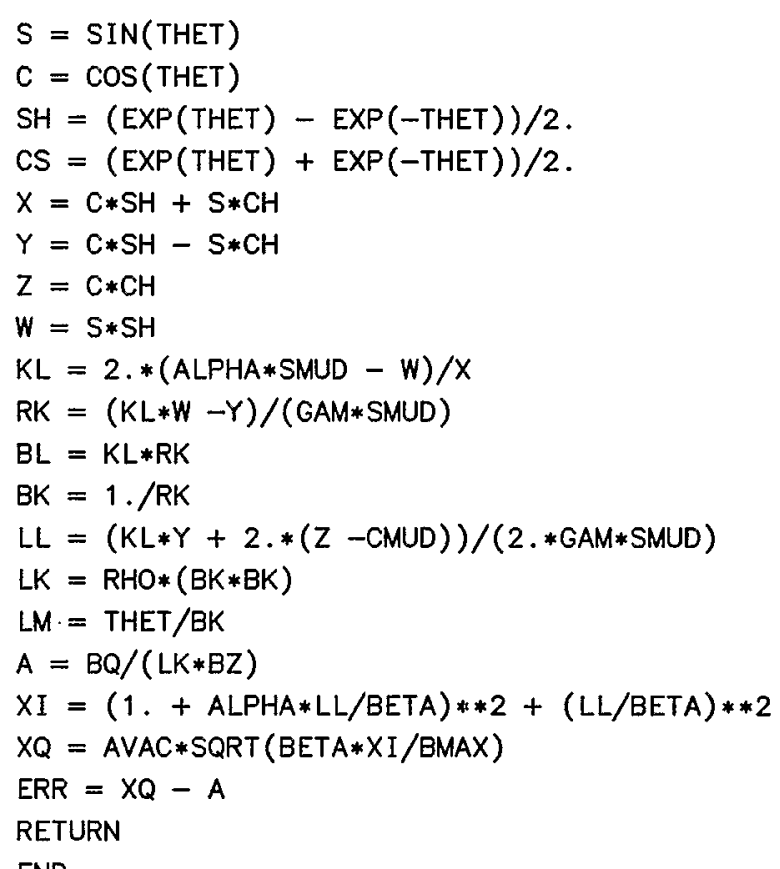

$\begin{array}{lr}\text { PARSR } & 2 \\ \text { PARSR } & 3 \\ \text { AGS } & 2 \\ \text { AGS } & 3 \\ \text { STR } & 2 \\ \text { STR } & 3 \\ \text { STR } & 4 \\ \text { PARSR } & 6 \\ \text { PARSR } & 7 \\ \text { PARSR } & 8 \\ \text { PARSR } & 9 \\ \text { PARSR } & 10 \\ \text { PARSR } & 11 \\ \text { PARSR } & 12 \\ \text { PARSR } & 13 \\ \text { PARSR } & 14 \\ \text { PARSR } & 15 \\ \text { PARSR } & 16 \\ \text { PARSR } & 17 \\ \text { PARSR } & 18 \\ \text { PARSR } & 19 \\ \text { PARSR } & 20 \\ \text { PARSR } & 21 \\ \text { PARSR } & 22 \\ \text { PARSR } & 23 \\ \text { PARSR } & 24 \\ \text { PARSR } & 25 \\ \text { PARSR } & 26 \\ \text { PARSR } & 27 \\ \text { PARSR } & 28\end{array}$


1

5

10

20

25

30

35

15
SUBROUT INE PBML(M)

C PRINT NAMED BEAM ELEMENTS WITH LENGTHS AND ACCUMULATED LENGTHS.

LEVEL 2, STORE, INFF, IWORK

COMMON STORE(48000), IWORK(10)

DIMENSION INFF $(24,2000)$

EQUIVALENCE (INFF,STORE)

COMMON/BMI 1/MI 1 ( 16000 )

COMMON/NELS1/NELS1

LEVEL 2,MI1

C

C

C

$\mathrm{NBCD}=1$

DO $100 \quad I=1, N B C D$

$\mathrm{NN}=\operatorname{MDAT}(\mathrm{M}, \mathrm{I})$

$M N M=\operatorname{MNAME}(N N)$

NDAT $=\operatorname{INFF}(17, N N)$

CALL MIFILL(NN, 1, NDAT, NELS1, MI1)

C

WRITE $(3,1)$ MNM

$E L T=\theta$.

C

DO $110 \mathrm{~J}=1$, NELS 1

$\mathrm{J} J=M I 1(\mathrm{~J})$

MNAM = MNAME $(J J)$

$E L=\operatorname{FDAT}(\mathrm{J} J, 1)$

$E L T=E L T+E L$

WRITE $(3,2)$ MNAM, EL, ELT

110 CONTINUE

100 CONTINUE

C

FORMAT ( $1 H 1,5 X, *$ ELEMENTS THROUGH BEAM LINE *,A5//6X, *NAME*,5X,

$1 *$, LENGTH*,6X,* ACC. LENGTH*)

2 FORMAT $(6 X, A 5,2 X, F 12.8,2 X, F 12.8)$

RETURN

END
PBMLL $\quad 2$

PBMLL 3

BLANK 2

BLANK 3

86MARSIZ 1

86MARSIZ 2

86MARSIZ 3

BLANK 5

NV3BMI 1

BMI1L 2

BMI 1L 3

BMI1L 4

BMI 13

PBMLL 6

PBMLL 7

$\begin{array}{ll}\text { PBMLL } & 8\end{array}$

PBMLL 9

PBMLL 10

PBMLL 11

PBMLL 12

PBMLL 13

PBMLL 14

PBMLL 15

PBMLL 16

PBMLL $\quad 17$

PBMLL $\quad 18$

PBMLL 19

PBMLL 20

PBMLL 21

PBMLL 22

PBMLL 23

PBMLL 24

PBMLL 25

PBMLL . 26

PBMLL 27

PBMLL 28

PBMLL 29

PBMLL 30

PBMLL 31 
* ROUND LIMITS FOR PLOT OF BETATRON FUNCTIONS. SET TICK INTERVALS. PLPAR

IF (PM.GT.5.) GO TO 43

PLPAR

$\mathrm{FL}=5$.

$\mathrm{IT}=1$

RETURN

10

43 IF (PM.GT.10.) GO TO 44

PLPAR 7

PLPAR 8

PLPAR 9

$F L=10$.

PLPAR 10

$\mathrm{IT}=2$

RETURN

44 IF (PM.GT.25.) GO TO 45

PLPAR 11

PLPAR 12

PLPAR 13

$F L=25$.

15

$I T=5$

RETURN

45 IF (PM.GT.50.) GO TO 46

$F L=50$.

$\mathrm{IT}=10$

20

RETURN

46 IF (PM.GT.100.) GO TO 471

PLPAR

PLPAR

PLPAR

PLPAR

PLPAR

PLPAR

PLPAR

PLPAR

PLPAR

$F L=100$.

PLPAR

$\mathrm{IT}=20$

RETURN

471 IF (PM.GT.150.) GO TO 47

PLPAR

PLPAR

PLPAR

$F L=150$.

IT $=30$

RETURN

47 IF (PM.GT.250.) GO TO 48

$F L=250$.

$\mathrm{IT}=50$

RETURN

48 IF (PM.GT.500.) GO TO 49

$\mathrm{FL}=500$.

$I T=100$ 
* PLOT BETAX, BETAY,XEQ, WICH WERE COMPUTED AND WRITTEN tO A FILE bY

PLTB682

* A BEST INSTRUCTION.

PLTB682

PLTB682

BLANK

LEVEL 2, STORE, INFF, IWORK

COMMON STORE (48000), IWORK(10)

DIMENSION INFF $(24,2000)$

EQUIVALENCE (INFF,STORE)

BLANK

86MARSIZ

86MARSIZ

86MARSIZ

BLANK

LEVEL 2, XX,Y1,Y2,Y3,Y4

COMMON XX(4000), Y1 (4000), Y2(4000), Y3(4000), Y4(4000)

COMMON/BPLTCOM/MN, KW, BXX, BYX, NPLT

COMMON/CCPOOL/XMIN, XMAX, YMIN, YMAX, CCXMIN, CCXMAX, CCYMIN, CCYMAX

COMMON/CCFACT/FACTOR

BETPTS

RENAM83

RENAM83

PL6683

PL6683

COMMON/BMI/MI (5000)

COMMON/NELS/NELS

COMMON/SVNAM/NAMRUN

DIMENSION IPRS(2), $\operatorname{PMX}(3), \operatorname{FLT}(3), \operatorname{PAR}(3), \operatorname{ITIK}(3)$

DIMENSION $X(6), Y(6), \operatorname{IFLT}(3), \operatorname{MOP}(8)$

DIMENSION XPL(4000), YPL(4000)

COMMON/TRKINT/G, EX, EY, OMSQ, SEND , VMX, VMN, DINT , BEG, DPR, JPR,

1 THET, PLT, NPT, NZ, PMAX, NSIZ, ITITLE(6)

LOGICAL BEG, DPR, PLT

INTEGER BDAT

LOGICAL LIMSW

LOGICAL PSW

DATA MOP/3HDRF, 3HMAG, 4HMAGV, 4HMAGS, 3HREF, 3HINV, 3HSHF, 3HEQU/

\section{**** INPUT PARAMETERS}

C KB - OPTION FOR UNITS OF BETA PLOT.

C $K B=O$ OR BLANK - PLOT SQ. RT. OF BETAS.

C KB.NE. O, PLOT BETAS

C KA IS MULTIPLE, NOT TO EXCEED 14, OF 10 INCHES FOR LENGTH OF

C FRAME DESIRED, I.E. X AXIS.

* NC is name of element that computed the betatron functions.

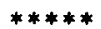

*** COMPUTED VARIABLES

* $\quad X X=$ LENGTH $S \quad Y 1=B E T A X, \quad Y 2=X E Q, Y 3=B E T A Y, Y 4=Y E Q$

* $\quad$ PMX = MAX OF BETA, MAX OF XEQ, YEQ, AND MIN OF XEQ, YEQ

* Flt = RAdJusted maX of betax, Betay, the READJusted maX of XEQ, and

* the readjusted Min of XeQ

LIMSW $=$.FALSE.

$B X M X=B X X$

IF(NPLT.EQ.0) CALL SETPLT

REWIND 98

NPLT $=$ NPLT+1

$B Y M X=B Y X$

BETPTS

BMIL

BMIL

BMIL

BMIL

BMI

SVNAM

PLTB682

PLTJL29

BMINV82

PLTB682

TRK INT

TRK INT

TRKINT

PLTB682

PLTB682

PLTB682

PLTB682

PLTB682

PLTB682

PLTB682

PLTB682

PLTB682

PLTB682

PLTB682

PLTB682

PLTB682

PLTB682

PLTB682

PLTB682

PLTB682

PLTB682

PLTB682

PLTB682

PLOTBEV

PLOTBEV

PLT

PLT

PLT

PLOTBEV 37

PLOTBEV 
60

65
GET INPUT DATA

$$
\text { INS }=0
$$$$
K A=\operatorname{INFF}(4, M)
$$$$
K B=\operatorname{INFF}(5, M)
$$$$
\text { IF (KA.EQ. } 0) K A=1
$$$$
N C=\operatorname{BDAT}(M, 1)
$$$$
\operatorname{NAM}=\operatorname{MDAT}(M, 1)
$$$$
N N O P=\operatorname{INFF}(1, N A M)
$$$$
\text { IF (NNOP . EQ . 4HFXPT) NAM=MDAT }(N A M, 2)
$$$$
\mathrm{KO}=2
$$$$
\text { CALL DATA }(M, 3,1,2, \text { IPRS })
$$$$
\text { CALL DATA }(M, 1,1,3, \text { PAR })
$$

NDAT $=\operatorname{INFF}(17$, NAM $)$

CALL MIFILL(NAM, 1 , NDAT, NELS, MI)

$\mathrm{NK}=\mathrm{NELS}$

\section{REWIND 12}

C SEEK CORRECT FILE OF BETATRON FUNCTIONS AND READ IT

30 READ (12) MN, KW

IF(MN.EQ.NC) GO TO 35

$\operatorname{READ}(12)$

$$
\operatorname{READ}(12)
$$

$$
\text { GO TO } 30
$$

\section{CONTINUE}

$\operatorname{READ}(12)(X X(I), Y 1(I), Y 2(I), Y 3(I), Y 4(I), I=1, K W)$

READ (12) BXMX, BYMX

$36 \quad \mathrm{NPT}=\mathrm{KW}$

$N Z=K W-1$

IF (KB.NE. O) GO TO 33

TAKE SQUARE ROOTS OF BETAX, BETAY

$B X M X=\operatorname{SQRT}(B X M X)$

BYMX = SQRT (BYMX)

DO $32 I=1, K W$

$Y 1(I)=\operatorname{SQRT}(Y 1(I))$

$Y 3(I)=\operatorname{SQRT}(Y 3(I))$

32 CONTINUE

33 CONTINUE

* determine paper coordinates

* Length of paper may nOt exCEed 120 inches

IF (KA.GT.14) KA=14

ALLOW SPACE FOR RIGHT AND LEFT MARGINS.

$\operatorname{CCXMIN~}=70$.

$\mathrm{FKA}=\mathrm{KA}$

IF (KA.LE. 1) FKA=1.4

CCXMAX $=($ FKA-1. $) * 850 .+745$.

$T 1=100 . / 8$.

$\mathrm{T} 2=100 . / 16$.
PLOTBEV 39

PLDRF

PLOTBEV 40

PLOTBEV 41

PLOTBEV 42

PLOTBEV 43

PLOTBEV 44

PLBAG17 1

PLBAG17 2

PLOTBEV 45

PLOTBEV 46

PLOTBEV 47

PLOTBEV 48

PLOTBEV 49

PLOTBEV 50

PLOTBEV 51

PLOTBEV 53

NOV $279 \quad 15$

NOV279 16

NoV279 17

NoV $279 \quad 18$

NoV $279 \quad 19$

NoV $279 \quad 20$

NOV $279 \quad 21$

NOV279 22

NOV $279 \quad 23$

NOV $279 \quad 24$

PLOTBEV 64

PLOTBEV 65

PLOTBEV 66

PLOTBEV 67

PLOTBEV 68

PLOTBEV 69

PLOTBEV 70

PLOTBEV 71

PLOTBEV 72

PLOTBEV 73

PLOTBEV 74

PLOTBEV 75

PLOTBEV 76

PLOTBEV 77

PLOTBEV 78

PLOTBEV 79

PLOTBEV 80

PLOTBEV 81

PLOTBEV 82

PLOTBEV 83

DEC85PLB 1

PLOTBEV 84

PLOTBEV 85

PLOTBEV 86

PLOTBEV 87

PLOTBEV 88

PLOTBEV 89

PLOTBEV 90

PLOTBEV 91

PLOTBEV 92 
56 IF $((\operatorname{PMX}(3) \cdot E Q \cdot Q M N) \cdot O R \cdot(P M X(3) \cdot E Q \cdot \theta))$ GO TO 59 GO TO 57

C IF ABS (LARGEST NEG) . LT. .05 XMAX PLOT BELOW X AXIS, RETAIN SCALE

59 ONEP $=.05 * F L T(2)$

IF (ABP.GT.ONEP) GO TO 60

PSW $=$. TRUE.

PLTB682 35

PLTB682 36

PLTB682 37

PLOTBEV 115

PLOTBEV 116

PLOTBEV 117

PLOTBEV 118

PLOTBEV 119

PLOTBEV 120

PLTB682 38

PLOTBEV 121

PLOTBEV 122

PLTB682 39

PLTB682 40

PLTB682 41 .

PLTB682 42

PLTB682 43

PLTB682 44

PLTB682 45

DEC85PLB 2

DEC85PLB 3

PLTB682 49

PLTB682 50

PLTB682 51

PLTB682 52

PLTB682 53

PLTB682 54

PLTB682 55

PLTB682 56

PLTB682 57

PLTB682 58

PLTB682 59

PLTB682 60

61 CONTINUE

PLTB682 61

57 IF (ABP.GT.10.) GO TO 62 
$\mathrm{IP}=\mathrm{ABP}$

PLOTBEV

$A P P=I P$

PLOTBEV

133

IF (ABP.GT.APP) $I P=I P+1$

PLOTBEV

134

$F L T(3)=-I P$

PLOTBEV

135

GO TO 65

$62 \quad \mathrm{IP}=\mathrm{ABP} / 10$.

$A B P=(I P+1) * 10$

PLOTBEV 136

$\operatorname{FLT}(3)=-A B P$

180

GO TO 65

$63 \quad \mathrm{FLT}(3)=0$.

$\operatorname{PMX}(3)=0$.

65 CONTINUE

DO $66 \mathrm{I}=1,3$

185

$\operatorname{IFLT}(\mathrm{I})=\mathrm{FLT}(\mathrm{I})$

66 CONTINUE

PLOTBEV 137

PLOTBEV 138

PLOTBEV 139

PLOTBEV 140

PLOTBEV 141

PLOTBEV 142

PLOTBEV 143

PLOTBEV 144

PLOTBEV 145

PLOTBEV 146

DETERMINE LIMITS IN $\times$ SCALE

PLOTBEV 147

PLOTBEV 148

IF (IPRS(1).GT.IPRS(2)) GO TO 205

PLOTBEV 149

190

IF (IPRS(2).NE.0.) GO TO 70

PLOTBEV 150

** deFAULT - entiRE beAM LINE

PLOTBEV

151

$\operatorname{IPRS}(1)=0$

$\operatorname{IPRS}(2)=\mathrm{NZ}$

PLOTBEV

152

PLOTBEV

153

PLOTBEV

154

195

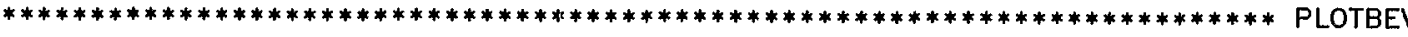

155

ORIGINALLY SET UP TO LOOP FROM HERE TO 200 TO PLOT MULTIPLE FRAMES

* INPUT CHANGED TO REQUIRE CARD INPUT FOR EACH fRAME.

PLOTBEV

156

70 DO $200 \mathrm{KK}=1, \mathrm{KO}, 2$

PLOTBEV

157

200

$N 1=\operatorname{IPRS}(K K)+1$

PLOTBEV

158

PLOTBEV

159

$\mathrm{NBT}=\operatorname{IPRS}(K K+1)+1$

PLOTBEV

160

$\mathrm{NBE}=\mathrm{NBT}-1$

PLOTBEV

161

$\mathrm{NP}=\mathrm{NBT}-\mathrm{N} 1+1$

PLOTBEV

162

PLOTBEV

163

205

$S 1=X X(N 1)$

LIMITS OF $X$ FROM $S 1$ TO SND

PLOTBEV

164

$\mathrm{SND}=\mathrm{XX}(\mathrm{NBT})$

PLOTBEV

165

$\mathrm{J} 1=\mathrm{N} 1$

PLOTBEV

166

PLOTBEV

167

CCYMAX $=950$

$210 \quad$ CCYMIN $=5$

PLFORM

168

PLOTBEV

PLOTBEV 171

* $\quad$ PRINT titLe FROM RUN CARD

PLOTBEV 172

$\operatorname{CCX}=$ CCXMIN -75 .

PLOTBEV

173

$\mathrm{CCY}=$ CCYMIN +150 .

215

CALL CCLTR(CCX, CCY, 1, 2, ITITLE, 40)

C PRINT ON BOTTOM OF PAGE

PLOTBEV 174

PLDRF

PLOTBEV 177

$\operatorname{CCX}=(\operatorname{CCXMAX}+\operatorname{CCXMIN}) / 2 .-112.5$

PLOTBE

$C C Y=C C Y M I N+5$.

PLDRF

CALL CCLTR(CCX, CCY, 0,2,15HPATH LENGTH $(M), 15)$

220

DRAW BOUNDARIES

PLT

PLOTBEV

PLOTBEV

PLOTBEV

PLFORM

TOP $=850$.

PBOT $=60$.

PLOTBEV

PLOTBEV

PLOTBEV

PLOTBEV

PLOTBEV 
$C Y B=((P B O T-C C Y M I N) *(Y M A X-Y M I N)) /(C C Y M A X-C C Y M I N)+Y M I N$

PLOTBEV

CYT $=(($ PTOP-CCYMIN $) *(Y M A X-Y M I N)) /($ CCYMAX-CCYMIN $)+Y M I N$

PLOTBEV

$X(1)=s 1$

$X(2)=S 1$

PLOTBEV

$Y(1)=C Y B$

PLOTBEV

$Y(2)=C Y T$

$X(3)=X(2) \$ Y(3)=Y(2)$

PLOTBEV

PLOTBEV

CALL CCPLOT $(X, Y, 3,4$ HJOIN, $1,1,1 \mathrm{HI})$

$Y(1)=C Y T$

$X(2)=$ SND

$X(1)=$ SND

240

$Y(2)=C Y B$

$X(3)=X(2) \$ Y(3)=Y(2)$

CALL CCPLOT $(X, Y, 3,4$ HJOIN, $1,1,1 \mathrm{HI})$

$X(2)=S 1$

$Y(1)=C Y B$

$X(3)=X(2) \$ Y(3)=Y(2)$

CALL CCPLOT $(X, Y, 3,4$ HJOIN, $1,1,1 \mathrm{H}-)$

PLFORM

PLFORM

PLOTBEV

PLOTBEV

PLOTBEV

PLOTBEV

PLFORM

PLFORM

PLOTBEV

PLOTBEV

PLFORM

PLFORM

C WRITE NAME OF ARRAY

CALL CCLTR(CCX,PTOP-90, ,0,1,NC, 5)

MAY1179

PLFORM

PLOTBEV 214

190

191

192

193

194

195

5

6

199

200

204

205

7
8

209

210

9

10

8

PLOTBEV 215

PLOTBEV 216

PLOTBEV 217

PLOTBEV 218

PLOTBEV 219

PLOTBEV 220

PLOTBEV 221

PLOTBEV 222

PLBNV82 1

PLBNV82 2

PLOTBEV 223

PLOTBEV 224

PLOTBEV 225

PLOTBEV 226

PLOTBEV 227

PLOTBEV 228

PLOTBEV

229

PLTJL29

PLOTBEV

PLOTBEV

SYFTN

PLOTBEV

3

230

231

GO TO IGO $(110,116)$

110 GO TO $(133,117,117,112,112,112,112,112)$ JN

207

233

234

PLOTBEV 235

PLOTBEV

236

* OTHERWISE tREAT It as a cOMPOSite.

PLOTBEV

237

NAMOP $=\operatorname{INFF}(1, N N)$

PLOTBEV

238

GO TO 105

116 GO TO $(133,117,117,109,109,109,109,109)$ JN

PLOTBEV

239

PLOTBEV

240

COMPOSITE. DRAW BOX LIKE BENDING MAGNET, BUT MUCH SMALLER.

280

109

$\mathrm{PCT}=\mathrm{PTOP}+5$.

$P C B=P T O P-5$.

$T Y=P T O P+7$.

GO TO 125

PLOTBEV

241

PLOTBEV

242

PLOTBEV

243

PLOTBEV

244

PLOTBEV

245

117 CONTINUE

PLOTBEV

246

PLOTBEV 
290

295

300

305

310

315

320

325

330

335
DRAW MAGNET ABOVE OR BELOW THE LINE FOR FOCCUSSING OR

* Defocussing gRAdient.

* straddle the line if zero gradient.

$G=\operatorname{FDAT}(N N, 2)$

IF (G) $113,115,120$

DEFOCUSSING

113 PCT = PTOP

$P C B=P T O P-50$.

GO TO 121

$$
\begin{array}{ll}
\text { * } & \text { FOCUSSING } \\
120 & \text { PCT }=\text { PTOP }+50 . \\
& \text { PCB }=\text { PTOP } \\
121 & \text { TY }=\text { PCB }+20 . \\
& \text { GO TO } 125
\end{array}
$$

\section{ZERO GRADIENT}

115 PCT $=$ PTOP +25 .

$P C B=P T O P-25$.

$\mathrm{TY}=\mathrm{PTOP}+5$.

GO TO 125

$125 \mathrm{PT}=(($ PCT - CCYMIN $) *($ YMAX-YMIN $)) /($ CCYMAX-CCYMIN $)+$ YMIN

$P B=((P C B-C C Y M I N) *(Y M A X-Y M I N)) /($ CCYMAX-CCYMIN $)+Y M I N$ DRAW MAGNETS

$X(1)=S$

$X(2)=S$

$Y(1)=P T$

$Y(2)=P B$

DO $126 \quad I=1,3$

$X(3)=X(2) \$ Y(3)=Y(2)$

CALL CCPLOT $(X, Y, 3,4$ HJOIN, $1,1,1 \mathrm{H}$.

126 CONTINUE

$X(2)=S E$

$Y(1)=P B$

DO $128 \quad I=1,3$

$X(3)=X(2) \$ Y(3)=Y(2)$

128 CONTINUE

$127 X(1)=S E$

$Y(2)=P T$

DO $130 \quad I=1,3$

$X(3)=X(2) \$ Y(3)=Y(2)$

CALL CCPLOT $(X, Y, 3,4$ HJOIN, $1,1,1 \mathrm{H}$.

130 CONTINUE

$X(2)=S$

$Y(1)=P T$

DO $132 \quad I=1,3$

$X(3)=X(2) \quad \$ Y(3)=Y(2)$

$\operatorname{CALL} \operatorname{CCPLOT}(X, Y, 3,4$ HJOIN, $1,1,1 \mathrm{H}$.

132 CONTINUE

GO TO 135

$$
\text { * }
$$

133 TY $=$ PTOP +5.

\section{SET PAPER POSITION FOR DRIFT}

\begin{tabular}{|c|c|}
\hline & \\
\hline _OTBEV & \\
\hline _OTBEV & \\
\hline PLOTBEV & \\
\hline PLOTBEV & \\
\hline PLOTBEV & \\
\hline PLOTBEV & \\
\hline PLOTBEV & \\
\hline PLOTBEV & \\
\hline PLOTBEV & \\
\hline PLOTBEV & \\
\hline PLOTBEV & \\
\hline PLOTBEV & \\
\hline PLOTBEV & \\
\hline PLOTBEV & \\
\hline PLOTBEV & \\
\hline PLOTBEV & \\
\hline PLOTBEV & \\
\hline PLOTBEV & \\
\hline PLOTBEV & \\
\hline PLOTBEV & \\
\hline PLOTBEV & 26 \\
\hline PLOTBEV & 27 \\
\hline PLOTBEV & \\
\hline PLOTBEV & \\
\hline PLOTBEV & 27 \\
\hline PLOTBEV & 27 \\
\hline PLOTBEV & \\
\hline PLFORM & \\
\hline PLFORM & \\
\hline PLOTBEV & \\
\hline PLOTBEV & 278 \\
\hline PLOTBEV & 27 \\
\hline PLOTBEV & \\
\hline PLFORM & \\
\hline PLFORM & \\
\hline PLOTBEV & \\
\hline PLOTBEV & \\
\hline PLOTBEV & 8 \\
\hline PLOTBEV & 8 \\
\hline PLFORM & \\
\hline PLFORM & \\
\hline PLOTBEV & 8 \\
\hline PLOTBEV & \\
\hline PLOTBEV & \\
\hline LOTBEV & 9 \\
\hline PLFORM & \\
\hline PLFORM & \\
\hline PLOTBEV & \\
\hline PLOTBEV & \\
\hline PLOTBEV & \\
\hline PLOTBEV & 9 \\
\hline PLDRF & \\
\hline PLDRF & \\
\hline PLDRF & \\
\hline PLDRF & \\
\hline PLDRF & \\
\hline
\end{tabular}

C IF DRIFT LONGER THAN 1 METER, SUBDIVIDE

$N Q=0$

IF(EL.LE.1.0) GO TO 1339

$N Q=E L \$ N Q P=N Q+1$

$K P L=K+I N S$ 
$E L P=E L / N Q P$

$D X P=(Y 2(K P L+1)-Y 2(K P L)) / N Q P$

PLDRF

$D Y P=(Y 4(K P L+1)-Y 4(K P L)) / N Q P$

PLDRF

9

$S S=0$.

PLDRF 11

IF (KB.NE. O) GO TO 1330

PLDRF

DO $1340 \mathrm{JJ}=\mathrm{KPL}, \mathrm{KW}$

JUN680

$Y_{1}(J J)=Y_{1}(J J) * * 2$

350

$1340 Y 3(\mathrm{JJ})=Y 3(\mathrm{JJ}) * * 2$

1330 CONTINUE

$B X S=S Q R T(Y 1(K P L) * Y 1(K P L+1)-E L * * 2)$

$B Y S=S Q R T(Y 3(K P L) * Y 3(K P L+1)-E L * * 2)$

$B \times 3=Y 1(K P L)-B X S \$ B X 4=Y 1(K P L+1)-B X S$

355

$B Y 3=Y 3(K P L)-B Y S \$ B Y 4=Y 3(K P L+1)-B Y S$

$G X=(B X 3+B X 4) / E L * * 2 \$ G Y=(B Y 3+B Y 4) / E L * * 2$

$\mathrm{SSX}=\mathrm{BX} 3 / \mathrm{GX} / \mathrm{EL} \$ \mathrm{SSY}=\mathrm{BY} 3 / \mathrm{GY} / \mathrm{EL}$

$K W W=K W-1$

* MOVE DATA ARRAYS

DO $1331 \mathrm{KJ}=\mathrm{KPL}, \mathrm{KWW}$

$K N=K W+K P L+N Q-K J$

$X X(K N)=X X(K N-N Q)$

$Y 1(K N)=Y 1(K N-N Q)$

$Y 2(K N)=Y 2(K N-N Q)$

365

$Y 3(K N)=Y 3(K N-N Q)$

$1331 Y 4(K N)=Y 4(K N-N Q)$

INS $=$ INS+NQ

$K W=K W+N Q$

$N P=N P+N Q$

* insert INTERPOlated dATA

DO $1332 \mathrm{~J} J=1$, NQ

$K N=K P L+J J$

$S S=S S+E L P$

$X X(K N)=X X(K P L)+S S$

375

$Y 2(K N)=Y 2(K N-1)+D X P$

$Y 4(K N)=Y 4(K N-1)+D Y P$

$Y 1(K N)=(S S-S S X) * * 2 * G X+1 . / G X$

$1332 Y 3(\mathrm{KN})=(\mathrm{SS}-\mathrm{SSY}) * * 2 * G Y+1 . / \mathrm{GY}$

IF (KB.NE. . ) GO TO 1339

380

DO $1349 \mathrm{~J} J=K P L, K W$

$Y 1(J J)=S Q R T(Y 1(J J))$

$1349 Y 3(\mathrm{JJ})=\operatorname{SQRT}(Y 3(\mathrm{JJ}))$

1339 CONTINUE

$S=S E$

385

GO TO 140

135 CONTINUE

C BY PASS LETTERING OF BEAM SYSTEM

136 CONTINUE

390

* pOSITION PAPER COORDinates to PRINT NAME at tOP

$\operatorname{DECODE}(10,3010$, NAMK) CHAR

IF (CHAR.NE.1H") GO TO 3020

3010 FORMAT $(A 1,9 X)$

DECODE $(10,3011$, NAMK) NAMK

395

3011 FORMAT $(1 X A 4,5 X)$

3020 TY=PCB-20.

WRITE $(98,2)$ NAMK

C CENTER POINT

$C E N T=S+E L / 2$.

C FIND PAPER COORDINATE

JUN680

JUN680 3

JUN680 4

JUN680 5

PLDRF 13

PLDRF 14

PLDRF 15

PLDRF $\quad 16$

PLDRF $\quad 17$

PLDRF 18

PLDRF $\quad 19$

PLDRF 20

PLDRF 21

PLDRF 22

PLDRF 23

PLDRF 24

PLDRF 25

PLDRF 26

PLDRF 27

PLDRF 28

PLDRF 29

PLDRF 30

PLDRF 31

PLDRF $\quad 32$

PLDRF 33

PLDRF 34

PLDRF 35

PLDRF 36

PLDRF 37

PLDRF 38

PLDRF 39

JUN680 6

JUN680 7

JUN680 8

JUN680 9

PLDRF $\quad 40$

PLT 133

PLT 134

PLOTBEV 297

PLOTBEV 299

PLOTBEV 300

PLOTBEV 301

JUN680 10

JUN680 11

JUN680 12

JUN680 13

JUN680 14

PLTB682 63

PLOTBEV 302

PLOTBEV 303

PLOTBEV 304

PLOTBEV 305 
CCY $=$ CCYMIN -15.

PLOTBEV 318

PLTB682 65

$\operatorname{CCX}=\operatorname{CCXMIN}-20$.

PLTB682 66

WRITE $(98,6)$ S1

PLTB682 67

CALL CCLTR(CCX, CCY-25.,0,1)

PLTB682

PLBNV82

WRITE $(98,3)$ SND

PLTB682

PLTB682

$\operatorname{CCX}=\operatorname{CCXMAX}-20$

PLBNV82

CALL CCLTR(CCX, CCY-25, , 0,1$)$

$\operatorname{CCX}=\operatorname{CCXMIN}-20$.

PLTB682

IF (KB.NE. $\theta)$ GO TO 142

PLTB682

PLTB682

$C C Y=460$

PLTB682

PLTB682

$C C Y=571$

PLTB682 78

$\operatorname{CCX}=\operatorname{CCX}-18$.

CALL CCLTR(CCX, CCY, 1,1,5H 1/2)

PLBNV82

GO TO 143

142 CONTINUE

$\mathrm{CCY}=500$.

CALL CCLTR(CCX, CCY, 1, 2, 4HBETA)

PLTB682

PLTB682

PLTB682

PLTB682

143 CONTINUE

$\mathrm{CCX}=$ CCXMAX +50 .

CALL CCLTR(CCX, CCY, 1, 2, 3HXEQ)

PLTB682

PLTB682

PLTB682

$Y$ AXIS

$\mathrm{CCY}=\mathrm{CCYMIN}$

$\operatorname{CCX}=\operatorname{CCXMIN}-15$.

CALL CCLTR(CCX, CCY, $0,1,1 \mathrm{H} 0)$

$\mathrm{CCY}=\mathrm{CCYMIN}$

PLTB682

PLTB682

PLTB682

PLTB682

PLTB682

PLTB682

PLTB682

PLTB682

PLTB682

PLOTBEV

PLOTBEV

PLOTBEV

PLBNV82

PLOTBEV

PLOTBEV

PLOTBEV

PLOTBEV

PLOTBEV

PLOTBEV

PLOTBEV

PLOTBEV

PLOTBEV 
YMIN $=0$.

PLOTBEV 370

YMAX $=$ FLT (1)

PLOTBEV 371

* For EACH 10 INCHES OF PAPER LENGTH, ALLOW ABOUT 5 INTERVALS

PLOTBEV 372

460

$\mathrm{CCY}=\mathrm{CCYMIN}+\mathrm{T} 1$

PLOTBEV 373

CYTK1 $=0$.

CYTK2 $=($ CCY-CCYMIN $) *($ YMAX-YMIN $) /($ CCYMAX-CCYMIN $)+$ YMIN

PLOTBEV 374

$Y(1)=$ CYTK1

$Y(2)=$ CYTK2

PLOTBEV 375

PLOTBEV 376

$\mathrm{DS}=($ SND-S1) $/ \mathrm{FKA}$

PLOTBEV 377

CALL PLPAR(DS, FM, ITK)

PLOTBEV 378

FTK = ITK

IS1 $=S 1 / F T K$

PLOTBEV 379

PLOTBEV 380

IS1 $=(I S 1+1) * I T K$

470

IEND = SND

PLOTBEV

381

PLOTBEV 382

$C C Y=$ CCYMIN -15.

DO 165 I=IS1, IEND, ITK

PLOTBEV

383

$X(1)=I$

$X(2)=I$

CALL CCPLOT $(X, Y, 2,4$ HJOIN)

$\operatorname{CCX}=\operatorname{CCXMIN}+(X(1)-X M I N) *(\operatorname{CCXMAX-CCXMIN}) /($ XMAX-XMIN $)$

WRITE $(98,9)$ I

165 CONTINUE

CALL CCLTR(CCX-26. , CCY, 0,1$)$

480

CCYMIN = PBOT

****** PLOT TICK MARKS ON LEFT Y AXIS FOR BETAX AND BETAY.

* PLOT TICK MARKS ON RIGHT Y AXIS FOR XEQ.

CCXMIN $=$ CCXMIN $-\mathrm{T} 2$

$J=1$

YMIN $=0$.

166 YMAX $=$ FLT $(\mathrm{J})$

$\operatorname{CCX}=\operatorname{CCXMIN}+\mathrm{T1}$

CXTK1 $=X X(J 1)$

CXTK2 $=($ CCX - CCXMIN $) *($ XMAX-XMIN $) /($ CCXMAX-CCXMIN $)+X M I N$

PLOTBEV

384

PLOTBEV 385

PLOTBEV 386

PLOTBEV 387

PLOTBEV 388

PLOTBEV 389

PLOTBEV 390

PLTB682 105

PLOTBEV 392

PLOTBEV 393

PLOTBEV 394

PLOTBEV 395

PLOTBEV 396

PLOTBEV 397

PLOTBEV 398

PLOTBEV 399

PLOTBEV 400

PLOTBEV 401

PLOTBEV 402

PLOTBEV 403

PLOTBEV 404

PLTB682 106

$X(2)=$ CXTK2

IF (.NOT.LIMSW) GO TO 164

PLTB682

107

$\mathrm{TIK}=\mathrm{YMAX} / 5$.

495

NTK=0 \$IF (YMIN. LT.も.) NTK=-YMIN/TIK

PLTB682

108

$T K=-N T K * T I K \quad \$ N T T=N T K+4$

DO $167 \mathrm{I}=1$,NTT

PLBNV82

PLBNV82

PLBNV82

$\mathrm{TK}=\mathrm{TK}+\mathrm{TIK}$

PLTB682

$Y(1)=T K$

500

$Y(2)=T K$

CALL CCPLOT $(X, Y, 2,4$ HJOIN)

IF (.NOT.LIMSW) GO TO 167

$F L I=(I-N T K) * F L T(J) / 5.0$

WRITE $(98,6) \quad F L I$

505

$C C Y=C C Y M I N+(Y(1)-Y M I N) *(C C Y M A X-C C Y M I N) /(Y M A X-Y M I N)$

CALL CCLTR(CCX,CCY, 0,1)

167 CONTINUE

GO TO 169

$164 \quad \operatorname{IS} 1=\operatorname{ITIK}(\mathrm{J})$

$I S M=Y M A X$

$I S M=I S M$ - IS1

CCXS $=\mathrm{CCX} \$ \mathrm{CCYS}=\mathrm{CCY}$

CCX $=$ CCXS-70.

PLTB682

PLTB682

PLTB682

PLBNV82

PLBNV82

PLBNV82

PLBNV82

PLBNV82

PLTB682

PLTB682

PLTB682

PLTB682

PLOTBEV

PLDRF

PLDRF 
515

520

525

530

535

540

545

550

555

*

$$
\begin{aligned}
& Y(1)=0 . \\
& Y(2)=0 . \\
& X(3)=X(2) \$ Y(3)=Y(2)
\end{aligned}
$$$$
\operatorname{CALL} \operatorname{CCPLOT}(X, Y, 3,4 \mathrm{HJOIN}, 1,1,1 \mathrm{H}-)
$$$$
I S M=A B S \text { (YMIN) }
$$$$
\text { DO } 171 \text { I=IS1, ISM, IS1 }
$$$$
Y(1)=-I
$$$$
Y(2)=-I
$$$$
X(3)=X(2) \$ Y(3)=Y(2)
$$$$
\text { CALL CCPLOT }(X, Y, 3,4 H J O I N, 1,1,1 \mathrm{H}-)
$$$$
\text { IM }=-I
$$$$
\text { WRITE }(98,9) \text { IM }
$$$$
\text { CCY }=\text { CCYMIN +(Y (1)-YMIN }) *(C C Y M A X-C C Y M I N) /(Y M A X-Y M I N)
$$$$
\text { CALL } \operatorname{CCLTR}(\operatorname{CCX}, \operatorname{CCY}, \theta, 1)
$$

171 CONTINUE

CONT INUE

CCX $=$ CCXS $\$$ CCY $=$ CCYS

CCXMAX = CCXMAX $-\mathrm{T} 2$

CCXMIN $=$ SAVXMN

$\begin{array}{lr}\text { PLDRF } & 47 \\ \text { PLOTBEV } & 409 \\ \text { PLOTBEV } & 410 \\ \text { PLOTBEV } & 411\end{array}$

PLFORM 20

PLFORM 21

PLDRF 48

PLDRF $\quad 49$

PLDRF 50

PLOTBEV 413

PLTB682 119

PLTB682 120

PLOTBEV 414

PLOTBEV 415

PLOTBEV 416

PLOTBEV 417

PLOTBEV 418

PLOTBEV 419

PLOTBEV 420

PLOTBEV 421

PLOTBEV 422

PLBNV82 15

PLOTBEV 423

PLOTBEV 424

PLOTBEV 425

PLFORM 22

PLFORM 23

PLTB682 121

PLOTBEV 430

PLOTBEV 431

PLOTBEV 432

PLFORM 24

PLFORM 25

PLDRF 51

PLDRF 52

MAY1179 10

PLDRF 53

PLOTBEV 434

PLOTBEV 435

PLDRF 54

PLOTBEV 436

PLOTBEV 437

PLOTBEV 438

PLOTBEV 439

PLOTBEV 440

PLOTBEV 441

PLOTBEV 442

PLOTBEV 443

PLOTBEV . 444

PLOTBEV 445

PLOTBEV 446

PLOTBEV 447

PLOTBEV 448

PLOTBEV 449

PLOTBEV 450

PLOTBEV 451

PLOTBEV 452 
575

580

585

590

595

600

605

610

615

620

625

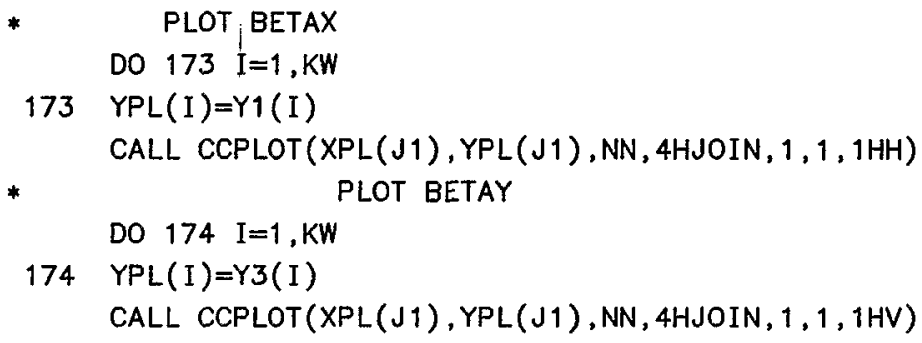

* find paper coORdinates of MAXIMUM POINTS

$\mathrm{M} 1=\mathrm{J} 1$

$M 2=J 1$

$M 3=J 1$

$M 4=J 1$

$J \mathrm{~J}=\mathrm{J} 1+1$

$Y 1 M X=Y 1(J 1)$

$Y 2 M X=Y 2(J 1)$

$Y 3 M X=Y 3(J 1)$

$Y 4 M X=Y 4(J 1)$

$\mathrm{JN}=\mathrm{J} 1+\mathrm{NN}-1$

DO $184 \mathrm{~J}=\mathrm{J} J, \mathrm{JN}$

IF $(Y 1(J) . L E . Y 1 M X)$ GO TO 178

$Y 1 M X=Y 1(J)$

$M 1=\mathrm{J}$

178 IF (Y2(J).LE.Y2MX) GO TO 179

$Y 2 M X=Y 2(J)$

$M 2=J$

179 IF (Y3(J).LE.Y3MX) GO TO 182

$Y 3 M X=Y 3(J)$

$M 3=J$

182 IF (Y4(J).LE.Y4MX) GO TO 184

$Y 4 M X=Y 4(J)$

$M 4=J$

184 CONTINUE

$P Y=Y 1(M 1)$

IF (PY.GT.YMAX) GO TO 183

CCY $=$ CCYMIN $+(P Y-Y M I N) *(C C Y M A X-C C Y M I N) /(Y M A X-Y M I N)$

$\mathrm{PX}=\mathrm{XX}(\mathrm{M1})$

CCX $=$ CCXMIN $+(P X-X M I N) *($ CCXMAX-CCXMIN $) /(X M A X-X M I N)$

IF (M1.EQ.J1) $\quad \operatorname{CCX}=\mathrm{CCX}+8$.

IF (M1.EQ.JN) $\operatorname{CCX}=\operatorname{CCX}-10$.

IF (KB.NE. $\theta$ ) GO TO 181

CALL CCLTR $(\operatorname{CCX}-5, \operatorname{CCY}+11,0,1,1 \mathrm{HW})$

CALL CCLTR(CCX , CCY $+7,0,0,2 \mathrm{HX})$

GO TO 183

181 CONTINUE

CALL CCLTR $(C C X-5 ., C C Y+12,, 0,1,5$ HBETAX)

183 CONTINUE

$P Y=Y 3(M 3)$

IF (PY.GT.YMAX) GO TO 187

$C C Y=C C Y M I N+(P Y-Y M I N) *(C C Y M A X-C C Y M I N) /(Y M A X-Y M I N)$

$P X=X X(M 3)$

$C C X=C C X M I N+(P X-X M I N) *(C C X M A X-C C X M I N) /(X M A X-X M I N)$

IF (M3.EQ.J1) $\quad \mathrm{CCX}=\mathrm{CCX}+8$.
PLOTBEV

PLOTBEV

PLOTBEV

PLOTBEV

PLBNV82

PLOTBEV

PLOTBEV

PLOTBEV

PLB882

PLOTBEV

PLOTBEV

PLOTBEV

PLOTBEV

PLOTBEV

PLOTBEV

PLOTBEV

PLOTBEV

PLOTBEV

PLOTBEV

PLOTBEV

PLOTBEV

PLOTBEV

PLOTBEV

PLOTBEV

PLOTBEV

PLOTBEV

PLOTBEV

PLOTBEV

PLOTBEV

PLOTBEV

PLOTBEV

PLOTBEV

PLOTBEV

PLOTBEV

PLOTBEV

PLOTBEV

PLOTBEV

PLOTBEV

PLOTBEV

PLOTBEV

PLOTBEV

PLOTBEV

PLOTBEV

PLOTBEV

PLOTBEV

PLOTBEV

PLBNV82

PLOTBEV

PLOTBEV

PLTB682

PLOTBEV

PLOTBEV

PLOTBEV

PLOTBEV

PLOTBEV

PLOTBEV

PLOTBEV
453

455

456

457

16

461

462

463

2

467

468

469

470

471

472

473

474

475

476

477

478

479

480

481

482

483

484

485

486

487

488

489

490

491

492

493 
630

640

645

650

655

660

665

670

675

680
IF (M3.EQ.JN) $\quad \operatorname{cCX}=\operatorname{cCX}-10$.

IF (KB.NE.O) GO TO 186

CALL CCLTR(CCX-5.,CCY+11, ,0,1,1HW)

CALL CCLTR(CCX , $\mathrm{CCY}+7,0,0,2 \mathrm{H} \mathrm{Y})$

GO TO 187

186 CONTINUE

CALL CCLTR(CCX-5.,CCY+12, ,0,1,5HBETAY)

187 CONTINUE

PLOT XEQ, YEQ

$Y M A X=F L T(2)$

$Y M I N=F L T(3)$

C IF $Y E Q=0$, DO NOT PLOT IT

IF(M4.EQ.J1.AND.Y4MX.EQ.0.) GO TO 1891

DO $189 \mathrm{I}=1, \mathrm{KW}$

$189 \mathrm{YPL}(\mathrm{I})=Y 4(\mathrm{I})$

CALL CCPLOT(XPL(J1), YPL(J1), NN, 4HJOIN, 1, 1, 1HY)

1891 CONTINUE

IF (.NOT.PSW) GO TO 191

C READJUST LOWER LIMITS FOR SMALL NEGATIVE VALUE

SVCYMIN = CCYMIN

CCY $=($ CCYMAX-CCYMIN $) * .01$

CCYMIN = CCYMIN - CCY

YMIN = YMIN - ONEP

191 DO $188 \mathrm{I}=1, \mathrm{KW}$

$188 \mathrm{YPL}(\mathrm{I})=\mathrm{Y} 2(\mathrm{I})$

CALL CCPLOT(XPL(J1), YPL(J1), NN, 4HJOIN $1,1,1 \mathrm{HX}$ )

DRAW $X$ AXIS

IF (.NOT.PSW) GO TO 195

C RESTORE PREVIOUS LIMITS

YMIN = FLT(3)

CCYMIN = SVCYMIN

GO TO 185

195 IF (YMIN.EQ.0.) GO TO 185

$X(1)=X M I N$

$X(2)=X M A X$

$Y(1)=0$.

$Y(2)=0$.

CALL $\operatorname{CCPLOT}(X, Y, 2,4$ HJOIN)

185 CONTINUE

$P Y=Y 2(M 2)$

IF (PY.GT.YMAX) GO TO 190

$C C Y=C C Y M I N+(P Y-Y M I N) *(C C Y M A X-C C Y M I N) /(Y M A X-Y M I N)$

$\mathrm{PX}=\mathrm{XX}(\mathrm{M} 2)$

$\operatorname{CCX}=\operatorname{CCXMIN}+(P X-X M I N) *($ CCXMAX-CCXMIN $) /(X M A X-X M I N)$

IF (M2.EQ.J1) $\quad \mathrm{CCX}=\mathrm{CCX}+8$.

IF (M2.EQ.JN) $\operatorname{CCX}=\operatorname{CCX}-15$.

CALL CCLTR(CCX-5., CCY+12, ,0,1,3HXEQ)

190

CONTINUE

$P Y=Y 4(M 4)$

IF (PY.GT.YMAX) GO TO 192

IF (M4.EQ.J1.AND.Y4MX.EQ.0.) GO TO 192

$\mathrm{CCY}=\mathrm{CCYMIN}+(\mathrm{PY}-Y M I N) *(C C Y M A X-C C Y M I N) /(Y M A X-Y M I N)$

$\mathrm{PX}=\mathrm{XX}(\mathrm{M4})$

$\mathrm{CCX}=\mathrm{CCXMIN}+(\mathrm{PX}-\mathrm{XMIN}) *($ CCXMAX-CCXMIN $) /($ XMAX-XMIN $)$

IF (M4.EQ.J1) $\quad \mathrm{CCX}=\mathrm{CCX}+8$.
PLOTBEV 515

PLOTBEV 516

PLOTBEV 517

PLBNV82 18

PLOTBEV 519

PLOTBEV 520

PLTB682 135

PLOTBEV 522

PLOTBEV 523

PLOTBEV 524

PLOTBEV 525

PLOTBEV 526

MAY1179. 11

MAY1179 12

PLOTBEV 530

PLOTBEV 531

PRCH 8

MAY1179 13

PLTB682 136

PLTB682 137

PLTB682 138

PLTB682 139

PLTB682 140

PLTB682 141

PLTB682 142

PLTB682 143

PLTB682 144

PLTB682 145

PLTB682 146

PLTB682 147

PLTB682 148

PLTB682 149

PLTB682 150

PLTB682 151

PLTB682 152

PLTB682 153

PLTB682 154

PLTB682 155

PLTB682 156

PLTB682 157

PLTB682 158

PLTB682 159

PLTB682 160

PLTB682 161

PLTB682 162

PLTB682 163

PLTB682 164

PLTB682 165

PLTB682 166

PLOTBEV 551

PLOTBEV 552

PLOTBEV 553

MAY1179 14

PLOTBEV 554

PLOTBEV 555

PLOTBEV 556

PLOTBEV 557 
IF (M4.EQ.JN) $\quad \operatorname{cCX}=\operatorname{cCX}-15$.

CALL CCLTR $(C C X-5,, C C Y+12,, 0,1,3$ HYEQ $)$

PLTB682 167

CONT INUE

PLOTBEV

CALL CCNEXT

PLOTBEV

561

200 CONTINUE

690

*

IF PLOTTED W, RETURN ARRAYS TO BETA

PLOTBEV

562

IF (KB.NE.0) RETURN

PLOTBEV

563

DO $202 \mathrm{I}=1, \mathrm{KW}$

PLOTBEV

564

$Y 1(I)=Y 1(I) * Y 1(I)$

PLOTBEV

565

$Y 3(I)=Y 3(I) * Y 3(I)$

PLOTBEV

566

695

202 CONTINUE

PLOTBEV

567

RETURN

PLOTBEV

568

PLOTBEV 569

* $\quad$ error in INPUT Plot positions

PLOTBEV

570

205 PRINT 10, IPRS

1 FORMAT (8A10)

PLOTBEV

571

700

2 FORMAT (A4)

3 FORMAT (F8.2)

4 FORMAT (6A10)

5 FORMAT (F8.1)

6 FORMAT (F7.2)

PLOTBEV

572

PLOTBEV 573

PLOTBEV 574

: PLOTBEV 575

PLTB682 168

705

8 FORMAT (I3)

PLBNV82 19

PLOTBEV 576

PLOTBEV 577

10

FORMAT (I5)

CANNOT PLOT FROM POSITION *, I5, * TO POSITION*, PLOTBEV

578

1 I5)

END

PLOTBEV 579

PLOTBEV

580

\begin{tabular}{|c|c|}
\hline 402 & I \\
\hline 416 & I \\
\hline 419 & I \\
\hline 423 & I \\
\hline 426 & I \\
\hline 430 & I \\
\hline 433 & I \\
\hline 437 & I \\
\hline 442 & I \\
\hline 448 & I \\
\hline 475 & I \\
\hline 478 & I \\
\hline 501 & I \\
\hline 506 & I \\
\hline 522 & I \\
\hline 550 & I \\
\hline 616 & I \\
\hline 617 & I \\
\hline 620 & I \\
\hline 630 & I \\
\hline 631 & I \\
\hline 634 & I \\
\hline 666 & I \\
\hline 676 & I \\
\hline 686 & I \\
\hline
\end{tabular}

ARGUMENT COUNT INCONSISTENT WITH PRIOR USAGE. ARGUMENT COUNT INCONSISTENT WITH PRIOR USAGE. ARGUMENT COUNT INCONSISTENT WITH PRIOR USAGE. ARGUMENT COUNT INCONSISTENT WITH PRIOR USAGE. ARGUMENT COUNT INCONSISTENT WITH PRIOR USAGE. ARGUMENT COUNT INCONSISTENT WITH PRIOR USAGE. ARGUMENT COUNT INCONSISTENT WITH PRIOR USAGE. ARGUMENT COUNT INCONSISTENT WITH PRIOR USAGE. ARGUMENT COUNT INCONSISTENT WITH PRIOR USAGE. ARGUMENT COUNT INCONSISTENT WITH PRIOR USAGE. ARGUMENT COUNT INCONSISTENT WITH PRIOR USAGE. ARGUMENT COUNT INCONSISTENT WITH PRIOR USAGE. ARGUMENT COUNT INCONSISTENT WITH PRIOR USAGE. ARGUMENT COUNT INCONSISTENT WITH PRIOR USAGE. ARGUMENT COUNT INCONSISTENT WITH PRIOR USAGE. ARGUMENT COUNT INCONSISTENT WITH PRIOR USAGE. ARGUMENT COUNT INCONSISTENT WITH PRIOR USAGE. ARGUMENT COUNT INCONSISTENT WITH PRIOR USAGE. ARGUMENT COUNT INCONSISTENT WITH PRIOR USAGE. ARGUMENT COUNT INCONSISTENT WITH PRIOR USAGE. ARGUMENT COUNT INCONSISTENT WITH PRIOR USAGE. ARGUMENT COUNT INCONSISTENT WITH PRIOR USAGE. ARGUMENT COUNT INCONSISTENT WITH PRIOR USAGE. ARGUMENT COUNT INCONSISTENT WITH PRIOR USAGE. ARGUMENT COUNT INCONSISTENT WITH PRIOR USAGE. 
LEVEL 2, STORE, INFF, IWORK

COMMON STORE (48000), IWORK (10)

DIMENSION INFF $(24,2000)$

EQUIVALENCE (INFF, STORE)

BLANK

86MARSIZ

86MARSIZ

86MARSIZ

COMMON/SWTCH/BEND, MSIZE, CYCSWT, VCSW, PV, MSSW, VPR, MHS, MODS, MINZER

BLANK

SWTCH

LOGICAL CYCSWT, MSSW, VCSW, PV, VPR, MHS, MODS

10

LOGICAL BEGIN

DIMENSION NAM(7),V(10,7), IH(12), CV(70)

EQUIVALENCE $(C V, V)$

INTEGER ELNUM, BDAT

SWTCH

SWTCH 4

PRINTV 5

PRINTV 6

PRINTV 7

PRINTV 8

PRINTV 9

PRINTV 10 12H11,2H12/

DATA IM, IB/1H-, $1 \mathrm{H} /$

BEGIN $=$. TRUE.

$J J=0$

DO $100 \quad \mathrm{~J} J=1, K$

IF (PV) GO TO 13

$N M=\operatorname{BDAT}(M, I J)$

25

$M 1=\operatorname{ELNUM}(N M)$

PR INTV

PRINT:

PRINTV

PRINTV

PRINTV

PRINTV

PRINTV

PRINTV

PRINTV

PRINTV

PRINTV

PRINTV

PRINTV

PRINTV

PRINTV

PRINTV

PRINTV

PRINTV

PRINTV

PRINTV

PRINTV

PRINTV

PRINTV

PRINTV

PRINTV

PRINTV

PRINTV

PRINTV

PRINTV

PRINTV

PRINTV

PRINTV

PRINTV

PRINTV

PRINTV

PRINTV

PRINTV

PRINTV

PRINTV

PRINTV

PRINTV

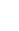

2 3 1 3 5 2 4 
20 CONTINUE

IF (NTYPE.NE.4HSNGL) GO TO 35

PRINTV $\quad 52$

60 BEGIN $=$.FALSE.

PRINTV 53

IF (ISUB.EQ.7.OR.IJ.EQ.K) GO TO 22

PRINTV 54

NROW1 = NROW

GO TO 100

21 ASSIGN 15 TO IRET

65

$\mathrm{NR}=$ NROW1

GO TO 25

22 ASSIGN 100 TO IRET

$\mathrm{NR}=\mathrm{NROW}$

70

C TIME TO PRINT

75

25 WRITE $(3,1)$

CALL DASH

WRITE $(3,4)(\operatorname{NAM}(J), J=1, J J)$

DO $30 \mathrm{~L}=1$, NR

WRITE $(3,5)(V(L, J), J=1, J J)$

30 CONTINUE

GO TO 40

C PRINT COMPLEX OR DOUBLE PRECISION VECTORS

35 WRITE $(3,1)$

CALL DASH

80

IF (NTYPE.EQ. 4HCPLX) GO TO 37

WRITE $(3,9)$ NAM(1)

GO TO 38

37 WRITE $(3,8)$ NAM(1)

38 WRITE $(3,10) \quad(\mathrm{CV}(\mathrm{J}), \mathrm{J}=1, \mathrm{NC})$

ASSIGN 100 TO IRET

85

$40 \quad$ BEGIN $=$. TRUE.

$J J=\theta$

GO TO IRET $(15,100)$

90

100 CONTINUE

95

CALL DASH

RETURN

FORMAT $(/ /)$

FORMAT $(4 X, 7(9 X, A 7))$

FORMAT $(4 X, 7(2 X, F 14,8))$

FORMAT $(1 X, I 1,8 X)$

FORMAT (A4, A1 , A2, 3X)

FORMAT (16H COMPLEX VECTOR, A5)

FORMAT (25H DOUBLE PRECISION VECTOR , A5)

100

FORMAT $(30 X, 2(E 14.8,5 X))$

END

PRINTV 55

PRINTV $\quad 56$

PRINTV 57

PRINTV 58

PRINTV 59

PRINTV 60

PRINTV 61

PRINTV 62

PRINTV 63

PRINTV 64

PRINTV 65

PRINTV 66

PRINTV 67

PRINTV 68

PRINTV 69

PRINTV 70

PRINTV 71

PRINTV 72

PRINTV 73

PRINTV 74

PRINTV $\quad 75$

PRINTV $\quad 76$

PRINTV 77

PRINTV 78

PRINTV $\quad 79$

PRINTV 80

PRINTV . 81

PRINTV 82

PRINTV 83

PRINTV 84

PRINTV 85

PRINTV $\quad 86$

PRINTV 87

PRINTV 88

PRINTV $\quad 89$

PRINTV 90

PRINTV 91

PRINTV 92

PRINTV 93

PRINTV 94 
SUBROUT INE PRNT (M)

PRNT

C PRINTS THE KA-TH PARAMETER OF TYPE KB FOR SPECIFIED ELEMENTS.

PRNT

C DEFAULTS ARE PARAMETER 1 OF TYPE 1

PRNT

BLANK

LEVEL 2, STORE, INFF, IWORK

COMMON STORE (48000), IWORK(10)

DIMENSION INFF $(24,2000)$

EQUIVALENCE (INFF, STORE)

10

COMMON/BMI/MI (5000)

COMMON/NELS/NELS

LEVEL 2,MI

15

C

DIMENSION VAL(100), IVAL(100), NAMTYPE(8), NAM(100)

EQUIVALENCE (VAL, IVAL)

INTEGER BDAT, ELNUM

20

C

DATA LIM, IBLNK/10,1H /

DATA NAMTYPE/5HFL PT, 3HBCD, 3HINT, $1 \mathrm{H}, 2 \mathrm{HLQ}, 10 \mathrm{HFL}$ STORAGE,

C

1 3HLQ2, 3HLQ3/

C RETRIEVE ELEMENTS, PARAMETER AND TYPE. SET DEFAULTS.

$\operatorname{NUM}=\operatorname{INFF}(17, M)$

$\operatorname{KNDX}=\operatorname{INFF}(4, M)$

$K T Y P E=\operatorname{INFF}(5, M)$

IF (KNDX.EQ.0) KNDX $=1$

IF (KTYPE.EQ.0) KTYPE $=5$

30

$J=0$

DO $10 \quad I=1$, NUM

NAME $=\operatorname{BDAT}(M, I)$

IF (NAME.EQ.IBLNK) GO TO 10

$J=J+1$

$\operatorname{NAM}(J)=$ NAME

$M I(J)=\operatorname{ELNUM}(N A M E)$

10 CONTINUE -

NUM $=\mathrm{J}$

40

C PRINT HEADING

45

WRITE $(3,100)$ KNDX, KTYPE, NAMTYPE (KTYPE)

DO $18 \mathrm{I}=1$, NUM

$\mathrm{NK}=\mathrm{MI}(\mathrm{I})$

GO TO $(15,12,13,14,15,15,15,15)$ KTYPE

$12 \operatorname{IVAL}(I)=\operatorname{BDAT}(\mathrm{NK}, \mathrm{KNDX})$

GO TO 18

$13 \operatorname{IVAL}(\mathrm{I})=\operatorname{IDAT}(\mathrm{NK}, \mathrm{KNDX})$

GO TO 18

14 RETURN

15 CALL DATA (NK, KTYPE, KNDX, 1, VAL (I))

50

18 CONTINUE

C

C WRITE NAMES OF ELEMENTS WITH VALUES UNDERNEATH, 10 TO A LINE.

$\mathrm{NM}=0$

$20 \quad \mathrm{I} 1=\mathrm{NM}+1$

$N M=N M+L I M$

IF (NM.GT.NUM) NM = NUM

WRITE $(3,105)$ (NAM(I), I=I $1, N M)$

BLANK

86MARSIZ

86MARSIZ

86MARSIZ

BLANK

BMIL

BMIL

BMIL

BMIL

BMI

PRNT

PRNT

PRNT

PRNT

PRNT

PRNT

PRNT

PRNT

PRNT

PRNT

PRNT

PRNT

PRNT

PRNT

PRNT

PRNT

PRNT

PRNT

PRNT

PRNT

PRNT

PRNT

PRNT

PRNT

PRNT

PRNT

PRNT

PRNT

PRNT

PRNT

PRNT

PRNT

PRNT

PRNT

PRNT

PRNT

PRNT

PRNT

PRNT

PRNT

PRNT

PRNT

PRNT

2

3

4

2

3

1

2

3

5

1

2

3 
60

70

75

65
GO TO $(21,22,23,24,21,21,21,21)$ KTYPE

21

WRITE $(3,106) \quad(\operatorname{VAL}(\mathrm{I}), I=I 1, \mathrm{NM})$

GO TO 25

22

WRITE $(3,107)$ (IVAL (I), I=I $1, N M)$

GO TO 25

23

WRITE $(3,108)$ (IVAL(I), I=I $1, N M)$

GO TO 25

24

25

100

RETURN

IF (NM.NE.NUM) GO TO 20

WRITE $(3,110)$

RETURN

105 FORMAT $(/ 1 X, 10($

106 FORMAT $(2 X, 10(F 12.6))$

107 FORMAT $(10(8 X, A 4))$

108 FORMAT $(10(2 X, I 10))$

110 FORMAT $(1 \times, 130(1 \mathrm{H}-))$

END

$\begin{array}{ll}\text { PRNT } & 50 \\ \text { PRNT } & 51 \\ \text { PRNT } & 52 \\ \text { PRNT } & 53 \\ \text { PRNT } & 54 \\ \text { PRNT } & 55 \\ \text { PRNT } & 56 \\ \text { PRNT } & 57 \\ \text { PRNT } & 58 \\ \text { PRNT } & 59 \\ \text { PRNT } & 60 \\ \text { PRNT } & 61 \\ \text { PRNT } & 62 \\ \text { PRNT } & 63 \\ \text { PRNT } & 64 \\ \text { PRNT } & 65 \\ \text { PRNT } & 66 \\ \text { PRNT } & 67 \\ \text { PRNT } & 68 \\ \text { PRNT } & 69\end{array}$


LEVEL 2, STORE, INFF, IWORK

COMMON STORE(48000), IWORK(10)

5

DIMENSION INFF $(24,2000)$

EQUIVALENCE (INFF, STORE)

BLANK

BLANK

EQUIVALENCE (IVAR, FVAR)

DIMENSION NAM(10), NCOL (10), F(10)

10

INTEGER ELNUM

LOGICAL PRES

86MARSIZ

86MARSIZ

86MARSIZ

BLANK

PRNTAB

PRNTAB

PRNTAB

PRNTAB

PRNTAB

C IF $M$ IS INDEX OF TAB INSTRUCTION, PRINT THAT ONE ONLY

PRNTAB

PRES $=$.FALSE.

C CALLED FROM TABULATE WHEN VECTOR FILLED

PRES $=$. TRUE.

$\mathrm{IK}=1$

$\operatorname{NAM}(1)=\operatorname{INFF}(2, M)$

200 CONTINUE

$K A=\operatorname{INFF}(4, M)$

$\mathrm{IK}=\mathrm{KA}$

C PICK UP TAB NAMES

CALL DATA $(M, 2,1, K A, N A M)$

205 CONTINUE

DO $220 \mathrm{I}=1, \mathrm{IK}$

C GET INDEX OF TABLE NAME AND NUMBER OF TABLE ENTRIES

$\operatorname{INDX}=\operatorname{ELNUM}(\operatorname{NAM}(\mathrm{I}))$

NUM $=\operatorname{INFF}(4, \operatorname{INDX})$

C GET NAMES FOR COLUMN HEADINGS AND PRINT THEM

CALL DATA(INDX, 2,1,NUM, NCOL)

WRITE $(3,1)(\operatorname{NCOL}(J), J=1, N U M)$

C GET CURSOR. COMPUTE NUMBER OF ROWS TO BE PRINTED

PRNTA

PRNTAB

PRNTAB

PRNTAB

PRNTAB

PRNTAB

PRNTAB

PRNTAB

PRNTAB

PRNTAB

PRNTAB

PRNTAB

PRNTAB

PRNTAB

PRNTAB

PRNTAB

PRNTAB

PRNTAB

PRNTAB

PRNTAB

PRNTAB

$L Q=\operatorname{INFF}(10, \operatorname{INDX})$

FVAR = STORE (LQ)

$L C=I V A R$

$N C=(L C-2) / N U M$

$\mathrm{IC}=2$

40

DO $210 \mathrm{~J}=1, \mathrm{NC}$

CALL DATA (INDX, 5, IC, NUM, F)

IAST $=55 \mathrm{~B}$

NK $=0$

DO $209 \mathrm{~K}=1$, NUM

45

209 IF (F(K).EQ.0.) NK $=\mathrm{NK}+1$

IF (NK.EQ.NUM) IAST $=47 B$

WRITE $(3,2)$ IAST, $(F(K), K=1, N U M)$

$I C=I C+N U M$

50

210 CONTINUE

C RESET CURSOR

I $V A R=2$

$\operatorname{STORE}(L Q)=F V A R$

220 CONTINUE

C IF CALLED FROM TAB, RESTORE PAGE.

100 FORMAT (1H1)

RETURN

PRNTAB 31

PRNTAB 32

PRNTAB 33

PRNTAB 34

PRNTAB 35

PRNTAB 36

PRNTAB 37

PRNTAB 38

PRNTAB 39

PRNTAB 40

PRNTAB 41

PRNTAB 42

PRNTAB 43

PRNTAB 44

PRNTAB $\quad 45$

PRNTAB $\quad 46$

PRNTAB $\quad 47$

PRNTAB 48

PRNTAB $\quad 49$

PRNTAB 50

PRNTAB 51

PRNTAB 52

PRNTAB 53 
1 FORMAT (1H1,6X,10(7X,A5)//)

PRNTAB 54

2 FORMAT ( $4 X, A 1,2 X, 10 F 12.8)$

PRNTAB

55

60 
LEVEL 2, STORE, INFF, IWORK

PRNTV7 3

COMMON STORE(48000), IWORK(10)

BLANK 2

DIMENSION INFF $(24,2000)$

EQUIVALENCE (INFF, STORE)

BLANK 3

COMMON/SWTCH/BEND, MSIZE, CYCSWT, VCSW, PV, MSSW, VPR, MHS, MODS, MINZER

LOGICAL PH

DIMENSION $V(7)$

INTEGER ELNUM,BDAT

SWTCH

SWTCH

PRNTV7

PRNTV7

PRNTV7

C PV IS LOGICAL VARIABLE THAT TELLS PRINTV IT HAS BEEN CALLED BY PRNTV7

PRNTV7

C AND SO DOES NOT HAVE LIST OF DIFFERENT VECTORS TO PRINT.

PRNTV7 10

C PH CONTROLS COLUMN HEADING. IF PRINTV WAS CALLED, NEW HEADING

PRNTV7

PRNTV7

C IS NEEDED.

$\mathrm{PH}=$. FALSE.

CALL DASH

DO $100 \quad I=1, K$

$P V=$. FALSE.

LOC $=1$

$$
\mathrm{NM}=\operatorname{BDAT}(M, I)
$$

$M 1=\operatorname{ELNUM}(N M)$

NROW $=\operatorname{INFF}(22, M 1)$

$N T Y P E=I N F F(21, M 1)$

PRNTV7 13

PRNTV7 14

PRNTV7 15

PRNTV7 16

PRNTV7 17

PRNTV7 18

PRNTV7 19

PRNTV7 20

PRNTV7 21

PRNTV7 22

C IF VECTOR IS NOT 7 ROW OR IF COMPLEX OR DOUBLE,CALL OTHER PRINT ROUT.

IF (NROW.EQ.7.AND.NTYPE.EQ.4HSNGL) GO TO 20

PRNTV7 23

$\mathrm{PV}=$. TRUE.

PRNTV7 24

PRNTV7 25

CALL PRINTV $(M 1,1)$

PRNTV7 26

$P V=$. FALSE.

PRNTV7 27

$\mathrm{PH}=$. FALSE.

GO TO 100

PRNTV7 28

C PARTICLE VECTOR WITH 7 ROWS

PRNTV7 29

PRNTV7 30

$20 \mathrm{NCOL}=\operatorname{INFF}(23, \mathrm{M1})$

$\mathrm{LOC}=1$

PRNTV7 31

IF (.NOT.VCSW) GO TO 25

40

DECODE $(10,2, N M)$ NUM

PRNTV7

PRNTV7 33

$L O C=(N U M-1) * 7+1$

PRNTV7

PRNTV7 35

$\mathrm{NCOL}=1$

25 IF (.NOT.PH) WRITE $(3,10)$

PRNTV7 36

DO $30 \mathrm{~J}=1, \mathrm{NCOL}$

CALL DATA (M1,5, LOC $, 7, V)$

PRNTV7 37

PRNTV7 38

PRNTV7 39

28

$\mathrm{J} J=\mathrm{J}$

IF (VCSW) J J=NUM

WRITE $(3,13)$ NM, JJ, $(V(L), L=1,7)$

PRNTV7 40

PRNTV7 41

PRNTV7 42

$29 \quad \mathrm{LOC}=\mathrm{LOC}+7$

30 CONTINUE

PRNTV7 43

$\mathrm{PH}=$. TRUE.

PRNTV7 44

PRNTV7 45

100 CONTINUE

PRNTV7 46

CALL DASH

PRNTV7 47

WRITE $(3,14)$ 
14HDP/P)

13 FORMAT (5X, A4, I $2,2 X, 7(F 10.5,1 X))$ 
1

5

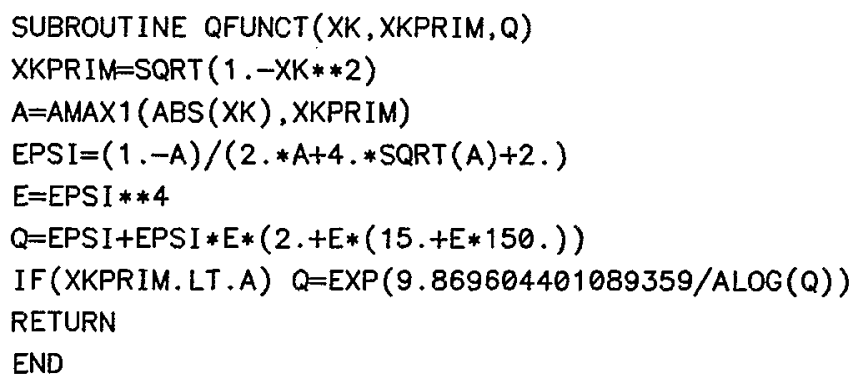

$\begin{array}{lr}\text { QFUNCT } & 2 \\ \text { QFUNCT } & 3 \\ \text { QFUNCT } & 4 \\ \text { QFUNCT } & 5 \\ \text { QFUNCT } & 6 \\ \text { QFUNCT } & 7 \\ \text { QFUNCT } & 8 \\ \text { QFUNCT } & 9 \\ \text { QFUNCT } & 10\end{array}$

CARD NR. SEVERITY DETAILS DIAGNOSIS OF PROBLEM CONSTANT TOO LONG. HIGH ORDER DIGITS RETAINED, BUT SOME PRECISION LOST. 
1

QFUNCT

QUEX

SUBROUTINE QUEX (X,DQX)

QUEX

$1, \mathrm{~K} 1, \mathrm{~K} 2$

5

COMMON/GRR/I ERR, BSW, VSW, RSW, QSW, XSW

LOGICAL IERR, BSW, VSW, RSW, QSW, XSW

QUEX

GRR

GRR

EXTERNAL QUEY

IF (IERR) RETURN

10

$V_{1}=X$

$\operatorname{CALL} \operatorname{GRTB}(1, \mathrm{~V} 2,7, \mathrm{QUEY})$

$\mathrm{DQX}=(\mathrm{CQX}-\mathrm{CQXZ}) * * 2+\mathrm{G} * \mathrm{G}$

RETURN

END

GRR

QUEX

QUEX

QUEX

QUEX

QUEX

QUEX

QUEX

2


$1, \mathrm{~K} 1, \mathrm{~K} 2$

COMMON/GRR/IERR, BSW, VSW, RSW, QSW, XSW

QUEY

LOGICAL IERR, BSW, VSW, RSW, QSW, XSW

GRR

GRR

GRR

INTEGER P1,P2

DIMENSION RX(2,3), RY( 2,3$), \operatorname{RW}(3)$

QUEY

QUEY

DIMENSION RXRY (12), V(7), BXBY(20)

QUEY

QUEY

$\mathrm{V} 2=\mathrm{Y}$

CALL REPFLT (M1,P1, V1)

CALL REPFLT (M2,P2,V2)

QUEY

QUEY

QUEY

QUEY

CALL DOIT(MSR)

15

IF (BSW) GO TO 3

QUEY

QUEY

IF (VSW) GO TO 4

IF (RSW) GO TO 8

QUEY

QUEY

CALL RXY (MC, RX, RY, RW)

QUEY

QUEY

$\mathrm{CQY}=(\operatorname{RY}(1,1)+\operatorname{RY}(2,2)) * .5$

GO TO 5

CALL BET(MC, BXBY (1), $\operatorname{BXBY}(11), 0)$

QUEY

QUEY

QUEY

QUEY

QUEY

QUEY

QUEY

QUEY

QUEY

QUEY

QUEY

QUEY

QUEY

QUEY

QUEY

QUEY

QUEY

QUEY

$\mathrm{DQY}=\mathrm{CQY}-\mathrm{CQYZ}$

$G=D Q Y$

RETURN

END 
1

SUBROUTINE RDINF(INF, M)

C READS ONE BLOCK OF INFF(M) INTO INF

5

10
LEVEL 2, STORE, INFF, IWORK

COMMON STORE(48000), IWORK(10)

DIMENSION INFF $(24,2000)$

EQUIVALENCE (INFF, STORE)

DIMENSION INF(1)

DO $20 I=1,24$

$20 \operatorname{INF}(I)=\operatorname{INFF}(I, M)$

RETURN

END

$\begin{array}{ll}\text { RDINF } & 2 \\ \text { RDINF } & 3 \\ \text { BLANK } & 2 \\ \text { BLANK } & 3 \\ \text { 86MARSIZ } & 1 \\ \text { 86MARSIZ } & 2 \\ \text { 86MARSIZ } & 3 \\ \text { BLANK } & 5 \\ \text { RDINF } & 5 \\ \text { RDINF } & 6 \\ \text { RDINF } & 7 \\ \text { RDINF } & 8 \\ \text { RDINF } & 9\end{array}$


C PROCESSES FIRST CARD OF NEW INSTRUCTION WHICH IS CURRENTLY IN CARD

(8) RDINST

C BY STORING NAME, OPNAME.

RDINST

COMMON/INSTR/OPNAME, NAME, OP, KA, KB, OBJA, OBJB, NXTM, TRA, LQ, NQ, LIN,

INSTR

5 NIN, LFL, NFL, LBC, NBC, NTOT , IX, KIND, TYPE, ROWS, COLS, EXTR, INSTR

$M, N F, N B, N I$, MSUBR, ISIGN
INTEGER OPNAME, OP, OBJA, OBJB, TRA, TYPE, ROWS, COLS, EXTR

INSTR

INSTR

COMMON/STORE/LMAX, LINF, LFI LE, MAX, MIN, ICARD (11)

* lmaX aND MAX ARE DiMENSIONS of STORE AND INFF

INSTR

STORE

STORE

COMMON/CONTRL/ERROR, MODE, RSRV , STOR, XEQ, TRASW, NFSW, EMPTY, INDEF,

1 LDFLG, FIN

LOGICAL ERROR, RSRV, STOR, XEQ, TRASW, NFSW, EMPTY, INDEF, LDFLG, FIN

STORE

CONTRL

CONTRL

CONTRL

COMMON /DIM/ LEND, MEND, ISAV, ISAV7, M7END, KADD, KADDR, MUNIT , MSYMPL

CONTRL

DIM

INTEGER OPNUM, HPERIOD

DIMENSION IC(24)

EQUIVALENCE( IC(1), OPNAME )

DATA HPERIOD, NULL/1H. , 4HNULL/

DIM

RDINST

RDINST

RDINST

RDINST

IF(MIN.GE.1) GO TO 7

RDINST

WRITE $(3,200)$

RDINST

RDINST

200 FORMAT $(6 \mathrm{H} 0 * * * * *, *$ ELEMENTS OVERFLOWED. INCREASE INFF ARRAY AND *,

$1 *$ MAX.*)

NSTO = LMAX - LFILE

NINFF $=$ MAX - MIN

WRITE $(3,201)$ LMAX, LFILE, NSTO, MAX, NINFF, M

RDINST

RDINST

RDINST

RDINST

RDINST

201 FORMAT(/1X*CORE USE SUMMARY*,27X,*MAXIMUM*,17X,*USED*,8X, *UNUSED*/ RDINST

$134 X, *$ STORE (ELEMENT STORAGE)*,14X, I10,1X,*(LMAX)*,4X, I10,5X, I10/ RDINST

$234 \mathrm{X}, *$ INFF (ELEMENT DEFINITIONS) *,10X, I10,1X,*(MAX)*,5X, I10,5X,

3 I 10)

ERROR $=$. TRUE.

RDINST 23

RDINST 24

RDINST 25

RETURN

7 CONTINUE

C

C ZERO COMMON BLOCK/INSTR/,BUT DONT ZERO M

DO $10 \quad I=1,24$

$10 \mathrm{IC}(\mathrm{I})=0$

IC $(21)=4$ HSNGL

$\mathrm{NFSW}=$.FALSE.

IF (. NOT. LDFLG) EMPTY=.FALSE.

RDINST 26

RDINST 27

RDINST 28

RDINST 29

RDINST $\quad 30$

RDINST 31

RDINST 32

RDINST 33

LDFLG $=$. FALSE.

RDINST 34

RDINST 35

IF (EMPTY) GO TO 4

RDINST

EMPTY $=$. TRUE.

$\operatorname{READ}(2,5)(\operatorname{ICARD}(I W), I W=1,8)$

RDINST

RDINST

RDINST

RDINST

RDINST

RDINST

3 FORMAT (A1,9X)

42

* IF ISIGN = P OR $C$, IT IS PAGE OR COMMENT AND NEEDS SPECIAL HANDLING IF (ISIGN.EQ.1HP) GO TO 100

RDINST 43

IF (ISIGN.EQ.1HC) GO TO 100

RDINST $\quad 44$

IF (ISIGN.EQ.HPERIOD) GO TO 100

RDINST

RDINST

RDINST

DECODE $(20,2$, ICARD $)$ ISIGN, NAME, OPNAME, KA, KB

RDINST 
2 FORMAT ( $A 1, A 5,1 X, A 5, I 3,1 X, I 3,1 X)$

RDINST $\quad 49$

C KA,KB BECOME - $\theta$ IF BLANK. RESET TO + 0 .

RDINST

IF (KA.EQ.0) $K A=0$

RDINST

IF (KB.EQ.0) KB=0

RDINST

RDINST

$\mathrm{OP}=$ OPNUM (OPNAME)

RDINST

IF (OPNAME. EQ. 3HRUN) $M=$ M7END -ISAV7

RDINST

CALL WRTINF ( IC,M )

RDINST

RDINST

RDINST

RDINST

RDINST

RDINST 
1

10
FUNCTION REALNUM(LOCI)

C

LEVEL 2, STORE, INFF, IWORK

COMMON STORE (48000), IWORK (10)

DIMENSION INFF $(24,2000)$

EQUIVALENCE (INFF, STORE)

COMMON/FLTN/IFL(15)

COMMON/TSW/TRSW, MCY (20), JM, LOCC

LOGICAL TRSW

COMMON/CONTRL/ERROR, MODE, RSRV, STOR, XEQ, TRASW, NFSW, EMPTY, INDEF,

1

LOGICAL ERROR, RSRV, STOR, XEQ, TRASW, NFSW, EMPTY, INDEF, LDFLG , FIN

C

INTEGER ELNUM, HRAND, RTBLNK

LOGICAL NEG, INEG

DIMENSION $Y(2)$

EQUIVALENCE (IVAR, FVAR), (NMVAR, FMVAR)

EQUIVALENCE (LOC, LOCC)

C

DATA MASK/00007 777777777777777 B/

DATA MASK1/77777 777770000000000 B/

DATA MASKF/77000 $000000000000000 \mathrm{~B} /$

DATA RTBLNK/00000 000005555555555 B/

C

DATA HRAND/4HRAND/

NEG $=$. FALSE.

$\mathrm{ITR}=0$

$\mathrm{LOC}=\mathrm{LOCI}$

C

$20 \quad$ FVAR $=$ STORE $(L O C)$

21 REALNUM=FVAR

C TEST FOR SYMBOLIC

NR $=$ IVAR. AND. MASK

$\mathrm{NL}=$ IVAR.AND. . NOT.MASK

IF(NL.NE.0.OR.NR.EQ.0) GO TO 80

C SYMBOLIC. NESTING CANNOT EXCEED 10

25 ITR $=$ ITR + 1

IF (ITR.GT.10) GO TO 90

C GET ELEMENT NAME

$\operatorname{DECODE}(10,110$, IVAR) NMVAR

IF (NMVAR.NE.1H\$) GO TO 28

REALNUM = FMVAR

RETURN

28 DECODE $(5,100$, IVAR $)$ NPT

100 FORMAT $(2 X, I 3)$

MSK $=$ MASKF. AND $\cdot$ NMVAR

C CHECK FOR +, - IN ELEMENT NAME

IF (MSK.NE.IFL(1)) GO TO 29

NEG $=($. NOT. NEG)

29 IF (MSK.NE.IFL(1).AND.MSK.NE.IFL(12)) GO TO 30

$\operatorname{DECODE}(6,105$, NMVAR) NMVAR

105 FORMAT ( 1 XA5)

$30 \quad M N=E L N U M(N M V A R)$
REALNUM 2

REALNUM 3

BLANK 2

BLANK 3

86MARSIZ 1

86MARSIZ 2

86MARSIZ 3

BLANK 5

FLTN 2

FLTN 3

NOV3TSW 1

TSW 3

CONTRL 2

CONTRL 3

CONTRL 4

CONTRL 5

REALNUM 7

REALNUM 8

REALNUM 9

REALNUM 10

REALNUM 11

NOV3RLNM 2

REALNUM 12

REALNUM 13

REALNUM 14

REALNUM 15

REALNUM 16

REALNUM 17

REALNUM 18

REALNUM 19

REALNUM 20

REALNUM ' 21

REALNUM 22

REALNUM 23

RLNTIM 1

REALNUM 25

REALNUM 26

REALNUM 27

RLNTIM 2

REALNUM $\quad 33$

REALNUM 34

REALNUM 35

RLNTIM 3

RLNTIM 4

REALNUM 40

REALNUM 41

REALNUM $\quad 42$

RLNTIM 5

REALNUM $\quad 45$

REALNUM 46

REALNUM 47

REALNUM 48

RLRAND 1

REALNUM 52

RLNTIM 6

RLNTIM 7

REALNUM 56 


\section{LQ IS DEFAULT}

60

$$
\text { IF (NPT.EQ.0) NPT }=-1
$$

IF (NPT.LT.O) GO TO 50

$\operatorname{LFMN}=\operatorname{INFF}(14, \mathrm{MN})$

$L O C=L F M N-1+N P T$

GO TO 20

65

70

75

80

85

90

$$
\text { RETRIEVE FROM LQ }
$$

C GET LOCC TO PASS THRU COMMON BLOCK FOR CHROM CORRECTION

$50 \quad \operatorname{LOCC}=\operatorname{INFF}(10, \mathrm{MN})-N P T-1$

REALNUM=FLDAT (MN, -NPT)

FVAR=REALNUM

$\operatorname{IF}(\operatorname{INFF}(1, M N)$.NE.HRAND) GO TO 21

C RANDOM VALUE $=V_{1}<-(V 2 / 2)$

DO $58 \mathrm{I}=1,2$

FVAR = FLDAT $(M N, I)$

$N R=$ IVAR . AND . MASK

$\mathrm{NL}=$ IVAR.AND. .NOT.MASK

IF (NL.EQ.O.AND.NR.NE.0) GO TO 56

$Y(I)=$ FVAR

GO TO 58

56 DECODE $(10,110$, NR) NAM2

110 FORMAT $(5 X, A 5)$

$Y(I)=\operatorname{FLDAT}(\operatorname{ELNUM}($ NAM2 $), 1)$

58 CONTINUE

REALNUM $=Y(1)+Y(2) *($ RANF $(D)-.5)$

80 CONTINUE

IF (NEG) REALNUM=-REALNUM

RETURN

C EXCEEDED DEPTH OF SYMBOLIC

90 WRITE $(3,115)$

115 FORMAT (5H ****,* SYMBOLIC FLOATING POINT VARIABLE EXCEEDED *

1 *ALLOWABLE NESTING DEPTH OF 10.*)

ERROR $=$. TRUE.

RETURN

END

\begin{tabular}{lr} 
& \\
REALNUM & 57 \\
REALNUM & 58 \\
REALNUM & 59 \\
REALNUM & 60 \\
REALNUM & 61 \\
REALNUM & 62 \\
NOV3RLNM & 3 \\
NOV3RLNM & 4 \\
NOV3RLNM & 5 \\
NOV3RLNM & 6 \\
REALNUM & 65 \\
RLRAND & 2 \\
REALNUM & 69 \\
REALNUM & 70 \\
REALNUM & 71 \\
REALNUM & 72 \\
REALNUM & 73 \\
REALNUM & 74 \\
REALNUM & 75 \\
REALNUM & 76 \\
REALNUM & 77 \\
REALNUM & 78 \\
REALNUM & 79 \\
REALNUM & 80 \\
REALNUM & 81 \\
REALNUM & 82 \\
REALNUM & 83 \\
REALNUM & 84 \\
REALNUM & 85 \\
REALNUM & 86 \\
REALNUM & 87 \\
REALNUM & 88 \\
REALNUM & 89 \\
REALNUM & 90 \\
REALNUM & 91 \\
& \\
\hline
\end{tabular}


SUBROUTINE REF $(M, N)$

C COMPUTES MATRIX REFLECTION

REFLECT MATRIX AS $7 \times 7$, THEN STORE ACCORDING TO KIND COMMON /DIM/ LEND, MEND, ISAV, ISAV7; M7END, KADD, KADDR, MUNIT, MSYMPL

LEVEL 2, STORE, INFF, IWORK

COMMON STORE(48000), IWORK(10)

DIMENSION INFF $(24,2000)$

10

EQUIVALENCE (INFF, STORE)

DIMENSION RW(3), T(49), TK(7,7), RWT(3)

DIMENSION ML(3)

COMMON /MM/ML \$ LEVEL 2,ML

15

$$
\text { DATA TK } 49 * 0.0 /
$$

COMPUTE REFLECTION BY MULTIPLYING MATRICES

$$
K \quad M(I N V) \quad K(I N V)
$$

WHERE $K$ IS MATRIX TK AND $K=K$ (INV)

REF 2

REF 3

REF 4

DIM 2

DIM 3

BLANK 2

BLANK 3

86MARSIZ 1

86MARSIZ 2

86MARSIZ 3

BLANK 5

REF 7

REF 8

REFLL 1

REFLL 2

REF 9

REF 10

REF 11

REF 12

REF 13

REF 14

* Get INVERse of MATRIX

REF 15

$\mathrm{NN}=-\mathrm{N}$

CALL RTRV7(NN, T,RW)

25

CALL STOR7 (M7END-2,T, RW)

REF

REF

REF

FORM $K$ MATRIX AND MULTIPLY

REF

$\operatorname{TK}(1,1)=1$.

$\operatorname{TK}(2,2)=-1$.

$\operatorname{TK}(3,3)=1$.

30

$\operatorname{TK}(4,4)=-1$.

$\operatorname{TK}(5,5)=-1$.

$\operatorname{TK}(6,6)=1$.

$\operatorname{TK}(7,7)=1$.

DO $1 \mathrm{I}=1,3$

35

$1 \quad \mathrm{RWT}(\mathrm{I})=0$.

NT $=$ MTEND -1

CALL STOR7 (NT, TK, RWT)

$M L(1)=N T$

$M L(2)=M 7 E N D-2$

REF

REF

REF

REF

REF

REF

REF

REF

REF

REF

REF

REF

REF

$M L(3)=N T$

$\operatorname{CALL} \operatorname{MMM}(M, 3, M L)$

C LENGTH SHOULD BE SAME AS FIRST MATRIX, NOT THAT OF INVERSE.

C REVERSE SIGN OF LENGTH

45

$$
R W(1)=-R W(1)
$$

$R W(2)=-R W(2)$

$\mathrm{RW}(3)=-\mathrm{RW}(3)$

$\operatorname{CALL} \operatorname{STDAT}(M, 5,1,3, \mathrm{RW})$

RETURN

REF

REF

REF

REF

REF

REF

REF

REF

REF

END

REF 
1

5

10
SUBROUTINE REPBCD $(M, J, B)$

C REPLACES BCD DATA FROM B INTO INDEX $J$ OF ELEMENT M BCD DATA

LEVEL 2, STORE, INFF, IWORK

COMMON STORE (48000), IWORK(10)

DIMENSION INFF $(24,2000)$

EQUIVALENCE (INFF,STORE)

$L O C=\operatorname{INFF}(16, M)+J-1$

STORE $(L O C)=B$

RETURN

END

$\begin{array}{ll}\text { REPBCD } & 2 \\ \text { REPBCD } & 3 \\ \text { BLANK } & 2 \\ \text { BLANK } & 3 \\ \text { 86MARSIZ } & 1 \\ \text { 86MARSIZ } & 2 \\ \text { 86MARSIZ } & 3 \\ \text { BLANK } & 5 \\ \text { REPBCD } & 5 \\ \text { REPBCD } & 6 \\ \text { REPBCD } & 7 \\ \text { REPBCD } & 8\end{array}$


1

C REPLACES FLOATING POINT DATA FROM F INTO INDEX $J$ OF ELEMENT $M$

5

10
LEVEL 2, STORE, INFF, IWORK

COMMON STORE(48000), IWORK(10)

DIMENSION INFF $(24,2000)$

EQUIVALENCE (INFF,STORE)

$L O C=\operatorname{INFF}(14, M)+J-1$

STORE $($ LOC $)=F$

RETURN

END
REPFLT 2

REPFLT 3

BLANK 2

BLANK 3

86MARSIZ 1

86MARSIZ 2

86MARSIZ 3

BLANK 5

REPFLT 5

REPFLT 6

REPFLT 7

REPFLT 8 
REPLACE A VALUE IN A PREVIOUSLY DEFINED INSTRUCTION

LEVEL 2, STORE, INFF, IWORK

COMMON STORE(48000), IWORK(10)

10

DIMENSION INFF $(24,2000)$

EQUIVALENCE (INFF, STORE)

COMMON/CONTRL/ERROR, MODE, RSRV, STOR, XEQ, TRASW, NFSW, EMPTY, INDEF, 1 LDFLG, FIN

LOGICAL ERROR, RSRV, STOR, XEQ, TRASW, NFSW, EMPTY, INDEF, LDFLG, FIN

20

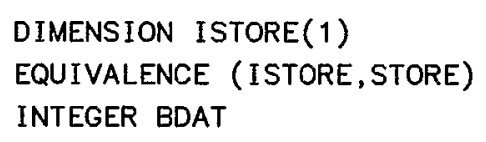

$\mathrm{NM}=\operatorname{BDAT}(M, 1)$

$N=\operatorname{MDAT}(M, 1)$

$K T=\operatorname{BDAT}(M, 2)$

$K=\operatorname{INFF}(4, M)$

* IF KT IS BLANK, ASSUME FLOATING POINT.

IF (KT.EQ.1H ) GO TO 100

IF (KT.EQ.1HF) GO TO 100

IF (KT.EQ.2HSF) GO TO 105

IF (KT.EQ.1HH) GO TO 110

IF (KT.EQ.1HI) GO TO 115

IF (KT.EQ.2HKA) GO TO 95

IF (KT.EQ.1HQ) GO TO 117

IF (KT.NE.2HKB) GO TO 120

$\mathrm{IK}=5$

GO TO 96

$95 \quad \mathrm{IK}=4$

$96 \quad \operatorname{IP}=\operatorname{IDAT}(M, 1)$

$\operatorname{INFF}(I K, N)=I P$

IF (MODE.EQ.3) WRITE $(3,97) \mathrm{KT}, \mathrm{NM}, \mathrm{IP}$

97 FORMAT $(6 \mathrm{H} * * *, 8 X, *$ REPLACE *,A3,* OF *,A5,* WITH *, I5)

RETURN

\section{FLOATING POINT}

* if SYMBolic Fl pt VARIABLE, insert as store in REPL

$100 \operatorname{LOCM}=\operatorname{INFF}(14, M)$

$\operatorname{LOCN}=\operatorname{INFF}(14, N)-1+K$

$\operatorname{STORE}($ LOCN $)=$ STORE $($ LOCM)

IF (MODE.EQ.3) WRITE $(3,101) \mathrm{K}, \mathrm{NM}, \mathrm{F}$

101 FORMAT $(6 \mathrm{H} * * *, 8 X, *$ REPLACE *, I3,* OF *,A5,* WITH *,F 10.6) RETURN

$$
\text { * } 105
$$

SYMBOLIC FLOATING POINT - INSERT AS IS

$\operatorname{LOCM}=\operatorname{INFF}(14, M)$

$\operatorname{LOCN}=\operatorname{INFF}(14, N)-1+K$

ISTORE (LOCN) = ISTORE (LOCM)

RETURN

\begin{tabular}{|c|c|}
\hline REPL & \\
\hline REPL & \\
\hline REPL & \\
\hline REPL & \\
\hline REPL & \\
\hline REPL & \\
\hline BLANK & \\
\hline BLANK & \\
\hline 86MARSIZ & \\
\hline 86MARSIZ & \\
\hline 86MARSIZ & \\
\hline BLANK & \\
\hline CONTRL & \\
\hline CONTRL & \\
\hline CONTRL & \\
\hline CONTRL & \\
\hline REPL & $1 e$ \\
\hline REPL & 1 \\
\hline REPL & 12 \\
\hline REPL & 1 \\
\hline REPL & 1 \\
\hline REPL & 15 \\
\hline REPL & 1 \\
\hline REPL & 17 \\
\hline REPL & 18 \\
\hline REPL & 15 \\
\hline REPL & 2 \\
\hline REPL & 2 \\
\hline REPL & 22 \\
\hline REPL & 23 \\
\hline REPL & 2 \\
\hline REPL & 25 \\
\hline REPL & 26 \\
\hline REPL & 27 \\
\hline REPL & 28 \\
\hline REPL & 25 \\
\hline REPL & $3 e$ \\
\hline REPL & 3 \\
\hline REPL & 32 \\
\hline REPL & 3 \\
\hline REPL & 3 \\
\hline REPL & 35 \\
\hline REPL & 36 \\
\hline REPL & 3 \\
\hline REPL & 38 \\
\hline REPL & 3 \\
\hline REPL & $4 e$ \\
\hline REPL & 41 \\
\hline REPL & 42 \\
\hline REPL & 43 \\
\hline REPL & 44 \\
\hline REPL & 4 \\
\hline REPL & 46 \\
\hline REPL & 4 \\
\hline REPL & 48 \\
\hline REPL & $4 S$ \\
\hline REPL & $5 e$ \\
\hline
\end{tabular}


60

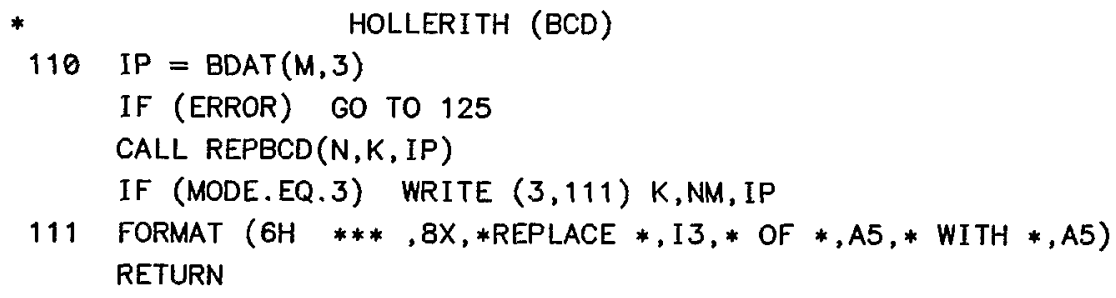

65

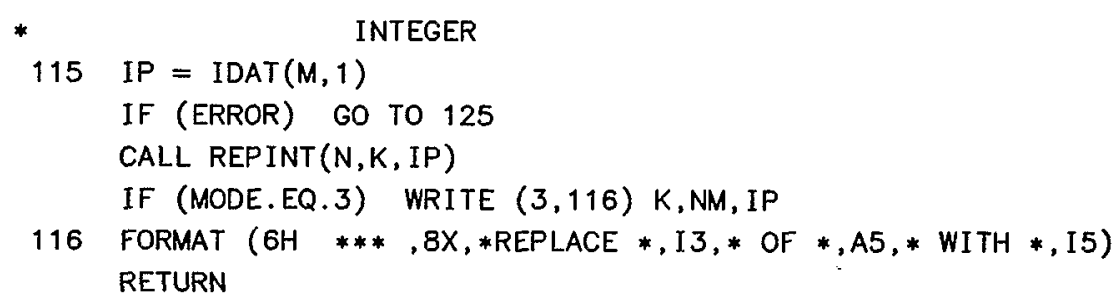

REPL

REPL $\quad 52$

REPL 53

REPL $\quad 54$

REPL 55

REPL 56

REPL 57

REPL 58

REPL $\quad 59$

REPL 60

REPL 61

REPL 62

REPL 6.3

REPL $\quad 64$

REPL 65

REPL $\quad 66$

REPL 67

REPL 68

REPL 69

REPL $\quad 70$

REPL 71

REPL $\quad 72$

REPL 73

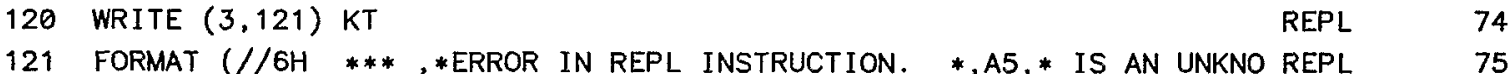
IWN TYPE,*)

REPL $\quad 76$

130 ERROR $=$. TRUE.

REPL $\quad 77$

85 RETURN

REPL $\quad 78$

125 WRITE $(3,126) \mathrm{KT}$

REPL 79

126 FORMAT $(/ / 6 \mathrm{H}$ *** ,*ERROR IN REPL INSTRUCTION. NO VALUES OF TYPE*,

1 A5,*.*)

RETURN

90 END

REPL 81

REPL $\quad 82$

REPL 83 
1

$$
\begin{aligned}
& \text { SUBROUT INE REPLQ }(M, L, J, F) \\
& \text { C PLACES F INTO INDEX } J \text { OF LQ STORAGE OF ELEMENT } M \\
& \text { C } \quad L=1 \text { FOR LQ } \quad L=2 \text { FOR } L Q 2 \quad L=3 \text { FOR } L Q 3
\end{aligned}
$$

5

LEVEL 2, STORE, INFF, IWORK

COMMON STORE (48000), IWORK (10)

DIMENSION INFF $(24,2000)$

EQUIVALENCE (INFF, STORE)

10

15

20
$L M=10$

IF (L.EQ.2) $L M=19$

IF (L.EQ.3) $L M=24$

$12 L O C=\operatorname{INFF}(L M, M)+J-1$

C IF LQ3 OR LQ2 HAVE NOT BEEN SET, GET LQ2 OR LQ IF (LOC.NE.O) GO TO 15

IF (LM.EQ.10) GO TO 15

IF (LM.EQ.24) $L M=19$

IF (LM.EQ.19) $L M=10$

GO TO 12

15 STORE $($ LOC $)=F$

RETURN

END

$\begin{array}{lr}\text { REPLQ } & 2 \\ \text { REPLQ } & 3 \\ \text { REPLQ } & 4 \\ \text { BLANK } & 2 \\ \text { BLANK } & 3 \\ \text { 86MARSIZ } & 1 \\ \text { 86MARSIZ } & 2 \\ \text { 86MARSIZ } & 3 \\ \text { BLANK } & 5 \\ \text { REPLQ } & 6 \\ \text { REPLQ } & 7 \\ \text { REPLQ } & 8 \\ \text { REPLQ } & 9 \\ \text { REPLQ } & 10 \\ \text { REPLQ } & 11 \\ \text { REPLQ } & 12 \\ \text { REPLQ } & 13 \\ \text { REPLQ } & 14 \\ \text { REPLQ } & 15 \\ \text { REPLQ } & 16 \\ \text { REPLQ } & 17 \\ \text { REPLQ } & 18\end{array}$


1

SUBROUTINE REPINT $(M, J, F I)$

REPINT

REPINT

REPINT

REPINT

BLANK

LEVEL 2, STORE, INFF, IWORK

COMMON STORE(48000), IWORK(10)

DIMENSION INFF $(24,2000)$

10

EQUIVALENCE (INFF,STORE)

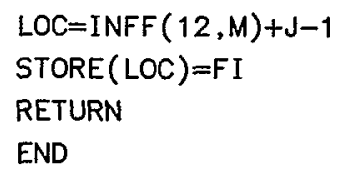




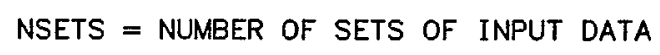

LEVEL 2, STORE, INFF, IWORK COMMON STORE (48000), IWORK(10)

DIMENSION INFF $(24,2000)$

EQUIVALENCE (INFF,STORE)

$$
1
$$

2 $M, N F, N B, N I, M S U B R$, ISIGN 1 LDFLG, FIN

LOGICAL ROWSW, COLSW, NQSW, CYSW 13HPRD, 4HSCAL, $1 \mathrm{H} /$

C

IF (MODE.EQ.3) RETURN

IF (. NOT.RSRV) RETURN

NQSW $=$, FALSE.

ROWSW $=$. FALSE.

COLSW $=$. FALSE

IF (ROWS. NE. . ) ROWSW=. TRUE.

IF (COLS.NE. . ) COLSW=. TRUE.

CALL ARGINP(ND, I, NSETS,

.)

- N1,I1,N2,I2,N3,I3,N4, I 4

$8 \quad$ NIN $=0$

NFL $=0$
COMMON/INSTR/OPNAME, NAME, OP , KA, KB, OBJA, OBJB, NXTM, TRA, LQ, NQ, LIN, NIN, LFL, NFL, LBC, NBC, NTOT, IX, KIND, TYPE, ROWS, COLS, EXTR,

INTEGER OPNAME, OP, OBJA, OBJB, TRA, TYPE, ROWS, COLS, EXTR

COMMON/STORE/LMAX, LINF , LFI LE, MAX, MIN, ICARD(11)

* lMaX aNd maX aRE dimensions of STORE AND INFF

COMMON/CONTRL/ERROR, MODE, RSRV, STOR, XEQ, TRASW, NFSW, EMPTY, INDEF,

LOGICAL ERROR, RSRV, STOR, XEQ, TRASW, NFSW, EMPTY, INDEF, LDFLG, FIN

COMMON /DIM/ LEND, MEND, ISAV, ISAV7, M7END, KADD, KADDR, MUNIT, MSYMPL

DIMENSION ND(10), I (10), NVAR(10), NKIND(10), INF(24)

DATA (NKIND(J), J=1, 10)/3HMXY, 3HPVC, 3HMAT, 2HM6, 2HR7, 3HROT, 3HMAP,

C STORE DATA SPECIFICATION NUMBERS IN ARRAYS ND(J) AND I $(J)$

$\mathrm{NBC}=0$

$L Q=L F I L E+1$ 


$$
\begin{aligned}
& L Q 2=L Q \\
& L Q 3=L Q
\end{aligned}
$$

C IF IQ.GE, $\theta, N Q=I Q=N O$. LOCATIONS RESERVED STARTING AT LQ

RESRV

C IF IQ.EQ. -0 , NQ WILL BE CALCULATED ACCORDING TO KIND

RESRV

C IF IQ.LE. -1, ABS(IQ) REFERS TO THE IQ-TH SET OF DATA.

RESRV

C $L Q$ WILL BE SET = LIN=LBC,OR LFL, DEPENDING ON DATA TYPE

RESRV IF (IQ.EQ.0) GO TO 10

RESRV IF (IQ.GE.O) GO TO 15

RESRV

C IQ IS NEGATIVE NUMBER.

C NQ WILL BE DETERMINED BY THE IQ-TH SET OF INPUT DATA

RESRV

$N Q=0$

GO TO 16

C DETERMINE SIGN OF ZERO

$10 \quad I Z=I Q . A N D .1$

IF (IZ.NE.O) NQSW = .TRUE.

IF (NQSW) GO TO 18

C IQ IS POSITIVE NUMBER. SET $N Q=I Q$

$15 N Q=I Q$

C IF KIND NOT SPECIFIED, SET IT TO OPNAME

16 IF (KKND.EQ.0) $\operatorname{KIND}=\operatorname{INFF}(1, M 0)$

IF (KIND.EQ.0) KIND = NKIND (KKND)

IF (OPNAME.NE. 4HFXPT) GO TO 30

C FXPT. SET UP 3 LQ LOCATIONS

RESRV

RESRV

RESRV

RESRV

RESRV

RESRV

RESRV

RESRV

RESRV

RESRV

RESRV

RESRV

RESRV

$L Q 2=L Q+K A D D+49$

$\mathrm{LQ3}=\mathrm{LQ} 2+35$

$N Q=L Q 3+15-L Q$

GO TO 30

C IQ IS - 0 . NQ DETERMINED BY KIND

18 IF (KKND.NE.4H ) GO TO 181

RESRV

RESRV

RESRV

RESRV

RESRV

RESRV

RESRV

C KIND IS UNKNOWN. SET NQ $=0$. LOAD WILL SET LQ AND NQ AFTER LOADING.

RESRV

RESRV

KIND $=$ KKND

$N Q=0$

GO TO 30

C KIND IS KNOWN. FIND OUT WHICH ONE.

181 NK $=1$

DO $19 \mathrm{~J}=1,10$

IF (KKND.EQ.NK) GO TO 20

95

$\mathrm{NK}=\mathrm{NK}+1$

19 CONTINUE

WRITE $(3,110)$

110 FORMAT $(/ 5 X, 25 \mathrm{H}$ ILLEGAL KIND ENCOUNTERED $/ /)$

ERROR $=$. TRUE.

100

\section{RETURN}

$20 \quad \mathrm{KIND}=\mathrm{NKIND}(\mathrm{NK})$

GO TO $(21,22,23,24,25,26,25,25,29)$, NK

RESRV

RESRV

RESRV

RESRV

RESRV

RESRV

RESRV

RESRV

RESRV

RESRV

RESRV

RESRV

RESRV

RESRV

C KIND IS MXPMY. MATRIX PAIRS RX AND RY

$21 \mathrm{NQ}=12+\mathrm{KADDR}$

IF (. NOT. ROWSW) ROWS $=2$

IF (.NOT. COLSW) COLS $=7$

GO TO 30

C KIND IS PVECS, PARTICLE VECTOR

C IF NOT OTHERWISE INDICATED, 7 ROWS BY KA COLUMNS

RESRV 89

RESRV 90

RESRV 91

RESRV 92

RESRV 93

RESRV 94

RESRV 95

RESRV 96

RESRV 97

RESRV 98

RESRV 99

RESRV 100

\footnotetext{
C KIND IS SCALAR
} 
IF (TYPE.NE.4HSNGL) NQ $=2$

RESRV

GO TO 30

RESRV

103

C KIND IS MAT, MATRIX OF KA ROWS AND KB COLUMNS

RESRV

23 GO TO 28

RESRV

105

120

C KIND IS 6X6 MATRIX

RESRV

106

$24 N Q=22+K A D D$

28 IF (.NOT.ROWSW) ROWS $=$ KA

RESRV

107

IF (.NOT.COLSW) COLS=KB

RESRV

108

GO TO 30

C KIND IS R7, MAP, OR PRD (7X7 MATRIX REPRESENTATION)

RESRV

109

RESRV 110

$25 N Q=49+K A D D$

ROWS $=7$

$\mathrm{COLS}=7$

GO TO 30

RESRV

111

RESRV 112

RESRV 113

RESRV 114

130

C KIND IS ROT, ROTATION

$26 \mathrm{NQ}=\mathrm{KADD}$

$K I N D=N K I N D(N K)$

C SET LFMX FOR NSETS $=0$ CASE. IF NSETS NOT $\theta$, LFMX COMPUTED BELOW.

30 LFMX $=$ LQ+NQ-1

IF(NSETS.EQ.0) GO TO 60

IF (I (NSETS).GE. 0 ) GO TO 31

C NUMBER OF DATA IN LAST SET IS INDEFINITE.

INDEF $=$. TRUE.

$I($ NSETS) $=-I$ (NSETS)

140

31 IF (I (1).NE.0) GO TO 32

C STORE VARIABLE-INPUT-LENGTH NUMBERS IN NVAR(I). DECODE $(50,100, \operatorname{ICARD}(3))(\operatorname{NVAR}(K), K=1, N 1)$

100 FORMAT (10I5)

C CALCULATE NUMBER OF DATA OF EACH TYPE.

32 DO $44 \quad J=1$, NSETS

$N A B=\operatorname{IABS}(N D(J))$

IF (ND(J).LT.0) ND(J) = NVAR(NAB)

$I J=I(J)+1$

GO TO $(44,41,42,43,44)$, IJ

150

$41 \quad \mathrm{NFL}=\mathrm{NFL}+\mathrm{ND}(\mathrm{J})$

GO TO 44

$42 \quad \mathrm{NBC}=\mathrm{NBC}+\mathrm{ND}(\mathrm{J})$

GO TO 44

$43 \quad N I N=N I N+N D(J)$

15544 CONTINUE

C IF A CYX INSTRUCTION, SET UP LQ2

CYSW $=$. FALSE.

IF ((OPNAME. EQ. 3HCYB) . OR. (OPNAME. EQ. 3HCYC)) CYSW = . TRUE.

RESRV

115

RESRV $\quad 116$

RESRV 117

RESRV 118

RESRV 119

RESRV 120

RESRV 121

RESRV 122

RESRV 123

RESRV 124

RESRV 125

RESRV 126

RESRV 127

RESRV 128

RESRV 129

RESRV 130

RESRV 131

RESRV 132

RESRV 133

RESRV 134

RESRV 135

RESRV 136

RESRV 137

RESRV 138

RESRV 139

RESRV 140

RESRV 141

RESRV 142

RESRV 143

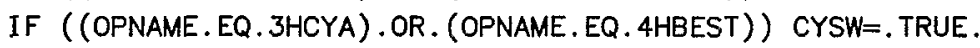

RESRV

144

160

IF (.NOT.CYSW) GO TO 45

RESRV

145

$L Q 2=L Q+N Q$

RESRV

146

$N Q=N Q+10$

RESRV

147

45 CONTINUE

RESRV

148

RESRV

IF (OPNAME. NE. 3HTAB) GO TO 46

C SETS UP EXTRA LQ FOR TABLE USE

RESRV

149

RESRV

$L Q 2=L Q+N Q$

$\mathrm{LQ3}=\mathrm{LQ} 2+10$

RESRV

151

$N Q=N Q+20$

RESRV

RESRV 


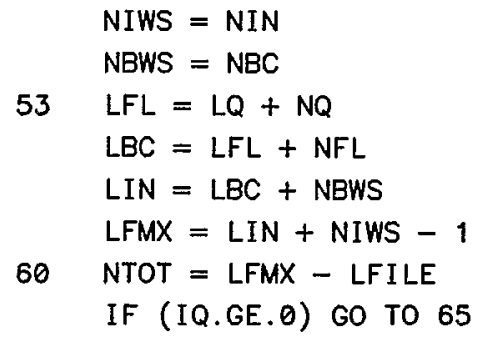

C RESET NQ. RESET LQ ACCORDING TO TYPE OF DATA IN IQ-TH SET

C IQ REFERS TO THE ABS IQ-TH SET OF INPUT DATA

$$
\begin{aligned}
& I Q=-I Q \\
& N Q=N D(I Q) \\
& I N=I(I Q)
\end{aligned}
$$

$61 \quad L Q=L F L$

$$
\begin{aligned}
& N Q=N F L \\
& \text { GO TO } 65
\end{aligned}
$$

\begin{tabular}{|c|c|}
\hline RESRV & 158 \\
\hline RESRV & 159 \\
\hline RESRV & 160 \\
\hline RESRV & 161 \\
\hline RESRV & 162 \\
\hline RESRV & 163 \\
\hline RESRV & 164 \\
\hline RESRV & 165 \\
\hline RESRV & 166 \\
\hline RESRV & 167 \\
\hline RESRV & 168 \\
\hline RESRV & 169 \\
\hline RESRV & 170 \\
\hline RESRV & 171 \\
\hline RESRV & 172 \\
\hline RESRV & 173 \\
\hline RESRV & 174 \\
\hline RESRV & 175 \\
\hline RESRV & 176 \\
\hline RESRV & 177 \\
\hline RESRV & 178 \\
\hline RESRV & 179 \\
\hline RESRV & 180 \\
\hline RESRV & 181 \\
\hline RESRV & 182 \\
\hline RESRV & 183 \\
\hline RESRV & 184 \\
\hline RESRV & 185 \\
\hline RESRV & 186 \\
\hline RESRV & 187 \\
\hline RESRV & 188 \\
\hline RESRV & 189 \\
\hline RESRV & 190 \\
\hline RESRV & 191 \\
\hline RESRV & 192 \\
\hline RESRV & 193 \\
\hline RESRV & 194 \\
\hline RESRV & 195 \\
\hline RESRV & 196 \\
\hline RESRV & 197 \\
\hline RESRV & 198 \\
\hline RESRV & 199 \\
\hline RESRV & 200 \\
\hline RESRV & 201 \\
\hline RESRV & 202 \\
\hline RESRV & 203 \\
\hline RESRV & 204 \\
\hline RESRV & 205 \\
\hline RESRV & 206 \\
\hline RESRV & 207 \\
\hline RESRV & 208 \\
\hline RESRV & 209 \\
\hline RESRV & 210 \\
\hline RESRV & 211 \\
\hline RESRV & 212 \\
\hline RESRV & 213 \\
\hline RESRV & 214 \\
\hline
\end{tabular}

190

$$
62 \quad L Q=L B C
$$

$63 L Q=L I N$

C PACK LOCATIONS AND LENGTHS INTO INFO $(I, N)$.

$$
\text { IF (NFL.EQ. } 0) \quad L F L=0
$$$$
\operatorname{IF}(N B C . E Q . \theta) \quad \angle B C=\theta
$$$$
\text { DO } 70 \mathrm{~J}=1,9
$$$$
70 \operatorname{INF}(J)=1 \mathrm{H} *
$$$$
\operatorname{INF}(10)=L Q
$$$$
\operatorname{INF}(11)=N Q
$$$$
\operatorname{INF}(12)=\operatorname{LIN}
$$$$
\operatorname{INF}(13)=N I N
$$$$
\operatorname{INF}(14)=\operatorname{LFL}
$$$$
\operatorname{INF}(15)=N F L
$$$$
\operatorname{INF}(16)=\mathrm{LBC}
$$$$
\operatorname{INF}(17)=\mathrm{NBC}
$$$$
\operatorname{INF}(18)=\text { NTOT }
$$$$
\operatorname{INF}(19)=L Q 2
$$$$
\operatorname{INF}(20)=K \operatorname{IND}
$$$$
\text { INF }(21)=\text { TYPE }
$$$$
\operatorname{INF}(22)=\text { ROWS }
$$$$
\operatorname{INF}(23)=\text { COLS }
$$$$
\operatorname{INF}(24)=L Q 3
$$$$
\text { IF (MO.GT.0) CALL WRTINF (INF, MO) }
$$

C RESET BOTTOM OF FILE, LFILE, TO BOTTOM OF MTH SECTION OF THE FILE.

$$
\text { LFI LE }=\text { LFMX }
$$

C CHECK FOR STORAGE OVERFLOW

NSTO = LMAX - LFILE

NINFF = MAX - MIN

IF (LFILE. LE. LMAX) GO TO 80

WRITE $(3,201)$

WRITE $(3,202)$ LMAX, LFILE, NSTO, MAX, NINFF, MO

ERROR $=$. TRUE.

BO IF (MO.GT.O) RETURN

WRITE $(3,200)$

IF (.NOT.ERROR) WRITE $(3,202)$ LMAX, LFILE, NSTO, MAX, NINFF, MO

ERROR $=$. TRUE.

FORMAT $(6 H 0 * * * * * *$ ELEMENTS OVERFLOWED. INCREASE INFF ARRAY AND * 
230

201 FORMAT $(6 H \theta * * * * * *$ STORE OVERFLOWED. INCREASE STORE ARRAY AND LMAX* RESRV 1

202 FORMAT $(/ 1 X *$ CORE USE SUMMARY *,27X,*MAXIMUM*,17X,*USED*,8X,*UNUSED $* /$ RESRV

$134 X, *$ STORE (ELEMENT STORAGE) *, 14X, I10,1X,*(LMAX)*,4X, I 10,5X, I 10/ RESRV

$234 \mathrm{X}, *$ INFF (ELEMENT DEFINTIONS)*,10X,I10,1X,*(MAX)*,5X,I10,5X,

RESRV

219

3 I 10)

RESRV

220

235 RETURN

RESRV

221

END

RESRV

222

CARD NR. SEVERITY DETAILS DIAGNOSIS OF PROBLEM 
1 SUBROUTINE REVMAT $(N, M, V)$

5

DIMENSION INFF $(24,2000)$

EQUIVALENCE (INFF,STORE)

DATA RADEG/.0174532925/

$N O P=\operatorname{INFF}(1, M)$

C MOVE

CALL MOVE(M,N,V)

RETURN

100 DO $2 \mathrm{I}=1,7$

$U(I)=V(I)$

$2 \quad V O(I)=V(I)$

C SEXTUPOLE

CALL $\operatorname{DATA}(M, 1,1,4$, PAR $)$

$\operatorname{INFF}(4, N)=\operatorname{INFF}(4, M)$

$\operatorname{INFF}(5, N)=\operatorname{INFF}(5, M)$

CALL $\operatorname{SXTP}(N, V, P A R)$

RETURN

CALL DATA $(M, 1,1,6$, PAR $)$

GRAD=PAR (2)

$B Z=P A R(4)$
LEVEL 2, STORE, INFF, IWORK

COMMON STORE(48000), IWORK(10)

COMMON /DIM/ LEND, MEND, ISAV, ISAV7, M7END , KADD , KADDR, MUNIT, MSYMPL

COMMON /MN/ML \$ LEVEL 2, ML

COMMON/CORB/TH(84), TV(84), NMON, NNH, NNV, I IH, I IV, NAMONH, NAMONV, NACORH, NACORV

DIMENSION PAR(6), T(7,7), RW(3),ML(3),V(1),VO(7),W(7),U(7)

EQUIVALENCE (ANG1, IANG1), (ANG2, IANG2), (BR, PAR(3))

IF (NOP.NE. 4HMOVE) GO TO 100

IF (NOP.NE. 4HSXTP) GO TO 1

C MAGNET DEFINED BY MAG INSTRUCTION

1 IF (NOP.NE.5HMAG) GO TO 7

IRECT $=0$

ANG 1=PAR (5)
REVMAT 2

REVMAT 3

REVMAT 4

REVMAT 5

REVMAT 6

REVMAT 7

REVMAT 8

REVMAT 9

REVMAT 10

REVMAT 11

REVMAT 12

REVMAT 13

REVMAT 14

REVMAT 15

REVMAT 16

REVMAT 17

BLANK 2

BLANK 3

86MARSIZ 1

86MARSIZ 2

86MARSIZ 3

BLANK 5

DIM 2

DIM 3

REVMATL 1

CORB613 1

CORB613 2

REVMAT 20

REVMAT 21

REVMAT 22

REVMAT 23

REVMAT 24

REVMAT 25

REVMAT 26

REVMAT 27

REVMAT 28

REVMAT 29

REVMAT 30

REVMAT 31

REVMAT 32

REVMAT 33

REVMAT $\quad 34$

REVMAT 35

REVMAT 36

REVMAT 37

REVMLTP 1

REVMLTP 2

REVMAT 38

REVMAT 39

REVMAT 40

REVMAT 41

REVMAT 42

REVMAT 43

REVMAT 44

REVMAT $\quad 45$

REVMAT $\quad 46$

REVMAT 47 
ANG2=PAR (6)

IF ( (IANG1 $, E Q, 1 H \$), O R \cdot($ IANG2, EQ $1 H \$)$ ) IRECT=1

IF ( (GRAD.NE.0.).OR. (IRECT.EQ.0).OR.(BZ.EQ.0.) ) GO TO 11

$\begin{array}{lr} & \\ \text { REVMAT } & 48 \\ \text { REVMAT } & 49 \\ \text { REVMAT } & 50 \\ \text { REVMAT } & 51 \\ \text { REVMAT } & 52 \\ \text { REVMAT } & 53 \\ \text { REVMAT } & 54 \\ \text { REVMAT } & 55 \\ \text { REVMAT } & 56 \\ \text { REVMAT } & 57 \\ \text { REVMAT } & 58 \\ \text { REVMAT } & 59 \\ \text { REVMAT } & 60 \\ \text { REVMAT } & 61 \\ \text { REVMAT } & 62 \\ \text { REVMAT } & 63 \\ \text { REVMAT } & 64 \\ \text { REVMAT } & 65 \\ \text { REVMAT } & 66 \\ \text { REVMAT } & 67 \\ \text { REVMAT } & 68 \\ \text { REVMAT } & 69 \\ \text { REVMAT } & 70 \\ \text { REVMAT } & 71 \\ \text { REVMAT } & 72 \\ \text { REVMAT } & 73 \\ \text { REVMAT } & 74 \\ \text { REVMAT } & 75 \\ \text { REVMAT } & 76 \\ \text { REVMAT } & 77 \\ \text { REVMAT } & 78 \\ \text { REVMAT } & 79 \\ \text { REVMAT } & 80 \\ \text { REVMAT } & 81 \\ \text { REVMAT } & 82 \\ \text { REVMAT } & 83 \\ \text { REVMAT } & 84 \\ \text { REVMAT } & 85 \\ \text { REVMAT } & 86 \\ \text { REVMAT } & 87 \\ \text { REVMAT } & 88 \\ \text { REVMAT } & 89 \\ \text { REVMAT } & 90 \\ \text { REVMAT } & 91 \\ \text { REVMAT } & 92 \\ \text { REVMATL } & 2 \\ \text { REVMATL } & 3 \\ \text { REVMATL } & 4 \\ \text { REVMAT } & 96 \\ \text { REVMAT } & 97 \\ \text { REVMAT } & 98 \\ \text { REVMAT } & 99 \\ \text { REVMAT } & 100 \\ \text { REVMAT } & 101 \\ \text { REVMAT } & 102 \\ \text { REVMAT } & 103 \\ \text { REVMAT } & 104 \\ & \\ \end{array}$

\$ CALL DRIFT(MEND-5,D2)

C RECTANGULAR, ZERO-GRADIENT BENDING MAGNET

CALL BEND $(N, V, P A R, 2)$

GO TO 9

65

11 CONTINUE

IF (BZ.NE.Q.) GO TO 111

C QUADRUPOLE

CALL MODQ $(M, N, V, W)$

GO TO 45

70

$111 \quad B R=B R *(1 .+V(6))$

CALL MAGNET (N,PAR)

CALL RTRV7( $N, T, R W)$

$\operatorname{CALL} \operatorname{MXVZ}(T, V, W)$

105

75

80

85

90

95

100

110
C DRIFT

7 IF(NOP.NE.3HDRF) GO TO 8

C BENDING MAGNET

THETA $=$ RW(2)

IF (IRECT.EQ.1) GO TO 3

$E 1=\operatorname{PAR}(5) * R A D E G$

$E 2=\operatorname{PAR}(6) * R A D E G$

$3 \quad \mathrm{E} 1=.5 *$ THETA

$E 2=E 1$

$4 \quad E 1 T=E 1+V(2)$

$E 2 T=E 2-W(2)$

$\mathrm{X} 1=\mathrm{V}(1)$

$X 2=W(1)$

IF (BZ.GE.0.) GO TO 40

$E 1 T=E 1-V(2)$

$E 2 T=E 2+W(2)$

$x_{2}=-x_{2}$

$\operatorname{PAR}(5)=E 1 T / R A D E G$

$\operatorname{PAR}(6)=E 2 T / R A D E G$

$D 1=X 1 * \operatorname{SIN}(E 1) / \operatorname{COS}(E 1 T)$

$D 2=X 2 * \operatorname{SIN}(E 2) / \operatorname{COS}(E 2 T)$

$D L=V(5)-W(5)$

$\operatorname{PAR}(1)=\operatorname{PAR}(1)+D L-D 1-D 2$

THETA $=$ THETA + V(2) $-W(2)$

RHO $=$ PAR( 1$) /$ THETA

$\operatorname{PAR}(4)=B R / R H O$

DO $5 \mathrm{I}=1,3$

$5 \quad M L(I)=$ MEND $-2-I$

CALL DRIFT(MEND-3, D1)

CALL MAGNET(MEND-4,PAR)

CALL MMM(N, 3,ML)

45 DO $50 \quad I=1,5$

$50 \quad V(I)=W(I)$

GO TO 9

$E L=\operatorname{FDAT}(M, 1)$ 
120

8 IF (NOP.NE.4HKICK) GO TO 82 NAKICK $=\operatorname{INFF}(2, M)$

IF (NAKICK.NE.NACORH) GO TO 81

$T K H=T H(I I H)$

$\operatorname{CALL} \operatorname{STDAT}(M, 1,3,1$, TKH $)$

CALL KICK(M)

$I \mathrm{IH}=\mathrm{IIH}+1$

IF (IIH.GT.NNH) IIH=1

GO TO 10

81 IF (NAKICK.NE.NACORV) GO TO 10

$T K V=T V(I I V)$

$\operatorname{CALL} \operatorname{STDAT}(M, 1,3,1$, TKV $)$

CALL KICK $(M)$

I IV = IIV + 1

IF (IIV.GT.NNV) IIV=1

GO TO 10

C OTHER LINEAR ELEMENTS-MMM, KICK, ETC.

82 CONTINUE

10 CALL RTRV7 $(M, T, R W)$

CALL $M X V 7(T, V, V)$

$140 \quad$ CALL STOR7 $(N, T, R W)$

RETURN

9 CALL RTRV7(N,T,RW)

$91 \operatorname{CALL} \operatorname{MXV7}(T, V \theta, W)$

145

$$
\text { DO } 12 \mathrm{I}=1,5
$$

$12 \quad T(I, 7)=V(I)-W(I)$

CALL STOR7 $(N, T, R W)$

RETURN

150

END
REVMAT 108

RVMT $683 \quad 2$

RVMT683 3

RVMT683 4

REVMAT613 1

RVMT683 6

RVMT683 7

RVMT683 8

RVMT683 9

RVMT683 10

RVMT683 11

REVMAT613 2

REVMAT613 3

REVMAT613 4

RVMT683 15

RVMT683 16

RVMT683 17

RVMT683 18

REVMAT 109

RVMT683 19

REVMAT 111

REVMAT 112

REVMAT 113

REVMAT 114

REVMAT 115

REVMAT 116

REVMAT 117

REVMAT 118

REVMAT 119

REVMAT 120

REVMAT 121

REVMAT 122

REVMAT 123

CARD NR. SEVERITY DETAILS DIAGNOSIS OF PROBLEM

$\begin{array}{rrr}70 & \text { I } & \text { V } \\ 83 & \text { I } & \text { V } \\ 88 & \text { I } & \text { V } \\ 96 & \text { I } & \text { V } \\ 98 & \text { I } & \text { V } \\ 115 & \text { I } & \text { V } \\ 115 & \text { I } & \text { V }\end{array}$

ARRAY REFERENCE OUTSIDE DIMENSION BOUNDS. ARRAY REFERENCE OUTSIDE DIMENSION BOUNDS. ARRAY REFERENCE OUTSIDE DIMENSION BOUNDS. ARRAY REFERENCE OUTSIDE DIMENSION BOUNDS. ARRAY REFERENCE OUTSIDE DIMENSION BOUNDS. ARRAY REFERENCE OUTSIDE DIMENSION BOUNDS. ARRAY REFERENCE OUTSIDE DIMENSION BOUNDS. 
1

5

10

15

20

25

30
SUBROUT INE ROTATE(THETA, T, TR, RW)

ROTATE MATRIX T BY THETA RADIANS AND FORM NEW MATRIX TR.

COMMON /DIM/ LEND, MEND, ISAV, ISAV7, M7END, KADD, KADDR, MUNIT, MSYMPL

COMMON /MO/ML(3) $\$$

LEVEL 2, ML

EQUIVALENCE(ML, MI)

DIMENSION MI (3)

DIMENSION T(1), TR(1), RW(1), RW1 (3)

DATA RW $1 / 3 * 0.0 /$

COMPUTE ROTATION MATRIX R+ AND R-

THETM $=-$ THETA

CALL ROTZ(THETA,TR)

$M 1=M 7 E N D-1$

CALL STOR7 (M1,TR, RW1)

$M I(1)=M 1$

$M 1=M 1-1$

CALL STOR7 $(M 1, T, R W)$

$M I(2)=M 1$

$M 1=M 1-1$

CALL ROTZ(THETM,TR)

CALL STOR7 (M1,TR, RW1)

$M I(3)=M 1$

$M 1=M 7 E N D$

$\operatorname{CALL~MMM}(M 1,3, M I)$

CALL RTRV7 (M1,TR, RW)

RETURN

END

$\begin{array}{lr}\text { ROTATE } & 2 \\ \text { ROTATE } & 3 \\ \text { ROTATE } & 4 \\ \text { ROTATE } & 5 \\ \text { DIM } & 2 \\ \text { DIM } & 3 \\ \text { ROTLL } & 1 \\ \text { ROTLL } & 2 \\ \text { ROTLL } & 3 \\ \text { ROTATE } & 7 \\ \text { ROTATE } & 8 \\ \text { ROTATE } & 9 \\ \text { ROTATE } & 10 \\ \text { ROTATE } & 11 \\ \text { ROTATE } & 12 \\ \text { ROTATE } & 13 \\ \text { ROTATE } & 14 \\ \text { ROTATE } & 15 \\ \text { ROTATE } & 16 \\ \text { ROTATE } & 17 \\ \text { ROTATE } & 18 \\ \text { ROTATE } & 19 \\ \text { ROTATE } & 20 \\ \text { ROTATE } & 21 \\ \text { ROTATE } & 22 \\ \text { ROTATE } & 23 \\ \text { ROTATE } & 24 \\ \text { ROTATE } & 25 \\ \text { ROTATE } & 26 \\ \text { ROTATE } & 27 \\ \text { ROTATE } & 28\end{array}$

CARD NR. SEVERITY DETAILS DIAGNOSIS OF PROBLEM

EQV/COMM I MO NOT ALL ITEMS IN THIS COMMON BLOCK OCCUR IN LEVEL STATEMENTS. 
1

SUBROUTINE ROTM(M, MB, THETA)

ROTM

ROTM

2

* Rotate matrix mb by theta Radians and store in matrix M.

ROTM

ROTM

ROTM

ROTM

ROTM

CALL RTRV7 (MB, T, RW)

CALL ROTATE (THETA,T,TR,RW)

ROTM

ROTM

ROTM

ROTM

ROTM

RETURN

END 
10

15

20
SUBROUTINE ROTZ(THET, T)

* DEFine a matrix REPRESEnting a rotation about the Z-AXIS

THET IS IN RADIANS

DIMENSION $T(7,7)$

DO $10 \quad I=1,7$

DO $10 \mathrm{~J}=1,7$

$T(I, J)=0$.

10 CONTINUE

$S N=\operatorname{SIN}(T H E T)$

$\mathrm{CS}=\cos (\mathrm{THET})$

$T(1,1)=C S$

$T(2,2)=\mathrm{CS}$

$T(3,3)=C S$

$\mathrm{T}(4,4)=\mathrm{CS}$

$T(1,3)=S N$

$\mathrm{T}(2,4)=\mathrm{SN}$

$\mathrm{T}(3,1)=-\mathrm{SN}$

$\mathrm{T}(4,2)=-\mathrm{SN}$

$T(5,5)=1$.

$T(6,6)=1$.

$T(7,7)=1$.

RETURN

END

$\begin{array}{lr}\text { ROTZ } & 2 \\ \text { ROTZ } & 3 \\ \text { ROTZ } & 4 \\ \text { ROTZ } & 5 \\ \text { ROTZ } & 6 \\ \text { ROTZ } & 7 \\ \text { ROTZ } & 8 \\ \text { ROTZ } & 9 \\ \text { ROTZ } & 10 \\ \text { ROTZ } & 11 \\ \text { ROTZ } & 12 \\ \text { ROTZ } & 13 \\ \text { ROTZ } & 14 \\ \text { ROTZ } & 15 \\ \text { ROTZ } & 16 \\ \text { ROTZ } & 17 \\ \text { ROTZ } & 18 \\ \text { ROTZ } & 19 \\ \text { ROTZ } & 20 \\ \text { ROTZ } & 21 \\ \text { ROTZ } & 22 \\ \text { ROTZ } & 23 \\ \text { ROTZ } & 24 \\ \text { ROTZ } & 25\end{array}$


1

SUBROUTINE ROW5(T)

$\begin{array}{lr}\text { ROW5 } & 2 \\ \text { ROW5 } & 3 \\ \text { ROW5 } & 4 \\ \text { ROW5 } & 5 \\ \text { ROW5 } & 6 \\ \text { ROW5 } & 7 \\ \text { ROW5 } & 8 \\ \text { ROW5 } & 9 \\ \text { ROW5 } & 10\end{array}$

CARD NR. SEVERITY DETAILS DIAGNOSIS OF PROBLEM

$7 \quad \mathrm{I} \quad \mathrm{T}$

7 I $T$

7 I $\quad T$

$\begin{array}{lll}7 & \mathrm{I} & \mathrm{T}\end{array}$
C COMPUTES ROW 5 OF $7 \times 7$ MATRIX T

DIMENSION $T(7,1)$

DO $10 \quad I=1,4$

$10 \quad T(5, I)=T(2, I) * T(1,6)-T(1, I) * T(2,6)+T(4, I) * T(3,6)-T(3, I) * T(4,6)$ RETURN

END 
* Reserves space for ka matrices; beginning with INDEX $M-1$ COMMON/INSTR/OPNAME, NAME, OP, KA, KB, OBJA, OBJB, NXTM, TRA, LQ, NQ, LIN, RSVMAT 4 INSTR 2 1 NIN, LFL, NFL, LBC, NBC, NTOT, IX, KIND, TYPE, ROWS, COLS, EXTR, 2 $M, N F, N B, N I, M S U B R, I S I G N$ INTEGER OPNAME, OP, OBJA, OBJB, TRA, TYPE, ROWS, COLS, EXTR

INSTR INTEGER OPNAME, OP, OBJA, OBJB,TRA, TYPE, ROWS, COLS, EXTR COMMON/CONTRL/ERROR, MODE, RSRV, STOR, XEQ, TRASW, NFSW, EMPTY, INDEF, INSTR

10 1 LDFLG, FIN

CONTRL 2
LOGICAL ERROR, RSRV, STOR, XEQ, TRASW, NFSW, EMPTY, INDEF, LDFLG, FIN CONTRL CONTRL DIMENSION LIST(25) CONTRL 5 RSVMAT 7

15

$I G=0$ IF (OPNAME. EQ . 3HDRF) IG $=1$ IF (OPNAME.EQ. 3HMAG) IG $=2$

IF (IG.LT.1) GO TO 25

* GENERATE NUMBERED NAMES

20 CALL GENNUM(NAME, KA, LIST) RESERVE ACCORDING TO OPNAME

RSVMAT

RSVMAT

RSVMAT

RSVMAT

RSVMAT

RSVMAT

RSVMAT

RSVMAT

$M 1=M-1$

$\mathrm{K} 1=0$

RSVMAT

RSVMAT

$K 2=0$

25

DO $20 \mathrm{I}=1, \mathrm{KA}$

GO TO $(10,15)$ IG

10 CALL DRFRSV(M1, LIST(I),K1,K2,

GO TO 18

RSVMAT

RSVMAT

RSVMAT

RSVMAT

RSVMAT

RSVMAT

RSVMAT

RSVMAT

RSVMAT

RSVMAT

RSVMAT

RSVMAT

RSVMAT

RSVMAT

RSVMAT

RSVMAT END FORMAT (5X,3H***,1X,*ERROR*,1X,A5, 1X,*IS NOT A DRF OR MAG.*/ $19 X, *$ ROUTINE RSVMAT CANNOT RESERVE MATRIX SPACE FOR IT.*) RETURN

CARD NR. SEVERITY DETAILS DIAGNOSIS OF PROBLEM 
SUBROUTINE RTRV7(M,TT, RW)

RTRV7

RTRV7

C RETRIEVES $7 \times 7$ MATRIX ELEMENTS

C IF ELEMENT NUMBER IS -, RETURN INVERSE

RTRV7

BLANK

LEVEL 2, STORE, INFF, IWORK

COMMON STORE(48000), IWORK(10)

BLANK

DIMENSION INFF $(24,2000)$

EQUIVALENCE (INFF,STORE)

COMMON/CONTRL/ERROR, MODE, RSRV, STOR , XEQ, TRASW, NFSW, EMPTY, INDEF,

1 LDFLG, FIN

LOGICAL ERROR, RSRV, STOR, XEQ, TRASW, NFSW, EMPTY, INDEF, LDFLG, FIN

DIMENSION TT( (1), TM(49), RW(3), RX(2,3), RY(2,3),T(7,7), RPQ(4)

15

COMMON /DIM/ LEND, MEND, ISAV, ISAV7, M7END, KADD, KADDR, MUNIT, MSYMPL

86MARSIZ 1

86MARSIZ 2

86MARSIZ 3

BLANK 5

CONTRL 2

CONTRL 3

CONTRL $\quad 4$

CONTRL 5

RTRV7 7

DIM 2

DIM 3

EQUIVALENCE (T, TM)

- LOGICAL INV

DATA RADEG/.0174532925/

RTRV7 9

RTRV7 10

RTRV7 11

c

INV $=$. FALSE.

DO $4 \mathrm{I}=1,3$

$4 \quad \mathrm{RW}(\mathrm{I})=\theta$.

IF (M) 5,6,6

25

$5 \quad M=-M$

INV $=$. TRUE.

$6 \quad \operatorname{KIND}=\operatorname{INFF}(20, M)$

CALL DATA $(M, 5,1,3, \mathrm{RW})$

C IS KIND R7 OR ROT

IF (KIND.EQ.3HROT) GO TO 40

IF (KIND.NE.3HMXY) GO TO 28

C KIND IS MXY. RETURN $2 \times 3$ MATRIX

CALL RXY (M, RX, RY, RW)

CALL DATA $(M, 5,4,4, R P Q)$

35

$\mathrm{KD}=7$

CALL CONVMAT (KD, T, RX, RY, RW, RPQ)

RTRV7 12

RTRV7 13

RTRV7 14

RTRV7 15

RTRV7 16

RTRV7 17

RTRV7 18

RTRV7 19

RTRV7 20

RTRV7 21

RTRV7 22

RTRV7 23

RTRV7 24

RTRV7 25

RTRV7 26

RTRV7 27

RTRV7 28

GO TO 50

C KIND IS R7

$28 L Q=\operatorname{INFF}(10, M)$

RTRV7 29

RTRV7 30

RTRV7 31

40

$N Q=\operatorname{INFF}(11, M)$

C CHECK STORAGE

RTRV7 32

RTRV7 33

$\mathrm{NNQ}=49+\mathrm{KADD}$

IF (NNQ.GT.NQ) GO TO 45

RTRV7 34

$N J=L Q+K A D D-1$

RTRV7 35

RTRV7 36

D035 I $=1,49$

RTRV7 37

$T M(I)=S T O R E(N J+I)$

RTRV7

RTRV7

RTRV7

RTRV7

RTRV7

CONTINUE

RTRV7

RTRV7

RTRV7

RTRV7

RTRV7

RTRV7

RTRV7

THETA = THETA*RADEG 
65

DO $41 \mathrm{I}=1,49$

RTRV7

$T M(I)=0$.

RTRV7

60

41 CONTINUE

RTRV7

CALL ROTZ(THETA;T)

RTRV7

50 IF (.NOT. INV) GO TO 52

RTRV7

CALL TINV7 (TT, TM)

RTRV7

$\mathrm{RW}(1)=-\mathrm{RW}(1)$

RTRV7

$R W(2)=-R W(2)$

RTRV7

GO TO 56

RTRV7

RTRV7

RTRV7

RTRV7

RTRV7

RTRV7

RTRV7

52 DO $55 \mathrm{I}=1,49$

$T T(I)=T M(I)$

55 CONTINUE

$56 \mathrm{RW}(3)=\mathrm{TT}(40)$

RETURN

END"

70

CARD NR. SEVERITY DETAILS : DIAGNOSIS OF PROBLEM

70 I TT ARRAY REFERENCE OUTSIDE DIMENSION BOUNDS. 
10

15

SUBROUTINE RXRY(M,DAT)

RXRY

RXRY

C READS MATRIX RX AND RY,DIMENSIONED $(2,3)$ FROM CARDS.

RXRY

RXRY

DIMENSION RX(2,3), RY $(2,3), \operatorname{DAT}(12), \operatorname{RW}(3)$

RXRY

RXRY

RXRY

RXRY

RXRY

RXRY

RXRY

RXRY

RXRY

RXRY

RXRY

RXRY

RXRY

RXRY 
1

10

15

25

30

35

40

45

50

55

SUBROUTINE RXY(M, RX, RY, RW)

RXY

RXY

C

C RETRIEVES $X$ AND $Y$ MATRICES FROM LQ $(M)$. DIMENSIONS ARE $(2,3)$ FOR EACH. C RETURN RX AND RY

C IF ELEMENT NUMBER IS -, RETURN INVERSE

LEVEL 2, STORE, INFF, IWORK

COMMON STORE( 48000$), \operatorname{IWORK}(10)$

DIMENSION INFF $(24,2000)$

EQUIVALENCE (INFF, STORE)

COMMON/CONTRL/ERROR, MODE, RSRV, STOR, XEQ, TRASW, NFSW, EMPTY, INDEF,

1 LDFLG, FIN

LOG ICAL ERROR, RSRV, STOR, XEQ, TRASW, NFSW, EMPTY, INDEF, LDFLG, FIN

COMMON/SWTCH/BEND, MS IZE, CYCSWT, VCSW, PV, MSSW, VPR, MHS, MODS, MINZER LOGICAL CYCSWT, MSSW, VCSW, PV, VPR, MHS, MODS

COMMON /DIM/ LEND, MEND, ISAV, ISAV7, M7END, KADD, KADDR, MUNIT , MSYMPL

LOGICAL INV

DIMENSION $\operatorname{RX}(2,3), \operatorname{RY}(2,3), \operatorname{AX}(2,3), \operatorname{AY}(2,3), \operatorname{RW}(3)$

DIMENSION TT $(49), T(7,7), R P Q(4)$

EQUIVALENCE $(T, T T)$

C

$\mathrm{INV}=$. FALSE.

IF (M.GE.O) GO TO 5

$4 \quad M=-M$

INV $=$. TRUE.

$5 \quad \operatorname{LQ}=\operatorname{INFF}(10, M)$

$N Q=\operatorname{INFF}(11, M)$

DO $7 \mathrm{~J}=1,3$

DO $3 \mathrm{I}=1,2$

$R X(I, J)=0$.

$\operatorname{RY}(I, J)=\theta$.

3 CONTINUE

$\operatorname{RW}(J)=0$.

7 CONTINUE

KIND $=\operatorname{INFF}(20, M)$

RXY $\quad 5$

RXY $\cdot 6$

BLANK 2

BLANK 3

86MARSIZ 1

86MARSIZ 2

86MARSIZ 3

BLANK 5

CONTRL 2

CONTRL 3

CONTRL 4

CONTRL 5

SWTCH 2

SWTCH 3

SWTCH 4

DIM 2

DIM 3

RXY 11

RXY 12

RXY 13

RXY 14

RXY 15

RXY 16

RXY $\quad 17$

RXY 18

RXY 19

RXY 20

RXY 21

RXY 22

RXY 23

RXY 24

RXY 25

RXY 26

RXY 27

RXY 28

RXY 29

RXY 30

RXY 31

RXY 32

RXY 33

RXY 34

RXY $\quad 35$

RXY $\quad 36$

RXY $\quad 37$

RXY 38

RXY $\quad 39$

RXY $\quad 40$

RXY $\quad 41$

RXY $\quad 42$

RXY $\quad 43$

RXY $\quad 44$

RXY $\quad 45$

RXY $\quad 46$

RXY $\quad 47$

C CHECK STORAGE 
6

CONT INUE

$N N Q=12+K A D D R$

60

IF (NNQ.GT.NQ) GO TO 20

RXY

RXY

48

$N J=L Q+K A D D R$

RXY

49

$N I=N J+6$

DO $10 \mathrm{I} J=1,3$

DO 10 I $I=1,2$

65

$R X(I I, I J)=S T O R E(N J)$

RXY

50

RY (I I , I J) $=S T O R E(N I)$

RXY

51

$\mathrm{NJ}=\mathrm{NJ}+1$

$\mathrm{NI}=\mathrm{NI}+1$

70

10

CONT INUE

RXY

52

RXY

RXY

RXY

RXY

RXY

RXY

RXY

RXY

RXY

RXY

RXY

RXY

RXY

RXY

RXY

RXY

RXY

RXY

RXY

RXY

RXY

RXY

RXY

RXY

RXY

RXY

RXY

RXY

RXY

RXY

ERROR

RETRIEVAL REQUEST

$1 \mathrm{E}$ AT INDEX I5)

RXY

ERROR=. TRUE.

RXY

RXY

RXY

END 
1

10 C

15
SUBROUTINE SETALPH (M)

LEVEL 2, STORE, INFF, IWORK

COMMON STORE (48000), IWORK(10)

DIMENSION INFF $(24,2000)$

EQUIVALENCE (INFF, STORE)

COMMON/CDERIV/IFLAG, MR, NR, DP, NV(11), DAT(20), UZ, STOT, 1 DAT2(7), MP2FLG, NF, HO, LOCALPH, NUMALPH

$\operatorname{MALPH}=\operatorname{MDAT}(M, 1)$

LOCALPH $=\operatorname{INFF}(10$, MALPH $)-1$

NUMALPH $=\operatorname{INFF}(11, \mathrm{MALPH})$

$\mathrm{HO}=\operatorname{DAT}(5)$

RETURN

END

$\begin{array}{lr}\text { SETALPH } & 2 \\ \text { BLANK } & 2 \\ \text { BLANK } & 3 \\ 86 M A R S I Z & 1 \\ \text { 86MARSIZ } & 2 \\ \text { 86MARSIZ } & 3 \\ \text { BLANK } & 5 \\ \text { CDERIV } & 2 \\ \text { CDERIV } & 3 \\ \text { SETALPH } & 5 \\ \text { SETALPH } & 6 \\ \text { SETALPH } & 7 \\ \text { SETALPH } & 8 \\ \text { SETALPH } & 9 \\ \text { SETALPH } & 10 \\ \text { SETALPH } & 11\end{array}$


DIMENSION RX(2,3), RY(2,3),RW(3),T(49)

LEVEL 2, STORE, INFF, IWORK

COMMON STORE(48000), IWORK(10)

DIMENSION INFF $(24,2000)$

EQUIVALENCE (INFF, STORE)

COMMON /DIM/ LEND, MEND, ISAV, ISAV7, M7END, KADD , KADDR, MUNIT, MSYMPL

$K N=\operatorname{INFF}(20, M)$

$N T 1=$ MEND - 1

GET M OF INPUT ELEMENT AND ITS SYNCH OPNAME

GO TO 55

CONTINUE

GO TO $(11,15,20)$ ITP

* IF IT IS A CELL TYPE, MATRIX IS IN STORAGE AND MI LIST IS AT LIN 11 IF (KA.GT.12) GO TO 65

CALL DATA (MPR, 3,1,12, MI)

GO TO 30
SETCYB

CONTRL

CONTRL

CONTRL

CONTRL

BMIL

BMIL

BMIL

BMIL

BMI

SETCYB

SETCYB

BLANK

BLANK

86MARSIZ

86MARSIZ

86MARSIZ

BLANK

DIM

DIM

SETCYB

SETCYB

SETCYB

SETCYB

SETCYB

SETCYB

SETCYB

SETCYB

SETCYB

SETCYB

SETCYB

SETCYB

SETCYB

SETCYB

SETCYB

SETCYB

SETCYB

SETCYB

SETCYB

SETCYB

SETCYB

SETCYB

SETCYB

SETCYB

SETCYB

SETCYB

SETCYB

SETCYB

SETCYB

SETCYB

SETCYB

SETCYB
2

3

4

5

6

7

2

3

4

5

1

2

3

4

3

10

11

2

3

1

2

3

5

\section{RETURN}

C ** SYNCH INSTR. GET M, DETERMINE TYPE AND FORM MI LIST. 
60

65

70

75

80

85

90

95

100

105

110
$M M=\operatorname{MDAT}(M P R, 1)$

$N B=\operatorname{INFF}(1, M M)$

ASSIGN 25 TO NGO

GO TO 55

25

IF (ITP.GT.2) GO TO 61

GO TO $(26,27)$ ITP

26 IF (KA.GT.12) GO TO 65

CALL DATA (MM, 3,1,12,MI)

GO TO 30

27 CONTINUE

$\mathrm{KNB}=\operatorname{INFF}(4, \mathrm{MM})$

IF (KA.GT.KNB) GO TO 65

CALL MLIST (MM, $1, K N B, M I)$

30 CONTINUE

* IF KN IS 5, IT IS AN R7 MATRIX.

IF (KN.EQ.5) GO TO 50

* TRANSFER MATRIX FROM INDEX MPR TO WORKING STORAGE WITH INDEX MEND-1.

CALL RXY (MPR, RX, RY, RW)

CALL STXY (NT1,RX,RY,RW)

RETURN

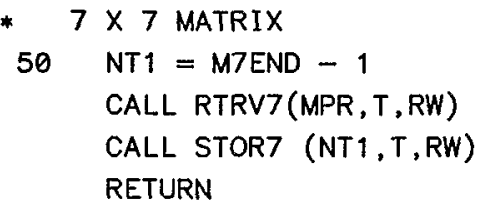

CONTINUE

SETCYB $\quad 46$

SETCYB $\quad 47$

SETCYB 48

SETCYB $\quad 49$

SETCYB $\quad 50$

SETCYB $\quad 51$

SETCYB $\quad 52$

SETCYB 53

SETCYB 54

SETCYB $\quad 55$

SETCYB $\quad 56$

SETCYB $\quad 57$

SETCYB 58

SETCYB . 59

SETCYB 60

SETCYB 61

SETCYB $\quad 62$

SETCYB 63

SETCYB 64

SETCYB 65

SETCYB 66

SETCYB $\quad 67$

SETCYB 68

SETCYB 69

SETCYB 70

SETCYB 71

SETCYB $\quad 72$

SETCYB 73

SETCYB $\quad 74$

SETCYB 75

SETCYB 76

SETCYB 77

SETCYB 78

SETCYB 79

SETCYB 80

SETCYB 81

SETCYB 82

SETCYB 83

SETCYB 84

SETCYB $\quad 85$

SETCYB 86

SETCYB 87

SETCYB 88

SETCYB $\quad 89$

SETCYB 90

SETCYB 91

SETCYB 92

$$
\text { GO TO NGO }(10,25)
$$

SETCYB 92

SETCYB 93

SETCYB 94

SETCYB 95

SETCYB 96

SETCYB 97

SETCYB 98

SETCYB 99

SETCYB 100

SETCYB 101

SETCYB 102 
115

$$
\text { GO TO } 70
$$

1 FORMAT $(5 X, 5 H * * *, *$ ERROR $*, 1 H *, *$ CYB WANTS *, I5, * ELEMENTS FOR SETCYB ICYCLING. *,A5,* HAS ONLY *,I5)

SETCYB

37 I

51 I

64 I
AN IF STATEMENT MAY BE MORE EFFICIENT THAN A 2 OR 3 BRANCH COMPUTED GO TO STATEMENT. AN IF STATEMENT MAY BE MORE EFFICIENT THAN A 2 OR 3 BRANCH COMPUTED GO TO STATEMENT. AN IF STATEMENT MAY BE MORE EFFICIENT THAN A 2 OR 3 BRANCH COMPUTED GO TO STATEMENT. 
* SETS UP LIST OF SYNCH INSTRUCTIONS.

SETOPS 3

* OPNUM ROUTINE WILL USE INDEX TO SET OP NUMBERS

5

COMMON/OPLIST/LIST(200)

DATA LIST/200(1H )/

10

15

20

25

30

35

40

45

50

55
$\operatorname{LIST}(1)=4 H S T O P$

$\operatorname{LIST}(2)=4 \mathrm{HFIN}$

$\operatorname{LIST}(3)=4 \mathrm{HRUN}$

$\operatorname{LIST}(4)=4 \mathrm{HSUB}$

$\operatorname{LIST}(5)=4 H E N D$

$\operatorname{LIST}(6)=4 \mathrm{HCALL}$

$\operatorname{LIST}(7)=4 H I N C R$

$\operatorname{LIST}(8)=4 \mathrm{HREPL}$

$\operatorname{LIST}(9)=4 H M E S H$

$\operatorname{LIST}(10)=4 H V P A R$

$\operatorname{LIST}(11)=4 \mathrm{H}=$

$\operatorname{LIST}(12)=4 \mathrm{HCRD}$

$\operatorname{LIST}(13)=4 \mathrm{HBML}$

$\operatorname{LIST}(14)=4 \mathrm{HDRF}$

$\operatorname{LIST}(15)=4 H M A G$

$\operatorname{LIST}(16)=4 \mathrm{HMAGV}$

$\operatorname{LIST}(17)=4 \mathrm{HEQU}$

$\operatorname{LIST}(18)=4 \mathrm{HINV}$

$\operatorname{LIST}(19)=4 \mathrm{HSHF}$

$\operatorname{LIST}(20)=4 \mathrm{HREF}$

$\operatorname{LIST}(21)=4 \mathrm{H} * *$

$\operatorname{LIST}(22)=4 H M M M$

$\operatorname{LIST}(23)=4 H C Y A$

$\operatorname{LIST}(24)=4 \mathrm{HCYB}$

$\operatorname{LIST}(25)=4 \mathrm{HCYC}$

$\operatorname{LIST}(26)=4 \mathrm{HCELL}$

$\operatorname{LIST}(27)=4 H A L T C$

$\operatorname{LIST}(28)=4 H T A B$

LIST $(29)=4 \mathrm{HPTAB}$

$\operatorname{LIST}(30)=4 H F I T Q$

$\operatorname{LIST}(31)=4 \mathrm{HFITB}$

$\operatorname{LIST}(32)=4 H F I T R$

$\operatorname{LIST}(33)=4 H F I T V$

$\operatorname{LIST}(34)=$ 4HPAGE

$\operatorname{LIST}(35)=4 \mathrm{HREM}$

$\operatorname{LIST}(36)=4 H W M A$

$\operatorname{LIST}(37)=4 \mathrm{HSIZE}$

$\operatorname{LIST}(38)=4$ HWBE

$\operatorname{LIST}(39)=4 \mathrm{HFIT}$

$\operatorname{LIST}(40)=4 \mathrm{HCOPY}$

$\operatorname{LIST}(41)=4 \mathrm{HNCPY}$

$\operatorname{LIST}(42)=4 \mathrm{HBEST}$

$\operatorname{LIST}(43)=3 \mathrm{HBEP}$

$\operatorname{LIST}(44)=4$ HBETA

$\operatorname{LIST}(45)=4 \mathrm{HKICK}$

$\operatorname{LIST}(46)=4 \mathrm{HRAND}$

$\operatorname{LIST}(47)=4 \mathrm{HSHF7}$

$\operatorname{LIST}(48)=3 \mathrm{HSOL}$
SETOPS

SETOPS

SETOPS

OPLIST

OPLIST

SETOPS

SETOPS

SETOPS

SETOPS

SETOPS

SETOPS

SETOPS

SETOPS

SETOPS

SETOPS

SETOPS

SETOPS

SETOPS

SETOPS

SETOPS

SETOPS

SETOPS

SETOPS

SETOPS

SETOPS

SETOPS

SETOPS

SETOPS

SETOPS

SETOPS

SETOPS

SETOPS

SETOPS

SETOPS

SETOPS

SETOPS

SETOPS

SETOPS

SETOPS

SETOPS

SETOPS

SETOPS

SETOPS

SETOPS

SETOPS

SETOPS

SETOPS

SETOPS

SETOPS

SETOPS

SETOPS

SETOPS

SETOPS

SETOPS

SETOPS 
60

$$
\begin{aligned}
& \operatorname{LIST}(49)=3 H V A R \\
& \operatorname{LIST}(50)=4 H P R N T
\end{aligned}
$$

SETOPS $\quad 58$

SETOPS $\quad 59$

SETOPS 60

SETOPS 61

$\operatorname{LIST}(51)=4 \mathrm{HSUM}$

SETOPS

62

$\operatorname{LIST}(53)=4 H D E L E$

SETOPS

63

$\operatorname{LIST}(54)=4 \mathrm{HSCOP}$

SETOPS

64

65

$\operatorname{LIST}(55)=4$ HTEST

SETOPS

65

$\operatorname{LIST}(56)=4 \mathrm{HWFL}$

SETOPS 66

$\operatorname{LIST}(57)=4$ HMAGS

SETOPS 67

$\operatorname{LIST}(58)=4 \mathrm{HCELC}$

SETOPS 68

$\operatorname{LIST}(59)=4 \mathrm{HCFD}$

70

$\operatorname{LIST}(60)=4 H D C F D$

$\operatorname{LIST}(61)=4 H S T R 2$

SETOPS 69

SETOPS 70

$\operatorname{LIST}(62)=4$ HSTR4

SETOPS 71

$\operatorname{LIST}(63)=4$ HSTRP

$\operatorname{LIST}(64)=4 H S T R N$

75

80

$\operatorname{LIST}(65)=4 \mathrm{HFXPT}$

$\operatorname{LIST}(66)=4 \mathrm{HTRK}$

$\operatorname{LIST}(67)=4 H P R D$

$\operatorname{LIST}(68)=4 H S X T P$

SETOPS 72

SETOPS 73

SETOPS 74

SETOPS 75

SETOPS 76

SETOPS 77

SETOPS 78

SETOPS 79

SETOPS 80

SETOPS 81

$\operatorname{LIST}(70)=4 \mathrm{HBMIS}$

SETOPS 82

$\operatorname{LIST}(71)=4 \mathrm{HEMIS}$

SETOPS 83

SETOPS 84

SETOPS 85

SETOPS 86

SETOPS 87

SETOPS 88

SETOPS 89

SETOPS 90

SETOPS 91

STPS683 1

SETOPS 92

$\operatorname{LIST}(100)=4 \mathrm{HPBML}$

95

$\operatorname{LIST}(101)=4 \mathrm{HMAT}$

$\operatorname{LIST}(102)=4 H V E C$

$\operatorname{LIST}(103)=4 H P V E C$

$\operatorname{LIST}(104)=4 \mathrm{HMXV}$

$\operatorname{LIST}(105)=4 H C P L X$

100

$\operatorname{LIST}(106)=4 H E Q I L$

$\operatorname{LIST}(107)=4$ HEVEC

$\operatorname{LIST}(108)=4 \mathrm{HPRTV}$

$\operatorname{LIST}(109)=4 \mathrm{HPRV} 7$

$\operatorname{LIST}(110)=4$ HCVEC

105

$\operatorname{LIST}(111)=4 \mathrm{HLIST}$

$\operatorname{LIST}(112)=4 \mathrm{HSIN}$

$\operatorname{LIST}(113)=4 \mathrm{HCOS}$

$\operatorname{LIST}(114)=4 \mathrm{HEXP}$

$\operatorname{LIST}(115)=4 H A L O G$

110

$\operatorname{LIST}(116)=4 H S Q$

$\operatorname{LIST}(117)=4 H S Q R T$

$\operatorname{LIST}(118)=4 \mathrm{HTAN}$

$\operatorname{LIST}(119)=4 \mathrm{HASIN}$

MY3STOP 1

SETOPS 93

SETOPS 94

SETOPS 95

SETOPS 96

SETOPS 97

SETOPS 98

SETOPS 99

SETOPS 100

SETOPS 101

SETOPS 102

SETOPS 103

SETOPS 104

SETOPS 105

SETOPS 106

SETOPS 107

SETOPS 108

SETOPS 109

SETOPS 110

SETOPS 111

$\operatorname{LIST}(120)=4 \mathrm{HACOS}$

SETOPS 112 


$$
\begin{aligned}
& \operatorname{LIST}(121)=4 \mathrm{HATAN} \\
& \operatorname{LIST}(122)=4 \mathrm{HABS} \\
& \operatorname{LIST}(123)=4 \mathrm{HCALC} \\
& \operatorname{LIST}(151)=4 \mathrm{HBEAM} \\
& \operatorname{LIST}(152)=4 \mathrm{HDKE} \\
& \operatorname{LIST}(153)=4 \mathrm{HEDRF} \\
& \operatorname{LIST}(155)=4 \mathrm{HCYEM} \\
& \operatorname{LIST}(156)=4 \mathrm{HBVAL} \\
& \operatorname{LIST}(157)=4 \mathrm{HCYAE}
\end{aligned}
$$
SETOPS

SETOPS

$\operatorname{LIST}(161)=4 \mathrm{HTRKB}$ SETOPS

$$
\begin{aligned}
& \operatorname{LIST}(166)=4 \mathrm{HIBET} \\
& \operatorname{LIST}(163)=4 \mathrm{HSMIN} \\
& \operatorname{RETURN} \\
& \operatorname{END}
\end{aligned}
$$

86SETOPS 2

86SETOPS 3

SETOPS 126

SETOPS 127

SETOPS 
* SETS UP FIT INPUT dATA FOR FIT ROUTINE.

* INPUT - NAME FITX KA KB SUB C QF QD I1 I2 A1 A2

* lmax aNd maX aRe dimensions of store aND INFF

LOGICAL FSW

INTEGER BDAT

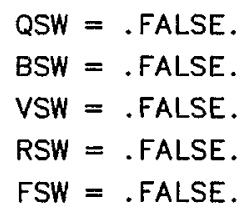

$F S W=. F A L S E$.

.)

IF (.NOT.RSW) GO TO 110

* in fitr case, ka and kb each contain 2 element numbers, which are

CONTRL

CONTRL

CONTRL

CONTRL

STORE

STORE

STORE

SETFIT

SETFIT

SETFIT

SETFIT

SETFIT

SETFIT

SETFIT

SETFIT

SETFIT

SETFIT

SETFIT

SETFIT

SETFIT

SETFIT

SETFIT

SETFIT

SETFIT

SETFIT

SETFIT

SETFIT

SETFIT

SETFIT

SETFIT

SETFIT

SETFIT

SETFIT

SETFIT

SETFIT

SETFIT

SETFIT

SETFIT

SETFIT
2 
$\operatorname{CALL} \operatorname{REPBCD}(M, 6, \mathrm{~K} 2)$

SETFIT $\quad 48$

SETFIT $\quad 49$

60

110 IF (MODE.EQ.2) RETURN

SETFIT 50

* XSW SUPPRESSES EXECUTION

SETFIT

50

115 IF (XSW) RETURN

SETFIT 52

116 CONTINUE

SETFIT 53

65

CALL DATA $(M, 2,3,2, M V)$

SETFIT

53

$\operatorname{CALL} \operatorname{DATA}(M, 3,1,2, \mathrm{IV})$

SETFIT $\quad 55$

$\operatorname{CALL} \operatorname{DATA}(M, 1,1,2, Q)$

SETFIT

$\operatorname{MSR}=\operatorname{MDAT}(M, 1)$

SETFIT

56

$\operatorname{MHZ}=\operatorname{MDAT}(M, 2)$

SETFIT

57

70

$K 1=K A$

SETFIT

58

$K 2=K B$

SETFIT

59

IF (.NOT.RSW) GO TO 117

SETF IT

60

$K 1=\operatorname{BDAT}(M, 5)$

SETFIT

61

$K 2=\operatorname{BDAT}(M, 6)$

SETFIT

62

IF (FSW) GO TO 120

SETFIT

SETFIT

SETFIT

SETFIT

SETFIT

SETFIT

SETFIT

SETFIT

SETFIT

CALL FITT(MSR, MHZ, MV, IV, Q, K1 ,K2)

RETURN

80

120 CALL FITE(MSR, MHZ, MV, IV, Q, K1,K2)

RETURN

END 
1

SUBROUTINE SETMAT(MT)

C ROUTINE SETS UP AND STORES UNIT AND TRANSPOSE MATRICES DIMENSION T $(7,7), R W(3)$

ASSIGN 6 TO NEXT

5

$$
\text { DO } 3 \quad I=1,3
$$

$3 \quad \mathrm{RW}(\mathrm{I})=0$.

4 DO $5 \mathrm{~J}=1,7$

DO $5 \mathrm{I}=1,7$

$5 \quad T(I, J)=0$.

10 GO TO NEXT. $(6,10)$

C COMPUTE UNIT MATRIX NAMED

6 DO $7 \quad \mathrm{I}=1,7$

$7 \quad T(I, I)=1$. $R W(3)=T(5,6)$

15

CALL STOR7 (MT, T, RW)

$M T=M T-1$

ASSIGN 10 TO NEXT

GO TO 4

C COMPUTE TRANSPOSE NAMED (S)

20

10 DO $12 \mathrm{I}=1,5,2$

$T(I, I+1)=-1$.

$12 T(I+1, I)=+1$.

$T(7,7)=+1$.

$\mathrm{RW}(3)=\mathrm{T}(5,6)$

25

CALL STOR7 (MT, T, RW)

RETURN

END

$\begin{array}{lr}\text { SETMAT } & 2 \\ \text { SETMAT } & 3 \\ \text { SETMAT } & 4 \\ \text { SETMAT } & 5 \\ \text { SETMAT } & 6 \\ \text { SETMAT } & 7 \\ \text { SETMAT } & 8 \\ \text { SETMAT } & 9 \\ \text { SETMAT } & 10 \\ \text { SETMAT } & 11 \\ \text { SETMAT } & 12 \\ \text { SETMAT } & 13 \\ \text { SETMAT } & 14 \\ \text { SETMAT } & 15 \\ \text { SETMAT } & 16 \\ \text { SETMAT } & 17 \\ \text { SETMAT } & 18 \\ \text { SETMAT } & 19 \\ \text { SETMAT } & 20 \\ \text { SETMAT } & 21 \\ \text { SETMAT } & 22 \\ \text { SETMAT } & 23 \\ \text { SETMAT } & 24 \\ \text { SETMAT } & 25 \\ \text { SETMAT } & 26 \\ \text { SETMAT } & 27 \\ \text { SETMAT } & 28\end{array}$


LEVEL 2, STORE, INFF, IWORK

BLANK

COMMON STORE (48000), IWORK(10)

86MARSIZ

DIMENSION INFF $(24,2000)$

86MARSIZ

EQUIVALENCE (INFF,STORE)

8GMARSIZ

DIMENSION RX(2,3),RY(2,3),PAR(4), RW(3)

BLANK

DATA (RW(I), I=1,3)/3*0.0/

SHF

SHF

SHF

SHF

$\operatorname{RX}(1,1)=1$.

$R \times(1,2)=0$.

$R \times(1,3)=\operatorname{PAR}(1)$

$\operatorname{RX}(2,1)=0$.

$\operatorname{RX}(2,2)=1$.

$\operatorname{RX}(2,3)=\operatorname{PAR}(2)$

$\operatorname{RY}(1,1)=1$.

$\operatorname{RY}(1,2)=0$.

$\operatorname{RY}(1,3)=\operatorname{PAR}(3)$

$\operatorname{RY}(2,1)=0$.

$\operatorname{RY}(2,2)=1$.

$\operatorname{RY}(2,3)=\operatorname{PAR}(4)$

$K B=\operatorname{INFF}(5, M)$

IF (KB. LE. O) GO TO 2

IF (KB.GE.1) ERRORS ARE RANDOM WITH GIVEN RANGE

DO1 $K=1,2$

$R X(K, 3)=R X(K, 3) *(R A N F(D)-.5)$

$1 \quad \operatorname{RY}(K, 3)=\operatorname{RY}(K, 3) *(\operatorname{RANF}(D)-.5)$

30

2 CONTINUE

CALL STXY (M,RX,RY,RW)

RETURN

END

SHF

SHF

SHF

SHF

SHF

SHF

SHF

SHF

SHF

SHF

SHF

SHF

SHF

SHF

SHF

SHF

SHF

SHF

SHF

SHF

SHF 
1

SUBROUT INE SKIP1

SKIP1

SKIP1

C SKIPS ONE PRINT LINE

SKIP1

SKIP1

FORMAT (1H )

SKIP1

SKIP1 
1

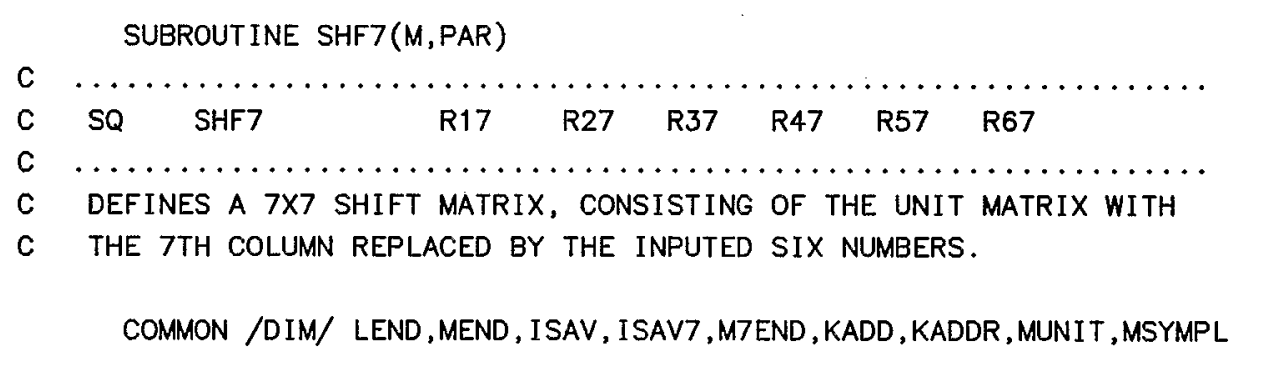

10 DIMENSION $T(7,7), \operatorname{RW}(3), \operatorname{PAR}(6)$

15

DIMENSION T(7,7), RW(3)
CALL RTRV7(MUNIT, T, RW)
DO $10 \quad I=1,6$
$10 \quad$ T $(I, 7)=$ PAR $(I)$
CALL STOR7 $(M, T, R W)$
RETURN
END


LEVEL 2, STORE, INFF, IWORK

COMMON STORE(48000), IWORK (10)

5

DIMENSION INFF $(24,2000)$

EQUIVALENCE (INFF, STORE)

SMIN

BLANK

BLANK

86MARSIZ

86MARSIZ 2

86MARSIZ 3

COMMON/CONTRL/ERROR, MODE, RSRV, STOR, XEQ, TRASW, NFSW, EMPTY, INDEF, 1 LDFLG, FIN

COMMON /MATCH2/ AA(30), BB(30), KBTS, SMINCAL, 1 $\operatorname{DWORD}(10), \operatorname{DWORD} 2(10), \operatorname{DWORD} 3(10), \operatorname{DWORD} 7(7,10), \mathrm{MINCOM}$ LOGICAL SMINCAL

BLANK 5

CONTRL $\quad 2$

CONTRL $\quad 3$

CONTRL 4

CONTRL 5

MATCH2 2

MATCH2 3

$\mathrm{MATCH} 2 \quad 4$

SMIN 6

SMIN 7

SMIN 8

C INSTRUCTION SMIN — SET COMMANDS FOR MINUIT

SMIN $\quad 9$

C INPUT HAS SAME STRUCTURE AS MINUIT COMMANDS, BUT SHIFTED TO START

SMIN $\quad 10$

20

C IN COLUMN 21, WITH FIVE WORDS OF FLOATING POINT DATA PER COMMAND.

SMIN

SMIN

SMIN

SMIN

C FIT SMIN 4 PRINTOUT 2.

$$
\mathrm{C}
$$

c

c

0.1

SIMPLEX 1500.

END RETURN

SMIN

SMIN

SMIN

SMIN

SMINCAL $=$.TRUE.

$\operatorname{KA}=\operatorname{INFF}(4, M)$

30

35

40

SMIN

SMIN

SMIN

SMIN

SMIN

SMIN

SMIN

SMIN

SMIN

SMIN

SMIN

SMIN

SMIN

SMIN

SMIN

SMIN

SMIN

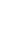
3

1

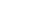

RETURN 
C FORMS A MATRIX REPRESENTING A SOLENOID.

SOL

$\mathrm{BO}$

SOL

SOL

$$
\mathrm{c}
$$$$
\text { (BLANK) }
$$

BRHO

SOL

SOL

C $L=$ LENGTH OF SOLENOID

c BRHO = B*RHO (THE RIGIDITY OF THE PARTICLE)

C $\mathrm{BO}=$ LONGITUDINAL FIELD IN SOLENOID

10

C

REAL K, L

DIMENSION PAR( 1$), T(7,7), \operatorname{RW}(3)$

c

DO $11 \mathrm{~J}=1,7$

15

DO $10 \mathrm{I}=1,7$

$10 \quad T(I, J)=0$.

11 CONTINUE

DO $12 I=1,3$

20

$12 \mathrm{RW}(\mathrm{I})=0$.

25

c

$L=\operatorname{PAR}(1)$

BRHO $=$ PAR (3)

$B O=\operatorname{PAR}(4)$

$K=B O /(2 . * B R H O)$

$C=\cos (K * L)$

$S=\operatorname{SIN}(K * L)$

$\mathrm{SC}=\mathrm{S} * \mathrm{C}$

$S S Q=S * S$

$\mathrm{CSQ}=\mathrm{C} * \mathrm{C}$

30

$\mathrm{T}(1,1)=\mathrm{CSQ}$

$T(2,1)=-K * S C$

$T(3,1)=-S C$

$T(4,1)=K * S S Q$

$T(1,2)=S C / K$

35

$T(2,2)=\operatorname{CS} Q$

$T(3,2)=-S S Q / K$

$T(4,2)=-S C$

$T(1,3)=S C$

$T(2,3)=-K * S S Q$

40

$T(3,3)=$ CSQ

$T(4,3)=-K * S C$

$T(1,4)=S S Q / K$

$T(2,4)=S C$

$T(3,4)=S C / K$

45

$T(4,4)=$ CSQ

$T(5,5)=1$.

$T(6,6)=1$.

C

$T(7,7)=1$.

50

$R W(1)=L$

CALL STOR7 $(M, T, R W)$

RETURN

END

SOL

SOL

SOL

SOL

SOL

SOL

SOL

SOL

SOL

SOL

SOL

SOL

SOL

SOL

SOL

SOL

SOL

SOL

SOL

SOL

SOL

SOL

SOL

SOL

SOL

SOL

SOL

SOL

SOL

SOL

SOL

SOL

SOL

SOL

SOL

SOL

SOL

SOL

SOL

SOL

SOL

SOL

SOL

SOL

SOL

SOL 
CARD NR. SEVERITY DETAILS

DIAGNOSIS OF PROBLEM

22 I PAR

ARRAY REFERENCE OUTSIDE DIMENSION BOUNDS.

23 I PAR ARRAY REFERENCE OUTSIDE DIMENSION BOUNDS. 
LEVEL 2, STORE, INFF, IWORK

COMMON STORE (48000), IWORK(10)

DIMENSION INFF $(24,2000)$

EQUIVALENCE (INFF, STORE)

COMMON/BMI 1/MI 1 (16000)

COMMON/NELS1/NELS1

LEVEL 2 , MI 1
C 1
COMMON/CONTRL/ERROR, MODE, RSRV, STOR, XEQ, TRASW, NFSW, EMPTY, INDEF,

BLANK 2

BLANK 3

86MARSIZ 1

86MARSIZ 2

86MARSIZ 3

BLANK 5

NV3BMI 1

BMI1L 2

BMI 1L 3

BMI1L 4

BMI 1

CONTRL 2

CONTRL 3

CONTRL 4

CONTRL 5

COMMONT 2

COMMONT 4

1/MINERR/ ERP(30) $\quad \operatorname{ERN}(30)$

2/PARINT/ $X(15) \quad X T(15)$

3/PAREXT/ U(30) ,NAM(30) ,WERR(30) ,MAXEXT ,NU

4/LIMITS/ $\operatorname{ALIM}(30), \operatorname{BLIM}(30), \operatorname{LCODE}(30), \operatorname{LCORSP}(30), \operatorname{LIMSET}$

5/VARIAN/ V $(15,15)$

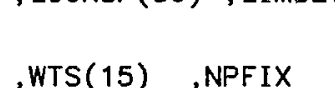

COMMONT

COMMONT

COMMONT

COMMONT

COMMONT

COMMONT

COMMONT

C/CASC/ JH, JL, Y(16)

F/DERIVA/ G(30)

, $\mathrm{G} 2(30)$

G/SIMVEC/ P(15,16) ,PSTAR(15) ,PSTST(15) ,PBAR(15) ,PRHO(15)

J/VARIAT/ VT $(15,15)$

$6 /$ UNIT / ISYSRD

, ISYSWR ,ISYSPU

, PRHO(15) COMMONT

COMMONT

COMMONT

,NBLOCK

, ISW(7)

8/TITLE / TITLE(13)

,DATE(2)

9/CONVER/ EPSI ,APSI

A/CARD / CWORD

. VTEST

B/MINIMA/ AMIN

,CWORD2

, NSTEPQ

, NFCN

, CWORD3

, WORD7 (7)

,UP NEWMIN

, ITAUR

,CNAME (25), CNAM2 (25), CNAM3 (25)

, NFCNMX

COMMONT

COMMONT

COMMONT

, SIGMA

COMMONT

COMMONT

MATCH

MATCH

MATCH

MATCH

MATCH

SOLV

SOLV

SOLV

SOLV

SOLV

SOLV

SOLV

SOLV

SOLV

MATCH2

MATCH2

MATCH2

MATCH3

MATCH3

SOLV

SOLV

SOLV

SOLV

SOLV

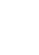

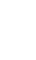

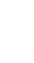

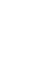

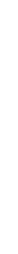


DATA MO/O/

DATA LIM1, LIM2/7HINITIAL,7H FINAL/

DATA MINZER/77777 7777777777 77777B/

DATA MASK1/77000 $000000000000000 \mathrm{~B} /$

DATA IBLNK1/550000000000000 00000B/

DATA NPR/6/

DATA IBLNK/1H /

C INSTRUCTION SOLV - BETATRON FUNCTION FITTING USING MINUIT

C $X X$ SOLV KA KB//SR TR IP1 IP2 ITS TOL PRN SAV PRI LAMDA SOLV

C (CONSTR CD 1) BNAM TYPE POS1 POS2 POS3 POS4 POS5 POS6 BFIT SIG SOLV

$C$ (CONSTR CD 2) BNAM TYPE POS1 POS2 POS3 POS4 POS5 POS6 BFIT SIG SOLV

SOLV

C (CONSTR CD KA) BNAM TYPE POS1 POS2 POS3 POS4 POS5 POS6 BFIT SIG SOLV

C (VARIABLE CD 1) PAR I1 I2 I3: I4 I5 LOWER UPPER STP SOLV

C (VARIABLE CD 2) PAR I1 I2 I3 I4 I5 LOWER UPPER STP SOLV

SOLV

C (VARIABLE CD KB) PAR I1 I2 I3 I4 I5 LOWER UPPER STP SOLV

C 2ND FORMAT FOR VARIABLE CARDS —

C $\ldots \ldots \ldots \ldots \ldots \ldots \ldots \ldots \ldots \ldots \ldots \ldots \ldots \ldots \ldots \ldots \ldots \ldots \ldots \ldots \ldots$ SOLV

C PAR1 PAR1 PAR3 PAR4 PAR5 I LOWER UPPER STO SOLV

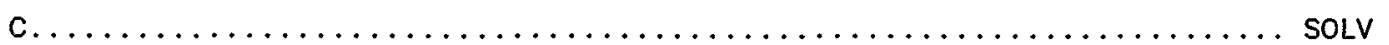

C PARK IS NAME OF KTH ELEMENT WHOSE ITH INPUT NUMBER IS TO BE VARIED..

\section{SOLV}

SOLV

SOLV

FIRST CARD —

$K A=$ NUMBER OF CONSTRAINT CARDS

SOLV

$K B=$ NUMBER OF VARIABLE CARDS

SR = NAME OF SYNCH SUBROUTINE WITH VARIABLES AND A TRKB INSTRUCTION

TR = NAME OF TRKB INSTRUCTION IN SR. TR MAY ALSO BE A CYC INSTRUCTION, WITH ITS KA SET TO 21 , OR AN MMM OR EQUIVALENT INSTRUCTION THAT DEFINES A SINGLE TRANSFER MATRIX FOR THE LATTICE PERIOD.

IP2 $=$ END POSITION OF TRKB RUN. IF ZERO, USE VALUES ON TRKB CARD. ITS = MAX NO. OF CALLS TO FCN BY MINUIT

TOL = DESIRED TOLERANCE ON FCN ( FCN . LE. 10**TOL )-AN INTEGER

PRN = PRINTOUT LEVEL FROM MINUIT ( 0 GIVES LEAST OUTPUT )

SOLV

SOLV

SOLV

SOLV

SOLV

SOLV

SOLV

SOLV

SOLV

SOLV

SOLV

SOLV

SAV - INITIALIZED TO 0 BY SRUN. IF SAV DIFFERENT FROM PREVIOUS VALUE, CURRENT VARIABLE VALUES ARE SAVED IN ARRAY V.

SOLV

SOLV SOLV ALWAYS TAKES INITIAL VARIABLE VALUES FROM $V$.

SOLV NORMALLY SET SAV=0, THEN VALUES FROM PREVIOUS FIT ARE USED

SOLV

SOLV FOR STARTING VALUES. SET SAV $=1$ TO ALWAYS START FROM SAME INITIAL VALUES.

$\mathrm{C}$

C

PRI $=0$ (OR BLANK) — PRINT BETAS BEFORE AND AFTER FITTING

SOLV

SOLV

- PRINT BETAS AFTER FITTING ONLY

SOLV

C 2

110

C $10,11,12$

- DO NOT PRINT BETAS

SOLV

C LAMDA - SEE BELOW UNDER VARIABLES 
C

C

C

C

C

C

C

C

C

C

C

C

C

C

C

C

C

c.

C

C

c

C

c

c

C

C

c

$$
\text { C }
$$

c

c

c

C

C

C

c

C

C C

C

c

C

$c$

$$
c
$$

\section{$c$}

c$$
\text { C }
$$$$
\text { C }
$$$$
\begin{aligned}
& \text { PASS ITS, TOL, PRN TO MINUIT } \\
& \text { IF (SMINCAL) GO TO } 5
\end{aligned}
$$

C

SAVE DEFAULT VALUES

$S=$ PATH LENGTH, THET = HORIZONTAL BEND ANGLE,

WST COMBINES THREE CONSTRAINTS AX, AY, AND $X$

MTRX — CONSTRAIN MATRIX ELEMENT I1, I2 OF MATRIX CORRESPONDING TO TR.

GAMT - TRANSITION GAMMA (TR MUST BE A CYC 21 INSTR.)

TYPE = STD OR BLANK -BETAS SHOULD FIT SPECIFIED VALUES

TYPE $=$ DIF — DIFFERENCE OF BETAS BETWEEN SUCCESSIVE POSITIONS SUM - SUM (DITTO) SHOULD FIT SPECIFIED VALUE BFIT.

WHEN BFIT $=S$, DIF AFFORDS A WAY TO OBTAIN GIVEN SEPARATIONS BETWEEN SPECIFIED POSITIONS.

TYPE = BFIT - BNAM IS THE NAME OF A PREVIOUSLY DEFINED MATRIX; THE BETA FUNCTIONS OF THAT MATRIX ARE CALCULATED; THEIR VALUES ARE THOSE THAT SOLV SEEKS TO FIT.

TYPE $=$ NO PASS OVER THIS CONSTRAINT

I1, I2, ... I5 ARE POSITIONS AT WHICH THE CONSTRAINT APPLIES.

THE POSITIONS CAN BE ENTERED SYMBOLICALLY IN THE FORM AAANN,

WHERE AAA IS THE NAME OF A BEAMLINE ELEMENT, AND NN DENOTES

WHICH OCCURRENCE OF AAA IN THE BEAMLINE IS MEANT.

IF NN IS BLANK, THE FIRST OCCURENCE OF THE NAME IS UNDERSTOOD.

SIGMA $=$ GOODNESS OF FIT DESIRED (INVERSE WEIGHT).

IF BNAM IS NOT A RECOGNIZED NAME, CONSTRAINT IGNORED, BUT POSITIONS CAN BE USED TO MARK FIT POINTS FOR SUBSEQUENT CONSTRAINT CARDS. IF A CONSTRAINT CARD HAS NO POSITIONS, THE POSITIONS ON THE NEAREST PREVIOUS CARD WITH POSITIONS PUNCHED WILL APPLY.

FCN = FUNCTION TO BE MINIMIZED BY SUBROUTINE FCN IS

FCN $=$ SUM $[($ (VALUE-FITVALUE)/TOLERANCE $) * * 2)] /($ NUMBER OF CONSTRAINTS)

VARI ABLE CARDS -

$K B=$ NUMBER OF VARIABLE CARDS, EACH MAY HAVE 1 PARAMETER NAME AND UP TO 5 PARAMETER INDICES.

SECOND FORMAT —UP TO 5 PARAMETER NAMES FOLLOWED BY 1 INDEX.

IF A VARIABLE CARD HAS NO POSITIONS, THE POSITIONS ON THE NEAREST

PREVIOUS CARD WITH POSITIONS PRESENT WILL APPLY.

IF STEPSIZ NOT ENTERED, DEFAULT IS (UPRBOUND-LOWBOUND) $/ 2$.

IF STEPSIZ IS NEGATIVE, VARIABLE ENTERS INTO FCN WITH

FIT VALUE $=($ UPPER+LOWER $) / 2$, TOLERANCE $=$ LAMDA $*($ UPPER-LOWER $) / 2$

ISV1 $=\operatorname{DWORD7}(1,1)$

$\operatorname{ISV} 2=\operatorname{DWORD} 7(1,2)$
SIG SOLV

SOLV

SOLV

SOLV

SOLV

SOLV

SOLV

SOLV

SOLV

SOLV

SOLV

SOLV

SOLV

SOLV

SOLV

SOLV

SOLV

SOLV

SOLV

SOLV

SOLV

SOLV

SOLV

SOLV

SOLV

SOLV

SOLV

SOLV

SOLV

SOLV

SOLV

SOLV

SOLV

SOLV

SOLV

SOLV

SOLV

SOLV

SOLV

SOLV

SOLV

SOLV

SOLV

SOLV

SOLV

SOLV

SOLV

SOLV

SOLV

SOLV

SOLV

SOLV

SOLV

SOLV

SOLV

SOLV

SOLV 
175

180

185

190

195

200

205

210

215

220
ISV3 $=\operatorname{DWORD7}(2,2)$
$\operatorname{ITS}=\operatorname{IDAT}(M, 1)$
$\operatorname{IF}(\operatorname{ITS}$. EQ.0) GO TO 3

DWORD7 $(1,2)=I$ TS

$3 \quad$ ITOL $=\operatorname{IDAT}(M, 2)$

$\operatorname{DWORD7}(2,2)=10 . * * \operatorname{ITOL}$

$4 \operatorname{IPR}=\operatorname{IDAT}(M, 3)$

IF (IPR.EQ.0) GO TO 5

$\operatorname{DWORD7}(1,1)=\operatorname{IPR}$

5 CONTINUE

IVSAVO=IVSAV

$\operatorname{IVSAV}=\operatorname{IDAT}(M, 4)$

$\operatorname{IPP}=\operatorname{IDAT}(M, 5)$

$M I F L G=. F A L S E$.

MATFLG $=$.FALSE.

GLOBAL $=$.FALSE.

IF (IPP.LT.10) GO TO 52

MIFLG $=$. TRUE.

$I P P=I P P-10$

$\operatorname{KAT}=\operatorname{INFF}(4, M)$

KAR=KAT

$K B T=\operatorname{INFF}(5, M)$

$K B R=K B T$

$K B T S=K B T$

$\operatorname{MSR}=\operatorname{MDAT}(M, 1)$

$\operatorname{MTR}=\operatorname{MDAT}(M, 2)$

KATR $=0$

OPTR $=\operatorname{INFF}(1$, MTR $)$

IF ((OPTR.NE.HTRKB).AND. (OPTR.NE.HCYC)) GO TO 55

MBML $=$ MDAT $($ MTR , 1)

NDAT $=\operatorname{INFF}(17$, MBML $)$

CALL MIFILL(MBML, 1 , NDAT, NELS1, MI1)

NELS2 $=$ NELS1

$\mathrm{KBTR}=\operatorname{INFF}(5, \mathrm{MTR})$

IF (OPTR.NE.HTRKB) GO TO 53

KATR $=\operatorname{INFF}(4$, MTR $)$

CALL DATA $(M, 2,3,2, N I P)$

CALL LOCS(NIP, 2,NELS1, MI 1, IP)

$I P 1=I P(1)$

$I P 2=I P(2)$

IF (IP1.EQ.0.AND.IP2.EQ.0) GO TO 54

CALL REPINT (MTR, 1, IP1)

CALL REPINT (MTR, 2, IP2)

GO TO 54

53 IF (KBTR.LT, O) NELS2 = NELS1 + 1

$54 \operatorname{LQTR}=\operatorname{INFF}(24$, MTR $)$

LTRTAB = LQTR - 14*KATR

GO TO 51

55 MATFLG $=$.TRUE.

C 51 NASR $=\operatorname{INFF}(2$, MSR $)$

51 CONTINUE

$X \operatorname{LAMDA}=\operatorname{FDAT}(M, 1)$

\begin{tabular}{|c|c|}
\hline SOLV & 138 \\
\hline SOLV & 139 \\
\hline SOLV & 140 \\
\hline SOLV & 141 \\
\hline SOLV & 142 \\
\hline SOLV & 143 \\
\hline SOLV & 144 \\
\hline SOLV & 145 \\
\hline SOLV & 146 \\
\hline SOLV & 147 \\
\hline SOLV & 148 \\
\hline SOLV & 149 \\
\hline SOLV & 150 \\
\hline SOLV & 151 \\
\hline SOLV & 152 \\
\hline SOLV & 153 \\
\hline SOLV & 154 \\
\hline SOLV & 155 \\
\hline SOLV & 156 \\
\hline SOLV & 157 \\
\hline SOLV & 158 \\
\hline SOLV & 159 \\
\hline SOLV & 160 \\
\hline SOLV & 161 \\
\hline SOLV & 162 \\
\hline SOLV & 163 \\
\hline SOLV & 164 \\
\hline SOLV & 165 \\
\hline SOLV & 166 \\
\hline SOLV & 167 \\
\hline SOLV & 168 \\
\hline SOLV & 169 \\
\hline SOLV & 170 \\
\hline SOLV & 171 \\
\hline SOLV & 172 \\
\hline SOLV & 173 \\
\hline SOLV & 174 \\
\hline SOLV & 175 \\
\hline SOLV & 176 \\
\hline SOLV & 177 \\
\hline SOLV & 178 \\
\hline SOLV & 179 \\
\hline SOLV & 180 \\
\hline SOLV & 181 \\
\hline SOLV & 182 \\
\hline SOLV & 183 \\
\hline SOLV & 184 \\
\hline SOLV & 185 \\
\hline SOLV & 186 \\
\hline SOLV & 187 \\
\hline SOLV & 188 \\
\hline SOLV & 189 \\
\hline SOLV & 190 \\
\hline SOLV & 191 \\
\hline SOLV & 192 \\
\hline SOLV & 193 \\
\hline SOLV & 194 \\
\hline
\end{tabular}


230

235

240

245

250

255

260

265

270

63

62 DO $6 \quad I=1,42$

6 IF (NCONK.EQ.NAMBFC(I)) ICON(K)=I

DO $2 \mathrm{~N}=1, \mathrm{KAR}$

$K=K+1$

$\operatorname{ICON}(K)=0$

NTYPE $=\operatorname{BDAT}(M, 8 * N-2)$

$\operatorname{ITYPE}(K)=0$

IF (NTYPE.EQ. 5HNO ) ITYPE(K) $=-1$

IF(NTYPE. EQ.5HSTD ) ITYPE(K) $=0$

IF(NTYPE.EQ.5HDIF ) ITYPE(K) $=1$

IF (NTYPE.EQ.5HSUM ) ITYPE(K)=2

NCONK $=\operatorname{BDAT}(M, 8 * N-3)$

IF (NTYPE.NE.5HBFIT) GO TO 62

$\operatorname{ITYPE}(K)=3$

GO TO 63

$I C O N K=I C O N(K)$

IF $(I C O N(K) \cdot G E \cdot 34 \cdot A N D \cdot I C O N(K) \cdot L E \cdot 37) \quad$ MIFLG $=$.TRUE.

$\operatorname{IF}(\operatorname{ICON}(K) . E Q . \theta)$ ITYPE $(K)=-1$

LPOSK $=7+8 *(\mathrm{~N}-1)$

IF (ICON(K).LE.14.OR. ICON(K).GE.34) GO TO 65

$\operatorname{IPOS}(1, K)=\operatorname{NELS} 2$

$\operatorname{NPOS}(K)=1$

GLOBAL $=$. TRUE.

GO TO 8

65 CONTINUE

CALL DATA (M, 2, LPOSK, 6, NAIPOS)

CALL LOCS(NAIPOS, $6, \operatorname{NELS} 1, M I 1, \operatorname{IPOS}(1, K))$

$\operatorname{NPOS}(K)=0$

7

$I=0$

$\mathrm{I}=\mathrm{I}+1$

IF (NAIPOS(I).NE. IBLNK) GO TO 72

NP $1=0$

$I 1=I$

DO $71 \quad \mathrm{I} I=\mathrm{I} 1,6$

$I P O S(I I, K)=I P O S(I I+1, K)$

71 IF (NAIPOS(I).NE. IBLNK) NP1 $=\mathrm{NP} 1+1$

275

280
$I=I-1$

IF(NP1.EQ.0) GO TO 73

GO TO 7

72

$\operatorname{NPOS}(K)=\operatorname{NPOS}(K)+1$

IF (I.LT.6) GO TO 7

73

CONT INUE

IF(NPOS(K).NE. $\theta)$ GO TO 8

IF (ICON(K).NE.0) GO TO 74

$\mathrm{K}=\mathrm{K}-1$

GO TO 2

\begin{tabular}{|c|c|}
\hline SOLV & 195 \\
\hline SOLV & 196 \\
\hline SOLV & 197 \\
\hline SOLV & 198 \\
\hline SOLV & 199 \\
\hline SOLV & 200 \\
\hline SOLV & 201 \\
\hline SOLV & 202 \\
\hline SOLV & 203 \\
\hline SOLV & 204 \\
\hline SOLV & 205 \\
\hline SOLV & 206 \\
\hline SOLV & 207 \\
\hline SOLV & 208 \\
\hline SOLV & 209 \\
\hline SOLV & 210 \\
\hline SOLV & 211 \\
\hline SOLV & 212 \\
\hline SOLV & 213 \\
\hline SOLV & 214 \\
\hline SOLV & 215 \\
\hline SOLV & 216 \\
\hline SOLV & 217 \\
\hline SOLV & 218 \\
\hline SOLV & 219 \\
\hline SOLV & 220 \\
\hline SOLV & 221 \\
\hline SOLV & 222 \\
\hline SOLV & 223 \\
\hline SOLV & 224 \\
\hline SOLV & 225 \\
\hline SOLV & 226 \\
\hline SOLV & 227 \\
\hline SOLV & 228 \\
\hline SOLV & 229 \\
\hline SOLV & 230 \\
\hline SOLV & 231 \\
\hline SOLV & 232 \\
\hline SOLV & 233 \\
\hline SOLV & 234 \\
\hline SOLV & 235 \\
\hline SOLV & 236 \\
\hline SOLV & 237 \\
\hline SOLV & 238 \\
\hline SOLV & 239 \\
\hline SOLV & $24 e$ \\
\hline SOLV & 241 \\
\hline SOLV & 242 \\
\hline SOLV & 243 \\
\hline SOLV & 244 \\
\hline SOLV & 245 \\
\hline SOLV & 246 \\
\hline SOLV & 247 \\
\hline SOLV & 248 \\
\hline SOLV & 249 \\
\hline SOLV & 250 \\
\hline SOLV & 251 \\
\hline
\end{tabular}


290

295

300

310

315

320

325

330

335
74 CONTINUE

IF (K.GT.1) GO TO 10

NUMCON $=1$

GO TO 32

$10 \quad \operatorname{NPOS}(K)=N P O S(K-1)$

DO $9 \mathrm{I}=1,6$

$9 \operatorname{IPOS}(\mathrm{I}, \mathrm{K})=\mathrm{IPOS}(\mathrm{I}, \mathrm{K}-1)$

8 CONTINUE

NUMCON=NPOS $(K)$

32

CONT INUE

IF (ITYPE(K),EQ. -1) NUMCON=0

IF ( (ITYPE(K).EQ.1).OR. (ITYPE(K).EQ.2) ) NUMCON=NUMCON-1

$\operatorname{LFPK}=2 *(\mathrm{~N}-1)+1$

$\operatorname{BETO}(K)=\operatorname{FDAT}(M, \operatorname{LFPK}+1)$

$\operatorname{SIG}(K)=\operatorname{FDAT}(M, \operatorname{LFPK}+2)$

$\operatorname{IF}(\operatorname{SIG}(K) . E Q .0.) \operatorname{SIG}(K)=1 . E 20$

$W T(K)=1 \cdot / \operatorname{SIG}(K)$

IF (ICON(K).LT.38) GO TO 13

$\mathrm{LL}=1$

IF (ICONK.NE.40. AND. ICONK.NE.39) GO TO 28

$\mathrm{LL}=2$

$\operatorname{ICON}(K)=3$

$\operatorname{ICON}(K+1)=6$

$\operatorname{ICON}(K+2)=9$

IF (ICONK.EQ.40) GO TO 27

$\mathrm{LL}=3$

$I \operatorname{CON}(K+3)=37$

BETOF = BETO $(K)$

$\mathrm{BETO}(\mathrm{K})=0$.

GO TO 27

28

IF (ICONK.NE.41) GO TO 29

$I \operatorname{CON}(K)=5$

$\operatorname{ICON}(K+1)=6$

GO TO 27

29 IF (ICONK. NE.42. AND. ICONK.NE.38) GO TO 27

$I C O N(K)=3$

$\operatorname{ICON}(K+1)=9$

IF (ICONK.EQ.42) GO TO 27

$\mathrm{LL}=2$

$I C O N(K+2)=37$

$B E T O F=B E T O(K)$

$\operatorname{BETO}(K)=0$.

27

CONT INUE

$\mathrm{KL}=\mathrm{K}$

DO $11 L=1, L L$

$K L=K L+1$

$\operatorname{ITYPE}(K L)=\operatorname{ITYPE}(K)$

$\operatorname{NPOS}(K L)=\operatorname{NPOS}(K)$
SOLV 252

SOLV 253

SOLV 254

SOLV 255

SOLV 256

SOLV 257

SOLV 258

SOLV 259

SOLV 260

SOLV 261

SOLV 262

SOLV 263

SOLV 264

SOLV 265

SOLV 266

SOLV 267

SOLV 268

SOLV 269

SOLV 270

SOLV 271

SOLV 272

SOLV 273

SOLV 274

SOLV 275

SOLV 276

SOLV 277

SOLV 278

SOLV 279

SOLV 280

SOLV 281

SOLV 282

SOLV 283

SOLV 284

SOLV 285

SOLV 286

SOLV 287

SOLV 288

SOLV 289

SOLV 290

SOLV 291

SOLV 292

SOLV 293

SOLV 294

SOLV 295

SOLV 296

SOLV 297

SOLV 298

SOLV 299

SOLV 300

SOLV 301

SOLV $\quad 302$

SOLV 303

SOLV $\quad 304$

SOLV 305

SOLV $\quad 306$

SOLV $\quad 307$

SOLV $\quad 308$ 
345

350

355

360

365

370

375

380

385

390

395

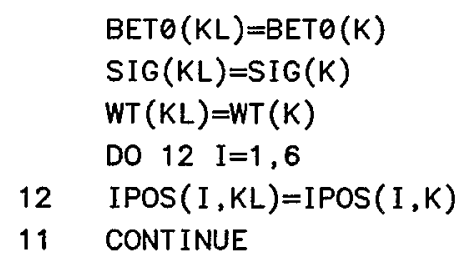

IF (ICONK.EQ . 38.OR. ICONK.EQ. 39) BETO $(K L)=B E T O F$ NUMCON $=(L L+1) * N U M C O N$

$\mathrm{K}=\mathrm{KL}$

$K A T=K A T+L L$

GO TO 131

13 CONTINUE

IF ((ICON(K).NE.35).AND. (ICON(K).NE.36)) GO TO 14 $\operatorname{NPOS}(K)=4$

14

NUMCON $=1$

IF $(\operatorname{IPOS}(3, K) \cdot E Q \cdot \theta) \quad \operatorname{IPOS}(3, K)=1$

$\operatorname{IF}(\operatorname{IPOS}(4, K), E Q \cdot \theta) \quad \operatorname{IPOS}(4, K)=1$

CONTINUE

IF (ICON(K).NE.36) GO TO 24

$\mathrm{NN}=\mathrm{IPOS}(1, \mathrm{~K}) / 2$

$I S G N=1$

IF $(\operatorname{IPOS}(2, \mathrm{~K}) \cdot$ LT. $\theta) \quad$ ISGN $=-1$

$\operatorname{IPOS}(2, K)=0$

$\operatorname{ICON}(K)=35$

$\mathrm{KK}=\mathrm{K}$

DO 25 I $I=1, N N$

$\mathrm{KK}=\mathrm{KK}+1$

DO $26 \quad J J=3,4$

26

$\operatorname{IPOS}(J J, K K)=\operatorname{IPOS}(J J, K)$

$\operatorname{ICON}(K K)=35$

$\operatorname{NPOS}(K K)=4$

$\operatorname{SIG}(K K)=\operatorname{SIG}(K)$

$\operatorname{BETO}(K K)=\operatorname{BETO}(K)$

$W T(K K)=W T(K)$

$\operatorname{IPOS}(1, K K)=\operatorname{IPOS}(1, K K-1)-2$

$\operatorname{IPOS}(2, K K)=\operatorname{IPOS}(2, K K-1)+2 * \operatorname{ISGN}$

25

CONT INUE

$\mathrm{K}=\mathrm{K}+\mathrm{NN}$

$\mathrm{KAT}=\mathrm{KAT}+\mathrm{NN}$

NUMCON $=N N+1$

24

CONT INUE

IF (ITYPE(K).NE.3) GO TO 131

MBET $=E L N U M$ (NCONK)

CALL BET (MBET , BXI, BYI, $\theta$ )

$\operatorname{ICON}(K)=2$

$\operatorname{ICON}(K+1)=3$

$\operatorname{ICON}(K+2)=5$

$\operatorname{ICON}(K+3)=6$

$\operatorname{ICON}(K+4)=8$

$\operatorname{ICON}(K+5)=9$

$\operatorname{BETO}(K \quad)=\operatorname{BXI}(2)$

$\operatorname{BETO}(K+1)=\operatorname{BXI}(3)$

$\operatorname{BET} \theta(K+2)=\operatorname{BXI}(5)$

\begin{tabular}{|c|c|}
\hline SOLV & 309 \\
\hline SOLV & 310 \\
\hline SOLV & 311 \\
\hline SOLV & 312 \\
\hline SOLV & 313 \\
\hline SOLV & 314 \\
\hline SOLV & 315 \\
\hline SOLV & 316 \\
\hline SOLV & 317 \\
\hline SOLV & 318 \\
\hline SOLV & 319 \\
\hline SOLV & 320 \\
\hline SOLV & 321 \\
\hline SOLV & 322 \\
\hline SOLV & 323 \\
\hline SOLV & 324 \\
\hline SOLV & 325 \\
\hline SOLV & 326 \\
\hline SOLV & 327 \\
\hline SOLV & 328 \\
\hline SOLV & 329 \\
\hline SOLV & 330 \\
\hline SOLV & 331 \\
\hline SOLV & 332 \\
\hline SOLV & 333 \\
\hline SOLV & 334 \\
\hline SOLV & 335 \\
\hline SOLV & 336 \\
\hline SOLV & 337 \\
\hline SOLV & 338 \\
\hline SOLV & 339 \\
\hline SOLV & 340 \\
\hline SOLV & 341 \\
\hline SOLV & 342 \\
\hline SOLV & 343 \\
\hline SOLV & 344 \\
\hline SOLV & 345 \\
\hline SOLV & 346 \\
\hline SOLV & 347 \\
\hline SOLV & 348 \\
\hline SOLV & 349 \\
\hline SOLV & 350 \\
\hline SOLV & 351 \\
\hline SOLV & 352 \\
\hline SOLV & 353 \\
\hline SOLV & 354 \\
\hline SOLV & 355 \\
\hline SOLV & 356 \\
\hline SOLV & 357 \\
\hline SOLV & 358 \\
\hline SOLV & 359 \\
\hline SOLV & 360 \\
\hline SOLV & 361 \\
\hline SOLV & 362 \\
\hline SOLV & 363 \\
\hline SOLV & 364 \\
\hline SOLV & 365 \\
\hline
\end{tabular}


400

$$
\begin{aligned}
& \operatorname{BETO}(K+3)=\operatorname{BXI}(6) \\
& \operatorname{BETO}(K+4)=\operatorname{BYI}(2) \\
& \operatorname{BETO}(K+5)=\operatorname{BYI}(3)
\end{aligned}
$$

SOLV

SOLV

SOLV

DO $132 L=1,5$

405

$\operatorname{ITYPE}(K+L)=\operatorname{ITYPE}(K)$

SOLV

SOLV

SOLV

$\operatorname{NPOS}(K+L)=\operatorname{NPOS}(K)$

SOLV

$\operatorname{SIG}(K+L)=S \operatorname{IG}(K)$

SOLV

$W T(K+L)=W T(K)$

SOLV

SOLV

SOLV

SOLV

SOLV

SOLV

SOLV

SOLV

SOLV

SOLV

SOLV

SOLV

SOLV

SOLV

SOLV

SOLV

SOLV

SOLV

SOLV

SOLV

SOLV

SOLV

SOLV

SOLV

SOLV

SOLV

SOLV

SOLV

SOLV

SOLV

SOLV

C GET DATA ON VARIABLES AND LOAD COMMON BLOCKS FOR FCN AND MIDATA

SOLV

SOLV

SOLV

SOLV

VARWTS $=$. FALSE

NVARWT $=0$

IF (XLAMDA.EQ. 0.$) \quad X L A M D A=1.0$

SOLV

SOLV

SOLV

SOLV

SOLV

DO $1 \mathrm{~J}=1, \mathrm{KBR}$

$\mathrm{L} 2=1$

$\lfloor O C J=2 * K A R+3 * J-2$

SOLV

SOLV

$A A J=F D A T(M, L O C J+1)$

$B B J=F D A T(M, L O C J+2)$

SOLV

SOLV

$W R J=F D A T(M, L O C J+3)$

SOLV

$V A L J=(B B J+A A J) / 2$.

$D E L=A B S(B B J-A A J) / 2$. 
460

465

470

480

485

490

495

17 CONTINUE

\section{FIND OUT FORMAT TYPE}

IFORM=1

$L I=L+1$

DO $17 \mathrm{I}=1,5$

475

$E R P R=$. FALSE.

MPARI $=$ MDAT $(M$, LVARJ $+I)$

IF (MPARI.EQ.MINZER) GO TO 16

IFORM $=2$

$\mathrm{L} 2=\mathrm{L} 2+1$

$L I=L I+1$

$\operatorname{MPAR}(L I)=$ MPARI

$\operatorname{NAMPAR}(L 2)=\operatorname{BDAT}(M$, LVARJ $+I)$

GO TO 17

16 NAIND (I) $=$ BDAT (M, LVARJ+I)

$\operatorname{DECODE}(5,100, \operatorname{NAIND}(\mathrm{I})) \operatorname{IND}(I)$

100 FORMAT (I5)

C TO MAKE INPUT COMPATIBLE WITH VAX VERSION $\operatorname{DECODE}(5,2000, \operatorname{NAIND}(\mathrm{I})) \mathrm{LL}$

2000 FORMAT (4XA1)

$\operatorname{IF}(L L . E Q .1 H) \operatorname{IND}(\mathrm{I})=\operatorname{IND}(\mathrm{I}) / 10$

IF(IND(I).EQ.O) GO TO 17

NAIND2 =NAIND (I)

IND2 $=\operatorname{IND}(\mathrm{I})$

\author{
$L \theta=L+1$ \\ IF(IFORM.EQ.2) GO TO 18 \\ C FORMAT 1
}

500

510

DO $15 \quad I=1,5$

$\operatorname{IF}(\operatorname{IND}(\mathrm{I}) \cdot \mathrm{EQ} .0)$ GO TO 15

$\operatorname{ENCODE}(10,101, \operatorname{NAMI}(\mathrm{I})) \operatorname{NAMPAR}(1), \operatorname{NAIND}(\mathrm{I})$

101 FORMAT (2A5)

$L=L+1$

505

$\operatorname{NAM}(L)=N$ NAMI $(I)$

$\operatorname{MPAR}(L)=$ MPARJ

$\operatorname{IPAR}(L)=\operatorname{IND}(\mathrm{I})$

15 CONTINUE

C IF NO INDEX WAS PRESENT, USE PREVIOUSLY DEFINED ONE.

IF (L.GE.LO) GO TO 20

$L=L+1$

ENCODE $(10,101, \operatorname{NAM}(L)) \quad \operatorname{NAMPAR}(1), \operatorname{NAIND} 2$

$\operatorname{MPAR}(L)=\operatorname{MPARJ}$

\begin{tabular}{|c|c|}
\hline SOLV & 423 \\
\hline SOLV & 424 \\
\hline SOLV & 425 \\
\hline SOLV & 426 \\
\hline SOLV & 427 \\
\hline SOLV & 428 \\
\hline SOLV & 429 \\
\hline SOLV & 430 \\
\hline SOLV & 431 \\
\hline SOLV & 432 \\
\hline SOLV & 433 \\
\hline SOLV & 434 \\
\hline SOLV & 435 \\
\hline SOLV & 436 \\
\hline SOLV & 437 \\
\hline SOLV & 438 \\
\hline SOLV & 439 \\
\hline SOLV & 440 \\
\hline SOLV & 441 \\
\hline SOLV & 442 \\
\hline SOLV & 443 \\
\hline SOLV & 444 \\
\hline SOLV & 445 \\
\hline SOLV & 446 \\
\hline SOLV & 447 \\
\hline SOLV & 448 \\
\hline SOLV & 449 \\
\hline SOLV & 450 \\
\hline SOLV & 451 \\
\hline SOLV & 452 \\
\hline 86SOLV & 1 \\
\hline 86SOLV & 2 \\
\hline 86SOLV & 3 \\
\hline 86SOLV & 4 \\
\hline SOLV & 453 \\
\hline SOLV & 454 \\
\hline SOLV & 455 \\
\hline SOLV & 456 \\
\hline SOLV & 457 \\
\hline SOLV & 458 \\
\hline SOLV & 459 \\
\hline SOLV & 460 \\
\hline SOLV & 461 \\
\hline SOLV & 462 \\
\hline SOLV & 463 \\
\hline SOLV & 464 \\
\hline SOLV & 465 \\
\hline SOLV & 466 \\
\hline SOLV & 467 \\
\hline SOLV & 468 \\
\hline SOLV & 469 \\
\hline SOLV & 470 \\
\hline SOLV & 471 \\
\hline SOLV & 472 \\
\hline SOLV & 473 \\
\hline SOLV & 474 \\
\hline SOLV & 475 \\
\hline
\end{tabular}


515

$$
\operatorname{IPAR}(L)=\operatorname{IND} 2
$$

$$
\text { GO TO } 20
$$

\section{FORMAT 2}

$18 \quad L L 2=L 2$

520

530

535

540

545

550

560

565

CALL MINNEW

MINFLG $=1$

CALL DASH

MIFLG $=$.FALSE.

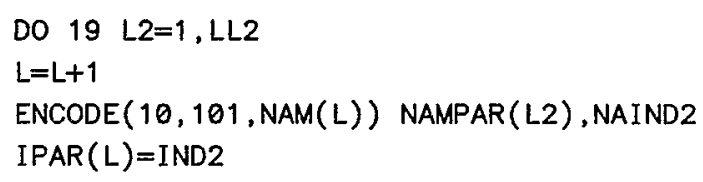

WRITE $(3,105)$ LIM2, (I,NAM(I),U(I), I=1,NPAR)

CALL FCN(NPAR, $G, F, U, 3$ )

\begin{tabular}{|c|c|}
\hline SOLV & 476 \\
\hline SOLV & 477 \\
\hline SOLV & 478 \\
\hline SOLV & 479 \\
\hline SOLV & 480 \\
\hline SOLV & 481 \\
\hline SOLV & 482 \\
\hline SOLV & 483 \\
\hline SOLV & 484 \\
\hline SOLV & 485 \\
\hline SOLV & 486 \\
\hline SOLV & 487 \\
\hline SOLV & 488 \\
\hline SOLV & 489 \\
\hline SOLV & 490 \\
\hline SOLV & 491 \\
\hline SOLV & 492 \\
\hline SOLV & 493 \\
\hline SOLV & 494 \\
\hline SOLV & 495 \\
\hline SOLV & 496 \\
\hline SOLV & 497 \\
\hline SOLV & 498 \\
\hline SOLV & 499 \\
\hline SOLV & 500 \\
\hline SOLV & 501 \\
\hline SOLV & 502 \\
\hline SOLV & 503 \\
\hline SOLV & 504 \\
\hline SOLV & 505 \\
\hline SOLV & 506 \\
\hline SOLV & 507 \\
\hline SOLV & 508 \\
\hline SOLV & 509 \\
\hline SOLV & 510 \\
\hline SOLV & 511 \\
\hline SOLV & 512 \\
\hline SOLV & 513 \\
\hline SOLV & 514 \\
\hline SOLV & 515 \\
\hline SOLV & 516 \\
\hline SOLV & 517 \\
\hline SOLV & 518 \\
\hline SOLV & 519 \\
\hline SOLV & 520 \\
\hline SOLV & 521 \\
\hline SOLV & 522 \\
\hline SOLV & 523 \\
\hline SOLV & 524 \\
\hline SOLV & 525 \\
\hline SOLV & 526 \\
\hline SOLV & 527 \\
\hline SOLV & 528 \\
\hline SOLV & 529 \\
\hline SOLV & 530 \\
\hline SOLV & 531 \\
\hline SOLV & 532 \\
\hline
\end{tabular}

C RESTORE DEFAULTS IF SAVED 
IF (SMINCAL) RETURN

SOLV

533

$\operatorname{DWORD} 7(1,1)=$ ISV1

SOLV

534

$\operatorname{DWORD} 7(1,2)=\operatorname{ISV} 2$

SOLV

535

$\operatorname{DWORD} 7(2,2)=\operatorname{ISV} 3$

SOLV

536

RETURN

SOLV

537

102 FORMAT (10X, *- SOLV - BETA-FUNCTION FITTING *)

SOLV

538

105 FORMAT ( $1 \mathrm{H} 0,5 X, A 7, *$ VALUES OF VARIABLES*/

SOLV

539

$1(7 X, I 5,3 X, A 5,5 X, E 15.8))$

SOLV

540

SOLV

541

580

END

SOLV

542 
* infut has P or c in col. 1, followed by hollerith data

* constructs a page or ReM synch instiructión

* if not in mOde 2, exECUTES page and/OR REMARK

* When in mOde 3, it is handled noRmally by SWITCH

INTEGER HPERIOD, HREM

DATA IB, HPERIOD, HREM/1H , $1 \mathrm{H} ., 3$ HREM/

DATA MASK1, MASK2/00777777777777777777B,55000000000000000000B/

INS $=1 \mathrm{H}$

IF (ISIGN.EQ.1HP) OPNAME=4HPAGE

IF (ISIGN.EQ. 1HC) OPNAME = 4HREM

IF (ISIGN.EQ. HPERIOD) OPNAME = HREM

CALL STINFO $(M, I B$, OPNAME, $\theta, \theta, \theta)$

$\operatorname{DECODE}(80,2, \operatorname{ICARD}(1))(\operatorname{ICOM}(\mathrm{I}), \mathrm{I}=1,16)$

MASK OFF FIRST CHARACTER

$\operatorname{ICOM}(1)=I \operatorname{COM}(1) \cdot \operatorname{AND} \cdot \operatorname{MASK} 1$

$\operatorname{ICOM}(1)=\operatorname{ICOM}(1) \cdot \mathrm{OR} \cdot \operatorname{MASK} 2$

CALL RESRV $(M, \theta, \theta$,

- $16,2,0,0,0,0,0,0$

.)

$\operatorname{CALL} \operatorname{STDAT}(M, 2,1,16, \operatorname{ICOM})$

IF (MODE.EQ.2) GO TO 10

IF (.NOT.CPYSW) RETURN

IF (MODE.EQ.1) GO TO 10

IF (ISIGN.EQ.1HP) INS = ISIGN

GO TO 12

10 IF (ISIGN.EQ.1HP) WRITE $(3,1)$

12 WRITE $(3,3)$ INS, $(\operatorname{ICOM}(I), I=1,16)$

RETURN

FORMAT (1H1)

FORMAT (16A5)

FORMAT $(4 X, A 4,16 A 5)$

END 
LEVEL 2, STORE, INFF, IWORK

COMMON STORE (48000), IWORK (10)

COMMON/INSTR/OPNAME, NAME, OP, KA, KB, OBJA, OBJB, NXTM, TRA, LQ, NQ, LIN, NIN, LFL, NFL, LBC, NBC, NTOT, IX, KIND, TYPE, ROWS, COLS, EXTR,

COMMON /DIM/ LEND, MEND, ISAV, ISAV7, M7END, KADD, KADDR, MUNIT, MSYMPL COMMON/FLTN/IFL(15)

COMMON/TRKINT/G, EX, EY, OMSQ, SEND, VMX, VMN, DINT , BEG , DPR , JPR,

1 THET, PLT, NPT, NZ, PMAX, NSIZ, ITITLE(6)

LOGICAL BEG, DPR, PLT

COMMON /MATCH/ KAT, KBT, MPAR(30), IPAR(30), MSR, MTR, LTRTAB, FNORM, $1 \operatorname{ICON}(30), \operatorname{ITYPE}(30), \operatorname{NPOS}(30), \operatorname{IPOS}(7,30), \operatorname{BETO}(30), \operatorname{WT}(30)$,

$2 \operatorname{Sig}(30), \operatorname{NAMBFC}(42), \operatorname{ERPR}, \operatorname{IVSAV}, \operatorname{LVAR}(30), \operatorname{WTVAR}(30), \operatorname{VAL}(30)$,

3 VARWTS, IPRPOS $(6,30)$

LOGICAL ERPR, LVAR, VARWTS

INTEGER R7, INAM(2)

COMMON/GRR/IERR, BSW, VSW, RSW, QSW, XSW

LOGICAL IERR, BSW, VSW, RSW, QSW, XSW

EQUIVALENCE (MIN, MS)

COMMON/SVNAM/NAMRUN

DATA IBLNK/1H /,MXPMY, R7/1,5/, ( INAM(I), I=1,2)/3H(1), 3H(S)/

DATA IBLNK /1H /

DATA MXPMY,R7/1,5/

INSTR

INSTR

INSTR

CONTRL

CONTRL

CONTRL

CONTRL

DIM

DIM

FLTN

FLTN

STORE

STORE

STORE

SWTCH

SWTCH

SWTCH

TRK INT

TRKINT

TRKINT

MATCH

MATCH

MATCH

MATCH

MATCH

SRUN

GRR

GRR

GRR

SRUN

SVNAM

SRUN

SRUN

SRUN

SRUN

SRUN

IF (OP.LT. $\theta$ ) RETURN

SRUN

SRUN

SRUN

SRUN

SRUN

SRUN

SRUN

SRUN

SRUN

SRUN

SRUN

SRUN 
60

DO $25 \mathrm{I}=1$, LMAX

DO $26 \mathrm{I}=1, \mathrm{MAX}$

\section{$26 \operatorname{INFF}(J, I)=0$}

EMPTY $=$. FALSE.

INDEF $=$, FALSE.

65

$X E Q=$. TRUE.

SRUN

SRUN

$S T O R=$. TRUE.

RSRV $=$. TRUE.

SRUN

SRUN

ERROR=. FALSE.

CYCSWT = . FALSE.

IERR=. FALSE.

$P V=$. FALSE.

MSSW $=$.FALSE .

VPR $=$. FALSE.

SRUN

$M H S=$. FALSE.

MODS =. FALSE.

SRUN

SRUN

$E R P R=. T R U E$.

IVSAV $=0$

MODE $=1$

$N F=0$

80

$\mathrm{NB}=0$

$N I=0$

NAMRUN=NAME

$B E N D=1$.

LFILE $=1$

MS $=$ MEND

SRUN

SRUN

SRUN

SRUN

SRUN

SRUN

SRUN

SRUN

SRUN

SRUN

SRUN

DO 3 I=1, ISAV

CALL RESRV(MS, MXPMY, MINZER,

.)

90

$0,0,0,0,0,0,0,0$

SRUN

SRUN

SRUN

SRUN

$\operatorname{INFF}(1, M S)=\operatorname{IBLNK}$
$\operatorname{INFF}(2, M S)=\operatorname{IBLNK}$

SRUN

$M S=M S-1$

3 CONTINUE

MSIZE $=37$

95

I7 = ISAV7 - 2

IF (ISAV7.EQ.0) GO TO 7

DO $10 \quad I=1, I 7$

CALL RESRV(MS, R7, MINZER,

SRUN

SRUN

SRUN

SRUN

SRUN

SRUN

SRUN

.)

$0,0,0,0,0,0,0,0$

100

$\operatorname{INFF}(1, M S)=$ IBLNK

SRUN

SRUN

SRUN

SRUN

SRUN

$M S=M S-1$

SRUN

SRUN

SRUN

SRUN

SRUN

SRUN

SRUN

SRUN

SRUN

SRUN

SRUN

.)

CALL RESRV(MS, R7, MINZER,

DO $15 \mathrm{I}=1,2$

( 
115

$$
15 \begin{aligned}
& M S=M S-1 \\
& M S Y M P L=M S
\end{aligned}
$$

C DEFINE AND STORE MATRICES

CALL SETMAT(MT)

* Restore info for RUN

SRUN

120 RETURN

C END OF RUN

FIN $=$. TRUE, FORMAT (/6H $* * *, 1 X, A 5,2 X, A 5,1 X, 13,1 X, 13,1 X, 3 H / /, 1 X$,

CARD NR. SEVERITY DETAILS DIAGNOSIS OF PROBLEM 
SUBROUTINE SSQMIN(M,N,F,X,E, ESCALE, IPRINT, MAXFUN)

SSQMIN

2

RETURN

SSQMIN

3

END

SSQMIN

4 
1

5

10

15
SUBROUTINE PLOTT $(N, C, X, Y)$

DIMENSION $A(112,51), Z(6), X(1), Y(1)$

COMMON/SAML/A, Z, XLOW, XRANGE, YLOW, YRANGE

IF ( $N$. LT. 1 ) RETURN

DO $100 \mathrm{~K}=1, \mathrm{~N}$

$X M U L T=(X(K)-X L O W) / X R A N G E$

IF( XMULT .LT. 0.0$)$ GO TO 100

YMULT $=(Y(K)-Y L O W) /$ YRANGE

IF( YMULT . LT. 0.0 ) GO TO 100

$I T=\operatorname{INT}(110.0 *$ XMULT +0.5$)+1$

$I=I T$

IF( I .GT. 111 )GO TO 100

$J T=\operatorname{INT}(50.0 *$ YMULT +0.5$)+1$

$\mathrm{J}=\mathrm{JT}$

IF ( J .GT. 51 )GO TO 100

$A(I, J)=C$

CONT INUE

RETURN

END
TRKP683 3

MY3TKPL 84

MY3TKPL $\quad 85$

MY3TKPL $\quad 86$

MY3TKPL $\quad 87$

MY3TKPL 88

MY3TKPL $\quad 89$

MY3TKPL 90

MY3TKPL 91

MY3TKPL 92

MY3TKPL 93

MY3TKPL 94

MY3TKPL 95

MY3TKPL 96

MY3TKPL 97

MY3TKPL 98

MY3TKPL $\quad 99$

MY3TKPL 100

MY3TKPL 101 
1

5

10

15

25

30

35

40

20
SUBROUTINE PAGTRKP(IC,K1)

DIMENSION $A(112,51), Z(6), X(1), Y(1)$

COMMON/SAML/A, $Z, X L O W, X R A N G E, Y L O W, Y R A N G E$

WRITE $(3,5555) \mathrm{K} 1$

5555 FORMAT ( 1 H0, *PART ICLE NUMBER*, I5)

DO $250 \mathrm{~K}=1,51$

$I=52-K$

$\mathrm{IP}=\mathrm{MOD}(\mathrm{I}-1,5)$

IF ( IP ) $210,220,210$

220 CONTINUE

DEL=YRANGE $/ 10.00$

$\mathrm{IM}=\mathrm{I} / 5$

$F M=I M$

$X X=Y L O W+F M * D E L$

IF ( IC ) $222,221,222$

221 WRITE $(3,1221) X X,(A(J, I), J=1,112)$

1221 FORMAT $(4 X, F 10.3,2 \mathrm{H}+, 112 \mathrm{~A} 1$ )

GO TO 250

222 WRITE $(3,1222) \times X,(A(J, I), J=1,112)$

1222 FORMAT $(4 X, E 10.3,2 \mathrm{H}+, 112 \mathrm{~A} 1)$

GO TO 250

210 WRITE $(3,1210)(A(J, I), J=1,112)$

1210 FORMAT $(15 X, 1 \mathrm{HI}, 112 \mathrm{~A} 1)$

250 CONTINUE

WRITE $(3,1230)$

1230 FORMAT $(15 \mathrm{X}, 112(1 \mathrm{H}-))$

WRITE $(3,1235)$

1235 FORMAT $(16 \mathrm{X}, 1 \mathrm{HI}, 5(21 \mathrm{X}, 1 \mathrm{HI}))$

$D E L=X R A N G E / 5.0$

DO $300 \mathrm{~J}=1,6$

$Z(J)=X L O W+D E L * F L O A T(J-1)$

300 CONTINUE

IF (IC) $400,500,400$

500 WRITE $(3,1500)(Z(I), I=1,6)$

1500 FORMAT $(10 X, F 10.3,5(12 X, F 10.3))$

GO TO 1499

400 WRITE $(3,1400)(Z(I), I=1,6)$

1400 FORMAT $(10 X, E 10.3,5(12 X, E 10.3))$

1499 RETURN

END

\begin{tabular}{lr} 
86PAG & 1 \\
MY3TKPL & 103 \\
MY3TKPL & 104 \\
MY3TKPL & 105 \\
MY3TKPL & 106 \\
MY3TKPL & 107 \\
MY3TKPL & 108 \\
MY3TKPL & 109 \\
MY3TKPL & 110 \\
MY3TKPL & 111 \\
MY3TKPL & 112 \\
MY3TKPL & 113 \\
MY3TKPL & 114 \\
MY3TKPL & 115 \\
MY3TKPL & 116 \\
MY3TKPL & 117 \\
MY3TKPL & 118 \\
MY3TKPL & 119 \\
MY3TKPL & 120 \\
MY3TKPL & 121 \\
MY3TKPL & 122 \\
MY3TKPL & 123 \\
MY3TKPL & 124 \\
MY3TKPL & 125 \\
MY3TKPL & 126 \\
MY3TKPL & 127 \\
MY3TKPL & 128 \\
MY3TKPL & 129 \\
MY3TKPL & 130 \\
MY3TKPL & 131 \\
MY3TKPL & 132 \\
MY3TKPL & 133 \\
MY3TKPL & 134 \\
MY3TKPL & 135 \\
MY3TKPL & 136 \\
MY3TKPL & 137 \\
MY3TKPL & 138 \\
MYTKPL & 139 \\
MYKPL & 140 \\
\hline$Y 31$
\end{tabular}


COMMON/BMI $1 / M I 1$ ( 16000 )

COMNON/NELS1/NELS1

LEVEL 2 , MI 1

C

DIMENSION MI (1)

EQUIVALENCE (MI, MI1), (NELS, NELS1)

COMMON/CONTRL/ERROR, MODE, RSRY, STOR, XEQ, TRASW, NFSW, EMPTY, INDEF , 1 LDFLG, FIN

COMMON /MATCH3/NOPR, NONU, FLAG, IPR, IPP, MINFLG, MIFLG, MATFLG, GLOBAL LOGICAL FLAG, NOPR, NONU, MIFLG, MATFLG, GLOBAL COMMON/CDERIV/IFLAG,MR, NR, DP,NV(11), DAT(20), UZ, STOT,

1 DAT2(7), MP2FLG, NF, HO, LOCALPH, NUMALPH

DIMENSION BXI (9), BYI (9), VO(6),VI (6),VF(7),T(49)

DIMENSION BX(6),BY(6),BXO(6),BYO (6), RX(2,3),RY(2,3),RW(3),BETF(14) DIMENSION $P(8), V(7)$

C

DATA IBLANK/4H /

C INSTRUCTION TRKB-TRACK BETATRON FUNCTIONS

C T TRKB IR1 IR2//BLIN BETO VO

IT1 IT2 ITBL IRAD SO THO

C RESERV A TABLE FOR STORAGE OF BETA FUNCTIONS FROM POSITION IR1

C THROUGH POSITION IR2 OF BETO.

$\mathrm{C}$

C TRACK BETA FUNCTIONS DEFINED BY [ BETO IBET $/ / \ldots$ ]

C THROUGH BEAM LINE DEFINED BY [ BLIN BML $/ / \ldots$ ] FROM IT1 TO IT2.

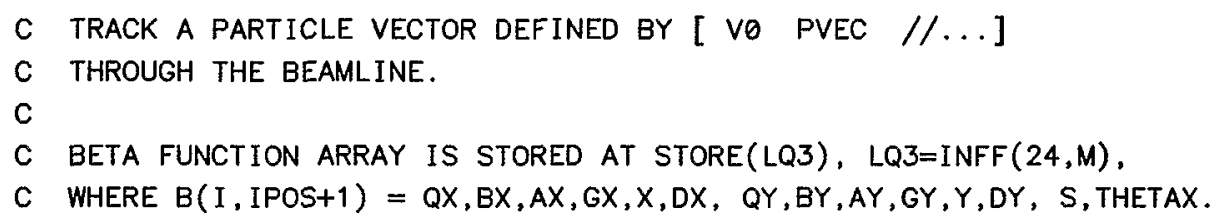


ON 1ST CALL, AND/OR IF IT1=0, FROM IBET INPUT.

86TRKB $\quad 36$

C BETO = /NAME/ - INITIAL BETAS FROM ARRAY/NAME/, FOR AN IBET, AND FROM BETA-FUNCTIONS OF /NAME/, IF A MATRIX.

IN UNITS OF 2PI

86TRKB $\quad 40$

86TRKB 41

$\begin{array}{lll}\text { DEFAULTS - } & \text { 86TRKB } & 42\end{array}$

65

$$
c
$$

c

C IF $K A=K B=0$ (OR BLANK), A TABLE IS RESERVED SUFFICIENT TO STORE 86TRKB $\quad 43$

C

$\mathrm{C}$

$\mathrm{C}$

IF $K A=-1$ THE COMPLETE BEAM LINE.

86TRKB

86TRKB

NO TABLE IS RESERVED, NO BETAS ARE STORE 86TRKB

86TRKB

IF $K T 1=I T 2=0$ (OR BLANK), TRACKING IS DONE THROUGH COMPLETE BEAM 86TRKB

86TRKB

86TRKB

RADFLG $=$.FALSE.

86TRKB

IF (MINFLG.NE.4) GO TO 23

86TRKB

IF (MIFLG) GO TO 152

86TRKB

GO TO 4

86TRKB

86TRKB

86TRKB

86TRKB

86TRKB

86TRKB

86TRKB

86TRKB

86TRKB

86TRKB

86TRKB

86TRKB

151 LTABO = LQ3 - NCOL*KA - 1

86TRKB

86TRKB

86TRKB

86TRKB

86TRKB

86TRKB

86TRKB

86TRKB

86TRKB

86TRKB

86TRKB

86TRKB

86TRKB

86TRKB

86TRKB

86TRKB

86TRKB

86TRKB

86TRKB

86TRKB

86TRKB

86TRKB

86TRKB

86TRKB

86TRKB

86TRKB

86TRKB

CALL DATA(MVEC, $1,1,6, V I)$

160 CONTINUE

IF (NAMVEC. EQ. IBLANK) GO TO 160 
C 10 IF ((ICALL.EQ.0).OR. (IP1.EQ.0) ) GO TO 12

C INITIAL BETAS FROM BETA FUNCTION TABLE OF TRKB INSTRUCTION,

C EXCEPT THAT FOR FIRST CALL, OR IF IP $1=0$, THEY ARE TAKEN FROM IBET OR

\section{MBET $=M$}

86TRKB

104

$$
\text { NTYP }=8
$$

CALL DATA(MBET, NTYP, LOCBET+12, 1, SI)

86TRKB

105

86TRKB

106

CALL DATA(MBET, NTYP, LOCBET+13,1, TI)

86TRKB

107

IF(VECFLAG) CALL DATA (MBET, NTYP, LOCBET $+14,6, \mathrm{VI}$ )

86TRKB

86TRKB GO TO 13

86TRKB

86TRKB

86TRKB

C INITIAL BETAS FROM ARRAY SPECIFIED BY NAMBET

$\operatorname{MBET}=\operatorname{MDA} T(M, 2)$

$N A M O P=I N F F(1$, MBET $)$

86TRKB

86TRKB

IF (NAMOP.EQ. 4HIBET) GO TO 13

C INITIAL BETAS ARE BETAS OF MATRICES MBET.

CALL BET (MBET, BXI, BYI , 1)

$\operatorname{BXI}(1)=0$.

86TRKB

86TRKB

$\operatorname{BYI}(1)=0$.

86TRKB

86TRKB

GO TO 16

C

16 CONTINUE

$\operatorname{CALL} \operatorname{STDAT}(M, 3,3,1$, ICALL $)$

$S=S I$

$\mathrm{TH}=\mathrm{TI}$

86TRKB 
CALL DASH

LNUM $=0$

86TRKB

185

\section{7 \\ ELNAME=ELNAMEI \\ CONTINUE \\ $I 1=I P 1+1$ \\ $\mathrm{I} 2=\mathrm{IP} 2$}

190

195

200

205

210

215

220

225

IPOS $=$ IBS -1

WRITE $(3,1004)$

WRITE $(3,1002)$

LNUM $=0$

LNUM = LNUM + 1

GO TO 6 WRITE $(3,1004)$

WRITE $(3,1000)$

LNUM $=0$

192

1

$6 \quad N E L=M I$ ( IBS)

DO $11 \mathrm{I}=1,14$

DO $110 \quad I=15,20$

8 CONTINUE
IF (BX(4).EQ.0.) $\mathrm{BX}(4)=(1 .+\mathrm{BX}(3) * \mathrm{BX}(3)) / \mathrm{BX}(2)$

IF $(B Y(4), E Q .0) \quad B Y.(4)=(1 .+B Y(3) * B Y(3)) / B Y(2)$

C LOOP OVER POSITIONS

19 DO 1 IBS $=I 1,12$

IF (NOPR) GO TO 6

IF (.NOT.VECFLAG) GO TO 190

IF (MOD(LNUM, 45).NE.0.OR. LNUM.EQ.0) GO TO 191

191 IF (MOD(LNUM, 5) , EQ.0.AND. LNUM.NE.0) WRITE $(3,1010)$

WRITE $(3,1003)$ IPOS, ELNAME, $,(B X(I), I=1,3), B X(5), B X(6)$, $(B Y(I), I=1,3), B Y(5), B Y(6),(V(I), I=1,4)$

190 IF (MOD(LNUM, 45).NE. O.OR. LNUM.EQ.0) GO TO 192

IF (MOD (LNUM, 5) , EQ.0.AND. LNUM.NE. 0) WRITE $(3,1010)$

WRITE $(3,1001)$ IPOS, ELNAME, $S,(B X(I), I=1,3), B X(5), B X(6)$, $(B Y(I), I=1,3), B Y(5), B Y(6)$

LNUM $=$ LNUM +1

IF (NOSTOR) GO TO 8

$J=L T A B O+N C O L *$ IPOS

$11 \operatorname{STORE}(J+I)=B E T F(I)$

IF (.NOT. VECFLAG) GO TO 8

$110 \operatorname{STORE}(J+I)=V F(I)$

ELNAME $=$ MNAME (NEL)

STOT $=\mathrm{S}$
86TRKB

86TRKB

86TRKB

86TRKB

86TRKB

86TRKB

86TRKB

86TRKB

86TRKB

86TRKB

86 TRKB

86TRKB

86TRKB

86TRKB

86TRKB

86TRKB

86TRKB

86TRKB

86TRKB

86TRKB

86TRKB

86TRKB

86TRKB

86TRKB

86TRKB

86TRKB

86TRKB

86TRKB

86TRKB

86TRKB

86TRKB

86TRKB

86TRKB

86TRKB

86TRKB

86TRKB

86TRKB

86TRKB

86TRKB

86TRKB

86TRKB

86TRKB

86TRKB

86TRKB

86TRKB

86TRKB

86TRKB

86TRKB

86TRKB

86TRKB
157

158

159

160

161

162

163

164

165

166

167

168

169

170

171

172

173

174

175

176

177

178

179

180

181

182

183

184

185

186

187

188

189

190

191

192

193

194

195

196

197

198

199

200

201

202

203 
86TRKB

86TRKB

86TRKB

86TRKB

86TRKB

86TRKB

86TRKB

86TRKB

86TRKB

86TRKB

86TRKB

86TRKB

86TRKB

86TRKB

86TRKB

86TRKB

86TRKB

86TRKB

86TRKB

86TRKB

86TRKB

86TRKB

86TRKB

86TRKB

86TRKB

86TRKB

86TRKB

86TRKB

86TRKB

86TRKB

86TRKB

86TRKB

86TRKB

86TRKB

86TRKB

86TRKB

86TRKB

86TRKB

86TRKB

86TRKB

86TRKB

86TRKB

86TRKB

86TRKB

86TRKB

86TRKB

86TRKB

86TRKB

86TRKB

86TRKB

86TRKB

86TRKB

86TRKB

86TRKB

86TRKB

86TRKB

86TRKB 
290

$\operatorname{COSINE}=R Y(1,1) * B E R A T-B Y O(3) * \operatorname{SINE}$

86TRKB

DNU = ATAN2 (SINE, COSINE)/DNOM

86TRKB

265

IF (DNU.LT.0) DNU = DNU + CCON

86TRKB

266

$B Y(1)=B Y O(1)+D N U$

86TRKB

267

86TRKB 268

183 CONTINUE

86TRKB

269

86TRKB 270

IF (. NOT. VECFLAG) GO TO 1

86TRKE

271

CALL RTRV7(NEL,T,RW)

86TRKB

272

CALL MXV7 (T,VO,V)

86TRKB

273

86TRKB 274

1 CONTINUE

86TRKB

275

17 IPOS $=12$

300

$M P 2 F L G=0$

IF(NOSTOR) GO TO 5

86TRK

276

86TRKB 277

86TRKB 278

86TRKB 279

$\mathrm{J}=\mathrm{J} . \mathrm{NCOL}$

DO $14 \mathrm{I}=1,14$

305

$14 \operatorname{STORE}(\mathrm{J}+\mathrm{I})=\operatorname{BETF}(\mathrm{I})$

IF (.NOT.VECFLAG) GO TO 5

DO $140 \quad I=15,20$

$140 \operatorname{STORE}(J+I)=\operatorname{VF}(I)$

86TRKB 280

86TRKB 281

86TRKB 282

86TRKB 283

86TRKB 284

86TRKB 285

86TRKB 286

86TRKB 287

310

5 CONTINUE

IF (NOPR) RETURN

86TRKB 288

86TRKB 289

86TRKB 290

IF (. NOT. VECFLAG) GO TO 150

315

WRITE $(3,1003)$ IPOS, ELNAME, S, (BX(I), I=1,3), BX(5),BX(6),

$1 \quad(B Y(I), I=1,3), B Y(5), B Y(6),(V(I), I=1,4)$

GO TO 154

86TRKB

291

86TRKB 292

86TRKB 293

86TRKB 294

86TRKB 295

86TRKB 296

150 WRITE $(3,1001)$ IPOS, ELNAME, $,(B X(I), I=1,3), B X(5), B X(6)$,

86TRKB 297

86TRKB 298

86TRKB 299

154 CALL DASH

86TRKB

RETURN

86TRKB

86TRKB

86TRKB

1000 FORMAT (" POS", 11X, "S", 7X, "QX", 10X, "BX" , 8X, "AX" , 8X, "X", 9X,

86TRKB

1 "DX", 14X, "QY", 10X, "BY", 8X, "AY", 8X, "Y", 9X, "DY")

86TRKB

86TRKB

1001 FORMAT (I4, $1 \mathrm{X}, \mathrm{A} 4,1 \mathrm{X}, \mathrm{F} 10.4,2(\mathrm{~F} 10.6, \mathrm{~F} 11.4, \mathrm{~F} 11.6, \mathrm{~F} 10.6, \mathrm{~F} 10.6,6 \mathrm{X}))$

86TRKB

86TRKB

1002 FORMAT (" POS", 10X, "S", 7X, "QX", 7X, "BX", 7X, "AX", 6X, "DX", 6X,

86TRKB

86TRKB

86TRKB

86TRKB

86TRKB

86TRKB

86TRKB

86TRKB

300

301

302

303

304

305

306

307

308

309

310

311

312

313

314

1004 FORMAT (1H1)

1010 FORMAT (1H)

315

END 
CARD NR. SEVERITY DETAILS

DIAGNOSIS OF PROBLEM

NOT ALL ITEMS IN THIS COMMON BLOCK OCCUR IN LEVEL STATEMENTS. 
LEVEL 2, STORE, INFF, IWORK

COMMON STORE (48000), IWORK (10)

DIMENSION INFF $(24,2000)$

EQUIVALENCE (INFF,STORE)

COMMON/BMI 1/MI 1 ( 16000 )

COMMON/NELS1/NELS1

LEVEL 2 , MI 1

15

20

25

30

35

40

45

50
DIMENSION MI (1)

EQUIVALENCE (MI, MI1), (NELS, NELS1)

COMMON/CONTRL/ERROR, MODE, RSRV, STOR, XEQ, TRASW, NFSW, EMPTY, INDEF,

1 LDFLG, FIN

LOGICAL ERROR, RSRV, STOR, XEQ, TRASW, NFSW, EMPTY, INDEF, LDFLG, FIN

COMMON /MATCH3/NOPR, NONU, FLAG, IPR, IPP, MINFLG, MIFLG, MATFLG, GLOBAL LOGICAL FLAG, NOPR, NONU, MIFLG, MATFLG, GLOBAL

COMMON/CDERIV/IFLAG, MR, NR, DP, NV(11), DAT(20), UZ, STOT,

1 DAT2(7), MP2FLG, NF, HO, LOCALPH, NUMALPH

DIMENSION BXI (9), BYI (9), VO(6), VI (6), VF(7), T (49)

DIMENSION BX(6), BY(6), BXO(6), BYO (6), RX(2,3), RY(2,3),RW(3),BETF(14) DIMENSION $P(8), V(7)$

EQUIVALENCE (BX,BETF), (BY,BETF(7)), (S,BETF (13)), (TH,BETF(14))

EQUIVALENCE (EPX,P(1)), (EPY,P(2)), (EPL,P(3)), (DPP,P(4)), (V,VF(7)) INTEGER BDAT, ELNAMEI, ELNAME

EXTERNAL DER2, DER3, DER4, DELQ

LOGICAL NOSTOR, MPFLG, VECFLAG

LOGICAL RADFLG

DATA DZERO/O.0/

DATA MP2FLG/O/

C

DATA IBLANK/" "/

C INSTRUCTION TRKE— CTRACK BEAM ENVELOPES

C T TRKE IR1 IR2//BLIN BETO VO EPS IT1 IT2 ITBL IADD EXCO

C RESERV A TABLE FOR STORAGE OF BETA FUNCTIONS FROM POSITION IR1

C THROUGH POSITION IR2 OF BETO.

C

C

TRACK BETA FUNCTIONS DEFINED BY [ BETO IBET $/ / \ldots$ ]

THROUGH BEAM LINE DEFINED BY [ BLIN BML / ...] FROM IT1 TO IT2,

CALCULATE AND DISPLAY BEAM ENVELOPES.

THE EMITTANCES ARE DEFINED BY [ EPS BVAL $/ / \ldots$ ].

TRACK A PARTICLE VECTOR DEFINED BY [ VO PVEC $/ / \ldots$ ]

THROUGH THE BEAMLINE.

C

C BETA FUNCTION ARRAY IS STORED AT $\operatorname{STORE(LQ3),~} \operatorname{LQ3}=\operatorname{INFF}(24, M)$,

C WHERE $B(I, I P O S+1)=Q X, B X, A X, G X, X, D X, Q Y, B Y, A Y, G Y, Y, D Y, S, T H E T A X$.
86TRKE 2

86TRKE 3

86TRKE 4

86TRKE 5

BLANK 2

BLANK 3

86MARSIZ 1

86MARSIZ 2

86MARSIZ 3

BLANK 5

NV3BMI 1

BMI1L 2

BMI 1L 3

BMI1L 4

BMI 1

86TRKE 8

86TRKE 9

CONTRL 2

CONTRL 3

CONTRL 4

CONTRL 5

MATCH3 2

MATCH3 3

CDERIV 2

CDERIV 3

86TRKE 13

86TRKE 14

86TRKE 15

86TRKE 16

86TRKE 17

86TRKE 18

86TRKE 19

86TRKE 20

86TRKE 21

86TRKE 22

86TRKE 23

86TRKE 24

86TRKE 25

86TRKE 26

86TRKE 27

86TRKE 28

86TRKE 29

86TRKE 30

86TRKE 31

86TRKE 32

86TRKE 33

86TRKE 34

86TRKE 35

86TRKE 36

86TRKE 37

86TRKE 38

86TRKE 39

86TRKE 40

86TRKE 41

86TRKE 42

86TRKE 43

86TRKE 44 
60

C

C

C

C

C

C

65

90

95

105

110

C

C

C

C

C
ITBL $=1-$ INPUT BETAS FROM TRKB TABLE, AT IT1, EXCEPT ON 1ST CALL, AND/OR IF IT1=0, FROM IBET INPUT.

BETO = /NAME/ - INITIAL BETAS FROM ARRAY/NAME/, FOR AN IBET, AND FROM BETA-FUNCTIONS OF /NAME/, IF A MATRIX. IADD $=0-A D D \times B$ AND XP IN QUADRATURE, ETC. $I A D D=1-A D D X B$ AND XP ALGEBRAICALLY, ETC.

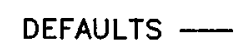

IF $K A=K B=0($ OR BLANK $)$, THE COMPLETE BEAM LINE.
NO TABLE IS RESERVED, NO

$$
\text { IF } K A=-1
$$

NO TABLE IS RESERVED, NO BETAS ARE STORE IF $K T 1=I T 2=0($ OR BLANK $)$ TRAC

$$
\text { RADFLG }=\text {.FALSE. }
$$

IF (MINFLG.NE.4) GO TO 23

IF (MIFLG) GO TO 152

GO TO 4

$$
\text { NOSTOR }=\text {.FALSE. }
$$$$
\text { TPI }=4 \text {. *ACOS (DZERO) }
$$

$$
\text { ELNAMEI }=I B L A N K
$$

$$
K B=\operatorname{INFF}(5, M)
$$$$
K A=\operatorname{INFF}(4, M)
$$$$
\operatorname{LQ3}=\operatorname{INFF}(24, M)
$$$$
\mathrm{NCOL}=\operatorname{IDAT}(M, 5)
$$

IF (KA.GT.-1) GO TO 151

NOSTOR $=$. TRUE.

GO TO 152

151 LTABO $=$ LQ3 - NCOL*KA -1

$152 \mathrm{MBML}=\operatorname{MDAT}(\mathrm{M}, 1)$

$$
\begin{array}{ll}
100 & \\
& \\
& I G=0.0 \\
& I F(I A D D \cdot E Q \cdot 0) \quad I G=2 \\
& D N O M=T P I \\
& C C O N=1 .
\end{array}
$$$$
\text { NDAT }=\operatorname{INFF}(17, \mathrm{MBML})
$$$$
\text { CALL MIFILL(MBML, } 1 \text {, NDAT, NELS, MI) }
$$$$
\text { CALL MMM(M,NELS, MI) }
$$$$
\text { IF (MINFLG.EQ.4) GO TO } 4
$$$$
\operatorname{IP1}=\operatorname{IDAT}(M, 1)
$$$$
\operatorname{IP2}=\operatorname{IDAT}(M, 2)
$$$$
\operatorname{ICALL}=\operatorname{IDAT}(M, 6)
$$$$
\operatorname{IADD}=\operatorname{IDAT}(M, 4)
$$$$
S I=0.0
$$

C GET VECTOR

NAMVEC $=\operatorname{BDAT}(M, 3)$

VECFLAG $=$.FALSE.

IF (NAMVEC. EQ. IBLANK) GO TO 160

VECFLAG $=$. TRUE.

MVEC $=\operatorname{MDAT}(M, 3)$

CALL DATA(MVEC, 1, 1, 6, VI)

CONT INUE
86TRKE 45

86TRKE $\quad 46$

86TRKE 47

86TRKE 48

86TRKE 49

86TRKE 50

86TRKE 51

86TRKE 52

86TRKE 53

86TRKE 54

86TRKE 55

86TRKE 56

86TRKE 57

86TRKE 58

86TRKE 59

86TRKE 60

86TRKE 61

86TRKE 62

86TRKE 63

B6TRKE 64

86TRKE 65

86TRKE 66

86TRKE 67

86TRKE 68

86TRKE 69

86TRKE 70

86TRKE 71

86TRKE 72

86TRKE 73

86TRKE 74

86TRKE 75

86TRKE 76

86TRKE 77

86TRKE 78

86TRKE 79

86TRKE 80

86TRKE 81

86TRKE 82

86TRKE 83

86TRKE 84

86TRKE 85

86TRKE 86

86TRKE 87

86TRKE 88

86TRKE 89

86TRKE 90

86TRKE 91

86TRKE 92

86TRKE 93

86TRKE 94

86TRKE 95

86TRKE 96

86TRKE 97

86TRKE 98

86TRKE 99

86TRKE 100

86TRKE 101 
MBVAL $=\operatorname{MDAT}(M, 4)$

86TRKE

CALL DATA(MBVAL , 5, 1,4,P)

86TRKE

$\operatorname{NAMBET}=\operatorname{BDAT}(M, 2)$

86TRKE

$\operatorname{NAME}=\operatorname{INFF}(2, M)$

86TRKE

$\operatorname{ITABLE}=\operatorname{IDAT}(M, 3)$

86TRKE

C 10 IF ((ICALL.EQ.0).OR. (IP1.EQ.0)) GO TO 12

86TRKE

86TRKE

C INITIAL BETAS FROM BETA FUNCTION TABLE OF TRKB INSTRUCTION,

86TRKE

C EXCEPT THAT FOR FIRST CALL, OR IF IP $1=0$, THEY ARE TAKEN FROM IBET OR LOCBET $=$ NCOL $*(I P 1-K A)+1$

86TRKE $M B E T=M$

86TRKE

CALL DATA(MBET, NTYP, LOCBET+12,1, SI)

86TRKE

CALL DATA(MBET, NTYP, LOCBET+13,1,TI)

86TRKE

IF (VECFLAG) CALL DATA(MBET, NTYP, LOCBET+14,6,VI)

86TRKE

GO TO 13

86TRKE

86TRKE

C INITIAL BETAS FROM ARRAY SPECIFIED BY NAMBET

12 LOCBET $=1$

NTYP=1

$\operatorname{MBET}=\operatorname{MDAT}(M, 2)$

86TRKE

$N A M O P=\operatorname{INFF}(1, M B E T)$

86TRKE

C INITIAL BETAS ARE BETAS OF MATRICES MBET.

86TRKE

CALL BET (MBET, BXI, BYI , 1)

86TRKE

$B X I(1)=0$.

86TRKE

$\operatorname{BYI}(1)=0$.

86TRKE

GO TO 16

86TRKE

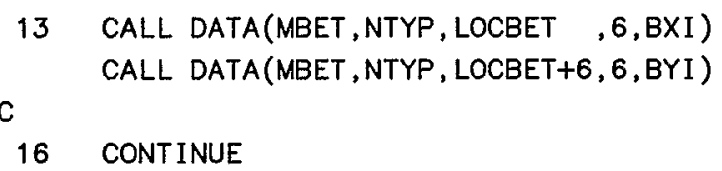

C GET EMITTANCES

$\operatorname{EPXCO}=\operatorname{FDAT}(M, 1)$

EPYCO $=\operatorname{FDAT}(M, 2)$

86TRKE 
4 CONTINUE

$$
S=S I
$$

$T H=T I$

DO $3 \mathrm{I}=1,6$

$B X(I)=B X I(I)$

$B Y(I)=B Y I(I)$

$V(I)=V I(I)$

3 CONTINUE

$I 1=I P 1+1$

$\mathrm{I} 2=\mathrm{IP} 2$

IF (NOPR) GO TO 7

LNUM $=0$

IF $($ I $2 . G T .40)$ WRITE $(3,4004)$

CALL DASH

190

WRITE(3, 4001) $P(1), \operatorname{EPXCO}, P(3), P(2), \operatorname{EPYCO}, P(4)$

195

404 WRITE $(3,4006)$

GO TO 406

405 WRITE $(3,4008)$

IF (.NOT. VECFLAG) GO TO 406

WRITE $(3,4011)$

GO TO 407

406 WRITE $(3,4010)$

407 CONTINUE

200

205

210

215

C

IF (BX(4).EQ.0.) $\mathrm{BX}(4)=(1 .+\mathrm{BX}(3) * \mathrm{BX}(3)) / \mathrm{BX}(2)$

ELNAME=ELNAMEI

7 CONTINUE

IF $(B Y(4) . E Q .0) \quad B Y.(4)=(1 .+B Y(3) * B Y(3)) / B Y(2)$

$X=S Q R T(E P X * B X(2))$

$Y=S Q R T(E P Y * B Y(2))$

$X P=B X(5) * D P P$

$Y P=B Y(5) * D P P$

$X C O=R A T X * X$

$Y C O=R A T Y * Y$

$X P R=S Q R T(E P X * B X(4))$

$Y P R=S Q R T(E P Y * B Y(4))$

$X P P R=B X(6) * D P P$

$Y P P R=B Y(6) * D P P$

$X C O P R=R A T X * X P R$

$Y C O P R=R A T Y * Y P R$

GO TO $(4116,4117)$ IG

$4116 \times$ XTOT $=X+A B S(X P)$

$X P R T O T=X P R+A B S(X P P R)$

YTOT $=Y+A B S$ (YP)

YPRTOT = YPR + ABS (YPPR)

GO TO 4118

225

4117 CONTINUE

$X T O T=\operatorname{SQRT}(X * X+X P * X P)$

$X P R T O T=S Q R T(X P R * X P R+X P P R * X P P R)$

$Y T O T=\operatorname{SQRT}(Y * Y+Y P * Y P)$

\begin{tabular}{|c|c|}
\hline 86TRKE & 159 \\
\hline 86TRKE & 160 \\
\hline 86TRKE & 161 \\
\hline 86TRKE & 162 \\
\hline 6TRKE & 163 \\
\hline 86TRKE & 164 \\
\hline 86TRKE & 165 \\
\hline 86TRKE & 166 \\
\hline 86TRKE & 167 \\
\hline 86TRKE & 168 \\
\hline 86TRKE & 169 \\
\hline 86TRKE & 170 \\
\hline 86TRKE & 171 \\
\hline 86TRKE & 172 \\
\hline 86TRKE & 173 \\
\hline 86TRKE & 174 \\
\hline 86TRKE & 175 \\
\hline 86TRKE & 176 \\
\hline 86TRKE & 177 \\
\hline 86TRKE & 178 \\
\hline 86TRKE & 179 \\
\hline 86TRKE & 180 \\
\hline 86TRKE & 181 \\
\hline 86TRKE & 182 \\
\hline 86TRKE & 183 \\
\hline 86TRKE & 184 \\
\hline 86TRKE & 185 \\
\hline 86TRKE & 186 \\
\hline 86TRKE & 187 \\
\hline 86TRKE & 188 \\
\hline 86TRKE & 189 \\
\hline 86TRKE & 190 \\
\hline 86TRKE & 191 \\
\hline 86TRKE & 192 \\
\hline 86TRKE & 193 \\
\hline 86TRKE & 194 \\
\hline 86TRKE & 195 \\
\hline 86TRKE & 196 \\
\hline 86TRKE & 197 \\
\hline 86TRKE & 198 \\
\hline 86TRKE & 199 \\
\hline 86TRKE & 200 \\
\hline 86TRKE & 201 \\
\hline 86TRKE & 202 \\
\hline 86TRKE & 203 \\
\hline 86TRKE & 204 \\
\hline 86TRKE & 205 \\
\hline 86TRKE & 206 \\
\hline 86TRKE & 207 \\
\hline 86TRKE & 208 \\
\hline 86TRKE & 209 \\
\hline 86TRKE & 210 \\
\hline 86TRKE & 211 \\
\hline 86TRKE & 212 \\
\hline 86TRKE & 213 \\
\hline 86TRKE & 214 \\
\hline 86TRKE & 215 \\
\hline
\end{tabular}


YPRTOT = SQRT $(Y P R * Y P R+Y P P R * Y P P R)$

86TRKE

4118 CONTINUE

86TRKE

$X T O T=X T O T+X C O$

86TRKE

$Y$ TOT $=Y T O T+Y C O$

86TRKE

XPRTOT $=X P R T O T+X C O P R$

86TRKE

YPRTOT $=$ YPRTOT+YCOPR

86TRKE

235

C LOOP OVER POSITIONS

86TRKE

19 DO 1 IBS $=I 1, I 2$

IPOS $=$ IBS -1

IF(NOPR) GO TO 6

240

IF (.NOT.VECFLAG) GO TO 190

IF (MOD (LNUM, 40).NE.0.OR. LNUM.EQ. $\theta)$ GO TO 191

WRITE $(3,4004)$

WRITE $(3,4011)$

LNUM $=0$

191 IF (MOD(LNUM, 40).NE.0.OR. LNUM.NE.0) WRITE $(3,4040)$

WRITE $(3,4012)$ IPOS, ELNAME, $S, X, X P, X C O, X T O T, Y, Y P, Y C O, Y T O T$,

86TRKE

86TRKE

86TRKE

86TRKE

1 XPRTOT, YPRTOT , $(V(I), I=1,4)$

LNUM $=$ LNUM +1

86TRKE

86TRKE

86TRKE

86TRKE

86TRKE

86TRKE

86TRKE

86TRKE

86TRKE

GO TO 6

250

190 IF (MOD(LNUM , 40).NE.0.OR.LNUM.EQ.0) GO TO 192

WRITE $(3,4004)$

WRITE $(3,4010)$

LNUM $=0$

192 IF (MOD(LNUM, 5).EQ.0.AND. LNUM.NE. 0$)$ WRITE $(3,4040)$

WRITE $(3,4012)$ IPOS, ELNAME, $S, X, X P, X C O, X T O T, Y, Y P, Y C O, Y T O T$, 1 XPR , XPPR , XPRTOT , YPR, YPPR, YPRTOT

86TRKE

86TRKE

86TRKE

86TRKE

86TRKE

86TRKE

86TRKE

86TRKE

LNUM $=$ LNUM +1

$6 \quad N E L=M I(I B S)$

86TRKE

IF(NOSTOR) GO TO 8

86TRKE

86TRKE

$J=L T A B O+N C O L * I P O S$

DO $11 I=1,14$

$11 \operatorname{STORE}(\mathrm{J}+\mathrm{I})=\mathrm{BETF}(\mathrm{I})$

IF (. NOT. VECFLAG) GO TO 8

DO $110 \mathrm{I}=15,20$

265

$110 \operatorname{STORE}(\mathrm{J}+\mathrm{I})=\mathrm{VF}(\mathrm{I})$

8 CONTINUE

ELNAME $=$ MNAME (NEL)

$S T O T=S$

270

C

18 CALL RXY(NEL, RX, RY, RW)

IF (.NOT. VECFLAG) GO TO 180

86TRKE

86TRKE

86TRKE

86TRKE

86TRKE

86TRKE

86TRKE

86TRKE

86TRKE

86TRKE

86TRKE

86TRKE

86TRKE

86TRKE

DO $181 \mathrm{I}=1,6$

86TRKE

$B \times 0(I)=B \times(I)$

86TRKE

$B Y O(I)=B Y(I)$

86TRKE

86TRKE

86TRKE

GO TO 182

86TRKE

86TRKE

86TRKE

86TRKE

86TRKE

86TRKE

86TRKE 
290

295

305

310

315

320

325

330

300

$$
\begin{aligned}
B \times(2)=R \times & (1,1) * R \times(1,1) * B \times \theta(2)-2, * R \times(1,1) * R \times(1,2) * B \times 0(3) \\
1 & +R \times(1,2) * R \times(1,2) * B \times \theta(4)
\end{aligned}
$$$$
\operatorname{BY}(2)=\mathrm{RY}(1,1) * \mathrm{RY}(1,1) * \mathrm{BY} \theta(2)-2 . * \mathrm{RY}(1,1) * \mathrm{RY}(1,2) * \mathrm{BY} \theta(3)
$$$$
1+\operatorname{RY}(1,2) * \operatorname{RY}(1,2) * B Y O(4)
$$$$
\mathrm{B} \times(3)=-\mathrm{R} \times(1,1) * \mathrm{R} \times(2,1) * \mathrm{~B} \times 0(2)
$$$$
1+(\mathrm{RX}(1,1) * \mathrm{RX}(2,2)+\mathrm{RX}(1,2) * \mathrm{RX}(2,1)) * \mathrm{BXO}(3)
$$$$
2-\mathrm{RX}(1,2) * \mathrm{R} \times(2,2) * B \times \theta(4)
$$

$$
\begin{aligned}
\operatorname{BY}(3)= & -\operatorname{RY}(1,1) * \operatorname{RY}(2,1) * \operatorname{BYO}(2) \\
1 & +(\operatorname{RY}(1,1) * \operatorname{RY}(2,2)+\operatorname{RY}(1,2) * R Y(2,1)) * \operatorname{BY} \theta(3) \\
2 & -\operatorname{RY}(1,2) * \operatorname{RY}(2,2) * \operatorname{BY} \theta(4)
\end{aligned}
$$

$$
\begin{aligned}
& \mathrm{BX}(4)=\mathrm{RX}(2,1) * \mathrm{RX}(2,1) * \mathrm{~B} \times 0(2)-2 . * \mathrm{R} \times(2,1) * \mathrm{R} \times(2,2) * \mathrm{~B} \times 0(3) \\
& 1+\operatorname{RX}(2,2) * R \times(2,2) * B \times \theta(4) \\
& \operatorname{BY}(4)=\operatorname{RY}(2,1) * \operatorname{RY}(2,1) * \operatorname{BYO}(2)-2 . * \operatorname{RY}(2,1) * \operatorname{RY}(2,2) * B Y O(3) \\
& 1+\operatorname{RY}(2,2) * \operatorname{RY}(2,2) * \operatorname{BYO}(4)
\end{aligned}
$$$$
B \times(5)=R \times(1,1) * B \times 0(5)+R \times(1,2) * B \times 0(6)+R \times(1,3)
$$$$
B Y(5)=R Y(1,1) * B Y O(5)+R Y(1,2) * B Y O(6)+R Y(1,3)
$$$$
\mathrm{BX}(6)=\mathrm{R} \times(2,1) * \mathrm{BX} 0(5)+\mathrm{R} \times(2,2) * \mathrm{BX} 0(6)+\mathrm{R} \times(2,3)
$$$$
\mathrm{BY}(6)=\operatorname{RY}(2,1) * \mathrm{BY} \theta(5)+\operatorname{RY}(2,2) * \mathrm{BY} \theta(6)+\operatorname{RY}(2,3)
$$

IF (NONU) GO TO 183

$$
\begin{aligned}
& \text { BETAV }=S Q R T(B X(2) * B X \theta(2)) \\
& \text { BERAT }=S Q R T(B X \theta(2) / B X(2)) \\
& \text { SINE }=\operatorname{RX}(1,2) / B E T A V
\end{aligned}
$$$$
\text { COSINE }=R \times(1,1) * B E R A T-B X O(3) * \operatorname{SINE}
$$

DNOM OR CCON SET TO TPI OR 1 . DEPENDING ON DEGREES OR RADIANS.

DNU = ATAN2 $($ SINE, COSINE) $/$ DNOM

IF (DNU. LT.0) DNU = DNU + CCON

$$
B X(1)=B X \theta(1)+D N U
$$

$$
\text { BETAV }=S Q R T(B Y(2) * B Y O(2))
$$$$
\text { BERAT }=S Q R T(B Y O(2) / B Y(2))
$$

$\operatorname{SINE}=R Y(1,2) / B E T A V$

COSINE $=R Y(1,1) * B E R A T-B Y O(3) * S I N E$

DNU $=$ ATAN2 (SINE, COSINE)/DNOM

IF (DNU.LT.O) DNU = DNU + CCON

$B Y(1)=B Y O(1)+D N U$

CONT INUE

IF (.NOT. VECFLAG) GO TO 184

CALL RTRV7(NEL, T, RW)

CALL MXV7 $(T, V \theta, V)$

184 CONT INUE
86TRKE

86TRKE

86TRKE

86TRKE

86TRKE

86TRKE

86TRKE

86TRKE

86TRKE

86TRKE

86TRKE

86TRKE

86TRKE

86TRKE

86TRKE

86TRKE

86TRKE

86TRKE

86TRKE

86TRKE

86TRKE

86TRKE

86TRKE

86TRKE

86TRKE

86TRKE

86TRKE

86TRKE

86TRKE

86TRKE

86TRKE

86TRKE

86TRKE

86TRKE

86TRKE

86TRKE

86TRKE

86TRKE

86TRKE

86TRKE

86TRKE

86TRKE

86TRKE

86TRKE

86TRKE

86TRKE

86TRKE

86TRKE

86TRKE

86TRKE

86TRKE

86TRKE

86TRKE

86TRKE

86TRKE

86TRKE

86TRKE
273

274

275

276

277

278

279

280

281

282

283

284

285

286

287

288

289

290

291

292

293

294

295

296

297

298

299

300

301

302

303

304

305

306

307

308

309

310

311

312

313

314

315

316

317

318

319

320

321

322 
$Y=S Q R T(E P Y * B Y(2))$

86TRKE

$X P=B X(5) * D P P$

86TRKE 331

$Y P=B Y(5) * D P P$

86TRKE

$X C O=R A T X * X$

86TRKE

333

$Y C O=R A T Y * Y$

86TRKE

334

$X P R=S Q R T(E P X * B X(4))$

86TRKE

335

$Y P R=S Q R T(E P Y * B Y(4))$

86TRKE 336

350

$X P P R=B X(6) * D P P$

$Y P P R=B Y(6) * D P P$

86TRKE 337

$X C O P R=R A T X * X P R$

86TRKE

338

$Y C O P R=R A T Y * Y P R$

86TRKE

339

GO TO $(5116,5117)$ IG

86TRKE

340

355

$5116 \times$ XTOT $=X+A B S(X P)$

$X P R T O T=X P R+A B S(X P P R)$

86TRKE

341

YTOT $=Y+A B S(Y P)$

86TRKE

342

YPRTOT $=$ YPR + ABS (YPPR)

86TRKE

343

86TRKE

344

GO TO 5118

360

5117 CONTINUE

XTOT $=\operatorname{SQRT}(X * X+X P * X P)$

86TRKE

345

86TRKE

346

86TRKE

347

$X P R T O T=S Q R T(X P R * X P R+X P P R * X P P R)$

86TRKE

348

$Y T O T=\operatorname{SQRT}(Y * Y+Y P * Y P)$

86TRKE

349

YPRTOT = SQRT (YPR*YPR + YPPR*YPPR)

365

5118 CONTINUE

$X T O T=X T O T+X C O$

$Y T O T=Y T O T+Y C O$

XPRTOT $=X P R T O T+X C O P R$

YPRTOT $=$ YPRTOT $+Y C O P R$

370

375

1 CONTINUE

86TRKE

350

86TRKE

351

86TRKE

352

86TRKE

353

86TRKE

354

86TRKE

86TRKE

355

86TRKE

86TRKE

86TRKE

86TRKE

86TRKE

86TRKE

86TRKE

86TRKE

$J=J+N C O L$

86TRKE

DO $14 I=1,14$

380

$14 \operatorname{STORE}(J+I)=B E T F(I)$

86TRKE

86TRKE

IF (.NOT. VECFLAG) GO TO 5

86TRKE

86TRKE

86TRKE

86TRKE

86TRKE

86TRKE

86TRKE

86TRKE

86TRKE

86TRKE

356

357

358

359

360

361

362

363

364

365

366

367

368

369

370

371

372

373

374

IF (. NOT. VECFLAG) GO TO 150

375

376

WRITE $(3,4012)$ IPOS, ELNAME, S, X, XP, XCO, XTOT , Y, YP, YCO, YTOT,

86TRKE

377

1

XPRTOT , YPRTOT , $(V(I), I=1,4)$

GO TO 153

395

150 WRITE $(3,4012)$

1

IPOS, ELNAME, S, X, XP , XCO, XTOT , Y, YP, YCO, YTOT ,

86TRKE

378

86TRKE 380

86TRKE 381

86TRKE 382

86TRKE 383

153 CALL DASH

XPR, XPPR , XPRTOT, YPR, YPPR, YPRTOT

86TRKE

384

RETURN

86TRKE. 385

86TRKE $\quad 386$ 
400

4001 FORMAT ( $3 X$, "BEAM ENVELOPES (MM, MRAD)"

86TRKE

$15 X$, "EMITTANCES (MM-MRAD) - EPSX $=$ ",F10.6,5X,"EPSXCO = ",

86TRKE

$2 F 10.6,5 X, " E P S L=", F 13,6 / 30 X, " E P S Y=", F 10,6,5 X, " E P S Y C O="$,

86TRKE

3 F10.6,5X,"SIGP $=$ ",F10.6," (0/00)")

86TRKE

405

4002 FORMAT $(1 \mathrm{H}+, 99 \mathrm{X}$, "DISPLACEMENT = ", F5.2, $1 \mathrm{H*}$, "SIGMA")

86TRKE

4006 FORMAT (5X, "SIGX $=X B+X P+X C O$, ETC." $/)$

86TRKE

4010 FORMAT (" POS", 10X, "S", 6X, "XB", 6X, "XP", 5X, "XCO", 4X, "SIGX", 3X,

86TRKE

410 "YB", 6X, "YP" , 5X, "YCO", 4X, "SIGY" , 4X,

4011 FORMAT(" POS", 10X, "S", 6X, "XB", 6X, "XP", 5X, "XCO", 4X, "SIGX" , 3X, "YB", 6X, "YP" , 5X, "YCO" , 4X, "SIGY" , 4X, "DSIGX" , 3X, "DSIGY" ,

CARD NR. SEVERITY DETAILS DIAGNOSIS OF PROBLEM

$\begin{array}{rll}\text { EQV/COMM } & \text { I } & \text { BMI 1 } \\ 190 & \text { I } & \\ 219 & \text { I } & \\ 354 & \text { I } & \end{array}$

NOT ALL ITEMS IN THIS COMMON BLOCK OCCUR IN LEVEL STATEMENTS.

AN IF STATEMENT MAY BE MORE EFFICIENT THAN A 2 OR 3 BRANCH COMPUTED GO TO STATEMENT AN IF STATEMENT MAY BE MORE EFFICIENT THAN A 2 OR 3 BRANCH COMPUTED GO TO STATEMENT AN IF STATEMENT MAY BE MORE EFFICIENT THAN A 2 OR 3 BRANCH COMPUTED GO TO STATEMENT 
COMMON/BMI 1/MI 1 ( 16000)

COMMON/NELS1/NELS1

LEVEL 2 , MI 1

DIMENSION MI (1)

EQUIVALENCE (MI, MI1), (NELS, NELS1)

COMMON/CONTRL/ERROR, MODE, RSRV, STOR, XEQ, TRASW, NFSW, EMPTY, INDEF,

1 LDFLG, FIN

COMMON /MATCH3/NOPR, NONU, FLAG, IPR, IPP, MINFLG, MIFLG, MATFLG, GLOBAL LOGICAL FLAG, NOPR, NONU, MIFLG, MATFLG, GLOBAL COMMON/CDERIV/I FLAG, MR, NR, DP, NV(11), DAT (20), UZ, STOT,

1 DAT2 (7), MP2FLG, NF, HO, LOCALPH, NUMALPH

DIMENSION BXI (9), BYI (9), VO(6), VI (6), VF(7),T(49)

DIMENSION BX(6),BY(6),BXO(6), BYO (6), RX(2,3),RY(2,3), RW(3),BETF(14) DIMENSION $P(8), V(7)$

$$
\text { DATA IBLANK/" "/ }
$$

C INSTRUCTION TRKM-TRACK THROUGH ELEMENTS DEFINED BY MAP STATEMENTS

\section{T TRKM IR1 IR2//BLIN BETO MAPK PAR IT1 IT2 ITBL IRAD HSTEP}

\section{RESERV A TABLE FOR STORAGE OF BETA FUNCTIONS FROM POSITION IR1}

C THROUGH POSITION IR2 OF BETO.

C

$$
\text { TRACK BETA FUNCTIONS DEFINED BY [ BETO IBET } / / \ldots \text { ] }
$$

86TRKM

B6TRKM

86 TRKM

86TRKM

BLANK

BLANK

86MARSIZ

86MARSIZ

86MARSIZ

BLANK

NV3BMI 1

BMI $1 \mathrm{~L}$

BMI $1 L$

BMI $1 L$

BMI 1

86TRKM

86TRKM

CONTRL

CONTRL

CONTRL

CONTRL

MATCH3

MATCH3

CDERIV

CDERIV

86TRKM

86TRKM

86TRKM

86TRKM

86TRKM

86TRKM

86TRKM

86TRKM

86TRKM

86TRKM

86TRKM

86TRKM

86TRKM

86TRKM

86TRKM

86TRKM

86TRKM

86TRKM

86TRKM

86TRKM

86TRKM

86TRKM

86TRKM

86TRKM

86TRKM

86TRKM

86TRKM

86TRKM

86TRKM

86TRKM

86TRKM

86TRKM 
INITIAL BETAS FROM ARRAY/NAME/, FOR AN IBET,

86TRKM $\quad 45$ AND FROM BETA-FUNCTIONS OF /NAME/, IF A MATRIX.

86TRKM $\quad 46$

c IRAD $=1-$ OUTPUT PHASE ADVANCES IN RADIANS

86TRKM 47

c

$$
=0
$$

IN UNITS OF $2 P I$

86TRKM

48

DEFAULTS -

86TRKM

C

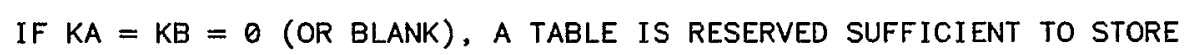

RADFLG $=$. FALS்E.

IF (MINFLG.NE.4) GO TO 23

IF (MIFLG) GO TO 152

GO TO 4 :

23 NOSTOR $=$.FALSE.

TPI =4 * *ACOS (DZERO)

ELNAME I $=I B L A N K$

86TRKM

86TRKM

86TRKM

86TRKM

86TRKM

86TRKM

86TRKM

86TRKM

$K B=\operatorname{INFF}(5, M)$

80

$\operatorname{KA}=\operatorname{INFF}(4, \mathrm{M})$

$L Q 3=\operatorname{INFF}(24, M)$

NCOL $=\operatorname{IDAT}(M, 5)$

IF (KA.GT. -1) GO TO 151

86TRKM

86TRKM

86TRKM

86TRKM

86TRKM

NOSTOR $=$. TRUE.

GO TO 152

151 LTABO $=$ LQ3 - NCOL $* K A-1$

$152 \operatorname{MBML}=\operatorname{MDAT}(M, 1)$

$$
\text { NDAT }=\operatorname{INFF}(17, M B M L)
$$

86TRKM 70

86TRKM 71

86TRKM 72

86TRKM 73

86TRKM 74

86TRKM $\quad 75$

86TRKM 76

CALL MIFILL(MBML, 1 , NDAT, NELS, MI)

86TRKM 77

CALL MMM(M,NELS, MI)

IF (MINFLG.EQ.4) GO TO 4

86TRKM 78

$\operatorname{IP1}=\operatorname{IDAT}(M, 1)$

$\operatorname{IP2}=\operatorname{IDAT}(M, 2)$

95

100

105

$\operatorname{ICALL}=\operatorname{IDAT}(M, 6)$

86TRKM 79

86TRKM 80

86TRKM 81

$S I=\operatorname{FDAT}(M, 2)$

86TRKM 82

$T I=\theta$.

86TRKM 83

$H O=\operatorname{FDAT}(M, 1)$

86TRKM 84

$\operatorname{DAT2}(7)=H \theta$

86TRKM 85

MAPK $=\operatorname{BDAT}(M, 3)$

86TRKM 86

$\operatorname{IRAD}=\operatorname{IDAT}(M, 4)$

86TRKM 87

86TRKM 88

C SET UP CONSTANTS FOR CALCULATING PSI IN DEGREES OR RADIANS

86TRKM 89

IF (IRAD.EQ. 1) RADFLG=.TRUE.

86TRKM 90

$\mathrm{DNOM}=\mathrm{TPI}$

86TRKM 91

$\mathrm{CCON}=1$.

86TRKM 92

IF (.NOT.RADFLG) GO TO 153

86TRKM 93

DNOM $=1$.

86TRKM 94

$\mathrm{CCON}=\mathrm{TPI}$

86TRKM 95

153 CONTINUE

86TRKM 96

86TRKM 97

$\operatorname{NAMBET}=\operatorname{BDAT}(M, 2)$

86TRKM 98

NAMVEC $=\operatorname{BDAT}(M, 3)$

86TRKM 99

NAMPAR $=\operatorname{BDAT}(M, 4)$

86TRKM 100

$\operatorname{NAME}=\operatorname{INFF}(2, M)$ 


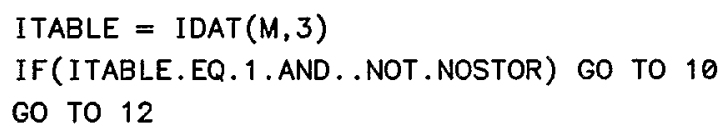

C 10 IF ((ICALL.EQ.0).OR.(IP1.EQ.0) ) GO TO 12 10 CONT INUE

C INITIAL BETAS FROM BETA FUNCTION TABLE OF TRKB INSTRUCTION,

C EXCEPT THAT FOR FIRST CALL, OR IF IP1=0, THEY ARE TAKEN FROM IBET OR LOCBET $=$ NCOL $*($ IP $1-K A)+1$ 
205

C MAP4 - TRANSVERSE ENVELOPES

$29 \quad M R=4$

C LOOP OVER POSITIONS

210

19 DO 1 IBS $=I 1, I 2$

IPOS $=$ IBS -1

IF (NOPR) GO TO 6

215

220

190 IF (MOD(LNUM, 45).NE.0.OR.LNUM.EQ.0) GO TO 191 WRITE $(3,1004)$ WRITE $(3,1000)$ LNUM $=0$

191 IF (MOD(LNUM, 5).EQ.0.AND.LNUM.NE.0) WRITE $(3,1003)$ WRITE $(3,1001)$ IPOS, ELNAME, $,(B X(I), I=1,3), B X(5), B X(6)$,

86TRKM

86TRKM 167

86TRKM 169

86TRKM 170

86TRKM 171

86TRKM 172

86TRKM 173

86TRKM 174

86TRKM 175

86TRKM 176

86TRKM 177

86TRKM 178

86TRKM 179

86TRKM 180

86TRKM 181

86TRKM 182

86TRKM 183

86TRKM 184

86TRKM 185

86TRKM 186

86TRKM 187

86TRKM 188

86TRKM 189

86TRKM 190

86TRKM 191

86TRKM 192

86TRKM 193

86TRKM 194

86TRKM 195

B6TRKM 196

86TRKM 197

86TRKM 198

86TRKM 199

86TRKM 200

86TRKM 201

86TRKM 202

86TRKM 203

86TRKM 204

86TRKM 205

86TRKM 206

86TRKM 207

$1 \quad(B Y(I), I=1,3), \operatorname{BY}(5), \operatorname{BY}(6)$

86TRKM 208

86TRKM 209

86TRKM 210

86TRKM 211

86TRKM 212

86TRKM 213

$11 \operatorname{STORE}(\mathrm{J}+\mathrm{I})=\operatorname{BETF}(\mathrm{I})$

8 CONTINUE 
C

MAPK2 $=\operatorname{INFF}(1, N E L)$

86TRKM 218

IF (MAPK2. EQ.MAPK) GO TO 20

86TRKM

219

MAPK=MAPK2

86TRKM 220

IF (MAPK.EQ.3HELQ) GO TO 22

86TRKM

221

NUM=4

GO TO 32

86TRKM

222

86TRKM 223

22 NUM=7

GO TO 36

240

20 IF (NUM.EQ.7) GO TO 36

IF (NUM-3) $30,31,32$

86TRKM

224

86TRKM 225

86TRKM 226

86TRKM 227

86TRKM 228

86TRKM 229

c MAP2

$30 \quad P(1)=B \times(2)$

245

$P(2)=B \times(3)$

$P(3)=B X(5)$

$P(4)=B X(6)$

$P(5)=B Y(2)$

$\mathrm{P}(6)=\mathrm{BY}(3)$

250

$\mathrm{P}(7)=\mathrm{BY}(5)$

$P(8)=B Y(6)$

CALL DIFEQ(NEL,P,DER2)

86TRKM

230

86TRKM 231

86TRKM 232

86TRKM 233

86TRKM 234

86TRKM 235

86TRKM 236

86TRKM 237

86TRKM 238

$B X(2)=P(1)$

$B X(3)=P(2)$

255

$B X(5)=P(3)$

$B X(6)=P(4)$

$B Y(2)=P(5)$

$\mathrm{BY}(3)=P(6)$

$B Y(5)=P(7)$

260

$\operatorname{BY}(6)=P(8)$

GO TO 33

86TRKM

239

86TRKM

240

86TRKM

241

86TRKM 242

86TRKM 243

86TRKM 244

86TRKM 245

86TRKM 246

86TRKM 247

86TRKM 248

C MAP3

86TRKM

249

86TRKM 250

$31 \quad P(1)=B \times(2)$

265

$P(2)=B X(3)$

$P(3)=B Y(2)$

86TRKM

251

86TRKM 252

$P(4)=B Y(3)$

86TRKM

253

$P(5)=B X(5)$

86TRKM

254

$P(6)=B X(6)$

86TRKM

255

270

$P(7)=0$.

86TRKM

256

CALL DIFEQ(NEL, P, DER3)

86TRKM

257

$B X(2)=P(1)$

$B X(3)=P(2)$

86TRKM

258

86TRKM 259

$B Y(2)=P(3)$

86TRKM

260

$B Y(3)=P(4)$

86TRKM

261

275

$B X(5)=P(5)$

86TRKM

262

$B X(6)=P(6)$

86TRKM

263

GO TO 33

86TRKM

264

86TRKM 265

86TRKM 266

280

C ELQ

$36 \quad P(1)=B X(2)$

86TRKM

267

86TRKM 268

$P(2)=B X(3)$

86TRKM

269

$P(3)=B Y(2)$

86TRKM

270

$P(4)=B Y(3)$

86TRKM

271

CALL DIFEQ(NEL,P,DELQ) 
GO TO 37

\begin{tabular}{|c|c|}
\hline 86TRKM & 273 \\
\hline 86TRKM & 27 \\
\hline 86TRKM & 275 \\
\hline 86TRKM & 276 \\
\hline 86TRKM & 277 \\
\hline 86TRKM & 278 \\
\hline 86TRKM & 279 \\
\hline 86TRKM & 281 \\
\hline 86TRKM & 28 \\
\hline 86TRKM & 282 \\
\hline 86TRKM & 283 \\
\hline 86TRKM & 284 \\
\hline 86TRKM & 285 \\
\hline 86TRKM & 286 \\
\hline 86TRKM & 287 \\
\hline 86TRKM & 288 \\
\hline 86TRKM & 289 \\
\hline 86TRKM & 290 \\
\hline 86TRKM & 291 \\
\hline 86TRKM & 292 \\
\hline 86TRKM & 293 \\
\hline 86TRKM & 29 \\
\hline 86TRKM & 29 \\
\hline 86TRKM & 296 \\
\hline 86TRKM & 297 \\
\hline 86TRKM & 298 \\
\hline 86TRKM & 299 \\
\hline 86TRKM & 300 \\
\hline 86TRKM & 301 \\
\hline 86TRKM & 302 \\
\hline 86TRKM & 303 \\
\hline 86TRKM & 304 \\
\hline 86TRKM & 305 \\
\hline 86TRKM & 306 \\
\hline 86TRKM & 307 \\
\hline 86TRKM & 308 \\
\hline 86TRKM & 309 \\
\hline 86TRKM & 310 \\
\hline 86TRKM & 311 \\
\hline 86TRKM & 312 \\
\hline 86TRKM & 313 \\
\hline 86TRKM & 314 \\
\hline 86TRKM & 315 \\
\hline 86TRKM & 316 \\
\hline
\end{tabular}

CARD NR. SEVERITY DETAILS DIAGNOSIS OF PROBLEM

EQV/COMM I BMI1 NOT ALL ITEMS IN THIS COMMON BLOCK OCCUR IN LEVEL STATEMENTS. 
SUBROUTINE TRKCN ( MFXPT, NEL, P, NP, IOP )

C A ROUTINE TO CONTROL TRACKING THROUGH LINEAR-, SINGLE NON-LINEAR-,

C OR PRD-TYPE PRODUCTS OF TRANSFORMATIONS.

C MFXPT = INDEX OF FXPT INSTRUCTION. HERE IT IS USED TO REFERENCE

C THE $7 X 7$ MATRIX REPRESENTING THE LINEARIZED BEAM SYSTEM RELATIVE TO

C THE REFERENCE RAY PP. ON EACH CALL TO TRKCN, MFXPT = NEL * MFXPT.

C NEL = INDEX OF ELEMENT OF BEAM SYSTEM

C $P P=$ PARTICLE VECTOR

C NP = PARTICLE INDEX

COMMON / DIM/ LEND, MEND, ISAV, ISAV7, M7END, KADD, KADDR, MUNIT , MSYMPL COMMON/CONTRL/ERROR, MODE, RSRV, STOR, XEQ, TRASW, NFSW, EMPTY, INDEF, 1 LDFLG, FIN

LOGICAL ERROR, RSRV, STOR, XEQ, TRASW, NFSW, EMPTY, INDEF, LDFLG, FIN

LEVEL 2, STORE, INFF, IWORK

COMMON STORE(48000), IWORK (10)

DIMENSION INFF $(24,2000)$

EQUIVALENCE (INFF, STORE)

COMMON/MQ/ ML \$LEVEL 2,ML

EXTERNAL DER1, DER2, DER4 , DER5, DER6, DER7

EXTERNAL DER3

DIMENSION P(7,1),T(49), PAR(25),ML(2)

DIMENSION RW(3)

INTEGER RET, OPNAME, HSXTP, HMOVE, HNPOL DATA RW, HSXTP, HMOVE/3*0.0, 4HSXTP, 4HMOVE/

DATA HNPOL/4HNPOL/

$N F=0$

IF (IOP.NE. 1) NF=M7END-5

$\mathrm{NN}=\mathrm{NEL}$

$\mathrm{KIND}=\operatorname{INFF}(20, N N)$

IF (KIND.EQ.4HEDRF) GO TO 35

IF (KIND.EQ.4HDKE) GO TO 36

IF (KIND.EQ.4HSOL) GO TO 37

IF (KIND.EQ.3HMAP) GO TO 20

IF (KIND.NE.3HPRD) GO TO 30

C PRD-TYPE PRODUCT

$K=\operatorname{INFF}(4, N N)$

ASSIGN 21 TO RET

$I=1$

$M M=N E L$

GO TO 22

C SINGLE LINEAR TRANSFORMATION

30 ASSIGN 45 TO RET

GO TO 40

C SINGLE NON-LINEAR TRANSFORMATION

20 ASSIGN 45 TO RET
TRKCN 2

TRKCN

TRKCN

TRKCN

TRKCN

TRKCN

TRKCN

TRKCN

TRKCN

TRKCN

TRKCN

TRKCN

DIM

DIM

CONTRL

CONTRL

CONTRL

CONTRL

BLANK

BLANK

86MARSIZ

86MARSIZ

86MARSIZ

BLANK

TRKCNL

TRKCNL

TRKCN

TRKCN

TRKCN

TRKCN

TRKCN

TRKCN

TRKCN

TRKCN

TRKCN

TRKCN

TRKCN

TRKCN

TRKCN

TRKCN

TRKCN

TRKCN

TRKCN

TRKCN

TRKCN

TRKCN

TRKCN

TRKCN

TRKCN

TRKCN

TRKCN

TRKCN

TRKCN

TRKCN

TRKCN

TRKCN

TRKCN 
60

IF (IOP.GT.1.AND. IOP.NE.5) ASSIGN 43 TO RET

GO TO 221

\begin{tabular}{|c|c|}
\hline TRKCN & 48 \\
\hline TRKCN & 49 \\
\hline TRKCN & 50 \\
\hline TRKCN & 51 \\
\hline TRKCN & 52 \\
\hline TRKCN & 53 \\
\hline TRKCN & 54 \\
\hline TRKCN & 55 \\
\hline TRKCN & 56 \\
\hline TRKCN & 57 \\
\hline TRKCN & 58 \\
\hline TRKCN & 59 \\
\hline TRKCN & 60 \\
\hline TRKCN & 61 \\
\hline TRKCN & 62 \\
\hline TRKCN & 63 \\
\hline TRKCN & 64 \\
\hline TRKCN & 65 \\
\hline TRKCN & 66 \\
\hline TRKCN & 67 \\
\hline TRKCN & 68 \\
\hline TRKCN & 69 \\
\hline TRKCN & 70 \\
\hline TRKCN & 71 \\
\hline TRKCN & 72 \\
\hline TRKCN & 73 \\
\hline TRKCN & 74 \\
\hline TRKCN & 75 \\
\hline TRKCN & 76 \\
\hline TRKCN & 77 \\
\hline TRKCN & 78 \\
\hline TRKCN & 79 \\
\hline TRKCN & 80 \\
\hline TRKCN & 81 \\
\hline TRKCN & 82 \\
\hline TRKCN & 83 \\
\hline TRKCN & 84 \\
\hline TRKCN & 85 \\
\hline TRKCN & 86 \\
\hline TRKCN & 87 \\
\hline TRKCN & 88 \\
\hline TRKCN & 89 \\
\hline TRKCN & 90 \\
\hline TRKCN & 91 \\
\hline TRKCN & 92 \\
\hline TRKCN & 93 \\
\hline TRKCN & 94 \\
\hline TRKCN & 95 \\
\hline TRKCN & 96 \\
\hline TRKCN & 97 \\
\hline TRKCN & 98 \\
\hline TRKCN & 99 \\
\hline TRKCN & 100 \\
\hline TRKCN & 101 \\
\hline TRKCN & 102 \\
\hline TRKCN & 103 \\
\hline TRKCN & 104 \\
\hline
\end{tabular}

C LOOP OVER PRD FACTORS

$21 \quad I=I+1$

IF (I.GT.K) GO TO 45

65

$22 \mathrm{NN}=\operatorname{MDAT}(\mathrm{MM}, \mathrm{I})$

$\mathrm{KIND}=\operatorname{INFF}(20, N N)$

IF (KIND.NE.3HPRD) GO TO 222

$\mathrm{N} 2=\mathrm{NN}$

$\mathrm{K} 2=0$

ASSIGN 321 TO RET

70

$\mathrm{KN}=\operatorname{INFF}(4, \mathrm{~N} 2)$

$321 \mathrm{~K} 2=\mathrm{K} 2+1$

$\mathrm{NN}=\operatorname{MDAT}(\mathrm{N} 2, \mathrm{~K} 2)$

$\mathrm{KIND}=\operatorname{INFF}(20, N N)$

75

322 IF (K2.EQ.KN) ASSIGN 21 TO RET

IF (KIND.NE. 3HPRD) GO JO 222

$N 3=N N$

$\mathrm{K} 3=0$

ASSIGN 421 TO RET

$\mathrm{KN} 3=\operatorname{INFF}(4, \mathrm{~N} 3)$

80

$421 K 3=K 3+1$

$\mathrm{NN}=\operatorname{MDAT}(\mathrm{N} 3, \mathrm{~K} 3)$

$K \operatorname{IND}=\operatorname{INFF}(20, N N)$

IF (K3.EQ.KN3) ASSIGN 321 TO RET

IF (K2.EQ.KN.AND.K3.EQ.KN3) ASSIGN 21 TO RET

85

IF (KIND.NE. 3HPRD) GO TO 222

WRITE $(3,1000)$

1000 FORMAT ( $*$ PRD INSTRUCTIONS NESTED MORE THAN THREE DEEP - ERROR*)

ERROR $=$. TRUE.

RETURN

90

222 IF (KIND.NE. 3HMAP) GO TO 40

$*$

221

PICK UP SUBROUTINE NAME

95

100

OPNAME $=\operatorname{INFF}(1, N N)$

IF (OPNAME.NE.HSXTP) GO TO 223

$\mathrm{NUM}=11$

GO TO 9

223 IF (OPNAME. EQ. HMOVE) GO TO 27

IF (OPNAME.NE. HNPOL) GO TO 25

NUM $=12$

GO TO 9

STRIP OFF MAP SUBROUTINE NUMBER

25 DECODE $(10,100$, OPNAME) NUM

100 FORMAT $(3 X, I 1,6 X)$

IF (NUM.EQ.0) NUM $=+0$

$\mathrm{NUM}=\mathrm{NUM}+1$

*

$9 \quad \mathrm{NFL}=\operatorname{INFF}(15, \mathrm{NN})$

GET DATA OF MAP INSTRUCTION. TRANSMIT IN ARGUMENT.

CALL DATA(NN, $1,1, N F L, P A R)$

110

GO TO $(10,11,12,13,14,15,16,17,18,19,23,28)$ NUM

10 CALL MAP (P,PAR)

GO TO RET, $(21,43,45,321,421)$

C MAP1 - WIGGLER MAGNET

11 CALL DIFEQ(NN,P,DER1)

TRKCN

104 


\begin{tabular}{|c|c|}
\hline TRKCN & 105 \\
\hline TRKCN & 106 \\
\hline TRKCN & 107 \\
\hline TRKCN & 108 \\
\hline TRKCN & 109 \\
\hline TRKCN & 110 \\
\hline TRKCN & 111 \\
\hline TRKCN & 112 \\
\hline TRKCN & 113 \\
\hline TRKCN & 114 \\
\hline TRKCN & 115 \\
\hline TRKCN & 116 \\
\hline TRKCN & 117 \\
\hline TRKCN & 118 \\
\hline TRKCN & 119 \\
\hline TRKCN & 120 \\
\hline TRKCN & 121 \\
\hline TRKCN & 122 \\
\hline TRKCN & 123 \\
\hline TRKCN & 124 \\
\hline TRKCN & 125 \\
\hline TRKCN & 126 \\
\hline TRKCN & 127 \\
\hline TRKCN & 128 \\
\hline TRKCN & 129 \\
\hline TRKMPL & 1 \\
\hline TRKMPL & 2 \\
\hline TRKMPL & 3 \\
\hline TRKCN & 131 \\
\hline TRKCN & 132 \\
\hline TRKCN & 133 \\
\hline TRKCN & 134 \\
\hline TRKCN & 135 \\
\hline TRKCN & 136 \\
\hline TRKCN & 137 \\
\hline TRKCN & 138 \\
\hline TRKCN & 139 \\
\hline TRKCN & 140 \\
\hline TRKCN & 141 \\
\hline TRKCN & 142 \\
\hline TRKCN & 143 \\
\hline TRKCN & 144 \\
\hline TRKCN & 145 \\
\hline TRKCN & 146 \\
\hline TRKCN & 147 \\
\hline TRKCN & 148 \\
\hline TRKCN & 149 \\
\hline TRKCN & 150 \\
\hline TRKCN & 151 \\
\hline TRKCN & 152 \\
\hline TRKCN & 153 \\
\hline TRKCN & 154 \\
\hline TRKCN & 155 \\
\hline TRKCN & 156 \\
\hline TRKCN & 157 \\
\hline TRKCN & 158 \\
\hline TRKCN & 159 \\
\hline
\end{tabular}


CARD NR. SEVERITY DETAILS DIAGNOSIS OF PROBLEM

137 I P

ARRAY REFERENCE OUTSIDE DIMENSION BOUNDS.

161 I $P$ ARRAY REFERENCE OUTSIDE DIMENSION BOUNDS. 
SUBROUTINE WBE $(K, L)$

C WRITES BETATRON FUNCTIONS OF ELEMENTS $M I(I), I=1, K$

5

10

15

20

25

30

35

40

45
LEVEL 2, STORE, INFF, IWORK

COMMON STORE (48000), IWORK (10)

DIMENSION INFF $(24,2000)$

EQUIVALENCE (INFF, STORE)

COMMON/BMI/MI (5000)

COMMON/NELS/NELS

LEVEL 2,MI

DIMENSION BETX(9), BETY(9)

CALL HED

IF (L.NE.O) GO TO 40

WRITE $(3,51)$

GO TO 42

40 WRITE $(3,52)$

42 DO $45 \quad \mathrm{I}=1, \mathrm{~K}$

$M 1=M I(I)$

$N=\operatorname{INFF}(2, M 1)$

IF (L.NE.O) GO TO 50

CALL BET (M1, BETX, BETY, 1)

$\operatorname{BETX}(1)=\operatorname{BETX}(1) / 6.283185307$

$\operatorname{BETY}(1)=\operatorname{BETY}(1) / 6.283185307$

WRITE $(3,53) N, \operatorname{BETX}(1), \operatorname{BETX}(2), \operatorname{BETX}(3), \operatorname{BETX}(5), \operatorname{BETX}(6), \operatorname{BETX}(7)$,

$1 \operatorname{BETY}(1), \operatorname{BETY}(2), \operatorname{BETY}(3), \operatorname{BETY}(5), \operatorname{BETY}(6), \operatorname{BETY}(7)$

GO TO 45

50 CALL BET (M1, BETX, BETY, 0)

$\operatorname{BETX}(1)=\operatorname{BETX}(1) / 6.283185307$

$\operatorname{BETY}(1)=\operatorname{BETY}(1) / 6.283185307$

WRITE $(3,53)$ N,BETX(1), BETX (2), BETX(3), BETX (5), BETX(6), BETX (9),

$1 \operatorname{BETY}(1), \operatorname{BETY}(2), \operatorname{BETY}(3), \operatorname{BETY}(5), \operatorname{BETY}(6), \operatorname{BETY}(9)$

45 CONTINUE

CALL HED

RETURN

51 FORMAT( $5 X, 19 H B E T A T R O N$ FUNCTIONS.)

$\begin{array}{llccr}1 X / 2 P I & B E T A X & \text { ALPHAX } & \text { XEQ } & \text { DXEQ } \\ 22 P I & \text { BETAY } & \text { ALPHAY } & \text { YEQ } & \text { DYEQ }\end{array}$

52 FORMAT( $5 X, 19 H B E T A T R O N$ FUNCTIONS./1HO, 131HNAME

1 ALPHAX XEQ DXEQ XTRACE $/ 2$

2 ALPHAY YEQ DYEQ YTRACE/2/1H0)

53 FORMAT( $1 X, A 5, F 12.8, F 10.5,4 F 10.6,2 X, F 12.8, F 10.5,4 F 10.6)$

END

\begin{tabular}{|c|c|}
\hline TRKCN & 162 \\
\hline WBE & \\
\hline WBE & \\
\hline BLANK & \\
\hline BLANK & \\
\hline 86MARSIZ & \\
\hline 86MARSIZ & \\
\hline 86MARSIZ & \\
\hline BLANK & \\
\hline BMIL & \\
\hline BMIL & \\
\hline BMIL & \\
\hline BMIL & \\
\hline $\mathrm{BMI}$ & 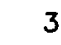 \\
\hline WBE & 6 \\
\hline WBE & - \\
\hline WBE & 8 \\
\hline WBE & 9 \\
\hline WBE & 10 \\
\hline WBE & 11 \\
\hline WBE & 12 \\
\hline WBE & 13 \\
\hline WBE & 14 \\
\hline WBE & 15 \\
\hline WBE & 16 \\
\hline WBE & 17 \\
\hline WBE & 18 \\
\hline WBE & 19 \\
\hline WBE & 20 \\
\hline WBE & 21 \\
\hline WBE & 22 \\
\hline WBE & 23 \\
\hline WBE & 24 \\
\hline WBE & 25 \\
\hline WBE & 26 \\
\hline WBE & 27 \\
\hline WBE & 28 \\
\hline WBE & 29 \\
\hline WBE & 30 \\
\hline WBE & 31 \\
\hline WBE & 32 \\
\hline WBE & 33 \\
\hline WBE & 34 \\
\hline WBE & 35 \\
\hline WBE & 36 \\
\hline WBE & 37 \\
\hline
\end{tabular}


C PRINTS CONTENTS OF $\operatorname{INFF}(\mathrm{J}, \mathrm{I}), \mathrm{J}=1,24$ AND I=MIN, MAX.

C IF ROUTINE CALLED BECAUSE OF ERROR, ONLY INFF IS PRINTED.

C IF KB OF WFL IS NOT $\theta$, STORE USED BY INSTRUCTIONS IS PRINTED.

LEVEL 2, STORE, INFF, IWORK

COMMON STORE (48000), IWORK(10)

DIMENSION INFF $(24,2000)$

COMMON/INSTR/OPNAME, NAME, OP, KA, KB, OBJA, OBJB, NXTM, TRA, LQ, NQ, LIN,

1 NIN, LFL, NFL, LBC, NBC, NTOT, IX, KIND, TYPE, ROWS, COLS, EXTR, 2

$$
M, N F, N B, N I, M S U B R, I S I G N
$$

INSTR

INTEGER OPNAME, OP, OBJA, OBJB, TRA, TYPE, ROWS, COLS, EXTR

COMMON/CONTRL/ERROR, MODE, RSRV, STOR, XEQ, TRASW, NFSW, EMPTY, INDEF, 1 LDFLG, FIN LOGICAL ERROR, RSRV, STOR, XEQ, TRASW, NFSW, EMPTY, INDEF, LDFLG, FIN

COMMON/STORE/LMAX, LINF, LFILE, MAX, MIN, ICARD (11)

* lmaX aNd maX aRE dimENSIONS of STORE AND INFF

COMMON /DIM/ LEND, MEND, ISAV, ISAV7, M7END, KADD, KADDR, MUNIT, MSYMPL

LOGICAL ENDL

C

IF (MODE.EQ.3) GO TO 16

$I S G N=1 H$

35 FORMAT ( $6 \mathrm{H} * * *, A 1, A 5,2 X, A 5,1 X, I 3,1 X, I 3,5 H / /, 6 A 10)$

IF (MODE.EQ.2) RETURN

IF (OP.LT.O) RETURN

24 FORMAT ( $3 X, *$ CONTENTS OF *, 5X,*INFF $(1, M) *$,

1 18X, *OPNAME*, 17X, *NAME*, 19X,*OP*,19X,*KA*/ 
60

65

70

75

80

85

90

95
WRITE $(3,30) J 1, J 2,(\operatorname{INFF}(J, M X), J=J 1, J 2)$

$\mathrm{J} 1=\mathrm{J} 2+1$

30 FORMAT $(21 \mathrm{X}, 5 \mathrm{H}(\mathrm{I}=, \mathrm{I} 2,1 \mathrm{H}, \mathrm{I} 2,1 \mathrm{H}), 5 \mathrm{X}, 4(2 \mathrm{X}, 020))$

31 CONTINUE

$M X=M X-1$

10 CONTINUE

C PRINT STOR

IF (ERROR) RETURN

IF (KB.EQ.0) RETURN

LX=LFILE

$L=I S A V *(14+K A D D)$

$\operatorname{IF}(K A . E Q .1) \quad L=1$

C ENDL IS SWITCH TO TERMINATE PRINTING

$E N D L=. F A L S E$.

WRITE $(3,28)$

28 FORMAT ( $31 \mathrm{H} 1$ CONTENTS OF STORE IN OCTAL//

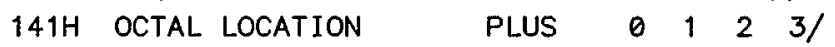

$241 \mathrm{H}$

$17 \quad J=L+3$

IF (J.LT.LX) GO TO 18

$J=L X$

ENDL $=$. TRUE.

18 WRITE $(3,25)$ L, (STORE(I), I=L, J)

25 FORMAT $(5 X, 06,5 X, 4(020,2 X))$

IF (ENDL) GO TO 20

$L=J+1$

$J=L+3$

IF (J.LT.LX) GO TO 19

$J=L X$

ENDL=. TRUE.

19 WRITE $(3,26)$ (STORE(I), I=L, J)

26 FORMAT $(16 X, 4(020,2 X) / /)$

IF (ENDL) GO TO 20

$L=J+1$

GO TO 17

20 WRITE $(3,27)$

27 FORMAT (1H1)

RETURN

END

$\begin{array}{ll}\text { WFLSR } & 44 \\ \text { WFLSR } & 45 \\ \text { WFLSR } & 46 \\ \text { WFLSR } & 47 \\ \text { WFLSR } & 48 \\ \text { WFLSR } & 49 \\ \text { WFLSR } & 50 \\ \text { WFLSR } & 51 \\ \text { WFLSR } & 52 \\ \text { WFLSR } & 53 \\ \text { WFLSR } & 54 \\ \text { WFLSR } & 55 \\ \text { WFLSR } & 56 \\ \text { WFLSR } & 57 \\ \text { WFLSR } & 58 \\ \text { WFLSR } & 59 \\ \text { WFLSR } & 60 \\ \text { WFLSR } & =61 \\ \text { WFLSR } & 62 \\ \text { WFLSR } & 63 \\ \text { WFLSR } & 64 \\ \text { WFLSR } & 65 \\ \text { WFLSR } & 66 \\ \text { WFLSR } & 67 \\ \text { WFLSR } & 68 \\ \text { WFLSR } & 69 \\ \text { WFLSR } & 70 \\ \text { WFLSR } & 71 \\ \text { WFLSR } & 72 \\ \text { WFLSR } & 73 \\ \text { WFLSR } & 74 \\ \text { WFLSR } & 75 \\ \text { WFLSR } & 76 \\ \text { WFLSR } & 77 \\ \text { WFLSR } & 78 \\ \text { WFLSR } & 79 \\ \text { WFLSR } & 80 \\ \text { WFLSR } & 81 \\ \text { WFLSR } & 82 \\ & \end{array}$


SUBROUTINE WMA(KA)

C WRITES MATRICES OF ELEMENTS MI (I), I=1,KA.

LEVEL 2, STORE, INFF, IWORK

COMMON STORE(48000), IWORK(10)

DIMENSION INFF $(24,2000)$

EQUIVALENCE (INFF,STORE)

COMMON/SWTCH/BEND, MSI ZE, CYCSWT, VCSW, PV , MSSW, VPR , MHS, MODS, MINZER

COMMON/BMI/MI (5000)

COMMON/NELS/NELS

LEVEL 2,MI

DIMENSION RX(2,3), $\operatorname{RY}(2,3), \operatorname{RW}(3), T(7,7)$

C PRINT ALL MATRICES AS DEFINED BY SIZE

C IF SIZE ARBITRARY, PRINT IN $7 \times 7$ IF ANY ARE $7 \times 7$

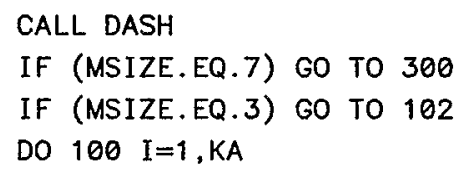

C PRINT HEADING FOR TRANSFER MATRICES-NO MISALIGNMENT

C PRINT ALL AS $7 \times 7$ MATRICES

300 CONTINUE

WRITE $(3,36)$

DO $350 \quad I=1, K A$

$M 1=M I(I)$

$N=\operatorname{MNAME}(M 1)$

\begin{tabular}{|c|c|}
\hline WMA & \\
\hline WMA & \\
\hline BLANK & \\
\hline BLANK & \\
\hline 86MARSIZ & \\
\hline 86MARSIZ & \\
\hline 86MARSIZ & \\
\hline BLANK & \\
\hline SWTCH & \\
\hline SWTCH & \\
\hline SWTCH & \\
\hline BMI L & \\
\hline BMIL & \\
\hline BMIL & \\
\hline BMIL & \\
\hline BMI & \\
\hline WMA & \\
\hline WMA & \\
\hline WMA & \\
\hline WMA & 1 \\
\hline WMA & 1 \\
\hline WMA & 1 \\
\hline WMA & 1 \\
\hline WMA & 1 \\
\hline WMA & 1 \\
\hline WMA & 1 \\
\hline WMA & 1 \\
\hline WMA & 1 \\
\hline WMA & 1 \\
\hline WMA & 2 \\
\hline WMA & 2 \\
\hline WMA & 2 \\
\hline WMA & 2 \\
\hline WMA & 2 \\
\hline WMA & 2 \\
\hline WMA & 2 \\
\hline WMA & 2 \\
\hline WMA & 2 \\
\hline WMA & 2 \\
\hline WMA & 3 \\
\hline WMA & 3 \\
\hline WMA & 3 \\
\hline WMA & 3 \\
\hline WMA & 3 \\
\hline WMA & 3 \\
\hline WMA & 3 \\
\hline WMA & 3 \\
\hline WMA & 3 \\
\hline WMA & 3 \\
\hline WMA & 4 \\
\hline WMA & 4 \\
\hline WMA & 4 \\
\hline WMA & 4 \\
\hline WMA & 4 \\
\hline WMA & 4 \\
\hline WMA & 4 \\
\hline WMA & \\
\hline
\end{tabular}


60

65

70

75

80

85
CALL RTRV7(M1,T, RW)

DO 305 I I=1,3

WRITE $(3,34)(T(I I, J), J=1,7)$

305 CONTINUE

WRITE $(3,35) \mathrm{N},(\mathrm{T}(4, \mathrm{~J}), \mathrm{J}=1,7)$

DO $310 \quad I I=5,7$

WRITE $(3,34)(T(I I, J), J=1,7)$

310 CONTINUE

WRITE $(3,15) \operatorname{RW}(1), \operatorname{RW}(2)$

350 CONTINUE

355 CALL DASH

RETURN

FORMAT (20H

$134 X, 1 H X, 11 X, 2 H D X, 11 X, 1 H 1,20 X, 1 H Y, 12 X, 2 H D Y, 11 X, 1 H 1 / 9 X, 7 H E L E M E N T)$

12 FORMAT (27X, 3F 13.8,8X,3F13.8)

13 FORMAT ( $10 \mathrm{X}, \mathrm{A5}, 12 \mathrm{X}, 3 \mathrm{~F} 13.8,8 \mathrm{X}, 3 \mathrm{~F} 13.8 / 30 \mathrm{X}, 2 \mathrm{HO}, 11 \mathrm{X}, 2 \mathrm{HO},, 11 \mathrm{X}, 2 \mathrm{H} 1, ., 19 \mathrm{X}$ WMA

$1,2 \mathrm{HO}, 11 \mathrm{X}, 2 \mathrm{HO}, ., 11 \times, 2 \mathrm{H} 1 . /)$

15 FORMAT ( $/ 30 X, 9 H L E N G T H=, F 13.8,5 X, 9 H$ THETA $=, F 13.8 / / /)$

21

FORMAT (20H TRANSFER MATRICES, 29X, 7HRX $(I, J), 35 X, 7 H R Y(I, J) / /$

$134 \mathrm{X}, 1 \mathrm{HX}, 9 \mathrm{X}, 5 \mathrm{HDX} / \mathrm{DS}, 9 \mathrm{X}, 3 \mathrm{H}-\mathrm{DS}, 8 \mathrm{X}, 4 \mathrm{HDP} / \mathrm{P}, 15 \mathrm{X}, 1 \mathrm{HY}, 10 \mathrm{X}, 5 \mathrm{HDY} / \mathrm{DS} /$

29X, 7HELEMENT)

22

FORMAT $(27 X, 2 F 13.8,3 X, 2 \mathrm{HO}, .8 X, F 13.8,3 X, 2 F 13.8)$

23

24

FORMAT (1OX, A5, 12X, 2F13.8,3X,2HO. 8X,F13.8,3X, 2F13.8)

FORMAT $(27 \mathrm{X}, 2 \mathrm{~F} 13.8,3 \mathrm{X}, 10 \mathrm{H} 1.00000000, \mathrm{~F} 13.8 / 30 \mathrm{X}, 2 \mathrm{HO}, 11 \mathrm{X}, 2 \mathrm{HO}, .11 \mathrm{X}$,

$12 \mathrm{HO}, 111 \mathrm{X}, 10 \mathrm{H} 1.00000000)$

34 FORMAT (30X,7F13.8)

35

36
FORMAT $(10 \mathrm{X}, \mathrm{A} 5,15 \mathrm{X}, 7 \mathrm{~F}$

FORMAT (20H TRANSFER MATRICES, 53X,6HR( $\mathrm{I}, \mathrm{J}) / / 36 \mathrm{X}, 1 \mathrm{HX}, 10 \mathrm{X}, 5 \mathrm{HDX} / \mathrm{DS}$, $110 X, 1 H Y, 10 X, 5 H D Y / D S, 9 X, 3 H-D S, 9 X, 4 H D P / P, 10 X, 1 H 1 / 9 X, 7 H E L E M E N T$ ) END

\begin{tabular}{|c|c|}
\hline WMA & 48 \\
\hline WMA & 49 \\
\hline WMA & 50 \\
\hline WMA & 51 \\
\hline WMA & 52 \\
\hline WMA & 53 \\
\hline WMA & 54 \\
\hline WMA & 55 \\
\hline WMA & 56 \\
\hline WMA & 57 \\
\hline WMA & 58 \\
\hline WMA & 59 \\
\hline WMA & 60 \\
\hline WMA & 61 \\
\hline WMA & 62 \\
\hline WMA & 63 \\
\hline WMA & 64 \\
\hline WMA & 65 \\
\hline WMA & 66 \\
\hline WMA & 67 \\
\hline WMA & 68 \\
\hline WMA & 69 \\
\hline WMA & 70 \\
\hline WMA & 71 \\
\hline WMA & 72 \\
\hline WMA & 73 \\
\hline WMA & 74 \\
\hline WMA & 75 \\
\hline WMA & 76 \\
\hline WMA & 77 \\
\hline
\end{tabular}


1

$$
\begin{aligned}
& \text { SUBROUTINE WRTINF }(\text { INF, M) } \\
& \text { C WRITES ONE COLUMN OF INF INTO INFF (M) }
\end{aligned}
$$

5

10

15
LEVEL 2, STORE, INFF, IWORK

COMMON STORE (48000), IWORK(10)

DIMENSION INFF $(24,2000)$

EQUIVALENCE (INFF, STORE)

DIMENSION INF(1)

DO $20 \mathrm{I}=1,24$

IF (INF(I).EQ.1H*) GO TO 20

$\operatorname{INFF}(I, M)=\operatorname{INF}(I)$

20 CONTINUE

RETURN

END

$\begin{array}{lr}\text { WRT INF } & 2 \\ \text { WRT INF } & 3 \\ \text { BLANK } & 2 \\ \text { BLANK } & 3 \\ \text { 86MARSIZ } & 1 \\ \text { 86MARSIZ } & 2 \\ \text { 86MARSIZ } & 3 \\ \text { BLANK } & 5 \\ \text { WRT INF } & 5 \\ \text { WRTINF } & 6 \\ \text { WRTINF } & 7 \\ \text { WRTINF } & 8 \\ \text { WRTINF } & 9 \\ \text { WRT INF } & 10 \\ \text { WRTINF } & 11\end{array}$


C CONTROL EXECUTION OF SYNCH INSTRUCTION BE ACT OR DELE

C IF $K B=1$, KA ELEMENTS SHOULD BE CHANGED BEGINNING WITH ONE INPUT

$X E Q C O N$

C IF $K B=0$, INPUT CONTAINS KA ELEMENTS

5

10

20

25

30

35

40

10

$$
\begin{aligned}
& \text { LEVEL 2, STORE, INFF, IWORK } \\
& \text { COMMON STORE }(48000), \operatorname{IWORK}(10) \\
& \text { DIMENSION INFF }(24,2000) \\
& \text { EQUIVALENCE (INFF, STORE) }
\end{aligned}
$$

$$
\begin{aligned}
& \text { LOGICAL SEQ,ACT } \\
& \text { INTEGER ELNUM } \\
& \text { DIMENSION NDAT }(50) \\
& \text { DATA IBLNK } / 1 \mathrm{H} /
\end{aligned}
$$

15

$$
\begin{aligned}
& \mathrm{SEQ}=. \operatorname{FALSE} . \\
& \mathrm{KA}=\operatorname{INFF}(4, M) \\
& \mathrm{KB}=\operatorname{INFF}(5, M) \\
& \operatorname{NAM}=\operatorname{INFF}(1, M)
\end{aligned}
$$$$
\text { ACT }=\text {. TRUE. }
$$$$
\text { IF (NAM. EQ . 4HDELE) ACT = .FALSE. }
$$$$
\text { IF (KB.EQ.1) SEQ = .TRUE. }
$$$$
K C=K A
$$$$
\text { IF (SEQ) } K C=1
$$$$
\text { CALL DATA }(M, 2,1, K C, N D A T)
$$$$
\text { IF(SEQ) GO TO } 15
$$

C CHANGE EXECUTION MODE OF ALL ELEMENTS IN NDAT ARRAY

$$
\text { DO } 10 I=1, K A
$$$$
\text { IF (NDAT(I).EQ.IBLNK) GO TO } 10
$$$$
N N=\operatorname{ELNUM}(N D A T(I))
$$$$
\operatorname{IOP}=\operatorname{IABS}(\operatorname{INFF}(3, \mathrm{NN}))
$$$$
\text { IF (.NOT.ACT) IOP }=-I O P
$$$$
\operatorname{INFF}(3, N N)=\operatorname{IOP}
$$$$
\text { CONTINUE }
$$$$
\text { RETURN }
$$

CONTINUE

$$
N N=\operatorname{ELNUM}(\operatorname{NDAT}(1))
$$$$
\text { DO } 20 \mathrm{I}=1, \mathrm{KA}
$$$$
I O P=\operatorname{IABS}(\operatorname{INFF}(3, N N))
$$$$
\text { IF (. NOT. ACT) IOP }=-I O P
$$$$
\operatorname{INFF}(3, N N)=1 O P
$$$$
N N=\operatorname{INFF}(8, N N)
$$

20

$$
\text { CONTINUE }
$$$$
\text { RETURN }
$$

45 
SUBROUT INE ZAMO(NO, X, DERI , Y,F,T, HPRO)

COMMON /INTC/ IPMX, AREF, EMAX, SSSR, HFAC, SWAM, SWEX

COMMON /INTP/ HPR, XX, N, EUB, ELB, IP, IT, NRKS, SWIN

DIMENSION $Y(1), F(1), T(8,1)$

5

LOGICAL SWAM, SWEX, SWIN

INTEGER HFAC

DOUBLE PRECISION T, HPRO, HPR, XX

DATA IPMX, AREF, EMAX, SSSR, HFAC, SWAM, SWEX

$\$ \quad / 1024,1.0,1.0 \mathrm{E}-6,100.0,2,$. TRUE. . . TRUE./

10

c

$$
\begin{aligned}
& H P R=H P R O \\
& X X=D B L E(X) \\
& N=N O \\
& E U B=E M A X
\end{aligned}
$$

15

$$
\mathrm{IP}=1
$$

I $T=0$

SWIN=SWEX 


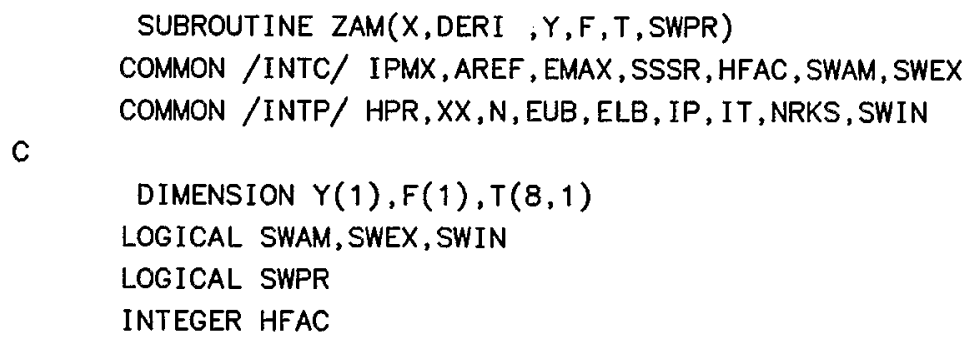


60

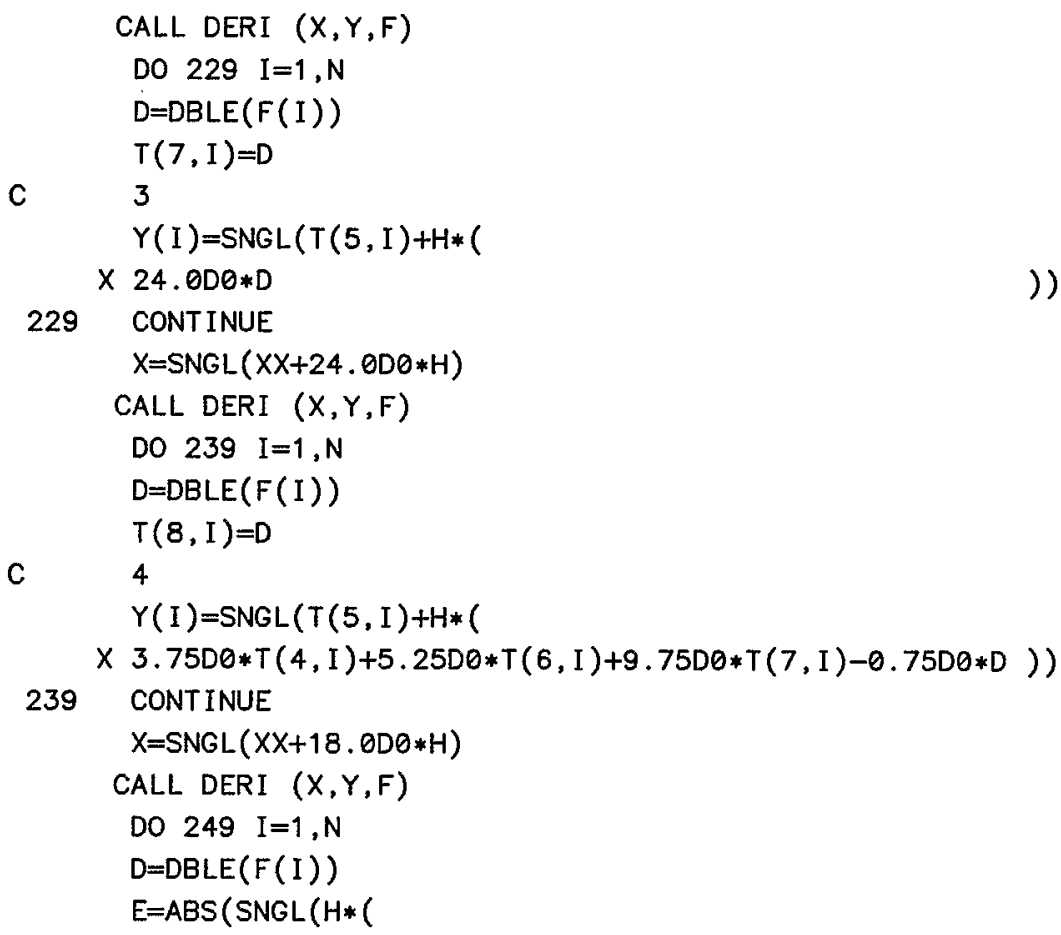

)

80

$X-16.0 \mathrm{D} 0 * T(4, \mathrm{I})+48.0 \mathrm{D} 0 * T(6, \mathrm{I})+48.0 \mathrm{D} 0 * T(7, \mathrm{I})+48.0 \mathrm{DO} * \mathrm{~T}(8, \mathrm{I})$

$X-128.000 * D \quad)))$

C 5

$D=(T(5, I)+H *($

85

$X 4.0 D 0 * T(4, I)+8.000 * T(6, I)+8.0 D 0 * T(7, I)+4.0 D 0 * T(8, I)$

$x))$

$T(6, I)=D$

TEST $=$ AMAX $1(E / A M A X 1(A R E F, A B S(S N G L(D))), T E S T)$

249 CONTINUE

C

90

95

c

C

100

110

105

C c 300

BOTH ADAMS-MOULTON AND ZONNEVELD METHODS CONTINUE FROM HERE.

CONTINUE

$X=\operatorname{SNGL}(X X+24.0 D \theta * H)$

IF (TEST . LE. EUB) GO TO 310

IF (IP*HFAC .GT. IPMX) GO TO 309

REPEAT STEP WITH SMALLER H.

NRKS $=0$

$I P=I P * H F A C$

$I T=I T * H F A C$

DO $305 I=1, N$

$Y(I)=\operatorname{SNGL}(T(5, I))$

$F(I)=\operatorname{SNGL}(T(4, I))$

305

CONT INUE

GO TO 1

C CANNOT DECREASE H BECAUSE OF HMIN.

309

CONT INUE

IF (.NOT. SWIN) GO TO 310

PRINT 6000, X, IPMX

SWIN=.FALSE.

C

310 CONTINUE

C

C
ZAM

ZAM

ZAM

ZAM

ZAM

ZAM

ZAM

ZAM

ZAM

ZAM

ZAM

ZAM

ZAM

ZAM

ZAM

ZAM

ZAM

ZAM

ZAM

ZAM

ZAM

ZAM

ZAM

ZAM

ZAM

ZAM

ZAM 


\begin{tabular}{|c|c|c|c|c|}
\hline \multirow[t]{5}{*}{115} & c & ACCEPT CURRENT STEP. & ZAM & 116 \\
\hline & c & & ZAM & 117 \\
\hline & c & XX STILL HAS NOT BEEN CHANGED SINCE ENTRY. & ZAM & 118 \\
\hline & c & YY $(X X)$ IS STILL IN $T(5)$, & ZAM & 119 \\
\hline & c & $F(Y Y)$ IS IN $T(4)$, & ZAM & 120 \\
\hline \multirow[t]{5}{*}{120} & c & & ZAM & 121 \\
\hline & & $I T=I T+1$ & ZAM & 122 \\
\hline & & $X X=X X+H P R / D B L E(F L O A T(I P))$ & ZAM & 123 \\
\hline & & NRKS $=$ MINO $($ NRKS $+1,4)$ & ZAM & 124 \\
\hline & & DO $319 I=1, N$ & ZAM & 125 \\
\hline \multirow[t]{5}{*}{125} & & $D=T(6, I)$ & ZAM & 126 \\
\hline & & $T(5, I)=D$ & ZAM & 127 \\
\hline & & $Y(I)=S N G L(D)$ & ZAM & 128 \\
\hline & 319 & CONTINUE & ZAM & 129 \\
\hline & & $X=\operatorname{SNGL}(X X)$ & ZAM & 130 \\
\hline \multirow[t]{5}{*}{130} & & CALL DERI $(X, Y, F)$ & ZAM & 131 \\
\hline & & IF (IT .LT. IP) GO TO 320 & ZAM & 132 \\
\hline & c & & ZAM & 133 \\
\hline & $\mathrm{C}$ & $X$ IS A MULTIPLE OF HPRINT. & ZAM & 134 \\
\hline & & SWPR $=$. TRUE & ZAM & 135 \\
\hline \multirow[t]{5}{*}{135} & & $I T=I T-I P$ & ZAM & 136 \\
\hline & c & & ZAM & 137 \\
\hline & 320 & CONTINUE & ZAM & 138 \\
\hline & & IF (TEST .GE. ELB) GO TO 330 & ZAM & 139 \\
\hline & & IF (MOD (IP, HFAC)+MOD (IT,HFAC).NE. 0$)$ GO TO 330 & ZAM & 140 \\
\hline \multirow[t]{5}{*}{140} & c & & ZAM & 141 \\
\hline & C & PROCEED TO NEXT STEP WITH LARGER $H$, USING ZONNEVELD METHOD. & ZAM & 142 \\
\hline & & NRKS $=0$ & ZAM & 143 \\
\hline & & $I P=I P / H F A C$ & ZAM & 144 \\
\hline & & $\mathrm{I} T=\mathrm{IT} / \mathrm{HFAC}$ & ZAM & 145 \\
\hline \multirow[t]{5}{*}{145} & & RETURN & ZAM & 146 \\
\hline & c & & ZAM & 147 \\
\hline & $\mathrm{c}$ & & ZAM & 148 \\
\hline & $\mathrm{c}$ & PROCEED TO NEXT STEP WITH SAME $H$. & ZAM & 149 \\
\hline & 330 & CONTINUE & ZAM & 150 \\
\hline \multirow[t]{5}{*}{150} & & DO $339 \quad I=1, N$ & ZAM & 151 \\
\hline & & $T(1, I)=T(2, I)$ & ZAM & 152 \\
\hline & & $T(2, I)=T(3, I)$ & ZAM & 153 \\
\hline & & $T(3, I)=T(4, I)$ & ZAM & 154 \\
\hline & 339 & CONTINUE & ZAM & 155 \\
\hline \multirow[t]{2}{*}{155} & & RETURN & ZAM & 156 \\
\hline & & END & ZAM & 157 \\
\hline
\end{tabular}


SUBROUT INE MINUITS

MINUITS

CC AUG. 17,1975

MINUITS

CALL MINNEW

MINUITS

RETURN

MINUITS

END

MINUITS

3

4

9

5

10 
1

10

15

20

25

30

35

5
FUNCTION CALFCN(PVEC)

CALLED ONLY FROM IMPROV. TRANSFORMS THE FUNCTION FCN BY DIVIDING OUT THE QUADRATIC PART IN ORDER TO FIND FURTHER MINIMA.

CALCULATES (F-FMIN $) /(X-X M I N) * V *(X-X M I N)$

CC

$\mathrm{CC}$

CC

COMMON

1/MINERR/ ERP(30) , ERN $(30)$

2/PARINT/ $X(15)$

XT(15)

,DIRIN(15)

, NAM(30)

, WERR (30)

, MAXINT

, LCODE (30)

, MAXEXT

,XTS(15)

, LCORSP (30)

5/VARIAN/ $V(15,15)$

, BLIM(30)

,WTS(15) ,NPFIX

C/CASC/ JH, JL, $Y(16)$

F/DERIVA/ $G(30)$

,$X S(15)$

,$G 2(30)$

G/SIMVEC/ $P(15,16)$

,PSTAR( 15$)$

$, \operatorname{PSTST}(15) \quad, \operatorname{PBAR}(15)$

J/VARIAT/ VT $(15,15)$

6/UNIT / ISYSRD

, ISYSWR , ISYSPU

8/TITLE / TITLE(13)

,DATE (2)

, ISW(7)

9/CONVER/ EPSI ,APSI

A/CARD / CWORD

,VTEST

, NSTEPQ

B/MINIMA/ AMIN

. CWORD2

, CWORD3

, UP

, NEWMIN

, CNAME (25)

, CNAM2 25

DIMENSION PVEC (15)

CALL INTOEX(PVEC)

CALL FCN(NPAR, G, F,U, 4)

$\mathrm{NFCN}=\mathrm{NFCN}+1$

DO $200 I=1$, NPAR

$G(I)=\theta$.

DO $200 \mathrm{~J}=1$, NPAR

$200 G(I)=G(I)+\operatorname{VT}(I, J) *(X T(J)-\operatorname{PVEC}(J))$

$\mathrm{DENOM}=0$.

DO $210 \mathrm{I}=1$, NPAR

210 DENOM $=$ DENOM $+\mathrm{G}(\mathrm{I}) *(\mathrm{XT}(\mathrm{I})-\mathrm{PVEC}(\mathrm{I}))$

IF (DENOM . LE. O.) ISW(2) $=0$

IF (DENOM .LE. 0.) DENOM $=1.0$

CALFCN $=(F-A P S I) /$ DENOM

RETURN

END
CALFCN 2

CALFCN 3

CALFCN 4

CALFCN 5

CALFCN 6 .

COMMONU 2

COMMONU 4

COMMONU 5

COMMONU 6

COMMONU 7

COMMONU 8

COMMONU 9

COMMONU 10

COMMONU 11

COMMONU 12

COMMONU 13

COMMONU 27

COMMONU 28

COMMONU 29

COMMONU 30

COMMONU 31

COMMONU 32

CALFCN 8

CALFCN 9

CALFCN 10

CALFCN 11

CALFCN 12

CALFCN 13

CALFCN 14

CALFCN 15

CALFCN 16

CALFCN 17

CALFCN 18

CALFCN 19

CALFCN 20

CALFCN 21

CALFCN 22

CALFCN 23 
SUBROUTINE COMAND

READS THE COMMAND CARDS AND TAKKES APPROPRIATE ACTION, EITHER DIRECTLY BY SKIPPING TO THE CORRESPONDING CODE IN COMAND, OR BY SETTING UP A CALL TO A SUBROUTINE

CC

$\mathrm{CC}$

CC

COMMON

$1 /$ MINERR/ ERP $(30) \quad$,ERN $(30)$

$\begin{array}{lllll}2 / \text { PARINT/ } X(15) & , X T(15) & \text {,DIRIN(15) } & \text {,MAXINT } & \text {,NPAR } \\ 3 / \text { PAREXT/ U(30) } & \text {,NAM(30) } & \text {,WERR(30) } & \text {,MAXEXT } & \text {,NU }\end{array}$

4/LIMITS/ $\operatorname{ALIM}(30), \operatorname{BLIM}(30), \operatorname{LCODE}(30), \operatorname{LCORSP}(30), \operatorname{LIMSET}$

5/VARIAN/ V $(15,15)$

7/FIX / IPFIX(15)

, XS (15)

,XTS(15)

,WTS(15) ,NPFIX

C/CASC/ JH, JL, Y(16)

F/DERIVA/ G(30)

,G2(30)

G/SIMVEC/ P(15,16) ".PSTAR(15) ,PSTST(15) ,PBAR(15) ,PRHO(15)

J/VARIAT/ VT $(15,15)$

$6 /$ UNIT / ISYSRD

8/TITLE / TITLE(13)

9/CONVER/ EPSI ,APSI

A/CARD / CWORD

B/MINIMA/ AMIN

, ISYSWR ,ISYSPU

,DATE(2) , ISW(7)

,VTEST

,NSTEPQ

. CWORD2

,CWORD3

, UP

, NEWMIN

, CNAME (25)

CNAM2 (25)

\section{, NBLOCK}

, NFCN

,WORD7 (7)

, ITAUR

, CNAM3 (25)

COMMON /MATCH2/ AA(30), BB(30), KBTS, SMINCAL, 1 DWORD (10), DWORD2 (10), DWORD3 (10), DWORD7 $(7,10), \operatorname{MINCOM}$ LOGICAL SMINCAL

DIMENSION WORD8(8), GF (30)

EQUIVALENCE (WORD8 (1), CWORD3)

DATA AGOOD, ABAD, ANONE/4HGOOD, 4H BAD, 4HNONE /

IKARD $=0$

50 IKARD $=$ IKARD +1

CWORD $=$ DWORD $($ IKARD $)$

CWORD2=DWORD2 (IKARD)

CWORD3=DWORD3 (IKARD)

DO $51 \mathrm{I}=1,7$

$51 \operatorname{WORD7}(\mathrm{I})=\operatorname{DWORD7}(\mathrm{I}, \mathrm{IKARD})$

$\mathrm{K}=1$

DO $60 \mathrm{I}=1,7$

IF (WORD7(I)) $58,60,58$

$58 \mathrm{~K}=\mathrm{I}+1$

60 CONTINUE

IF (CWORD .EQ. CNAME(5)) $K=2$

WRITE (ISYSWR, 5001) IKARD, CWORD, CWORD2, (WORD8 (I), I=1,K)

WRITE (ISYSWR, 5004)

NFCNMX $=$ WORD7 $(1)+0.5$

IF (NFCNMX .LE. O) NFCNMX $=1000$

EPSI $=$ WORD7 (2)

IF (EPSI .LE. 0.) EPSI $=0.1 *$ UP

NEWMIN $=\theta$

ITAUR $=0$

$\operatorname{ISW}(1)=0$

DO $80 I=1$, NNAME

IF (CWORD . EQ. CNAME(I)) GO TO 90

80 CONTINUE

WRITE (ISYSWR, 5006)

GO TO 50

90 Go To $(100,200,300,400,500,600,700,1100,900,1000,1100,1200,1300$,

* $1400,1500,1600,1700,1800,1402,2000,2100,2200,2300)$. I
COMAND 2

COMAND 3

COMAND 4

COMAND 5

COMAND 6

COMMONU 2

COMMONU 4

COMMONU 5

COMMONU 6

COMMONU 7

COMMONU 8

COMMONU 9

COMMONU 10

COMMONU 11

COMMONU 12

COMMONU 13

COMMONU 27

COMMONU 28

COMMONU 29

COMMONU 30

COMMONU 31

COMMONU 32

MATCH4 2

$\mathrm{MATCH} 4$

MATCH4 4

COMAND 10

COMAND $\quad 15$

COMAND $\quad 16$

COMAND 17

COMAND 18

COMAND 19

COMAND $\quad 20$

COMAND 21

COMAND 22

COMAND 23

COMAND 24

COMAND 25

COMAND 26

COMAND 27

COMAND 28

COMAND 29

COMAND 30

COMAND 31

COMAND 32

COMAND 34

COMAND 39

COMAND 40

COMAND $\quad 41$

COMAND 42

COMAND 43

COMAND 44

COMAND 45

COMAND 46

COMAND 47

COMAND $\quad 48$

COMAND $\quad 49$

COMAND 50 
60

65

70

75

80

85

90

95

100

105

110
C

95 WRITE (ISYSWR, 5011)

ITAUR $=0$

EPSI $=0.1 * U P$

C

$100 \mathrm{NF}=\mathrm{NFCN}$

CALL SIMPLX

IF (ISW(1) .GE. 1) GO TO 50

NFCNMX $=$ NFCNMX + NF - NFCN

150 VTEST $=0.04$

GO TO 460

C

200 CALL SEEK

GO TO 50

C

300 CALL SIMPLX

GO TO 50

C

400 VTEST $=$ WORD7 $(3)$

IF (VTEST . LE. 0.0 ) VTEST $=0.01$

$460 \mathrm{NF}=\mathrm{NFCN}$

APSI = EPSI

CALL MIGRAD

IF (ISW(2) .GT. 2) GO TO 50

IF (ISW(1) .EQ. 1) GO TO 50

NFCNMX $=$ NFCNMX $+N F-N F C N$

$\mathrm{NF}=\mathrm{NFCN}$

CALL SIMPLX

IF (ISW(1) .EQ. 1) GO TO 50

NFCNMX $=$ NFCNMX + NF - NFCN

CALL MIGRAD

GO TO 50

C

500 IF (ISW(2) . LT. 1) GO TO 550

EPSI $=0.1 * \mathrm{UP}$

VTEST $=0.10$

CALL MINOS

IF (NEWMIN . LT. 1) GO TO 50

GO TO 95

550 WRITE (ISYSWR, 5006)

555 WRITE (ISYSWR, 5007)

GO TO 50

C

600 CALL MPUNCH

GO TO 50

C

$700 \operatorname{ISW}(5)=\operatorname{WORD} 7(1)+0.5$

GO TO 50

C

900 IT $=$ WORD7 $(1)+0.5$

IF (IT . LE. O .OR. IT .GT. NU) GO TO 905

IF (LCORSP(IT) . LE. O) GO TO 905

CALL FIXPAR(IT, $\theta$, ILAX)

IF (ISW(2) .GT. 1) CALL MPRINT(1,AMIN)

GO TO 50

905 WRITE (ISYSWR, 5006)

WRITE (ISYSWR, 5012)
COMAND 51

COMAND 52

COMAND 53

COMAND 54

COMAND 55

COMAND 56

COMAND 57

COMAND 58

COMAND 59

COMAND 60

COMAND 61

COMAND 62

COMAND 63

COMAND 64

COMAND 65

COMAND 66

COMAND 67

COMAND 68

COMAND $\quad 69$

COMAND 70

COMAND 71

COMAND 72

COMAND 73

COMAND 74

COMAND 75

COMAND $\quad 76$

COMAND $\quad 77$

COMAND 78

COMAND 79

COMAND 80

COMAND 81

COMAND 82

COMAND 83

COMAND 84

COMAND 85

COMAND 86

COMAND 87

COMAND 88

COMAND 89

COMAND 90

COMAND 91

COMAND 92

COMAND 93

COMAND 94

COMAND 95

COMAND 96

COMAND 97

COMAND 98

COMAND 99

COMAND 100

COMAND 101

COMAND 102

COMAND 103

COMAND 104

COMAND 105

COMAND 106

COMAND 107 
c

$1000 \mathrm{IT}=$ WORD7 $(1)+0.5$

RESTORE

CALL RESTOR(IT)

GO TO 50

C

1100 IT $=$ WORD7 $(1)+0.5$

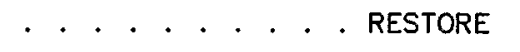

COMAND

109

COMAND

110

COMAND

111

COMAND

112

COMAND

113

IF (ISW(4) .EQ. 1 .OR. IT .GT. 0) GO TO 1150

END, EXIT

COMAND

114

CSY IFLAG $=3$

CSY WRITE (ISYSWR, 5009)

125

CSY CALL FCN(NPAR, G, F,U, IFLAG)

NFCN $=$ NFCN +1

1150 IF ( I .EQ. 11) STOP

RETURN

C

$1200 \operatorname{ISW}(3)=1$

IF (WORD7(1) .GT. 0.0) GO TO 50

DO $1230 I=1$, NU

$1230 \mathrm{GF}(\mathrm{I})=0.0$

CALL INTOEX $(X)$

CALL FCN(NPAR, GF, AMIN,U, 2)

$\mathrm{NFCN}=\mathrm{NFCN}+1$

CALL DERIVE(GF,G2)

$\operatorname{ISW}(3)=0$

CALL DERIVE(G, G2)

140

WRITE (ISYSWR, 5013)

$\operatorname{ISW}(3)=1$

DO $1250 \mathrm{I}=1$, NU

$\mathrm{LC}=\operatorname{LCORSP}(\mathrm{I})$

IF (LC .EQ. O) GO TO 1250

GRADIENT

COMAND

115

COMAND

116

COMAND 117

COMAND 118

COMAND 119

COMAND 120

COMAND 121

COMAND 122

COMAND 123

COMAND $\quad 124$

COMAND 125

COMAND 126

COMAND $\quad 127$

COMAND 128

COMAND 129

COMAND 130

COMAND 131

COMAND 132

COMAND 133

COMAND 134

COMAND 135

COMAND 136

145

$A W D=A G O O D$

IF $(A B S(G F(L C)-G(L C)) \quad . G T \cdot A B S(G 2(L C))) \quad A W D=A B A D$

COMAND

137

IF (GF(LC) .EQ. O.) AWD = ANONE

IF (AWD, NE. AGOOD) ISW(3) $=0$

WRITE( ISYSWR, 5014) I, NAM(I), GF(LC), G(LC), G2(LC), AWD

1250 CONTINUE

IF (ISW(3) .EQ. Ө) WRITE (ISYSWR, 5015)

GO TO 50

C

1300 IFLAG $=$ WORD7 $(1)$

CALL FCN

COMAND

COMAND

COMAND

COMAND

COMAND

COMAND

COMAND

COMAND

COMAND

COMAND

COMAND

COMAND

COMAND

COMAND

COMAND

COMAND

COMAND

COMAND

COMAND

COMAND

COMAND

COMAND

MATOUT

COMAND

COMAND

HESSE

COMAND

COMAND

COMAND

138

139

140

141

142

143

144

145

146

147

148

149

150

151

152

153

154

155

156

157

158

159

160

161

C

1402 CALL HESSE

CALL MPRINT (1, AMIN) 
175

180

185

190

195

200

205

210

215

220

225
1405 CALL MATOUT $(0.0,1)$

GO TO 50

C

1500 NRAPE $=$ WORD7 $(1)+0.5$

IF (NRAPE .NE. NPAR) GO TO 1550

READ (ISYSRD, 5002) ((V(I,J), I=1,NRAPE), J $=1$, NRAPE)

$\operatorname{ISW}(2)=3$

$\operatorname{CALL} \operatorname{MATOUT}(0.0,1)$

CALL MPRINT $(1$, AMIN)

GO TO 50

1550 WRITE (ISYSWR, 5006)

WRITE (ISYSWR, 5008)

NRAP2 = NRAPE $* * 2$

READ (ISYSRD, 5002) (G(1), I=1, NRAP2)

GO TO 50

C

1600 CONTINUE

IF (ISW(2) .LT. 2) GO TO 550

CALL IMPROV

IF (NEWMIN .EQ. 1) GO TO 150

GO TO 50

C

1700 CONTINUE

UP $=$ WORD7 (1)

IF (UP . LE. O.) UP $=1.0$

IF (ISW(2) .GE. 1) CALL MPRINT(1,AMIN)

GO TO 50

C

1800 WRITE (ISYSWR, 5005)

GO TO 50

C

2000 CONTINUE

IF (ISW(2) .LT. 1) GO TO 550

$\mathrm{KE} 1=\operatorname{WORD7}(1)$

$K E 2=$ WORD7 (2)

IF (KE1 .LE. O .OR, KE2 . LE. O) GO TO 905

IF (KE1 .GT. NU .OR. KE2 .GT. NU) GO TO 905

IF (LCORSP(KE1) . LE, 0 .OR. LCORSP(KE2) .LE. O) GO TO 905

NFCNMX $=1000$

CALL CONTOU

CALL MPRINT $(1$, AMIN)

IF (NEWMIN .LE. $\theta$ ) GO TO 50

GO TO 95

C

2100 CALL STAND

GO TO 50

$\mathrm{C}$

2200 CONTINUE

DO 2220 IRL $=1,7$

$K R L=$ WORD7 (IRL)

IF (KRL .EQ. 0) GO TO 50

$K R L=-I A B S(K R L)$

CALL RESTOR(KRL)

2220 CONTINUE

GO TO 50

C

2300 CONTINUE
COMAND $\quad 165$

COMAND $\quad 166$

COMAND 167

COMAND 168

COMAND 169

COMAND 170

COMAND 171

COMAND $\quad 172$

COMAND 173

COMAND 174

COMAND 175

COMAND $\quad 176$

COMAND $\quad 177$

COMAND 178

COMAND 179

COMAND 180

COMAND 181

COMAND 182

COMAND 183

COMAND 184

COMAND 185

COMAND 186

COMAND 187

COMAND 188

COMAND 189

COMAND 190

COMAND 191

COMAND 192

COMAND 193

COMAND 194

COMAND 195

COMAND 196

COMAND 197

COMAND 198

COMAND 199

COMAND 200

COMAND 201

COMAND 202

COMAND 203

COMAND 204

COMAND 205

COMAND 206

COMAND 207

COMAND 208

COMAND 209

COMAND 210

COMAND 211

COMAND 212

COMAND 213

COMAND 214

COMAND 215

COMAND 216

COMAND 217

COMAND 218

COMAND $\quad 219$

COMAND 220

COMAND 221 
GO TO 50

5000 FORMAT (2A4, A2, 7F10.0)

COMAND

5001 FORMAT (1H 10(1H*)/1H 3H*** I3,4H****,2A4,A2,7F15.5)

COMAND

5002 FORMAT (7F10.0,10X)

COMAND 224

5003 FORMAT $(13 E 10.2)$

COMAND 225

5004 FORMAT $(1 \mathrm{H} 10(1 \mathrm{H} *))$

COMAND

5005 FORMAT (1H1)

COMAND 227

5006 FORMAT ( $1 \mathrm{H}+10 \times$ 17H(COMMAND IGNORED))

COMAND 228

5007 FORMAT ( $118 \mathrm{HOTHE}$ ABOVE COMMAND CANNOT

COMAND

INCE MATRIX WAS NEITHER CALCULATED NOR SUPPLIED, OR WAS DESTROYED./

COMAND 2/)

COMAND

5008 FORMAT ( $102 \mathrm{H}$ SIZE OF COVARIANCE MATRIX TO BE READ DOES NOT CORRESP

COMAND 1OND TO NUMBER OF CURRENTLY VARIABLE PARAMETERS./)

COMAND 234

5009 FORMAT ( 28HOCALL TO FCN WITH IFLAG $=3 /$ )

COMAND 235

5010 FORMAT ( $51 \mathrm{H}$ NEW START POINT ASSUMED COVA

COVARIANCE MATRIX LOST) COMAND

5011 FORMAT ( 5OHONEW MINIMUM FOUND. GO BACK TO MINIMIZATION STEP./1H COMAND $60(1 \mathrm{H}=) / 60 \times 1 \mathrm{HV} / 60 \times 1 \mathrm{HV} / 60 \times 1 \mathrm{HV} / 57 \times 7 \mathrm{HVVVVVV} / 58 \times 5 \mathrm{HVVVVV} / 59$ COMAND

5012 FORMAT ( 91HOTHE ABOVE COMMAND CANNOT BE EXECUTED BECAUSE IT REQU COMAND 240 1 ESTS A PARAMETER THAT IS NOT VARIABLE.//)

5013 FORMAT ( 37HOCHECK OF GRADIENT CALCULATION IN FCN 12X 9HPARAME COMAND 1TER $6 X \quad 9 H G$ (IN FCN) $3 X \quad 9 H G$ (MINUIT) $4 X$ 5HERROR 5X

COMAND 2 SHAGREEMENT)

5015 FORMAT ( 54 HOMINUIT DOES NOT ACCEPT DERIVATIVE CALCULATIONS BY FC COMAND 246 1N) 
SUBROUTINE CONTOU

CONTOU

CC

$\mathrm{CC}$

CC

5

CC

COMMON

1/MINERR/ ERP(30)

2/PARINT/ $X(15)$

3/PAREXT/ U(30)

, $\operatorname{ERN}(30)$

4/LIMITS/ ALIM(30)

5/VARIAN/ $V(15,15)$

7/FIX / IPFIX(15)

C/CASC/JH, JL, Y(16)

F/DERIVA/ G(30)

$$
\text { , XT( 15) }
$$

, NAM(30)

,DIRIN(15) ,MAXINT ,NPAR

, $\operatorname{BLIM}(30)$

, LCODE $(30)$

- MAXEXT

,NU

G/SIMVEC/ $P(15,16)$

J/VARIAT/VT $(15,15)$

6/UNIT / ISYSRD

8/TITLE / TITLE(13)

9/CONVER/ EPSI ,APSI

A/CARD / CWORD

B/MINIMA/ AMIN

K/COMMND/ NNAME

COMMON /CONPTS/

DIMENSION DIG(5)

DATA CROSS, ANEWMN, DIG / 1HX, $1 \mathrm{H} * 1 \mathrm{H} 1,1 \mathrm{H} 2,1 \mathrm{H} 3,1 \mathrm{H} 4,1 \mathrm{H} 5 /$

DATA NSTMX, THMAX, MINPT, TEN, NPTDIM / 10, 0.4, 10, 100., $298 /$

ALLOW(IEXT) $=($ U(IEXT)-ALIM(IEXT) $) *(B L I M($ IEXT $)-U($ IEXT $))$

$\mathrm{KE1}=$ WORD7 (1)

KE2 $=$ WORD7 (2)

NCON $=$ WORD7 (3)

IF (NCON.LE. 0 ) $\quad N C O N=2$

IF (NCON .GT , 5) NCON=5

$\operatorname{SPT}(1,1)=U(K E 1)$

$\operatorname{SPT}(2,1)=U(K E 2)$

$\operatorname{SPT}(3,1)=1 \mathrm{H}$

NSPT $=1$

NEWMIN $=0$

$\mathrm{KI} 1=\operatorname{LCORSP}(\mathrm{KE} 1)$

$K I 2=\operatorname{LCORSP}(K E 2)$

IF (ISW(2) . LT. 2) GO TO 40

CALL UCOPY $(V, V T, M A X I N T * * 2)$

CALL VERMIN(VT, MAXINT, MAXINT, NPAR, IERR)

IF (IERR . LE. O) GO TO 60

C MATRIX NOT POSITIVE-DEFINITE

WRITE (ISYSWR, 35)

35 FORMAT ( 40 HOCOVARIANCE MATRIX NOT POSITIVE-DEFINITE)

40 WRITE (ISYSWR, 42)

42 FORMAT ( 26HOONLY DIAGONAL ERRORS USED)

$A 11=V(K I 1, K I 1) * U P$

$A 22=V(K I 2, K I 2) * U P$

$\mathrm{A} 12=0.0$

GO TO 75

C INVERT $2 \times 2$ SUBMATRIX BY HAND

60 CONTINUE

$D E T R=V T(K I 1, K I 1) * V T(K I 2, K I 2)-V T(K I 1, K I 2) * * 2$

IF (DETR . LE, 0.0$)$ GO TO 40

DETR = UP/DETR
CONTOU

CONTOU 4

CONTOU 5

CONTOU 6

COMMONU 2

COMMONU 4

COMMONU 5

COMMONU 6

COMMONU 7

COMMONU 8

COMMONU 9

COMMONU 10

COMMONU 11

PRHO (15) COMMONU 12

COMMONU 13

COMMONU 27

COMMONU 28

COMMONU 29

COMMONU 30

COMMONU $\quad 31$

COMMONU 32

CONTOU 8

CONTOU 9

CONTOU 10

CONTOU 11

CONTOU 12

CONTOU 13

CONTOU 14

CONTOU 15

CONTOU 16

CONTOU 17

CONTOU 18

CONTOU 19

CONTOU 20

CONTOU 21

CONTOU 22

CONTOU 23

CONTOU 24

CONTOU 25

CONTOU 26

CONTOU 27

CONTOU 28

CONTOU 29

CONTOU 30

CONTOU 31

CONTOU 32

CONTOU 33

CONTOU $\quad 34$

CONTOU 35

CONTOU 36

CONTOU 37

CONTOU 38

CONTOU 39

CONTOU 40

CONTOU $\quad 41$

CONTOU 42 
EXIN2 $=1.0$

GO TO 125

120 CONTINUE

EXIN2 $=0.5 *(B L I M(K E 2)-A L I M(K E 2)) * \operatorname{COS}(X(K I 2))$

$A 11=A 11 *$ EXIN1**2

$A 22=A 22 *$ EXIN $2 * * 2$

$A 12=A 12 * A B S(E X I N 1 * E X I N 2)$

CIRCUM $=4.0 *$ SQRT $(A 11+A 22)$

IFLAG $=4$

- calculate the contours

CONTOU

CONTOU

CONTOU

CONTOU

CONTOU

CONTOU

CONTOU

CONTOU

DO 800 ICON $=1$, NCON

CONTOU

$S Y M=\operatorname{DIG}(I C O N)$

ANP $=50-5 *(N C O N-I C O N)$

$D C A=6.2832 / A N P$

RAVE = FLOAT (ICON) * CIRCUM / ANP

CONTOU

CONTOU

CONTOU

CONTOU

RMAXAB $=3.0 *$ RAVE

CONTOU

CONTOU

DIFF $=$ UP* FLOAT $(I C O N * * 2)$

$A I M=A M I N+D I F F$

TOLERN $=0.05 *$ DIFF

NPTMX $=200$

IF (NPTDIM-NSPT .LT. NPTMX) NPTMX = NPTDIM-NSPT

CONTOU

CONTOU

CONTOU

CONTOU

CONTOU

IF (NPTMX .LT. 1) GO TO 810

WRITE (ISYSWR, 1000) KE1, KE2, SYM,DIFF

CONTOU

CONTOU - SET UP FIRST POINT LONG ARM CONTOU 


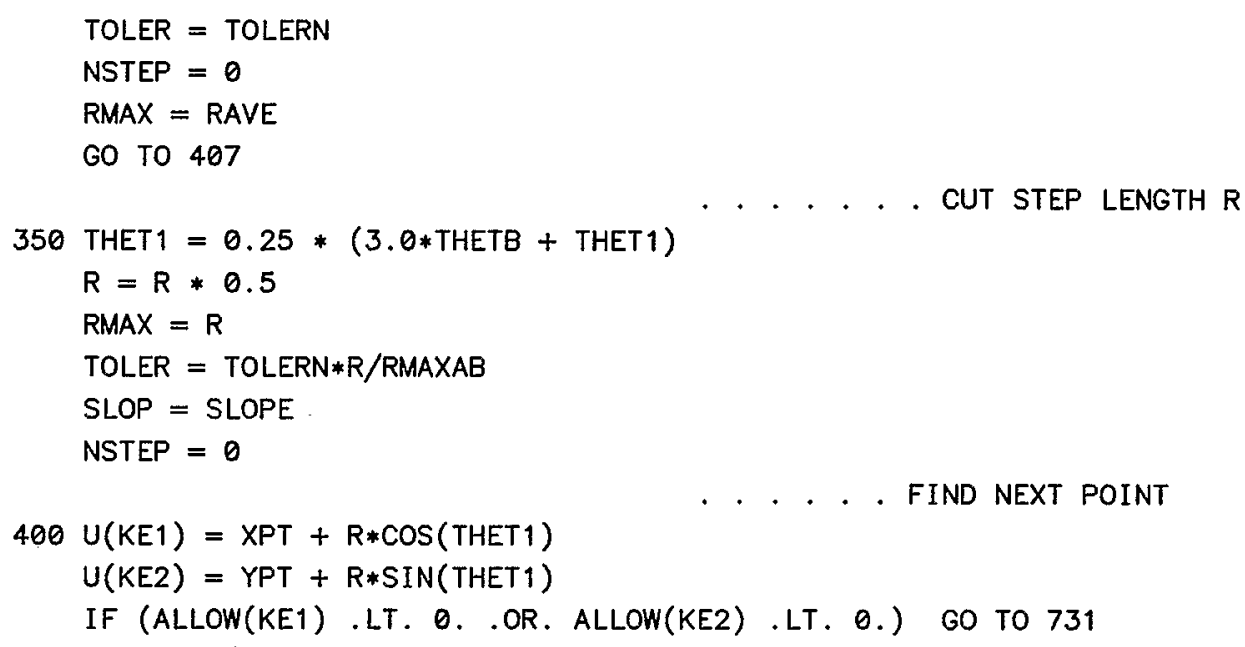

CONTOU 100

CONTOU $\quad 101$

CONTOU 102

CONTOU 103

CONTOU 104

CONTOU 105

CONTOU 106

CONTOU 107

CONTOU 108

CONTOU 109

CONTOU 110

CONTOU 111

CONTOU 112

CONTOU 113

CONTOU 114

CONTOU $\quad 115$

CONTOU $\quad 116$

CONTOU 117

- CHECK IF TWO TRIES ARE NEEDED CONTOU 118

C

IF (F2 . LE. AMIN) GO TO 741

CONTOU

$\mathrm{F} 1=\mathrm{F} 2$

CONTOU

119

THET2 = THET1

$A F A=A B S(F 2-A I M)$

IF (AFA .GT, 10.*TOLER . AND, R .GT. RMIN) GO TO 350

CONTOU

120

IF (AFA . LT. 0.2*TOLER) GO TO 420

CONTOU

CONTOU

CONTOU

CONTOU

CONTOU

CONTOU

$\begin{array}{llll}\text { IF (ABS(DTHET) } & . G T . & \text { THMAX) } & \text { DTHET }=S I G N(T H M A X, D T H E T) \\ \text { IF (ABS(DTHET) } & . \text { LT. THMIN) } & \text { DTHET }=\text { SIGN(THMIN,DTHET) }\end{array}$

C - SUBSEQUENT TRIALS FOR NEXT PT 410 CONTINUE

CONTOU

CONTOU

CONTOU

CONTOU

CONTOU

CONTOU

CONTOU

CONTOU

CONTOU

CONTOU

CONTOU

CONTOU

CONTOU

CONTOU

CONTOU

CONTOU

CONTOU

CONTOU

CONTOU

CONTOU

CONTOU

CONTOU

CONTOU

CONTOU

CONTOU

CONTOU

CONTOU

CONTOU 
C

GO TO 410

. . ACCEPT NEW POINT

CONTOU

157

$430 \times P T=X P T+R * \cos ($ THETA $)$

CONTOU

158

$Y P T=Y P T+R * S I N(T H E T A)$

CONTOU

159

$U(K E 1)=X P T$

$U(K E 2)=$ YPT

CONTOU

160

CONTOU 161

IF (ALLOW(KE1) . LT. O. .OR. ALLOW(KE2) . LT. 0.) GO TO 731

CONTOU

162

THETAB $=$ ABS (THETA-THETB)

NSTEP $=0$

440 IF (NPT .GT. 1) GO TO 445

THBEG $=$ THETA

XBEG $=$ XPT

YBEG $=$ YPT

445 CONTINUE

$\mathrm{NPT}=\mathrm{NPT}+1$

NSPT $=$ NSPT +1

$\operatorname{SPT}(1, \mathrm{NSPT})=\mathrm{XPT}$

$\operatorname{SPT}(2$, NSPT $)=$ YPT

190

$\operatorname{SPT}(3, N S P T)=S Y M$

$X P M=X P T-X M I N$

$Y P M=Y P T-Y M I N$

IF (ISW(5) .LT. 2) GO TO 450

THDEG $=$ THETA*180. / 3.14159265

195

THDEG $=A M O D(T H D E G, 360.0)$

WRITE (ISYSWR, 901) NPT , XPT, YPT, XPM, YPM, DELF, R, THDEG, NFCN

450 CONTINUE

c

IF (NPT . LT. MINPT) GO TO 500

200

205

210

215

220

C 500 IF (NPT .GE. NPTMX) GO TO 761

RINV $=$ THETAB**2/(R*DCA**2)

$\mathrm{R} 2=\mathrm{RMAX}$

GO TO 540

$522 \mathrm{R} 2=1.0 / \mathrm{RINV}$

IF (R2 . LT. RMIN) R2 = RMIN

540 CHANG $=R 2 * R I N V * D C A$

THET 1 = THETA + CHANG

THETB = THETA

$\mathrm{R}=\mathrm{R} 2$

$\operatorname{RMAX}=\operatorname{AMIN1}(2.0 * \mathrm{R}, \operatorname{RMAXAB})$

TOLER $=$ TOLERN * R/RMAXAB

$S L O P=S L O P E$

GO TO 400

C

600 WRITE (ISYSWR, 605) SYM, NPT GO TO 790

C
IF ((YPT-YBEG)**2+(XPT-XBEG)**2.GT. R**2) GO TO 500

IF (COS(THETA-THBEG) .GT. 0.5) GO TO 600

ESTIMATE NEW POINT

IF (RINV .GT. 1.0/RMAX) GO TO 522

IF (CHANG .GT. THMAX) CHANG = THMAX

IF (THETB .GT. THETA) CHANG $=-$ CHANG

- CONTOUR COMPLETED NORMALLY

605 FORMAT ( 9HOCONTOUR A3, 18H IS COMPLETED WITHI5, 7H POINTS//)

731 WRITE (ISYSWR, 735) U(KE1), U(KE2)

. . ERROR RETURNS

735 FORMAT ( 34 HOCONTOUR LEAVING ALLOWED REGION AT2E16.7)

GO TO 780

741 WRITE (ISYSWR, 745)
CONTOU 163

CONTOU 164

CONTOU 165

CONTOU 166

CONTOU 167

CONTOU 168

CONTOU 169

CONTOU 170

CONTOU 171

CONTOU 172

CONTOU 173

CONTOU 174

CONTOU 175

CONTOU 176

CONTOU $\quad 177$

CONTOU 178

CONTOU 179

CONTOU 180

CONTOU 181

CONTOU 182

CONTOU 183

CONTOU 184

CONTOU 185

CONTOU 186

CONTOU 187

CONTOU 188

CONTOU 189

CONTOU 190

CONTOU 191

CONTOU 192

CONTOU 193

CONTOU 194

CONTOU 195

CONTOU 196

CONTOU 197

CONTOU 198

CONTOU 199

CONTOU 200

CONTOU 201

CONTOU 202

CONTOU 203

CONTOU 204

CONTOU 205

CONTOU 206

CONTOU 207

CONTOU 208

CONTOU 209

CONTOU 210

CONTOU 211

CONTOU 212

CONTOU 213 
230

235

240

245

250

255

260
745 FORMAT ( 38HONEW ABSOLUTE MINIMUM FOUND BY CONTOUR/)

NEWMIN $=1$

NSPT $=$ NSPT +1

$\operatorname{SPT}(1, N S P T)=U(K E 1)$

$\operatorname{SPT}(2, N S P T)=U(K E 2)$

$\operatorname{SPT}(3$, NSPT $)=$ ANEWMN

$A M I N=F 2$

CALL EXTOIN $(X)$

CALL MPRINT $(\theta, A M I N)$

GO TO 810

751 WRITE (ISYSWR, 755)

755 FORMAT ( $39 H O T O O$ MANY ITERATIONS TO FIND NEXT POINT) GO TO 780

761 WRITE (ISYSWR, 765)

765 FORMAT ( 45HOMEMORY OVERFLOW. TOO MANY POINTS IN CONTOUR)

780 WRITE (ISYSWR, 1001) NPT

$790 \mathrm{U}(\mathrm{KE1})=\mathrm{XMIN}$

$U(K E 2)=Y M I N$

800 CONTINUE

C

810 CONTINUE

WRITE (ISYSWR, 1002) KE1, KE2,UP, KE2

CALL PLTCON(NSPT, SPT)

WRITE（ISYSWR, 1003) KE1

RETURN

900 FORMAT ( $8 X \quad 2 H P T$ 9X 1HX 13X 1HY 10X

1-YMIN 7X 8HACCURACY $4 X$ 10HSEPARATION $5 X$ 2LLS)

901 FORMAT (I10,2F14.7, 2X,2F12.7,F13.6,2X, F12.7, F10.1, I8)

$6 H X-X M I N 6 X$

5HTHETA $3 X$

8HCONTOUR A3,

1000 FORMAT (15HOPARAMETERS NO.I4, 4H ANDI4,10X

$1 H \quad F=$ FMIN +F11.6)

1001 FORMAT ( $55 \mathrm{H}$ CONTOUR NOT COMPLETED BECAUSE OF ABOVE ERROR CONDITI $10 \mathrm{~N} / 1 \times 54(1 \mathrm{H} *), \mathrm{I} 5,43 \mathrm{H}$ POINTS ON THE CONTOUR HAVE BEEN DETERMINED/ 2/)

1002 FORMAT(36H1 FUNCTION CONTOURS FOR PARAMETERS 2I4, 20X,

$120 \mathrm{H}$ CONTOUR $1=$ FMIN + F10.5/12HO PARAMETER I3)

1003 FORMAT (25Ht

END
CONTOU

CONTOU

CONTOU

CONTOU

CONTOU

CONTOU

CONTOU

CONTOU

CONTOU

CONTOU

CONTOU

CONTOU

CONTOU

CONTOU

CONTOU

CONTOU

CONTOU

CONTOU

CONTOU

CONTOU

CONTOU

CONTOU

CONTOU

CONTOU

CONTOU

GHY CONTOU

5HCA CONTOU

CONTOU

CONTOU

2 CONTOU

CONTOU

CONTOU

CONTOU

CONTOU

CONTOU

CONTOU

CONTOU

CONTOU
214

215

216

217

218

219

220

221

222

223

224

225

226

227

228

229

230

231

232

233

234

235

236

237

238

239

240

241

242

243

244

245

246

247

248

249

250

251 
1

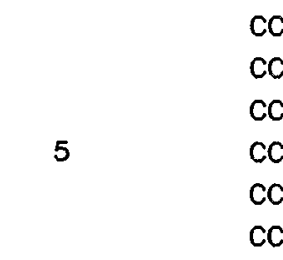

10

C

C

C
SUBROUTINE DERIVE(GG,GG2)

CALCULATES THE FIRST DERIVATIVES OF FCN (GG), EITHER BY FINITE DIFFERENCES OR BY TRANSFORMING THE USERSUPPLIED DERIVATIVES TO INTERNAL COORDINATES, ACCORDING TO WHETHER ISW(3) IS ZERO OR ONE. IF ISW(3) $=0$, AN ERROR ESTIMATE GG2 IS AVAILABLE COMMON

$1 /$ MINERR/ ERP (30) , ERN (30)

2/PARINT/ $\times(15) \quad, X T(15) \quad$,DIRIN(15) ,MAXINT ,NPAR

3/PAREXT/ U(30) ,NAM(30) ,WERR(30) ,MAXEXT ,NU

4/LIMITS/ $\operatorname{ALIM}(30), \operatorname{BLIM}(30), \operatorname{LCODE}(30), \operatorname{LCORSP}(30), \operatorname{LIMSET}$

5/VARIAN/ V(15,15)

7/FIX / IPFIX(15)

C/CASC/ JH, JL, Y(16)

,$x S(15)$

, XTS(15)

.WTS(15) ,NPFIX

F/DERIVA/ G(30)

, $\mathrm{G} 2(30)$

G/SIMVEC/ $P(15,16)$

J/VARIAT/ VT $(15,15)$

6/UNIT / ISYSRD

PSTAR (15)

,PSTST (15) ,PBAR( 15)

,PRHO(15)

8/TITLE / TITLE(13)

, ISYSWR ,ISYSPU

9/CONVER/ EPSI, APSI

A/CARD / CWORD

B/MINIMA/ AMIN

,DATE(2) , ISW(7)

,VTEST ,NSTEPQ

, CWORD2

, CWORD3

, NBLOCK

, UP

, NEWMIN

, NFCN

K/COMMND/NNAME, $\operatorname{CNAME(25)}$

, CNAM2 (25), CNAM3 (25)

IF (ISW(3) .EQ. 1) GO TO 100

IFLAG $=4$

DO $46 \quad I=1, N P A R$

$\mathrm{EPS}=0.1 * \operatorname{ABS}(\mathrm{DIRIN}(\mathrm{I}))$

IF (ISW(2) .GE. 1) EPS $=$ EPS + $0.005 * \operatorname{SQRT}(V(I, I) * U P)$

IF (EPS . LT. 1.0E-8*ABS $(X(I))$ ) EPS $=1.0 E-8 * X(I)$

$X T F=X(I)$

$X(I)=X T F+E P S$

CALL INTOEX(X)

CALL FCN(NPAR,GY, FS1,U, IFLAG)

$\mathrm{NFCN}=\mathrm{NFCN}+1$

$X(I)=X T F-$ EPS

CALL INTOEX(X)

CALL FCN(NPAR, GY, FS2, U, IFLAG)

$N F C N=N F C N+1$

$G G(I)=(F S 1-F S 2) /(2.0 * E P S)$

$G G 2(I)=(F S 1+F S 2-2.0 * A M I N) /(2.0 * E P S)$

$X(I)=X T F$

46 CONTINUE

CALL INTOEX $(X)$

GO TO 200

100 DO $150 \mathrm{I}=1$, NU

DERIVATIVES CALC BY FCN

$L C=L C O R S P(I)$

IF (LC . LT. 1) GO TO 150

IF (LCODE (I) .GT . 1) GO TO 120

$G G(L C)=G G(I)$

GO TO 150

$120 \mathrm{DD}=(\mathrm{BLIM}(\mathrm{I})-\mathrm{ALIM}(\mathrm{I})) * 0.5 * \cos (X(\mathrm{LC}))$

$G G(L C)=G G(I) * D D$
DERIVE 2

DERIVE 3

DERIVE 4

DERIVE 5

DERIVE 6

DERIVE 7

DERIVE 8

COMMONU 2

COMMONU 4

COMMONU 5

COMMONU 6

COMMONU 7

COMMONU 8

COMMONU 9

COMMONU 10

COMMONU 11

COMMONU 12

COMMONU 13

COMMONU 27

COMMONU 28

COMMONU 29

COMMONU 30

COMMONU 31

COMMONU 32

DERIVE 11

DERIVE 16

DERIVE 17

DERIVE 18

DERIVE 19

DERIVE 20

DERIVE 21

DERIVE 22

DERIVE 23

DERIVE 24

DERIVE 25

DERIVE 26

DERIVE 27

DERIVE 28

DERIVE 29

DERIVE 30

DERIVE 31

DERIVE 32

DERIVE 33

DERIVE 34

DERIVE 35

DERIVE 36

DERIVE 37

DERIVE 38

DERIVE 39

DERIVE 40

DERIVE 41

DERIVE 42

DERIVE 43

DERIVE 44

DERIVE 45

DERIVE 46

DERIVE 47 
150 CONT INUE

200 RETURN

60
DERIVE $\quad 48$

DERIVE 49

DERIVE 50 
1

5

10

15

20

25

30
SUBROUTINE EXTOIN(PINT)

TRANSFORMS THE EXTERNAL PARAMETER -VALUES $X$ TO INTERNAL

VALUES IN THE DENSE ARRAY PINT. FUNCTION PINTF IS USED.

$\mathrm{CC}$

$\mathrm{CC}$

$$
\text { COMMON }
$$

1/MINERR/ ERP(30) , ERN(30)

2/PARINT/ $\times(15) \quad$,XT(15) ,DIRIN(15) ,MAXINT $\quad$,NPAR

3/PAREXT/ U(30)

, NAM(30)

, WERR (30)

- MAXEXT

,$N U$

4/LIMITS/ ALIM(30)

, $B \operatorname{LIM}(30)$

, LCODE (30)

, LCORSP (30) , LIMSET

7/FIX / IPFIX(15)

,$x S(15)$

, $\operatorname{XTS}(15)$

,WTS(15) ,NPF IX

C/CASC/ JH, JL, Y(16)

F/DERIVA/ $G(30)$

, $\mathrm{G} 2(30)$

G/SIMVEC/ $P(15,16)$

J/VARIAT/VT $(15,15)$

6/UNIT / ISYSRD

,PSTAR( 15$)$

,PSTST ( 15)

,PBAR (15)

, ISYSWR , ISYSPU

8/TITLE / TITLE(13)

,DATE(2)

, ISW(7)

, NSTEPQ

VTEST

A/CARD / CWORD

,CWORD2

, CWORD3

, NBLOCK

, NFCN

B/MINIMA/ AMIN

, UP

, NEWMIN

. WORD7(7)

, ITAUR

. CNAME (25)

, CNAM2 (25)

, CNAM3 (25)

DIMENSION PINT(2)

LIMSET $=0$

DO $100 \quad \mathrm{I}=1, \mathrm{NU}$

$J=\operatorname{LCORSP}(\mathrm{I})$

IF ( J ) $100,100,50$

$50 \operatorname{PINT}(\mathrm{J})=\operatorname{PINTF}(U(\mathrm{I}), \mathrm{I})$

100 CONTINUE

RETURN

END

$\begin{array}{lr}\text { EXTOIN } & 2 \\ \text { EXTOIN } & 3 \\ \text { EXTOIN } & 4 \\ \text { EXTOIN } & 5 \\ \text { COMMONU } & 2 \\ \text { COMMONU } & 4 \\ \text { COMMONU } & 5 \\ \text { COMMONU } & 6 \\ \text { COMMONU } & 7 \\ \text { COMMONU } & 8 \\ \text { COMMONU } & 9 \\ \text { COMMONU } & 10 \\ \text { COMMONU } & 11 \\ \text { COMMONU } & 12 \\ \text { COMMONU } & 13 \\ \text { COMMONU } & 27 \\ \text { COMMONU } & 28 \\ \text { COMMONU } & 29 \\ \text { COMMONU } & 30 \\ \text { COMMONU } & 31 \\ \text { COMMONU } & 32 \\ \text { EXTOIN } & 7 \\ \text { EXTOIN } & 8 \\ \text { EXTOIN } & 9 \\ \text { EXTOIN } & 10 \\ \text { EXTOIN } & 11 \\ \text { EXTOIN } & 12 \\ \text { EXTOIN } & 13 \\ \text { EXTOIN } & 14 \\ \text { EXTOIN } & 15 \\ & \\ \end{array}$


10

15
SUBROUTINE FIXPAR( I2, KODE, ILAX) REMOVES PARAMETER I2 FROM THE INTERNAL (VARIABLE) PARAMETER LIST, AND ARRANGES THE REST OF THE LIST TO FILL THE HOLE. IF $K O D E=0$, I 2 IS AN EXTERNAL NUMBER, OTHERWISE INTERNAL. ILAX IS RETURNED AS THE EXTERNAL NUMBER OF THE PARAMETER.

$\mathrm{CC}$ $\mathrm{CC}$

\section{COMMON}

\begin{tabular}{|c|c|c|c|c|}
\hline 1/MINERR/ ERP(30) &,$E R N(30)$ & & & \\
\hline 2/PARINT/ $\times(15)$ &,$x T(15)$ & ,DIRIN(15) & , MAXINT & , NPAR \\
\hline 3/PAREXT/ U(30) & . $\operatorname{NAM}(30)$ & , WERR (30) & , MAXEXT & , NU \\
\hline 4/LIMITS/ ALIM(30) & , BLIM(30) &, $\operatorname{LCODE}(30)$ &, $\operatorname{LCORSP}(30)$ & , LIMSET \\
\hline \multicolumn{5}{|l|}{$5 /$ VARIAN/ V $(15,15)$} \\
\hline $7 / F I X \quad /$ IPFIX(15) & $X S(15)$ &,$X T S(15)$ & ,WTS(15) & , NPF IX \\
\hline \multicolumn{5}{|l|}{ C/CASC/ JH, JL, Y(16) } \\
\hline F/DERIVA/ G(30) &, $\mathrm{G} 2(30)$ & & & \\
\hline G/SIMVEC/ $P(15,16)$ & ,PSTAR (15) & ,PSTST (15) &, $\operatorname{PBAR}(15)$ & , PRHO ( 15 \\
\hline \multicolumn{5}{|l|}{ J/VARIAT/ VT $(15,15)$} \\
\hline 6/UNIT / ISYSRD & , ISYSWR & , ISYSPU & & \\
\hline 8/TITLE / TITLE(13) & .DATE(2) & $\operatorname{ISW}(7)$ & , NBLOCK & \\
\hline 9/CONVER/ EPSI ,APSI & , VTEST & , NSTEPQ & NFCN & , NFCNMX \\
\hline A/CARD / CWORD & ,CWORD2 & , CWORD3 & WORD7(7) & \\
\hline B/MINIMA/ AMIN & ,UP & , NEWMIN & , ITAUR & , SIGMA \\
\hline K/COMMND/ NNAME & . CNAME (25) & , CNAM2 (25) & . CNAM3 (25) & \\
\hline \multicolumn{5}{|c|}{ DIMENSION V1(2), YY(15) } \\
\hline \multicolumn{5}{|c|}{ EQUIVALENCE $(V(1,1), V 1(1))$} \\
\hline IF (KODE) $250,50,150$ & & & & \\
\hline
\end{tabular}

C

$50 I=I 2$

IF (I .GT. NU) GO TO 70

EXT PARAM No. SPECIFIED

IF (I .LT. 1) GO TO 70 C

60 IF $(\operatorname{LCORSP}(\mathrm{I})) \quad 70,70,80$

70 I LAX $=0$

WRITE (ISYSWR, 500) I

GO TO 300

$80 L C=\operatorname{LCORSP}(I)$

$I T=L C$

$\operatorname{LCORSP}(I)=0$

$I L A X=I$

NPAR $=$ NPAR -1

NPFIX $=$ NPFIX +1

$\operatorname{IPFIX}(\mathrm{NPFIX})=\mathrm{I}$

$X S(N P F I X)=X(L C)$

$X T S(N P F I X)=X T(L C)$

ERROR RETURN PARAM ALREADY FIXED

$\mathrm{EPS}=\operatorname{ABS}(\mathrm{DIRIN}(L C)) * 10$.

IF (ISW(2) .GE, 1) EPS = EPS + SQRT (ABS $(V(L C, L C)) * U P)$

IF (EPS . LT. 1.0E-10*ABS $(X(L C))) \quad$ EPS $=1.0 \mathrm{E}-8 * X(L C)$

WTS $($ NPFIX) $=$ EPS $* 0.1$

DO $100 \quad I K=I, N U$

IF (LCORSP(IK)) $100,100,85$

$85 \operatorname{LC}=\operatorname{LCORSP}(\mathrm{IK})-1$

$\operatorname{LCORSP}(\mathrm{IK})=\mathrm{LC}$

$X(L C)=X(L C+1)$

$X T(L C)=X T(L C+1)$

$\operatorname{DIRIN}(L C)=\operatorname{DIRIN}(L C+1)$

100 CONTINUE

IF (ISW(2) .GT. 1) GO TO 250
FIXPAR 2

FIXPAR 3

FIXPAR 4

FIXPAR 5

FIXPAR 6

FIXPAR 7

COMMONU 2

COMMONU 4

COMMONU 5

COMMONU 6

COMMONU 7

COMMONU 8

COMMONU 9

COMMONU 10

COMMONU 11

COMMONU 12

COMMONU 13

COMMONU 27

COMMONU 28

COMMONU 29

COMMONU 30

COMMONU 31

COMMONU 32

FIXPAR 10

FIXPAR 15

FIXPAR 16

FIXPAR 17

FIXPAR 18

FIXPAR 19

FIXPAR 20

FIXPAR 21

FIXPAR 22

FIXPAR 23

FIXPAR 24

FIXPAR 25

FIXPAR 26

FIXPAR 27

FIXPAR 28

FIXPAR 29

FIXPAR 30

FIXPAR 31

FIXPAR 32

FIXPAR 33

FIXPAR 34

FIXPAR 35

FIXPAR 36

FIXPAR $\quad 37$

FIXPAR 38

FIXPAR $\quad 39$

FIXPAR $\quad 40$

FIXPAR $\quad 41$

FIXPAR $\quad 42$

FIXPAR $\quad 43$

FIXPAR 44

FIXPAR $\quad 45$

FIXPAR $\quad 46$

FIXPAR $\quad 47$ 
60

70

75

80

85

90

95

100
$\operatorname{ISW}(2)=0$

GO TO 300

C 150 CONTINUE

DO $200 I Q=1$, NU

IF (LCORSP(IQ) .NE. I2) GO TO 200

$I=I Q$

GO TO 60

200 CONTINUE

GO TO 70

C REMOVE ONE ROW AND ONE COLUMN FROM VARIANCE MATRIX $250 \mathrm{KON}=0$

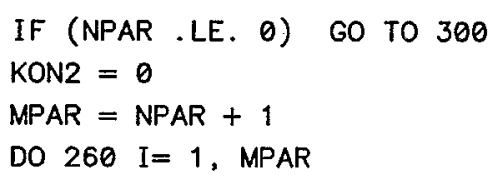

FIXPAR $\quad 48$

FIXPAR $\quad 49$

FIXPAR $\quad 50$

FIXPAR $\quad 51$

FIXPAR $\quad 52$

FIXPAR $\quad 53$

FIXPAR 54

FIXPAR 55

FIXPAR $\quad 56$

FIXPAR $\quad 57$

FIXPAR $\quad 58$

FIXPAR $\quad 59$

FIXPAR $\quad 60$

FIXPAR $\quad 61$

FIXPAR $\quad 62$

FIXPAR 63

FIXPAR $\quad 64$

FIXPAR $\quad 65$

FIXPAR $\quad 66$

FIXPAR $\quad 67$

FIXPAR $\quad 68$

FIXPAR $\quad 69$

FIXPAR $\quad 70$

FIXPAR $\quad 71$

FIXPAR $\quad 72$

FIXPAR $\quad 73$

FIXPAR $\quad 74$

FIXPAR $\quad 75$

FIXPAR $\quad 76$

FIXPAR $\quad 77$

FIXPAR $\quad 78$

FIXPAR $\quad 79$

FIXPAR $\quad 80$

FIXPAR $\quad 81$

FIXPAR $\quad 82$

FIXPAR $\quad 83$

FIXPAR 84

FIXPAR $\quad 85$

501 FORMAT ( 72HOCOVARIANCE MATRIX WAS ILL-CONDITIONED AND HAS BEEN D FIXPAR 88 1ESTROYED BY FIXPAR./) $\quad$ FIXPAR 89 END 
1

$\mathrm{CC}$

$\mathrm{CC}$

$\mathrm{CC}$

5
FUNCTION FTIME(BIDON)

GIVES THE ELAPSED JOB TIME IN FLOATING-POINT MINUTES

BY CALLING SOME INSTALLATION-DEPENDENT SUBROUTINE

CALL SECOND(SEC)

FTIME $=$ SEC

RETURN

END
FIXPAR $\quad 91$

FIXPAR $\quad 92$

FIXPAR 93

FIXPAR 94

FIXPAR 95

FIXPAR $\quad 96$

FIXPAR $\quad 97$

FIXPAR 98 
1

10

15

5

SUBROUTINE HESSE CALCULATES THE FULL SECOND-DERIVATIVE MATRIX OF FCN BY TAKING FINITE DIFFERENCES. INCLUDES SOME SAFEGUARDS AGAINST NON-POSITIVE-DEFINITE MATRICES, AND IT MAY SET

HESSE

$\mathrm{CC}$ OFF-DIAGONAL ELEMENTS TO ZERO IN ATTEMPT TO FORCE POSITIVENESS HESSE

$\mathrm{CC}$ $\mathrm{CC}$

COMMON

1/MINERR/ ERP(30) ,ERN(30)

2/PARINT / $X(15)$

3 PAREXT/U(30)

.XT(15)

, DIRIN(15)

, MAXINT

, NAM(30)

, WERR (30)

, MAXEXT

, LCODE (30)

, LCORSP (30) , LIMSET

4/LIMITS/ ALIM(30)

, $B \operatorname{LIM}(30)$

, XTS (15)

,WTS(15) ,NPFIX

7/FIX / IPFIX(15)

C/CASC/ JH, JL, Y(16)

.XS(.15)

F/DERIVA/ G(30)

, G2(30)

G/SIMVEC/ P $(15,16)$

J/VARIAT/ VT $(15,15)$

6/UNIT / ISYSRD

,PSTAR(15)

,PSTST(15) ,PBAR(15)

, ISYSWR ,ISYSPU

8/TITLE / TITLE(13)

,DATE(2)

, ISW(7)

9/CONVER/ EPSI ,APSI

A/CARD / CWORD

B/MINIMA/ AMIN

-VTEST

.CWORD2

,NSTEPQ

.CWORD3

- NEWMIN

K/COMMND/ NNAME

UP

CNAME(25), ,CNAM2(25), , CNAM3(25)

DIMENSION YY(15), GY(30)
,NBLOCK

, NFCN

,WORD7 (7)

, ITAUR . CNAM3 (25)

HESSE

COMMONU

COMMONU

COMMONU

COMMONU

COMMONU

COMMONU

COMMONU

COMMONU

COMMONU

,PRHO(15) COMMONU

COMMONU

COMMONU

COMMONU

.NFCNMX COMMONU

COMMONU

.SIGMA COMMONU

COMMONU

HESSE

DATA DFWANT, DFZERO, DFMIN, DFMAX/ $0.01,0.00000001,0.001,0.1 /$ HESSE

WRITE (ISYSWR, 500)

IFLAG $=4$

NPFN $=$ NFCN

$N P A R D=N P A R$

HESSE 16

HESSE 17

HESSE 18

HESSE $\quad 19$

HESSE 20

MDIAG $=0$

DO 100 ID $=1$, NPARD

HESSE

HESSE

HESSE

$I=I D+N P A R-N P A R D$

HESSE

$D=0.02 * A B S(D I R I N(I))$

IF (ISW(2) .GE. 1) $D=0.02 * \operatorname{SQRT}(A B S(V(I, I)) * U P)$

HESSE

IF (D .LT. 1.0E-8*ABS $(X(I))) \quad D=1.0 E-8 * A B S(X(I))$

HESSE

HESSE

HESSE

HESSE

HESSE

HESSE

HESSE

HESSE

HESSE

HESSE

HESSE

HESSE

HESSE

HESSE

HESSE

CHECK IF STEP SIZES APPROPRIATE HESSE

$\mathrm{ICYC}=\mathrm{ICYC}+1$

HESSE

IF (ICYC .GE. 4) GO TO 55

HESSE

$D F=\operatorname{AMAX1}(A B S(F S 1-A M I N), A B S(F S 2-A M I N)) / U P$

HESSE

IF (DF .GT. DFMIN) GO TO 45

HESSE

IF (DF .GT. DFZERO) GO TO 50

HESSE

$D=D * 1000$.

HESSE 
60

65

70

75

80

85

90

95

100

105

110
GO TO 40

45 IF (DF .LT. DFMAX) GO TO 55

50 CHAN $=$ SQRT (DFWANT/DF)

IF (CHAN . LT. 0.001) CHAN $=0.001$

$D=D * C H A N$

GO TO 40

55 CONTINUE
C

$G(I)=(F S 1-F S 2) /(2.0 * D)$

$G 2(I)=(F S 1+F S 2-2.0 * A M I N) / D * * 2$

$Y Y(I)=F S 1$

IF (ABS $(G(I))+\operatorname{ABS}(G 2(I)) . G T \cdot 1.0 E-30)$ GO TO 80

C

IF (ITAUR .GE. 1) GO TO 85

FIX A PARAMETER IF $G=G 2=0.0$.

$\operatorname{ISW}(2)=0$

CALL FIXPAR(I, 1, IFIX)

WRITE (ISYSWR, 460) IFIX, NAM(IFIX), G(I), G2(I)

IF (NPAR .EQ. 0 ) MDIAG $=1$

GO TO 100

80 IF (G2(I) .GT $1.0 \mathrm{E}-30)$ GO TO 90

85 MDIAG $=1$

WRITE (ISYSWR, 510) I

$90 V(I, I)=G 2(I)$

100 CONTINUE

CALL INTOEX $(X)$

IF (MDIAG .EQ. 1) GO TO 390

$\operatorname{ISW}(2)=1$

C

IF (NPAR .EQ. 1) GO TO 214

NPARM $1=$ NPAR -1

DO $200 \mathrm{I}=1$, NPARM 1

$\mathrm{IP} 1=\mathrm{I}+1$

DO $180 \mathrm{~J}=$ IP1, NPAR

IF (NFCNMX-NFCN+NPFN .LT. NPAR) GO TO 210

$X T I=X(I)$

$X T J=X(J)$

$X(I)=X T I+D I R I N(I)$

$x(J)=X T J+\operatorname{DIRIN}(J)$

CALL INTOEX $(X)$

CALL FCN(NPAR, GY, FS1, U, IFLAG)

$\mathrm{NFCN}=\mathrm{NFCN}+1$

$X(I)=X T I$

$X(J)=X T J$

$\operatorname{ELEM}=(F S 1+A M I N-Y Y(I)-Y Y(J)) /(\operatorname{DIRIN}(I) * \operatorname{DIRIN}(J))$

IF (ELEM**2 .LT. G2(I)*G2(J)) GO TO 170

ELEM $=0$.

WRITE (ISYSWR, 470) I,J

$170 V(I, J)=$ ELEM

$V(J, I)=E L E M$

180 CONTINUE

200 CONTINUE

GO TO 214

$210 \mathrm{~J}=\mathrm{J}-1$

WRITE (ISYSWR, 490) I, J

214 CALL INTOEX $(X)$

CALL VERMIN( $V$, MAXINT, MAXINT, NPAR, IFAIL)

IF (IFAIL. . LT. 1) GO TO 222
HESSE $\quad 48$

HESSE $\quad 49$

HESSE $\quad 50$

HESSE $\quad 51$

HESSE $\quad 52$

HESSE 53

HESSE $\quad 54$

HESSE $\quad 55$

HESSE $\quad 56$

HESSE 57

HESSE $\quad 58$

HESSE $\quad 59$

HESSE 60

HESSE 61

HESSE 62

HESSE 63

HESSE 64

HESSE 65

HESSE 66

HESSE $\quad 67$

HESSE 68

HESSE 69

HESSE $\quad 70$

HESSE $\quad 71$

HESSE $\quad 72$

HESSE $\quad 73$

HESSE $\quad 74$

HESSE $\quad 75$

HESSE $\quad 76$

HESSE $\quad 77$

HESSE $\quad 78$

HESSE $\quad 79$

HESSE $\quad 80$

HESSE $\quad 81$

HESSE 82

HESSE $\quad 83$

HESSE $\quad 84$

HESSE $\quad 85$

HESSE $\quad 86$

HESSE $\quad 87$

HESSE $\quad 88$

HESSE $\quad 89$

HESSE 90

HESSE 91

HESSE 92

HESSE 93

HESSE 94

HESSE 95

HESSE 96

HESSE 97

HESSE 98

HESSE $\quad 99$

HESSE 100

HESSE $\quad 101$

HESSE 102

HESSE $\quad 103$

HESSE 104 
C

216 WRITE (ISYSWR, 540)

HESSE 105

$\operatorname{ISW}(2)=1$ . Diagonal MATRIX ONLY . . HESSE 106

DO $220 \mathrm{I}=1$, NPAR

HESSE 107

120

DO $218 \mathrm{~J}=1$, NPAR

HESSE 108

$$
218 V(I, J)=0
$$

$220 V(I, I)=1.0 / G 2(I)$

MDIAG $=0$

GO TO 223

222 WRITE (ISYSWR, 480)

HESSE 109

HESSE $\quad 110$

HESSE 111

HESSE $\quad 112$

HESSE 113

$\operatorname{ISW}(2)=2$

HESSE $\quad 114$

HESSE 115

C

223 DO $225 \mathrm{I}=1$, NPAR

HESSE $\quad 116$

DO $225 \mathrm{~J}=1$, NPAR

..... CAlCulate E D M HESSE 117

HESSE $\quad 118$

130

$225 V(I, J)=2.0 * V(I, J)$

HESSE 119

SIGMA $=0$.

HESSE 120

DO $250 \mathrm{I}=1$, NPAR

HESSE 121

IF (V(I,I) .GT. O.) GO TO 228

HESSE 122

WRITE (ISYSWR, 510) I

HESSE 123

135

MDIAG $=1$

HESSE $\quad 124$

HESSE 125

$228 R=0$.

HESSE $\quad 126$

DO $240 \mathrm{~J}=1$, NPAR

IF (I .EQ. J) GO TO 230

HESSE 127

IF $(V(I, J) * * 2 . L T$. ABS $(V(I, I) * V(J, J)))$ GO TO 230

HESSE 128

140

WRITE (ISYSWR, 470) I, J

HESSE $\quad 129$

$V(I, J)=0$.

HESSE 130

$V(J, I)=0$.

HESSE 131

230 CONTINUE

$240 R=R+V(I, J) * G(J)$

145

250 SIGMA $=$ SIGMA $+0.5 * R * G(I)$

HESSE $\quad 132$

HESSE $\quad 133$

HESSE $\quad 134$

IF (MDIAG .EQ. 1) GO TO 390

HESSE $\quad 135$

IF (SIGMA .GT. O.) GO TO 400

HESSE 136

WRITE (ISYSWR, 530)

HESSE 137

GO TO 216

$390 \operatorname{ISW}(2)=0$

400 RETURN

HESSE

138

HESSE 139

460 FORMAT ( $10 \mathrm{HOPA}$

HESSE

140

- HESSE

141

1T DERIVATIVE IS E11.3/1X40(1H*), $8 X$ 2OHSECOND DERIVATIVE IS 11.3 HESSE

142 2/)

HESSE

470 FORMAT ( $71 \mathrm{H}$ COVARIANCE MATRIX NOT POSITIVE-DEFINITE.

FAULTY E HESSE

HESSE

1LEMENT IN POSITION2I3)

480 FORMAT ( $35 \mathrm{H}$ SECOND DERIVATIVE MATRIX INVERTED )

HESSE

490 FORMAT ( 76HOCALL LIMIT IN HESSE. OFF-DIAGONAL ELEMENTS CALCULAT HESSE

IED ONLY UP TO POSITION2I3/)

500 FORMAT ( 37 H START SECOND DERIVATIVE CALCULATION )

HESSE

510 FORMAT ( $17 \mathrm{H}$ DIAGONAL ELEMENT I5, 20H IS ZERO OR NEGATIVE)

HESSE

520 FORMAT ( 23H MATRIX INVERSION FAILS)

HESSE

HESSE

530 FORMAT ( $29 H$ MATRIX NOT POSITIVE-DEFINITE)

540 FORMAT ( $3 O H$ ONLY DIAGONAL MATRIX PRODUCED)

HESSE

HESSE

HESSE

143

144

145

146

147

148

149

150

151

152

153

END

154

155 
1

10

15

20
SUBROUTINE IMPROV

ATTEMPTS TO IMPROVE ON A GOOD LOCAL MINIMUM BY FINDING A BETTER ONE. THE QUADRATIC PART OF FCN IS REMOVED BY CALFCN AND THIS TRANSFORMED FUNCTION IS MINIMIZED USING THE SIMPLEX METHOD FROM SEVERAL RANDOM STARTING POINTS.

REF. - GOLDSTEIN AND PRICE, MATH.COMP. 25, 569 (1971)

COMMON

1/MINERR/ ERP $(30), \operatorname{ERN}(30)$

2/PARINT/ X(15) XT (15)

3/PAREXT/ U(30)

XT (15)

,DIRIN $(15)$

, $N A M(30)$

, WERR (30)

, MAXINT

, LCODE (30)

- MAXEXT

, NPAR

4/LIMITS/ ALIM(30)

, $\operatorname{BLIM}(30)$

, LCORSP(30) , LIMSET

5/VARIAN/ V $(15,15)$

.$x 5(15)$

, XTS(15)

,WTS(15) ,NPFIX

C/CASC/ JH, JL, Y(16)

F/DERIVA/ G(30)

, G2 (30)

G/SIMVEC/ $P(15,16)$

J/VARIAT/ VT $(15,15)$

6/UNIT / ISYSRD

, PSTAR( 15$)$

,PSTST (15)

,PBAR (15)

, ISYSWR ,ISYSPU

8/TITLE / TITLE(13)

, DATE (2)

. ISW(7)

, VTEST

A/CARD / CWORD

, CWORD2

, NSTEPQ

,CWORD3

,UP ,NEWMIN

B/MINIMA/ AMIN , CNAME (25)

, CNAM2 (25)

NBLOCK

, NFCN

, WORD7 (7)

, ITAUR

,CNAM3 (25)

DATA ALPHA, BETA, GAMMA / $1.0,0.5,2.0 /$

IF (NPAR . LE. $\theta$ ) RETURN

ITAUR $=1$

EPSI $=0.1 *$ UP

$N P F N=N F C N$

NLOOP $=$ WORD7 (2)

IF (NLOOP .LE. O) $\quad \mathrm{NLOOP}=\mathrm{NPAR}+4$

NPARP $1=$ NPAR+1

$W G=1.0 / F L O A T$ (NPAR)

IFLAG $=4$

SIGSAV = SIGMA

APSI $=$ AMIN

DO $2 I=1$, NPAR

$X T(I)=X(I)$

$\mathrm{G} 2(\mathrm{I})=\operatorname{SQRT}(V(I, I) * \mathrm{UP})$

DO $2 J=1$, NPAR

$2 \operatorname{VT}(\mathrm{I}, \mathrm{J})=\mathrm{V}(\mathrm{I}, \mathrm{J})$

CALL VERMIN(VT, MAXINT, MAXINT, NPAR, IFAIL)

IF (IFAIL . GE. 1) GO TO 280

LOOP $=0$

3 DO $4 I=1$, NPAR

$\operatorname{DIRIN}(I)=2.0 * G 2(I)$

$4 X(I)=X T(I)+2.0 * \operatorname{DIRIN}(I) *(\operatorname{RGEN}(I)-0.5)$

LOOP $=$ LOOP +1

REG $=2.0$

WRITE (ISYSWR, 1040) LOOP

5 AMIN $=\operatorname{CALFCN}(X)$

C

$J L=$ NPARP 1

$J H=$ NPARP1

. . . Set up RANDOM Simplex

$Y($ NPARP1) $=$ AMIN

AMAX = AMIN

DO $15 \mathrm{I}=1$, NPAR
IMPROV 2

IMPROV

IMPROV

IMPROV

IMPROV

IMPROV

IMPROV

COMMONU

COMMONU

COMMONU

COMMONU

COMMONU

COMMONU

COMMONU

COMMONU

COMMONU

, $\mathrm{PRHO}(15)$

COMMONU

COMMONU

COMMONU

COMMONU

, NFCNMX

COMMONU

COMMONU

COMMONU 31

COMMONU 32

IMPROV

IMPROV

IMPROV

IMPROV

IMPROV

IMPROV

IMPROV

IMPROV

IMPROV

IMPROV

IMPROV

IMPROV

IMPROV

IMPROV

IMPROV

IMPROV

IMPROV

IMPROV

IMPROV

IMPROV

IMPROV

IMPROV

IMPROV

IMPROV

IMPROV

IMPROV

IMPROV

IMPROV

IMPROV

IMPROV

IMPROV

IMPROV

IMPROV

4

5 
60

65

70

$c$
$X I=X(I)$

$X(I)=X I-D I R I N(I) *(\operatorname{RGEN}(I)-0.5)$

$Y(I)=\operatorname{CALFCN}(X)$

IF (Y(I) .GE. AMIN) GO TO 7

AMIN $=Y(I)$

$\mathrm{JL}=\mathrm{I}$

7 IF (Y(I) . LE. AMAX) GO TO 8

AMAX $=Y(I)$

$\mathrm{JH}=\mathrm{I}$

8 CONTINUE

DO $10 \mathrm{~J}=1$, NPAR

$10 P(J, I)=X(J)$

$P(I, N P A R P I)=X I$

$15 X(I)=X I$

SIGMA = AMIN

SIG2 = SIGMA

50 CONTINUE

IF (AMIN . LT. O.) GO TO 95

IF (ISW(2) . LT. 2) GO TO 280

$E P=0.1 * A M I N$

IF (SIG2 . LT. EP .AND. SIGMA.LT.EP ) GO TO 100

SIG2 = SIGMA

C

IF ((NFCN-NPFN) .GT. NFCNMX) GO TO 300

DO $60 \mathrm{I}=1$, NPAR

$\mathrm{PB}=0$.

DO $59 \mathrm{~J}=1$, NPARP1

$59 \mathrm{~PB}=\mathrm{PB}+\mathrm{WG} * \mathrm{P}(\mathrm{I}, \mathrm{J})$

$\operatorname{PBAR}(I)=P B-W G * P(I, J H)$

$60 \operatorname{PSTAR}(\mathrm{I})=(1 .+\mathrm{ALPHA}) * \operatorname{PBAR}(\mathrm{I})-\mathrm{ALPHA} * \mathrm{P}(\mathrm{I}, \mathrm{JH})$

YSTAR = CALFCN(PSTAR)

C

IF (YSTAR.GE. AMIN) GO TO 70

POINT * BETTER THAN JL, CALCULATE NEW POINT **

DO $61 \mathrm{I}=1, \mathrm{NPAR}$

$61 \operatorname{PSTST}(I)=$ GAMMA*PSTAR ( I ) +(1 . -GAMMA ) *PBAR (I)

YSTST $=$ CALFCN (PSTST)

66 IF (YSTST.LT. Y(JL)) GO TO 67

CALL RAZZIA(YSTAR, PSTAR)

GO TO 50

67 CALL RAZZIA(YSTST,PSTST)

GO TO 50

C

POINT * IS NOT AS GOOD AS JL

70 IF (YSTAR .GE, Y(JH)) GO TO 73

JHOLD $=\mathrm{JH}$

CALL RAZZIA(YSTAR, PSTAR)

IF (JHOLD .NE. JH) GO TO 50

C CALCULATE NEW POINT **

73 DO $74 I=1$, NPAR

$74 \operatorname{PSTST}(\mathrm{I})=\mathrm{BETA} * P(\mathrm{I}, \mathrm{JH})+(1 .-B E T A) * \operatorname{PBAR}(\mathrm{I})$

YSTST $=$ CALFCN (PSTST)

IF(YSTST.GT.Y(JH)) GO TO 5

C POINT ** IS BETTER THAN JH

IF (YSTST .LT. AMIN) GO TO 67

CALL RAZZIA(YSTST, PSTST)

GO TO 50

C
IMPROV $\quad 43$

IMPROV $\quad 44$

IMPROV $\quad 45$

IMPROV $\quad 46$

IMPROV 47

IMPROV $\quad 48$

IMPROV $\quad 49$

IMPROV 50

IMPROV $\quad 51$

IMPROV 52

IMPROV 53

IMPROV $\quad 54$

IMPROV 55

IMPROV $\quad 56$

IMPROV 57

IMPROV $\quad 58$

IMPROV 59

IMPROV 60

IMPROV 61

IMPROV 62

IMPROV 63

IMPROV 64

IMPROV 65

IMPROV $\quad 66$

IMPROV 67

IMPROV 68

IMPROV 69

IMPROV 70

IMPROV 71

IMPROV 72

IMPROV $\quad 73$

IMPROV 74

IMPROV 75

IMPROV $\quad 76$

IMPROV 77

IMPROV $\quad 78$

IMPROV $\quad 79$

IMPROV 80

IMPROV 81

IMPROV 82

IMPROV 83

IMPROV 84

IMPROV $\quad 85$

IMPROV $\quad 86$

IMPROV 87

IMPROV 88

IMPROV 89

IMPROV 90

IMPROV 91

IMPROV 92

IMPROV 93

IMPROV 94

IMPROV 95

IMPROV 96

IMPROV 97

IMPROV 98

IMPROV 99 
115

120

125

130

135

140

145

150

155

160

165
95 WRITE (ISYSWR, 1000)

$\mathrm{REG}=0.1$

C

100 CALL INTOEX $(X)$

CALL FCN(NPAR, G, AMIN,U, 4)

$\mathrm{NFCN}=\mathrm{NFCN}+1$

DO $120 \mathrm{I}=1$, NPAR

$\operatorname{DIRIN}(I)=R E G * G 2(I)$

IF $(A B S(X(I)-X T(I))$.GT. DIRIN(I)) GO TO 150

120 CONTINUE

GO TO 230

150 NFCNMX $=$ NFCNMX + NPFN - NFCN

NPFN $=$ NFCN

CALL SIMPLX

IF (AMIN .GE. APSI) GO TO 325

DO $220 \mathrm{I}=1$, NPAR

$\operatorname{DIRIN}(\mathrm{I})=0.1 * \mathrm{G} 2(\mathrm{I})$

IF (ABS(X(I)-XT(I)) .GT. DIRIN(I)) GO TO 250

220 CONTINUE

230 IF (AMIN .LT. APSI) GO TO 350

GO TO 325

C

250 NEWMIN $=1$

$\operatorname{ISW}(2)=0$

ITAUR $=0$

NFCNMX = NFCNMX + NPFN - NFCN

WRITE (ISYSWR, 1030)

RETURN

C

280 WRITE (ISYSWR, 1020)

$\operatorname{ISW}(2)=0$

GO TO 325

$300 \operatorname{ISW}(1)=1$

325 DO $330 \mathrm{I}=1$, NPAR

$\operatorname{DIRIN}(I)=0.01 * \mathrm{G} 2(\mathrm{I})$

$330 \times(I)=X T(I)$

AMIN = APSI

SIGMA = SIGSAV

350 CALL INTOEX(X)

WRITE (ISYSWR, 1010)

IF (ISW(2) .LT. 2) GO TO 380

IF (LOOP . LT. NLOOP .AND. ISW(1) .LT. 1) GO TO 3

$I S W(2)=3$

380 CALL MPRINT (1, AMIN)

RETURN

. . . . TRULY NEW MINIMUM

FORMAT 1D)

1010 FORMAT ( $51 \mathrm{H}$ IMPROVE HAS RETURNED TO REGION OF ORIGINAL MINIMUM) 1020 FORMAT ( 44HOCOVARIANCE MATRIX WAS NOT POSITIVE-DEFINITE)

1030 FORMAT (38HOIMPROVE HAS FOUND A TRULY NEW MINIMUM/1H $37(1 \mathrm{H*}) /$ )

1040 FORMAT ( 18 HOSTART ATTEMPT NO.I2, 20H TO FIND NEW MINIMUM) END
IMPROV 100

IMPROV 101

IMPROV 102

IMPROV 103

IMPROV 104

IMPROV 105

IMPROV 106

IMPROV 107

IMPROV 108

IMPROV 109

IMPROV $\quad 110$

IMPROV 111

IMPROV 112

IMPROV 113

IMPROV $\quad 114$

IMPROV $\quad 115$

IMPROV 116

IMPROV 117

IMPROV 118

IMPROV $\quad 119$

IMPROV 120

IMPROV 121

IMPROV 122

IMPROV 123

IMPROV 124

IMPROV 125

IMPROV 126

IMPROV $\quad 127$

IMPROV 128

IMPROV 129

IMPROV 130

IMPROV 131

IMPROV 132

IMPROV 133

IMPROV 134

IMPROV 135

IMPROV $\quad 136$

IMPROV 137

IMPROV 138

IMPROV 139

IMPROV 140

IMPROV 141

IMPROV 142

IMPROV 143

IMPROV 144

IMPROV 145

IMPROV 146

IMPROV 147

IMPROV 148

IMPROV 149

IMPROV 150

IMPROV 151 
1

10

15

20

25

30
SUBROUTINE INTOEX (PINT)

CC

$\mathrm{CC}$

$\mathrm{CC}$

10 COMMON

\section{TRANSFORMS FROM INTER} PARAMETERS (U) THE MINIMIZING ROUTINES WHICH WORK IN INTERNAL COORDINATES CALL THIS ROUTINE BEFORE CALLING FCN.

$$
\text { 1/MINERR/ ERP(30) ,ERN(30) }
$$

INTOX

INTOX

INTOX

INTOX

COMMONU

COMMONU

COMMONU

COMMONU

COMMONU

COMMONU

COMMONU

COMMONU

COMMONU

COMMONU 12

COMMONU 13

COMMONU 27

COMMONU 28

COMMONU 29

COMMONU 30

COMMONU 31

COMMONU 32

INTOX 7

INTOX 8

INTOX 9

INTOX 10

INTOX 11

INTOX 12

INTOX 13

INTOX $\quad 14$

INTOX 15

INTOX 16

INTOX 17

INTOX 18

INTOX 19


1

5

10

15

20

25

30

35

40

45

50

SUBROUTINE MATOUT(TRACE, KODE)

PRINTS THE COVARIANCE MATRIX $V$. CALCULATES AND PRINTS THE INDIVIDUAL CORRELATION COEFFICIENTS AND GLOBAL CORRELATIONS.

$\mathrm{CC}$

CC COMMON

\begin{tabular}{|c|c|c|c|c|}
\hline $1 /$ MINERR/ ERP $(30)$ & , $\operatorname{ERN}(30)$ & & & \\
\hline 2/PARINT/ X(15) & $x T(15)$ &. $\operatorname{DIRIN}(15)$ & , MAXINT & , NPAR \\
\hline 3/PAREXT/ U(30) & , NAM(30) & ,WERR (30) & , MAXEXT &. $\mathrm{NU}$ \\
\hline 4/LIMITS/ ALIM(30) & . BLIM(30) &, $\operatorname{LCODE}(30)$ & , LCORSP $(30)$ & , LIMSET \\
\hline \multicolumn{5}{|l|}{ 5/VARIAN/ V $(15,15)$} \\
\hline $7 / F I X \quad / \operatorname{IPFIX}(15)$ & .XS(15) &.$X T S(15)$ & , WTS (15) & NPFIX \\
\hline \multicolumn{5}{|l|}{ C/CASC/ JH, JL, Y(16) } \\
\hline F/DERIVA/ G(30) &., $62(30)$ & & & \\
\hline G/SIMVEC/ $P(15,16)$ & , PSTAR(15) & ,PSTST(15) & .PBAR(15) & ,PRHO(15) \\
\hline \multicolumn{5}{|l|}{ J/VARIAT/ VT $(15,15)$} \\
\hline 6/UNIT / ISYSRD & , ISYSWR & . ISYSPU & & \\
\hline 8/TITLE / TITLE(13) & , DATE(2) &. $\operatorname{ISW}(7)$ & , NBLOCK & \\
\hline 9/CONVER/ EPSI ,APSI & VTEST & , NSTIEPQ & NFCN & , NFCNMX \\
\hline A/CARD / CWORD & . CWORD2 & . CWORD3 & . WORD7 (7) & \\
\hline B/MINIMA/ AMIN & , UP & , NEWMIN & ITAUR & . SIGMA \\
\hline K/COMMND/ NNAME & , CNAME (25) & . CNAM2 (25) & , $\operatorname{CNAM} 3(25)$ & \\
\hline \multicolumn{5}{|l|}{ DIMENSION VLINE(15) } \\
\hline IF（ISW(2) . LT, 2) & & & & \\
\hline
\end{tabular}

WRITE (ISYSWR, 600)

IF (TRACE .NE, 0.0) WRITE (ISYSWR, 610) TRACE

IF (NPAR .EQ. 0 ) GO TO 250

ISWTR $=$ ISW(5) - ITAUR

IF (ISWTR .LT. 2) GO TO 120

c

DO $100 \mathrm{I}=1$, NPAR

100 WRITE (ISYSWR, 620) $\quad(V(I, J), J=1, I)$

WRITE (ISYSWR, 630)

120 CONTINUE

. INTERNAL COVARIANCE MATRIX

IF (KODE .LT. 1) GO TO 500

IF (NPAR .LE. 1) GO TO 500

WRITE (ISYSWR, 650)

NPARM $=$ MINO (NPAR-1, 18)

WRITE (ISYSWR, 690) (ID, ID $=1$, NPARM)

DO $200 \mathrm{I}=2$, NPAR

$\mathrm{IM}=\mathrm{I}-1$

DO $170 \mathrm{~J}=1$, IM

$170 \operatorname{VLINE}(J)=V(I, J) / \operatorname{SQRT}(\operatorname{ABS}(V(I, I) * V(J, J)))$

200 WRITE (ISYSWR, 660) I, (VLINE(IZ), IZ=1, IM)

250 CONTINUE

WRITE (ISYSWR, 630)

C

CALL UCOPY $(V, P, M A X I N T * * 2)$

GLOBAL CORRELATION COEFFS ...

CALL VERMIN (P, MAXINT, MAXINT, NPAR, IERR)

IF(IERR .GT . O) RETURN

WRITE (ISYSWR, 670)

DO $400 \mathrm{I}=1$, NU

$\mathrm{L}=\operatorname{LCORSP}(\mathrm{I})$

IF (L.EQ. O) GO TO 400

$G C C=1.0-1.0 /(V(L, L) * P(L, L))$

WRITE(ISYSWR, 680) I, NAM(I), GCC

400 CONTINUE

CORRELATION COEFFS. .

MATOUT

MATOUT

MATOUT

MATOUT

COMMONU

COMMONU

COMMONU

COMMONU

COMMONU

COMMONU

COMMONU

COMMONU

COMMONU

COMMONU 12

COMMONU 13

COMMONU 27

COMMONU 28

COMMONU 29

COMMONU 30

COMMONU $\quad 31$

COMMONU $\quad 32$

MATOUT 8

MATOUT 13

MATOUT 14

MATOUT 15

MATOUT 16

MATOUT 17

MATOUT 18

MATOUT 19

MATOUT 20

MATOUT 21

MATOUT 22

MATOUT 23

MATOUT 24

MATOUT 25

MATOUT 26

MATOUT 27

MATOUT 28

MATOUT $\quad 29$

MATOUT 30

MATOUT 31

MATOUT 32

MATOUT 33

MATOUT $\quad 34$

MATOUT 35

MATOUT 36

MATOUT 37

MATOUT 38

MATOUT 39

MATOUT 40

MATOUT $\quad 41$

MATOUT 42

MATOUT $\quad 43$

MATOUT 44

MATOUT 45

MATOUT $\quad 46$

MATOUT $\quad 47$ 
60

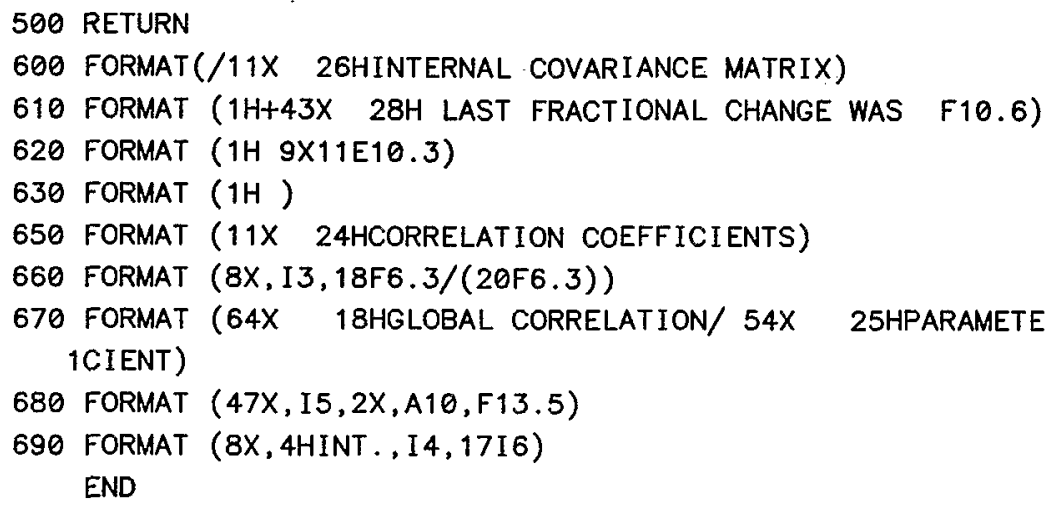

\begin{tabular}{|c|c|}
\hline & MATOUT \\
\hline & MATOUT \\
\hline & MATOUT \\
\hline & MATOUT \\
\hline & MATOUT \\
\hline & MATOUT \\
\hline & MATOUT \\
\hline COEFFI & MATOUT \\
\hline & MATOUT \\
\hline & MATOUT \\
\hline & MATOUT \\
\hline & MATOUT \\
\hline
\end{tabular}


SUBROUTINE MIDATA

CC

$\mathrm{CC}$

CC

5

CC

\section{COMMON}

1/MINERR/ ERP(30)

2/PARINT/ $X(15)$

3/PAREXT/ U(30)

4/LIMITS/ ALIM(30)

5/VARIAN/ V $(15,15)$

7/FIX / IPFIX(15)

C/CASC/JH, JL, $Y(16)$

F/DERIVA/ $G(30)$

$$
\begin{aligned}
& \text {, XT ( 15) } \\
& \text {, NAM(30) }
\end{aligned}
$$

, MAXINT

G/SIMVEC/ $P(15,16)$

J/VARIAT/ VT $(15,15)$

6/UNIT / ISYSRD

8/TITLE / TITLE(13)

9/CONVER/ EPSI ,APSI

A/CARD / CWORD

B/MINIMA/ AMIN

K/COMMND/ NNAME

COMMON /MATCH2/ AA(30), BB(30), KBTS, SMINCAL,

1 LOGICAL SMINCAL

C

NBLOCK $=$ NBLOCK +1

VERSN $=1.75$

WRITE (ISYSWR, 1004) VERSN, NBLOCK

WRITE (ISYSWR, 1005)

DO $50 \mathrm{I}=1,7$

$50 \operatorname{ISW}(\mathrm{I})=0$

SIGMA $=0$.

WRITE (ISYSWR, 1005)

NPFIX $=0$

NINT $=0$

$\mathrm{NU}=0$

NPAR $=0$

IFATAL $=0$

WRITE (ISYSWR, 1005)

DO $100 \mathrm{I}=1$, MAXEXT

$\operatorname{ERP}(I)=0.0$

$\operatorname{ERN}(\mathrm{I})=0.0$

$\operatorname{LCODE}(\mathrm{I})=0$

$100 \operatorname{LCORSP}(\mathrm{I})=0$

$\mathrm{UP}=1.0$

$\operatorname{ISW}(5)=1$

C

DO $200 \mathrm{~K}=1$, KBTS

NAMK $=N A M(K)$

$\mathrm{UK}=\mathrm{U}(\mathrm{K})$

WK=WERR $(K)$

$A=A A(K)$

$\mathrm{B}=\mathrm{BB}(\mathrm{K})$

$N U=\operatorname{MAXO}(N U, K)$

IF (K . LE. MAXEXT) GO TO 115

IFATAL $=$ IFATAL +1
, $\operatorname{ERN}(30)$

,DIRIN(15)

, $B \operatorname{LIM}(30)$

, WERR (30)

, LCODE (30)

, MAXEXT

, LCORSP (30) , LIMSET

, XTS (15)

,WTS(15) ,NPFIX

,$x S(15)$

, $62(30)$

MIDATA 2

MIDATA 3

MIDATA 4

MIDATA 5

MIDATA 6

COMMONU 2

COMMONU 4

COMMONU 5

COMMONU 6

COMMONU 7

COMMONU 8

COMMONU 9

COMMONU 10

COMMONU 11

,PSTAR(15) ,PSTST(15) ,PBAR(15) ,PRHO(15) COMMONU 12

, ISYSWR , ISYSPU

,DATE(2) ,ISW(7) ,NBLOCK

,NSTEPQ ,NFCN ,NFCNMX COMMONU

,CWORD2 ,CWORD3 ,WORD7(7)

,UP ,NEWMIN ,ITAUR

, CNAM3(25)

, SIGMA

COMMONU

COMMONU

COMMONU

MATCH4

$\mathrm{MATCH} 4$

MATCH4

- INITIALIZE NEW DATA BLOCK - MIDATA

MIDATA

MIDATA

MIDATA

MIDATA

MIDATA

MIDATA

MIDATA

MIDATA

MIDATA

MIDATA

MIDATA

MIDATA

MIDATA

MIDATA 23

MIDATA 24

MIDATA 25

MIDATA 26

MIDATA 27

MIDATA 28

MIDATA 29

MIDATA 30

MIDATA 31

MIDATA 32

MIDATA 33

MIDATA 34

MIDATA 35

MIDATA $\quad 36$

MIDATA 37

MIDATA 38

MIDATA 39

MIDATA 40 
68

65

105

110
WRITE (ISYSWR, 1009) K, MAXEXT

WRITE（ISYSWR, 1002） K,NAMK, UK, WK, A,B

GO TO 160

115 CONTINUE

IF (WK .GT. 0.0) GO TO 122

WRITE (ISYSWR, 1002) K, NAMK, UK

$\operatorname{LCODE}(K)=0$

GO TO 160

C

122 WRITE (ISYSWR, 1002) K, NAMK, UK, WK, A, B

NINT $=$ NINT +1

IF (A) $140,130,140$

130 IF (B) $140,135,140$

$135 \operatorname{LCODE}(K)=1$

GO TO 160

140 IF (B-A) $145,142,150$

142 IFATAL = IFATAL + 1

WRITE (ISYSWR, 1010)

GO TO 150

$145 \mathrm{SAV}=\mathrm{B}$

$B=A$

$A=S A V$

$150 A \operatorname{ALIM}(K)=A$

$\operatorname{BLIM}(K)=B$

$\operatorname{LCODE}(K)=4$

IF $((B-U(K)) *(U(K)-A)) \quad 153,155,160$

153 IFATAL = IFATAL + 1

WRITE (ISYSWR, 1011)

GO TO 160

155 WRITE (ISYSWR, 1006)

160 CONTINUE

200 CONTINUE

$\mathrm{C}$

C

250 WRITE (ISYSWR, 1005)

- IF (NINT . LE. MAXINT) GO TO 253

WRITE (ISYSWR, 1008) NINT, MAXINT

IFATAL = IFATAL + 1

253 IF (IFATAL . LE. 0) GO TO 280

WRITE (ISYSWR, 1013) IFATAL

STOP

280 NPAR $=0$

CALCULATE STEP SIZES DIRIN

- end parameter caRds

. . STOP IF FATAL ERROR

DO $300 \mathrm{~K}=1$, NU

IF (LCODE(K) . LE. 0) GO TO 300

$\mathrm{NPAR}=\mathrm{NPAR}+1$

$\operatorname{LCORSP}(K)=$ NPAR

$S A V=U(K)$

$X(N P A R)=P I N T F(S A V, K)$

$X T(N P A R)=X(N P A R)$

SAV2 $=$ SAV + WERR $(K)$

VPLU $=$ PINTF $($ SAV2,K $)-X(N P A R)$

SAV2 $=$ SAV - WERR $(K)$

VMINU $=$ PINTF $($ SAV2,K) $-X(N P A R)$

DIRIN(NPAR) $=0.5 *(A B S(V P L U)+A B S(V M I N U))$

300 CONTINUE
MIDATA $\quad 41$

MIDATA 42

MIDATA $\quad 43$

MIDATA 44

MIDATA $\quad 45$

MIDATA 46

MIDATA $\quad 47$

MIDATA 48

MIDATA $\quad 49$

MIDATA 50

MIDATA 51

MIDATA 52

MIDATA 53

MIDATA 54

MIDATA 55

MIDATA 56

MIDATA 57

MIDATA 58

MIDATA 59

MIDATA 60

MIDATA 61

MIDATA 62

MIDATA 63

MIDATA 64

MIDATA 65

MIDATA 66

MIDATA 67

MIDATA 68

MIDATA 69

MIDATA 70

MIDATA 71

MIDATA 72

MIDATA 73

MIDATA 74

MIDATA 75

MIDATA $\quad 76$

MIDATA 77

MIDATA 78

MIDATA 79

MIDATA 80

MIDATA 81

MIDATA 82

MIDATA 83

MIDATA 84

MIDATA 85

MIDATA 86

MIDATA 87

MIDATA 88

MIDATA 89

MIDATA 90

MIDATA 91

MIDATA 92

MIDATA 93

MIDATA 94

MIDATA 95

MIDATA 96

MIDATA 97 


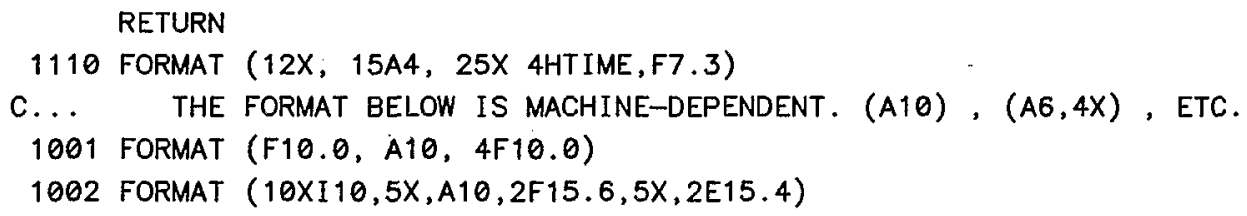


5

SUBROUTINE MIGRAD PERFORMS A LOCAL FUNCTION MINIMIZATION USING BASICALLY THE METHOD OF DAVIDON-FLETCHER-POWELL AS MODIFIED BY FLETCHER REF. - FLETCHER, COMP.J. 13,317 (1970) "SWITCHING METHOD"

$\mathrm{CC}$ cC

$$
\text { COMMON }
$$

$$
1 / \text { MINERR/ ERP(30) }
$$

2/PARINT/ $\times(15)$

3/PAREXT/ U(30)

$$
\begin{aligned}
& , \operatorname{ERN}(30) \\
& , X T(15) \\
& , \operatorname{NAM}(30)
\end{aligned}
$$

4/LIMITS/ ALIM(30)

MIGRAD $\quad 47$


60

65

70

75

80

85

90

95

100

105

110
CALL FCN(NPAR, G, FS2,U, 4)

$\mathrm{NFCN}=\mathrm{NFCN}+1$

$X(I)=X T F$

$G S(I)=(F S 1-F S 2) /(2.0 * D)$

$G 2(I)=(F S 1+F S 2-2.0 * A M I N) / D * * 2$

IF (G2(I) .GT. 1.0E-30) GO TO 10

C

WRITE (ISYSWR, 520)

NEGG2 $=$ NEGG2 +1

NTRY = NTRY + 1

IF (NTRY .GT. 4) GO TO 230

$D=50 . * A B S(D I R I N(I))$

$X B E G=X T F$

IF (GS(I) .LT. 0.) DIRIN(I) $=-\operatorname{DIRIN(I)}$

$K G=0$

$N F=0$

NS $=0$

$5 X(I)=X T F+D$

CALL INTOEX $(X)$

CALL FCN(NPAR, G, F,U,4)

$\mathrm{NFCN}=\mathrm{NFCN}+1$

IF (F. . LE. AMIN) GO TO 6

C FAILURE

IF (KG .EQ. 1) GO TO 8

$K G=-1$

$N F=N F+1$

$D=-0.4 * D$

IF (NF . LT. 10) GO TO 5

$D=1000 . * D$

GO TO 7

C SUCCESS

$6 \mathrm{XTF}=\mathrm{X}(\mathrm{I})$

$D=3.0 * D$

$A M I N=F$

$K G=1$

$\mathrm{NS}=\mathrm{NS}+1$

IF (NS.LT, 10) GO TO 5

IF (AMIN .LT. FS) GO TO 8

$D=0.001 * D$

7 XTF $=$ XBEG

$G 2(I)=1.0$

NEGG2 $=$ NEGG $2-1$

$8 \times(I)=X T F$

$\operatorname{DIRIN}(I)=0.1 * D$

$F S=A M I N$

10 CONTINUE

IF (NEGG2 .GE. 1) GO TO 4

NTRY $=0$

MATGD $=1$

C

IF (ISW(2) .GT. 1) GO TO 15

11 NTRY $=1$

MATGD $=0$

DO $13 \mathrm{I}=1$, NPAR

DO $12 \mathrm{~J}=1$, NPAR

$12 V(I, J)=0$.

$13 V(I, I)=2.0 / G 2(I)$
MIGRAD 48

MIGRAD $\quad 49$

MIGRAD 50

MIGRAD 51

MIGRAD 52

MIGRAD 53

MIGRAD $\quad 54$

MIGRAD $\quad 55$

MIGRAD $\quad 56$

MIGRAD $\quad 57$

MIGRAD 58

MIGRAD $\quad 59$

MIGRAD 60

MIGRAD 61

MIGRAD 62

MIGRAD 63

MIGRAD 64

MIGRAD 65

MIGRAD $\quad 66$

MIGRAD $\quad 67$

MIGRAD 68

MIGRAD $\quad 69$

MIGRAD 70

MIGRAD 71

MIGRAD $\quad 72$

MIGRAD $\quad 73$

MIGRAD $\quad 74$

MIGRAD $\quad 75$

MIGRAD $\quad 76$

MIGRAD $\quad 77$

MIGRAD $\quad 78$

MIGRAD $\quad 79$

MIGRAD 80

MIGRAD $\quad 81$

MIGRAD $\quad 82$

MIGRAD 83

MIGRAD 84

MIGRAD $\quad 85$

MIGRAD 86

MIGRAD $\quad 87$

MIGRAD 88

MIGRAD $\quad 89$

MIGRAD 90

MIGRAD 91

MIGRAD 92

MIGRAD 93

MIGRAD 94

MIGRAD 95

MIGRAD 96

MIGRAD 97

MIGRAD 98

MIGRAD 99

MIGRAD 100

MIGRAD 101

MIGRAD 102

MIGRAD 103

MIGRAD 104 
C

15 SIGMA $=0$.

DO $18 \mathrm{I}=1$, NPAR

IF (V(I,I).LE. O.) GO TO 11

$\mathrm{RI}=0$.

DO $17 \mathrm{~J}=1$, NPAR

$X X S(I)=X(I)$

$17 \mathrm{RI}=\mathrm{RI}+\mathrm{V}(\mathrm{I}, \mathrm{J}) * \mathrm{GS}(\mathrm{J})$

IF (SIGMA .GE. 0.) GO TO 20

WRITE (ISYSWR, 520)

IF (NTRY.EQ.0) GO TO 11

$\operatorname{ISW}(2)=0$

GO TO 230

$20 \operatorname{ISW}(2)=1$

ITER $=0$

CALL INTOEX(X)

C

24 CONTINUE

$\mathrm{GDEL}=0$.

DO $30 \quad I=1$, NPAR

$\mathrm{RI}=0$.

DO $25 \mathrm{~J}=1$, NPAR

$25 \mathrm{RI}=\mathrm{RI}+\mathrm{V}(\mathrm{I}, \mathrm{J}) * \mathrm{GS}(\mathrm{J})$

$\operatorname{DIRIN}(\mathrm{I})=-0.5 * \mathrm{RI}$

GDEL $=$ GDEL + DIRIN $(I) * G S(I)$

C

$30 X(I)=X X S(I)+D I R I N(I)$

CALL INTOEX $(X)$

CALL FCN (NPAR, G, $F, U, 4$ )

$N F C N=N F C N+1$

C

DENOM $=2.0 *(F-A M I N-G D E L)$

IF (DENOM .LE. O.) GO TO 35

SLAM = -GDEL/DENOM

GO TO 40

35 SLAM = SLAMAX

DO $45 \mathrm{I}=1$, NPAR

$45 X(I)=X X S(I)+\operatorname{SLAM} * \operatorname{DIRIN}(I)$

CALL INTOEX $(X)$

CALL FCN(NPAR, G, F2,U, 4)

$\mathrm{NFCN}=\mathrm{NFCN}+1$

C

$A A=F S / S L A M$

$B B=F /(1.0-S L A M)$

$C C=F 2 /(S L A M *(S L A M-1.0))$

DENOM $=2.0 *(A A+B B+C C)$

IF (DENOM .LE. O.) GO TO 48
18 SIGMA = SIGMA + GS(I) *RI $* 0.5$

IF (ISWTR .GE. 1) CALL MPRINT $(\theta$, AMIN)

IF (ISWTR .GE. 2) CALL MATOUT $(0.0,1)$

. QUADR INTERP USING SLOPE GDEL

IF (SLAM .GT. SLAMAX) GO TO 35

IF (SLAM . LT. SLAMIN) SLAM=SLAMIN

. . . START MAIN LOOP

. LINEAR SEARCH ALONG -VG . . .

40 IF (ABS(SLAM-1.0) . LT. 0.1) GO TO 70

- QUADR INTERP USING 3 POINTS

$T L A M=(A A *(S L A M+1.0)+B B * S L A M+C C) / D E N O M$

IF (TLAM .GT. TLAMAX) GO TO 48

IF (TLAM . LT. TLAMIN) TLAM=TLAMIN

GO TO 50
105

MIGRAD 106

MIGRAD $\quad 107$

MIGRAD $\quad 108$

MIGRAD $\quad 109$

MIGRAD $\quad 110$

MIGRAD 111

MIGRAD $\quad 112$

MIGRAD $\quad 113$

MIGRAD $\quad 114$

MIGRAD 115

MIGRAD $\quad 116$

MIGRAD $\quad 117$

MIGRAD 118

MIGRAD $\quad 119$

MIGRAD 120

MIGRAD 121

MIGRAD 122

MIGRAD 123

MIGRAD 124

MIGRAD $\quad 125$

MIGRAD $\quad 126$

MIGRAD $\quad 127$

MIGRAD $\quad 128$

MIGRAD $\quad 129$

MIGRAD 130

MIGRAD 131

MIGRAD $\quad 132$

MIGRAD 133

MIGRAD $\quad 134$

MIGRAD 135

MIGRAD 136

MIGRAD $\quad 137$

MIGRAD 138

MIGRAD $\quad 139$

MIGRAD 140

MIGRAD 141

MIGRAD 142

MIGRAD 143

MIGRAD $\quad 144$

MIGRAD 145

MIGRAD 146

MIGRAD 147

MIGRAD 148

MIGRAD 149

MIGRAD 150

MIGRAD 151

MIGRAD 152

MIGRAD 153

MIGRAD 154

MIGRAD 155

MIGRAD 156

MIGRAD 157

MIGRAD $\quad 158$

MIGRAD 159

MIGRAD $\quad 160$

MIGRAD $\quad 161$ 
CALL INTOEX $(X)$

CALL FCN(NPAR, G, F3,U,4)

MIGRAD 165

$N F C N=N F C N+1$

IF (F.GE.AMIN . AND. F2.GE.AMIN .AND. F3.GE.AMIN) GO TO 200

MIGRAD 166

MIGRAD $\quad 167$

IF (F . LT, F2 .AND. F . LT, F3) GO TO 61

MIGRAD

MIGRAD

$58 F=F 2$

GO TO 65

61 SLAM $=1.0$

65 DO 67 I= 1, NPAR

$\operatorname{DIRIN}(I)=D I R I N(I) * S L A M$

$67 X(I)=X X S(I)+\operatorname{DIRIN}(I)$

70 AMIN $=F$

$\operatorname{ISW}(2)=2$

IF (SIGMA+FS-AMIN . LT. ROSTOP) GO TO 170

IF (SIGMA+RHO2+FS-AMIN .GT. APSI) GO TO 75

IF (TRACE . LT. VTEST) GO TO 170

75 CONTINUE

IF (NFCN-NPFN .GE. NFCNMX) GO TO 190

ITER = ITER + 1

IF (ISWTR.GE. 3 .OR. (ISWTR.EQ. 2 .AND. MOD(ITER, 10) . EQ.1))

IF (ISW(3) .NE. 1) GO TO 80 GET GRADIENT AND SIGMA

MIGRAD

CALL INTOEX $(X)$

CALL FCN(NPAR, G, AMIN, U, IFLAG)

MIGRAD

$\mathrm{RHO} 2$ = SIGMA

MIGRAD

MIGRAD

196

SIGMA $=0$.

MIGRAD

GVG $=0$.

DELGAM $=0$.

MIGRAD 199

DO $100 \mathrm{I}=1$, NPAR

MIGRAD 200

$R I=\theta$.

MIGRAD

201

VGI $=0$.

MIGRAD 202

DO $90 \mathrm{~J}=1$, NPAR

$V G I=V G I+V(I, J) *(G(J)-G S(J))$

MIGRAD 203

MIGRAD 204

MIGRAD 205

$90 R I=R I+V(I, J) * G(J)$

$R(I)=R I * 0.5$

MIGRAD 206

$V G(I)=V G I * 0.5$

MIGRAD 207

$G A M I=G(I)-G S(I)$

GVG = GVG + GAMI $* V G(I)$

DELGAM $=$ DELGAM + DIRIN(I)*GAMI

MIGRAD

208

MIGRAD 209

MIGRAD 210

100 SIGMA $=$ SIGMA + G(I)*R(I)

MIGRAD 211

IF (SIGMA . LT. O.) GO TO 1

MIGRAD 212

IF (GVG .LE. O.) GO TO 105

MIGRAD 213

IF (DELGAM .LE. O.) GO TO 105

MIGRAD

214

GO TO 107

MIGRAD

215

MIGRAD 216

105 IF (SIGMA.LT. 0.1*ROSTOP) GO TO 170

GO TO 1 
$\mathrm{FS}=\mathrm{F}$

GO TO 24

C

170 WRITE (ISYSWR, 500)

$\operatorname{ISW}(2)=3$

IF(ISWTR .GE. $\theta$ ) CALL MPRINT(1-ITAUR, AMIN)

ISWTR $=$ ISWTR $-3 *$ ITAUR

IF (ISWTR . GT. O) CALL MATOUT (TRACE, 1)

IF (ITAUR .GT. O) GO TO 435

IF (MATGD . GT. O) GO TO 435

180 FORMAT ( 55H COVARIANCE MATRIX INACCURATE. MINUIT WILL RECALCULA 1TE)

CALL HESSE

CALL MPRINT (1, AMIN)

CALL MATOUT $(0.0,1)$

IF (ISW(2) .GE. 2) ISW(2) $=3$

GO TO 435

$190 \operatorname{ISW}(1)=1$

GO TO 230

200 WRITE (ISYSWR, 650)

CALL UCOPY (XXS, $X, N P A R$ )

$\operatorname{ISW}(2)=1$

CALL INTOEX $(X)$

CALL MPRINT(1-ITAUR, AMIN)

ISWTR $=$ ISW $(5)-I T A U R * 3$

IF (ISWTR .LT. 1) GO TO 435

IF (ISW(2) .LE. 1) GO TO 435

CALL MATOUT(TRACE, 1) 
1ENCE CRITERIA - ESTIMATED DISTANCE TO MINIMUM (EDM) .LT.E9.2/45 MIGRAD

$2 \times 11$ HOR EDM .LT.E9.2, 46H AND FRACTIONAL CHANGE IN VARIANCE MATR MIGRAD 
SUBROUTINE MINNEW

THIS IS THE MAIN PROGRAM, DISGUISED AS A SUBROUTINE FOR REASONS OF COMPATIBILITY BETWEEN SYSTEMS. IT INITIALIZES

MINNEW

MINNEW SOME CONSTANTS IN COMMON (INCLUDING THE LOGICAL I/O UNIT NOS.) WHICH WOULD HAVE TO BE IN BLOCK DATA FOR MANY COMPILERS. THEN VERIFIES THAT FCN GIVES THE SAME VALUE WHEN CALLED

MINNEW TWICE WITH THE SAME ARGUMENTS, AND PASSES CONTROL TO COMAND.

COMMON

$1 /$ MINERR/ $\operatorname{ERP}(30) \quad, \operatorname{ERN}(30)$

2/PARINT/ $X(15)$

2/PARINT/ $X(15)$

, XT(15)

,DIRIN(15)

.NAM(30)

, WERR (30)

, MAXINT

. $\operatorname{LCODE}(30)$

, MAXEXT

, NPAR

,NU

4/LIMITS/ ALIM(30)

. BLIM(30)

, LCORSP (30), LIMSET

5/VARIAN/ V(15, 15)

XTS (15)

.WTS(15) ,NPFIX

C/CASC/ JH, JL, Y(16)

F/DERIVA/ G(30)

.$x S(15)$

, $62(30)$

G/SIMVEC/ $P(15,16)$

J/VARIAT/ VT $(15,15)$

6/UNIT / ISYSRD

. $\operatorname{PSTAR}(15)$

,PSTST (15) ,PBAR(15)

, ISYSWR , ISYSPU

8/TITLE / TITLE(13)

,DATE(2)

, ISW(7)

,VTEST

, NSTEPQ

,NBLOCK

9/CONVER/ EPSI ,APSI

A/CARD / CWORD

,CWORD2

. CWORD 3

, NFCN

,UP ,NEWMIN ,ITAUR

.WORD7 (7)

K/COMMND/ NNAME

, CNAME (25)

, CNAM2 (25)

, CNAM3 (25)

MINNEW

MINNEW

MINNEW

MINNEW

COMMONU

COMMONU

COMMONU

COMMONU

COMMONU

COMMONU

COMMONU

COMMONU

COMMONU

.PRHO(15) COMMONU

COMMONU

COMMONU

COMMONU

,NFCNMX COMMONU

COMMONU

COMMONU

COMMONU

MINNEW

DATA RET1, RET2, RET3/ 4H RET, 4HRETU, 4HRETR /

4HMINI ,4HSEEK , 4HSIMP ,4HMIGR , 4HMINO , 4HPUNC 7

DATA CNAME

, 4HPRIN , 4HEND

, 4 HREST ,

4HEXIT , 4HGRAD , 4HCALL

4

, 4HMATO , 4HCOVA, 4HIMPR

, 4HERRO ,

, 4HSTAN, 4HRELE, $4 \mathrm{H}$

DATA CNAM2

4 HMIZE, $4 \mathrm{H}$

$, 4 \mathrm{H}, 4 \mathrm{HEND} /$

, 4 HLEX ,4HAD , $4 \mathrm{HS} \quad, 4 \mathrm{HH}$

,4HORE ,4H , 4HIENT , $4 \mathrm{H} \mathrm{FCN}$

7

, 4HTOUT , 4H , 4H

$4 \mathrm{HE}$

, 4HOUR

$, 4 \mathrm{H}, 4 \mathrm{HRETU} /$

,4HDARD, 4HASE, $4 \mathrm{H}$

DATA CNAM3 / $2 \mathrm{H}, 2 \mathrm{H}$

7

4

1

DATA NNAME, NBLOCK / $25,0 /$

DATA ISYSRD, ISYSWR, ISYSPU, MAXINT, MAXEXT $/ 2,3,98,15,30 /$

210 FORMAT $(5(8 X, 2 A 4, A 2))$

110 CONTINUE

$\mathrm{NFCN}=1$

CALL MIDATA

CALL INTOEX $(X)$

C WRITE (ISYSWR, 120)

120 FORMAT (/2OHOFIRST ENTRY TO FCN )

C CALL FCN(NPAR, G, AMIN, U, 1)

CALL FCN(NPAR, G, AMIN,U,4)

CALL MPRINT (1, AMIN)

CALL FCN(NPAR,G,F ,U,4)

IF ( $F$.NE. AMIN) GO TO 160

$\mathrm{NFCN}=3$

CALL COMAND

IF (CWORD2.EQ.RET1 .OR.CWORD2.EQ.RET2 .OR.CWORD2.EQ.RET3) RETURN

GO TO 110

160 CONTINUE
MINNEW 12

MINNEW 13

MINNEW 14

MINNEW $\quad 15$

MINNEW $\quad 16$

MINNEW 17

MINNEW 18

MINNEW $\quad 19$

MINNEW 20

MINNEW 21

MINNEW 22

MINNEW 23

MINNEW 24

MINNEW 26

MINNEW 27

MINNEW 28

MINNEW 29

MINNEW 30

MINNEW 31

MINNEW $\quad 32$

MINNEW 33

MINNEW $\quad 34$

MINNEW $\quad 35$

MINNEW 36

MINNEW 37

MINNEW 38

MINNEW 39

MINNEW 40

MINNEW $\quad 41$

MINNEW $\quad 42$

MINNEW $\quad 43$ 
WRITE' (ISYSWR, 880) AMIN, F

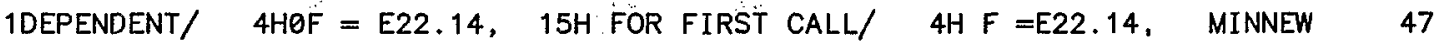
211H FOR SECOND) 
1

10
SUBROUTINE MINOS PERFORMS A MINOS ERROR ANALYSIS ON THOSE PARAMETERS FOR WHICH IT IS REQUESTED ON THE MINOS COMMAND CARD.

$\mathrm{CC}$

$\mathrm{CC}$

$5 \quad \mathrm{CC}$

$\mathrm{CC}$

$\mathrm{CC}$ THE PARAMETER IN QUESTION IS VARIED, ANND THE MINIMUM OF THE FUNCTION WITH RESPECT TO THE OTHER PARAMETERS IS FOLLOWED UNTIL IT CROSSES THE VALUE FMIN+UP.

COMMON

1/MINERR/ ERP $(30) \quad, \operatorname{ERN}(30)$

2/PARINT / X(15) XT(15)

$$
\text { XT (15) }
$$$$
\text { , NAM(30) }
$$

,DIRIN(15) ,MAXINT

, BLIM(30)

, WERR(30)

, MAXEXT

, LCODE $(30$

, LCORSP (30) , LIMSET

4/LIMITS/ ALIM(30)

, XTS (15)

,WTS(15) ,NPFIX

7/FIX / IPFIX(15)

,$x S(15)$

C/CASC/ JH, JL, Y(16)

F/DERIVA/ G(30)

, $62(30)$

G/SIMVEC/ $P(15,16)$

J/VARIAT/ VT $(15,15)$

6/UNIT / ISYSRD

,PSTAR( 15)

,PSTST (15) ,PBAR( 15)

, ISYSWR , ISYSPU

8/TITLE / TITLE(13)

9/CONVER/ EPSI ,APSI

A/CARD / CWORD

B/MINIMA/ AMIN

DATE (2)

, ISW(7)

,VTEST

,CWORD2

, NSTEPQ

,UP

,CWORD3

, NEWMIN

, CNAME(25) ,CNAM2(25) ,CNAM3 (25)

, CNAME(25) ,CNAM2(25) ,CNAM3 (25)

,PRHO(15)

K/COMMND/ NNAME

, NBLOCK

,NFCN

,WORD7 (7)

, ITAUR

, CNAM3 $(25)$

DIMENSION LMI (30), $\operatorname{KIRSCH}(5), \operatorname{XDEV}(15), W(30), \operatorname{SPT}(3,20)$

DATA APOS, ANEG, ADOT, ASTAR / 4HPOSI, 4HNEGA, $1 \mathrm{H}$, 1H*/

IF (NPAR . LE. O) GO TO 700

C

MARC $=0$

DO $5 \mathrm{I}=1,30$

$5 \operatorname{LMI}(\mathrm{I})=0$

$\mathrm{KNT}=0$

DO $20 I=2,7$

LIME $=$ WORD7 (I) +0.5

IF (LIME .EQ. $\theta$ ) GO TO 20

DO $10 \mathrm{~K}=1,5$

$\mathrm{K} 2=6-\mathrm{K}$

LEMON = MOD (LIME, 100)

$\mathrm{KIRSCH}(K 2)=$ LEMON

$10 \mathrm{LIME}=\mathrm{LIME} / 100$

DO $15 \mathrm{~J}=1,5$

LEMON $=$ KIRSCH $(J)$

IF (LEMON . GT. MAXEXT) GO TO 14

IF (LEMON . EQ. O) GO TO 15

IF (LCORSP(LEMON) .EQ. O) GO TO 14

$\mathrm{KNT}=\mathrm{KNT}+1$

LMI $(K N T)=$ LEMON

GO TO 15

14 MARC $=1$

15 CONTINUE

20 CONTINUE

IF (KNT .GT. O) GO TO 40

DO $30 \mathrm{I}=1$, MAXEXT

IF (LCORSP (I) .LT. 1) GO TO 30

$\mathrm{KNT}=\mathrm{KNT}+1$

$\operatorname{LMI}(K N T)=I$

IF (KNT .EQ. 30) GO TO 40
MINOS 2

MINOS

MINOS

MINOS

MINOS

MINOS

MINOS

COMMONU

COMMONU

COMMONU

COMMONU 6

COMMONU 7

COMMONU 8

COMMONU 9

COMMONU 10

COMMONU 11

COMMONU 12

COMMONU 13

COMMONU 27

COMMONU 28

COMMONU 29

COMMONU 30

COMMONU 31

COMMONU 32

MINOS 11

MINOS 16

MINOS 17

MINOS 18

MINOS 19

MINOS 20

MINOS 21

MINOS 22

MINOS 23

MINOS 24

MINOS 25

MINOS 26

MINOS 27

MINOS 28

MINOS $\quad 29$

MINOS $\quad 30$

MINOS 31

MINOS 32

MINOS 33

MINOS $\quad 34$

MINOS $\quad 35$

MINOS $\quad 36$

MINOS 37

MINOS $\quad 38$

MINOS $\quad 39$

MINOS 40

MINOS 41

MINOS $\quad 42$

MINOS 43

MINOS 44

MINOS $\quad 45$

MINOS $\quad 46$

MINOS $\quad 47$ 
65

70

75

80

85

90

95

100

105

110
30 CONTINUE

40 CONTINUE

60

IF (MARC .EQ. 1) WRITE (ISYSWR, 811)

WRITE (ISYSWR, 810) (LMI (IZ3), IZ3̈=1, KNT)

SIGSAV = SIGMA

TOLER = EPSI

APSI $=$ EPSI $* 0.5$

ITAUR $=1$

ABEST=AMIN

$A I M=A M I N+U P$

NSAVE = NFCNMX

MPAR=NPAR

DO $130 \mathrm{I}=1$, MPAR

$\mathrm{XT}(\mathrm{I})=\mathrm{X}(\mathrm{I})$

DO $125 \mathrm{~J}=1$, MPAR

$125 \mathrm{VT}(\mathrm{I}, \mathrm{J})=\mathrm{V}(\mathrm{I}, \mathrm{J})$

130 CONTINUE

DO $135 \mathrm{I}=1, \mathrm{NU}$

$\operatorname{ERP}(I)=0$.

$\operatorname{ERN}(I)=0$.

$135 W(I)=$ WERR $(I)$

$\mathrm{KNT}=0$

C

$150 \mathrm{KNT}=\mathrm{KNT}+1$

$\operatorname{ISW}(1)=0$

NLIMIT = NFCN + NSAVE

IF (KNT .GT. 30) GO TO 590

IF (LMI(KNT) .LT. 1) GO TO 590

$I L A X=L M I(K N T)$

$\operatorname{ERP}(I L A X)=0$.

$I T=\operatorname{LCORSP}(\mathrm{ILAX})$

$X T I T=X T(I T)$

CALL INTOEX $(X T)$

$\mathrm{UT}=\mathrm{U}(\mathrm{ILAX})$

$\operatorname{SPT}(1,1)=\mathrm{UT}$

$\operatorname{SPT}(2,1)=\operatorname{AIM}$

$\operatorname{SPT}(3,1)=1 \mathrm{H}$

$\operatorname{SPT}(1,2)=U T$

$\operatorname{SPT}(2,2)=A B E S T$

$\operatorname{SPT}(3,2)=A S T A R$

$\operatorname{SPT}(1,3)=U T$

$\operatorname{SPT}(2,3)=A I M+0.2 *(A I M-A B E S T)$

$\operatorname{SPT}(3,3)=A D O T$

NSPT $=3$

IF (LCODE (ILAX) .GT. 1) GO TO 160

$\operatorname{ALIM}($ I LAX) $=$ UT $-100 . * W($ I LAX)

$\operatorname{BLIM}($ ILAX $)=U T+100 . * W($ ILAX $)$

160 CONT INUE

XUNIT $=$ SQRT $(U P / V T(I T, I T))$

MARC $=0$

DO $162 I=1$, MPAR

IF (I .EQ. IT) GO TO 162

MARC $=$ MARC +1

$\operatorname{XDEV}($ MARC $)=\operatorname{XUNIT*VT}(I T, I)$

162 CONTINUE

CALL FIXPAR (IT, 1, ILAX)
- saVe AND PREPARE START VALS MINOS

MINOS $\quad 48$

MINOS $\quad 49$

MINOS 50

MINOS $\quad 51$

MINOS

MINOS 55

MINOS $\quad 56$

MINOS 57

MINOS 58

MINOS $\quad 59$

MINOS 60

MINOS 61

MINOS 62

MINOS 63

MINOS 64

MINOS 65

MINOS 66

MINOS 67

MINOS 68

MINOS 69

MINOS 70

. . . START MAIN LOOP . . MinOS 71

MINOS 72

MINOS 73

MINOS 74

MINOS 75

MINOS $\quad 76$

MINOS $\quad 77$

MINOS 78

MINOS $\quad 79$

MINOS 80

MINOS 81

MINOS 82

MINOS 83

MINOS 84

MINOS 85

MINOS 86

MINOS 87

MINOS $\quad 88$

MINOS $\quad 89$

MINOS 90

MINOS 91

MINOS 92

MINOS 93

MINOS 94

MINOS 95

MINOS 96

MINOS 97

MINOS 98

MINOS 99

MINOS 100

MINOS 101

MINOS 102

MINOS 103

MINOS 104 
115

120

125

130

135

140

145

150

155

160

165

$$
\begin{aligned}
& \text { SIG }=1.0 \\
& \text { ASIG = APOS } \\
& \text { DULIM = BLIM(ILAX) - UT } \\
& \operatorname{IF(ISW(2).LT.1)~GO~TO~} 460
\end{aligned}
$$

C 165 WRITE (ISYSWR, 806) ASIG, I LAX, NAM(I LAX)

SIG SIGN OF ERROR BEING CALCD MINOS

MINOS

ITER $=\theta$

LIMSET $=0$

DU1 $=S I G * W(I L A X)$

IF (ABS(DU1) . LE. DULIM) GO TO 180

LIMSET $=1$

DU1 $=$ SIG * DULIM

IF (DULIM . LT. $1.0 \mathrm{E}-3 * W($ ILAX)) GO TO 440

$180 \mathrm{U}(\mathrm{ILAX})=\mathrm{UT}+\mathrm{DU} 1$

IF (NPAR . EQ. O) GO TO 205

$F A C=D U 1 / W(I L A X)$

DO $185 I=1$, NPAR

$185 X(I)=X T(I)+F A C * \operatorname{XDEV}(I)$

200 CALL INTOEX (X)

205 WRITE (ISYSWR, 801) ILAX, UT, DU1, U(ILAX)

CALL FCN(NPAR, G, AMIN, U, 4)

$N F C N=N F C N+1$

NFCNMX = NLIMIT - NFCN

CALL MIGRAD

IF (AMIN . LT. ABEST) GO TO 650

IF (ISW(1) .GE. 1) GO TO 450

IF (ISW(2) .GE. 2) GO TO 240

NFCNMX $=$ NLIMIT - NFCN

CALL SIMPLX

IF (AMIN .LT. ABEST) GO TO 650

IF (ISW(1) .GE. 1) GO TO 450

NFCNMX $=$ NLIMIT - NFCN

CALL MIGRAD

IF (AMIN . LT. ABEST) GO TO 650

IF (ISW(1) .GE. 1) GO TO 450

IF (ISW(2) . LT. 2) GO TO 460

$240 \mathrm{CREM}=$ AMIN - ABEST

NSPT $=$ NSPT +1

$\operatorname{SPT}(1, N S P T)=U(I L A X)$

$\operatorname{SPT}(2, N S P T)=A M I N$

$\operatorname{SPT}(3, N S P T)=A S I G$

IF (CREM . LE. $0 . \theta$ ) GO TO 650

SQUC $=$ SQRT (UP/CREM)

IF (ABS(AMIN-AIM) . LT. TOLER) GO TO 400

C

ITER = ITER +1 - ANOTHER ITERATION NECESSARY

IF (ITER . GT. 6) GO TO 430

IF (ITER . EQ. 1) GO TO 270

C

CHECK PREVIOUS ITERATION TO AVOID OSCILLATING SQUC2 $=$ SQUC

IF $(($ SQUC2-1.0)*(SQUC1-1.0) .GT. 0.0$)$ GO TO 270

SQUC $=0.65 *$ SQUC2 +0.35

SQUC11 $=1.0 /$ SQUC 1

IF ((SQUC11-SQUC)*(SQUC-1.0) . .GT. 0.0) GO TO 270

WRITE (ISYSWR, 260)

260 FORMAT ( $44 H$ MINOS IS HAVING TROUBLE WITH THIS PARAMETER)

SQUC $=0.5 *$ SQUC11 +0.5
MINOS

MINOS

MINOS 
270 CONT INUE

SQUC1 $=$ SQUC

DU1 $=$ DU1 $*$ SQUC

180

IF (ABS(DU1) . LE. DULIM) GO TO 280

IF (LIMSET .EQ. 1) GO TO 440

LIMSET $=1$

DU1 $=$ SIG*DULIM

$280 U(I L A X)=U T+$ DU1

DO $290 I=1$, NPAR

$290 \times(\mathrm{I})=\mathrm{XT}(\mathrm{I})+\mathrm{SQUC} *(\mathrm{X}(\mathrm{I})-\mathrm{XT}(\mathrm{I}))$

GO TO 200

185

C

400 EROS $=$ DU1 $*$ SQUC

- ERROR SUCCESSFULLY CALCULATED

WRITE (ISYSWR, 808) ASIG, ILAX, NAM(ILAX), EROS

410 WRITE (ISYSWR, 812)

IF (SIG .GT. O.) GO TO 420

$\operatorname{ERN}(I L A X)=$ EROS

GO TO 500

190

$420 \operatorname{ERP}(I L A X)=$ EROS

SIG $=-1.0$

ASIG $=$ ANEG

DULIM = UT - ALIM(ILAX)

GO TO 165

195

C

430 WRITE (ISYSWR, 809)

EROS $=0$.

GO TO 410

440 WRITE (ISYSWR, 807) ASIG, ILAX, NAM(ILAX), DULIM

200

205

$\mathrm{EROS}=0$.

GO TO 410

450 WRITE (ISYSWR, 802) NSAVE

GO TO 500

460 WRITE (ISYSWR, 805)

C

500 CONTINUE

CALL RESTOR(1)

$\operatorname{ISW}(2)=3$

DO $560 \mathrm{I}=1$, MPAR

210

DO $550 \mathrm{~J}=1$, MPAR

. PARAMETER FINISHED. RESET $\vee$ MINOS 195

MINOS $\quad 162$

MINOS 163

MINOS $\quad 164$

MINOS $\quad 165$

MINOS $\quad 166$

MINOS $\quad 167$

MINOS $\quad 168$

MINOS $\quad 169$

MINOS 170

MINOS $\quad 171$

MINOS $\quad 172$

MINOS 173

MINOS $\quad 174$

MINOS $\quad 175$

MINOS $\quad 176$

MINOS 177

MINOS $\quad 178$

MINOS $\quad 179$

MINOS 180

MINOS 181

MINOS 182

MINOS 183

MINOS $\quad 184$

..... . FAILURE RETURNS MINOS 185

MINOS $\quad 186$

MINOS 187

MINOS 188

MINOS $\quad 189$

MINOS 190

MINOS 191

MINOS 192

MINOS 193

MINOS 194

MINOS 196

MINOS 197

MINOS 198

MINOS 199

$550 \mathrm{~V}(\mathrm{I}, \mathrm{J})=\mathrm{VT}(\mathrm{I}, \mathrm{J})$

MINOS

200

560 CONTINUE

IF (NSPT .LT. 9) GO TO 150

MINOS

201

NSPT3 $=$ NSPT -3

MINOS

202

203

215

WRITE (ISYSWR, 813) ILAX, NAM( I LAX), NSPT3

MINOS

204

CALL PLTCON(NSPT, SPT)

MINOS

205

WRITE(ISYSWR, 814) ILAX

MINOS

206

GO TO 150

MINOS

207

C

220

590 CALL UCOPY (XT, X, MPAR)

MINOS

208

209

MINOS

210

CALL INTOEX (XT)

MINOS

210

SIGMA = SIGSAV

MINOS

211

AMIN = ABEST

MINOS

212

CALL MPRINT $(2$, AMIN)

MINOS

213

CALL MATOUT $(0.0,1)$

MINOS

214

225

GO TO 700

MINOS

215

C

650 NEWMIN $=1$

.. NEW MINIMUM FOUND . . . MINOS 
801 FORMAT ( 1OHOPARAMETERI4, 7H SET TOE11.3,3H + E10.3,3H= E12.3) MINOS

802 FORMAT ( 87HOCALL LIMIT EXCEEDED. CALCULATION OF MINOS ERROR FOR MINOS

1 THIS PARAMETER REQUIRES MORE THANI5, $11 \mathrm{H}$ FCN CALLS./)

MINOS

805 FORMAT (/45X 46HMINOS ERROR NOT CALCULATED FOR THIS PARAMETER.) MINOS 806 FORMAT ( 18 HODETERMINATION OF A4, 3OHTIVE MINOS ERROR FOR PARAME MINOS 1TERI3, 2X A10)

MINOS

807 FORMAT (/45X4HTHE A4, 29HTIVE MINOS ERROR OF PARAMETERI3, 2H, A MINOS

110 , 9H, EXCEEDS E12.4)

MINOS

808 FORMAT (/45X4HTHE A4, 29HTIVE MINOS ERROR OF PARAMETERI3, 2H, A MINOS

110. $4 \mathrm{H}$, IS E12.4)

MINOS

811 FORMAT ( $55 \mathrm{H}$ THERE ARE MISTAKES IN THE MINOS COMMAND CARD JUST RE MINOS $1 A D)$ 
1

10
SUBROUTINE MPRINT (IKODE, FVAL) PRINTS THE VALUES OF THE PARAMETERS AT THE TIME OF THE CALL. ALSO PRINTS OTHER RELEVANT INFORMATION SUCH AS FUNCTION VALUE, ESTIMATED DISTANCE TO MINIMUM, PARAMETER ERRORS, STEP SIZES. ACCORDING TO THE VALUE OF IKODE, THE PRINTOUT IS LONG FORMAT, SHORT FORMAT, OR MINOS FORMAT $(0,1,2)$

CC

$\mathrm{CC}$

$\mathrm{CC}$

$\mathrm{cC}$

$\mathrm{CC}$

$$
\text { COMMON }
$$

1/MINERR/ ERP(30) , ERN(30)

2/PARINT/ $X(15) \quad X T(15)$

3/PARINT/ $\times(15)$

$$
\text { , XT (15) }
$$

, NAM(30)

4/LIMITS/ ALIM(30)

, $\operatorname{BLIM}(30)$

5/VARIAN/ V(15,15)

7/FIX / IPFIX(15)

C/CASC/ JH, JL, $Y(16)$

F/DERIVA/ $G(30)$

,$X S(15)$

, $\mathrm{G} 2(30)$

G/SIMVEC/ $P(15,16)$

J/VARIAT/ VT $(15,15)$

6/UNIT / ISYSRD

,PSTAR (15)

8/TITLE / TITLE(13)

$$
\text { , ISYSWR , ISYSPU }
$$

9/CONVER/ EPSI ,APSI

A/CARD / CWORD

B/MINIMA/ AMIN

,DATE(2)

, VTEST

,CWORD2

,CWORD3

, CNAME(25)

C

K/COMMND/ NNAME

TI = FTIME(BIDON)

IF (IKODE .EQ. 2) GO TO 10

WRITE (ISYSWR, 1000)

GO TO 18

C

10 WRITE (ISYSWR, 1006) TITLE, DATE

$18 \mathrm{E}=$ SIGMA

KOUNT $=0$

C

DO $200 \quad I=1, N U$

IF (NAM (I)) $20,200,20$

$20 \mathrm{~L}=\operatorname{LCORSP}(\mathrm{I})$

IF ( $L$. EQ. O) GO TO 55

C

VARIABLE PARAMETER.

IF (ISW(2) . LT. 1) GO TO 27

$D X=\operatorname{SQRT}(A B S(V(L, L) * U P))$

IF (LCODE(I) . LE. 1) GO TO 26

$A L=A L I M(I)$

$B A=B L I M(I)-A L$

$\mathrm{DU} 1=\mathrm{AL}+0.5 *(\operatorname{SIN}(X(L)+D X)+1.0) * B A-U(I)$

$\mathrm{DU} 2=\mathrm{AL}+0.5 *(\operatorname{SIN}(X(L)-D X)+1.0) * B A-U(I)$

IF $(D X . G T .1 .0)$ DU1 = BA

$D X=0.5 *(A B S(D U 1)+A B S(D U 2))$

26 WERR (I) $=\mathrm{DX}$

$27 \times 1=X(L)$

$\times 2=\operatorname{DIRIN}(L)$

IF (IKODE . LT. 2) GO TO 29

$X 1=\operatorname{ERP}(I)$

$X 2=\operatorname{ERN}(\mathrm{I})$

29 IF (KOUNT) $30,30,40$

30 KOUNT $=1$

WRITE (ISYSWR, 1001) FVAL, NFCN,TI,E, L, I,NAM(I),U(I),WERR(I),X1,X2
,DIRIN(15) , MAXINT ,NPAR

,WERR (30) ,MAXEXT

,NU

$, \operatorname{LCODE}(30), \operatorname{LCORSP}(30)$, LIMSET

, XTS(15)

.WTS(15) ,NPFIX

, ISW(7)

\section{,NSTEPQ}

,WORD7 (7)

,NEWMIN , ITAUR

,CNAM2 (25) ,CNAM3(25)

- GET TIME AND PRINT HEADINGS .

MPRINT

MPRINT

MPRINT

MPRINT

MPRINT

MPRINT

MPRINT

COMMONU

COMMONU

COMMONU

COMMONU

COMMONU

COMMONU

COMMONU

COMMONU

COMMONU

,PRHO (15) COMMONU

COMMONU

COMMONU

COMMONU

COMMONU

COMMONU

COMMONU

COMMONU

MPRINT

MPRINT

MPRINT

MPRINT

MPRINT

MINOS PRINTOUT HAS SPECIAL HEADINGS.

MPRINT

MPRINT

MPRINT

MPRINT

MPRINT

MPRINT

MPRINT

MPRINT

MPRINT

CALCULATE EXTERNAL ERROR IF $\vee$ EXISTS MPRINT

MPRINT

MPRINT

MPRINT

MPRINT

MPRINT

MPRINT

MPRINT

MPRINT

MPRINT

MPRINT

MPRINT

MPRINT

MPRINT

MPRINT

MPRINT

MPRINT

MPRINT

MPRINT
2

3

4

5

6

7

8

2

4

5

6

7

8

9

10

11

12

13

27

28

29

30

31

32

10

11

12

13

14

15

16

17

18

19

20

21

22

23

24

25

26

27

28

29

30

31

32

33

34 
60

65

70

75

80

85
GO TO 45

40 WRITE (ISYSWR, 1002)

$L, I, \operatorname{NAM}(I), U(I), W E R R(I), X 1, \times 2$

45 IF (LCODE(I) .LE. 1) GO TO 200

IF $(A B S(\cos (X(L)))$. LT. 0.001) WRITE (ISYSWR, 1004)

GO TO 200

C

FIXED PARAMETER, PRINT ONLY IF IKODE .GT. 0

55 IF (IKODE .EQ. Ө) GO TO 200

IF (KOUNT) $60,60,70$

60 KOUNT $=1$

WRITE (ISYSWR, 1001) FVAL, NFCN,TI,E, L, I, NAM(I),U(I)

GO TO 200

70 WRITE (ISYSWR, 1003)

200 CONTINUE

IF (IKODE.GE.1 .AND. ISW(2).GE, 1) WRITE (ISYSWR, 1005) UP RETURN

$$
I, \operatorname{NAM}(I), U(I)
$$

1000 FORMAT (/4X"FCN VALUE"5X"CALLS"4X"TIME"5X"EDM"7X"INT. EXT. PARAMETER 1 "5X"VALUE"12X"ERROR"6X"INTERN. VALUE INT.STEP SIZE")

1001 FORMAT(E15.7, I7, F9.3, E11.2, I6, I4, 1XA10, E17.8,3E14.5)

1002 FORMAT ( $42 X$

1003 FORMAT (1H 47X I4, SXA10,4E14.5

I6, I4, IXA10, E17.8,3E14.5)

1004 FORMAT (1H 52X 42HM:ARNING - - ABOVE PARAM:TER IS AT LIMIT.)

1005 FORMAT (/45X 40HEF:RORS CORRESPJND TO FUNCT:ON CHANGE O:- F10.4)

1006 FORMAT (1H140X36HRES:JLTS OF FULL MINOS ERROR ANALYSIS/41\%36(1H*)/

/20X 15A4//8E: 9 SHPARABC:.IC4X 26H..... MINOS ERRORS ....

2.. / 4X9HFCN VALUE5X5HCALLS4×4HTIME4X6H EDM 4X 75HIN MPRINT

3T.EXT. PARAMETER VALUE ERROR POSITIVE

4TIVE )

END
MPRINT 43

MPRINT 44

MPRINT $\quad 45$

MPRINT $\quad 46$

MPRINT $\quad 47$

MPRINT $\quad 48$

MPRINT $\quad 49$

MPRINT 50

MPRINT 51

MPRINT $\quad 52$

MPRINT 53

MPRINT 54

MPRINT $\quad 55$

MPRINT 56

MPRINT $\quad 57$

MAY117+ 16

MAY117+ 17

MAY117+ 18

MPRINT 63

MPRINT 64

MPRINT 65

MPRINT $\quad 66$

MPRINT 67

68

69

MPRINT $\quad 70$

MPRINT 71 
1
SUBROUTINE MPUNCH PUNCHES CURRENT PARAMETER VALUES AND STEP SIZES ONTO CARDS IN FORMAT WHICH CAN BE REREAD BY MINUIT FOR RESTARTING. THE COVARIANCE MATRIX IS ALSO PUNCHED IF IT EXISTS.

$\mathrm{CC}$

$\mathrm{CC}$

\section{COMMON}

1/MINERR/ $\operatorname{ERP}(30) \quad, \operatorname{ERN}(30)$

2/PARINT/ X(15) XT (15)

3/PAREXT/ U(30)

.XT(15) ,DIRIN(15) ,MAXINT ,NPAR

,NAM(30) ,WERR(30) ,MAXEXT ,NU

4/LIMITS/ ALIM(30)

, $\operatorname{BLIM}(30)$

$, \operatorname{LCODE}(30), \operatorname{LCORSP}(30)$, LIMSET

5/VARIAN/ V $(15,15)$

7/FIX / IPFIX(15)

C/CASC/ JH, JL, $Y(16)$

, XS ( 15)

, $\mathrm{XTS}(15)$

F/DERIVA/ $G(30)$

, $\mathrm{G} 2(30)$

G/SIMVEC/ $P(15,16)$

J/VARIAT/ VT $(15,15)$

6/UNIT / ISYSRD

, PSTAR (15)

,PSTST(15) ,PBAR(15)

, ISYSWR , ISYSPU

8/TITLE / TITLE(13)

,DATE(2) ,ISW(7)

9/CONVER/ EPSI ,APSI

A/CARD / CWORD

, VTEST

,NSTEPQ

,CWORD2 ,CWORD3

B/MINIMA/ AMIN

UP

K/COMMND/ NNAME

, CNAME(25)

, NEWMIN

DIMENSION VC(7)

$T I$ = FTIME(DUMMY)

WRITE (ISYSPU, 1002)

WRITE (ISYSPU, 1000) TITLE, DATE,TI

DO $200 I=1, \mathrm{NU}$

IF (NAM (I)) $20,200,20$

C (AVOID PUNCHING MINUS ZERO)

20 IF (WERR(I) .EQ. 0.0) WERR(I) $=0.0$

IF (LCODE(I) .GT. 1) GO TO 100

C PARAMETER WITHOUT LIMITS

WRITE (ISYSPU, 1001) I, NAM(I),U(I),WERR(I)

GO TO 200

C

100 CONTINUE

WRITE (ISYSPU, 1001) I, NAM(I),U(I),WERR(I), ALIM(I),BLIM(I)

200 CONTINUE

WRITE (ISYSPU, 1002)

IF (ISW(2) . LT. 3) GO TO 300

WRITE (ISYSPU, 1003) NPAR

$K=0$

$\mathrm{KC}=0$

DO $250 \mathrm{I}=1$, NPAR

DO $250 \mathrm{~J}=1$, NPAR

$\mathrm{K}=\mathrm{K}+1$

$V C(K)=V(I, J)$

IF (K.NE. 7) GO TO 250

$K=0$

$K C=K C+1$

WRITE(ISYSPU, 1004) VC,TI,NBLOCK, KC

250 CONTINUE

IF (K.EQ. 0) GO TO 300

$\mathrm{KP1}=\mathrm{K}+1$

DO $260 \mathrm{I}=\mathrm{KP} 1,7$

$260 \mathrm{VC}(\mathrm{I})=0$.

$K C=K C+1$
,WTS(15) ,NPFIX

MPUNCH

MPUNCH

MPUNCH

MPUNCH

MPUNCH

COMMONU

COMMONU

COMMONU

COMMONU

COMMONU

COMMONU

COMMONU

COMMONU

COMMONU

,PRHO(15) COMMONU

COMMONU

COMMONU

,NBLOCK

, NFCN

COMMONU

, NFCNMX

,WORD7 (7)

, I TAUR

, CNAM3 (25)

, SIGMA

COMMONU

COMMONU

.COMMONU

COMMONU

MPUNCH

MPUNCH

MPUNCH

MPUNCH

MPUNCH

MPUNCH

MPUNCH

MPUNCH

MPUNCH

MPUNCH

MPUNCH

MPUNCH

MPUNCH

MPUNCH

MPUNCH

MPUNCH

MPUNCH

MPUNCH

MPUNCH

MPUNCH

MPUNCH

MPUNCH

MPUNCH

MPUNCH

MPUNCH

MPUNCH

MPUNCH

MPUNCH

MPUNCH

MPUNCH

MPUNCH

MPUNCH

MPUNCH

MPUNCH

MPUNCH
2

3

4

5

6

2

4

5

6

7

8

9

10

11

12

13

27

28

29

30

31

32

8

9

10

11 
65

WRITE(ISYSPU, 1004) VC,TI, NBLOCK, KC

MPUNCH

MPUNCH

MPUNCH

MPUNCH

MPUNCH

MPUNCH

MPUNCH

MPUNCH
43

44

45

46

47

48

49

50 
10

15

20

25

30

35

40

45

50
FUNCTION PINTF (PEXTI, I) CALCULATES THE INTERNAL PARAMETER VALUE-PINTF CORRESPONDING TO THE EXTERNAL VALUE PEXTI FOR PARAMETER I.

CC $\mathrm{CC}$

COMMON

$1 /$ MINERR/ ERP(30) , ERN(30)

2/PARINT/ $X(15)$

.$X T(15)$

, NAM(30)

, DIRIN(15)

, MAXINT

, BLIM(30)

, WERR (30)

, MAXEXT

, NPAR

4/LIMITS/ ALIM(30)

5/VARIAN/ $V(15,15)$

, LCODE (30)

, LCORSP(30) ,LIMSET

7/FIX / IPFIX(15)

, XS(15)

,$X T S(15)$

,WTS(15) ,NPFIX

C/CASC/ JH, JL, Y(16)

F/DERIVA/ G(30)

, G2(30)

,PSTAR(15) ,PSTST(15) ,PBAR(15)

G/SIMVEC/ $P(15,16)$

J/VARIAT/ VT $(15,15)$

6/UNIT / ISYSRD

, ISYSWR , ISYSPU

8/TITLE / TITLE(13)

,DATE(2) ,ISW(7)

, NSTEPQ

, VTEST

, CWORD3

, NBLOCK

, NEWMIN

, WORD7(7)

A/CARD / CWORD

,CWORD2

.CNAME(25), CNAM2(25), CNAM3(25)

, ITAUR

K/COMMND/ NNAME

DATA BIG, SMALL / $1.570796326795,-1.570796326795$ /

$I G O=\operatorname{LCODE}(\mathrm{I})$

GO TO $(100,200,300,400), I 60$

C- $\quad$ IGO $=1$ MEANS NO LIMITS

100 PINTF $=$ PEXTI

GO TO 800

200 CONTINUE

300 CONTINUE

C-

IGO $=4$ MEANS THERE ARE TWO LIMITS

400 ALIMI $=$ ALIM(I)

BLIMI $=$ BLIM(I)

IF (PEXTI-ALIMI) $\quad 440,500,460$

$440 A=$ SMALL

450 PINTF $=A$

PEXTI $=A L I M I+0.5 *(B L I M I-A L I M I) *(\operatorname{SIN}(A)+1.0)$

LIMSET $=1$

C WRITE (ISYSWR, 241) I

GO TO 800

460 IF (BLIMI-PEXTI) $\quad 470,520,480$

$470 \mathrm{~A}=\mathrm{BIG}$

GO TO 450

$480 Y Y=2.0 *(P E X T I-A L I M I) /(B L I M I-A L I M I)-1.0$

PINTF $=\operatorname{ATAN}(Y Y / S Q R T(1 . \theta-Y Y * * 2))$

GO TO 800

500 PINTF $=$ SMALL

GO TO 800

520 PINTF $=$ BIG

800 RETURN

241 FORMAT ( $19 H$ WARNING - VARIABLEI3, 46H HAS BEEN BROUGHT BACK INS 1 IDE LIMITS BY PINTF.)

END
PINTF 2

PINTF 3

PINTF 4

PINTF 5

COMMONU 2

COMMONU 4

COMMONU 5

COMMONU 6

COMMONU 7

COMMONU 8

COMMONU 9

COMMONU 10

COMMONU 11

,PRHO(15) COMMONU 12

COMMONU 13

COMMONU 27

COMMONU 28

COMMONU 29

COMMONU 30

COMMONU 31

COMMONU 32

PINTF 7

PINTF 8

PINTF 9

PINTF 10

PINTF 11

PINTF 12

PINTF 13

PINTF 14

PINTF 15

PINTF 16

PINTF 17

PINTF 18

PINTF 19

PINTF 20

PINTF 21

PINTF 22

PINTF 23

PINTF 24

PINTF 25

PINTF 26

PINTF 27

PINTF 28

PINTF 29

PINTF $\quad 30$

PINTF 31

PINTF 32

PINTF 33

PINTF $\quad 34$

PINTF 35

PINTF $\quad 36$

PINTF 37 
1

$5 \quad \mathrm{CC}$

$\mathrm{CC}$

$\mathrm{CC}$

$\mathrm{CC}$

$\mathrm{CC}$

$c$

c
SUBROUTINE PLTCON(NSPT, SPT) PLOTS POINTS IN ARRAY SPT ONTO ONE PAGE WITH LABELLED AXES NSPT IS THE NUMBER OF POINTS TO BE PLOTTED $\operatorname{SPT}(1, I)=X$-COORD. OF ITH POINT $\operatorname{SPT}(2, I)=Y$-COORD. OF ITH POINT $\operatorname{SPT}(3, \mathrm{I})=$ CHARACTER TO BE PLOTTED AT THIS POSITION

COMMON

1/MINERR/ ERP(30) . ERN(30)

2/PARINT/ $X(15) \quad$ XT(15)

3/PAREXT/ U(30) , NTM ( 15$)$

,DIRIN(15) ,MAXINT ,NPAR .NU

4/LIMITS/ ALIM(30) , $\operatorname{BLIM}(30)$

,WERR (30) ,MAXEXT

, LCORSP (30) , LIMSET

5/VARIAN/ V $(15,15)$

, LCODE(30)

7/FIX / IPFIX(15)

$, X S(15) \quad, X T S(15) \quad, W T S(15) \quad, N P F I X$

C/CASC/ JH, JL, Y(16)

F/DERIVA/ G(30)

$.62(30)$

G/SIMVEC/ P $(15,16)$

J/VARIAT/ VT $(15,15)$

6/UNIT / ISYSRD

.PSTAR(15) ,PSTST(15) ,PBAR(15)

(1)

8/TITLE / TITLE(13)

,ISYSWR , ISYSPU

$9 /$ CONVER/ EPSI ,APSI

A/CARD / CWORD

B/MINIMA/ AMIN

K/COMMND/ NNAME

,DATE(2) ,ISW(7)

,VTEST ,NSTEPQ

.CWORD2 ,CWORD3

,UP ,NEWMIN

, CNAME(25) ,CNAM2(25)

, NBLOCK

, NFCN

,WORD7(7)

, ITAUR

, $\mathrm{PRHO}(15)$

DIMENSION $\operatorname{SPT}(3,2), \operatorname{ALINE}(100), \operatorname{SAV}(3)$

DIMENSION FMT(6), FMTIN(12)

DATA DOT, SLASH, AI, ZERO, ABLANK/1H., 1H/, 1HI, $1 \mathrm{HO}, 1 \mathrm{H} /$

DATA MAXNX, MAXNY/ 98, 56/

DATA (FMT (IU), IU=1,6)/4H(5X, 6HF15.7, ,1H ,6HF15.7, ,1H ,6HF15.7)/

DATA (FMTIN(IU), IU =1,12)/1H, 4H 5X, , 4H10X, , 4H15X, , 4H20X, .

* 4H25X, , 4H30X, , 4H35X, 4H40X, , 4H45X, , 4H50X, , 4H55X,/

IF (NSPT .LE. 1) RETURN

XBEST $=\operatorname{SPT}(1,1)$

YBEST $=\operatorname{SPT}(2,1)$

CHBEST $=\operatorname{SPT}(3,1)$

ORDER THE POINTS BY DECREASING $Y$

$\mathrm{KM} 1=$ NSPT -1

DO $150 \mathrm{I}=1, \mathrm{KM} 1$

$\mathrm{IQUIT}=0$

$\mathrm{NI}=\mathrm{NSPT}-\mathrm{I}$

DO $140 \mathrm{~J}=1, \mathrm{NI}$

IF $(\operatorname{SPT}(2, J)-\operatorname{SPT}(2, J+1)) \quad 120,140,140$

120 DO 130 I $3=1,3$

$\operatorname{SAV}(13)=\operatorname{SPT}(13, J)$

$\operatorname{SPT}(13, J)=\operatorname{SPT}(I 3, J+1)$

$130 \operatorname{SPT}(I 3, J+1)=\operatorname{SAV}(I 3)$

IQUIT $=1$

140 CONTINUE

IF (IQUIT) $160,160,150$

150 CONTINUE

160 CONTINUE

FIND EXTREME VALUES

$X \operatorname{MAX}=\operatorname{SPT}(1,1)$

$X M I N=X M A X$

DO $200 \mathrm{I}=1$, NSPT

IF $(\operatorname{SPT}(1, I) . G T . X M A X) \quad X M A X=\operatorname{SPT}(1, I)$

IF $(\operatorname{SPT}(1, I) . L T . \quad X M I N) \quad X M I N=\operatorname{SPT}(1, I)$
PLTCON 2

PLTCON 3

PLTCON 4

PLTCON 5

PLTCON 6

PLTCON 7

PLTCON 8

COMMONU 2

COMMONU 4

COMMONU 5

COMMONU 6

COMMONU 7

COMMONU 8

COMMONU 9

COMMONU 10

COMMONU 11

COMMONU 12

COMMONU 13

COMMONU 27

COMMONU 28

COMMONU 29

COMMONU 30

COMMONU 31

COMMONU 32

PLTCON 10

PLTCON 11

PLTCON 12

PLTCON 13

PLTCON 14

PLTCON 15

PLTCON 16

PLTCON 17

PLTCON 18

PLTCON 19

PLTCON 20

PLTCON 21

PLTCON 22

PLTCON 23

PLTCON 24

PLTCON 25

PLTCON 26

PLTCON 27

PLTCON 28

PLTCON 29

PLTCON 30

PLTCON 31

PLTCON 32

PLTCON 33

PLTCON $\quad 34$

PLTCON $\quad 35$

PLTCON $\quad 36$

PLTCON 37

PLTCON 38

PLTCON $\quad 39$

PLTCON 40

PLTCON 41

PLTCON 42 
200 CONTINUE

CALL BINSIZ(XMIN, XMAX, MAXNX, XMIN, XMAX, NX, BWIDX)

PLTCON

ANX $=N X$

YMAX $=\operatorname{SPT}(2,1)$

YMIN $=\operatorname{SPT}(2, N S P T)$

IF (YMAX .EQ. YBEST) YMAX=YBEST + 1.0

CALL BINSIZ(YMIN, YMAX, MAXNY, YMIN, YMAX, NY, BWIDY)

65

ANY $=$ NY

IF (CHBEST . EQ. ABLANK) GO TO 50

XBEST $=0.5 *(X M A X+X M I N)$

YBEST $=0.5 *($ YMAX+YMIN $)$

50 CONTINUE

70

C

FIND SCALE CONSTANTS

$A X=1 . \theta / B W I D X$

$A Y=1.0 / B W I D Y$

$B X=-A X * X M I N+2.0$

$B Y=-A Y * Y M I N-2.0$

C

DO $300 I=1$, NSPT

$N X B E S T=A X * X B E S T+B X$

PRINT THE CONTOURS

$N Y=A N Y+1$.

$N X=A N X+2$.

$I S P 1=1$

LINODD $=1$

DO $400 I=1, N Y$

DO 310 IBK $=1$, NX

310 ALINE (IBK) = ABLANK

$\operatorname{ALINE}(1)=D O T$

$\operatorname{ALINE}(N X)=$ DOT

ALINE (NXBEST) $=$ DOT

DO $315 \mathrm{~J}=1$, NX

315 ALINE $(J)=$ DOT

320 CONTINUE

DO $340 \mathrm{~K}=$ ISP 1 , NSPT

$\mathrm{KS}=\operatorname{SPT}(2, \mathrm{~K})$

$I X=\operatorname{SPT}(1, K)$

$340 \operatorname{ALINE}(I X)=\operatorname{SPT}(3, K)$

$\mathrm{ISP} 1=\mathrm{NSPT}+1$

GO TO 350

345 ISP $1=\mathrm{K}$

350 CONTINUE

LINODD $=1$

GO TO 400 CONVERT POINTS TO GRID POSITIONS

$\operatorname{SPT}(1, I)=A X * \operatorname{SPT}(1, I)+B X$

$300 \operatorname{SPT}(2, I)=A N Y-A Y * \operatorname{SPT}(2, I)-B Y$

NYBEST $=$ ANY $-A Y * Y B E S T-B Y$

IF (I.NE.1 .AND. I.NE.NYBEST .AND. I.NE.NY) GO TO 320

YPRT = YMAX - FLOAT $(I-1) * B W I D Y$

IF (ISP1 .GT. NSPT) GO TO 350

IF (KS .GT. I) GO TO 345

IF (LINODD .EQ. 1 .OR. I .EQ. NY) GO TO 380

WRITE (ISYSWR, 1007) (ALINE(IW), IW=1,NX)

380 WRITE (ISYSWR, 1001) YPRT, (ALINE(IW), IW=1, NX)

LINODD $=0$

400 CONTINUE

DO $410 \mathrm{IBK}=1, \mathrm{NX}$
PLTCON $\quad 44$

PLTCON 45

PLTCON 46

PLTCON 47

PLTCON 48

PLTCON 49

PLTCON 50

PLTCON 51

PLTCON 52

PLTCON 53

PLTCON 54

PLTCON 55

PLTCON 56

PLTCON 57

PLTCON 58

PLTCON 59

PLTCON 60

PLTCON 61

PLTCON 62

PLTCON 63

PLTCON 64

PLTCON 65

PLTCON 66

PLTCON 67

PLTCON 68

PLTCON 69

PLTCON 70

PLTCON 71

PLTCON 72

PLTCON 73

PLTCON 74

PLTCON 75

PLTCON 76

PLTCON 77

PLTCON 78

PLTCON 79

PLTCON 80

PLTCON 81

PLTCON 82

PLTCON 83

PLTCON 84

PLTCON 85

PLTCON 86

PLTCON 87

PLTCON 88

PLTCON 89

PLTCON 90

PLTCON 91

PLTCON 92

PLTCON 93

PLTCON 94

PLTCON 95

PLTCON 96

PLTCON 97

PLTCON 98

PLTCON 99 
$\operatorname{ALINE}(1)=$ SLASH

$\begin{array}{ll}\text { PLTCON } & 100 \\ \text { PLTCON } & 101 \\ \text { PLTCON } & 102 \\ \text { PLTCON } & 103 \\ \text { PLTCON } & 104 \\ \text { PLTCON } & 105 \\ \text { PLTCON } & 106 \\ \text { PLTCON } & 107 \\ \text { PLTCON } & 108 \\ \text { PLTCON } & 109 \\ \text { PLTCON } & 110 \\ \text { PLTCON } & 111 \\ \text { PLTCON } & 112 \\ \text { PLTCON } & 113 \\ \text { PLTCON } & 114 \\ \text { PLTCON } & 115 \\ \text { PLTCON } & 116 \\ \text { PLTCON } & 117 \\ \text { PLTCON } & 118 \\ \text { PLTCON } & 119 \\ \text { PLTCON } & 120 \\ \text { PLTCON } & 121 \\ \text { PLTCON } & 122 \\ & \end{array}$

120

$A L I N E(N X B E S T)=A I$

(ALINE(IW), IW=1,NX)

WRITE (ISYSWR, 1007)

$\mathrm{N} 1=\mathrm{NXBEST} / 5-1$

IF (N1 .LT. 1) $N 1=1$

IF (N1 .GT, 12) $N 1=12$

$N 2=N X / 5-N 1-3$

IF (N2 .GT. O) GO TO 420

125

IF (N1 .GT. 1) $N 1=N 1-1$

$\mathrm{N} 2=1$

420 IF (N2 .GT. 12) N2 $=12$

$\operatorname{FMT}(3)=\operatorname{FMTIN}(N 1)$

FMT (5) $=$ FMTIN(N2)

130

WRITE (ISYSWR, FMT ) XMIN, XBEST, XMAX

WRITE (ISYSWR, 1002) BWIDX

500 RETURN

1001 FORMAT (F15.7,3H ..,100A1)

1002 FORMAT $(40 X, 13$ HONE COLUMN $=$

F13.7)

135

1003 FORMAT (5XA10)

1007 FORMAT $(18 X, 100 A 1)$

END

PLTCON 
1
SUBROUTINE RAZZIA (YNEW, PNEW)

CC

CC

$\mathrm{CC}$
CALLED ONLY BY SIMPLEX (AND IMPROV) TO ADD A NEW POINT AND REMOVE AN OLD ONE FROM THE CURRENT SIMPLEX, AND GET THE ESTIMATED DISTANCE TO MINIMUM.
$5 \quad C C$

\section{COMMON}

1/MINERR/ ERP(30)

2/PARINT/ $X(15)$

3/PAREXT/ U(30)

4/LIMITS/ ALIM(30)

5/VARIAN/ $V(15,15)$

$7 / F I X$ / IPFIX(15)

C/CASC/ JH, JL, Y(16)

F/DERIVA/ G(30)

G/SIMVEC/ $P(15,16)$

J/VARIAT/ VT $(15,15)$

6/UNIT / ISYSRD

8/TITLE / TITLE(13)

9/CONVER/ EPSI ,APSI

A/CARD / CWORD

B/MINIMA/ AMIN

K/COMMND/ NNAME

DIMENSION PNEW(15)

DO $10 \mathrm{I}=1$, NPAR

$10 \quad P(I, J H)=P N E W(I)$

$Y(J H)=Y N E W$

IF(YNEW.GE.AMIN) GO TO 18

DO $15 \mathrm{I}=1$, NPAR

$15 X(I)=P N E W(I)$

CALL INTOEX $(X)$

AMIN $=$ YNEW

$J L=J H$

18 CONTINUE

$\mathrm{JH}=1$

NPARP $1=N P A R+1$

20 DO $25 \mathrm{~J}=2$, NPARP1

IF $(Y(J) . G T, Y(J H)) \quad J H=J$

25 CONTINUE

SIGMA $=Y(J H)-Y(J L)$

IF (SIGMA.LE. O.) GO TO 45

$U S=1.0 /$ SIGMA

DO $35 \mathrm{I}=1$, NPAR

$P B I G=P(I, 1)$

PLIT = PBIG

DO $30 \mathrm{~J}=2$, NPARP 1

IF $(P(I, J) . G T$. PBIG) PBIG $=P(I, J)$

IF $(P(I, J)$.LT. PLIT) PLIT $=P(I, J)$

30 CONTINUE

$\operatorname{DIRIN}(I)=$ PBIG - PLIT

35 CONTINUE

40 RETURN

45 WRITE (ISYSWR, 1000) NPAR GO TO 40

1000 FORMAT ( $59 H 0 * * * * *$ FUNCTION VALUE DOES NOT SEEM TO DEPEND ON ANY
RAZZIA

RAZZIA

RAZZIA

RAZZIA

RAZZIA

COMMONU

COMMONU

COMMONU

COMMONU

COMMONU

COMMONU

COMMONU

COMMONU

COMMONU

,PBAR(15) ,PRHO(15)

, ISYSWR ,ISYSPU

,DATE(2) ,ISW(7)

,VTEST ,NSTEPQ

,NBLOCK

,NFCN ,NFCNMX

, WORD7 (7)

, ITAUR

. CNAM3 (25) , SIGMA

COMMONU

COMMONU

COMMONU

COMMONU

COMMONU

COMMONU

COMMONU 31

COMMONU. $\quad 32$

RAZZIA 8

RAZZIA 9

RAZZIA 10

RAZZIA 11

RAZZIA 12

RAZZIA 13

RAZZIA 14

RAZZIA 15

RAZZIA 16

RAZZIA 17

RAZZIA 18

RAZZIA $\quad 19$

RAZZIA 20

RAZZIA 21

RAZZIA 22

RAZZIA 23

RAZZIA 24

RAZZIA 25

RAZZIA 26

RAZZIA 27

RAZZIA $\quad 28$

RAZZIA 29

RAZZIA 30

RAZZIA 31

RAZZIA $\quad 32$

RAZZIA $\quad 33$

RAZZIA $\quad 34$

RAZZIA $\quad 35$

RAZZIA $\quad 36$

RAZZIA $\quad 37$

RAZZIA $\quad 38$

RAZZIA $\quad 39$ 
SUBROUTINE RESTOR(K)

CC RESTORES A FIXED PARAMETER TO VARIABLE STATUS

$\mathrm{CC}$

$\mathrm{CC}$ $\mathrm{CC}$ APPROPRIATE PLACE.

RESTOR

RESTOR

RESTOR

RESTOR

RESTOR

COMMON

1/MINERR/ ERP(30)

, $\operatorname{ERN}(30)$

2/PARINT/ $X(15)$

3/PAREXT/ U(30)

, XT(15)

, NAM(30)

,DIRIN(15)

, WERR (30)

, MAXINT

- MAXEXT

, NPAR

, $B \operatorname{LIM}(30)$

, $\operatorname{LCODE}(30)$

, LCORSP(30) , LIMSET

5/VARIAN/ V $(15,15)$

, XTS(15)

,WTS(15) ,NPFIX

$7 / F I X / \operatorname{IPFIX}(15)$
C/CASC/ JH, JL, Y(16)

F/DERIVA/ G(30)

XS(15)

NPIX

,G2(30)

G/SIMVEC/ P $(15,16)$

J/VARIAT/ VT $(15,15)$

6/UNIT / ISYSRD

,PSTAR (15)

,PSTST(15) ,PBAR(15)

, $P R H O(15)$

8/TITLE / TITLE(13)

, ISYSWR , ISYSPU

9/CONVER/ EPSI ,APSI

A/CARD / CWORD

,DATE(2)

, $\operatorname{ISW}(7)$

,VTEST

, NSTEPQ

,NBLOCK

,CWORD2

,CWORD 3

, NFCN

,WORD7(7)

UP

, NEWMIN

, ITAUR

$\mathrm{K} / \mathrm{COMMND} / \mathrm{NNAME}, \mathrm{CNAME}(25)$, ,CNAM2(25)
$K=0$ MEANS RESTORE ALL PARAMETERS

,CNAM3 (25)

25

$\mathrm{c}-$

$K=0$ MEANS RESTORE ALL PARAMETERS

$\mathrm{C}$ -

$K=1$ MEANS RESTORE THE LAST PARAMETER FIXED

$K=-I$ MEANS RESTORE EXTERNAL PARAMETER I (IF POSSIBLE)

$I Q=F I X$-LOCATION WHERE INTERNAL PARAMETERS WERE STORED

$I R=$ EXTERNAL NUMBER OF PARAMETER BEING RESTORED

IS = INTERNAL NUMBER OF PARAMETER BEING RESTORED

IF (K .GT. 1) WRITE (ISYSWR, 510)

30

IF (NPFIX . LT. 1) WRITE (ISYSWR, 500)

IF (K.EQ.1 .OR. K.EQ.0) GO TO 50

C

RELEASE PARAMETER WITH SPECIFIED EXTERNAL NUMBER

$K A=\operatorname{IABS}(K)$

IF (LCORSP(KA) .EQ. O) GO TO 15

WRITE (ISYSWR, 540)

540 FORMAT (49HOERROR. PARAMETER SPECIFIED IS ALREADY VARIABLE. /) RETURN

15 IF (NPFIX . LT. 1) GO TO 21

DO $20 I K=1$, NPFIX

IF (IPFIX(IK) .EQ. KA) GO TO 24

20 CONTINUE

21 WRITE (ISYSWR, 530)

530 FORMAT (53HEERROR.

RETURN
C

24 IF (IK .EQ. NPFIX) GO TO 50

$$
\begin{aligned}
& \text { IPSAV }=\text { IPFIX }(\text { IK }) \\
& \text { XSSAV }=\text { XS }(\text { IK }) \\
& \text { XTSSAV }=\text { XTS }(\text { IK }) \\
& \text { WTSSAV }=\text { WTS }(\text { IK }) \\
& \text { IKPI }=\text { IK }+1 \\
& \text { DO } 30 I=\text { IKP1, NPFIX } \\
& \text { IPFIX (I-1) }=\text { IPFIX(I) } \\
& \text { XS }(I-1)=\text { XS (I) } \\
& \text { XTS }(I-1)=X T S(I) \\
& 30 \text { WTS }(I-1)=\text { WTS (I) } \\
& \text { IPFIX(NPFIX) }=\text { IPSAV }
\end{aligned}
$$

COMMONU 2

COMMONU 4

COMMONU 5

COMMONU 6

COMMONU 7

COMMONU 8

COMMONU 9

COMMONU 10

COMMONU 11

COMMONU 12

COMMONU 13

COMMONU 27

COMMONU 28

COMMONU 29

COMMONU 30

COMMONU 31

COMMONU 32

RESTOR 8

RESTOR 9

RESTOR 10

RESTOR 11

RESTOR 12

RESTOR 13

RESTOR 14

RESTOR 15

RESTOR 16

RESTOR 17

RESTOR 18

RESTOR 19

RESTOR 20

RESTOR 21

RESTOR 22

RESTOR 23

RESTOR 24

RESTOR 25

RESTOR 26

RESTOR 27

RESTOR 28

RESTOR 29

RESTOR 30

RESTOR 31

RESTOR 32

RESTOR 33

RESTOR $\quad 34$

RESTOR 35

RESTOR 36

RESTOR $\quad 37$

RESTOR 38

RESTOR 39

RESTOR 40

RESTOR 41

RESTOR 42 
60

65

70

75

80

85

90
$X S(N P F I X)=X S S A V$

$X T S(N P F I X)=X T S S A V$

WTS $($ NPFIX) $=$ WTSSAV

RESTORE LAST PARAMETER IN LIST - IPFIX(NPFIX)

C

50 CONTINUE

IF (NPFIX .LT. 1) GO TO 300

$I R=\operatorname{IPFIX}($ NPFIX)

IS $=0$

DO $100 \mathrm{IJ}=\mathrm{IR}, \mathrm{NU}$

$I K=N U+I R-I J$

IF (LCORSP(IK)) $100,100,85$

$85 \operatorname{LC}=\operatorname{LCORSP}(I K)+1$

IS $=$ LC -1

$\operatorname{LCORSP}(I K)=L C$

$X(L C)=X(L C-1)$

$X T(L C)=X T(L C-1)$

$\operatorname{DIRIN}(L C)=\operatorname{DIRIN}(L C-1)$

1.00 CONTINUE

NPAR $=$ NPAR +1

IF (IS .EQ. O) IS = NPAR

$\operatorname{LCORSP}(I R)=I S$

$I Q=N P F I X$

$X(I S)=X S(I Q)$

$X T(I S)=X T S(I Q)$

$\operatorname{DIRIN}(I S)=W T S(I Q)$

NPFIX $=$ NPFIX -1

$\operatorname{ISW}(2)=0$

IF (ITAUR .LT. 1) WRITE(ISYSWR, 520) IR, NAM(IR)

IF (K.EQ.0) GO TO 50

300 RETURN

500 FORMAT ( 61 HOERROR IN CALL TO RESTOR IRAMETERS/)

510 FORMAT ( 52 HOERROR IN CALL TO RESTOR. ARGUMENT GREATER THAN ONE/ RES 1)

520 FORMAT (20X, 9HPARAMETER, $14,2 \mathrm{H}$, ,A10, 22H RESTORED TO VARIABLE. 1) END
RESTOR 43

RESTOR 44

RESTOR $\quad 45$

RESTOR 46

RESTOR $\quad 47$

RESTOR $\quad 48$

RESTOR $\quad 49$

RESTOR $\quad 50$

RESTOR 51

RESTOR $\quad 52$

RESTOR 53

RESTOR 54

RESTOR 55

RESTOR 56

RESTOR 57

RESTOR 58

RESTOR $\quad 59$

RESTOR $\quad 60$

RESTOR $\quad 61$

RESTOR 62

RESTOR 63

RESTOR $\quad 64$

RESTOR $\quad 65$

RESTOR 66

RESTOR $\quad 67$

RESTOR 68

RESTOR $\quad 69$

RESTOR 70

RESTOR 71

RESTOR $\quad 72$

RESTOR 73

RESTOR $\quad 74$

RESTOR 70

RESTOR $\quad 77$

RESTOR $\quad 78$

RESTOR $\quad 79$ 
1

5

10

15

20

25

30

35

40

45

50

55

SUBROUTINE SEEK

SEEK

CC. PERFORMS A ROUGH-MINIMIZATION BY MONTE CARLO SEARCH.

SEEK EACH TIME A NEW MINIMUM IS FOUND, THE SEARCH AREA IS SHIFTED

SEEK TO BE CENTERED AT THE BEST VALUE.

$\mathrm{CC}$ UNIFORMLY OVER A HYPERCUBE DETERMINED BY CURRENT STEP SIZES.

$\mathrm{CC}$

COMMON

$1 /$ MINERR/ $\operatorname{ERP}(30) \quad, \operatorname{ERN}(30)$

2/PARINT/ $X(15) \quad, X T(15)$

3/PAREXT/ U(30)

4/LIMITS/ ALIM(30)

5/VARIAN/ V(15,15)

7/FIX / IPFIX(15)

C/CASC/JH, JL, Y(16)

, NAM(30)

, $B \operatorname{LIM}(30)$

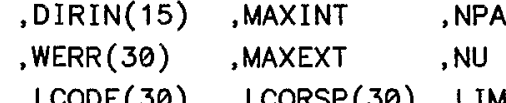

$, \operatorname{LCODE}(30), \operatorname{LCORSP}(30), \operatorname{LIMSET}$

F/DERIVA/ G(30)

G/SIMVEC/ $P(15,16)$

J/VARIAT/ VT $(15,15)$

6/UNIT / ISYSRD:

8/TITLE / TITLE(13)

9/CONVER/ EPSI ,APSI

A/CARD / CWORD

B/MINIMA/ AMIN

K/COMMND/ NNAME

DIMENSION AMID(15), N(15)

WRITE (ISYSWR, 1000)

NUMBER $=$ WORD7 $(1)+0.5$

IF (NUMBER . LE. $\theta$ ) NUMBER $=10 *$ NPAR

IFLAG $=4$

$c-$

INITIAL VALUES

DO $100 \mathrm{~J}=1$, NU

$N I=\operatorname{LCORSP}(J)$

IF (NI .LE. O) GO TO 100

$N(N I)=\rfloor$

$\operatorname{AMID}(N I)=U(J)$

100 CONTINUE

$\mathrm{NCYCL}=0$

C

MONTE CARLO SEA
DO 650 INUM $=1$, NUMBER

DO $200 \mathrm{I} 2=1$, NPAR

$\mathrm{I} 3=\mathrm{N}(\mathrm{I} 2)$

C

$180 X P L S=2.0 *(\operatorname{RGEN}(12)-0.5)$

$U(I 3)=$ AMID (I2) + XPLS*WERR (I3)

IF (LCODE(I3) .LE. 1) GO TO 200

,XTS(15) ,WTS(15) ,NPFIX

,PSTAR(15) ,PSTST(15) ,PBAR(15) ,PRHO(15)

, ISYSWR , ISYSPU

DATE(2) , ISW(7)

VTEST

, NSTEPQ

$\begin{array}{ll}\text {, NEWMIN } & \text {,I ITAUR } \\ \text { CNAM2(25) } & \text {, CNAM3 (25) }\end{array}$

IF (U(I3) .GT. BLIM(I3) .OR. U(I3) .LT. ALIM(I3)) GO TO 180

200 CONTINUE

CALL FCN(NPAR, G, F,U, IFLAG)

$N F C N=N F C N+1$

IF (F .GE. AMIN) GO TO 650

$A M I N=F$

DO $500 \mathrm{I}=1$, NPAR

$J=N(I)$

$500 \operatorname{AMID}(I)=U(\mathrm{~J})$

$\mathrm{NCYCL}=\mathrm{NCYCL}+1$

IF (ISW(5) . LT. 2) GO TO 650

CALL EXTOIN(X)

IF (ISW(5).GE. 3 .OR. MOD(NCYCL, 10).EQ.1) CALL MPRINT( 0, AMIN)
RANDOM POINTS ARE CHOSEN

, NBLOCK

, NFCN

,WORD7(7)

, NFCNMX

SEEK

SEEK

SEEK

COMMONU

COMMONU

COMMONU

COMMONU

COMMONU

COMMONU

COMMONU

COMMONU

COMMONU

COMMONU

COMMONU

COMMONU

COMMONU

COMMONU

COMMONU

COMMONU

COMMONU

SEEK

SEEK

SEEK

SEEK

SEEK

SEEK

SEEK

SEEK

SEEK

SEEK

SEEK

SEEK

SEEK

SEEK

SEEK

SEEK

SEEK

RANDOM POINTS IN UNIFORM DISTR. SEEK

SEEK

SEEK

SEEK

SEEK

SEEK

SEEK

SEEK

SEEK

SEEK

SEEK

SEEK

SEEK

SEEK

SEEK

SEEK

SEEK
2

3

4

5

6 
650 CONTINUE

SEEK 48

C

DO $800 \mathrm{I}=1$, NPÁR

SEARCH FINISHED. SET U TO BEST VALUES SEEK

60

$N I=N(I)$

SEEK 50

$800 U(N I)=\operatorname{AMID}(I)$

SEEK

51

CALL EXTOIN(X)

SEEK

52

WRITE (ISYSWR, 1005)

SEEK 53

65 CALL MPRINT ( 1, AMIN)

SEEK RETURN

SEEK

1000 FORMAT ( $35 \mathrm{H}$ SEEK - MONTE CARLO MINIMUM SEARCH )

SEEK

1005 FORMAT ( 28HOBEST VALUE FOUND IN SEEK IS )

SEEK 
1

10

15

20

25

30

35

40

45

50

55
SUBROUTINE SIMPLX

CC

CC

$\mathrm{CO}$

5

10

15

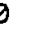
.

\section{COMMON}

1/MINERR/ ERP(30)

2/PARINT/ $X(15)$

, ERN(30)

3/PAREXT/ U(30)

, XT(15)

, NAM(30)

,DIRIN(15) ,MAXINT

, $\operatorname{BLIM}(30)$

,WERR(30) ,MAXEXT

, LCODE(30) , LCORSP(30) , LIMSET

4/LIMITS/ ALIM(30)

5/VARIAN/ $V(15,15)$

7/FIX / IPFIX(15)
C/CASC/ JH, JL, Y(16)

,XS(15)

, $\mathrm{XTS}(15)$

,WTS(15) ,NPFIX

F/DERIVA/ G(30)

, $62(30)$

G/SIMVEC/ $P(15,16)$

,PSTAR (15)

J/VARIAT/ VT $(15,15)$

6/UNIT / ISYSRD

, ISYSWR ,ISYSPU

8/TITLE / TITLE(13)

,DATE(2)

, ISW(7)

9/CONVER/ EPSI ,APSI

A/CARD / CWORD

,VTEST

,NSTEPQ

, CWORD2

,CWORD3

,UP

,NEWMIN

B/MINIMA/ AMIN

,CNAME (25)

, CNAM2 (25)

DIMENSION XBEST(15)

LOGICAL CONVER

DATA ALPHA, BETA, GAMMA, RHOMIN, RHOMAX / 1.0, $0.5,2.0,4.0,8.0 /$

IF (NPAR .LE. O) RETURN

$\mathrm{NPFN}=\mathrm{NFCN}$

NPARP $1=$ NPAR +1

$\mathrm{RHO} 1=1.0+$ ALPHA

$\mathrm{RHO} 2=\mathrm{RHO} 1+$ ALPHA $*$ GAMMA

$W G=1.0 / F L O A T$ (NPAR)

IFLAG $=4$

WRITE(ISYSWR, 100) EPSI

DO $2 I=1$, NPAR

IF (ISW(2) .GE. 1) $\operatorname{DIRIN(I)}=\operatorname{SQRT}(V(I, I) * U P)$

IF (ABS(DIRIN(I)) .LT. 1.0E-10*ABS(X(I))) DIRIN(I)=1.0E-8*X(I)

IF(ITAUR. LT. 1) $V(I, I)=\operatorname{DIRIN(I)**2/UP}$

2 CONTINUE

IF (ITAUR .LT. 1) ISW(2) $=1$

C** CHOOSE THE INITIAL SIMPLEX USING SINGLE-PARAMETER SEARCHES

1 CONTINUE

CONVER $=$. TRUE.

YNPP1 $=$ AMIN

$J L=N P A R P 1$

$Y($ NPARP1 $)=A M I N$

$A B S M I N=A M I N$

DO $10 I=1$, NPAR

AMING $=$ AMIN

$\operatorname{PBAR}(I)=X(I)$

BESTX $=X(I)$

$K G=0$

$\mathrm{NS}=0$

$\mathrm{NF}=0$

$4 X(I)=B E S T X+D I R I N(I)$

CALL INTOEX $(X)$

CALL FCN(NPAR, G, F, U, 4)

$\mathrm{NFCN}=\mathrm{NFCN}+1$

IF ( $F$. LE. AMING) GO TO 6
SIMPLX 2

SIMPLX 3

SIMPLX $\quad 4$

SIMPLX 5

COMMONU 2

COMMONU 4

COMMONU 5

COMMONU 6

COMMONU 7

COMMONU 8

COMMONU 9

COMMONU 10

COMMONU 11

COMMONU 12

COMMONU 13

COMMONU 27

COMMONU 28

COMMONU 29

COMMONU 30

COMMONU $\quad 31$

COMMONU $\quad 32$

SIMPLX 7

SIMPLX 8

SIMPLX $\quad 9$

SIMPLX 10

SIMPLX 11

SIMPLX $\quad 12$

SIMPLX 13

SIMPLX $\quad 14$

SIMPLX $\quad 15$

SIMPLX $\quad 16$

SIMPLX $\quad 17$

SIMPLX $\quad 18$

SIMPLX 19

SIMPLX 20

SIMPLX 21

SIMPLX 22

SIMPLX 23

SIMPLX 24

SIMPLX 25

SIMPLX 26

SIMPLX 27

SIMPLX 28

SIMPLX 29

SIMPLX $\quad 30$

SIMPLX 31

SIMPLX $\quad 32$

SIMPLX 33

SIMPLX $\quad 34$

SIMPLX $\quad 35$

SIMPLX $\quad 36$

SIMPLX $\quad 37$

SIMPLX $\quad 38$

SIMPLX $\quad 39$

SIMPLX $\quad 40$

SIMPLX $\quad 41$

SIMPLX $\quad 42$ 
C

60

65

70

105

110

c

C

C

C

\section{FAILURE}

IF (KG .EQ. 1) GO TO 8

$K G=-1$

$N F=N F+1$

$\operatorname{DIRIN}(I)=\operatorname{DIRIN}(I) *(-0.4)$

IF (NF .LT. 3) GO TO 4

$\mathrm{NS}=6$

6 BESTX $=X(I)$

$\operatorname{DIRIN}(I)=\operatorname{DIRIN}(I) * 3.01$

AMING $=F$

$K G=1$

$N S=N S+1$

IF (NS .LT. 6) GO TO 4

LOCAL MINIMUM FOUND IN ITH DIRECTION

$8 Y(I)=$ AMING

IF (AMING .LT. ABSMIN) JL $=I$

IF (AMING.$L T$. ABSMIN) ABSMIN = AMING

$X(I)=B E S T X$

XBEST $(I)=B E S T X$

DO $9 K=1$, NPAR

$9 P(K, I)=X(K)$

10 CONTINUE

$\mathrm{JH}=\mathrm{NPARP} 1$

AMIN $=Y(J L)$

CALL RAZZIA(YNPP1, PBAR)

DO $20 I=1$, NPAR

$20 \times(I)=P(I, J L)$

CALL INTOEX $(X)$

IF (ISW(5) .GE. 1) CALL MPRINT(0,AMIN)

SIGMA = SIGMA *10.

SIG2 = SIGMA

IGNAL $=0$

NCYCL $=0$

50 CONTINUE

IF (IGNAL .GE. 10) GO TO 1

IF (SIG2 .LT. EPSI .AND. SIGMA.LT.EPSI) GO TO 76

SIG2 = SIGMA

IF ((NFCN-NPFN) .GT. NFCNMX) GO TO 78 CALCULATE NEW POINT * BY REFLECTION

DO $60 \mathrm{I}=1$, NPAR

$\mathrm{PB}=0$.

DO $59 \mathrm{~J}=1$, NPARP1

$59 \mathrm{~PB}=\mathrm{PB}+\mathrm{WG} * \mathrm{P}(\mathrm{I}, \mathrm{J})$

$\operatorname{PBAR}(I)=P B-W G * P(I, J H)$

$60 \operatorname{PSTAR}(I)=(1 .+\operatorname{ALPHA}) * \operatorname{PBAR}(\mathrm{I})-\operatorname{ALPHA} * \mathrm{P}(\mathrm{I}, \mathrm{JH})$

CALL INTOEX(PSTAR)

CALL FCN(NPAR, G, YSTAR, $U, 4)$

$N F C N=N F C N+1$

IF (YSTAR.GE.AMIN) GO TO 70

C POINT * BETTER THAN JL, CALCULATE NEW POINT **

DO $61 \mathrm{I}=1$, NPAR

$61 \operatorname{PSTST}(\mathrm{I})=$ GAMMA $* \operatorname{PSTAR}(\mathrm{I})+(1 .-G A M M A) * \operatorname{PBAR}(\mathrm{I})$

CALL INTOEX(PSTST)

CALL FCN(NPAR, G, YSTST, $U, 4)$

$N F C N=N F C N+1$
SIMPLX $\quad 43$

SIMPLX $\quad 44$

SIMPLX $\quad 45$

SIMPLX $\quad 46$

SIMPLX 47

SIMPLX $\quad 48$

SIMPLX $\quad 49$

SIMPLX $\quad 50$

SIMPLX $\quad 51$

SIMPLX $\quad 52$

SIMPLX 53

SIMPLX $\quad 54$

SIMPLX $\quad 55$

SIMPLX $\quad 56$

SIMPLX $\quad 57$

SIMPLX $\quad 58$

SIMPLX $\quad 59$

SIMPLX 60

SIMPLX $\quad 61$

SIMPLX $\quad 62$

SIMPLX 63

SIMPLX $\quad 64$

SIMPLX $\quad 65$

SIMPLX $\quad 66$

SIMPLX $\quad 67$

SIMPLX $\quad 68$

SIMPLX $\quad 69$

SIMPLX $\quad 70$

SIMPLX $\quad 71$

SIMPLX $\quad 72$

SIMPLX 73

SIMPLX $\quad 74$

SIMPLX $\quad 75$

SIMPLX $\quad 76$

SIMPLX 77

SIMPLX $\quad 78$

SIMPLX $\quad 79$

SIMPLX $\quad 80$

SIMPLX $\quad 81$

S.IMPLX $\quad 82$

SIMPLX $\quad 83$

SIMPLX $\quad 84$

SIMPLX $\quad 85$

SIMPLX $\quad 86$

SIMPLX $\quad 87$

SIMPLX $\quad 88$

SIMPLX $\quad 89$

SIMPLX $\quad 90$

SIMPLX $\quad 91$

SIMPLX $\quad 92$

SIMPLX $\quad 93$

SIMPLX $\quad 94$

SIMPLX $\quad 95$

SIMPLX $\quad 96$

SIMPLX $\quad 97$

SIMPLX $\quad 98$

SIMPLX $\quad 99$ 
$Y 1=(Y S T A R-Y(J H)) * R H O 2$

SIMPLX 101

$Y 2=(Y S T S T-Y(J H)) * R H O 1$

SIMPLX 102

$\mathrm{RHO}=0.5 *(\mathrm{RHO} 2 * Y 1-\mathrm{RHO} 1 * \mathrm{Y} 2) /(\mathrm{Y} 1-\mathrm{Y} 2)$

SIMPLX 103

IF (RHO .LT. RHOMIN) GO TO 66

SIMPLX 104

120

IF (RHO .GT. RHOMAX) RHO = RHOMAX

SIMPLX 105

DO $64 \mathrm{I}=1$, NPAR

$64 \mathrm{PRHO}(\mathrm{I})=\mathrm{RHO} * \mathrm{PSTAR}(\mathrm{I})+(1,-\mathrm{RHO}) * \mathrm{P}(\mathrm{I}, \mathrm{JH})$

SIMPLX 106

CALL INTOEX(PRHO)

CALL FCN(NPAR, G, YRHO, U, 4)

$\mathrm{NFCN}=\mathrm{NFCN}+1$

IF (YRHO .LT. Y(JL) .AND. YRHO .LT. YSTST) GO TO 65

IF (YSTST .LT. Y(JL)) GO TO 67

IF (YRHO .GT. Y(JL)) GO TO 66

C

ACCEPT MINIMUM POINT OF PARABOLA, PRHO

65 CALL RAZZIA (YRHO,PRHO)

IGNAL $=$ MAXO $($ IGNAL-2, 0$)$

GO TO 68

66 IF (YSTST .LT. Y (JL)) GO TO 67

$I G N A L=$ MAXO $(I G N A L-1,0)$

CALL RAZZIA(YSTAR,PSTAR)

SIMPLX 107

SIMPLX 108

SIMPLX 109

SIMPLX 110

SIMPLX 111

SIMPLX 112

SIMPLX 113

SIMPLX 114

SIMPLX 115

SIMPLX $\quad 116$

SIMPLX 117

SIMPLX 118

SIMPLX 119

SIMPLX 120

GO TO 68

67 IGNAL $=$ MAXO $($ IGNAL-2, 0$)$

675 CALL RAZZIA(YSTST,PSTST)

$68 \mathrm{NCYCL}=\mathrm{NCYCL+1}$

IF (ISW(5) .LT, 2) GO TO 50

SIMPLX 121

SIMPLX 122

SIMPLX 123

SIMPLX 124

IF (ISW(5) .GE. 3. OR. MOD(NCYCL, 10).EQ. 0$)$ CALL MPRINT(0,AMIN)

GO TO 50

SIMPLX 125

SIMPLX 126

SIMPLX 127

C POINT * IS NOT AS GOOD AS JL

SIMPLX 128

70 IF (YSTAR .GE. Y(JH)) GO TO 73

SIMPLX 129

145

JHOLD $=$ JH

CALL RAZZIA(YSTAR,PSTAR)

IF (JHOLD. NE. JH) GO TO 50

C

CALCULATE NEW POINT **

150

73 DO $74 \mathrm{I}=1$, NPAR

$74 \operatorname{PSTST}(\mathrm{I})=\mathrm{BETA} * \mathrm{P}(\mathrm{I}, \mathrm{JH})+(1,-\mathrm{BETA}) * \operatorname{PBAR}(\mathrm{I})$

CALL INTOEX (PSTST)

CALL FCN(NPAR, G, YSTST, U, 4)

$N F C N=N F C N+1$

IF (YSTST.GT.Y(JH)) GO TO 1

155 C POINT ** IS BETTER THAN JH

IF (YSTST .LT. AMIN) GO TO 675

$I G N A L=I G N A L+1$

CALL RAZZIA(YSTST, PSTST)

GO TO 50

160

C

76 WRITE( ISYSWR, 120)

GO TO 80

78 WRITE( ISYSWR, 130)

$\operatorname{ISW}(1)=1$

CONVER $=$. FALSE.

80 DO $82 \mathrm{I}=1$, NPAR

$\mathrm{PB}=0$.

DO $81 \mathrm{~J}=1$, NPARP1

$81 \mathrm{~PB}=\mathrm{PB}+\mathrm{WG} * \mathrm{P}(\mathrm{I}, \mathrm{J})$

$82 \operatorname{PBAR}(\mathrm{I})=\mathrm{PB}-W \mathrm{~W} * \mathrm{P}(\mathrm{I}, \mathrm{JH})$

CALL INTOEX(PBAR)

SIMPLX 130

SIMPLX 131

SIMPLX 132

SIMPLX 133

SIMPLX 134

SIMPLX 135

SIMPLX 136

SIMPLX 137

SIMPLX 138

SIMPLX 139

SIMPLX 140

SIMPLX 141

SIMPLX 142

SIMPLX 143

SIMPLX $\quad 144$

SIMPLX $\quad 145$

SIMPLX $\quad 146$

SIMPLX 147

SIMPLX 148

SIMPLX $\quad 149$

SIMPLX 150

SIMPLX 151

SIMPLX $\quad 152$

SIMPLX 153

SIMPLX 154

SIMPLX 155

SIMPLX 156 
CALL FCN(NPAR, G, YPBAR,U, IFLAG)

SIMPLX $\quad 157$

- $N F C N=N F C N+1$

SIMPLX 158

IF (YPBAR . LT. AMIN) CALL RAZZIA(YPBAR, PBAR)

SIMPLX 159

CALL INTOEX $(X)$

SIMPLX 160

IF (NFCNMX+NPFN-NFCN . LT. 3*NPAR) GO TO 90

SIMPLX 161

IF (SIGMA .GT . 2.0*EPSI) GO TO 1

SIMPLX 162

90 IF (CONVER) GO TO 902

SIMPLX 163

CALL INTOEX(XBEST)

180

902 CALL MPRINT(1-ITAUR, AMIN)

SIMPLX 164

SIMPLX $\quad 165$

RETURN

SIMPLX $\quad 166$
100 FORMAT (
37H START SIMPLEX MINIMIZATION

8X 65HCON SIMPLX

167

IVERGENCE CRITERION - ESTIMATED DISTANCE TO MINIMUM (EDM) .LT.E10. 22 )

185

120 FORMAT(1H, 34HSIMPLEX MINIMIZATION HAS CONVERGED)

130 FORMAT(1H , 38HSIMPLEX TERMINATES WITHOUT CONVERGENCE) END
SIMPLX 169

SIMPLX 170

SIMPLX 171

SIMPLX 172 
SUBROUTINE STAND

CC

CC

CC
OPTIONAL USER COMMAND "STANDARD" APPEARS.
3

4

5

6

7 
1

10

15

20

40

45

50

55
SUBROUT INE VERMIN(A,L,M,N, IFAIL) INVERTS A SYMMETRIC MATRIX. MATRIX IS -FIRST SCALED TO HAVE ALL ONES ON THE DIAGONAL (EQUIVALENT TO CHANGE OF UNITS) BUT NO PIVOTING IS DONE SINCE MATRIX IS POSITIVE-DEFINITE.

$\mathrm{CC}$

$\mathrm{CC}$

$\mathrm{CC}$

COMMON

1/MINERR/ ERP(30) ,ERN(30)

3/PAREXT/ U(30)

$$
, X T(15)
$$

, $\operatorname{DIRIN}(15)$

, NAM(30)

, WERR(30)

, MAXINT

, $\operatorname{LCODE}(30)$

, MAXEXT

, $\operatorname{BLIM}(30)$

, LCORSP(30), LIMSET

5/VARIAN/ $V(15,15)$

$, X T S(15) \quad, W T S(15) \quad, N P F I X$

$7 / F I X / \operatorname{IPFIX}(15)$

.XS(15)

C

F/DERIVA/ G(30)

$.62(30)$

G/SIMVEC/ P $(15,16)$

J/VARIAT/ VT $(15,15)$

6/UNIT / ISYSRD

8/TITLE / TITLE(13)

9/CONVER/ EPSI ,APSI

A/CARD / CWORD

B/MINIMA/ AMIN

K/COMMND/ NNAME

, PSTAR (15)

,PSTST(15) ,PBAR(15)

,PRHO(15)

, ISYSWR , ISYSPU

,DATE(2) ,ISW(7)

, NSTEPQ

- NBLOCK

- VTEST

,CWORD3

, NFCN

.UP ,NEWMIN ,ITAUR

,WORD7 (7)

. CNAM3(25)

DIMENSION $A(L, M), P P(15), Q(15), S(15)$

IFAIL $=0$

IF (N.LT. 1) GO TO 100

IF (N.GT. MAXINT) GO TO 100

C

SCALE MATRIX BY SQRT OF DIAG ELEMENTS

DO $8 \quad I=1, N$

$\mathrm{SI}=\mathrm{A}(\mathrm{I}, \mathrm{I})$

IF (SI) $100,100,8$

$8 \mathrm{~S}(\mathrm{I})=1.0 / \mathrm{SQRT}(\mathrm{SI})$

DO $20 \mathrm{I}=1, \mathrm{~N}$

DO $20 \mathrm{~J}=1, \mathrm{~N}$

$20 A(I, J)=A(I, J) * S(I) * S(J)$

DO $65 \mathrm{I}=1, \mathrm{~N}$

START MAIN LOOP . . .

$\mathrm{K}=\mathrm{I}$

c

\section{PREPARATION FOR ELIMINATION STEP1}

$Q(K)=1 \cdot / A(K, K)$

$P P(K)=1.0$

$A(K, K)=0.0$

$\mathrm{KP} 1=\mathrm{K}+1$

$K M 1=K-1$

IF (KM1) $100,50,40$

40 DO $49 \mathrm{~J}=1, \mathrm{KM} 1$

$P P(J)=A(J, K)$

$Q(J)=A(J, K) * Q(K)$

$49 A(J, K)=0$.

$50 \operatorname{IF}(K-N) 51,60,100$

51 DO $59 J=K P 1, N$

$\operatorname{PP}(J)=A(K, J)$

$Q(J)=-A(K, J) * Q(K)$

$59 \quad A(K, J)=0.0$

C

60 DO $65 \mathrm{~J}=1, \mathrm{~N}$

DO $65 \mathrm{~K}=\mathrm{J}, \mathrm{N}$

$65 A(J, K)=A(J, K)+P P(J) * Q(K)$
VERMIN 2

VERMIN 3

VERMIN 4

VERMIN 5

VERMIN 6

COMMONU 2

COMMONU 4

COMMONU 5

COMMONU 6

COMMONU 7

COMMONU 8

COMMONU 9

COMMONU 10

COMMONU 11

COMMONU 12

COMMONU 13

COMMONU 27

COMMONU 28

COMMONU 29

COMMONU 30

COMMONU 31

COMMONU 32

VERMIN 9

VERMIN 14

VERMIN 15

VERMIN 16

VERMIN 17

VERMIN 18

VERMIN 19

VERMIN 20

VERMIN 21

VERMIN 22

VERMIN 23

VERMIN 24

VERMIN 25

VERMIN 26

VERMIN 27

VERMIN 28

VERMIN 29

VERMIN 30

VERMIN 31

VERMIN 32

VERMIN 33

VERMIN 34

VERMIN 35

VERMIN 36

VERMIN 37

VERMIN 38

VERMIN $\quad 39$

VERMIN 40

VERMIN 41

VERMIN 42

VERMIN 43

VERMIN 44

VERMIN 45

VERMIN 46

VERMIN 47 
C

60

DO $70 \mathrm{~J}=1, \mathrm{~N}$

DO $70 K=1, J$

VERMIN

$A(K, J)=A(K, J) * S(K) * S(J)$

VERMIN

$70 A(J, K)=A(K, J)$

VERMIN RETURN

VERMIN 53

C

FAILURE RETURN

VERMIN 54

65

100 IFAI $L=1$
RETURN
END

VERMIN

VERMIN 55

VERMIN 56 
1

SUBROUTINE UCOPY $(A, X, N)$

UCOPY

UCOPY

2

DIMENSION $A(N), X(N)$

C COMMENT COPIES $N$ WORDS FROM A INTO $X$, THE BEGINNING

UCOPY

UCOPY

UCOPY

UCOPY

UCOPY

UCOPY

UCOPY

UCOPY

UCOPY

END

$$
X(I)=A(I)
$$

10

100 CONTINUE

RETURN

12 
1

5
SUBROUTINE BINSIZ(A1, A2, NAA, BL, BH, NB, BWID)

SUBROUTINE TO DETERMINE REASONABLE HISTOGRAM INTERVALS GIVEN ABSOLUTE UPPER AND LOWER BOUNDS A1 AND A2 AND DESIRED MAXIMUM NUMBER OF BINS NAA PROGRAM MAKES REASONABLE BINNING FROM BL TO BH OF WIDTH BWID F. JAMES, AUGUST, 1974 TYPED BY E. CLOSE 9/75 FROM LISTING FROM CERN THAT CAME WITH THE CODE.

$A L=A M I N 1(A 1, A 2)$

$A H=A M A X 1(A 1, A 2)$

$I F(A L, E Q, A H) \quad A H=A L+1$.

C IF (NAA.EQ.-1), PROGRAM USES BWID INPUT FROM CALLING ROUTIINE IF (NAA.EQ. -1) GOTO 150

$10 \quad \mathrm{NA}=\mathrm{NAA}-1$

IF (NA.LT.1) NA= 1

C GET NOMINAL BIN WIDTH IN EXPON FORM

$20 \quad A W I D=(A H-A L) / F L O A T(N A)$

$L O G=A L O G 10(A W I D)$

IF (AWID. LE. 1.0) LOG $=\angle O G-1$

SIGFIG $=\operatorname{AWID} *(10.00 * *(-L O G))$

ROUND MANTISSA UP TO $2,2.5,5$, OR 10

IF(SIGFIG .GT . 2.0) GOTO 40

$S I G R N D=2.0$

GOTO 100

40 IF(SIGFIG.GT.2.5) GOTO 50

$S I G R N D=2.5$

GOTO 100

50 IF(SIGFIG.GT.5.0) GOTO 60

$S I G R N D=5.0$

GOTO 100

60 SIGRND $=1.0$

$L O G=L O G+1$

100 CONTINUE

BWID $=$ SIGRND $* 10.0 * *$ LOG

GOTO 200

C GET NEW BOUNDS FROM NEW WIDTH BWID

150 IF(BWID.LE.0.) - GOTO 10

200 CONTINUE

$A L B=A L / B W I D$

$L W I D=A L B$

IF (ALB.LT.0.0) LWID= LWID-1

$B L=B W I D * F L O A T$ (LWID)

$A L B=A H / B W I D+1.0$

$K W I D=A L B$

IF (ALB. LT. Ө.0) KWID = KWID-1

$B H=B W I D * F L O A T(K W I D)$

$\mathrm{NB}=K W I D-L W I D$

IF (NAA.GT.5) GOTO 240

IF (NAA.EQ. -1) RETURN

REQUEST FOR ONE BIN IS DIFFICULT CASE

IF (NAA.GT . 1 .OR. NB .EQ. 1) RETURN

$B W I D=B W I D * 2.0$

$\mathrm{NB}=1$

RETURN

240 IF (2*NB.NE. NAA) RETURN

$\mathrm{NA}=\mathrm{NA}+1$

GOTO 20
BINSIZ 2

BINSIZ 3

BINSIZ 4

BINSIZ 5

BINSIZ 6

BINSIZ 7

BINSIZ 8

BINSIZ 9

BINSIZ 10

BINSIZ 11

BINSIZ 12

BINSIZ 13

BINSIZ 14

BINSIZ 15

BINSIZ 16

BINSIZ 17

BINSIZ 18

BINSIZ 19

BINSIZ 20

BINSIZ 21

BINSIZ 22

BINSIZ 23

BINSIZ 24

BINSIZ 25

BINSIZ 26

BINSIZ 27

BINSIZ 28

BINSIZ 29

BINSIZ 30

BINSIZ 31

BINSIZ 32

BINSIZ 33

BINSIZ 34

BINSIZ 35

BINSIZ 36

BINSIZ 37

BINSIZ 38

BINSIZ $\quad 39$

BINSIZ 40

BINSIZ 41

BINSIZ 42

BINSIZ 43

BINSIZ 44

BINSIZ 45

BINSIZ 46

BINSIZ 47

BINSIZ 48

BINSIZ 49

BINSIZ 50

BINSIZ 51

BINSIZ 52

BINSIZ 53

BINSIZ 54

BINSIZ 55

BINSIZ 56

BINSIZ 57

BINSIZ 58 
B NL SCOPE $2.1 .5 \quad 5908 B$

SYS DEVICES 844/14/PF. FLS=200K FLL=1747K MXS=160K MXL=1301K MXB=1301B

HH.MM.SS CPU SECOND ORIGIN

16:47:41 CVX STATION SYNLSGU

30 "COURANT $":$ : DUA1: [COURANT.SYN.PROG]SYNLIST. OUT

16.50.17 00000.003 MFZ. -SYNLS, STMFZ,T77.

16.50.17 00000.003 JOB. -ACCOUNT, COURANT, 3,*.

16.50.2200000.027 JOB. -ATTACH, OLDPL, BSY86, ID=EDC.

16.50.25 $00000.003 \mathrm{MFZ} . \quad$-SJRSN, T400, P7000, SN.

16.50.40 00000.109 LOD. -DISPLAY,R1.

16.50 .4200000 .113 USR. $\theta \quad 0 B$

16.50.42 00000.113 LOD. -PRELOD. SET UP TO RETRIEVE FILE

16.50.44 00000.125 JOB. -LOADPF, RET.

RETRIEVE FILE

$16.50 .4500000 .129 \mathrm{MFZ}$.

$16.54 .32 \mathrm{MFC}$.

JM260 - STAGE NT IN LFN=DUMTAPE VSN=0X9242

16.54.32 MFC.

16.54.32 MFC.

16.48.33. VSN (DUMTAPE $=\times 9242)$

16. 49.33. REQUEST (DUMTAPE, NT, PE, US, NORING , U, E, S, VSN=0X9242)

16.54.32 MFC.

$16.54 .32 \mathrm{MFC}$.

16.49.33. ( NT 041 ASSIGNED)

16.49.33.-SP- PRE STAGING (PARTIAL)

16.54.32 MFC.

16.54.33 MFC.

16.54.33 STP.

16.54 .3500000 .133 USR.

16.54 .3500000 .156 USR.

16.54 .3500000 .157 USR.

16.54 .4300000 .312 USR.

16.54 .4300000 .312 USR.

16.54 .4300000 .313 USR.

16.54 .4300000 .313 USR.

16.49.33. NT:41 VOLUME SERIAL NUMBER IS $0 \times 9242$

16.52.16.-SP- SKIPPED 3234, STAGED 167, BLOCK(S)

16.52.16.NO. WORDS $=85478$ NO. OF EOR $=1$

JM511 - WORDS READ- ' 247254B

PFLOD - BEGIN FILE DUMTAPE RECOVERY

PFLOD - - LOAD BEGINS AGAIN $\longrightarrow$ OBT

PFLOD - LOADING BEGINS WITH FILE 112

PFLOD - $\quad$ EDC CYCLE 1 BSY86

PFLOD - 1 PERMANENT FILES LOADED

PFLOD - LOADPF ERROR COUNT 0

PFLOD - END LOADPF

16.54.4300000.314 LOD. -SET, R2=0.

16.54.43 00000.318 JOB. -EXIT(U)

16.54.52 $00000.038 \mathrm{MFZ}$.

16.54 .5200000 .038 LOD.

16.55 .0000000 .625 USR.

16.55.00 00000.627 USR.

16.55.01 00000.628 LOD.

16.58 .0400003 .759 USR.

16.58 .0400003 .760 LOD.

16.58 .4700008 .508 USR.

16.58 .4800008 .510 USR.

16.58.48 00008.511 LOD.

17.17 .2100041 .459 USR.

17.17.2100041.461 MFZ.

17.17.21 $00041.461 \mathrm{MFZ}$.

17.17.21 $00041.461 \mathrm{MFZ}$.

$17.17 .2100041 .461 \mathrm{MFZ}$.

$17.17 .2100041 .461 \mathrm{MFZ}$.

17.17.21 $00041.462 \mathrm{MFZ}$.

17.17.21 00041.462 MFZ.

17.17.21 $00041.462 \mathrm{MFZ}$.

17.17.21 $00041.462 \mathrm{MFZ}$.

17.17.21 00041.462 MFZ.

17.17.21 00041.463 MFZ.

17.17.21 $00041.463 \mathrm{MFZ}$.

$7.17 .2100041 .463 \mathrm{MFZ}$.

$17.17 .2100041 .463 \mathrm{MFZ}$.

$17.17 .2100041 .463 \mathrm{MFZ}$.

17.17.21 $00041.464 \mathrm{MFZ}$.
PF254 - CYCLE 1 ATTACHED FROM SN=SYSTEM

-UPDATE, $Q, L=1$.

3 NON-FATAL ERRORS

UPDATE COMPLETE.

$-F T N, I, P, R=0$.

3.127 CP SECONDS COMPILATION TIME

-UPDATE, $Q, L=1$.

3 NON-FATAL ERRORS

UPDATE COMPLETE.

$-F T N, I, P, R=0$.

32.945 CP SECONDS COMPILATION TIME

JM166 - MAXIMUM USER SCM

JM167 - MAXIMUM USER LCM

JM170 - MAXIMUM JS+IO LCM

RM770 - MAXIMUM ACTIVE FILES

RM771 - OPEN/CLOSE CALLS

RM772 - DATA TRANSFER CALLS

RM773 - CONTROL/POSITIONING CALLS

RM774 - BM DATA TRANSFER CALLS

RM775 - BM CONTROL/POSITIONING CALLS

$47000 B$ WORDS

20000B WORDS

103B BUFFERS

RM776 - QUEUE MANAGER CALLS

RM777 - RECALL CALLS

$\operatorname{SCM}$

827.165 KWS

LCM

19.912 KWS

I $/ O$

$1.619 \mathrm{MW}$

RMS

14.211 MWS

USER

$$
\begin{array}{r}
4 \\
70 \\
233,290 \\
3,433 \\
27,315 \\
2,006 \\
5,145 \\
4,620
\end{array}
$$


17.-17.21 00041.464 MFZ.

17.17.21 00041.464 MFZ.

17.17.21 $00041.464 \mathrm{MFZ}$.

17.17.21 $00041.464 \mathrm{MFZ}$.

17.17.21 $00041.465 \mathrm{MFZ}$.

2. 17.2100041.465 MFZ.

17.17.21, 00041.465 MFZ.

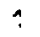

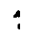

4

i

$\therefore$
JOB

DIO

RU

$14412.857 \mathrm{KW}$

BNL CCUS

108.287

CCUS COMPUTED AT UPPER TIER RATE

SC050 - 007376 SC/LC SWAPS

EJ END OF JOB - PNOOOOO3 
SUBROUTINE SSSR $(M, P, K F, K G)$

SETS UP MATRICES FOR A LONG(PI) OR COLLINS(PI/2) STRAIGHT SECTIONS MATRICES GENERATED AS FOLLOWS

QF = FOCUSSING MAGNET AT M-1.

$\mathrm{QD}=$ DEFICUSSING MAGNET AT M-2.

$\mathrm{QS}=$ END DRIFT SPACE (HALF CENTER DRIFT SPACE) AT M-3 (PI)

QS = HALF CENTER DRIFT SPACE AT M-4 (COLLINS), AND THE STRAIGHT SECTION MAGNET Q AT $M$.

QT = DRIFT SPACE BETWEEN F AND D MAGNETS AT M-4 (PI), AND THE STRAIGHT SECTION MAGNET Q AT $M$.

$\mathrm{QE}=$ END DRIFT SPACE AT $M-3$ (COLLINS)

$Q$ CAN TAKE EITHER OF TWO FORMS DEPENDING ON VALUE OF KF.

IF $K F=1, Q=$ PRODUCT OF ..QQS QD QT QF QS QS QD QT QF QS. (DFDF) IF $K F=2, Q=P R O D U C T$ OF ...QS QF QT QD QS QS QF QT QD QS. (FDFD) FOR LONG (PI) STRAIGHT SECTION FOR COLLINS STRAIGHT SECTION

IF $K F=1, Q=P R O D U C T$ OF $\ldots Q E$ QD QS QS QF QE, (DF)

IF $K F=2, Q=P R O D U C T$ OF $\ldots Q E$ QF $Q S$ QS QD QE. (FD)

COMMON/BMI/MI (5000)

COMMON/NELS/NELS

LEVEL 2,MI

EQUIVALENCE (E,T)

LOGICAL COLL

DIMENSION $P(6)$

C

COLL $=$.FALSE.

IF (KG.EQ.1) COLL $=$. TRUE.

C SET UP FOR PI SECTION FIRST

$S=P(4) / 2$.

$\mathrm{T}=\mathrm{P}(5)$

$P(2)=A B S(P(2))$

$P(4)=0$.

$P(5)=0$.

$P(6)=0$.

C SET UP QF

$$
\mathrm{L}=\mathrm{M}-1
$$

CALL STDAT $(L, 1,1,6, P)$

CALL MAGNET $(L, P)$

$M I(4)=L$

C SET UP QD

$$
L=M-2
$$

CALL STDAT $(L, 1,1,6, P)$

CALL EXCHM( $L+1, L)$

$M I(2)=L$

C SET UP QS

$$
L=M-3
$$

CALL DRIFT $(L, S)$

$M I(1)=L$

$M I(5)=L$

C SET UP QT OR QE (T IS REALLY E IF COLLINS)

$L=M-4$

CALL DRIFT $(L, T)$

$\mathrm{MI}(3)=\mathrm{L}$

IF (KF.EQ.1) GO TO 2
SSSR $\quad 2$

SSSR

SSSR

SSSR

SSSR

SSSR

SSSR

SSSR

SSSR

SSSR

SSSR

SSSR

SSSR

SSSR

SSSR

SSSR

SSSR

SSSR

SSSR

BMIL

BMIL

BMI L

BMIL

BMI

SSSR

SSSR

SSSR

SSSR

SSSR

SSSR

SSSR

SSSR

SSSR

SSSR

SSSR

SSSR

SSSR

SSSR

SSSR

SSSR

SSSR

SSSR

SSSR

SSSR

SSSR

SSSR

SSSR

SSSR

SSSR

SSSR

SSSR

SSSR

SSSR

SSSR

SSSR

SSSR

SSSR 
60

C AN FD OR FDFD

$$
\begin{aligned}
& M I(2)=M-1 \\
& M I(4)=M-2
\end{aligned}
$$

2 IF (COLL) GO TO 4

C COMPLETE MI LIST

DO $3 \mathrm{I}=1,5$

$3 \quad M I(I+5)=M I(I)$

65

$\mathrm{IM}=10$

GO TO 5

C MAKE CHANGES FOR COLLINS STRAIGHT SECTION

$4 \quad$ ISAV $=$ MI (1)

$\operatorname{MI}(1)=\operatorname{MI}(3)$

70

$M I(3)=I S A V$

$\mathrm{ISAV}=\mathrm{MI}(4)$

$\operatorname{MI}(4)=\operatorname{MI}(5)$

$M I(5)=$ ISAV

$M I(6)=M I(1)$

75

$I M=6$

$5 \quad L=L M$

CALL MMM(L, IM, MI)

C SAVE THE MILIST AS DATA FOR $Q$

$\begin{array}{ll}\text { SSSR } & 55 \\ \text { SSSR } & 56 \\ \text { SSSR } & 57 \\ \text { SSSR } & 58 \\ \text { SSSR } & 59 \\ \text { SSSR } & 60 \\ \text { SSSR } & 61 \\ \text { SSSR } & 62 \\ \text { SSSR } & 63 \\ \text { SSSR } & 64 \\ \text { SSSR } & 65 \\ \text { SSSR } & 66 \\ \text { SSSR } & 67 \\ \text { SSSR } & 68 \\ \text { SSSR } & 69 \\ \text { SSSR } & 70 \\ \text { SSSR } & 71 \\ \text { SSSR } & 72 \\ \text { SSSR } & 73 \\ \text { SSSR } & 74 \\ \text { SSSR } & 75 \\ \text { SSSR } & 76 \\ \text { SSSR } & 77 \\ \text { SSSR } & 78 \\ \text { SSSR } & 79 \\ \text { SSSR } & 80\end{array}$


SUBROUTINE STAR $(M, K A)$

C THE MATRIX $M=$ MATRIX $N$ RAISED TO POWER $K(M=N * * K)$

$\begin{array}{lr}\text { STAR } & 2 \\ \text { STAR } & 3 \\ \text { STAR } & 4 \\ \text { BMIL } & 1 \\ \text { BMIL } & 2 \\ \text { BMIL } & 3 \\ \text { BMIL } & 4 \\ \text { BMI } & 3 \\ \text { STAR } & 6 \\ \text { STAR } & 7 \\ \text { STAR } & 8 \\ \text { STAR } & 9 \\ \text { STAR } & 10 \\ \text { STAR } & 11 \\ \text { STAR } & 12\end{array}$

15

COMMON/BMI/MI (5000)

COMMON/NELS/NELS

LEVEL 2,MI

$N=\operatorname{MDAT}(M, 1)$

10

DO $20 \quad I=1, K A$

$M I(I)=N$

20

CONT INUE

$\operatorname{CALL} M M M(M, K A, M I)$

RETURN

END 
SUBROUTINE STDAT $(M, I, J, K, A)$

C REPLACES $K$ DATA OF TYPE I FROM ARRAY A INTO INFF $(J, M)$

STDAT

STDAT

STDAT

C IF I $=4$, SKIP. IF I=5, STORE AT LQ

IF $I=3$, INTEGER.

5

C IF $I=7$, STORE AT LQ2. IF $I=8$, STORE AT LQ3

STDAT

STDAT

LEVEL 2, STORE, INFF, IWORK

BLANK

BLANK

COMMON STORE (48000), IWORK (10)

86MARSIZ

DIMENSION INFF $(24,2000)$

10

EQUIVALENCE (INFF, STORE)

86MARSIZ

86MARSIZ

BLANK

DIMENSION $A(1), \operatorname{LOC}(10)$

STDAT

IF (I.EQ.4) RETURN

STDAT

$\operatorname{LOC}(1)=\operatorname{INFF}(14, M)$

STDAT

$\operatorname{LOC}(2)=\operatorname{INFF}(16, M)$

$\operatorname{LOC}(3)=\operatorname{INFF}(12, M)$

STDAT

$\operatorname{LOC}(5)=\operatorname{INFF}(10, M)$

STDAT

STDAT

$\operatorname{LOC}(7)=\operatorname{INFF}(19, M)$

STDAT

$\operatorname{LOC}(8)=\operatorname{INFF}(24, M)$

20

$\operatorname{LOCI}=\operatorname{LOC}(\mathrm{I})+\mathrm{J}-2$

STDAT

STDAT

DO $10 \mathrm{~L}=1, \mathrm{~K}$

STDAT

LOCI $=$ LOCI 1

$10 \operatorname{STORE}(L O C I)=A(L)$

RETURN

25

END

STDAT

STDAT

STDAT

STDAT

2
3
4
5
6
2
3
1
2
3
5
8
9
10
1
1
3
4
15
16
17
18
19
10
11




$\begin{array}{lr}\text { STINFO } & 2 \\ \text { STINFO } & 3 \\ \text { STINFO } & 4 \\ \text { STINFO } & 5 \\ \text { STINFO } & 6 \\ \text { STINFO } & 7 \\ \text { STINFO } & 8 \\ \text { STINFO } & 9 \\ \text { BLANK } & 2 \\ \text { BLANK } & 3 \\ \text { 86MARSIZ } & 1 \\ \text { 86MARSIZ } & 2 \\ \text { 86MARSIZ } & 3 \\ \text { BLANK } & 5 \\ \text { STINFO } & 11 \\ \text { STINFO } & 12 \\ \text { STINFO } & 13 \\ \text { STINFO } & 14 \\ \text { STINFO } & 15 \\ \text { STINFO } & 16 \\ \text { STINFO } & 17 \\ \text { STINFO } & 18 \\ \text { STINFO } & 19 \\ \text { STINFO } & 20 \\ \text { STINFO } & 21 \\ \text { STINFO } & 22 \\ \text { STINFO } & 23\end{array}$

LEVEL 2, STORE, INFF, IWORK

COMMON STORE(48000), IWORK(10)

DIMENSION INFF $(24,2000)$

EQUIVALENCE (INFF, STORE)

15

20

25
INTEGER OPNUM, OPNAMO

IF (MO) $2,1,1$

$1 \operatorname{INFF}(2, M \theta)=\operatorname{NAMEO}$

GO TO 3

$2 M \theta=-M \theta$

$\operatorname{ENCODE}(10,102, \operatorname{INFF}(2, \mathrm{MO}))$ NAME1, NAMEO

102 FORMAT (A1, A4, 5X)

$3 \quad \operatorname{INFF}(1, M O)=$ OPNAMO

$\operatorname{INFF}(3, M 0)=$ OPNUM(OPNAMO)

$\operatorname{INFF}(4, M \theta)=K A \theta$

$\operatorname{INFF}(5, M \theta)=K B \theta$

RETURN

END

8


20

$$
5 \text { CONTINUE }
$$

30

C CHECK STORAGE

35

LEVEL 2, STORE, INFF, IWORK

COMMON STORE (48000), IWORK(10)

DIMENSION INFF $(24,2000)$

EQUIVALENCE (INFF, STORE)

COMMON/CONTRL/ERROR, MODE, RSRV, STOR, XEQ, TRASW, NFSW, EMPTY, INDEF, LDFLG, FIN

LOGICAL ERROR, RSRV, STOR, XEQ, TRASW, NFSW, EMPTY, INDEF, LDFLG, FIN

COMMON /DIM/ LEND, MEND, ISAV, ISAV7, M7END, KADD, KADDR, MUNIT, MSYMPL

DIMENSION $T(49), \operatorname{TINV}(49), \operatorname{RX}(2,3), \operatorname{RY}(2,3), \operatorname{RW}(3), \operatorname{RPQ}(4)$

$$
\begin{array}{ll}
\operatorname{IF}(M) 4,10,10 \\
\\
M=-M \\
\text { CALL TINV7(TINV, T) }
\end{array}
$$

$$
\mathrm{RW}(1)=-\mathrm{RW}(1)
$$$$
\mathrm{RW}(2)=-\mathrm{RW}(2)
$$$$
\operatorname{RW}(3)=\operatorname{TINV}(40)
$$$$
\text { DO } 5 \mathrm{I}=1,49
$$$$
T(I)=\operatorname{TINV}(I)
$$

$10 L Q=\operatorname{INFF}(10, M)$

$N Q=\operatorname{INFF}(11, M)$

$\operatorname{KIND}=\operatorname{INFF}(20, M)$

IF (KIND.EQ.3HMXY) GO TO 11

$N Q Q=49+K A D D$

IF (NQQ.GT.NQ) GO TO 45

CALL STDAT $(M, 5,1,3, \mathrm{RW})$

$$
N J=L Q+K A D D-1
$$

STOR7

STOR7

BLANK

BLANK

86MARSIZ

86MARSIZ

86MARSIZ

BLANK

CONTRL

CONTRL

CONTRL

CONTRL

DIM

DIM

STOR7

STOR7

STOR7

STOR7

STOR7

STOR7

STOR7

STOR7

STOR7

STOR7

STOR7

STOR7

STOR7

STOR7

STOR7

STOR7

STOR7

STOR7

STOR7

STOR7

STOR7

STOR7

STOR7

STOR7

STOR7

STOR7

STOR7

REQUEST EXCEEDS STORAGE RESERV STOR7

STOR7

STOR7

STOR7

STOR7

STOR7

STOR7
3

2

3

1

2

3

5

2

3

4

5

2 
SUBROUTINE STRFCN ( $X$, BKDIF)

C FUNCTION EVALUATION FOR STR4 INSTRUCTION C

COMMON/WRK/BZ, BQ, AZ, DPSI , SM, SL, PSI , WW, WT , BETW,P, Q,

$$
1 \quad T, U, B K Z, B K Z P, C Z, S Z, C H Z B, S H Z B, P H I, B K, S A, B L, B M \text {, }
$$$$
2 A Q, R H O, B E T S, P S I W S, B K Z I, B K Z P I, G(6), A B, C, S, C H B \text {, }
$$$$
3 \text { SHB, E, EI , SZCB, SZBC, CZBC, CZCB, SZSB, SZBS, CZBS, CZSB, }
$$

C

$4 \quad Z W, Z T, B K I, Y, Z, Y 1, Y 2, Y 3, Y 4$

$$
\begin{aligned}
& \mathrm{PHI}=\mathrm{X} \\
& \mathrm{C}=\cos (\mathrm{PHI}) \\
& \mathrm{S}=\operatorname{SIN}(\mathrm{PHI}) \\
& \mathrm{E}=\operatorname{EXP}(\mathrm{PHI})
\end{aligned}
$$$$
\text { EQUIVALENCE (BKS, BZ) }
$$$$
E I=1.0 / E
$$$$
\mathrm{SHB}=0.5 *(E-E I)
$$$$
\mathrm{CHB}=0.5 *(E+E I)
$$

C

$$
\mathrm{SZCB}=\mathrm{SZ} * \mathrm{CHB}
$$$$
S Z B C=S H Z B * C
$$$$
\mathrm{CZBC}=\mathrm{CHZB} * \mathrm{C}
$$$$
\mathrm{CZCB}=\mathrm{CZ} * \mathrm{CHB}
$$$$
S Z S B=S Z * S H B
$$$$
S Z B S=S H Z B * S
$$$$
\mathrm{CZBS}=\mathrm{CHZB} * \mathrm{~S}
$$$$
\mathrm{CZSB}=\mathrm{CZ} * \mathrm{SHB}
$$

c

$$
\begin{aligned}
Z W= & B K Z * S Z S B+B K Z P * S Z B S * P-T *(C Z B S+B K Z P * S L * S Z B S) \\
Z T= & -(C Z S B+U * C Z B S)+S L *(B K Z * S Z S B-B K Z P * U * S Z B S) \\
& +Q * B K Z P * S Z B S
\end{aligned}
$$$$
1
$$$$
Y 1=B K Z * S Z C B-B K Z P * P * S Z B C+T *(C Z B C+B K Z P * S L * S Z B C)
$$$$
Y 2=C Z C B-U * C Z B C-S L *(B K Z * S Z C B+B K Z P * U * S Z B C)+Q * B K Z P * S Z B C
$$$$
Y 3=C Z S B-P * C Z B S+T *(B K Z P I * S Z B S+S L * C Z B S)
$$$$
Y 4=-B K Z I * S Z S B-B K Z P I * U * S Z B S-S L *(C Z S B+U * C Z B S)+Q * C Z B S
$$$$
B K=(Y 1 * Z T+Y 2 * Z W) /(Y 3 * Z T+Y 4 * Z W)
$$$$
B K I=1.0 / B K
$$$$
\begin{array}{rl}
S A & =(C Z S B-P * C Z B S-B K I *(B K Z * S Z C B-B K Z P * P * S Z B C)- \\
1 & T *(B K I * C Z B C-B K Z P I * S Z B S-S L * C Z B S+B K Z P * B K I * S L
\end{array}
$$$$
T *(B K I * C Z B C-B K Z P I * S Z B S-S L * C Z B S+B K Z P * B K I * S L * S Z B C))
$$$$
/ Z W
$$$$
2
$$$$
B L=(C Z C B-P * C Z B C-B K I *(B K Z * S Z S B+B K Z P * P * S Z B S)-
$$$$
S A *(B K Z * S Z C B+B K Z P * P * S Z B C)+T *(S Z B C * B K Z P I+B K I * C Z B S+
$$$$
(S L+S A) * C Z B C+B K Z P * B K I * S L * S Z B S+B K Z P * S L * S A * S Z B C)) /
$$

\begin{tabular}{|c|c|}
\hline STRFCN & \\
\hline STRFCN & \\
\hline WRK & \\
\hline WRK & \\
\hline WRK & \\
\hline WRK & \\
\hline WRK & \\
\hline WRK & \\
\hline WRK & \\
\hline STRFCN & \\
\hline STRFCN & \\
\hline STRFCN & \\
\hline STRFCN & \\
\hline STRFCN & \\
\hline STRFCN & \\
\hline STRFCN & \\
\hline STRFCN & \\
\hline STRFCN & \\
\hline STRFCN & \\
\hline STRFCN & \\
\hline STRFCN & \\
\hline STRFCN & \\
\hline STRFCN & \\
\hline STRFCN & \\
\hline STRFCN & \\
\hline STRFCN & \\
\hline STRFCN & \\
\hline STRFCN & \\
\hline STRFCN & \\
\hline STRFCN & \\
\hline STRFCN & \\
\hline STRFCN & \\
\hline STRFCN & \\
\hline STRFCN & \\
\hline STRFCN & \\
\hline STRFCN & \\
\hline STRFCN & \\
\hline STRFCN & \\
\hline STRFCN & \\
\hline STRFCN & \\
\hline STRFCN & \\
\hline STRFCN & \\
\hline STRFCN & \\
\hline STRFCN & \\
\hline STRFCN & \\
\hline STRFCN & \\
\hline STRFCN & \\
\hline STRFCN & \\
\hline STRFCN & \\
\hline STRFCN & \\
\hline
\end{tabular}$$
(B K *(B K I *(B K Z * S Z C B+B K Z P * P * S Z B C)-(C Z S B+P * C Z B S)
$$$$
+S A *(B K Z * S Z S B-B K Z P * P * S Z B S)+T *(-B K I * C Z B C+
$$$$
B K Z P I * S Z B S-B K I * B K Z P * S L * S Z B C+(S A+S L) * C Z B S+
$$$$
B K Z P * S A * S L * S Z B S)) \text { ) }
$$

$B M=P H I / B K$

$B K D I F=B K-B K S$

RETURN

END 
SUBROUTINE STRN( $M, N C E L L, P A R$ )

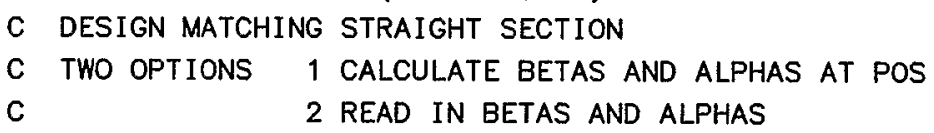

C

DIMENSION RW(3)

DIMENSION NCELL(3),PAR( (8), BX(9), BY(9), RX(2,3), RY(2,3), $\operatorname{PX}(2,2)$, 1 $\operatorname{PY}(2,2), U X(2,3), U Y(2,3)$

INTEGER C,BLBX,POS, BDAT

REAL LZ, LTOT

DATA EPSK, ITMX/.0000001,25/

DATA IFO, IDFO, IBL/9HFOCUSSING, 2HDE, 2H /

30

35

40

45

50
C

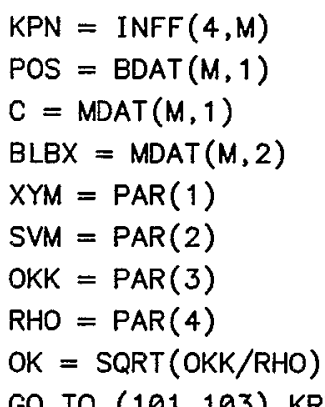

$101 \mathrm{CALL} B E T(C, B X, B Y, 0)$

GO TO 110

C GET BETAS AND ALPHAS FROM INPUT

$103 \mathrm{BX}(2)=\operatorname{PAR}(5)$

$\operatorname{BY}(2)=\operatorname{PAR}(6)$

$\operatorname{BX}(3)=\operatorname{PAR}(7)$

$\operatorname{Br}(3)=\operatorname{PAR}(8)$

$\mathrm{BX}(4)=(1 .+\mathrm{BX}(3) * \mathrm{BX}(3)) / \mathrm{BX}(2)$

$B Y(4)=B X(4)$

110 CALL HED

WRITE $(3,501)$ POS

WRITE $(3,502) \quad X Y M, O K K, R H O, B X(2), B Y(2), B X(3), B Y(3)$

$X Y M=X Y M * 6.283185307$

C XYM IS NOW PSI OF WRITE UP

$B X(1)=X Y M$

$B Y(1)=X Y M$

$\operatorname{CSI}=\cos (X Y M)$
STRN 2

STRN

STRN

STRN

STRN

\section{STRN}

BLANK

BLANK

86MARSIZ

86MARSIZ

86MARSIZ

BLANK

BMIL

BMIL

BMIL

BMIL

BMI

STRN

STRN

STRN

STRN

STRN

STRN

STRN

STRN

STRN

STRN

STRN

STRN

STRN

STRN

STRN

STRN

STRN

STRN

STRN

STRN

STRN

STRN

STRN

STRN

STRN

STRN

STRN

STRN

STRN

STRN

STRN

STRN

STRN

STRN

STRN

STRN 
SSI $=\operatorname{SIN}(X Y M)$

C COMPUT PX AND PY (P AND Q). PY IS $Q$ OF WRITE UP

RBX $=$ SQRT $(B X(2))$

RBY $=\operatorname{SQRT}(B Y(2))$

$\operatorname{PX}(1,1)=(\mathrm{RBY} *(\mathrm{CSI}+\mathrm{BX}(3) * \mathrm{SSI})) / \mathrm{RBX}$

$P X(1,2)=R B X * R B Y * S S I$

$\operatorname{PX}(2,1)=((B X(3) * B Y(3)-1) * S S I+.(B X(3)+B Y(3)) * C S I) /(R B X * R B Y)$

65

$\operatorname{PX}(2,2)=(\mathrm{RBX} *(\mathrm{CSI}+\mathrm{BY}(3) * \mathrm{SSI})) / \mathrm{RBY}$

$\operatorname{PY}(1,1)=\operatorname{PX}(2,2)$

$\operatorname{PY}(2,2)=\operatorname{PX}(1,1)$

$\operatorname{PY}(1,2)=\operatorname{PX}(1,2)$

$\operatorname{PY}(2,1)=\operatorname{PX}(2,1)$

C GET KIA (UX,UY) (U AND $V$ OF WRITE UP). STORE KIA IN $M-1$

CALL RXY (BLBX, RX,RY,RW)

$L=M-1$

C

OMEG $=0$.

CALL REPFLT $(L, 4$, OMEG)

CALL STXY(L,RX,RY,RW)

$L Z=R W(1)$

DO $160 \mathrm{~J}=1,2$

DO $158 \quad I=1,2$

80

$\operatorname{UX}(I, J)=\operatorname{RX}(I, J)$

$\operatorname{UY}(I, J)=\operatorname{RY}(I, J)$

158 CONTINUE

160 CONTINUE

C COMPUT KIB $=W I=W X, W Y(O L D, W I X, W I Y)$

85

$$
L=M-2
$$

CALL REF ( $L, B L B X)$

CALL RXY ( L, RX, RY, RW)

DO $164 \mathrm{I}=1,2$

DO $162 \mathrm{~J}=1,2$

90

$\operatorname{RXS}=\operatorname{RX}(I, J)$

$R X(I, J)=\operatorname{RY}(I, J)$

$162 \operatorname{RY}(I, J)=\operatorname{RXS}$

164 CONTINUE

CALL REPFLT $(L, 4$, OMEG)

95

CALL STXY (L,RX,RY,RW)

C SET UP ITERATION FOR LENGTH

$N I T=1$

$O M=S V M$

$\mathrm{DCS}=0$.

100

$D E L M=S V M / 5$.

$\mathrm{OMM}=0$.

SOK $=1$.

185

$\mathrm{PHIO}=\mathrm{OK}=\mathrm{OM}$

$\mathrm{CS}=\cos (\mathrm{PHIO})$

105

$S N=\operatorname{SIN}(P H I O)$

$E P=\operatorname{EXP}(\mathrm{PHIO})$

$E M=1 . / E P$

$C B=(E P+E M) / 2$.

$S B=C B-E M$

110

C COMPUTE $F$ AND $G$

C TRY D MAGNET FOR KA

SOK $=1$.

$F 1=\operatorname{SB} * U X(1,1)+P X(2,2) * \operatorname{SN} * U Y(1,1)$

$F 2=-P X(2,1) * \operatorname{SN} * \operatorname{UY}(1,1)$

STRN

STRN

STRN

STRN

STRN

STRN

STRN

STRN

STRN

STRN

STRN

STRN

STRN

STRN

STRN

STRN

STRN

STRN

STRN

STRN

STRN

STRN

STRN

STRN

STRN

STRN

STRN

STRN

STRN

STRN

STRN

STRN

STRN

STRN

STRN

STRN

STRN

STRN

STRN

STRN

STRN

STRN

STRN

STRN

STRN

STRN

STRN

STRN

STRN

STRN

STRN

STRN

STRN

STRN

STRN

STRN
50

51

52

53

54

55

56

57

58

59

60

61

62

63

64

65

66

.67

68

69

70

71

72

73

74

75

76

77

78

79

80

81

82

83

84

85

86

87

88

89

90

91

92

93

94

95

96

97

98

99

100

101

102

103

104

105

106 


$$
\begin{aligned}
& F 3=\operatorname{CB} * U X(2,1)-P X(2,2) * \operatorname{CS} * \operatorname{UY}(2,1) \\
& F 4=\operatorname{PX}(2,1) * \operatorname{CS} * \operatorname{UY}(2,1)
\end{aligned}
$$$$
G 1=\operatorname{SB} * U X(1,1)-P X(1,1) * \operatorname{SN} * \operatorname{UY}(1,1)+P X(2,1) * \operatorname{SN} * \operatorname{UY}(1,2)
$$$$
G 2=\operatorname{SB} * U X(1,2)+P X(2,2) * \operatorname{SN} * U Y(1,2)-P X(1,2) * \operatorname{SN} * U Y(1,1)
$$$$
\mathrm{G} 3=\mathrm{CB} * \operatorname{UX}(2,1)+P X(1,1) * \operatorname{CS} * \operatorname{UY}(2,1)-P X(2,1) * \operatorname{CS} * \operatorname{UY}(2,2)
$$

$207 \quad A F G=F 1 * G 1+F 2 * G 2$

$B F G=(F 1 * G 3+F 3 * G 1+F 2 * G 4+F 4 * G 2) * S O K$

$\mathrm{CFG}=\mathrm{F3} * \mathrm{G} 3+F 4 * \mathrm{G} 4$

$\mathrm{DCS}=\mathrm{BFG} * \mathrm{BFG}-4 . * A F G * C F G$

IF (SOK.LT.O..OR.DCS.GE.0.) GO TO 2085

C OTHERWISE TRY $F$ MAGNET FOR KA

STRN

$\mathrm{SS}=\mathrm{SN}$

130

$\mathrm{SN}=\mathrm{SB}$

$\mathrm{SB}=\mathrm{SS}$

$\mathrm{SS}=\mathrm{CS}$

$\mathrm{CS}=\mathrm{CB}$

$\mathrm{CB}=\mathrm{SS}$

135

GO TO 196

2085 DEN $=1 . * A F G$

IF (DCS.LT.O.) GO TO 2086

DIS = SQRT (DCS)

140

C COMPUTE K(MO) CALLED TK

STRN

STRN

$\mathrm{TK}=0$.

$T K P=(-B F G+D I S) / D E N$

IF (TKP.GE.0.) GO TO 2185

STRN

IF (SOK.LT.O.) GO TO 220

STRN

GO TO 2077

145

$2086 \mathrm{OMM}=\mathrm{OMM}+\mathrm{DELM}$

$O M=O M M$

$\mathrm{NIT}=\mathrm{NIT}+1$

GO TO 185

2185 TK $=$ TKP

150

$T K M=(-B F G-D I S) / D E N$

IF (TKM.LE.O.) GO TO 220

$D K P=A B S(O K-T K P)$

$D K M=A B S(O K-T K M)$

IF (DKM.LT.DKP) TK=TKM

155

C COMPARE TK AND OK,NIT AND ITMX

STRN

135

STRN

136

STRN

137

STRN

138

STRN

139

STRN

140

STRN

141

STRN

142

STRN

STRN

STRN

STRN

STRN

$220 \mathrm{DK}=\mathrm{TK}-\mathrm{OK}$

$A D=A B S(D K)$

IF (AD.LE.EPSK.OR.NIT.GE.ITMX) GO TO 313

STRN

STRN

STRN

STRN

STRN

STRN

STRN

STRN

STRN

STRN

STRN

STRN

311

IF (NIT.EQ.1) GO TO 311

STRN

STRN

STRN

STRN 
C COMPUTE MAGNET KB,KA. STORE IN $M-3, M-4$ $313 \mathrm{BX}(1)=\mathrm{OM}$

$3131 \mathrm{BX}(2)=$ SOK*OKK

$\mathrm{BX}(3)=\mathrm{RHO}$

$\mathrm{BX}(4)=0$.

$\mathrm{BX}(5)=0$.

$B X(6)=0$.

$\mathrm{L}=\mathrm{M}-3$

180

CALL STDAT $(L, 1,1,6, B X)$

CALL MAGNET (L,BX)

$B X(2)=-S O K * O K K$

$L=M-4$

CALL $\operatorname{STDAT}(L, 1,1,6, B X)$

185

CALL MAGNET ( $L, B X)$

$M I(1)=M-1$

$M I(2)=M-4$

$\mathrm{L}=\mathrm{M}-5$

CALL REPFLT (L, 4, OMEG)

CALL MMM $(L, 2, M I)$

C UX AND UY ARE NOW TO BE $X, Y$ PARTITIONS OF WD(OLD UIJ,VIJ)

CALL RXY ( L, UX,UY, RW)

STRN

STRN

STRN

STRN

STRN

STRN

STRN

STRN

STRN

STRN

STRN

STRN

$L=M-6$

$M I(1)=M-2$

STRN

$M I(2)=M-3$

STRN

CALL REPFLT (, 4, , OMEG)

STRN

CALL MMM(L, 2,MI)

STRN

ELE $=(\operatorname{UX}(2,1)+P X(1,1) * U Y(2,1)-P X(2,1) * U Y(2,2)) /(P X(2,1) * U Y(2,1))$

STRN

$\operatorname{DLD}=-\operatorname{UX}(2,1)+P X(1,1) * \operatorname{UY}(2,1)-P X(2,1) *(U Y(2,2)+\operatorname{ELE} * U Y(2,1))$

STRN

$\operatorname{ELD}=\operatorname{UX}(1,1)-P X(1,1) * \operatorname{UY}(1,1)+P X(2,1) *(\operatorname{UY}(1,2)+\operatorname{ELE} * \operatorname{UY}(1,1))$

STRN

200

205

210

$E L D=E L D / D L D$

DLD $=2 . * E L D$

$\mathrm{IPR}=\mathrm{IBL}$

IF (SOK.GT.O.) IPR = IDFO

STRN

STRN

STRN

STRN

WRITE $(3,504)$ IPR, IFO

STRN

STRN

$3317 L=M-7$

CALL DRIFT(L,ELE)

$\mathrm{L}=\mathrm{L}-8$

CALL DRIFT(L, ELD)

STRN

STRN

STRN

STRN

$M I(1)=M-7$

$M I(2)=M-1$

$M I(3)=M-4$

$M I(4)=M-8$

$M I(5)=M-8$

215

$M I(6)=M-3$

$M I(7)=M-2$

$M I(8)=M-7$

STRN

STRN

STRN

STRN

STRN

STRN

STRN

STRN

$\operatorname{CALL} \operatorname{MMM}(M, 8, M I)$

STRN

$D L H=D L D / 2$.

STRN

STRN

STRN

WRITE $(3,503)$ NIT

STRN 
230

$15 \mathrm{X}, *=*, \mathrm{~F} 13.8 / 2 \mathrm{X}, * \mathrm{BETAX}=*, \mathrm{~F} 13.8,5 \mathrm{X}, * \mathrm{BETAY}$

$=*, F 13.8$

504 FORMAT ( $2 X, * F I R S T$ MAGNET KA IS *,A2,A10)

CARD NR. SEVERITY DETAILS DIAGNOSIS OF PROBLEM 
C $B 0$ BQ AVAC LTO LSEP

C $N$ IS THE NUMBER OF THE LONG STRAIGHT SECTION.

C $M$ IS THE NUMBER OF THE CELL.

STRPI

C $K$ IS THE STRAIGHT-SECTION TYPE INDICATOR. ( $K=1$ FOR DFDF, $K=2$ FOR FDFD)

C J IS THE POINT OF INSERTION INTO THE CELL.

STRPI

C THIS ROUTINE ASSUMES THAT THE CELL MATRICES ARE STORED AS ...CELL,

STRPI

STRPI

C CELL1,CELL2,..., CELL12, AND THAT BETAMAX OCCURS AT CELL POSITION $S$.

C IF $M$ IS A SYMMETRIC CELL (CELL, ALTC), ITS RHO IS PARAMETER 3.

C IF $M$ IS ASYMMETRIC (CFD,DCFD), IS RHO IS PARAMETER 4.

LEVEL 2, STORE, INFF, IWORK

COMMON STORE(48000), IWORK(10)

DIMENSION INFF $(24,2000)$.

EQUIVALENCE (INFF, STORE)

COMMON/GRR/IERR, BSW, VSW, RSW, QSW, XSW

LOGICAL IERR, BSW, VSW, RSW, QSW, XSW

COMMON/AGS/ALPHA, BETA, RHO, BZ, AVAC, BMAX

C

COMMON/STR/GAM , A, LK, BK, F, LM, BL , LL, BQ, LTO, SMUD , CMUD

C

REAL LK, LM, LL, LTO

EXTERNAL LPAR

REAL LDRF

INTEGER $C, C I, P, G A M, B D A T, E L N U M, H C F D$

DIMENSION BX(9), BY(9), PAR(5)

EQUIVALENCE (PAR, BX)

DATA HCFD/3HCFD/

C

c NAME OF CELL FROM INPUT AND ITS INDEX

$$
\begin{aligned}
& C=\operatorname{BdAT}(M, 1) \\
& \mathrm{CI}=\operatorname{ELNUM}(\mathrm{C}) \\
& \mathrm{P}=\operatorname{INFF}(5, M) \\
& \mathrm{L}=\mathrm{CI}-\mathrm{P} \\
& \mathrm{KI}=\operatorname{INFF}(4, M)
\end{aligned}
$$

C RETRIEVE INPUT

CALL DATA $(M, 1,1,5$, PAR $)$

C SET BZERO, BQ, AVAC, LTO, LSEP

$$
\begin{aligned}
& B 0=\operatorname{PAR}(1) \\
& B Q=\operatorname{PAR}(2) \\
& \operatorname{AVAC}=\operatorname{PAR}(3) \\
& \operatorname{LTO}=\operatorname{PAR}(4) \\
& \operatorname{LSEP}=\operatorname{PAR}(5)
\end{aligned}
$$

CALL BET $(L, B X, B Y, 0)$

$A L P H A=B X(3)$

$B E T A=B X(2)$

C COMPUTE BETMAX FOR THE CELL

$L=C I-2$

CALL BET $(L, B X, B Y, \theta)$

BETMAX $=B X(2)$

C get the VAlue of RHO FOR THE CELL
STRPI

STRPI

STRPI

STRPI

STRPI

BLANK

BLANK

86MARSIZ

86MARSIZ

86MARSIZ

BLANK

GRR

GRR

GRR

AGS

AGS

STR

STR

STR

STRPI

STRPI

STRPI

STRPI

STRPI

STRPI

STRPI

STRPI

STRPI

STRPI

STRPI

STRPI

STRPI

STRPI

STRPI

STRP I.

STRP I

STRPI

STRPI

STRPI

STRP I

STRPI

STRP I

STRP I

STRP I

STRP I

STRP I

STRP I

STRPI 
70

75

80

85

90

95
$I O P=\operatorname{INFF}(1, \mathrm{CI})$

IF (IOP.EQ.HCFD) GO TO 51

C CELL, ALTC,DCFD

$$
I=3
$$

GO TO 52

C CFD

65

$$
51 \quad \mathrm{I}=4
$$

52 RHO $=$ FDAT $(C I, I)$

$B L=L T O / 2$.

$F=\operatorname{SQRT}($ LSEP $* B L)$

$S=L S E P+B L$

$A=A V A C$

$A S=A * S Q R T((B E T A / B E T M A X) *((1 .+A L P H A * S / B E T A) * * 2+(S / B E T A) * * 2))$

$L K=B Q /(A S * B \theta)$

$B K=\operatorname{SQRT}(L K / R H O)$

$L M=1 \cdot /(F * B K * * 2)$

THETA $=B K * L M$

GAM $=P$

CALL GRT (1, THETA, 1, LPAR)

IF (IERR) RETURN

LDRF $=2 . * B L$

$G R A D=B Q / A S$

CALL HED

WRITE $(3,3)$ C, ALPHA , BETA, BETMAX, RHO, BO, BQ, AVAC, AS, LM, LK, LDRF, LSEP,

1 LTO, BL, GRAD

CALL HED

$\operatorname{PAR}(1)=L M$

$\operatorname{PAR}(2)=L K$

$\operatorname{PAR}(3)=\mathrm{RHO}$

$\operatorname{PAR}(4)=$ LDRF

$\operatorname{PAR}(5)=$ LSEP

CALL SSSR (M,PAR, KI, O)

RETURN

3
FORMAT$$
1
$$

2

3

4

5

6

END

$\begin{array}{ll} & \\ \text { STRPI } & 49 \\ \text { STRPI } & 50 \\ \text { STRPI } & 51 \\ \text { STRPI } & 52 \\ \text { STRPI } & 53 \\ \text { STRPI } & 54 \\ \text { STRPI } & 55 \\ \text { STRPI } & 56 \\ \text { STRPI } & 57 \\ \text { STRPI } & 58 \\ \text { STRPI } & 59 \\ \text { STRPI } & 60 \\ \text { STRPI } & 61 \\ \text { STRPI } & 62 \\ \text { STRPI } & 63 \\ \text { STRPI } & 64 \\ \text { STRPI } & 65 \\ \text { STRPI } & 66 \\ \text { STRPI } & 67 \\ \text { STRPI } & 68 \\ \text { STRPI } & 69 \\ \text { STRPI } & 70 \\ \text { STRPI } & 71 \\ \text { STRPI } & 72 \\ \text { STRPI } & 73 \\ \text { STRPI } & 74 \\ \text { STRPI } & 75 \\ \text { STRPI } & 76 \\ \text { STRPI } & 77 \\ \text { STRPI } & 78 \\ \text { STRPI } & 79 \\ \text { STRPI } & 80 \\ \text { STRPI } & 81 \\ \text { STRPI } & 82 \\ \text { STRPI } & 83 \\ \text { STRPI } & 84 \\ \text { STRPI } & 85 \\ \text { STRPI } & 86 \\ \text { STRPI } & 87 \\ \text { STRP } & 89 \\ \text { ST } & \end{array}$


C DESIGN A COLLINS LONG STRAIGHT SECTION TO MATCH A SPECIFIED CELL.

C THE MU VALUE OF THE STRAIGHT SECTION MAY BE SPECIFIED BY THE USER.

STR2

C INPUT CARDS...

C $A(M)$ STR2 $K \quad A(N)$ BZ $\quad B Q \quad A V A C \quad M U / 2 P I$

C $M$ IS THE NUMBER OF THE FIRST CONSTITUENT MATRIX OF STRT. SECTION

STR2

STR2

C $N$ IS THE NUMBER OF THE CELL

STR2

c THIS ROUTINE ASSUMES THAT THE. CELL MATRICES ARE STORED AS ... CELL,

STR2

C CELL1,CELL2, ...,CELL12, AND THAT BETAMAX OCCURS AT CELL POSITION 2.

C

LEVEL 2, STORE, INFF, IWORK

COMMON STORE (48000), IWORK(10)

DIMENSION INFF $(24,2000)$

EQUIVALENCE (INFF, STORE)

STR2

STR2

STR2

BLANK

BLANK

COMMON/BMI/MI (5000)

COMMON/NELS/NELS

LEVEL 2,MI

COMMON/GRR/IERR, BSW, VSW, RSW, QSW, XSW

LOGICAL IERR, BSW, VSW, RSW, QSW, XSW

COMMON/AGS/ALPHA, BETA, RHO, BZ, AVAC, BMAX

C

COMMON/STR/GAM, A, LK, BK, F, LM, BL, LL, BQ, LTO, SMUD , CMUD

C

REAL LK, LM, LL, LTO

C

\section{EXTERNAL PARSR}

DIMENSION PAR(5),BX(9),BY(9)

EQUIVALENCE (LTO, LTOT), (E, PAR, BX)

INTEGER C, CI , BDAT, ELNUM, HCFD

REAL MUD, LTOT, LEN

DATA HCFD/3HCFD/

C NAME OF CELL FROM INPUT AND ITS INDEX

86MARSIZ

86MARSIZ

86MARSIZ

BLANK

BMI L

BMIL

BMI L

BMI L

BMI

GRR

GRR

GRR

AGS

AGS

STR

STR

STR

STR2

STR2

STR2

STR2

STR2

STR2

STR2

STR2

$C=\operatorname{BDAT}(M, 1)$

$C I=\operatorname{ELNUM}(C)$

$K I=\operatorname{INFF}(4, M)$

C RETRIEVE INPUT DATA

CALL DATA $(M, 1,1,4$, PAR)

STR2

STR2

STR2

STR2

STR2

STR2

STR2

STR2

STR2

STR2

STR2

STR2

STR2

STR2

STR2

$50 \quad$ INSP $=10$

STR2

STR2

STR2

STR2

STR2

$$
A L P H A=A B S(P A L P H)
$$

CALL BET (L,BX, BY, O) 
60

$$
B E T A=B \times(2)
$$

C COMPUTE BMAX FOR THE CELL

$\mathrm{L}=\mathrm{CI}-2$

CALL BET $(L, B X, B Y, \theta)$

STR2

$B M A X=B X(2)$

65

C GET VALUE OF RHO FOR THE CELL

STR2

$I O P=\operatorname{INFF}(1, \mathrm{CI})$

IF (IOP.EQ.HCFD) GO TO 51

STR2

STR2

C CELL, ALTC OR DCFD

STR2

$I=3$

GO TO 52

70

C CFD

$51 \quad \mathrm{I}=4$

$52 \mathrm{RHO}=\operatorname{FDAT}(\mathrm{CI}, \mathrm{I})$

SMUD $=\operatorname{SIN}($ MUD $)$

CMUD $=\cos ($ MUD $)$

$A=A V A C$

C COMPUTE FIRST GUESS FOR THETA

$L K=B Q /(A * B Z)$

$B K=S Q R T(L K / R H O)$

$F=A L P H A / G A M$

80

$$
L M=1 . /(F *(B K * B K))
$$

THET $=B K * L M$

C CALL ROOT FINDING ROUTINE

CALL GRT (1,THET, 1,PARSR)

IF (IERR) RETURN

85

LTOT $=B L+2 * L L+2, * L M$

$G=B Q / A$

CALL HED

WRITE(3, 1) C, PALPH, BETA, RHO, BZ, BQ, AVAC, LM, LK, BL, LL, LTOT , A, G

CALL HED

90

$\operatorname{PAR}(1)=L M$

$\operatorname{PAR}(2)=L K$

$\operatorname{PAR}(3)=$ RHO

$\operatorname{PAR}(4)=B L$

$\operatorname{PAR}(5)=L L$

STR2

STR2

STR2

STR2

STR2

STR2

STR2

STR2

STR2

STR2

STR2

STR2

STR2

STR2

STR2

STR2

STR2

STR2

STR2

STR2

STR2

STR2

STR2

STR2

STR2

STR2

STR2

STR2

CALL SSSR(M,PAR, KI 1 )

STR2

STR2

STR2

STR2

STR2

STR2

STR2

STR2

STR2

STR2

STR2

STR2

STR2

STR2

STR2

STR2

STR2

STR2

STR2

STR2

45

46

47

48

49

50

51

52

53

54

55

56

57

58

59

60

61

62

63

64

65

66

67

68

69 


$$
\begin{array}{ll} 
& M I(11)=L \\
& \text { IF (KI.EQ.2) GO TO } 53 \\
& M I(4)=M \\
& \text { GO TO } 54 \\
53 \quad & M I(10)=M \\
54 \quad & L=M-5 \\
& \text { CALL MMM }(L, 13, M I)
\end{array}
$$

120

C STORE THE MI LIST

$$
\begin{aligned}
& E=0 . \\
& J=0
\end{aligned}
$$

CONT INUE RETURN

$\begin{array}{ll}\text { STR2 } & 102 \\ \text { STR2 } & 103 \\ \text { STR2 } & 104 \\ \text { STR2 } & 105 \\ \text { STR2 } & 106 \\ \text { STR2 } & 107 \\ \text { STR2 } & 108 \\ \text { STR2 } & 109 \\ \text { STR2 } & 110 \\ \text { STR2 } & 111 \\ \text { STR2 } & 112 \\ \text { STR2 } & 113 \\ \text { STR2 } & 114 \\ \text { STR2 } & 115 \\ \text { STR2 } & 116 \\ \text { STR2 } & 117 \\ \text { STR2 } & 118 \\ \text { STR2 } & 119 \\ \text { STR2 } & 120 \\ \text { STR2 } & 121 \\ \text { STR2 } & 122 \\ \text { STR2 } & 123\end{array}$


LEVEL 2, STORE, INFF, IWORK

COMMON STORE(48000), IWORK(10)

DIMENSION INFF $(24,2000)$

EQUIVALENCE (INFF,STORE)

BLANK

BLANK

86MARSIZ

86MARSIZ

86MARSIZ

COMMON/GRR/IERR, BSW, VSW, RSW, QSW, XSW

LOGICAL IERR, BSW, VSW, RSW, QSW, XSW

COMMON/BMI/MI (5000)

COMMON/NELS/NELS

LEVEL 2,MI

15

C

C

COMMON/WRK/BZ, BQ , AZ, DPSI , SM , SL, PSI ,WW, WT , BETW, P, Q,

$1=\quad T, U, B K Z, B K Z P, C Z, S Z, C H Z B, S H Z B, P H I, B K, S A, B L, B M$,

$2 A Q, R H O, B E T S, P S I W S, B K Z I, B K Z P I, G(6), A B, C, S, C H B$,

3 SHB, E, EI , SZCB, SZBC , CZBC, CZCB, SZSB, SZBS, CZBS, CZSB,

$4 \quad Z W, Z T, B K I, Y, Z, Y 1, Y 2, Y 3, Y 4$

DIMENSION BX(9),BY(9),PAR(4), PARQ(7), PARR(7),NCELL(3)

EQUIVALENCE $(B X, G),(B Z, B K S)$

LOGICAL QMAG, RMAG

INTEGER PI , QI , RI , ELNUM, HMAG

EXTERNAL STRFCN

DATA HMAG/3HMAG/

C

$\begin{array}{lllllllll}C & \text { INPUT ...K } & \text { STR4 } & P & Q & R & M U S / 2 P I & L & M\end{array}$

C

$\operatorname{PSI}=\operatorname{PAR}(1) * 6.283185307$

$S L=\operatorname{PAR}(2)$

$S M=P A R(3)$

C FIND INDEX OF PREVIOUSLY DEFINED ELEMENTS

$P I=\operatorname{ELNUM}(\operatorname{NCELL}(1))$

QI $=\operatorname{ELNUM}(\operatorname{NCELL}(2))$

$R I=\operatorname{ELNUM}(\operatorname{NCELL}(3))$

INAME = MNAME $(M)$

QMAG $=$.FALSE.

RMAG $=$. FALSE.

C IF INPUT ELEMENTS Q AND R ARE MAGNETS, TURN ON MAG SWITCH

C IF NOT MAGNETS, IT IS ASSUMED THEY ARE CELLS

$\operatorname{IQP}=\operatorname{INFF}(1, \mathrm{QI})$

45

$\operatorname{IRP}=\operatorname{INFF}(1, \mathrm{RI})$

IF (IQP.EQ.HMAG) QMAG $=$.TRUE.

IF (IRP.EQ.HMAG) RMAG = .TRUE.

C GET PARAMETERS FOR OUTER AND INNER ELEMENTS-IN MAG SEQUENCE.

IF (QMAG) GO TO 9

C $Q$ IS CELL. PUT INTO MAG SEQUENCE

CALL DATA(QI , 1, 1,7,PARQ)

PARQ(4) = PARQ(7)

PARQ(5) $=$ PARQ (6)

$\operatorname{PARQ}(6)=\operatorname{PARQ}(5)$

55

GO TO 10

C Q IS MAGNET

9 CALL DATA(QI , 1, 1,6,PARQ) 
65

80

90

95

100

10 IF (RMAG) GO TO 15

CALL DATA(RI $1,1,7$, PARR)

$\operatorname{PARR}(4)=\operatorname{PARR}(7)$

$\operatorname{PARR}(5)=\operatorname{PARR}(6)$

$\operatorname{PARR}(6)=\operatorname{PARR}(5)$

GO TO 20

$$
\text { C R IS MAGNET }
$$

15 CALL DATA(RI , 1, 1, 6, PARR)

C COMPUTE BETATRON FUNCTIONS

20 IF (PAR(4).NE.0.) PARR(1) = PAR(4)

CALL BET (PI, BX, BY, 0)

$A L P H X=B X(3)$

$A L P H Y=B Y(3)$

$B E T W=B X(2)$

$B E T S=B Y(2)$

C

$$
=S Z=\operatorname{SIN}(P S I)
$$$$
C Z=\cos (P S I)
$$

C REQUIRED MATRIX ELEMENTS OF COMPLETE REPLACEMENT ARRAY

STR4

STR4

STR4

STR4

STR4

STR4

STR4

STR4

STR4

STR4

STR4

STR4

STR4

STR4

STR4

STR 4

STR4

STR4

C (HORIZONTAL PLANE) $M 11=P, M 12=0, M 21=T, M 22=U$

STR4

$\mathrm{P}=(\mathrm{CZ}+\mathrm{BX}(3) * \mathrm{SZ}) * \mathrm{BY}(7) / \mathrm{BX}(7)$

$Q=B X(7) * B Y(7) * S Z$

STR4

$T=-((1 .-B X(3) * B Y(3)) * S Z-(B X(3)+B Y(3)) * C Z) /(B X(7) * B Y(7))$

$U=(C Z+B Y(3) * S Z) * B X(7) / B Y(7)$

STR4

C

$$
B X(1)=A B S(P A R Q(2))
$$

$$
\mathrm{RHO}=\mathrm{PARQ}(3)
$$

$\mathrm{BX}(4)=1.0 \% \mathrm{RHO}$

$B K Z=\operatorname{SQRT}((B X(1)+B X(4)) / R H O)$

$B K Z P=\operatorname{SQRT}(A B S(-B X(1)+B X(4)) / R H O)$

$B K Z I=1.0 / B K Z$

$B K Z P I=1.0 / B K Z P$

C

$\mathrm{PHI}=\mathrm{BKZ} * \mathrm{SM}$

$C Z=\cos (P H I)$

$-S Z=\operatorname{SIN}(\mathrm{PHI})$

$Z=B K Z P * S M$

$E=\operatorname{EXP}(Z)$

$E I=1.0 / E$

$\mathrm{CHZB}=0.5 *(E+E I)$

$S H Z B=0.5 *(E-E I)$

C SET UP KOF AND KOD

$B \times(1)=S M$

DO $25 \mathrm{I}=2,6$

STR4

STR4

STR4

STR4

STR4

STR4

STR4

STR4

STR4

STR4

STR4

STR4

STR4

STR4

STR4

STR4

STR4

STR4

STR4

STR4

STR4

STR4

STR4

STR4

$B X(2)=A B S(B X(2))$

STR4

$\mathrm{L}=\mathrm{M}-3$

$\operatorname{CALL} \operatorname{STDAT}(L, 1,1,6, B X)$

STR 4

CALL MAGNET ( $L, B X)$

STR 4

$M I(2)=L$

STR4

$\mathrm{L}=\mathrm{M}-4$

STR4

$B X(2)=-B X(2)$

STR4

CALL STDAT $(L, 1,1,6, B X)$

STR4

CALL MAGNET ( $L, B X)$

STR4

$M I(9)=L$

STR4

STR4

42

43

44

45

46

47

48

49

50

51

52

53

54

55

56

57

58

59

60

61

62

63

64

65

66

67

68

69

70

71

72

73

74

75

76

77

78

79

80

81

82

83

84

85

86

87

88

89

90

91

92

93

94

95

96

C SET UP KE 
$\operatorname{PHAD}=\operatorname{PAR}(1) * 360$.

STR4

STR4

WRITE $(3,2)$ INAME, NCELL(1)

STR4

WRITE $(3,3)$ PAR (1), PHAD, INAME, INAME, NCELL(2), SM, PSI, INAME, INAME, STR4

FORMAT $(1 X, * 4-E L E M E N T$ ANTISYMMETRIC LONG STRAIGHT SECTION ARRAY *, STR4

1 A1,* , REPLACES LATTICE FROM *A3,* TO NEXT ANTI HOMOLOGOUS * STR 4

4 FORMAT $(4 X, * B E T A X=*, F 14.8,2 X, *$ ALPHAX $=*, F 14.8, *$ LEND $=*, F 14.8$, STR4

5 FORMAT $(4 X, *$ BETAY $=*, F 14.8, * \quad$ ALPHAY $=*, F 14.8, * \quad$ LSEP $=*, \quad$ STR4 153

1 F14.8,* LDRF $=*, F 14.8)$

RETURN 
1

C STORES RX AND RY MATRICES IN LQ(M). DIMENSIONS ARE $(2,3)$ FOR EACH.

C IF ELEMENT NUMBER IS -, STORE INVERSE

5

10

15

20

25

30

35

40

45

50

LEVEL 2, STORE, INFF, IWORK

COMMON STORE (48000), IWORK(10)

DIMENSION INFF $(24,2000)$

EQUIVALENCE (INFF, STORE)

COMMON/CONTRL/ERROR, MODE, RSRV, STOR, XEQ, TRASW, NFSW, EMPTY, INDEF, 1 LDFLG , FIN

LOGICAL ERROR, RSRV, STOR, XEQ, TRASW, NFSW, EMPTY, INDEF, LDFLG, FIN

COMMON/SWTCH/BEND, MSIZE, CYCSWT , VCSW, PV, MSSW, VPR, MHS, MODS, MINZER

LOGICAL CYCSWT, MSSW, VCSW, PV, VPR, MHS, MODS

COMMON /DIM/ LEND, MEND, ISAV, ISAV7, M7END, KADD, KADDR, MUNIT, MSYMPL

DIMENSION RX(2,3), RY(2,3),AX(2,3),AY(2,3), RW(3)

DIMENSION RPQ(4), T (49)

EQUIVALENCE $(P 1, R P Q(1)),(P 2, R P Q(2)),(Q 1, R P Q(3)),(Q 2, R P Q(4))$

C

IF (M.GE. O) GO TO 7

$4 \quad M=-M$

$\operatorname{AX}(2,1)=-R \times(2,1)$

$\operatorname{AX}(1,1)=\operatorname{RX}(2,2)$

$\operatorname{AX}(2,2)=\operatorname{RX}(1,1)$

$\operatorname{AX}(1,2)=-R \times(1,2)$

$\operatorname{AX}(1,3)=\operatorname{RX}(1,2) * \operatorname{RX}(2,3)-\operatorname{RX}(2,2) * R X(1,3)$

$\operatorname{AX}(2,3)=\operatorname{RX}(2,1) * \mathrm{RX}(1,3)-\mathrm{RX}(1,1) * \mathrm{RX}(2,3)$

$\operatorname{AY}(1,1)=\operatorname{RY}(2,2)$

$\operatorname{AY}(2,2)=\operatorname{RY}(1,1)$

$\operatorname{AY}(1,2)=-\operatorname{RY}(1,2)$

$\operatorname{AY}(2,1)=-\operatorname{RY}(2,1)$

$\operatorname{AY}(1,3)=\operatorname{RY}(1,2) * \operatorname{RY}(2,3)-\operatorname{RY}(2,2) * \operatorname{RY}(1,3)$

$\operatorname{AY}(2,3)=\operatorname{RY}(2,1) * \operatorname{RY}(1,3)-\operatorname{RY}(1,1) * \operatorname{RY}(2,3)$

DO $5 \mathrm{~J}=1,3$

DO $5 \mathrm{I}=1,2$

$\operatorname{RX}(I, J)=\operatorname{AX}(I, J)$

$\operatorname{RY}(I, J)=\operatorname{AY}(I, J)$

5 CONTINUE

$R W(1)=-R W(1)$

$R W(2)=-R W(2)$

IF (BEND.NE.0.) RW(3) $=-\mathrm{RW}(3)$

7 CONTINUE

$\mathrm{P} 1=\mathrm{RX}(1,3) * \mathrm{RX}(2,1)-\mathrm{R} \times(2,3) * \mathrm{RX}(1,1)$

$P 2=R X(1,3) * R X(2,2)-R X(2,3) * R X(1,2)$

$Q 1=\operatorname{RY}(1,3) * R Y(2,1)-\operatorname{RY}(2,3) * R Y(1,1)$

$Q 2=\operatorname{RY}(1,3) * R Y(2,2)-\operatorname{RY}(2,3) * R Y(1,2)$

$L Q=\operatorname{INFF}(10, M)$

$N Q=\operatorname{INFF}(11, M)$

$\operatorname{KIND}=\operatorname{INFF}(20, M)$

IF (KIND.NE.3HMXY) GO TO 11

C CHECK STORAGE REQUIREMENT

$N N Q=12+K A D D R$

IF (NNQ.GT.NQ) GO TO 20

\begin{tabular}{|c|c|}
\hline STXY & 2 \\
\hline STXY & 3 \\
\hline STXY & . \\
\hline BLANK & 2 \\
\hline BLANK & \\
\hline 86MARSIZ & \\
\hline 86MARSIZ & \\
\hline 86MARSIZ & 3 \\
\hline BLANK & 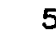 \\
\hline CONTRL & 2 \\
\hline CONTRL & 3 \\
\hline CONTRL & 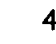 \\
\hline CONTRL & \\
\hline SWTCH & 2 \\
\hline SWTCH & 3 \\
\hline SWTCH & 4 \\
\hline DIM & 2 \\
\hline DIM & 3 \\
\hline STXY & \\
\hline STXY & 10 \\
\hline STXY & 11 \\
\hline STXY & 12 \\
\hline STXY & 13 \\
\hline STXY & 14 \\
\hline STXY & 15 \\
\hline STXY & 16 \\
\hline STXY & 17 \\
\hline STXY & 18 \\
\hline STXY & 19 \\
\hline STXY & $2 e$ \\
\hline STXY & 21 \\
\hline STXY & 22 \\
\hline STXY & 23 \\
\hline STXY & 24 \\
\hline STXY & 25 \\
\hline STXY & 26 \\
\hline STXY & 27 \\
\hline STXY & 28 \\
\hline STXY & 29 \\
\hline STXY & 36 \\
\hline STXY & 31 \\
\hline STXY & 32 \\
\hline STXY & 33 \\
\hline STXY & 34 \\
\hline STXY & 35 \\
\hline STXY & 36 \\
\hline STXY & 37 \\
\hline STXY & 38 \\
\hline STXY & 39 \\
\hline STXY & 46 \\
\hline STXY & 41 \\
\hline STXY & 42 \\
\hline STXY & 43 \\
\hline STXY & 4 \\
\hline STXY & 45 \\
\hline STXY & 46 \\
\hline STXY & 47 \\
\hline
\end{tabular}


60

$$
N J=L Q+K A D D R
$$

$\mathrm{NI}=\mathrm{NJ}+6$

STXY

DO $10 \mathrm{IJ}=1,3$

STXY

STXY

STORE $(N J)=R X(I I, I J)$
ST

STXY

STXY

$\operatorname{STORE}(N I)=R Y(I I, I J)$

STXY

STXY

STXY

STXY

STXY

STXY

STXY

STXY

STXY

STXY

STXY

STXY

STXY

STXY

STXY

STXY

STXY

$11 \mathrm{CALL}$ CONVMAT (7, T,RX,RY,RW, RPQ)

CALL $S T O R 7(M, T, R W)$

RETURN

67

68

80

END 
SUBROUTINE SUBR (IENTRY)
SUBROUTINE LOGIC. INSTRUCTION $/ 0 /$ SUB $/ .$. MEANS O IS THE NAME

STXY

SUBR

C OF A S/R CONSISTING OF INSTRUCTIONS FOLLOWING UP TO AN END

SUBR INSTRUCTION. Q MAY LATER BE REQUESTED BY...// /CALL/K/ $/ Q$.

LEVEL 2, STORE, INFF, IWORK

COMMON STORE(48000), IWORK(10)

DIMENSION INFF $(24,2000)$

COMMON/INSTR/OPNAME, NAME, OP, KA, KB, OBJA, OBJB, NXTM, TRA, LQ, NQ, LIN,

COMMON/CONTRL/ERROR, MODE, RSRV , STOR, XEQ, TRASW, NFSW, EMPTY, INDEF, 1 LDFLG, FIN

LOGICAL ERROR, RSRV, STOR, XEQ, TRASW, NFSW, EMPTY, INDEF, LDFLG, FIN

COMMON/SWTCH/BEND, MSIZE, CYCSWT, VCSW, PV, MSSW, VPR, MHS, MODS, MINZER LOGICAL CYCSWT, MSSW, VCSW, PV, VPR, MHS, MODS

COMMON/STORE/LMAX, LINF , LFILE, MAX, MIN, ICARD (11)

COMMON/COPY/CPYSW

LOGICAL CPYSW

c

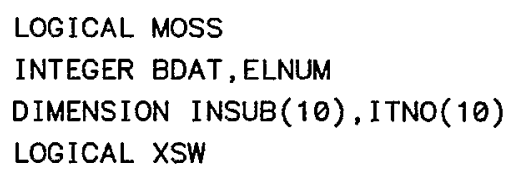

$$
\text { CALL HED }
$$

61 IF (MODE.EQ.1) GO TO 1

ERROR=, TRUE.

WRITE $(3,52)$

RETURN

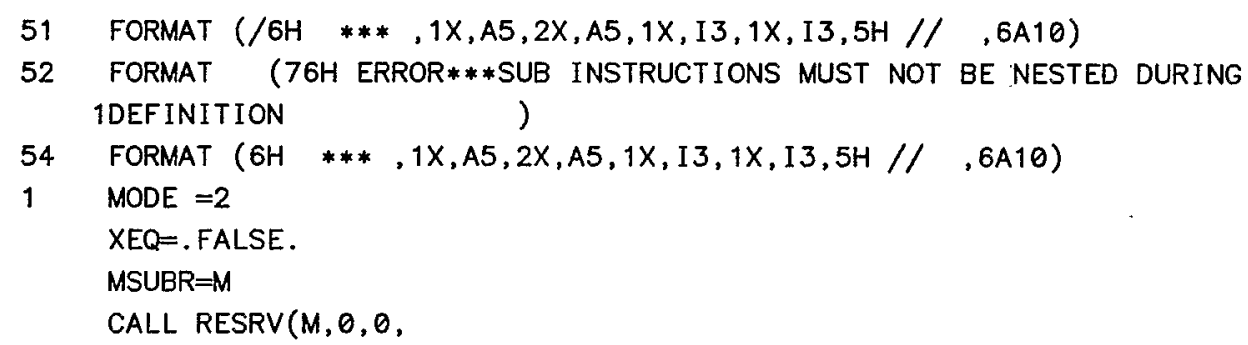

\section{INSTR}

INSTR

INSTR

CONTRL

CONTRLL

CONTRL

CONTRL

SWTCH

SWTCH

SWTCH

STORE

STORE

STORE

COPY

COPY

COPY

SUBR

SUBR

SUBR

SUBR

SUBR

SUBR

SUBR

SUBR

SUBR

SUBR

SUBR

SUBR

SUBR

SUBR

SUBR

SUBR

SUBR

SUBR

SUBR

SUBR

SUBR

SUBR

SUBR

SUBR

SUBR

SUBR 
60

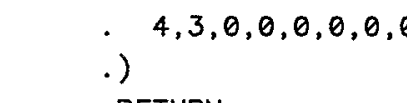

RETURN

C END ENTRY

70 CONTINUE

GO TO $(4,2,3)$, MODE

4 CONTINUE

65

70

75

80

85

90

95

100

105

110

IF (MSSW) GO TO 25

ERROR $=$. TRUE.

WRITE $(3,53)$

RETURN IUB INSTRUCTION.)

C SET TRA TO MSUBR

CALL HED

CALL SKIP1

30 MODE $=1$

$X E Q=. T R U E$.

RETURN

C VPAR ARE OPERATIVE.

25 MOSS $=$. TRUE.

$3 \quad$ MSUBR $=\operatorname{INFF}(9, M)$

$K T=\operatorname{IDAT}($ MSUBR, 3$)$

ICOUNT $=$ ICOUNT -1

$K T=K T+1$

NAMESR=MNAME(MSUBR)

IF ( ICOUNT-1) 5, 6, 7

ISNB=ISNB- 1

$M=M C A L L$

IF (MOSS) GO TO 8

IF (MODS) MODE $=1$

$8 \quad X E Q=$. TRUE.

$S T O R=$. TRUE.

RSRV $=$. TRUE.

RETURN

9 STOR=. TRUE.

RSRV $=$. TRUE .

$X E Q=. F A L S E$.

RETURN

10 STOR=.FALSE.

RSRV $=$. FALSE.
53 FORMAT (7OH ERROR****INSTRUCTION NAMED END MUST BE PRECEDED BY A $S$

2 CALL INFW(MSUBR, 9,M)

IF (.NOT.CPYSW) GO TO 30

WRITE $(3,54)$ NAME, OPNAME, KA, KB, (ICARD ( IW $), I W=3,8)$

C MOSS CONTROLS MODE TO ALLOW PROPER FLOW OF PROGRAM WHEN MESH OR

ICOUNT $=$ IDAT (MSUBR, 4)

CALL REPINT(MSUBR, 3, KT)

CALL REPINT (MSUBR , 4 , ICOUNT)

CALL REPINT(MSUBR, 1,0)

CALL REPINT(MSUBR, 2,0)

$5 \quad$ MCALL $=\operatorname{INFF}(9$, MSUBR $)$

IF (.NOT.MODS) MODE $=\operatorname{INFF}(19$, MCALL $)$

GO TO $(8,9,10)$, MODE

$X E Q=$. TRUE.

RETURN

6 CALL REPINT (MSUBR , 2, 1)

7 TRA $=$ MSUBR -1
SUBR $\quad 40$

SUBR $\quad 41$

SUBR $\quad 42$

SUBR $\quad 43$

SUBR 44

SUBR $\quad 45$

SUBR $\quad 46$

SUBR $\quad 47$

SUBR $\quad 48$

SUBR $\quad 49$

SUBR $\quad 50$

SUBR $\quad 51$

SUBR $\quad 52$

SUBR $\quad 53$

SUBR = 54

SUBR $\quad 55$

SUBR $\quad 56$

SUBR = 57

SUBR $\quad 58$

SUBR $\quad 59$

SUBR 60

SUBR $\quad 61$

SUBR 62

SUBR 63

SUBR 64

SUBR 65

SUBR $\quad 66$

SUBR $\quad 67$

SUBR 68

SUBR $\quad 69$

SUBR 70

SUBR 71

SUBR $\quad 72$

SUBR $\quad 73$

SUBR $\quad 74$

SUBR $\quad 75$

SUBR . 76

SUBR $\quad 77$

SUBR $\quad 78$

SUBR $\quad 79$

SUBR 80

SUBR 81

SUBR 82

SUBR 83

SUBR 84

SUBR 85

SUBR $\quad 86$

SUBR $\quad 87$

SUBR $\quad 88$

SUBR $\quad 89$

SUBR 90

SUBR $\quad 91$

SUBR 92

SUBR 93

SUBR 94

SUBR $\quad 95$

SUBR 96 
SUBR

SUBR

SUBR

SUBR

SUBR

SUBR

SUBR

SUBR

SUBR

SUBR

SUBR

SUBR

SUBR

SUBR

SUBR

SUBR

SUBR

SUBR

SUBR

SUBR

SUBR

SUBR

SUBR

SUBR

SUBR

SUBR

SUBR

SUBR

SUBR

SUBR

SUBR

SUBR

SUBR

SUBR

SUBR

SUBR

SUBR

SUBR

SUBR

SUBR

SUBR

SUBR

SUBR

SUBR

SUBR

SUBR

SUBR

SUBR

SUBR

SUBR

SUBR

SUBR

SUBR

SUBR

SUBR

SUBR

SUBR
IF (VPR) GO TO 55 


\begin{tabular}{|c|c|c|c|c|}
\hline & \multicolumn{2}{|r|}{$I S N B=I S N B+1$} & SUBR & 154 \\
\hline & & INSUB $($ ISNB $)=$ NAMESR & SUBR & 155 \\
\hline & & ITNO $($ ISNB $)=1$ & SUBR & 156 \\
\hline \multirow[t]{5}{*}{175} & 8 & CONTINUE & SUBR & 157 \\
\hline & & IF (MODE.NE.3) GO TO 55 & SUBR & 158 \\
\hline & & $\mathrm{IGO}=1$ & SUBR & 159 \\
\hline & & GO TO 200 & SUBR & 160 \\
\hline & & CALL INFW(M,9,MSUBR $)$ & SUBR & 161 \\
\hline \multirow[t]{5}{*}{180} & c & SETS SWITCH FOR INCREMENT TO RESTORE ORIGINAL VALUE & SUBR & 162 \\
\hline & & CALL REPINT(MSUBR, 2, 0 ) & SUBR & 163 \\
\hline & c & SET BEGINNING OF COUNT FOR ITERATION NUMBER PRINT & SUBR & 164 \\
\hline & & CALL REPINT(MSUBR, 3,1) & SUBR & 165 \\
\hline & c & SET TOTAL COUNT FOR ITERATIONS THROUGH THE SUBROUTINE & SUBR & 166 \\
\hline \multirow[t]{5}{*}{185} & & IF (KA.LT.1) KA $=1$ & SUBR & 167 \\
\hline & & CALL REPINT(MSUBR, $4, K A)$ & SUBR & 168 \\
\hline & & IF (KA.EQ.1) CALL REPINT(MSUBR, 2,1) & SUBR & 169 \\
\hline & & STOR=.FALSE. & SUBR & 170 \\
\hline & & RSRV $=$. FALSE. & SUBR & 171 \\
\hline \multirow[t]{5}{*}{190} & & TRASW $=$. TRUE & SUBR & 172 \\
\hline & & $T R A=M S U B R-1$ & SUBR & 173 \\
\hline & c & PUT MODE INTO IX OF INFO & SUBR & 174 \\
\hline & & IF (.NOT.MSSW) CALL INFW(MODE, 19,M) & SUBR & 175 \\
\hline & c & SET SWITCH FOR INCREMENT TO STORE ORIGINAL VALUE & SUBR & 176 \\
\hline \multirow[t]{4}{*}{195} & & CALL REPINT(MSUBR, 1, 1) & SUBR & 177 \\
\hline & & $M O D E=3$ & SUBR & 178 \\
\hline & & RETURN & SUBR & 179 \\
\hline & & END & SUBR & 180 \\
\hline
\end{tabular}

CARD NR. SEVERITY DETAILS DIAGNOSIS OF PROBLEM

$\begin{array}{rr}41 & \text { I } \\ 63 & \text { I } \\ 100 & \text { I } \\ 146 & \text { I }\end{array}$

AN IF STATEMENT MAY BE MORE EFFICIENT THAN A 2 OR 3 BRANCH COMPUTED GO TO STATEMENT. AN IF STATEMENT MAY BE MORE EFFICIENT THAN A 2 OR 3 BRANCH COMPUTED GO TO STATEMENT. AN IF STATEMENT MAY BE MORE EFFICIENT THAN A 2 OR 3 BRANCH COMPUTED GO TO STATEMENT. AN IF STATEMENT MAY BE MORE EFFICIENT THAN A 2 OR 3 BRANCH COMPUTED GO TO STATEMENT. 
1

SUBROUTINE SUM(K,S, SM)

DIMENSION $S(1)$

$S M=0$.

DO $10 \mathrm{I}=1, \mathrm{~K}$

$S M=S M+S(I)$

CONTINUE

RETURN

END

$\begin{array}{ll}\text { SUM } & 2 \\ \text { SUM } & 3 \\ \text { SUM } & 4 \\ \text { SUM } & 5 \\ \text { SUM } & 6 \\ \text { SUM } & 7 \\ \text { SUM } & 8 \\ \text { SUM } & 9\end{array}$


* CONTROLS CALLS to DifFERENT SWITCHES OR OVERLAYS, ACCORDiNG TO

SWITCH

* OP OF SYNCH INSTRUCTION.

SWITCH COMMON/INSTR/OPNAME, NAME, OP, KA, KB, OBJA, OBJB, NXTM, TRA, LQ, NQ, LIN,

INSTR $1 \quad$ NIN, LFL, NFL, LBC, NBC, NTOT, IX, KIND, TYPE, ROWS, COLS, EXTR, 2 $M, N F, N B, N I, M S U B R, I S I G N$ INTEGER OPNAME, OP, OBJA, OBJB, TRA, TYPE, ROWS, COLS, EXTR COMMON/CONTRL/ERROR, MODE, RSRV, STOR, XEQ. TRASW, NFSW, EMPTY, INDEF, 1 LDFLG, FIN

LOGICAL ERROR, RSRV, STOR, XEQ, TRASW, NFSW, EMPTY, INDEF, LDFLG, FIN

COMMON/GRR/IERR, BSW, VSW, RSW, QSW, XSW

* if opName=NUll, it is a P or C card. it nEEds special handling to

* construct a synch page or rem instruction. IF (OPNAME. NE. 4HNULL) GO TO 5

\section{CALL SPLOAD}

RETURN

5 CONTINUE $X S W=$.FALSE.

* if op is negative, execution is to be deleted. turn on XSW. IF (OP.LT.O) XSW = .TRUE. IF ((OP.LE. . ) . AND. (MODE. EQ.3)) RETURN

* turn op to positive if loAding is ReQuired IF (XSW) OP = -OP

* IF OP WAS ILLEGAL, IT WAS SET TO $\theta$ BY OPNUM. ERROR SWITCH IS ON IF (OP.EQ.O) RETURN

* if op is fin, incr or repl, ignore error. go to next case.

IF (OP.EQ.2.OR.OP.EQ.7.OR.OP.EQ.8) IERR = .FALSE.

IF (IERR) RETURN

$I E R R=$. FALSE

IF (OP.GT.50) GO TO 10

CALL OVERLAY ( 5 LSYNCH, $1,0,6$ HRECALL)

GO TO 20

10 IF (OP.GT.64) GO TO 11

CALL OVERLAY (5LSYNCH, $3,0,6$ HRECALL)

GO TO 20

11 IF (OP.GE. 100) GO TO 12

CALL OVERLAY ( 5 LSYNCH, 4, 0, 6HRECALL)

GO TO 20

12 IF (OP.GT.150) GO TO 14

CALL OVERLAY (5LSYNCH, 5, 0, 6HRECALL)

GO TO 20

14 IF (OP.GT.160) GO TO 15

CALL OVERLAY ( 5 LSYNCH, $6,0,6$ HRECALL)

GO TO 20

15 IF (OP.GT.200) GO TO 16

CALL OVERLAY (5LSYNCH, 7, 0,6 HRECALL)

20 RETURN

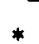

WRITE $(3,50)$ OP,OPNAME

$$
\text { IF OP.GT.200 IT IS AN ERROR }
$$




$\begin{array}{llllllll}C & S X & S X T P & K B & L & D 2 B & B R H O & \text { EXACT } \\ C\end{array}$

SXTP

(1)

C SX IS A SEXTUPOLE THAT ACTS AT A POINT. IT REPRESENTS THE EFFECT OF A SEXTUPOLE OF LENGTH L, WITH D2B $=$ D2B/DX2.

C IF KA.NE. $\theta$ SEXTUPOLE IS REPLACED BY GENERALIZED MULTIPOLE

$C$ OF ORDER KA, SKEW IF KB=1 (SEE NPOL WRITEUP)

COMMON /DIM/ LEND, MEND, ISAV, ISAV7, M7END, KADD, KADDR, MUNIT, MSYMPL

LEVEL 2, STORE, INFF, IWORK

COMMON STORE (48000), IWORK( 10$)$

DIMENSION INFF $(24,2000)$

EQUIVALENCE (INFF, STORE)

SXTP

SXTP

SXTP

SXTP

SXTP

SXTP

SXTP

SXTP

SXTP

SXTP

SXTP

SXTP

SXTP

SXTP

SXTMPOL

SXTMPOL

DIM

DIM

BLANK

BLANK

86MARSIZ

86MARSIZ 2

86MARSIZ 3

BLANK 5

COMMON /MP/ML \$ LEVEL 2, ML

DIMENSION $V(1), P A R(1), W(4), P(4), R(7,7), R W(3), M L(3), M A G(6)$

EQUIVALENCE $(X, W(1)),(P X, W(2)),(Y, W(3)),(P Y, W(4))$,

SXLL

SXLL

SXTP

SXTP

SXTP

SXTP

SXTP

SXTP

REAL L, LSX, LH, K, KP, MAG $($ LSX,MAG (1)), (K, MAG (2)), (ONE, MAG (3)), (CURV, MAG (4)),

DATA ONE, S3, RADEG/1.0,1.732050808, 0.0174532925/

DATA S32,SS3,S13,S23/.433012702,1.44224957,.33333333,3.464101616/

C

$\operatorname{IF}(\operatorname{INFF}(4, M) \cdot E Q .0)$ GO TO 15

CALL NPOL $(M, V, P A R)$

RETURN

CONTINUE

DO $1 \quad I=1,4$

$W(I)=V(I)$

$1 \quad P(I)=P A R(I)$

$\mathrm{DPP}=\mathrm{V}(6)$

$F A C=1 .+D P P$

$\mathrm{BR}=\mathrm{FAC} * \mathrm{BRHO}$

$F=D 2 B / B R$

$\mathrm{KP}=\mathrm{F}$

C IF KB" 0 , TURN OFF COLUMN 6

$\mathrm{KB}=\operatorname{INFF}(5, M)$

IF(X.EQ.Q. .AND.PX.EQ.Q..OR.KP.EQ.Q.) GO TO 11 IF $(P(4)$.NE. . .AND.L.NE.O..AND.Y.EQ.0..AND.PY.EQ.0.) GO TO 5

SXTP

SXTP

SXTP

SXTMPOL

SXTMPOL

SXTMPOL

SXTMPOL

SXTP

SXTP

SXTP

SXTP

SXTP

SXTP

SXTP

SXTP

SXTP

SXTP

SXTP

SXTP

SXTP

SXTP

SXTP

$\mathrm{LH}=\mathrm{L} / 2$.

SXTP

$X=X+P X * L H$

SXTP

$Y=Y+P Y * L H$ 
65

$$
F=L * F
$$

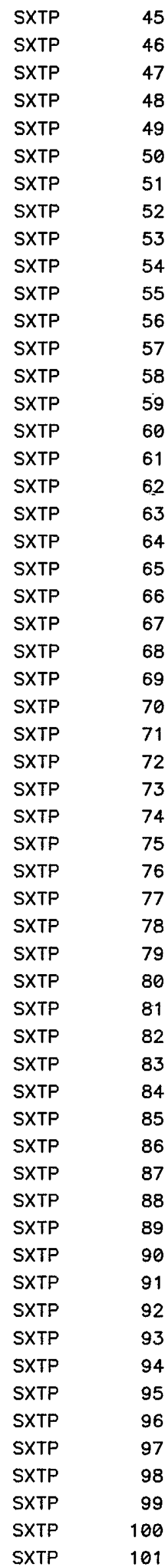


115

120

125

130

135

140

145

150

155

160

165

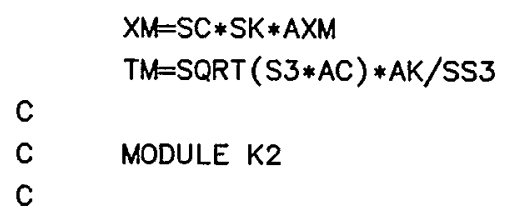

\begin{tabular}{|c|c|}
\hline SXTP & 102 \\
\hline SXTP & 103 \\
\hline SXTP & 104 \\
\hline SXTP & 105 \\
\hline SXTP & 106 \\
\hline SXTP & 107 \\
\hline SXTP & 108 \\
\hline SXTP & 109 \\
\hline SXTP & 110 \\
\hline SXTP & 111 \\
\hline SXTP & 112 \\
\hline SXTP & 113 \\
\hline SXTP & 114 \\
\hline SXTP & 115 \\
\hline SXTP & 116 \\
\hline SXTP & 117 \\
\hline SXTP & 118 \\
\hline SXTP & 119 \\
\hline SXTP & 120 \\
\hline SXTP & 121 \\
\hline SXTP & 122 \\
\hline SXTP & 123 \\
\hline SXTP & 124 \\
\hline SXTP & 125 \\
\hline SXTP & 126 \\
\hline SXTP & 127 \\
\hline SXTP & 128 \\
\hline SXTP & 129 \\
\hline SXTP & 130 \\
\hline SXTP & 131 \\
\hline SXTP & 132 \\
\hline SXTP & 133 \\
\hline SXTP & 134 \\
\hline SXTP & 135 \\
\hline SXTP & 136 \\
\hline SXTP & 137 \\
\hline SXTP & 138 \\
\hline SXTP & 139 \\
\hline SXTP & 140 \\
\hline SXTP & 141 \\
\hline SXTP & 142 \\
\hline SXTP & 143 \\
\hline SXTP & 144 \\
\hline SXTP & 145 \\
\hline SXTP & 146 \\
\hline SXTP & 147 \\
\hline SXTP & 148 \\
\hline SXTP & 149 \\
\hline SXTP & 150 \\
\hline SXTP & 151 \\
\hline SXTP & 152 \\
\hline SXTP & 153 \\
\hline SXTP & 154 \\
\hline SXTP & 155 \\
\hline SXTP & 156 \\
\hline SXTP & 157 \\
\hline SXTP & 158 \\
\hline
\end{tabular}


$100 V(1)=\times 1$

$V(2)=P \times 1$

175

180

185

IF (M.EQ.0) RETURN

C $\operatorname{MAG}(I)=L S X, K, 1,0$, CURV, EPSO, EPS1 $\mathrm{LSX}=\mathrm{L}$

THETA $=P X-P X 1$

$X A V=(X+X 1) / 2$. + THETA*L/12.

$K=K P * X A V$

CURV $=$ THETA $/ L$

EPSO $=P X / R A D E G$

EPS $1=-P X 1 / R A D E G$

CALL MAGNET (M,MAG)

RETURN

END
SXTP $\quad 159$

SXTP 160

SXTP 161

SXTP $\quad 162$

SXTP $\quad 163$

SXTP $\quad 164$

SXTP $\quad 165$

SXTP $\quad 166$

SXTP $\quad 167$

SXTP $\quad 168$

SXTP $\quad 169$

SXTP 170

SXTP $\quad 171$

SXTP 172

SXTP $\quad 173$

SXTP 174

CARD NR. SEVERITY DETAILS DIAGNOSIS OF PROBLEM

$\begin{array}{rlll}44 & \text { I } & \text { V } & \text { ARRAY REFERENCE OUTSIDE DIMENSION BOUNDS. } \\ 63 & \text { I } & \text { V } & \text { ARRAY REFERENCE OUTSIDE DIMENSION BOUNDS. } \\ 63 & \text { I } & \text { V } & \text { ARRAY REFERENCE OUTSIDE DIMENSION BOUNDS. } \\ 64 & \text { I } & \text { V } & \text { ARRAY REFERENCE OUTSIDE DIMENSION BOUNDS. } \\ 64 & \text { I } & \text { V } & \text { ARRAY REFERENCE OUTSIDE DIMENSION BOUNDS. } \\ 66 & \text { I } & V & \text { ARRAY REFERENCE OUTSIDE DIMENSION BOUNDS. } \\ 67 & \text { I } & \text { V } & \text { ARRAY REFERENCE OUTSIDE DIMENSION BOUNDS. } \\ 67 & \text { I } & \text { V } & \text { ARRAY REFERENCE OUTSIDE DIMENSION BOUNDS. } \\ 173 & \text { I } & \text { V } & \text { ARRAY REFERENCE OUTSIDE DIMENSION BOUNDS. }\end{array}$


SUBROUT INE TABULAT( $M$, TABSW)

SXTP

LEVEL 2, STORE, INFF, IWORK

COMMON STORE (48000), IWORK(10)

DIMENSION INFF $(24,2000)$

EQUIVALENCE (INFF, STORE)

COMMON/SWTCH/BEND, MSIZE, CYCSWT, VCSW, PV, MSSW, VPR, MHS, MODS, MINZER

EQUIVALENCE (IVAR, FVAR)

LOGICAL TABSW

DIMENSION INDX(10), NAM(10), JND(10), $F(10)$

$$
\begin{aligned}
& K A=\operatorname{INFF}(4, M) \\
& K B=\operatorname{INFF}(5, M) \\
& K S=K A * 60
\end{aligned}
$$

C IF KB.GT.0, SET IPR=KB, WHICH GOES TO INNF(6) OF CYX

C INSTRUCTION TO SUPPRESS PRINT.

$\mathrm{IPR}=0$

IF (KB.GT. $\theta)$ IPR $=K B$

IF (TABSW) GO TO 120

C FETCH BCD LIST

C SET UP TABLE OF INDICES

CALL DATA $(M, 2,1, K A, N A M)$

DO $100 \quad I=1, K A$

$\mathrm{NM}=\mathrm{NAM}(\mathrm{I})$

$\operatorname{INDX}(\mathrm{I})=\operatorname{ELNUM}(\mathrm{NM})$

$\operatorname{JND}(\mathrm{I})=1$

$L M=I N D X(I)$

IF (LM.NE. $\theta) \operatorname{INFF}(6, \operatorname{LM})=\operatorname{IPR}$

IF (.NOT.VCSW) GO TO 100

C WHEN VCSW IS ON, ELEMENT IS ONE OF A VECTOR

C SHIFT OFF LETTER NAME. SHIFT RIGHT FOR INTEGER NUMBER

LNUM $=$ NM.AND.007777777777777777777

LNUM= SHIFT $($ LNUM, -48$)$

$\operatorname{JND}(I)=$ LNUM

100 CONTINUE

CALL STDAT $(M, 7,1, K A, I N D X)$

$\operatorname{CALL} \operatorname{STDAT}(M, 8,1, \mathrm{KA}, J N D)$

RETURN

120 CONTINUE

$L Q=\operatorname{INFF}(10, M)$

C GET INDECES

CALL DATA( $M, 7,1, K A$, INDX)

$\operatorname{CALL} \operatorname{DATA}(M, 8,1, K A, J N D)$

C GET CURSOR FROM LQ

FVAR=STORE (LQ)

IC=IVAR

$I U P=I C+K A$

IF (IUP.GT.KS) GO TO 130

DO $125 \mathrm{I}=1, \mathrm{KA}$ 
60

65

70

80
$\operatorname{INDX}(I)=\operatorname{ELNUM}(N M)$

CALL REPLQ(M, 2, I , INDX(I))

$L M=I N D X(I)$

$\operatorname{INFF}(6, \operatorname{LM})=\operatorname{IPR}$

IF (.NOT.VCSW) GO TO 124

LNUM= SHIFT $(N M, 6)$

DECODE $(10,1$, LNUM) JND(I)

FORMAT $(I 1,9 X)$

CALL REPLQ(M, 3, I ,JND(I))

$124 \mathrm{IN}=\operatorname{INDX}(\mathrm{I})$

$J N=J N D(I)$

C FETCH VALUE

$$
\text { CALL FLQ(IN, 2,JN, } F(I))
$$

125 CONTINUE

C STORE

$\operatorname{CALL} \operatorname{STDAT}(M, 5, I C, K A, F)$

C RESET CURSOR

IVAR $=$ IUP

STORE (LQ) $=$ FVAR

RETURN

C STORAGE FILLED. PRINT TABLE AND CONTINUE

130 CALL PRNTAB(M)

GO TO 120

END
TABUL $\quad 51$

TABUL $\quad 52$

TABUL 53

TABUL $\quad 54$

TABUL $\quad 55$

TABUL $\quad 56$

TABUL $\quad 57$

TABUL 58

TABUL 59

TABUL 60

TABUL $\quad 61$

TABUL 62

TABUL 63

TABUL 64

TABUL 65

TABUL 66

TABUL $\quad 67$

TABUL 68

TABUL 69

TABUL 70

TABUL 71

TABUL $\quad 72$

TABUL $\quad 73$

TABUL $\quad 74$ 


$$
\begin{aligned}
& \text { FUNCTION } \operatorname{THETAI}(U, Q) \\
& A=\operatorname{SIN}(U)
\end{aligned}
$$$$
\text { GO TO } 1
$$

5

$$
\begin{aligned}
& \text { ENTRY THETA2 } \\
& \mathrm{A}=\operatorname{COS}(\mathrm{U})
\end{aligned}
$$

$1 \mathrm{QN}=2 . * \mathrm{Q} * * .25$

THETA $1=Q N * A$

IF (THETA1 . EQ. . .) RETURN

$C=(A+A) * * 2-2$.

10

$B=A$

$Q M=1$.

GO TO 3

ENTRY THETA3

$A=\cos (U+U)$

15

GO TO 2

ENTRY THETA4

$A=-\cos (U+U)$

$2 C=A+A$

$B=C * A-1$.

20

$Q M=Q * 3$

$Q N=Q M *(Q+Q)$

THETA $1=Q N * B+Q * C+1$.

IF (Q.EQ. ०.) RETURN

25

$3 Q Q=A M I N 1(Q * * 2,0.81)$

\section{$4 A=C * B-A$}

$B=C * A-B$

$Q M=Q Q * Q M$

$\mathrm{QN}=\mathrm{QM} * \mathrm{QN}$

THETA $1=Q N * A+T H E T A 1$

30

$\mathrm{QM}=\mathrm{QQ} * \mathrm{QM}$

$\mathrm{QN}=\mathrm{QM} * \mathrm{QN}$

THETA $1=Q N * B+T H E T A 1$

IF(QN.GE.1.E-14) GO TO 4 RETURN

35

END

$\begin{array}{lr}\text { THETA1 } & 2 \\ \text { THETA1 } & 3 \\ \text { THETA1 } & 4 \\ \text { THETA1 } & 5 \\ \text { THETA1 } & 6 \\ \text { THETA1 } & 7 \\ \text { THETA1 } & 8 \\ \text { THETA1 } & 9 \\ \text { THETA1 } & 10 \\ \text { THETA1 } & 11 \\ \text { THETA1 } & 12 \\ \text { THETA1 } & 13 \\ \text { THETA1 } & 14 \\ \text { THETA1 } & 15 \\ \text { THETA1 } & 16 \\ \text { THETA1 } & 17 \\ \text { THETA1 } & 18 \\ \text { THETA1 } & 19 \\ \text { THETA1 } & 20 \\ \text { THETA1 } & 21 \\ \text { THETA1 } & 22 \\ \text { THETA1 } & 23 \\ \text { THETA1 } & 24 \\ \text { THETA1 } & 25 \\ \text { THETA1 } & 26 \\ \text { THETA1 } & 27 \\ \text { THETA1 } & 28 \\ \text { THETA1 } & 29 \\ \text { THETA1 } & 30 \\ \text { THETA1 } & 31 \\ \text { THETA1 } & 32 \\ \text { THETA1 } & 33 \\ \text { THETA1 } & 34 \\ \text { THETA1 } & 35 \\ \text { THETA1 } & 36\end{array}$


1

SUBROUTINE TINV7 (TINV,T)
C INVERSE OF $7 \times 7$ SYMPLETIC MATRIX

THETA1 37

TINV7 2

c $6 \times 6$ MATRIX INVERSE OF $S=S C=-U \times S T R \times U$

TINV7 3

5

c (SC $-\mathrm{SC} \times \mathrm{k}$ )

$K=(T(I, 7), I=1,6) \quad T I N V 7$

c $7 \times 7$ MATRIX INVERSE OF $T=$

TINV7 5

c (0)

1)

TINV7

DIMENSION $\operatorname{TINV}(7,7), \operatorname{T}(7,7), \operatorname{STR}(6,6), U(6,6), \operatorname{SC}(6,6), \operatorname{VK}(6)$

10

C

C STR IS TRANSPOSE OF $6 \times 6$ OF $T$

TINV7

TINV7

TINV7

DO $10 \quad I=1,6$

DO $10 \mathrm{~J}=1,6$

$\operatorname{STR}(I, J)=T(J, I)$

10 CONTINUE

15

C SET UP U ARRAY

DO $15 \mathrm{~J}=1,6$

DO $15 \quad I=1,6$

$U(I, J)=0.0$

15 CONTINUE

20

DO $20 \quad I=1,5,2$

$U(I, I+1)=-1$.

$U(I+1, I)=+1$.

20 CONTINUE

CALL MXMG (STR,U,SC)

25

CALL MXMG $(U, S C, S C)$

TINV7

TINV7

TINV7

TINV7

TINV7

TINV7

TINV7

TINV7

TINV7

TINV7

TINV7

TINV7

TINV7

TINV7

TINV7

TINV7

TINV7

TINV7

TINV7

25 CONTINUE

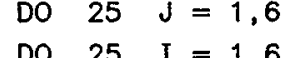

TINV7

TINV7

TINV7

TINV7

TINV7

TINV7

TINV7

TINV7

TINV7

TINV7

TINV7

TINV7

40

35

$\operatorname{TINV}(7, I)=0.0$

TINV7

RETURN 
LEVEL 2, STORE, INFF, IWORK NIN, LFL, NFL, LBC, NBC, NTOT, IX, KIND, TYPE, ROWS, COLS, EXTR, INSTR $M, N F, N B, N I, M S U B R, I S I G N$

INSTR

INTEGER OPNAME, OP, OBJA, OBJB, TRA, TYPE, ROWS, COLS, EXTR

INSTR

INSTR

C

C

15

$\mathrm{C}$

MATRIX STORED BY ROWS AT LFL TRANSPOSED AND STORED BY COLUMNS AT LQ

$I L=L Q$

$\mathrm{IRR}=\mathrm{LFL}-1$

c

DO $1 \mathrm{~J}=1, \mathrm{~KB}$

20

25
$I R R=I R R+1$

$I R=I R R$

DO $1 \mathrm{I}=1, \mathrm{KA}$

STORE(IL) $=$ STORE (IR)

$I L=I L+1$

$1 \quad I R=I R+K B$

RETURN

END
TRNSPOS

TRNSPOS

TRNSPOS

TRNSPOS

TRNSPOS

TRNSPOS

TRNSPOS

TRNSPOS

TRNSPOS

TRNSPOS

TRNSPOS

TRNSPOS

TRNSPOS

TRNSPOS

TRNSPOS
2

3

1

2

3

5

2

3

4

5

6

5

6

7

8

9

10

11

12

13

14

15

16

17

18

19 
LEVEL 2, STORE, INFF, IWORK

COMMON STORE (48000), IWORK(10)

DIMENSION INFF $(24,2000)$

EQUIVALENCE (INFF, STORE)

COMMON/BMI/MI (5000)

COMMON/NELS/NELS

LEVEL 2,MI

COMMON /DIM/ LEND, MEND, ISAV, ISAV7, M7END, KADD, KADDR, MUNIT, MSYMPL

COMMON/TRKINT/G, EX, EY, OMSQ, SEND, VMX, VMN, DINT , BEG, DPR, JPR,

1 THET, PLT, NPT, NZ, PMAX, NSIZ, ITITLE(6)

LOGICAL BEG, DPR, PLT

COMMON/CONTRL/ERROR, MODE, RSRV , STOR, XEQ, TRASW, NFSW, EMPTY, INDEF, 1

LDFLG, FIN

LOGICAL ERROR, RSRV, STOR, XEQ, TRASW, NFSW, EMPTY, INDEF, LDFLG, FIN

DIMENSION $V(7), V 0(7), U(56), B X(9), B Y(9), V V(7)$

LOGICAL DPRSAV

DIMENSION R7(7,7), RW(3),UO(7),UP(7),UM(7),UF(7)

EQUIVALENCE $(\operatorname{AXCOS}, V(1)),(\operatorname{AYCOS}, V(3)),(D P P, U(6))$

INTEGER BDAT

COMMON/CDERIV/IFLAG, MR, NR, DP, NV(11), DAT (20), UZ, STOT,

1 DAT2(7), MP2FLG, NF, HO, LOCALPH, NUMALPH

DIMENSION SCALE(4)

DATA MASK/77000 $000000000000000 \mathrm{~B} /$

DATA IBLNK, (RW(I), I=1,3)/1H, 3*0.0/

DATA DELP/1.E-08/

C REVISED FEB. 1976

C INSTRUCTION TRK-TRACK PARTICLES THROUGH LINEAR OR

C NON-LINEAR TRANSFORMATIONS

BLANK

BLANK

86MARSIZ

86MARSIZ

86MARSIZ

BLANK

BMIL

BMIL

BMIL

BMIL

BMI

DIM

DIM

TRKINT

TRKINT

TRKINT

CONTRL

CONTRL

CONTRL

CONTRL

TRK

TRK

TRK

TRK

TRK

CDERIV

CDERIV

MY3TRK

TRK

TRK

TRK

TRK

TRK

TRK

TRK

TRK

TRK

$T$ TRK NP IMP/N BLIN/MAT VO / NR IOF/IVSAV IMODE/IPR IPL/ / XMAX /DXMAX /YMAX JDYMAX

MY3TRK

MY3TRK

TRK

TRK

NP=NUMBER PARTICLES. THESE ARE STORED IN V, A PREVIOUS PVEC.

TRK

NR = NUMBER OF TRANSITS OF BLIN TO BE MADE BY EACH PARTICLE.

IF BLANK, BEAM LINE CURRENTLY STORED IN MI ARRAY IS USED.

TRK

TRK

TRK

TRK

TRK

TRK MAT = NAME OF MATRIX THAT GIVES BETAS AT PRINT POSITIONS. IOF = OUTPUT FREQUENCY $=$

$$
\begin{aligned}
& =00001, \text { EACH POSITION } \\
& =\text { OROOP, EVERY R?TH TRANSIT AT P?TH POSITION.. }
\end{aligned}
$$

C IMP $=$ INPUT OPTION

C IMP $=0 \quad V=X, D X, Y, D Y$ (METERS, RADIANS)

C IMP $=1 \quad V=E X(M-M R A D), P S I X(D E G), E Y, P S I Y$

TRK

TRK

TRK

TRK

TRK

TRK

TRK

TRK 
(CARD MUST ALWAYS BE INCLUDED)

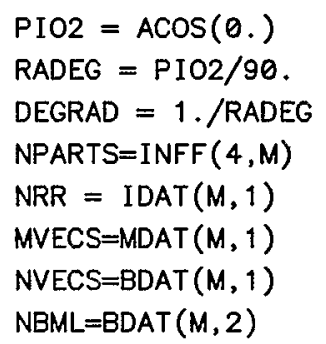

MY3TRK

TRK

TRK

TRK

TRK

TRK

TRK

TRK

TRK

TRK

TRK

TRK

TRK

TRK

TRK

TRK

TRK

TRK

TRK

TRK

TRK

TRK

MY3TRK

TRK

TRK

$$
\begin{aligned}
& \operatorname{IMP}=\operatorname{INFF}(5, M) \\
& \operatorname{IOF}=\operatorname{IDAT}(M, 2) \\
& \operatorname{IVSAV}=\operatorname{IDAT}(M, 3)
\end{aligned}
$$




$$
\begin{aligned}
& \operatorname{IMODE}=\operatorname{IDAT}(M, 4) \\
& \operatorname{IPR}=\operatorname{IDAT}(M, 5) \\
& I P L=\operatorname{IDAT}(M, 6) \\
& \text { IF (IMODE.EQ.0) IMODE }=1 \\
& \text { IF (IMODE. NE. 2) GO TO } 30
\end{aligned}
$$

$$
\text { NPARTS }=1
$$

CALL RTRV7(MUNIT,R7,RW)

CALL $\operatorname{STOR7}(M, R 7, R W)$

30 IF (NBML.NE. IBLNK) GO TO 402

NELS = IABS (NELS)

$$
\text { GO TO } 401
$$

402 CONTINUE

$M B M L=M D A T(M, 2)$

C PUT BEAM LIST IN MI ARRAY

NDAT $=\operatorname{INFF}(17$, MBML $)$

CALL MIFILL(MBML , 1 , NDAT, NELS, MI)

IF(ERROR) RETURN

C FIND BETAS FOR REFERENCE MATRIX MAT

401 NMAT=BDAT $(M, 3)$

IF (NMAT.EQ.5H ) GO TO 403

135

$M E L=\operatorname{MDAT}(M, 3)$

CALL BET (MEL, BX, BY, $\theta)$

403 CONTINUE

$P L T=. F A L S E$.

$D P R=, F A L S E$.

IF (IPR. LT.0) DPR = . TRUE.

\begin{tabular}{|c|c|}
\hline TRK & 94 \\
\hline TRK & \\
\hline TRK & 0 \\
\hline TRK & ك \\
\hline TRK & 9 \\
\hline TRK & 9 \\
\hline TRK & 10 \\
\hline TRK & 10 \\
\hline TRK & 102 \\
\hline TRK & 10 \\
\hline TRK & 104 \\
\hline TRK & 105 \\
\hline TRK & 10 \\
\hline TRK & 107 \\
\hline TRK & 108 \\
\hline TRK & 109 \\
\hline TRK & 110 \\
\hline TRK & 111 \\
\hline TRK & 112 \\
\hline TRK & 113 \\
\hline TRK & 114 \\
\hline TRK & 115 \\
\hline TRK & 116 \\
\hline TRK & 117 \\
\hline TRK & 118 \\
\hline TRK & 119 \\
\hline TRK & 120 \\
\hline TRK & 121 \\
\hline TRK & 122 \\
\hline TRK & 123 \\
\hline TRK & 124 \\
\hline TRK & 125 \\
\hline TRK & 126 \\
\hline TRK & 127 \\
\hline TRK & 128 \\
\hline TRK & 129 \\
\hline TRK & 130 \\
\hline TRK & 131 \\
\hline TRK & 132 \\
\hline TRK & 133 \\
\hline TRK & 134 \\
\hline TRK & 135 \\
\hline TRK & 136 \\
\hline TRK & 137 \\
\hline TRK & 138 \\
\hline TRK & 139 \\
\hline TRK & 140 \\
\hline TRK & 141 \\
\hline TRK & 142 \\
\hline TRK & 143 \\
\hline TRK & 144 \\
\hline TRK & 145 \\
\hline TRK & 146 \\
\hline TRK & 147 \\
\hline TRK & 148 \\
\hline TRK & 149 \\
\hline TRK & 15 \\
\hline
\end{tabular}

IF (IPL.GT.0) PLT $=$. TRUE.

$\mathrm{KREV}=\mathrm{IOF} / 1000$

$\mathrm{KPOS}=\operatorname{MOD}($ IOF , 1000)

IF (PLT) WRITE(5) NPARTS, NRR, NVECS, NBML, NMAT, KPOS, NVECO, 1

$(U(J), J=1,7)$ 
83 DO $5 \mathrm{~J}=1.7$

TRK

$$
U(J)=V \theta(J)+V(J)
$$

TRK

152

$$
5 \quad \mathrm{UO}(\mathrm{J})=U(\mathrm{~J})
$$

C $\quad N M=\operatorname{INFF}(2, M)$

IF (IOF.EQ.0) IOF $=1$

TRK

153

TRK

154

TRK

TRK

155

$$
\text { IF (NR.EQ.0) NR=1 }
$$

C TRACK ONE PARTICLE THROUGH THE BEAM SYSTEM.

180

C PRINT HEADING AND INITIAL CONDITIONS

TRK

156

IF(DPR) GO TO 51

TRK

TRK

TRK

IF (IOF.NE.1) GO TO 551

TRK

WRITE $(3,1001)$

GO TO 51

551 IF (NREF.EQ.1) WRITE $(3,1021)$

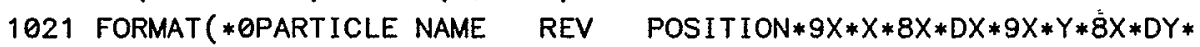
$16 X * D S * 6 X * D P / P \quad E X(M M-M R)$ EY $(M M-M R) *)$ IF (NREF.EQ.2) WRITE $(3,1011)$

190

51

$$
\text { CONTINUE }
$$

IRV $=1$

$I P O S=1$

C FIND NAME OF PARTICLE

IF (DPR) GO TO 52

IF (NREF.EQ.2) WRITE $(3,1012)$ NVECS, IP

195

$$
\text { IF (NREF.EQ.1) WRITE }(3,1002) \text { NVECS, IP }
$$

52 CONTINUE

C LOOP OVER REVOLUTIONS

55 DO 213 IRV = 1, NRR

IRM $=$ IRV -1

200

IF (.NOT.DPR.AND. IOF.EQ.1) WRITE $(3,1005)$ IRM

C LOOP THROUGH BEAM SYSTEM

NELF $=$ NELS

IF (IRV.EQ.NRR) NELF $=$ NELF +1

205

C RESET RANDOM SEED SO ERRORS REPEAT ON SUCCESSIVE TURNS CALL RANGET(NRN)

DO 212 IBS=1, NELF

NEL=MI (IBS)

IPOS $=$ IBS -1

210

C IF INTEGRATION STEPS ARE BEING PRINTED, OMIT TRK PRINT. IF (IOF.NE.01000) GO TO 61

IF (IBS.NE.1) GO TO 211

IF (NMAT.EQ.IBLNK) GO TO 214

GO TO 62

61 CONTINUE

215

62 DO $6 \quad J=1,4$

$6 \quad V(J)=1 . E 3 *(U(J)-V \theta(J))$

IF (IOF.EQ.1) GO TO 214

IF (NMAT.EQ.5H ) GO TO 214

IF (IPOS.NE.KPOS) GO TO 211

TRK

TRK

TRK

TRK

TRK

TRK

TRK

TRK

TRK

TRK

TRK

TRK

TRK

TRK

TRK

TRK

TRK

TRK

TRK

TRK

TRK

TRK

TRK

TRK

TRKAPR86

TRK

TRK

TRK

TRK

TRK

TRK

TRK

MY3TRK

MY3TRK

TRK

TRK

TRK

IF (MOD (IRV-1, KREV) . NE. 0 ) GO TO 211

$W X=B X(2) * V(2) * V(2)+2 * B X(3) * V(1) * V(2)+B X(4) * V(1) * V(1)$

TRK

TRK

TRK

TRK

TRK

TRK

TRK

TRK

TRK

C TIME FOR OUTPUT

IF (NREF.NE.1) GO TO 7 
$\mathrm{PHIX}=0$.

GO TO 72

$71 \quad$ AXSIN $=-B X(2) * V(2)-B X(3) * V(1)$

PHIX $=$ ATAN2 (AXSIN, AXCOS) *DEGRAD

72 IF (WY.NE.O.) GO TO 73

235

$\mathrm{PHIY}=0$.

GO TO 74

$73 \quad$ AYSIN $=-B Y(2) * V(4)-B Y(3) * V(3)$

PHIY = ATAN2 (AYSIN, AYCOS) *DEGRAD

74 CONTINUE

240

IF (DPR) GO TO 215

WRITE $(3,1014)$ IRM, IPOS,DPP,WX,PHIX,WY, PHIY, $(V(J), J=1,4)$

GO TO 215

214 CONTINUE

IF (IPR.NE.2) GO TO 216

245

MATI $=\operatorname{INFF}(2, N E L)$

IF ((MASK.AND.MATI).NE.1L") GO TO 215

216 CONTINUE

IF (DPR) GO TO 215

WRITE $(3,1004)$ IRM, IPOS, (U(J), $J=1,6)$

250

215 CONTINUE

IF (.NOT.PLT) GO TO 211

IF (IBS.GT.1) GO TO 211

WRITE(5) (U(J), J=1,4), (V(J), J=1,4), IP, IRM, IPOS

WRITE(6) (U(J), J=1,4), (V(J), J=1,4), IP, IRM, IPOS

255

C ADVANCE THROUGH BEAM SYSTEM

211 CONTINUE

IF (IBS.GT.NELS) GO TO 212

NEL = MI (IBS)

CALL TRKCN(M,NEL, U, $\theta$, IMODE)

260

212 CONTINUE

CALL RANSET (NRN)

213 CONTINUE

IF (IGO.EQ. $\theta$ ) GO TO 245

IF (IGO-2) $220,225,240$

265

$220 \mathrm{CALL} R T R V 7(M, R 7, R W)$

DPRSAV $=$ DPR

DPR=. TRUE.

$D L P=-D E L P$

DO $222 \quad I=1,7$

270

$222 \mathrm{UF}(\mathrm{I})=\mathrm{U}(\mathrm{I})$

GO TO 230

$225 \mathrm{DLP}=+$ DELP

DO $227 \mathrm{I}=1,7$

$227 U M(I)=U(I)$

275

230 DO $231 \mathrm{~J}=1,7$

$231 U(J)=V \theta(J)+V(J)$

$U(6)=U(6)+$ DLP

$I G O=I G O+1$

GO TO 55

280

240 DO $242 \quad I=1,7$

$242 \mathrm{UP}(\mathrm{I})=\mathrm{U}(\mathrm{I})$

DO $243 I=1,6$

$243 R 7(I, 6)=0.5 *(U P(I)-U M(I)) / D E L P$

CALL MXV7(R7, U日, U日)

DO $244 \mathrm{I}=1,5$

TRK

TRK

TRK

TRK

TRK

TRK

TRK

TRK

TRK

TRK

TRK

TRK

TRK

TRK

TRK

TRK

TRK

TRK

TRK

MY3TRK

TRK

TRK

TRKAPR8 6

MY3TRK 13

MY3TRK 14

TRK 231

TRK 232

TRK 233

TRK 234

TRK 235

TRK 236

TRK 237

TRK 238

TRK

TRK

TRK

TRK

TRK

TRK

TRK

TRK

TRK

TRK

TRK

TRK

TRK

TRK

TRK

TRK

TRK

TRK

TRK

TRK

TRK

TRK

TRK
209

210

211

12

13

215

216

217

18

20

221

222

223

224

25

26

12

28

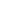

3

1

3

4

5


290

295

300

305

310

315
$244 \operatorname{R7}(I, 7)=U F(I)-\mathrm{U} 0(I)$

CALL STOR7(M,R7,RW)

CALL MXV7 (R7, VO, VO)

DO $246 \mathrm{~J}=1,7$

$246 \quad V(J)=U F(J)-V 0(J)$

DPR=DPRSAV

GO TO 248

245 CONTINUE

DO $247 \mathrm{~J}=1,4$

$247 V(J)=U(J)-V O(J)$

248 IF (IVSAV.EQ.1) CALL STDAT(MVECS, 1, IPS, 7,V)

250 CONTINUE

\section{CALL HED}

IF (.NOT.PLT) RETURN

END FILE 5

CALL TRKPLT(SCALE)

RETURN

1000 FORMAT (27H PARTICLE TRACKING THROUGH A5,1H.)

1001 FORMAT (106HOPARTICLE NAME REV. POSITION

1

$$
Y
$$

DY

DS

1002 FORMAT $(1 \mathrm{HO}, 3 \mathrm{X}, \mathrm{A5}, \mathrm{I3})$

1004 FORMAT $(16 \mathrm{X}, \mathrm{I} 3,6 \mathrm{X}, \mathrm{I} 3,5 \mathrm{X}, 6(1 \mathrm{X}, \mathrm{F} 12.8))$

1005 FORMAT $(16 X, I 3,6 X, I 3,5 X, 8(F 10.7))$

1006 FORMAT (10X,*TRACKING RELATIVE TO REFERENCE RAY *A5*, ( UNITS (X, DX TRK

$1, Y, D Y, D S, D P / P)=(M M, M R A D, M M, M R A D, M M, 0 / 0)) * / 20 X, A 5 *=* 7 F 10.5)$ TRK 1011 FORMAT $(1 \mathrm{HO}, *$ RAY REV. POS. DP/P EX(MM-MR)

1) $E Y(M M-M R) \quad$ PHIY(DEG) $X(M M)$

2MR)* )

1012 FORMAT ( $1 \mathrm{HO}, 3 \mathrm{X}, \mathrm{A} 5, \mathrm{I} 2)$

1014 FORMAT (10X,2I5, F11.3,8F12.5)

END

\begin{tabular}{lr} 
TRK & 262 \\
TRK & 263 \\
TRK & 264 \\
TRK & 265 \\
TRK & 266 \\
TRK & 267 \\
TRK & 268 \\
TRK & 269 \\
TRK & 270 \\
TRK & 271 \\
TRK & 272 \\
TRK & 273 \\
TRK & 274 \\
TRK & 275 \\
TRK & 276 \\
TRK & 277 \\
MY3TRK & 15 \\
TRK & 279 \\
TRK & 280 \\
TRK & 281 \\
TRK & 282 \\
TRK & 283 \\
MY3TRK & 16 \\
TRK & 285 \\
TRK & 286 \\
TRK & 287 \\
TRK & 288 \\
TRK & 289 \\
TRK & 290 \\
TRK & 291 \\
TRK & 292 \\
TRK & 293 \\
& \\
TRK & \\
TRK & 279 \\
\hline
\end{tabular}


SUBROUTINE TRKPLT(SCALE)

DIMENSION U(7), $V(7), T(3), \operatorname{SCALE}(1)$

DATA T/1HX, 1HY, 1H./

REWIND 5

REWIND 6

$X R 1=\operatorname{SCALE}(1)$

YU1 $=$ SCALE $(2)$

$X R 2=\operatorname{SCALE}(3)$

YU2 = SCALE (4)

READ (5) NPARTS, NR, NVECS, NBML, NMAT , KPOS, NVECO,U

DO $1 K 1=1$, NPARTS

NERR $=0$

NDX $=0$

NDY $=0$

DO $2 \mathrm{~K} 2=1, \mathrm{NR}$

$\operatorname{READ}(5)(U(J), J=1,4),(V(J), J=1,4), I P, I R M, I P O S$

IF (NERR.EQ.1) GO TO 2

IF (ABS(V(1)).LT.1.EO4.AND.ABS(V(2)).LT.1.EO4) GO TO 4433

$K 22=K 2-1$

WRITE $(3,777) \mathrm{K} 1, \mathrm{~K} 22$

777 FORMAT (1HO, *PARTICLE NUMBER*, I6, * WENT UNSTABLE ON REV*, I6)

NERR $=1$

GO TO 2

4433 CONTINUE

IF (K2.GT.1) GO TO 20

WRITE $(3,100)$

100

FORMAT $(1 \mathrm{H} 1,5 \mathrm{X}, * \mathrm{U} 1 *, 7 \mathrm{X}, * \mathrm{U} 2 *, 7 \mathrm{X}, * \mathrm{U} 3 *, 7 \mathrm{X}, * \mathrm{U} 4 *, 17 \mathrm{X}, * \mathrm{~V} 1 *, 7 \mathrm{X}, * \mathrm{~V} 2 *, 7 \mathrm{X}$, $* V 3 *, 7 X, * V 4 *, 7 X, *$ IPX IRM IPOS*)

WRITE $(3,101)(U(J), J=1,4),(V(J), J=1,4), I P, \operatorname{IRM}, \operatorname{IPOS}$

101 FORMAT (1H0,4X,4F9.6,6X,4F9.3,3I5)

$X L 1=-X R 1$

$Y L 1=-Y U 1$

CALL SETP(XL1,XR1, YL1, YU1)

20

CONT INUE

CALL PLOTT(1,T(1),V(1),V(2))

IF (K2.EQ.NR) CALL PAGE $(0, K 1)$

IF (K2.EQ.NR) CALL PAGTRKP $(0, K 1)$

IF (ABS(V(1)) .GT.XR1.OR.ABS(V(2)) .GT.YU1) NDX=NDX+1

IF (K2.EQ.NR) WRITE $(3,102)$ NDX

102 FORMAT(1HO, I6," PATICLES OUTSIDE DIAGRAM")

2 CONTINUE

DO $3 \mathrm{~K} 2=1$, NR

$\operatorname{READ}(6)(U(J), J=1,4),(V(J), J=1,4), I P, I R M, I P O S$

IF (NERR.EQ.1) GO TO 3

$\operatorname{IF}(A B S(V(3)) . L T .1 . E 4 . A N D . A B S(V(4)) . L T .1 . E 4)$ GO TO 4434

$\mathrm{K} 22=\mathrm{K} 2-1$

WRITE $(3,777) \mathrm{K} 1, \mathrm{~K} 22$

NERR $=1$

GO TO 3

4434 CONTINUE

IF (K2.GT.1) GO TO 30

WRITE $(3,100)$

WRITE $(3,101)(U(J), J=1,4),(V(J), J=1,4)$, IP, IRM, IPOS

$X L 2=-X R 2$

$Y L 2=-Y U 2$

CALL SETP(XL2, XR2, YL2, YU2)

CONT INUE
MY3TKPL 1

MY3TKPL 2

MY3TKPL 3

MY3TKPL 4

MY3TKPL 5

MY3TKPL 6

MY3TKPL 7

MY3TKPL 8

MY3TKPL 9

MY3TKPL 10

MY3TKPL 11

MY3TKPL 12

MY3TKPL 13

MY3TKPL 14

MY3TKPL 15

MY3TKPL 16

MY3TKPL 17

MY3TKPL 18

MY3TKPL 19

MY3TKPL 20

MY3TKPL 21

MY3TKPL 22

MY3TKPL 23

MY3TKPL 24

MY3TKPL 25

MY3TKPL 26

MY3TKPL 27

MY3TKPL 28

MY3TKPL 29

MY3TKPL 30

MY3TKPL 31

MY3TKPL 32

MY3TKPL 33

MY3TKPL 34

TRKP683 1

MY3TKPL 36

86TRKPL 1

86TRKPL 2

86TRKPL 3

86TRKPL 4

86TRKPL 5

86TRKPL 6

86TRKPL 7

86TRKPL 8

86TRKPL 9

86TRKPL 10

86TRKPL 11

86TRKPL 12

86TRKPL 13

86TRKPL 14

86TRKPL 15

86TRKPL 16

86TRKPL 17

86TRKPL 18

86TRKPL 19

86TRKPL 20

86TRKPL 21 
60

65
CALL PLOTT( $1, T(2), V(3), V(4))$

IF (K2.EQ.NR) CALL PAGTRKP $(0, K 1)$

IF (ABS (V(3)) .GT.XR1 . OR.ABS $(V(4)) \cdot G T \cdot Y U 2) \quad N D Y=N D Y+1$ IF(K2.EQ.NR) WRITE $(3,102)$ NDY

3 CONTINUE

1 CONTINUE

REWIND 5

REWIND 6

RETURN

END
86TRKPL 22

86TRKPL 23

86TRKPL 24

MY3TKPL 60

MY3TKPL 61

MY3TKPL $\quad 62$

MY3TKPL 63

MY3TKPL 64

MY3TKPL 65

MY3TKPL $\quad 66$

CARD NR. SEVERITY DETAILS DIAGNOSIS OF PROBLEM

$\begin{array}{llll}7 & \text { I } & \text { SCALE } & \text { ARRAY REFERENCE OUTSIDE DIMENSION BOUNDS. } \\ 8 & \text { I } & \text { SCALE } & \text { ARRAY REFERENCE OUTSIDE DIMENSION BOUNDS. } \\ 9 & \text { I } & \text { SCALE } & \text { ARRAY REFERENCE OUTSIDE DIMENSION BOUNDS. }\end{array}$


1

5

10

15
SUBROUTINE SETP (XL, XTOP, YL, YTOP) DIMENSION $A(112,51), Z(6), X(1), Y(1)$ COMMON/SAML/A, $Z, X L O W, X R A N G E, Y L O W$, YRANGE DATA BLANK/4H /

$X R=X T O P-X L$

$Y R=Y T O P-Y L$

$X L O W=X L$

XRANGE $=X R$

YLOW=YL

YRANGE $=Y R$

DO $10 \quad I=1,112$

DO $10 \mathrm{~J}=1,51$

$A(I, J)=B$ LANK

10
CONT INUE

RETURN

END
MY3TKPL 67

MY3TKPL 68

MY3TKPL 69

MY3TKPL 70

MY3TKPL $\quad 71$

MY3TKPL $\quad 72$

MY3TKPL 73

MY3TKPL $\quad 74$

MY3TKPL $\quad 75$

MY3TKPL $\quad 76$

MY3TKPL $\quad 77$

MY3TKPL $\quad 78$

MY3TKPL $\quad 79$

MY3TKPL 80

MY3TKPL 81

MY3TKPL 82 